An Exploration of the Assessment for Intervention Model in an Irish Educational Psychological Context

\author{
Carol Anne Slattery
}

A thesis submitted in fulfilment of the requirements of Mary Immaculate College for the degree of Professional Doctorate in Educational and Child Psychology

Supervised by Dr. Margaret Egan

Submitted to Mary Immaculate College August 2019 


\title{
Abstract \\ 'An Exploration of the Assessment for Intervention Model in an Irish Educational Psychological Context'
}

\section{Carol Slattery, DECPsy student, 2016-2019}

\begin{abstract}
Aims The current research sought to explore the utility of the Assessment for Intervention (AFI) model in bridging the gap between assessment and intervention, through the conceptual lens of Ecological Systems Theory (EST) and Social Constructivist Theory (SCT). AFI is a five-stage assessment model that aims to provide recommendations that are both useful and evidence-based (Pameijer, 2017).
\end{abstract}

Method An exploratory 'two case' case study was employed to answer the research question: 'Can the Assessment for Intervention model bridge the gap between assessment and intervention, through activity and interaction at the meso and microsystem levels?' The AFI framework was applied to two case referrals made to a school psychological service in Ireland. Case 1 included a student in $4^{\text {th }}$ class, his mother, class teacher and Special Education Teacher (SET) $(n=4)$. Case 2 comprised of a student in $6^{\text {th }}$ class, her mother and class teacher $(n=3)$. A pilot study was conducted to inform the research design and a case study protocol guided data collection and analysis of findings $(n=3)$. Participants rated Likert statements exploring case propositions, before and after application of the AFI model. Semi-structured interviews were also conducted to answer the research question and to address case propositions. AFI templates served as a researcher diary and were recorded before, during and after application of the model.

Results Thematic analysis and pattern-matching were employed to analyse the interview data. Changes in ratings on Likert statements were presented in tabular format and excerpts from the researcher diary were included in the discussion of findings. All data were analysed within the conceptual framework detailed. Results suggest some interesting implications for policy and practice, particularly for those in educational psychology.

Conclusions The findings suggest the AFI model, grounded in EST and SCT, can address the gap between assessment and intervention, discussed in the literature. 


\section{Declaration}

The work has not previously been accepted for any degree and is not being concurrently submitted for any degree.

This research is being submitted in fulfilment of the requirements of the Doctorate in Educational and Child Psychology.

I hereby declare that I am the sole author of this thesis. Where the use has been made of other people, it has been fully acknowledged and referenced.

I hereby give my permission for my thesis, if accepted, to be available for reading and interlibrary loans, and for the title and summary to be made available to outside organisations.

Name: Carol Slattery

Signature:

Date: 


\section{Acknowledgements}

Undertaking this doctoral programme has been a journey, with ups and downs along the way. They say it takes a village to raise a child, and I couldn't have gotten to this point without the support of so many.

To Margaret, my research supervisor, thank you for the countless emails, and hours of supervision and support you have provided me. I feel so fortunate to have benefited from your wisdom and guidance on this doctoral journey. Throughout the programme you have encouraged and inspired me to 'dig deep' and to realise my potential. Thank you, Margaret, for your enthusiasm and kindness.

To the DECPsy team, particularly Therese, Siobhan and Claire, thank you for giving me the opportunity to embark on this doctoral journey, and for the numerous learning opportunities along the way. To my placement supervisor Anne, who supervised my casework on placement, thank you for your guidance and genuine interest in my research. To the DECPsy class, what a journey we have travelled together! Thanks for all the shared messages of support, and friendships that have developed. Lainey, thank you for your words of encouragement during the research process. Alison, it is hard to believe the three years have gone past, thank you for your peer support and friendship throughout the programme.

To my parents, I am indebted to you both, for your patience, encouragement and support these past three years. I cannot thank you enough for welcoming me back home, for the kind, supportive words on the days that were tough, the delicious dinners that I never had to cook and for the jokes and laughs along the way. Thank you for instilling a love of learning in me from a very young age and for leading by example in achieving goals in life. To my godmother Anne, thank you for inspiring me to consider postgraduate study and for your constant kindness and support these past three years, and always. Thank you to my siblings for your patience and understanding and for indulging me with treats at the weekend. To my extended family, thank you Maureen and Colin for taking an interest in my research and for the words of advice along the way. Thank you to my friends, in particular Catherine, Mags, Ailbhe and Caitriona, for the calls, chats and laughs in the evenings and on weekends.

Finally, to my husband Will, I am lost for words when it comes to thanking you. Who would have thought when you were helping me with my maths homework in leaving cert, you would still be supporting my learning at doctoral level! Thank you from the bottom of my heart Will, for your boundless generosity, understanding and patience these past three years. Even though we travelled this doctoral journey thousands of miles apart, I always felt the presence of your support and love. Undertaking this doctorate was just as much your sacrifice as it was mine. Will I dedicate this thesis to our future and the path that lies ahead, wherever it may lead. I look forward to the learning that we will continue to encounter in life, and the joy it will bring. 


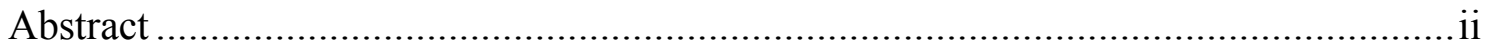

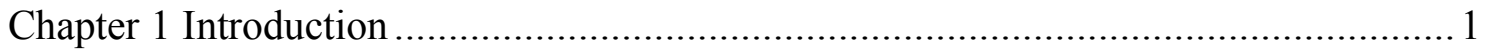

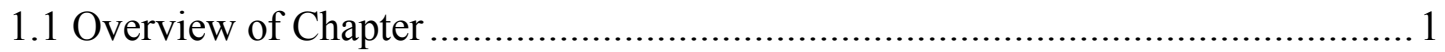

1.2 Assessment in EP practice: International Context ............................................ 1

1.3 Assessment in EP Practice: National Context .................................................. 2

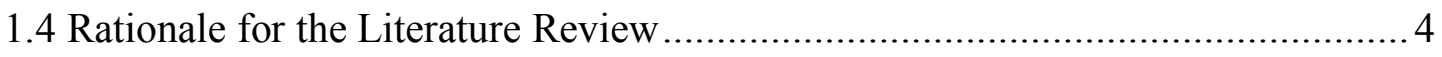

1.5 Aims and Methodology of the Research Project ............................................. 5

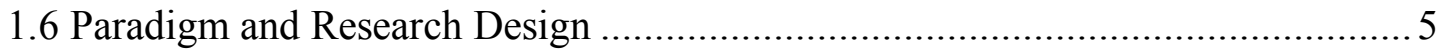

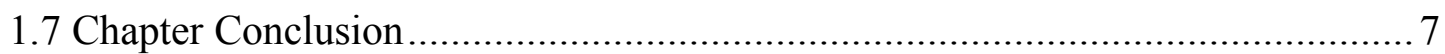

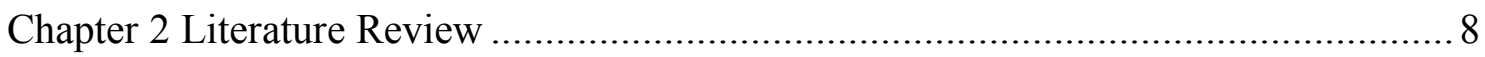

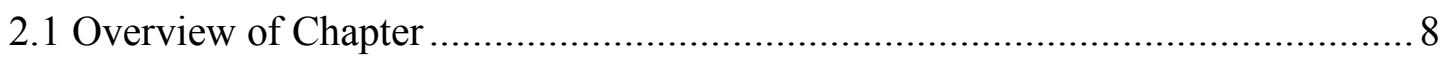

2.2 Phase 1 of the Literature Review: A Systematic Approach ............................... 8

2.3 Key Concepts and Terminology Defined ................................................... 9

2.4 Search Strategy/Literature Search............................................................... 10

2.5 Systematic Review of the Literature ............................................................ 12

2.5.1 Critical Analysis Framework .................................................................. 13

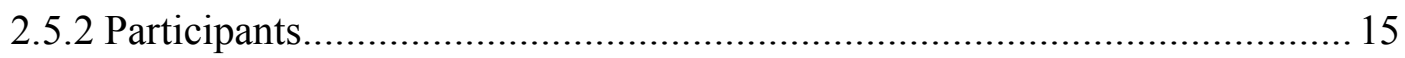

2.5.3 Critique of Design and Methodologies................................................... 21

2.6 A Synthesis of Literature on Assessment and Intervention Approaches Currently

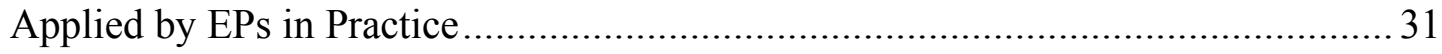

2.7 Review Question 1: What Assessment Approaches are Educational Psychologists

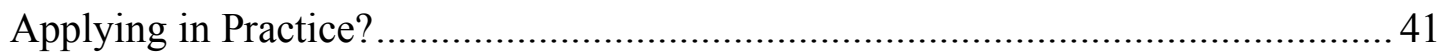

2.7.1 A Medical Model of Practice ..................................................................... 41

2.7.2 Inconsistencies in Assessment Practices ................................................... 42

2.7.3 Impact of Legislation on Assessment Approaches .................................. 42

2.7.4 Evidence of Alternative Assessment Approaches...................................... 43

2.8 Review Question 2: Are the Assessment Approaches Informing Appropriate

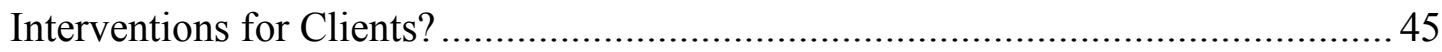

2.8.1 Assessment for Intervention (Pameijer, 2017) ........................................ 46

2.8.2 Dynamic Assessment (Lawrence \& Cahill, 2014) ................................... 47

2.8.3 The Voice of the Child ........................................................................ 47

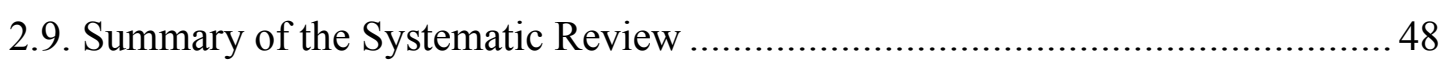

2.10 Limitations of the Systematic Review........................................................ 49

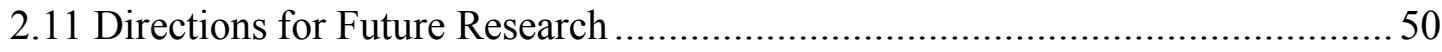


2.12 Phase 2 of the Literature Review: A Theoretical Exploration of the Literature. 51

2.13 Search Strategy/Literature Search.............................................................. 52

2.14 Ecological Systems Theory: Definition and Model …....................................53

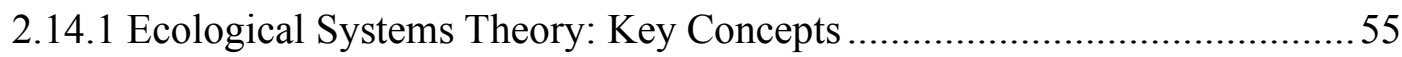

2.14.2 Ecological Systems Theory in Educational Psychological Practice............ 57

2.14.3 Ecological Transitions in the Context of Ecological Systems Theory ........ 58

2.15 Social Constructivist Theory: Definition and Concepts .................................59

2.15.1 Social Constructivist Theory in Educational Psychological Practice..........61

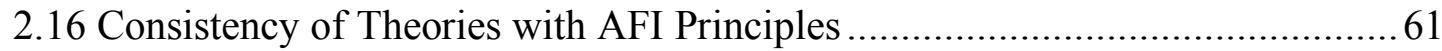

2.16.1 Conceptual Framework Development ....................................................64

2.16.2 Theoretical Statement and Research Question.......................................6 65

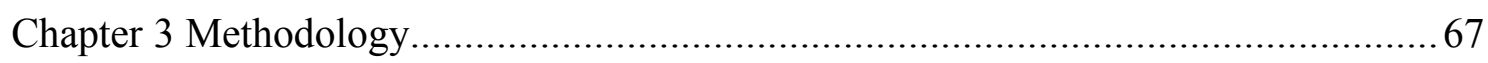

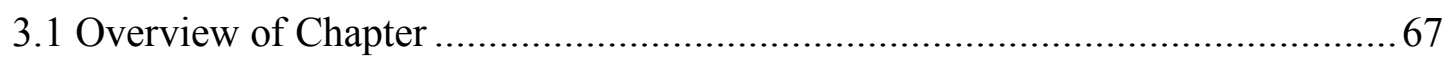

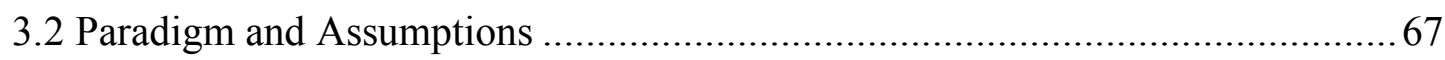

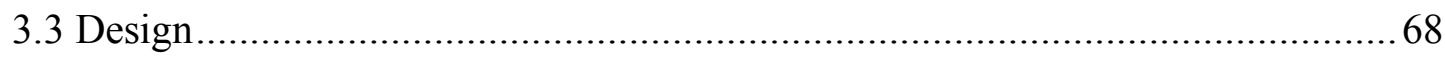

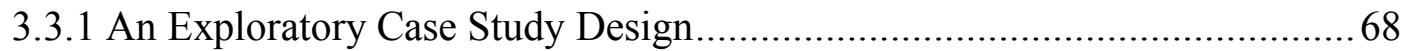

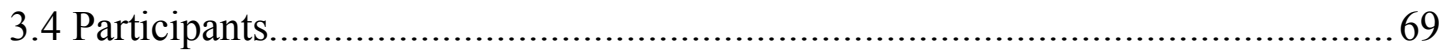

3.5 Components of a Case Study .................................................................. 70

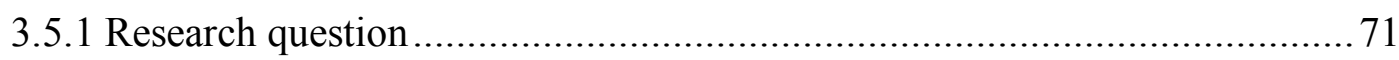

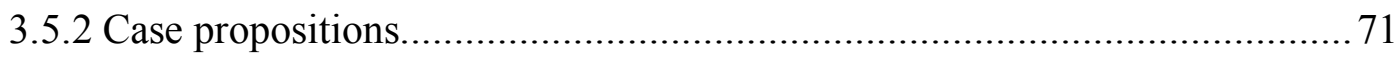

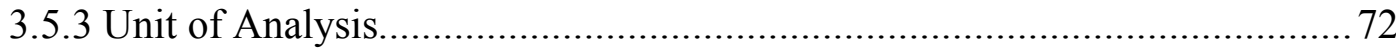

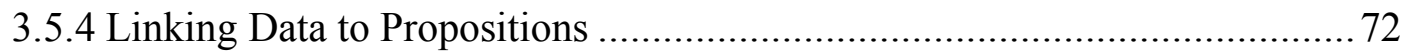

3.5.5 Criteria for Interpreting the Findings................................................ 72

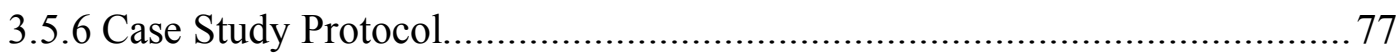

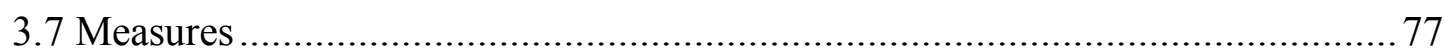

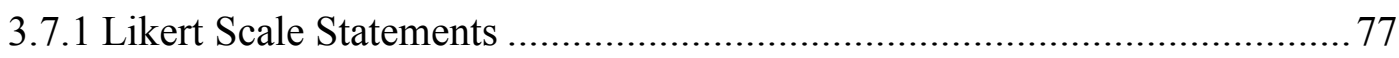

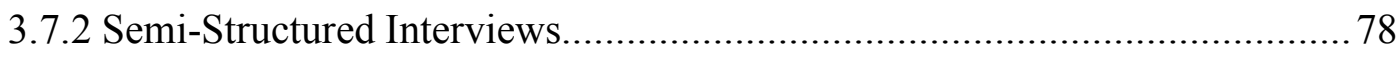

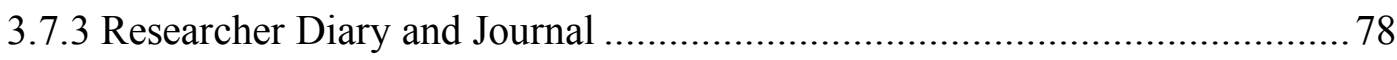

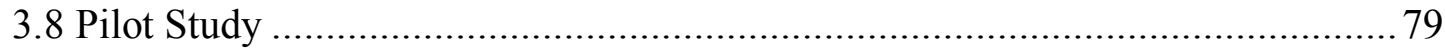

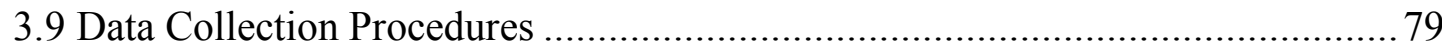

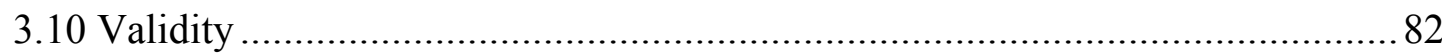

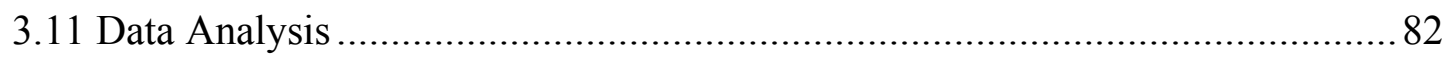

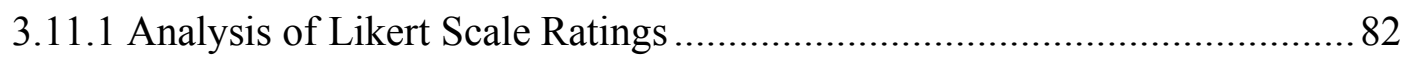

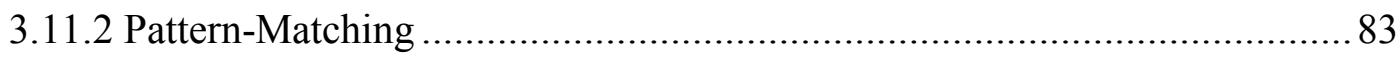

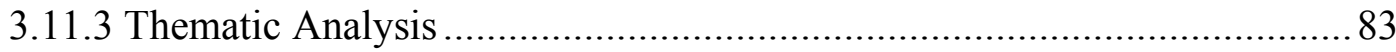




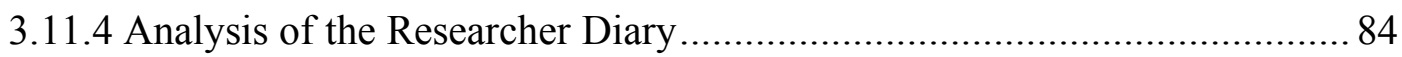

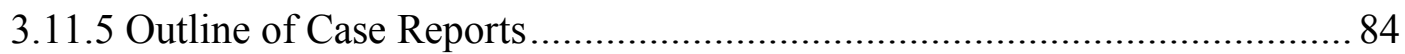

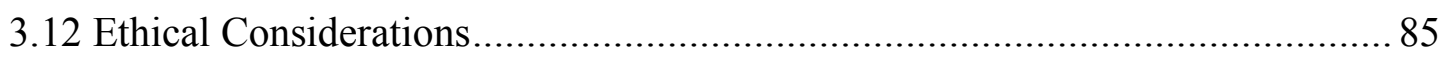

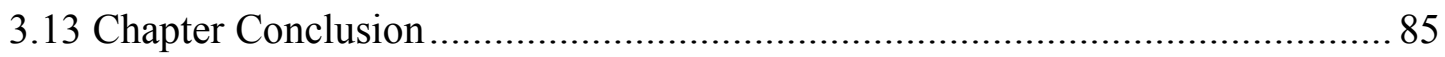

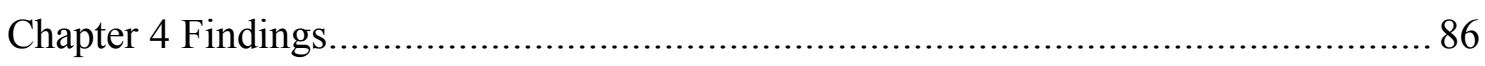

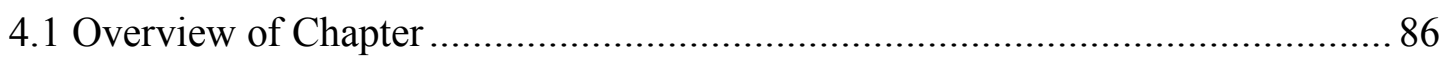

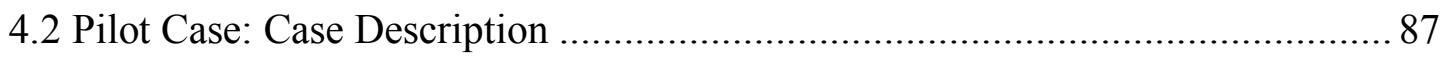

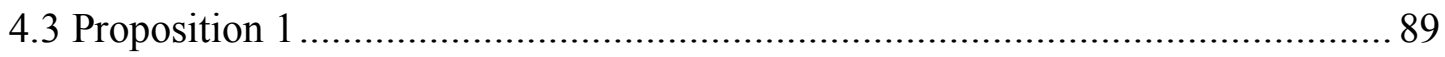

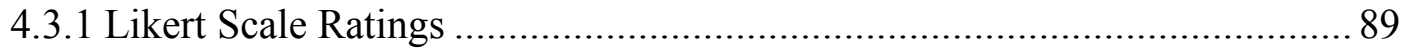

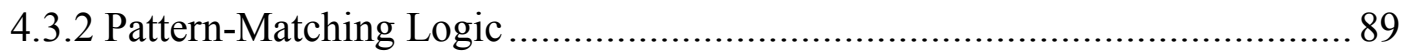

4.3.3 Researcher Diary and Thematic Analysis........................................... 90

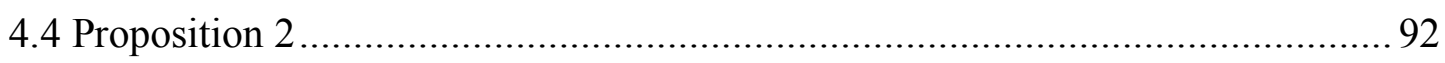

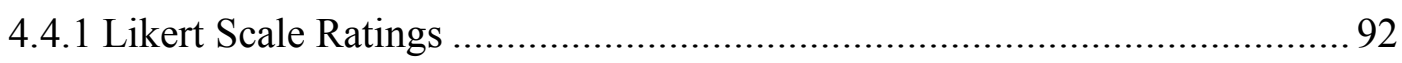

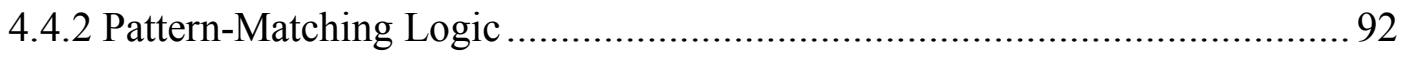

4.4.3 Researcher Diary and Thematic Analysis................................................ 93

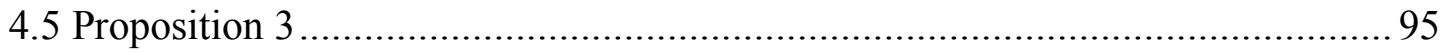

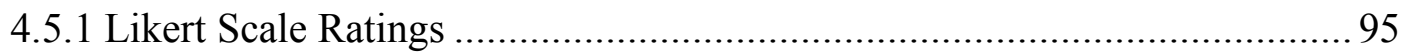

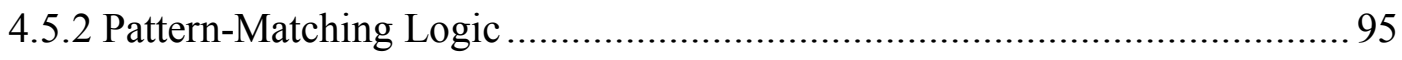

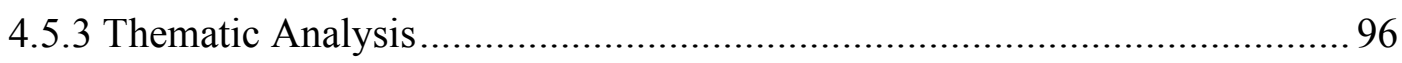

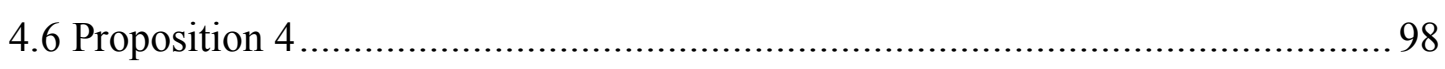

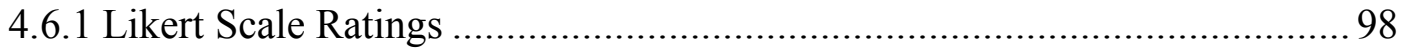

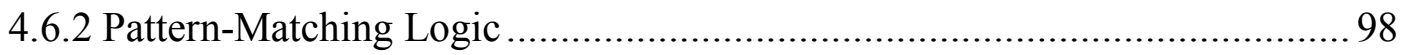

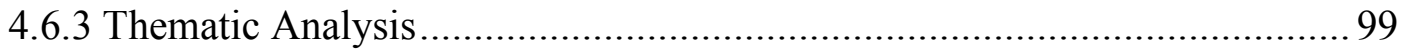

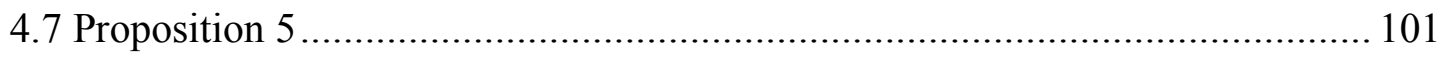

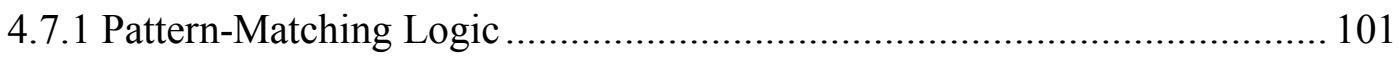

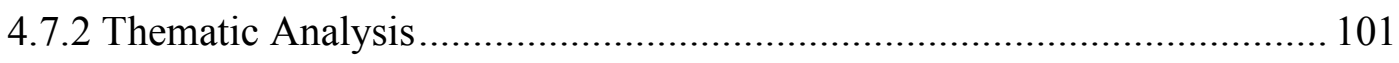

4.8 Summary of Pilot Case Proposition Findings............................................. 103

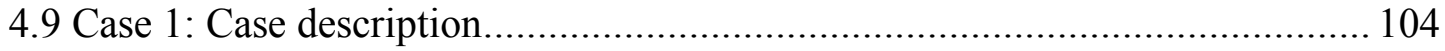

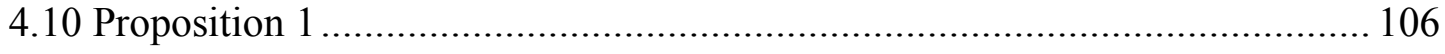

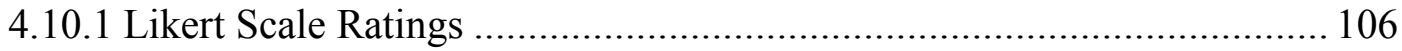

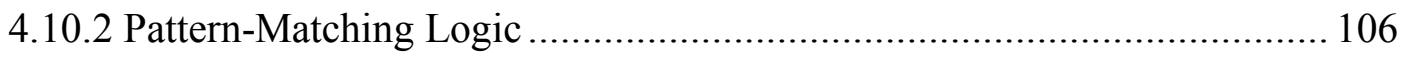

4.10.3 Researcher Diary and Thematic Analysis.......................................... 107

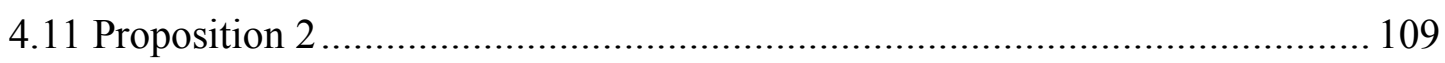

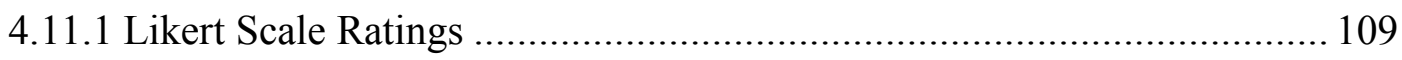

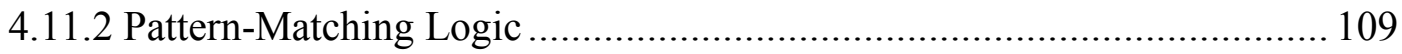


4.11.3 Researcher Diary and Thematic Analysis .......................................... 110

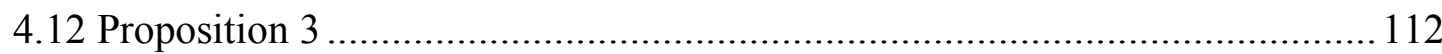

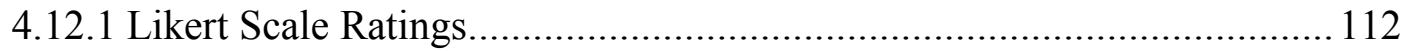

4.12.2 Pattern-Matching Logic ............................................................. 112

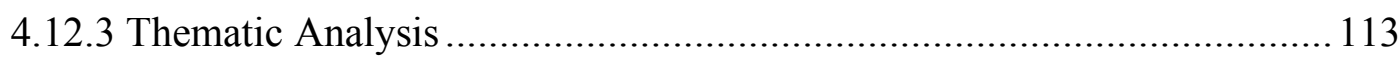

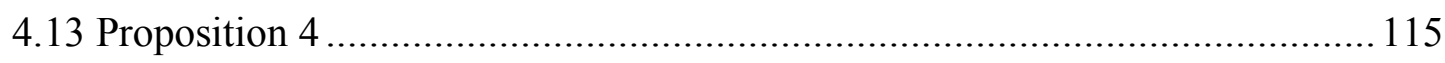

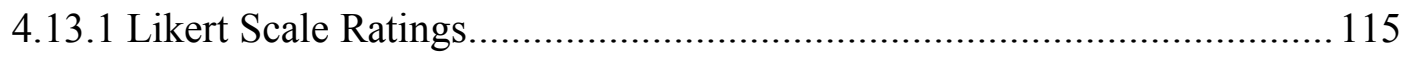

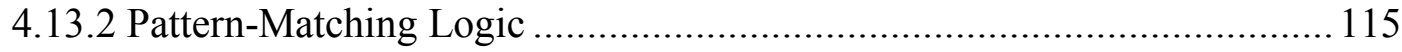

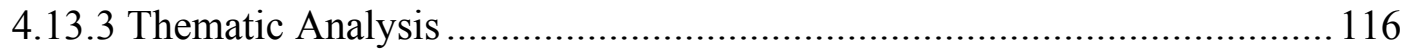

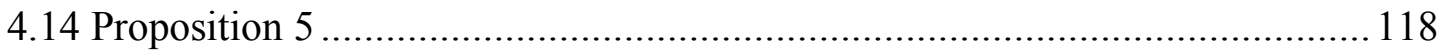

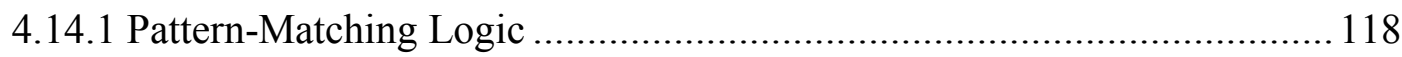

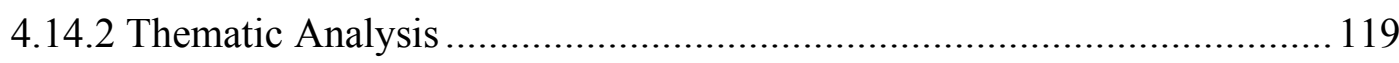

4.15 Summary of Case 1 Proposition Findings ................................................. 120

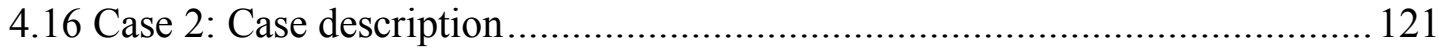

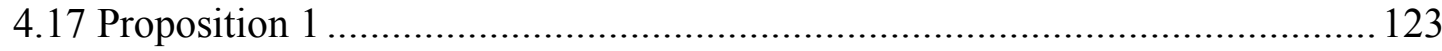

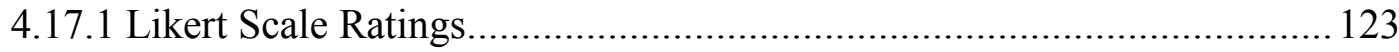

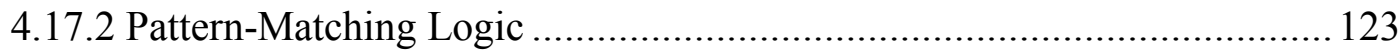

4.17.3 Researcher Diary and Thematic Analysis....................................... 124

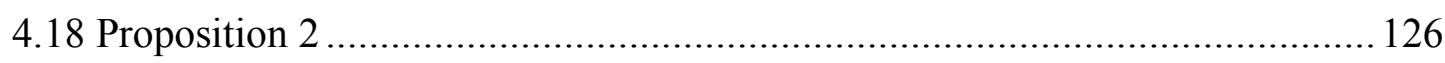

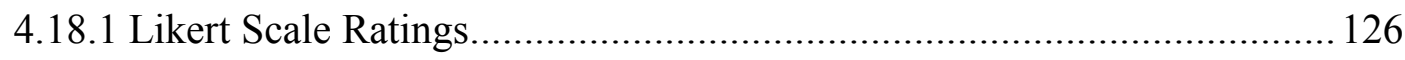

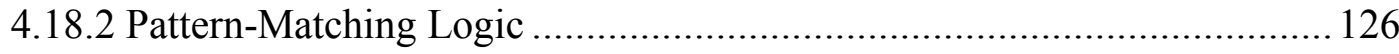

4.18.3 Researcher Diary and Thematic Analysis.......................................... 127

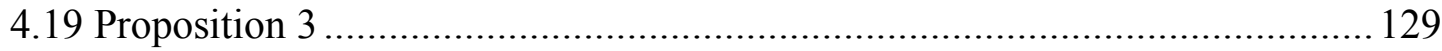

4.19.1 Likert Scale Ratings......................................................................... 129

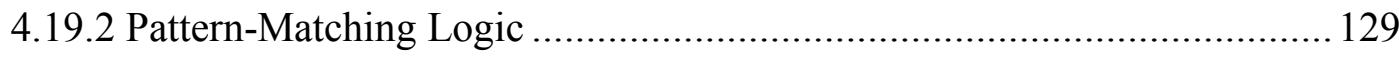

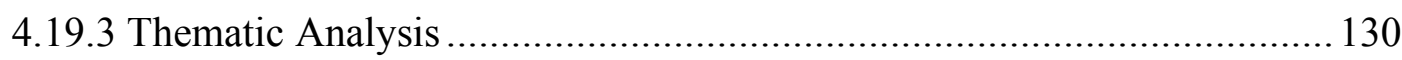

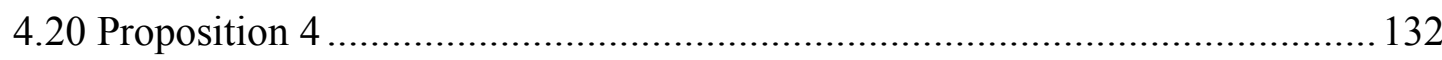

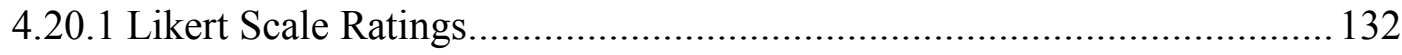

4.20.2 Researcher Diary and Thematic Analysis......................................... 132

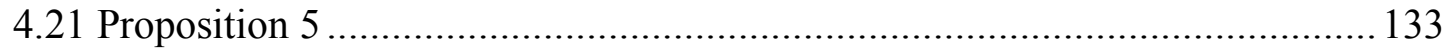

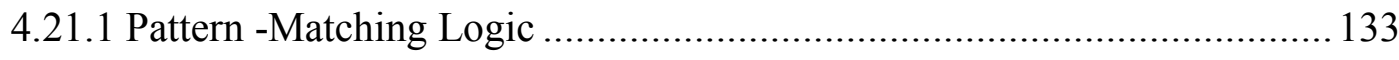

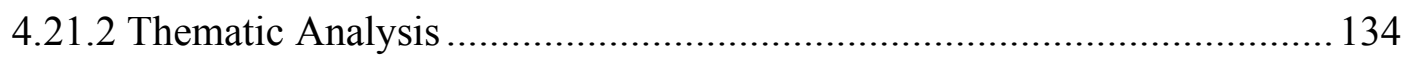

4.22 Summary of Case 2 Proposition Findings ................................................ 135

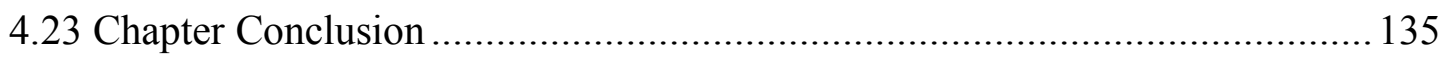

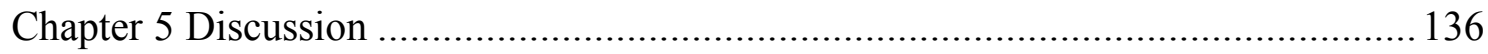




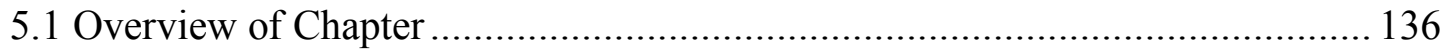

5.2 Discussion of Findings: Pilot Case ............................................................ 136

5.3 Views and Experiences of the AFI Model ................................................... 136

5.4 A Team Approach to Assessment …............................................................ 138

5.4.1 Active Roles and Communication......................................................... 138

5.4.2 Insight into Strengths, Needs and Perspectives ....................................... 139

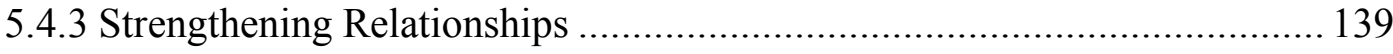

5.5 Perspectives on Barriers to Parental Engagement .......................................... 140

5.6 Factors Bridging the Gap between Assessment and Intervention .................... 141

5.6.1 Experiencing Intervention Effectiveness and Practical Interventions......... 141

5.6.2 Shared Awareness of Strengths, Needs and Goals.................................. 142

5.6.3 A Collaborative Approach to Implementing Interventions ....................... 143

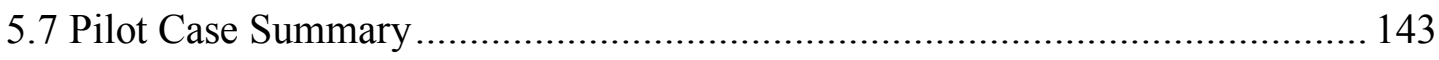

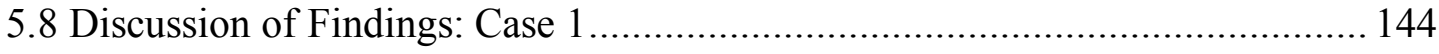

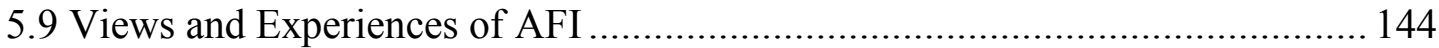

5.10 A Team Approach to Assessment ................................................................... 145

5.10.1 Active Roles and Communication........................................................... 146

5.10.2 Insight into Strengths, Needs and Perspectives....................................... 146

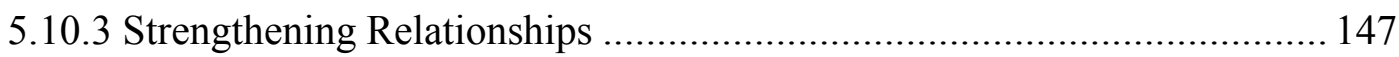

5.11 Perspectives on Barriers to Parental Engagement......................................... 148

5.12 Factors Bridging the Gap between Assessment and Intervention.................... 149

5.12.1 Experiencing Intervention Effectiveness and Practical Interventions....... 150

5.12.2 Shared Awareness of Strengths, Needs and Goals ............................... 150

5.12.3 A Collaborative Approach to Implementing Interventions ..................... 151

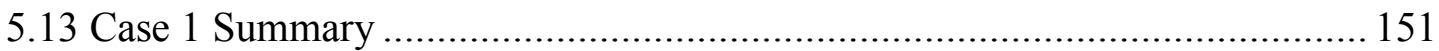

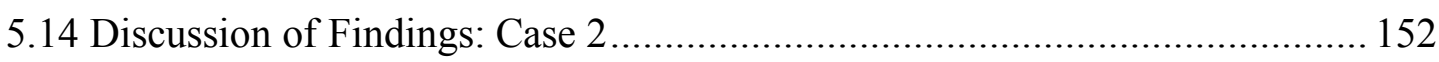

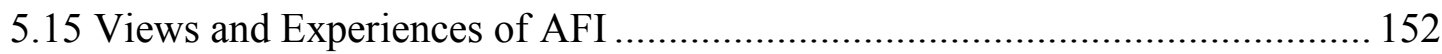

5.16 A Team Approach to Assessment ........................................................... 153

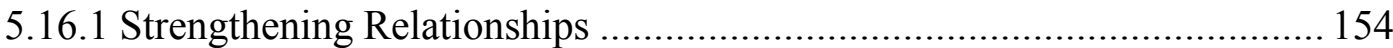

5.17 Perspectives on Barriers to Parental Involvement ........................................ 155

5.18 Factors Bridging the Gap between Assessment and Intervention................... 156

5.18.1 Experiencing Intervention Effectiveness and Practical Interventions....... 157

5.18.2 Shared Awareness of Strengths, Needs and Goals................................ 158

5.18.3 A Collaborative Approach to Implementing Interventions ..................... 158

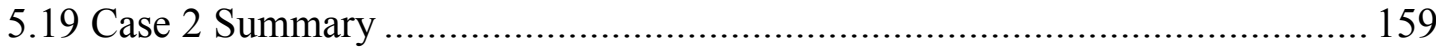


5.20 Cross-Case Analysis Discussion ........................................................... 160

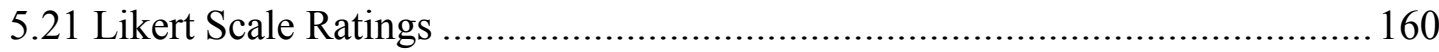

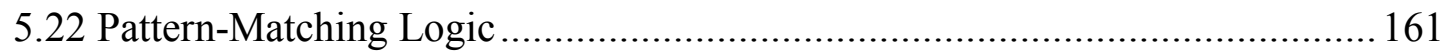

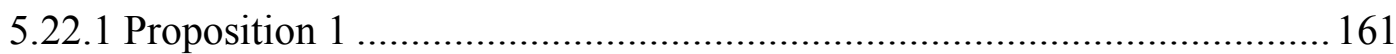

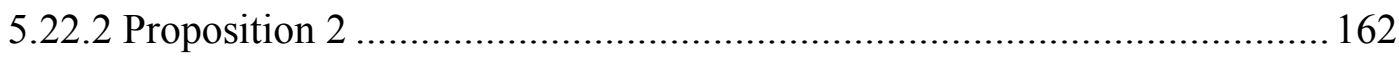

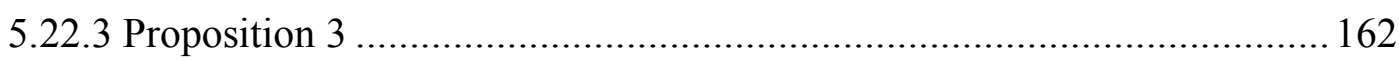

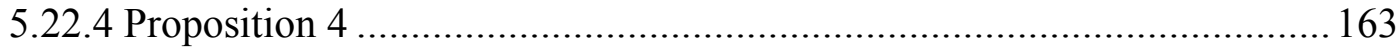

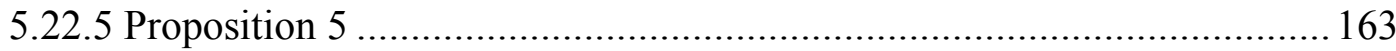

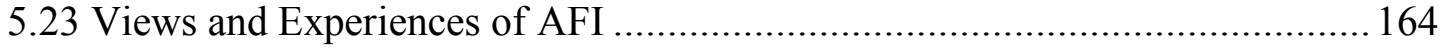

5.23.1 Applicability to Other Situations........................................................ 165

5.24 A Team Approach to Assessment ..................................................................... 165

5.25 Perspectives on Barriers to Parental Engagement........................................... 166

5.26 Factors Bridging the Gap Between Assessment and Intervention.................. 167

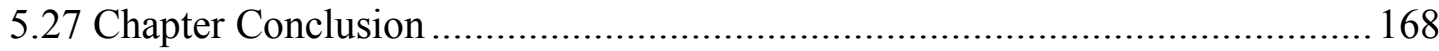

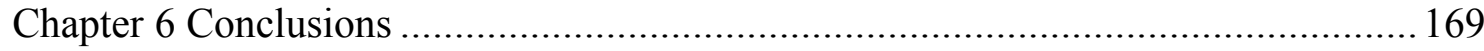

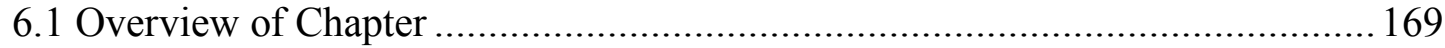

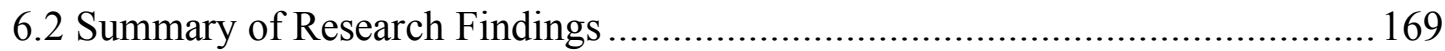

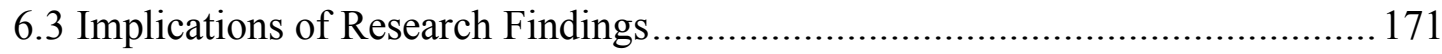

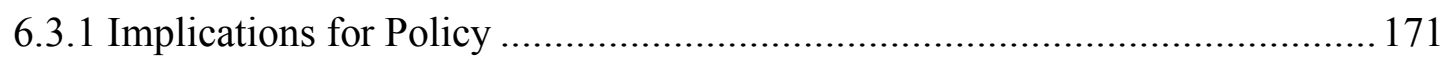

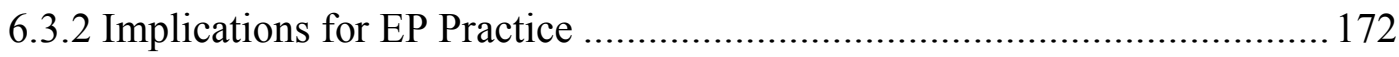

6.3.3 Implications for Educational Contexts ............................................... 173

6.3.4 Summary of Recommendations for Practice ........................................ 173

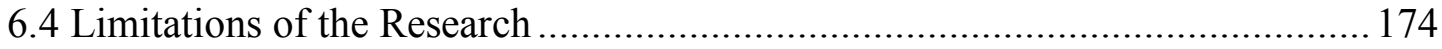

6.5 Scholarly Significance of the Research ......................................................... 175

6.6 Recommendations for Future Research...................................................... 177

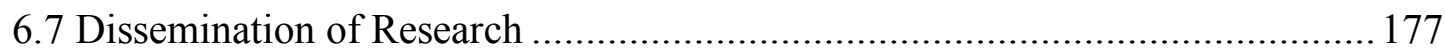

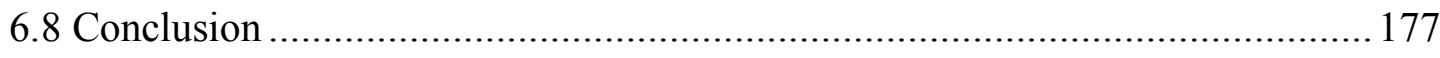

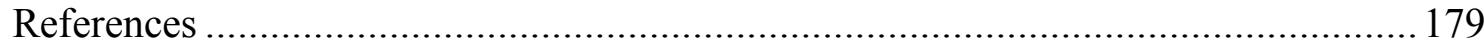




\section{List of Tables}

Table 2.1 Database Search Items Review Question 1 ........................................... 10

Table 2.2 Database Search Items Review Question 2 .............................................. 10

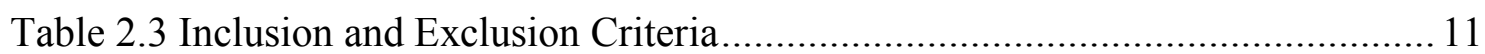

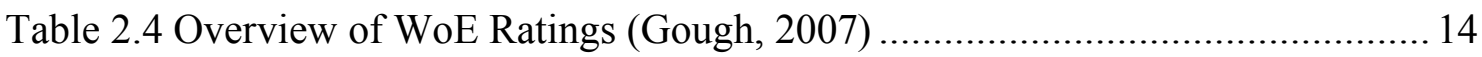

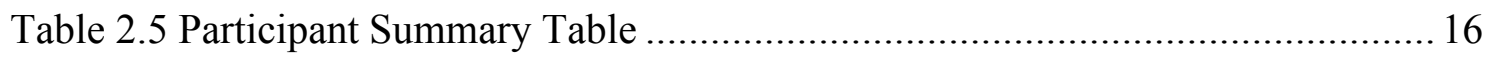

Table 2.6 Summary of Study Designs and Methodologies..................................... 22

Table 2.7 Summary of Systematic Review Findings................................................ 32

Table 2.8 Database Search Items Review Question 1 (Search 1) ...............................52

Table 2.9 Database Search Items Review Question 2 (Search 2) ...............................53

Table 2.10 Consistency of AFI Principles with EST and SCT .................................63

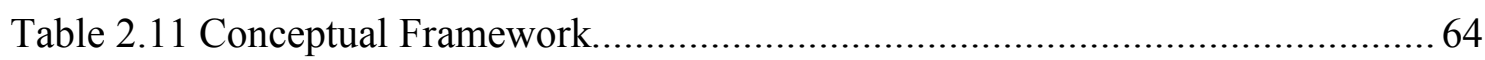

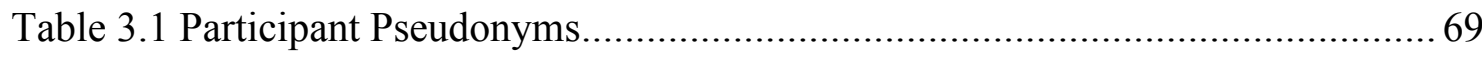

Table 3.2 Criteria for Interpreting the Findings ...................................................... 74

Table 3. 3 The Five Stages of the AFI Model .......................................................... 81

Table 4.1 Case Log of Actions Pilot Case ................................................................... 88

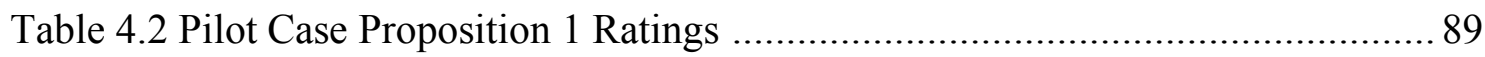

Table 4.3 Pilot Case Key Quotes Addressing Proposition 1....................................... 90

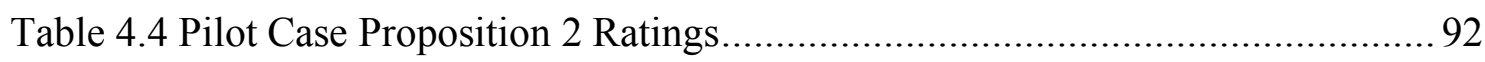

Table 4.5 Pilot Case Key Quotes Addressing Proposition 2 .................................... 93

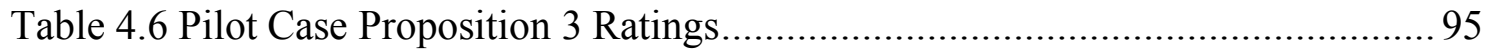

Table 4.7 Pilot Case Key Quotes Addressing Proposition 3.....................................96

Table 4.8 Pilot Case Proposition 4 Ratings................................................................ 98

Table 4.9 Pilot Case Key Quotes Addressing Proposition 4..................................... 99

Table 4.10 Pilot Case Key Quotes Addressing Proposition 5.................................. 101

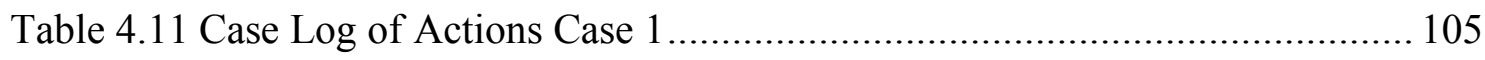

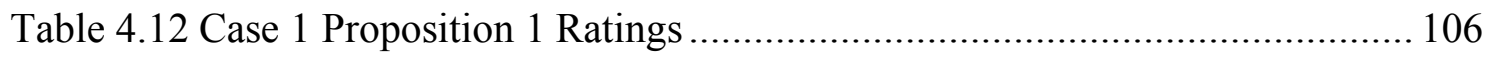

Table 4.13 Case 1 Key Quotes Addressing Proposition 1 …................................... 107

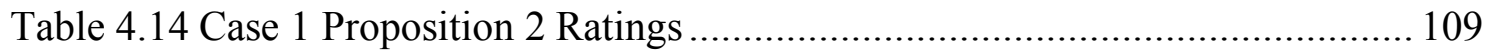

Table 4.15 Case 1 Key Quotes Addressing Proposition 2 ....................................... 110

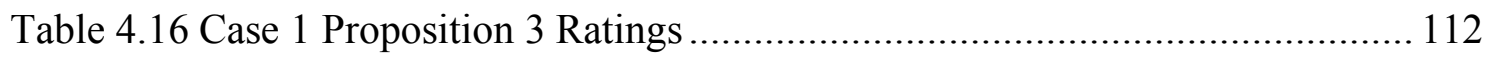

Table 4.17 Case 1 Key Quotes Addressing Proposition 3 ....................................... 113

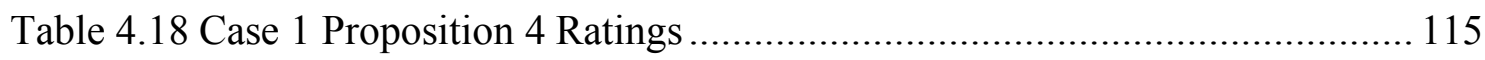

Table 4.19 Case 1 Key Quotes Addressing Proposition 4 ....................................... 116 
Table 4.20 Case 1 Key Quotes Addressing Proposition 5 ..................................... 118

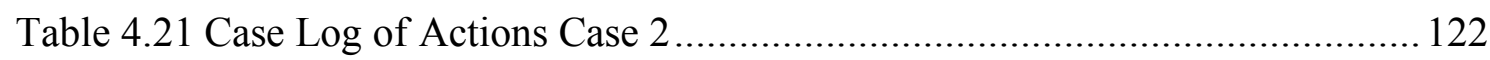

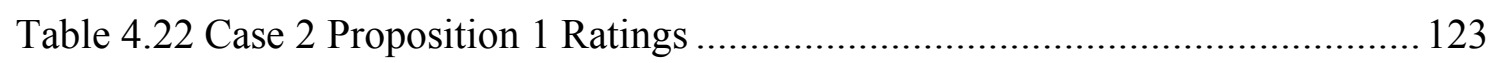

Table 4.23 Case 2 Key Quotes Addressing Proposition 1 ....................................... 124

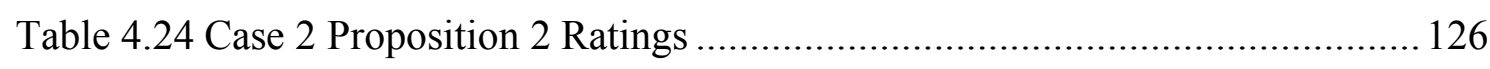

Table 4.25 Case 2 Key Quotes Addressing Proposition 2 .................................... 127

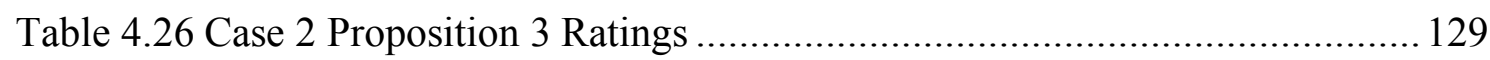

Table 4.27 Case 2 Key Quotes Addressing Proposition 3 ...................................... 130

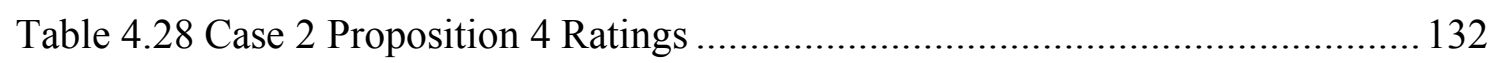

Table 4.29 Case 2 Key Quotes Addressing Proposition 5 ...................................... 133

Table 5.1 Participant Average Likert Scale Ratings.................................................. 161 


\section{List of Figures}

Figure 1.1. Research Project Flowchart............................................................ 7

Figure 2.1. Flowchart Delineating the Literature Search and Screening Process .......... 12

Figure 2.2. Ecological Systems Theory (Bronfenbrenner, 1979)...............................55

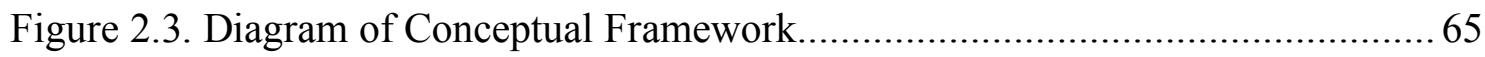

Figure 4.1. Pilot Case Proposition 1 Findings........................................................ 91

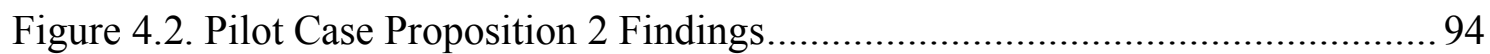

Figure 4.3. Pilot Case Proposition 3 Findings......................................................... 97

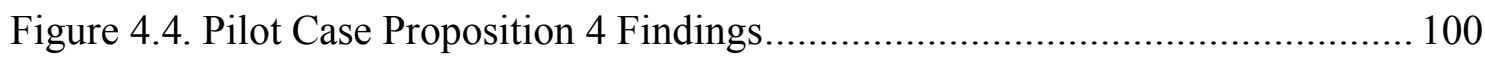

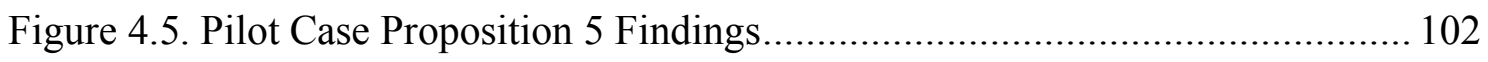

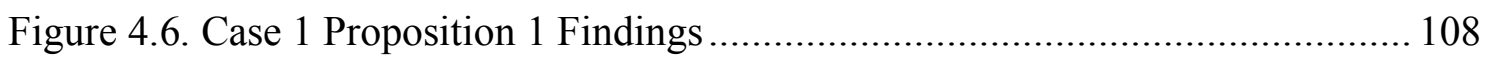

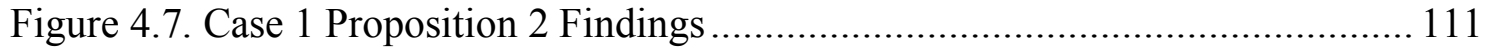

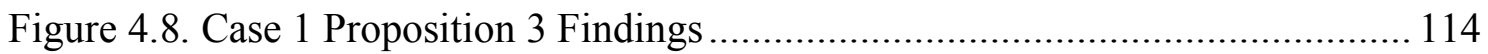

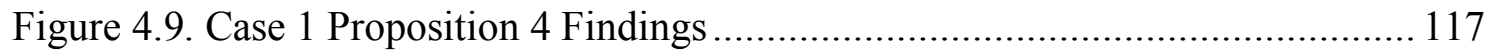

Figure 4.10. Case 1 Proposition 5 Findings .............................................................. 119

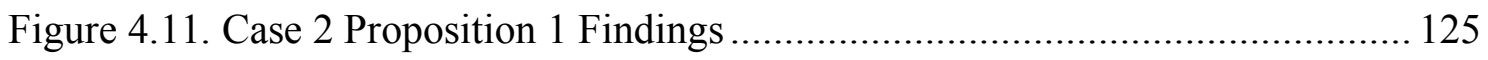

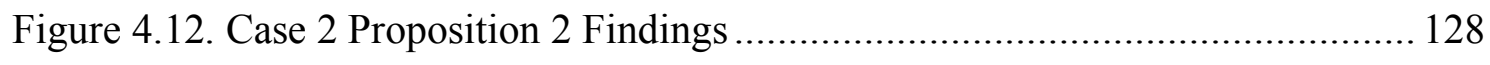

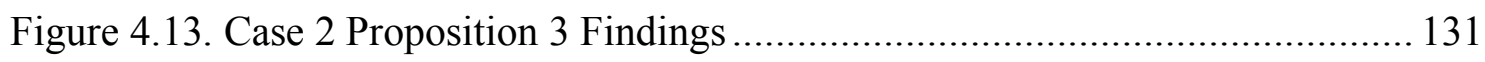

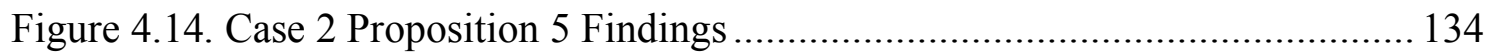




\section{List of Appendices}

Appendix 1 Summary of Included Studies 187

Appendix 2 Excluded Articles with Rationale and Full References (full text). 189

Appendix 3 WoE A Criteria and Scoring...... 193

Appendix 4 WoE B Criteria and Scoring........................................................ 208

Appendix 5 WoE C Criteria and Scoring.......................................................... 211

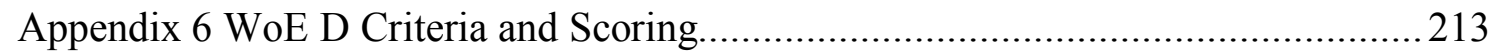

Appendix 7 Assessment Approaches Informing Appropriate Interventions ...............2 214

Appendix 8 The Five Stages of the AFI Model..........................................................2 216

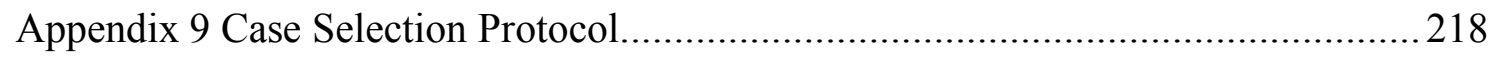

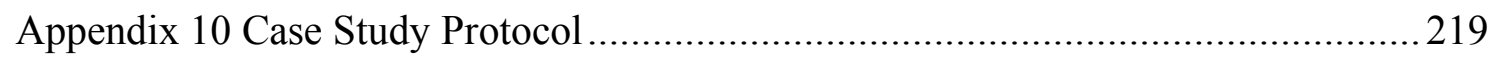

Appendix 11 Pilot and Revised Parent Likert Scale Statements .............................. 223

Appendix 12 Pilot and Revised Teacher Likert Scale Statements ...........................2225

Appendix 13 Student Likert Scale Statements .......................................................227

Appendix 14 Pilot and Revised Parent Interview Schedule.................................... 228

Appendix 15 Pilot and Revised Teacher Interview Schedule ....................................2232

Appendix 16 Pilot and Revised Student Interview Schedule...................................235

Appendix 17 Pilot and Revised Interview Guide .................................................237

Appendix 18 Documentation of Revisions Following Pilot Study ...........................239

Appendix 19 Detailed Procedure for Conducting Cases......................................... 241

Appendix 20 Phases of Thematic Analysis (Braun \& Clarke, 2006) .........................249

Appendix 21 15-Point Checklist of Criteria for Good Thematic Analysis ..................2250

Appendix 22 Parent/Guardian Information Sheet ................................................... 251

Appendix 23 Parent/Guardian Informed Consent Form .......................................256

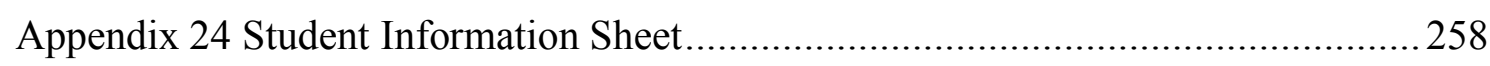

Appendix 25 Student Informed Assent Form.................................................... 261

Appendix 26 Procedures for Ethical Issues During the Research Process ................. 263

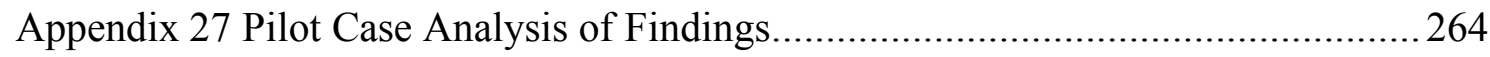

Appendix 28 Pilot Case Analysis of Findings: Proposition 1 ..................................266

Appendix 29 Pilot Case Analysis of Findings: Proposition 2 .................................. 274

Appendix 30 Pilot Case Analysis of Findings: Proposition 3 ..................................281

Appendix 31 Pilot Case Analysis of Findings: Proposition 4 .................................. 288

Appendix 32 Pilot Case Analysis of Findings: Proposition 5 ..................................294

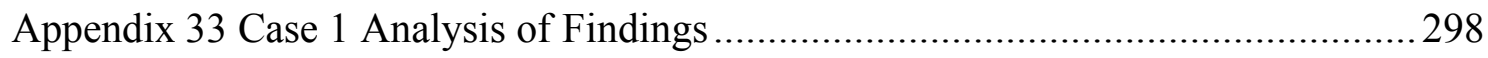


Appendix 34 Case 1 Analysis of Findings: Proposition 1 300

Appendix 35 Case 1 Analysis of Findings: Proposition 2

Appendix 36 Case 1 Analysis of Findings: Proposition 3

Appendix 37 Case 1 Analysis of Findings: Proposition 4 325

Appendix 38 Case 1 Analysis of Findings: Proposition 5 330

Appendix 39 Case 2 Analysis of Findings 336

Appendix 40 Case 2 Analysis of Findings: Proposition 1 338

Appendix 41 Case 2 Analysis of Findings: Proposition 2 348

Appendix 42 Case 2 Analysis of Findings: Proposition 3 355

Appendix 43 Case 2 Analysis of Findings: Proposition 4 359

Appendix 44 Case 2 Analysis of Findings: Proposition 5 363

Appendix 48 Devising Main Themes, Subthemes, Codes and Relevant Statements... 378 Appendix 49 Excerpt from Researcher Diary Demonstrating Progressing Understanding of Student's Situation (Pilot Case).... 412

Appendix 50 Excerpt from Researcher Diary Demonstrating Researcher's Knowledge of Intervention Supports (Pilot Case) 414

Appendix 51 Excerpt from Researcher Diary Demonstrating Progressing Understanding of Student's Situation (Case 1).

Appendix 52 Excerpt from Researcher Diary Demonstrating Researcher's Knowledge of Intervention Supports (Case 1)

Appendix 53 Excerpt from Researcher Diary Demonstrating Researcher's Understanding of the Student's Situation and Knowledge of Intervention Supports (Case 2)

Appendix 54 Empirical Paper 422 


\section{List of Abbreviations}

ADHD Attention Deficit Hyperactivity Disorder

AFI

Assessment for Intervention

AIM

Access and Inclusion Model

ANOVA Analysis of Variance

ASD

Autism Spectrum Disorder

BASC 2 Behaviour Assessment System for Children

BOM Board of Management

CBT Cognitive Behaviour Therapy

CHC Cattell Horn Carroll

CLD Culturally and Linguistically Diverse

CPD Continuing Professional Development

CSF Context Strength Finder

DA Dynamic Assessment

DCYA Department of Children and Youth Affairs

DES Department of Education and Skills

DSM IV Diagnostic and Statistical Manual

ED Emotional Disturbance

EP Educational Psychologist/ Educational Psychological

ESE Exceptional Student Education

EST Ecological Systems Theory

FAI Functional Assessment Interview

IDEIA Individuals with Disabilities Education Improvement Act

IQ Intelligence Quotient

NASP National Association of School Psychologists

NEPS National Educational Psychological Service

NCSE National Council for Special Education

PSI Psychological Society of Ireland

RTI Response to Intervention

SCT Social Constructivist Theory

SEN Special Educational Need

SET Special Education Teacher

SFBT Solution-Focused Brief Therapy

SLD Specific Learning Difficulty 
TEP Trainee Educational Psychologist

UK United Kingdom

WoE Weight of Evidence

ZAD Zone of Actual Development

ZPD Zone of Proximal Development 



\section{Chapter 1 Introduction}

\subsection{Overview of Chapter}

This chapter outlines the context, rationale and aims underpinning the current research project. Assessment in educational psychological (EP) practice is discussed within an international and national context and the rationale for conducting the literature review is outlined. An overview of the research is provided with the aims, methodology, measures and analysis applied. A flowchart is delineated, outlining the structure of the thesis.

\subsection{Assessment in EP practice: International Context}

Traditionally, the educational psychologist's (EP) distinctive role involved psychometric testing and diagnosis of a Special Educational Need (SEN) for categorisation and special education provision purposes (Farrell, 2010; Filter, Ebsen, \& Dibos, 2013). Over the decades, a global shift has occurred in assessment practices, from a traditional focus on within-child factors reflective of the medical model, towards consideration of social and environmental factors reflective of an interactionist, ecological approach (Davis \& Deponio, 2013; Kennedy, Frederickson, \& Monsen, 2008). International policy and legislative initiatives have been somewhat consistent with this reported shift in practice. In 2007, the European Agency for Special Needs and Inclusive Education commissioned a project examining assessment that informs teaching and learning in inclusive settings (Watkins, 2007). The concluding comments of the report state:

There has been a move from looking at individual pupils in isolation, to considering the context of pupil's learning. At the same time, the assessment process has moved away from a 'snapshot' approach involving professionals from outside the mainstream classroom, to an on-going process of mainstream teachers, parents and pupils themselves developing an understanding of not just what pupils learn, but also how they learn it. (p. 61)

The report endorses 'inclusive assessment', whereby policy and practice are designed to support the learning of all students and the allocation of support, placement and additional resourcing to meet a student's needs should be informed by, and not solely based upon initial identification or diagnostic procedures (Watkins, 2007). In the United Kingdom, a diagnosis of a disability or SEN is not required for access to additional resources in schools (National Council for Special Education [NCSE], 2013). 
Relevant to EP practice, Passenger (2013) reports the enactment of the Green Paper (2011) in the United Kingdom may have reduced the need for statutory assessment work with emphasis placed on developmental surveillance and intervention with children under the age of five. In the United States, the Individuals with Disabilities Education Improvement Act (IDEIA) prevails, requiring assessment and diagnosis of disability for access to special education resource provision (U.S. Department of Education, 2004). However, it is reported that school psychological practice has oriented towards a problem solving, Response to Intervention (RTI) approach (Desforges \& Lindsay, 2010). In practice, a survey study investigating school psychologists' continuing professional development (CPD) activities and preferences, indicated frequent engagement in RTI professional development activities, including academic, behavioural, and social-emotional interventions, academic screening and progress monitoring. Furthermore, respondents reported a high level of need for further CPD in these topic areas, and a low level of need for offerings in standardised assessment (Jose, Michael, Chappel, \& Cunningham, 2013). Continuing Professional Development activities and preferences reported in this study may be indicative of the types of professional activities that are being performed in actual practice.

\subsection{Assessment in EP Practice: National Context}

In Ireland, the traditional resource allocation system within mainstream schools reflected a medical model of practice, as assessment and diagnosis of disability and SEN were prerequisite to accessing limited resources (NCSE, 2014). In 2013, a NCSE policy advice paper exposed several inequities within the system including delays in accessing assessments and resource allocation contingent upon labelling and categorisation (NCSE, 2013). In 2017, the new model for allocation of special education teaching resources in mainstream schools was launched and stipulates resource allocation based on identified needs rather than diagnosis (Department of Education and Skills [DES], 2017a). Additional support should enhance the child's performance and participation in all school activities (DES, 2017), consistent with the aims of the European Agency for Special Needs and Inclusive Education (Watkins, 2007). According to national policy in Ireland and to ensure children's needs are being addressed, schools are encouraged to answer three pivotal questions; "how can we identify needs? how can we meet needs? [and] how can we monitor and report on progress?" (DES, 2017a, p. 3).The assessment approach advocated by the new model is consistent with other governmental initiatives, including the Access and Inclusion 
Model (AIM), which provides needs-based support to children in pre-school services (Department of Children and Youth Affairs [DCYA], 2016). Relevant to EP practice, Circular 0013/2017 and Circular 0014/2017 affirm the focus of professional assessment under the new allocation model in mainstream primary and post-primary schools, is on providing "understanding of a child's needs, the nature of difficulties, and to inform relevant interventions" (DES, 2017b, p. 14; DES, 2017c, p. 14).

Models of service delivery. In an international review of procedures used to diagnose a disability or SEN, Desforges and Lindsay (2010) put forward three dominant models of assessment and intervention; the medical model, social model and ecological model, also referred to as the 'bio-psychosocial' model (Desforges \& Lindsay, 2010, p. 3). The bio-psychosocial model evolved from ecological systems theory and proposes that presenting issues are a result of the systemic interaction between individual factors and factors at the micro, meso, exo and macrosystem levels (Bronfenbrenner, 1989, 1994; Desforges \& Lindsay, 2010). Key to EP practice, the international review recommends the bio-psychosocial model for informing SEN policy and identification and assessment of students with SEN (Desforges \& Lindsay, 2010). Within the Irish EP context, the National Educational Psychological Service (NEPS) adhere to the principles of the bio-psychosocial model and apply a problem solving approach to casework, critically exploring the interplay of biological, psychological and social factors (NEPS, 2007, 2010). The service supports the holistic development of children in primary and post-primary schools and implements a consultative 'Continuum of Support' model that guides assessment, intervention and review of a child's needs (NEPS, 2003, 2007). Consultation in EP practice is a process of working together with schools and families to identify solutions to presenting problems (Wagner, 2008). Wagner (2008) outlines three consultation frameworks for EP practice; 'Full Consultation' with teachers and staff in schools and centers, to explore what is currently successful, what progress would look like and what might contribute to that progress; 'Joint School-Family' consultation involving parents or guardians, to establish collaborative problem solving and solution finding between family and school systems; and 'Multi-Agency Meetings', where professionals from different agencies consult on finding strengths and solutions. Consultation as a framework for service delivery endeavors to move away from models that focus on individual difference, to problem solving and solution finding approaches (Clarke \& Jenner, 2006). In the context of the new model for allocation of special education teaching resources, the NEPS consultative Continuum of Support model operates under the guiding principle that 
students with the greatest level of need receive the greatest levels of support (DES, 2017 a).

\subsection{Rationale for the Literature Review}

The stipulations for professional assessment outlined in Circular 0013/2017 (DES, 2017b) and Circular 0014/2017 (DES, 2017c) directly impact upon EP practice in Ireland, specifically the shift in focus from assessment for diagnosis to a needs-based approach that informs appropriate interventions. Linking assessment to intervention is a presenting challenge in EP practice (Resing, Lauchlan, \& Elliott, 2017) and has been described as "the next frontier for school psychology" (VanDerHeyden, 2018, p. 51). As a trainee educational psychologist (TEP) who undertook a school psychology placement, and as a future practitioner in the field, the assertions regarding professional assessment in Circular 0013/2017 (DES, 2017b) and Circular 0014/2017 (DES, 2017c) hold particular significance. A systematic review of assessment approaches that EPs are currently applying in practice and whether these approaches are informing appropriate interventions for clients was conducted. The review provided evidence of assessment approaches consistent with the medical model of practice, with classification and diagnosis of an SEN or disability reported in 12 of the studies reviewed. Six studies provided evidence of assessment approaches that inform intervention for clients and discussion of findings indicated their alignment with elements of Ecological Systems Theory (EST) (Bronfenbrenner, 1979) and Social Constructivist Theory (SCT) (Palinscar, 1998; Vygotsky, 1978) which, following a second phase of the literature review, formed the conceptual framework for the overall study. Of all the assessment approaches reviewed, the Assessment for Intervention model (AFI) (Pameijer, 2017) was considered most consistent with the aims of Circular 0013/2017 (DES, 2017b) and Circular 0014/2017 (DES, 2017c), as it endeavours to offer a better understanding of the child and the support he/she needs.

AFI is a five-stage model of assessment and intervention and its aim is to bridge the gap between assessment and intervention in order to provide recommendations that are both scientifically sound and useful for the student, teacher and parent/guardian (Pameijer, 2017). The AFI model is implemented in mainstream and special education settings, predominantly in the Netherlands and Flanders and has been described by the European Agency for Special Needs and Inclusive Education as a model of 'best practice' (Pameijer, 2017, p. 73). Publications on the AFI model have been issued for school counsellors, mental health professionals, teachers in primary and secondary 
schools and parents, suggesting the applicability of the framework to various settings (Pameijer, 2017).

\subsection{Aims and Methodology of the Research Project}

An exploratory 'two case' case study was employed to answer the research question: 'Can the Assessment for Intervention model bridge the gap between assessment and intervention, through activity and interaction ${ }^{1}$ at the meso and microsystem levels?'. The research question sought to explore the utility of the AFI model in bridging the gap between assessment and intervention, through the conceptual lens of EST (Bronfenbrenner, 1979) and SCT (Palinscar, 1998; Vygotsky, 1978). Consistent with a case study design (Yin, 2009), five case propositions were devised, which emerged from the conceptual framework, research question and aims of AFI (Pameijer, 2016, 2017). The AFI framework was applied to two case referrals made to a school psychological service in Ireland. It was proposed that EST and SCT concepts could inform the five-stage process and that findings would provide interesting implications for policy and practice. Case 1 included a student in $4^{\text {th }}$ class, his mother, class teacher and Special Education Teacher (SET) $(n=4)$, referred to the service with learning needs. Case 2 comprised of a student in $6^{\text {th }}$ class, her mother and class teacher $(n=3)$, referred with learning and social-emotional needs. A pilot study which included a student in $4^{\text {th }}$ class, his mother and class teacher $(n=3)$, referred with behaviour needs, was conducted to inform the research design and a case study protocol. Due to an incomplete data set for Case 2, the pilot study was also analysed and presented as a case.

\subsection{Paradigm and Research Design}

The constructivist paradigm was considered consistent with the researcher's conceptual framework and qualitative measures were employed to address the research question and five case propositions. Participants rated Likert statements exploring case propositions, before and after engagement with the AFI model. Semi-structured interviews were also conducted to answer the research question and to explore case propositions. AFI templates served as a researcher diary and were recorded before, during and after application of the model. A reflective journal was also used to elucidate actions recorded and to reflect upon the process. Thematic analysis (Braun \& Clarke,

\footnotetext{
${ }^{1}$ Activity and interaction are those activities and interactions that resonate with EST and SCT concepts, including molar activities, dyadic relations, ZPD and tools and signs.
} 
2006) and pattern-matching (Yin, 2009) were employed to analyse the interview data and changes in ratings on Likert statements were presented in tabular format. All data were further analysed in conjunction with the conceptual framework in order to theorise from the data. Each case report was discussed in the context of the research question, conceptual framework and literature review, followed by a cross-case discussion. Several implications for EP practice were revealed and in line with Circular 0013/2017 (DES, 2017b) and Circular 0014/2017 (DES, 2017c), implications for the broader educational context were also identified. Figure 1 provides a flowchart to delineate the structure of the research project overleaf. 


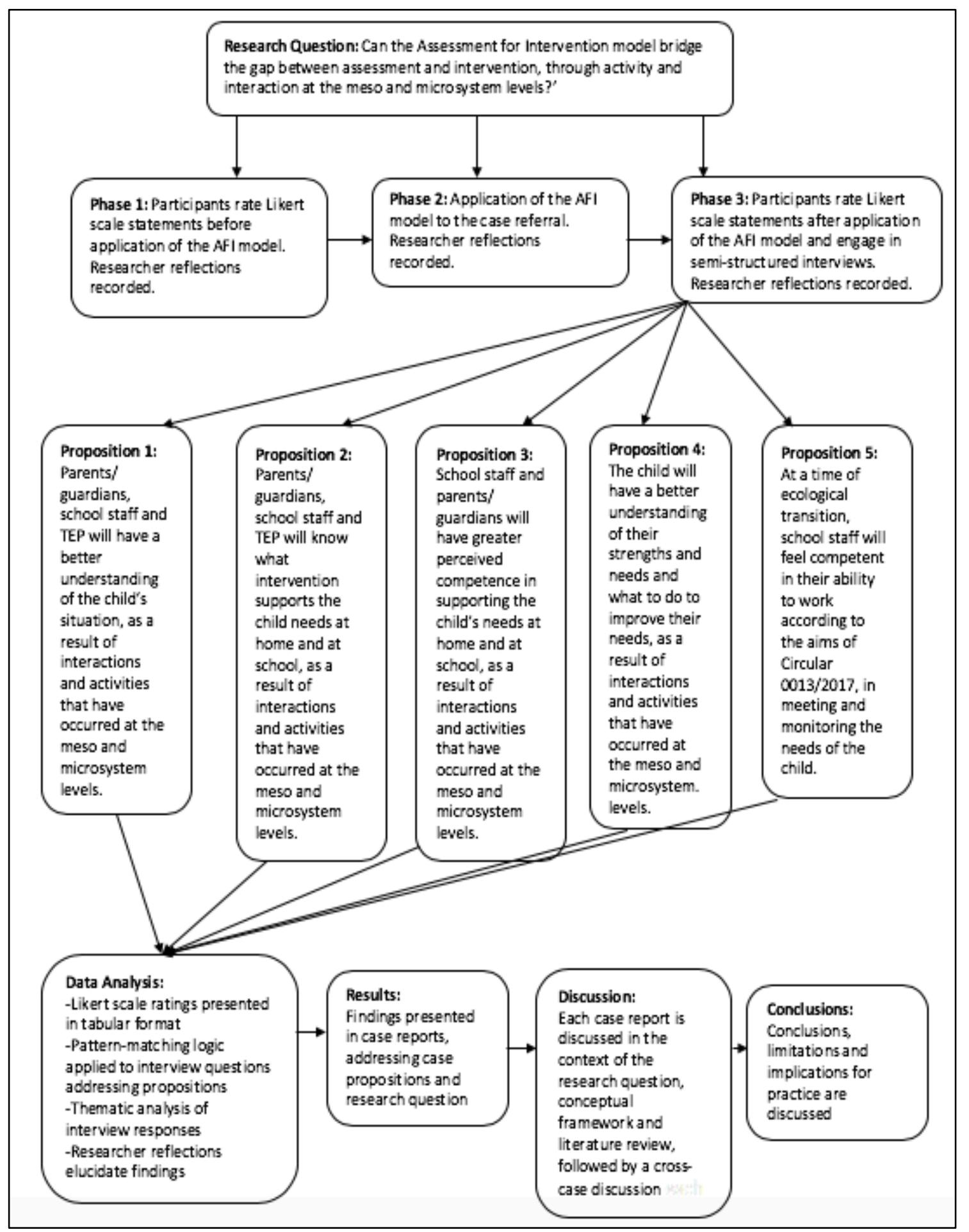

Figure 1.1. Research Project Flowchart

\subsection{Chapter Conclusion}

This introductory chapter described the rationale and aims underpinning the research project. The literature, pertinent to the topics under investigation, are reviewed and analysed in Chapter 2. This is followed by the Methodology (Chapter 3), Findings (Chapter 4), Discussion (Chapter 5) and finally, Conclusions Chapter (Chapter 6). 


\section{Chapter 2 Literature Review}

\subsection{Overview of Chapter}

This chapter provides a review of the literature pertaining to the research topic, setting the context and rationale for the current study. The review comprises two phases. The first phase is a systematic review which investigated the assessment approaches currently being applied by EPs in practice and whether these approaches are informing appropriate intervention recommendations for clients. The studies were critiqued both conceptually and methodologically and the findings identified assessment and intervention approaches consistent with the aims of Circular 0013/2017 (DES, 2017b) and Circular 0014/2017 (DES, 2017c). From a theoretical perspective, the findings were deemed to be in accordance with ECT (Bronfenbrenner, 1979) and SCT (Palinscar, 1998; Vygotsky, 1978). Directions for future research proposed further exploration of the AFI model (Pameijer, 2017) and a second phase of the literature review was conducted to examine how EST (Bronfenbrenner, 1979) and SCT (Palinscar, 1998; Vygotsky, 1978) inform EP assessment practices generally, and more specifically, the AFI model (Pameijer, 2016, 2017). The findings of this phase led to the development of a conceptual framework to address the research question for the current study.

The first phase of the review begins with an outline of the systematic approach applied to the review process and key concepts of the review questions. A description of Gough's Weight of Evidence (WoE) Framework (2007) is provided and is followed by a critical evaluation of the design, methodology and findings of the studies. A summary and limitations of the systematic review are presented along with directions for future research. The second phase of the review first outlines the search strategy applied. It then discusses EST (Bronfenbrenner, 1979) and SCT (Palinscar, 1998; Vygotsky, 1978) separately and expands upon their concepts and application in EP practice. This is followed by a discussion on how concepts within these theories resonate with AFI principles and a presentation of the conceptual framework for the research. The section concludes with a theoretical statement for the study and the research question.

\subsection{Phase 1 of the Literature Review: A Systematic Approach}

Systematic reviews "provide a meticulous way of finding relevant, high quality studies; and integrating their findings gives a clearer and more comprehensive picture than any single study can produce" (Gough, Oliver, \& Thomas, 2013, p. 5). They 
establish what is known in a particular area of research and inform decisions regarding future research (Gough et al., 2013). The current systematic review is two-staged and investigated the following questions:

1. What assessment approaches are educational psychologists applying in practice?

2. Are the assessment approaches informing appropriate interventions for clients?

\subsection{Key Concepts and Terminology Defined}

When conducting a systematic review, the conceptual assumptions implicit in the review question must be defined as this drives the methods of the review and how the question is answered (Gough, 2007). Concepts within the review questions are defined as follows.

Population. The new model for allocation of special education teaching resources provides for school age children in mainstream primary and secondary schools. The assessment approaches of qualified EPs working with children attending primary or secondary school were investigated. For consistency of language use throughout the systematic review, the title 'EP' is used and refers to school psychologists and psychologists working in the field of school/educational psychology.

Assessment approaches. The first review question investigated the current assessment approaches of EPs. The purpose of assessment is "to gather information in order to provide informed advice or recommendations concerning aspects of the student's educational and/or psychosocial functioning and attainment" (Bowles et al., 2016, p. 11). This definition guided the review process. Assessment 'approach' in this context refers to the practice, model or framework applied by the EP. The alignment of assessment approaches to theoretical models or frameworks was also considered.

Intervention. The second review question investigated whether the EP assessment approaches reported inform appropriate interventions for clients in practice. 'Client' refers to the individual(s) with the referral issue (e.g. parent/guardian or teacher) and the child or young person. In the current review 'intervention' refers to any intervention, recommendation, strategy or treatment arising from an EP assessment. An 'appropriate' intervention is one that has satisfactorily addressed the referral issue and has improved the situation, according to results from a response to intervention approach, feedback from the clients or self-reflection of the EP. Again, the alignment of interventions to specific theoretical models or frameworks was also considered. 


\subsection{Search Strategy/Literature Search}

An electronic database search of Academic Search Complete, PsycINFO and ERIC was conducted through EBSCO host in August, 2018. Keywords pertaining to review question 1 (Table 2.1) and review question 2 (Table 2.2) were searched. If available, a filter was applied so that only peer reviewed studies and studies written in English would be included. A limit on year of publication was also applied (2013-2018) to yield the most current assessment approaches applied in practice. An initial search of 2,027 titles was generated. Titles were screened against inclusion and exclusion criteria outlined in Table 2.3, reducing the search to 399 studies for screening of abstracts. A total of 43 articles remained for full text screening against inclusion and exclusion criteria and 21 articles were included for review. See Figure 2.1 for a flowchart delineating the literature search and screening process, Appendix 1 for a summary of included studies and Appendix 2 for the full list of excluded articles and rationale.

Table 2.1

Database Search Items Review Question 1

\begin{tabular}{ll}
\hline Databases & Search Terms \\
\hline Academic Search, ERIC, PsycINFO & "education* psycholog*” OR "school \\
& psycholog*" AND "assessment practice" \\
& OR "assessment approach" OR \\
& "assessment model” OR "assessment \\
& framework" \\
\hline
\end{tabular}

Table 2.2

Database Search Items Review Question 2

\begin{tabular}{ll}
\hline Databases & Search Terms \\
\hline Academic Search, ERIC, PsycINFO & "education* psycholog*” OR "school \\
& psychology*" AND "assessment \\
& practice" OR "assessment approach" OR \\
& "assessment model" OR "assessment \\
& framework" AND intervention OR \\
& recommendation OR strateg* OR \\
& treatment \\
& "education* psycholog* assessment" OR \\
Academic Search, ERIC, PsycINFO & "school psycholog* assessment" AND \\
& "intervention" OR "recommendation" OR \\
& "strateg* OR treatment" \\
\hline
\end{tabular}


Table 2.3

Inclusion and Exclusion Criteria

\begin{tabular}{|c|c|c|c|}
\hline Criteria & Inclusion & Exclusion & Rationale \\
\hline $\begin{array}{l}1 \text { Type of } \\
\text { publication }\end{array}$ & $\begin{array}{l}\text { Peer reviewed } \\
\text { paper }\end{array}$ & $\begin{array}{l}\text { Not a peer } \\
\text { reviewed paper }\end{array}$ & $\begin{array}{l}\text { Peer reviewed papers have } \\
\text { been independently assessed } \\
\text { for quality }\end{array}$ \\
\hline 2 Language & $\begin{array}{l}\text { Study must be } \\
\text { written in English }\end{array}$ & $\begin{array}{l}\text { Study is not } \\
\text { written in English }\end{array}$ & $\begin{array}{l}\text { For the study to be } \\
\text { understood, it must be written } \\
\text { in English }\end{array}$ \\
\hline $\begin{array}{l}3 \\
\text { Assessment } \\
\text { Approach }\end{array}$ & $\begin{array}{l}\text { An assessment } \\
\text { approach, } \\
\text { practice, model or } \\
\text { framework } \\
\text { applied by a } \\
\text { qualified EP }\end{array}$ & $\begin{array}{l}\text { Assessment } \\
\text { approaches that } \\
\text { are not applied by } \\
\text { an EP }\end{array}$ & $\begin{array}{l}\text { The current review is situated } \\
\text { in an educational } \\
\text { psychological context } \\
\text { therefore assessments carried } \\
\text { out by EPs only were } \\
\text { considered. }\end{array}$ \\
\hline $\begin{array}{l}4 \\
\text { Intervention }\end{array}$ & $\begin{array}{l}\text { An intervention, } \\
\text { recommendation, } \\
\text { strategy or } \\
\text { treatment arising } \\
\text { from an EP's } \\
\text { assessment }\end{array}$ & $\begin{array}{l}\text { Interventions, } \\
\text { recommendations, } \\
\text { strategies or } \\
\text { treatments that do } \\
\text { not arise from an } \\
\text { EP's assessment }\end{array}$ & $\begin{array}{l}\text { The current review is situated } \\
\text { in an educational } \\
\text { psychological context } \\
\text { therefore interventions arising } \\
\text { from an EP's assessment only } \\
\text { were considered. }\end{array}$ \\
\hline 5 Data & $\begin{array}{l}\text { The study } \\
\text { provides primary, } \\
\text { empirical data }\end{array}$ & $\begin{array}{l}\text { The study does } \\
\text { not provide } \\
\text { empirical data } \\
\text { (e.g. reviews, } \\
\text { commentaries) }\end{array}$ & $\begin{array}{l}\text { Empirical data allows the } \\
\text { reviewer to investigate the } \\
\text { assessment approaches } \\
\text { currently being applied by } \\
\text { EPs (review question 1) and } \\
\text { the appropriateness of } \\
\text { intervention } \\
\text { recommendations (review } \\
\text { question 2) arising from these } \\
\text { assessments. A variety of } \\
\text { measures may be employed in } \\
\text { studies including } \\
\text { questionnaires, interviews } \\
\text { and self-reflection. }\end{array}$ \\
\hline $\begin{array}{l}6 \\
\text { Participants }\end{array}$ & $\begin{array}{l}\text { Participants in } \\
\text { studies must be } \\
\text { parents, teachers, } \\
\text { children or EPs. } \\
\text { The child may or } \\
\text { may not have a } \\
\text { diagnosed SEN } \\
\text { and attends a } \\
\text { mainstream } \\
\text { primary or } \\
\text { secondary school. }\end{array}$ & $\begin{array}{l}\text { Participants in the } \\
\text { study are not } \\
\text { parents, teachers, } \\
\text { children or EPs } \\
\text { and the child does } \\
\text { not attend a } \\
\text { mainstream } \\
\text { primary or } \\
\text { secondary school. }\end{array}$ & $\begin{array}{l}\text { The new model for allocation } \\
\text { of special education teaching } \\
\text { resources in mainstream } \\
\text { schools provides for school } \\
\text { age children in mainstream } \\
\text { schools therefore studies of } \\
\text { students attending primary or } \\
\text { secondary school, their } \\
\text { parents/guardians, teachers, } \\
\text { EP were considered. }\end{array}$ \\
\hline
\end{tabular}




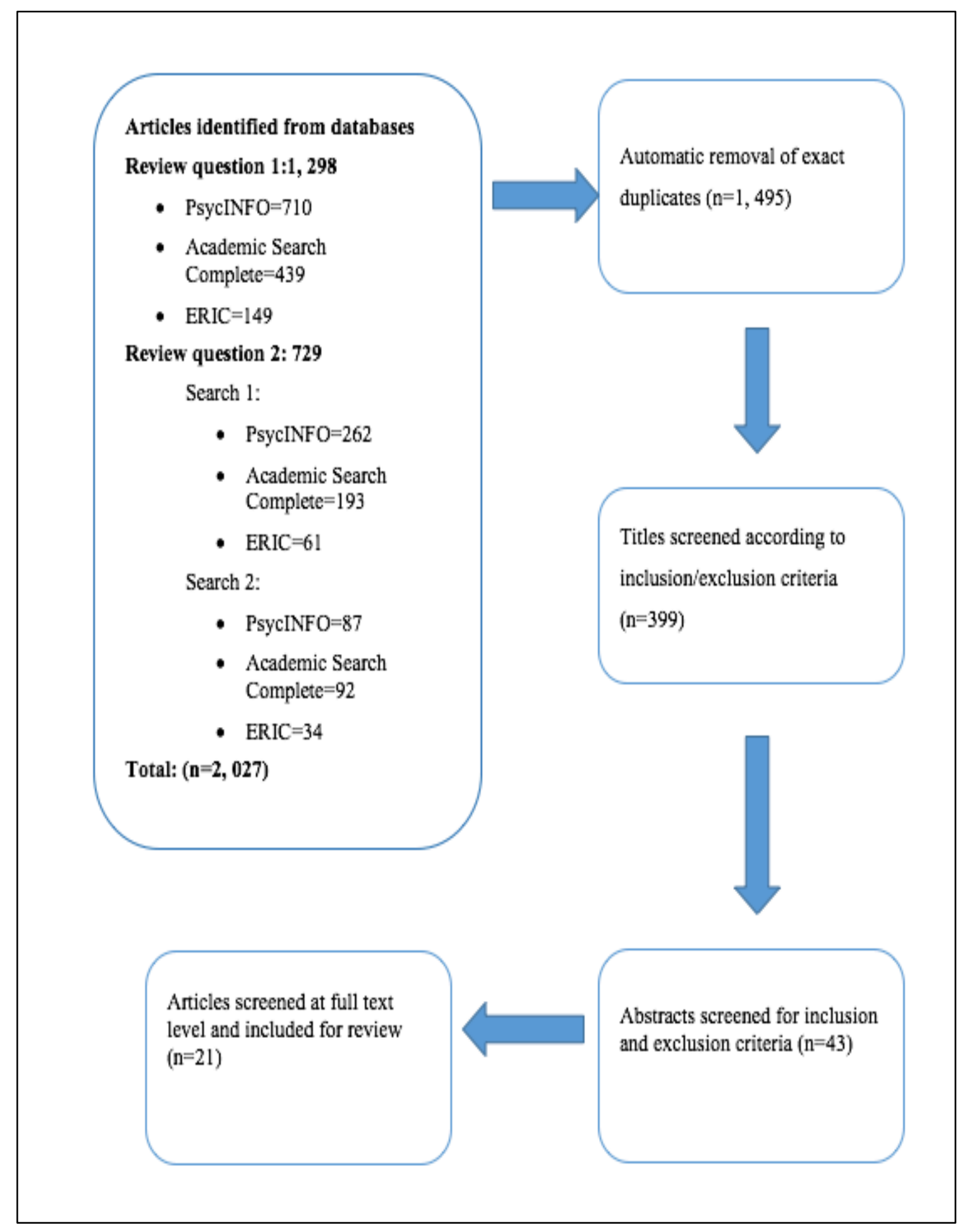

Figure 2.1. Flowchart Delineating the Literature Search and Screening Process

\subsection{Systematic Review of the Literature}

The following section provides an overview of Gough's (2007) WoE

Framework applied. A critical analysis of the studies' participants and design follows. A synthesis of findings relative to the review questions, conclusions and directions for future research are then outlined. 


\subsubsection{Critical Analysis Framework}

Each of the studies included for review were critically appraised using Gough's (2007) WoE Framework. There are four categories within the framework, Weight of Evidence A (WoE A), Weight of Evidence B (WoE B), Weight of Evidence C (WoE C) and Weight of Evidence D (WoE D). Studies were allocated a 'High', 'Medium' or 'Low' rating according to coding protocols and review criteria set by the researcher. WoE A measures the study's general quality of design and methodology. To ensure a reliable critique of the studies, published coding protocols and quality criteria checklists were applied. WoE B measures whether the design of the study was relevant to addressing the specified review questions (Gough, 2007). The criteria for WoE B weightings was devised in accordance with the quality criteria used for WoE A and inclusion and exclusion criteria. WoE $\mathrm{C}$ is also review specific, measuring the extent to which the study and its findings are relevant to answering the review question (Gough, 2007). The WoE C criteria was designed, with reference to stipulated inclusion and exclusion criteria. WoE D is a general overall weighting of the study, calculated by averaging the scores for WoE A, WoE B and WoE C (Gough, 2007). Appendices 3, 4, 5 and 6 outline the criteria for each of the coding categories and provide examples of coding protocols and calculation of weightings applied. Only two studies (Lawrence \& Cahill, 2014; Pameijer, 2017) received a 'High' overall WoE D rating, assigned by the researcher. Eighteen studies were allocated an overall 'Medium' rating and one study received an overall 'Low' rating. Table 2.4 provides an overview of the WoE ratings for each of the studies reviewed. 
Table 2.4

Overview of the WoE Ratings (Gough, 2007)

\begin{tabular}{|c|c|c|c|c|}
\hline Study & WoE A & WoE B & WoE C & WoE D \\
\hline $\begin{array}{l}\text { Lawrence \& } \\
\text { Cahill (2014) }\end{array}$ & 3 (High) & 3 (High) & 3 (High) & 3 (High) \\
\hline $\begin{array}{l}\text { Pameijer } \\
(2017)\end{array}$ & 3 (High) & 2 (Medium) & 3 (High) & 3 (High) \\
\hline Bozic (2013) & 3 (High) & 1 (Low) & 3 (High) & 2 (Medium) \\
\hline $\begin{array}{l}\text { Bahr, Leduc, } \\
\text { Hild, Davis, } \\
\text { Summers \& } \\
\text { McNeal (2017) }\end{array}$ & 3 (High) & 2 (Medium) & 2 (Medium) & 2 (Medium) \\
\hline $\begin{array}{l}\text { Sotelo-Dynega, } \\
\text { \& Dixon } \\
(2014)\end{array}$ & 3 (High) & 2 (Medium) & 1 (Low) & 2 (Medium) \\
\hline $\begin{array}{l}\text { Stothard, } \\
\text { Woods \& } \\
\text { Innoue (2018) }\end{array}$ & 3 (High) & 2 (Medium) & 1 (Low) & 2 (Medium) \\
\hline $\begin{array}{l}\text { Hill \& Turner } \\
\text { (2016) }\end{array}$ & 3 (High) & 2 (Medium) & 1 (Low) & 2 (Medium) \\
\hline $\begin{array}{l}\text { Sansosti \& } \\
\text { Sansosti (2013) }\end{array}$ & 3 (High) & 2 (Medium) & 1 (Low) & 2 (Medium) \\
\hline $\begin{array}{l}\text { Aiello, Ruble } \\
\& \text { Esler (2016) }\end{array}$ & 3 (High) & 2 (Medium) & 1 (Low) & 2 (Medium) \\
\hline $\begin{array}{l}\text { Cottrell \& } \\
\text { Barrett (2015) }\end{array}$ & 3 (High) & 2 (Medium) & 1 (Low) & 2 (Medium) \\
\hline $\begin{array}{l}\text { Ogg et al. } \\
(2013)\end{array}$ & 3 (High) & 2 (Medium) & 1 (Low) & 2 (Medium) \\
\hline Tobias (2017) & 3 (High) & 1 (Low) & 2 (Medium) & 2 (Medium) \\
\hline Cane (2016) & 3 (High) & 1 (Low) & 2 (Medium) & 2 (Medium) \\
\hline $\begin{array}{l}\text { Parker, Zaboski } \\
\text { \& Joyce- } \\
\text { Beaulieu } \\
(2016)\end{array}$ & 3 (High) & 1 (Low) & 2 (Medium) & 2 (Medium) \\
\hline $\begin{array}{l}\text { McCrimmon \& } \\
\text { Yule (2016) }\end{array}$ & 3 (High) & 1 (Low) & 1 (Low) & 2 (Medium) \\
\hline
\end{tabular}




\begin{tabular}{|c|c|c|c|c|}
\hline $\begin{array}{l}\text { Bourke \& } \\
\text { Dharan (2015) }\end{array}$ & 2 (Medium) & 2 ( Medium) & 2 (Medium) & 2 (Medium) \\
\hline $\begin{array}{l}\text { Filter, Ebsen \& } \\
\text { Dibos (2013) }\end{array}$ & 2 (Medium) & 2 ( Medium) & 2 (Medium) & 2 (Medium) \\
\hline $\begin{array}{l}\text { Hanchon \& } \\
\text { Allen (2013) }\end{array}$ & 2 (Medium) & 2 (Medium) & 1 (Low) & 2 (Medium) \\
\hline $\begin{array}{l}\text { Vega, Lasser \& } \\
\text { Afifi }(2015)\end{array}$ & 2 (Medium) & 2 ( Medium) & 1 (Low) & 2 (Medium) \\
\hline $\begin{array}{l}\text { Bozic, } \\
\text { Lawthom \& } \\
\text { Murray (2017) }\end{array}$ & 2 (Medium) & 1 (Low) & 2 (Medium) & 2 (Medium) \\
\hline $\begin{array}{l}\text { Harrison \& } \\
\text { McManus } \\
(2016)\end{array}$ & 2 (Medium) & 1 (Low) & 1 (Low) & 1 (Low) \\
\hline
\end{tabular}

\subsubsection{Participants}

According to stipulated inclusion and exclusion criteria, participants must be parents, teachers, children or EPs and the students attend a mainstream primary or secondary school, with or without a previously diagnosed SEN. Table 2.5 provides summary information of participants, the reason for referral/purpose of assessment, age range of students assessed and participants who reported on the appropriateness of interventions arising from the assessment where relevant. A critical discussion of participant demographics and assessment information follows. 
Table 2.5

Participant Summary Table

\begin{tabular}{|c|c|c|c|c|}
\hline Study & $\begin{array}{l}\text { Who were the participants in the study and } \\
\text { where were they from? }\end{array}$ & $\begin{array}{l}\text { Who carried } \\
\text { out the } \\
\text { assessment? }\end{array}$ & $\begin{array}{l}\text { What was the reason for } \\
\text { referral/purpose of assessment and } \\
\text { age range of students assessed? }\end{array}$ & $\begin{array}{l}\text { Who reported on } \\
\text { the utility of the } \\
\text { intervention? }\end{array}$ \\
\hline $\begin{array}{l}\text { Pameijer, N. } \\
\text { (2017) }\end{array}$ & $\begin{array}{l}\text { Teachers }(n=104) \text {, counsellors }(n=87), \text { parents } \\
(n=96) \text { and assessors }(n=99) \\
\text { Netherlands (geographical area not specified) }\end{array}$ & $\begin{array}{l}\text { School } \\
\text { psychologists } \\
\text { and external } \\
\text { school-coaches }\end{array}$ & $\begin{array}{l}\text { Children with learning and/or } \\
\text { behaviour difficulties in primary } \\
\text { school (ages 4-12). }\end{array}$ & $\begin{array}{l}\text { Teachers, } \\
\text { counsellors, } \\
\text { parents, students } \\
\text { and assessors }\end{array}$ \\
\hline $\begin{array}{l}\text { Ogg, Fefer, } \\
\text { Sundman- } \\
\text { Wheat, } \\
\text { McMahan, } \\
\text { Stewart, } \\
\text { Chappel \& } \\
\text { Bateman } \\
(2013)\end{array}$ & $\begin{array}{l}217 \text { school psychologists } \\
\text { United States (nationally representative } \\
\text { sample reported) }\end{array}$ & $\begin{array}{l}\text { School } \\
\text { psychologists }\end{array}$ & $\begin{array}{l}\text { Assessment of children presenting } \\
\text { with symptoms of Attention Deficit } \\
\text { Hyperactivity Disorder (ADHD) in } \\
\text { Pre-K, Elementary and Middle } \\
\text { Schools (ages 4-15 approx.) }\end{array}$ & N/A \\
\hline $\begin{array}{l}\text { Sotelo- } \\
\text { Dynega, \& } \\
\text { Dixon }(2014)\end{array}$ & $\begin{array}{l}323 \text { school psychologists } \\
\text { United States ( } 42 / 50 \text { states represented) }\end{array}$ & $\begin{array}{l}\text { School } \\
\text { psychologists }\end{array}$ & $\begin{array}{l}\text { Children referred for cognitive } \\
\text { assessment in Pre-K, Elementary, } \\
\text { Middle School, High School and } \\
\text { College (ages 3-21 approx.) }\end{array}$ & N/A \\
\hline $\begin{array}{l}\text { Bourke \& } \\
\text { Dharan (2015) }\end{array}$ & $\begin{array}{l}34 \text { psychologists working in Education } \\
\text { New Zealand (geographical area not } \\
\text { specified) }\end{array}$ & $\begin{array}{l}\text { Psychologists } \\
\text { working in } \\
\text { Education }\end{array}$ & $\begin{array}{l}\text { Children with behavioural, } \\
\text { emotional, social, and cognitive } \\
\text { difficulties. Age range not specified. }\end{array}$ & N/A \\
\hline
\end{tabular}




\begin{tabular}{|c|c|c|c|c|}
\hline $\begin{array}{l}\text { Bahr, Leduc, } \\
\text { Hild, Davis, } \\
\text { Summers \& } \\
\text { McNeal (2017) }\end{array}$ & $\begin{array}{l}175 \text { school psychology practitioners } \\
\text { United States (Missouri, Illinois, and Iowa) }\end{array}$ & $\begin{array}{l}\text { School } \\
\text { psychology } \\
\text { practitioners }\end{array}$ & $\begin{array}{l}\text { Reason for referral not described. } \\
\text { Children in Elementary, Middle } \\
\text { School and High School (ages 4-18 } \\
\text { approx.) }\end{array}$ & $\mathrm{N} / \mathrm{A}$ \\
\hline $\begin{array}{l}\text { Hanchon \& } \\
\text { Allen (2013) }\end{array}$ & $\begin{array}{l}214 \text { school psychologists } \\
\text { United States ( } 24 / 50 \text { states) }\end{array}$ & $\begin{array}{l}\text { School } \\
\text { psychologists }\end{array}$ & $\begin{array}{l}\text { Assessment of children presenting } \\
\text { with symptoms of Emotional } \\
\text { Disturbance (ED). Age range not } \\
\text { specified }\end{array}$ & N/A \\
\hline $\begin{array}{l}\text { Hill \& Turner } \\
(2016)\end{array}$ & $\begin{array}{l}136 \text { EPs } \\
\text { United Kingdom (geographically } \\
\text { representative sample reported) }\end{array}$ & EPs & $\begin{array}{l}\text { Assessment of school age children } \\
\text { presenting with symptoms of ADHD. }\end{array}$ & N/A \\
\hline $\begin{array}{l}\text { Sansosti \& } \\
\text { Sansosti } \\
(2013)\end{array}$ & $\begin{array}{l}978 \text { school psychologists and } 93 \text { trainers of } \\
\text { school psychology } \\
\text { United States ( } 20 / 50 \text { states represented) }\end{array}$ & $\begin{array}{l}\text { School } \\
\text { psychologists }\end{array}$ & $\begin{array}{l}\text { Assessment of children presenting } \\
\text { with symptoms of Autism Spectrum } \\
\text { Disorder (ASD) in Pre-K, } \\
\text { Elementary, Middle School, High } \\
\text { School and alternative educational } \\
\text { centres. }\end{array}$ & N/A \\
\hline $\begin{array}{l}\text { Aiello, Ruble } \\
\& \text { Esler (2016) }\end{array}$ & $\begin{array}{l}402 \text { school psychologists } \\
\text { United States ( } 43 / 50 \text { states represented) }\end{array}$ & $\begin{array}{l}\text { School } \\
\text { psychologists }\end{array}$ & $\begin{array}{l}\text { Assessment of children presenting } \\
\text { with symptoms of ASD ages } 0-21 \text {. }\end{array}$ & N/A \\
\hline $\begin{array}{l}\text { Vega, Lasser } \\
\& \text { Afifi }(2015)\end{array}$ & $\begin{array}{l}140 \text { school psychologists } \\
\text { United States (states not specified) }\end{array}$ & $\begin{array}{l}\text { School } \\
\text { psychologists }\end{array}$ & $\begin{array}{l}\text { Assessment of culturally and } \\
\text { linguistically diverse (CLD) students }\end{array}$ & N/A \\
\hline
\end{tabular}


(age range not specified) for special

education eligibility.

Cottrell \&

Barrett (2015)

471 school psychologists

United States (South, West, Mid-Western States)

Filter, Ebsen \& 216 school psychologists

Dibos (2013)

United States (41/50 states)

Lawrence \& Students (9), parents (8)

Cahill (2014) and teachers (7)

United Kingdom (London)

Stothard,

Woods \&

6 EPs

Innoue (2018) North of England (geographical area not reported)

Tobias (2017) Student and her family

United Kingdom (Brighton)

Bozic, $\quad 8$ children and young people

Lawthom \&

Murray (2017) United Kingdom (geographical area not specified)
EP

School

psychologists

Assessment of children presenting with symptoms of Specific Learning Disabilities (SLD) in Elementary,

Middle School and High School.

School psychologists

Reason for referral and age range is not specified.

EP

EPs

Assessment of the learning potential of children with special educational needs in primary and secondary schools (ages 7-14).

Assessment of children presenting with symptoms of literacy

difficulties/Dyslexia. Age range is not specified.

Assessment of child presenting with school refusal, aged 11 .

EP

Referrals included learning, social and behaviour difficulties (ages 6.919.2 years).
Students, parents and teachers

N/A

N/A 


\begin{tabular}{|c|c|c|c|c|}
\hline Bozic (2013) & $\begin{array}{l}6 \text { children and young people } \\
\text { United Kingdom (geographical area not } \\
\text { specified) }\end{array}$ & EP & $\begin{array}{l}\text { Referrals included emotional and } \\
\text { behaviour difficulties (ages 10.7-14.9 } \\
\text { years). }\end{array}$ & EP \\
\hline $\begin{array}{l}\text { Harrison \& } \\
\text { McManus } \\
(2016)\end{array}$ & $\begin{array}{l}\text { Student, parents, teachers } \\
\text { Canada (Victoria, British Columbia) }\end{array}$ & $\begin{array}{l}\text { School } \\
\text { psychologist }\end{array}$ & $\begin{array}{l}\text { Assessment of child presenting with } \\
\text { symptoms of Writing Disorder, aged } \\
13 .\end{array}$ & N/A \\
\hline $\begin{array}{l}\text { Parker, } \\
\text { Zaboski \& } \\
\text { Joyce-Beaulieu } \\
(2016)\end{array}$ & $\begin{array}{l}\text { Student, parent and teachers } \\
\text { United States (South-eastern region) }\end{array}$ & $\begin{array}{l}\text { School } \\
\text { psychologist }\end{array}$ & $\begin{array}{l}\text { Assessment of child with symptoms } \\
\text { of ADHD, oppositional behaviours, } \\
\text { and explosive anger symptomology, } \\
\text { aged } 14 \text {. }\end{array}$ & $\begin{array}{l}\text { Parent and } \\
\text { teachers }\end{array}$ \\
\hline $\begin{array}{l}\text { McCrimmon } \\
\& \text { Yule }(2016)\end{array}$ & $\begin{array}{l}\text { Student, parent, teachers } \\
\text { Canada (Calgary) }\end{array}$ & $\begin{array}{l}\text { School } \\
\text { psychologist }\end{array}$ & $\begin{array}{l}\text { Assessment of child presenting with } \\
\text { symptoms of ASD, aged } 12 \text {. }\end{array}$ & N/A \\
\hline Cane (2016) & $\begin{array}{l}\text { Student and school staff (SENCO and two } \\
\text { members of pastoral staff) } \\
\text { United Kingdom (Surrey) }\end{array}$ & EP & $\begin{array}{l}\text { Assessment of child presenting with } \\
\text { behavioural difficulties, aged } 12 \text {. }\end{array}$ & $\begin{array}{l}\text { Student and } \\
\text { school staff }\end{array}$ \\
\hline
\end{tabular}


Participant demographics. Participants in 11 of the studies were EPs. In two of the studies, participants included EPs, parents, students, teachers and other professionals (Pameijer, 2017; Sansosti \& Sansosti, 2013). Participants in eight of the studies were parents, students, teachers/school staff (Bozic, 2013; Bozic, Lawthom, \& Murray, 2017; Cane, 2016; Harrison \& McManus, 2016; Lawrence \& Cahill, 2014; McCrimmon \& Yule, 2016; Parker, Zaboski, \& Joyce-Beaulieu, 2016; Tobias, 2017). Five out of the 10 studies conducted in the United States demonstrated a geographically representative sample of EPs from 25 or more states (Aiello, Ruble, \& Esler, 2016; Filter et al., 2013; Ogg et al., 2013; Sotelo-Dynega \& Dixon, 2014), or large regional areas (Cottrell \& Barrett, 2015). One study conducted in the United Kingdom represented 70 local authorities from across the country, indicating a geographical representative sample (Hill \& Turner, 2016). Six studies did not specify the geographical location or region where the study was conducted having implications for the representativeness and generalisability of study findings.

Relevant assessment information. Assessors in all 21 studies were EPs and in one of the studies included EPs and external school-coaches (Pameijer, 2017). The students assessed were of school age, attending primary and/or secondary school. Three studies had an expanded age range, including children attending pre-school and/or college, having implications for the relevance of findings to the review question (Aiello et al., 2016; Ogg et al., 2013; Sansosti \& Sansosti, 2013; Sotelo-Dynega \& Dixon, 2014). The reasons for referral across studies included social-emotional, socialcommunication, learning, fine motor and behavioural difficulties. Four studies referred to at least one reason for referral (e.g. learning and behaviour difficulties), increasing generalisability of the reported assessment approaches to wider ranging populations (Bourke \& Dharan, 2015; Bozic, 2013; Bozic et al., 2017; Pameijer, 2017). Eleven studies specified the purpose of assessment was to determine eligibility for a diagnosis including ADHD, ASD, ED, SLD and Writing Disorder, limiting generalisability of findings to these specified populations. Three studies did not outline reasons for referral (Bahr et al., 2017; Filter et al., 2013; Sotelo-Dynega \& Dixon, 2014). Participants reporting on whether the assessment approach informed appropriate interventions for clients included EPs (Bozic, 2013; Tobias, 2017), teachers, counsellors, parents, students and assessors (Pameijer, 2017), students, parents and teachers (Lawrence \& Cahill, 2014), parent and teachers (Parker et al., 2016) and student and school staff (Cane, 2016). 


\subsubsection{Critique of Design and Methodologies}

Twenty-one studies with a quantitative, qualitative or mixed-methods design were reviewed. The studies were exploratory or descriptive in their design. Twelve were survey studies, seven were case studies and two of the studies utilised interview methods to explore the views of participants. Fifteen studies received a 'High' WoE A rating and six were allocated a 'Medium' WoE A rating for quality of design. One study received a 'High' WoE B rating, 13 received a 'Medium' rating and seven were allocated a 'Low' rating for relevance of the design to answering the review question. See Table 2.6 for a summary of design, measures and analysis applied, response rates (survey/ interview studies) and demonstration of case significance (case studies). This is followed by a critical discussion on the studies' design and methodologies. 
Table 2.6

Summary of Study Designs and Methodologies

\begin{tabular}{|c|c|c|c|c|c|}
\hline Study & $\begin{array}{l}\text { What was the } \\
\text { study design? }\end{array}$ & $\begin{array}{l}\text { What measures were } \\
\text { applied? }\end{array}$ & $\begin{array}{l}\text { What analysis } \\
\text { was applied? }\end{array}$ & $\begin{array}{l}\text { What was the } \\
\text { response rate? } \\
\text { (For survey/ } \\
\text { interview studies) }\end{array}$ & $\begin{array}{l}\text { Was the case study } \\
\text { significant? } \\
\text { (For case studies) }\end{array}$ \\
\hline $\begin{array}{l}\text { Pameijer, N. } \\
(2017)\end{array}$ & $\begin{array}{l}\text { Questionnaire } \\
\text { evaluation } \\
\text { study } \\
\text { (quantitative) }\end{array}$ & $\begin{array}{l}\text { Researcher-designed print- } \\
\text { based questionnaires } \\
\text { evaluating the AFI model, with } \\
105 \text { questions related to the } \\
\text { aims and principles of AFI, } \\
\text { mostly multiple choice and a } \\
\text { few open-ended. }\end{array}$ & $\begin{array}{l}\text { Descriptive } \\
\text { statistics }\end{array}$ & $\begin{array}{l}\text { A low overall response } \\
\text { rate was reported: } \\
\text { Teachers }(42 \%) \\
\text { Counsellors }(54 \%) \\
\text { Parents }(33 \%) \\
\text { Assessors }(76 \%)\end{array}$ & N/A \\
\hline $\begin{array}{l}\text { Ogg, Fefer, } \\
\text { Sundman- } \\
\text { Wheat, } \\
\text { McMahan, } \\
\text { Stewart, } \\
\text { Chappel \& } \\
\text { Bateman } \\
(2013)\end{array}$ & $\begin{array}{l}\text { Descriptive } \\
\text { survey study } \\
\text { (quantitative) }\end{array}$ & $\begin{array}{l}\text { Researcher-designed print- } \\
\text { based survey with } 60 \text { items } \\
\text { investigating participant } \\
\text { demographics, experiences } \\
\text { specific to ADHD, training, } \\
\text { assessment practices and their } \\
\text { response to a presented case } \\
\text { study. }\end{array}$ & $\begin{array}{l}\text { Descriptive and } \\
\text { parametric } \\
\text { statistics }\end{array}$ & $\begin{array}{l}\text { A low overall response } \\
\text { rate was reported } \\
(27 \%) .\end{array}$ & N/A \\
\hline $\begin{array}{l}\text { Sotelo- } \\
\text { Dynega, \& } \\
\text { Dixon } \\
(2014)\end{array}$ & $\begin{array}{l}\text { Descriptive } \\
\text { survey study } \\
\text { (quantitative) }\end{array}$ & $\begin{array}{l}\text { Researcher-designed online } \\
\text { survey with approximately } 30 \\
\text { multiple choice, yes/no, and } \\
\text { open-ended questions } \\
\text { investigating demographic and }\end{array}$ & $\begin{array}{l}\text { Descriptive and } \\
\text { nonparametric } \\
\text { statistics }\end{array}$ & $\begin{array}{l}\text { An overall response } \\
\text { rate of } 67 \% \text { was } \\
\text { reported, lower than } \\
\text { WoE B criteria set } \\
\text { (Johnson \& }\end{array}$ & N/A \\
\hline
\end{tabular}


personal background,

credentials and training, and

preferred cognitive assessment

practices.

$\begin{array}{lll}\begin{array}{l}\text { Bourke \& } \\ \text { Dharan } \\ (2015)\end{array} & \begin{array}{l}\text { Descriptive } \\ \text { survey study }\end{array} & \begin{array}{l}\text { Researcher-designed online } \\ \text { and print-based survey with 6 } \\ \text { items and 12 possible answers, } \\ \text { investigating the assessment } \\ \text { practices of EPs. }\end{array}\end{array}$

Bahr, Descriptive Researcher-designed online Leduc, Hild, survey study survey comprising of four

Davis,

Summers \& (quantitative)

McNeal

(2017)

Hanchon \& Descriptive

Allen (2013) survey study

(quantitative)

cher-designed online

survey titled 'ED Assessment

Practice Survey' with 32 items

requiring participants to rate

the frequency with which they

completed evaluation

procedures in the context of
Christensen, 2008,

cited in Mertens, 2015

p. 191).

Descriptive Response rate not N/A

statistics reported.

Descriptive and nonparametric

statistics

Low response rate

reported. The 31

N/A

Missouri, 69 Illinois,

and 75 Iowa

respondents,

respectively,

represented $19.37 \%$,

$12.43 \%$, and $26.31 \%$

of the possible

respondents by state.

Descriptive and

parametric

Response rate not

N/A

reported.

(ANOVAS) 
initial ED identification, the

perceived usefulness of

assessment tools and opinions

on the federal definition of ED.

\begin{tabular}{|c|c|c|c|c|}
\hline $\begin{array}{l}\text { Hill \& } \\
\text { Turner } \\
(2016)\end{array}$ & $\begin{array}{l}\text { Descriptive } \\
\text { survey study } \\
\text { (mixed } \\
\text { methods) }\end{array}$ & $\begin{array}{l}\text { Researcher-designed online } \\
\text { survey exploring the views of } \\
\text { EPs regarding the assessment, } \\
\text { diagnosis and treatment of } \\
\text { ADHD with Likert scale and } \\
\text { open-ended questions. }\end{array}$ & $\begin{array}{l}\text { Descriptive and } \\
\text { parametric/non- } \\
\text { parametric } \\
\text { statistics } \\
\text { Thematic } \\
\text { analysis }\end{array}$ & $\begin{array}{l}\text { Response rate not } \\
\text { reported. }\end{array}$ \\
\hline $\begin{array}{l}\text { Sansosti \& } \\
\text { Sansosti } \\
(2013)\end{array}$ & $\begin{array}{l}\text { Descriptive } \\
\text { survey study } \\
\text { (quantitative) }\end{array}$ & $\begin{array}{l}\text { Researcher-designed online } \\
\text { survey comprising of three } \\
\text { sections and } 15 \text { items } \\
\text { investigating the type of } \\
\text { assessment and intervention } \\
\text { practices school psychologists } \\
\text { currently employ for students } \\
\text { with ASD. Likert scale and } \\
\text { open-ended questions were } \\
\text { used. }\end{array}$ & $\begin{array}{l}\text { Descriptive and } \\
\text { parametric } \\
\text { statistics } \\
\text { (ANOVAS) }\end{array}$ & $\begin{array}{l}\text { Response rate not } \\
\text { reported. }\end{array}$ \\
\hline $\begin{array}{l}\text { Aiello, } \\
\text { Ruble \& } \\
\text { Esler (2016) }\end{array}$ & $\begin{array}{l}\text { Descriptive } \\
\text { survey study } \\
\text { (quantitative) }\end{array}$ & $\begin{array}{l}\text { Researcher-designed online } \\
\text { and paper-based survey titled } \\
\text { 'The Autism Survey for } \\
\text { School Psychologists' } \\
\text { investigating participant } \\
\text { demographics, services }\end{array}$ & $\begin{array}{l}\text { Descriptive and } \\
\text { parametric } \\
\text { statistics ( } \mathrm{t} \text { tests) } \\
\text { and chi-squared } \\
\text { test of } \\
\text { independence }\end{array}$ & $\begin{array}{l}\text { A low overall response } \\
\text { rate was reported } \\
(27 \%) \text {. }\end{array}$ \\
\hline
\end{tabular}


provided to students with

autism, autism knowledge and

training needs. Likert scale

questions and 'yes'/ 'no'

questions were asked.

\begin{tabular}{|c|c|c|c|c|}
\hline $\begin{array}{l}\text { Vega, } \\
\text { Lasser \& } \\
\text { Afifi }(2015)\end{array}$ & $\begin{array}{l}\text { Descriptive } \\
\text { survey study } \\
\text { (quantitative) }\end{array}$ & $\begin{array}{l}\text { Researcher-designed online } \\
\text { survey titled 'Culturally and } \\
\text { Linguistically Diverse } \\
\text { Assessment' with } 24 \text { items } \\
\text { investigating training and } \\
\text { practice related to the } \\
\text { assessment of CLD students } \\
\text { and participant demographics. } \\
\text { Multiple response and open- } \\
\text { ended questions were used. }\end{array}$ & $\begin{array}{l}\text { Descriptive } \\
\text { statistics }\end{array}$ & $\begin{array}{l}\text { Response rate not } \\
\text { reported. }\end{array}$ \\
\hline $\begin{array}{l}\text { Cottrell \& } \\
\text { Barrett } \\
(2015)\end{array}$ & $\begin{array}{l}\text { Descriptive } \\
\text { survey study } \\
\text { (quantitative) }\end{array}$ & $\begin{array}{l}\text { Researcher-designed online } \\
\text { questionnaire investigated } \\
\text { participant demographics, } \\
\text { preferred SLD practices, actual } \\
\text { SLD practices, and level of } \\
\text { assessment job satisfaction. } \\
\text { Likert scale questions were } \\
\text { used. }\end{array}$ & $\begin{array}{l}\text { Descriptive } \\
\text { statistics and } \\
\text { parametric } \\
\text { statistics } \\
\text { (multiple } \\
\text { regression) }\end{array}$ & $\begin{array}{l}\text { Response rate not } \\
\text { reported. }\end{array}$ \\
\hline $\begin{array}{l}\text { Filter, Ebsen } \\
\& \text { Dibos } \\
(2013)\end{array}$ & $\begin{array}{l}\text { Descriptive } \\
\text { survey study } \\
\text { (quantitative) }\end{array}$ & $\begin{array}{l}\text { Researcher-designed print- } \\
\text { based survey investigating } \\
\text { discrepancies experienced } \\
\text { between actual discrete }\end{array}$ & $\begin{array}{l}\text { Descriptive } \\
\text { statistics and } \\
\text { parametric } \\
\text { statistics ( } \mathrm{t} \text { tests }\end{array}$ & $\begin{array}{l}\text { A low overall response } \\
\text { rate was reported } \\
(21.6 \%) .\end{array}$ \\
\hline
\end{tabular}




$\begin{array}{ll}\text { Lawrence \& } & \text { Interview and } \\ \text { Cahill } & \text { focus group } \\ \text { (2014) } & \text { study }\end{array}$

(qualitative)

Stothard,

Woods \&

Exploratory

Innoue

(2018) survey design

(qualitative)

practices and preferred discrete

practices relative to several

domains of practice including

assessment, intervention,

meetings, and continuing

education. Participants

indicated the number of hours

they actually spend and would

prefer to spend on the various

professional activities.

Semi-structured interviews

with students, parents and

teachers (some teachers

participated in focus groups) to

explore their views regarding

the psychological reports

produced from dynamic

assessment. The researcher

aimed to be reflexive at all

points during the research and

used a reflexive journal to

facilitate this process.

In-depth interviews with EPs were conducted to explore

aspects of their current

attitudes, knowledge of and

and multiple

regression

analyses)

Thematic

analysis

Thematic

analysis

practice around literacy

learning and Dyslexia.
24 interviews were

conducted in total.

(reaching data

saturation-12

interviews) (Guest,

Bunce, \& Johnson,

2016).

6 interviews were
conducted (not
reaching data
saturation-12
interviews) (Guest et
al., 2016).

N/A

N/A 


\begin{tabular}{|c|c|c|c|c|c|}
\hline $\begin{array}{l}\text { Tobias } \\
(2017)\end{array}$ & $\begin{array}{l}\text { Descriptive } \\
\text { single case } \\
\text { study } \\
\text { (qualitative) }\end{array}$ & $\begin{array}{l}\text { A descriptive case study, } \\
\text { describing the assessment/ } \\
\text { intervention in the real life } \\
\text { context which it occurred. }\end{array}$ & $\begin{array}{l}\text { A narrative was } \\
\text { constructed to } \\
\text { give an account } \\
\text { of the case. }\end{array}$ & $\mathrm{N} / \mathrm{A}$ & $\begin{array}{l}\text { Yes- The assessment approach } \\
\text { (genogram) demonstrated } \\
\text { multiple benefits in its use with } \\
\text { children and young people, } \\
\text { including highlighting areas } \\
\text { for intervention. }\end{array}$ \\
\hline $\begin{array}{l}\text { Bozic, } \\
\text { Lawthom \& } \\
\text { Murray } \\
(2017)\end{array}$ & $\begin{array}{l}\text { Multiple case } \\
\text { study } \\
\text { (qualitative) }\end{array}$ & $\begin{array}{l}\text { Strength-Based Assessment } \\
\text { tools, diary and record sheets } \\
\text { were used to explore how a } \\
\text { contextualised strength-based } \\
\text { assessment worked with } \\
\text { children and young people. }\end{array}$ & $\begin{array}{l}\text { A storyboard } \\
\text { was constructed } \\
\text { to enable a } \\
\text { holistic } \\
\text { understanding of } \\
\text { the case to } \\
\text { emerge. A } \\
\text { narrative was } \\
\text { constructed to } \\
\text { give an account } \\
\text { of each case. }\end{array}$ & N/A & $\begin{array}{l}\text { Yes-The information yielded } \\
\text { by the strength-based } \\
\text { assessment approach could } \\
\text { stimulate interesting } \\
\text { hypotheses about the kinds of } \\
\text { social arrangements and } \\
\text { pedagogic strategies that might } \\
\text { suit an individual with often } \\
\text { quite complex needs. }\end{array}$ \\
\hline $\begin{array}{l}\text { Bozic } \\
(2013)\end{array}$ & $\begin{array}{l}\text { Explorative } \\
\text { multiple case } \\
\text { study } \\
\text { (qualitative) }\end{array}$ & $\begin{array}{l}\text { Strength-Based Assessment } \\
\text { tools were applied to cases to } \\
\text { demonstrate how they can be } \\
\text { integrated into school-based } \\
\text { educational psychological } \\
\text { work, to identify actual and } \\
\text { potential strengths at personal, } \\
\text { interpersonal and systems } \\
\text { levels. A number of measures } \\
\text { were used to monitor progress } \\
\text { (Target, Monitoring and }\end{array}$ & $\begin{array}{l}\text { Repeated } \\
\text { measures was } \\
\text { applied. } \\
\text { Participant } \\
\text { assessment } \\
\text { scores were } \\
\text { presented in } \\
\text { tabular format } \\
\text { and direct } \\
\text { quotations were } \\
\text { also presented. }\end{array}$ & N/A & $\begin{array}{l}\text { Yes- Use of strength-based } \\
\text { assessment tools can generate } \\
\text { information about pupil } \\
\text { perceived strengths, located } \\
\text { within the pupil and also } \\
\text { within different layers of the } \\
\text { surrounding ecology. } \\
\text { Information can be used to } \\
\text { inform intervention plans }\end{array}$ \\
\hline
\end{tabular}


Evaluation (TME) Scale and

the initial Child and

Adolescent Strengths

Assessment (CASA).

$\begin{array}{lll}\begin{array}{l}\text { Harrison \& } \\ \text { McManus } \\ \text { (2016) }\end{array} & \begin{array}{l}\text { Descriptive } \\ \text { case study }\end{array} & \begin{array}{l}\text { A descriptive case study } \\ \text { describing the multi-modal } \\ \text { assessment approach and } \\ \text { clinical reasoning of a school } \\ \text { psychologist, involved in the } \\ \text { assessment and intervention } \\ \text { planning for a child with a } \\ \text { writing disorder. }\end{array}\end{array}$

Parker,

Joyce-

Beaulieu

(2016)

study

(qualitative)

A single case study outlining the multi-modal assessment/

RTI approach used by a school psychologist to inform intervention for a child presenting with EBD. Several measures were used to monitor progress (BASC-2, the number of office discipline referrals and behaviour plan data).

McCrimmon Descriptive

$\&$ Yule case study

(2016) (qualitative)
A descriptive case study illustrating the clinical reasoning of a school psychologist conducting an assessment for ASD.
A narrative was constructed to

give an account

of the case.

Pre and post intervention data

(t-scores) were presented in graphical and tabular format and direct quotations were also presented.

A narrative was

N/A constructed to give an account of the case.

\section{N/A}

N/A
Yes- This case highlighted a multimodal assessment approach to inform intervention for a child with writing disorder.

Yes- The case demonstrated the efficacy of utilising a comprehensive, integrated, school-based intervention plan to address multiple behavioural needs.

Yes- The case outlined a school-based approach to assessment for ASD. This assessment could inform targeted and effective 
interventions/recommendations

that address the identified areas

of need and support the

student, their family, and their

school to promote the best

possible outcome for the

student.

\begin{tabular}{|c|c|c|c|c|}
\hline Cane (2016) & $\begin{array}{l}\text { Single case } \\
\text { study } \\
\text { (qualitative) }\end{array}$ & $\begin{array}{l}\text { A single case study outlining } \\
\text { the assessment approach used } \\
\text { by an EP to inform } \\
\text { intervention for a child } \\
\text { presenting with externalising } \\
\text { behavioural difficulties. } \\
\text { Measures were used to monitor } \\
\text { progress (Scaling and } \\
\text { qualitative feedback). }\end{array}$ & $\begin{array}{l}\text { Participant } \\
\text { assessment } \\
\text { scores were } \\
\text { presented in } \\
\text { tabular format. } \\
\text { Scaling was used } \\
\text { to monitor } \\
\text { intervention and } \\
\text { quotations were } \\
\text { also presented. }\end{array}$ & $\begin{array}{l}\text { Yes- This case emphasised the } \\
\text { value of systemic approaches } \\
\text { in educational psychology } \\
\text { casework; it highlighted the } \\
\text { role of school stakeholders in } \\
\text { promoting and enhancing } \\
\text { positive change with students. }\end{array}$ \\
\hline
\end{tabular}


Measures and analysis. All survey studies applied researcher-designed survey measures. Several survey studies provided detailed evidence of efforts to establish the validity of their researcher-designed measures. For example, surveys were developed based on best practice literature and previous research in the field (Filter et al., 2013; Ogg et al., 2013; Sansosti \& Sansosti, 2013), piloting and expert review of questions (Cottrell \& Barrett, 2015; Filter et al., 2013; Sotelo-Dynega \& Dixon, 2014) and topics discussed during focus group sessions (Hill \& Turner, 2016). Regarding interview studies, Stothard, Woods, and Innoue (2018) reported conducting focus group sessions with four academic psychologists, to provide a conceptual foundation for interviews with EPs. All survey studies applied descriptive analysis to present means and standard deviations of data gathered. Nine of these studies conducted parametric and nonparametric tests of analysis to detect differences between groups and variable effects. In the studies that utilised interview methods, thematic analysis was applied to the interview data gathered. Both inductive (Lawrence \& Cahill, 2014; Stothard et al., 2018) and deductive (Stothard et al., 2018) methods were applied, consistent with the researchers' paradigmatic position. Case studies applied various methods of analysis including scaling, narratives and repeated measures, outlined in Table 2.6.

Response rates and significance. Surveys were distributed in print (four studies) or online format (seven studies). Two studies utilised both methods of distribution (Aiello et al., 2016; Bourke \& Dharan, 2015). Research comparing mail and web-based survey response rates indicates higher response to mail surveys amongst medical doctors, school teachers and general consumers and higher response rates to online surveys amongst college students (Shih and Fan, 2008, cited in Mertens, 2015, p. 190). Distribution of mail or web-based surveys in the current review did not impact upon response rates, as most studies demonstrated low response rates in accordance with WoE B criteria set (Johnson \& Christensen, 2008, cited in Mertens, 2015 p. 191). Six studies did not report a response rate having implications for WoE A and $\mathrm{B}$ ratings applied. Three of these studies stated it was not possible to calculate the response rate as the online link to the survey was widely distributed and the total number of participants who received the link was unknown (Cottrell \& Barrett, 2015; Sansosti \& Sansosti, 2013; Vega, Lasser, \& Afifi, 2015). Two studies employed interview methods to explore participant views and experience and were allocated 'High' (Lawrence \& Cahill, 2014) and 'Medium' (Stothard et al., 2018) WoE B ratings according to whether data saturation (12 interviews) was demonstrated (Guest et al., 2016). All seven case studies were considered significant as they provided a detailed account of an assessment 
approach used by an EP in practice. Significant cases are described as unusual and of general public interest and the underlying issues are nationally important, either in theoretical terms or in policy or practical terms (Yin, 2009). Four of these studies presented evidence that the approach informed appropriate intervention for clients (Bozic, 2013; Cane, 2016; Parker et al., 2016; Tobias, 2017) or provided an outline of how the approach could inform intervention (Bozic et al., 2017; Harrison \& McManus, 2016; McCrimmon \& Yule, 2016), consistent with policy aims of Circular 0013/2017 (DES, 2017b) and Circular 0014/2017 (DES, 2017c).

\subsection{A Synthesis of Literature on Assessment and Intervention Approaches Currently Applied by EPs in Practice}

The systematic review sought to examine studies that provide empirical data, to investigate the assessment approaches currently being applied by EPs (review question 1) and the appropriateness of intervention recommendations arising from these assessments for clients (review question 2). This section provides a summary table outlining the assessment approach and context under which the assessment was undertaken, evidence of whether the approach informed relevant interventions for clients and resonating theories and models inferred by the researcher during the review process (Table 2.7). A discussion of findings relative to review question 1 and 2 follows. 
Table 2.7

Summary of Systematic Review Findings

\begin{tabular}{|c|c|c|c|c|}
\hline $\begin{array}{l}\text { Study/ } \\
\text { Geographical } \\
\text { Location }\end{array}$ & $\begin{array}{l}\text { What was the assessment approach/model } \\
\text { applied? }\end{array}$ & $\begin{array}{l}\text { In what context was } \\
\text { the assessment } \\
\text { carried out? }\end{array}$ & $\begin{array}{l}\text { Evidence of } \\
\text { assessment informing } \\
\text { appropriate } \\
\text { intervention? }\end{array}$ & $\begin{array}{l}\text { What theories or } \\
\text { models resonate with } \\
\text { this approach? }\end{array}$ \\
\hline $\begin{array}{l}\text { Pameijer } \\
\text { (2017) } \\
\text { Netherlands }\end{array}$ & $\begin{array}{l}\text { The Assessment for Intervention (AFI) } \\
\text { Model. AFI is a five-stage model of assessment } \\
\text { and intervention. The stages of the model } \\
\text { provided structure to the assessment from } \\
\text { beginning to end, all involved worked towards a } \\
\text { shared goal and outcomes were meaningful to } \\
\text { counsellors, teachers and parents as their } \\
\text { questions led the assessment. }\end{array}$ & $\begin{array}{l}\text { Twenty schools } \\
\text { participated in a three } \\
\text { year pilot in the } \\
\text { Netherlands, } \\
\text { implementing the AFI } \\
\text { model, to assess the } \\
\text { needs of children with } \\
\text { learning and/or } \\
\text { behaviour difficulties. }\end{array}$ & $\begin{array}{l}\text { Yes- The majority of } \\
\text { teachers reported that } \\
\text { not only did they better } \\
\text { understand the student, } \\
\text { they also knew how to } \\
\text { adjust their teaching } \\
\text { more to their student's } \\
\text { specific educational } \\
\text { needs. }\end{array}$ & $\begin{array}{l}\text { EST (Bronfenbrenner, } \\
\text { 1979, 1989) }\end{array}$ \\
\hline $\begin{array}{l}\text { Lawrence \& } \\
\text { Cahill (2014) } \\
\text { United } \\
\text { Kingdom }\end{array}$ & $\begin{array}{l}\text { Dynamic assessment was reported to impact } \\
\text { positively upon the child's emotional well- } \\
\text { being, self-perceptions, approach to learning and } \\
\text { social relationships, both directly, and through } \\
\text { the subsequent intervention of parents and } \\
\text { educators. Dynamic assessment, as well as } \\
\text { providing instructionally useful information for } \\
\text { parents and teachers, encouraged them to move } \\
\text { beyond locating the problem within the child, to } \\
\text { reconceptualising their special educational needs } \\
\text { in context. }\end{array}$ & $\begin{array}{l}\text { Assessment of the } \\
\text { learning potential of } \\
\text { children with special } \\
\text { educational needs. }\end{array}$ & $\begin{array}{l}\text { Yes- Student and } \\
\text { teacher comments } \\
\text { provided evidence of } \\
\text { utility of intervention in } \\
\text { the classroom context. } \\
\text { Parents also reported } \\
\text { that DA resulted in } \\
\text { attempts to manage } \\
\text { their child's behaviour } \\
\text { in a different and more } \\
\text { positive way. }\end{array}$ & $\begin{array}{l}\text { SCT (Palinscar, 1998; } \\
\text { Vygotsky, 1978) } \\
\text { EST (Bronfenbrenner, } \\
\text { 1979, 1989) } \\
\text { Structural Cognitive } \\
\text { Modifiability and } \\
\text { Mediated Learning } \\
\text { Experience } \\
\text { (Feuerstein, Rand, \& } \\
\text { Hoffman, 1979) }\end{array}$ \\
\hline
\end{tabular}




\begin{tabular}{|c|c|c|c|c|}
\hline $\begin{array}{l}\text { Tobias }(2017 \\
\text { United } \\
\text { Kingdom }\end{array}$ & $\begin{array}{l}\text { As an assessment tool, the genogram was able } \\
\text { to gather an extremely rich source of } \\
\text { information, and its co-construction with the } \\
\text { family facilitated a much deeper discussion than } \\
\text { would have normally been possible within the } \\
\text { constraints of an initial consultation. The } \\
\text { genogram is described as an assessment tool for } \\
\text { identifying behavioural patterns and } \\
\text { psychological aspects of family relationships, as } \\
\text { well as being part of the therapeutic intervention } \\
\text { itself. It is commonly used in systemic family } \\
\text { therapy. }\end{array}$ & $\begin{array}{l}\text { Assessment of child } \\
\text { presenting with school } \\
\text { refusal. }\end{array}$ & $\begin{array}{l}\text { Yes- The EP reported } \\
\text { the genogram was } \\
\text { helpful as part of an } \\
\text { initial intervention, } \\
\text { because it drew the } \\
\text { family's attention to } \\
\text { some of the repeated } \\
\text { patterns of behaviour } \\
\text { and unresolved, or } \\
\text { unvoiced, issues. }\end{array}$ & $\begin{array}{l}\text { EST (Bronfenbrenner, } \\
\text { 1979, 1989) } \\
\text { SCT (Palinscar, 1998; } \\
\text { Vygotsky, 1978) }\end{array}$ \\
\hline $\begin{array}{l}\text { Bozic (2013) } \\
\text { United } \\
\text { Kingdom }\end{array}$ & $\begin{array}{l}\text { A strength-based assessment approach was } \\
\text { applied. In each case, the child/young person } \\
\text { was interviewed using either the Child and } \\
\text { Adolescent Strengths Assessment (Lyons et al., } \\
\text { 2000) or Assets Interview (Morrison et al., } \\
\text { 2006). Information gained from the assessment } \\
\text { was then used to inform an intervention plan. } \\
\text { Findings indicated that strengths could be } \\
\text { identified at a range of ecological levels. } \\
\text { Strengths influenced action plans by affecting } \\
\text { the choice of strategy and/or target of } \\
\text { interventions. }\end{array}$ & $\begin{array}{l}\text { Assessments of } \\
\text { children with } \\
\text { emotional and } \\
\text { behaviour difficulties. }\end{array}$ & $\begin{array}{l}\text { Yes-There was } \\
\text { evidence of positive } \\
\text { change in a high } \\
\text { proportion of cases. In } \\
\text { four out of the five } \\
\text { cases where strength- } \\
\text { based information } \\
\text { influenced the } \\
\text { development of an } \\
\text { action plan, there was } \\
\text { evidence of positive } \\
\text { change when repeated } \\
\text { measures were taken. }\end{array}$ & $\begin{array}{l}\text { Positive Psychology } \\
\text { (Seligman, 2002) } \\
\text { EST (Bronfenbrenner, } \\
\text { 1979, 1989) }\end{array}$ \\
\hline $\begin{array}{l}\text { Parker, } \\
\text { Zaboski \& } \\
\text { Joyce- }\end{array}$ & $\begin{array}{l}\text { This case demonstrated a multi-modal } \\
\text { assessment approach to inform intervention, } \\
\text { including a developmental history; parent, }\end{array}$ & $\begin{array}{l}\text { Assessment of child } \\
\text { presenting with } \\
\text { symptoms of attention }\end{array}$ & $\begin{array}{l}\text { Yes- Treatment } \\
\text { included CBT sessions } \\
\text { over } 6 \text { months and a 9- }\end{array}$ & $\begin{array}{l}\text { Response to } \\
\text { Intervention Model }\end{array}$ \\
\hline
\end{tabular}




\begin{tabular}{|c|c|c|c|c|}
\hline $\begin{array}{l}\text { Beaulieu } \\
(2016) \\
\text { United States }\end{array}$ & $\begin{array}{l}\text { teacher, and student interviews; systematic } \\
\text { classroom observations; and BASC-2 and } \\
\text { Conners parent, teacher, and student social, } \\
\text { emotional, and behaviour rating reports. An RTI } \\
\text { approach indicated ratings of ADHD-type } \\
\text { behaviours and severe problematic behaviours } \\
\text { that no longer fell in the clinically significant } \\
\text { range. }\end{array}$ & $\begin{array}{l}\text { deficit hyperactivity } \\
\text { disorder (ADHD), } \\
\text { oppositional } \\
\text { behaviours, and } \\
\text { explosive anger } \\
\text { symptomology. }\end{array}$ & $\begin{array}{l}\text { week classroom Daily } \\
\text { Behaviour Report Card } \\
\text { plan. Outcome data } \\
\text { revealed a decrease in } \\
\text { office discipline } \\
\text { referrals, lower levels } \\
\text { of behaviour symptoms, } \\
\text { and an increase in } \\
\text { prosocial classroom } \\
\text { behaviours with } \\
\text { maintained } \\
\text { improvement into the } \\
\text { following school year. }\end{array}$ & $\begin{array}{l}\text { (U.S. Department of } \\
\text { Education, 2004) } \\
\text { EST (Bronfenbrenner, } \\
1979,1989)\end{array}$ \\
\hline $\begin{array}{l}\text { Cane (2016) } \\
\text { United } \\
\text { Kingdom }\end{array}$ & $\begin{array}{l}\text { A solution-focused assessment approach was } \\
\text { applied. The solution-focused model adopted a } \\
\text { strength-based approach, which seeks to identify } \\
\text { and foster the conditions necessary to achieve } \\
\text { the preferred state. Readiness to change was } \\
\text { assessed using the Sheffield Motivational } \\
\text { Interviewing card-sort activity. School staff and } \\
\text { student views were sought and intervention } \\
\text { goals devised accordingly. }\end{array}$ & $\begin{array}{l}\text { Assessment of child } \\
\text { presenting with } \\
\text { externalising } \\
\text { behavioural } \\
\text { difficulties. }\end{array}$ & $\begin{array}{l}\text { Yes-The use of scaling } \\
\text { illustrated how, over the } \\
\text { course of the SFBT } \\
\text { intervention, the child } \\
\text { rated himself as } \\
\text { progressing and } \\
\text { maintained positive } \\
\text { changes at follow up. } \\
\text { Qualitative feedback } \\
\text { from school staff } \\
\text { indicated improvements } \\
\text { in behaviour and } \\
\text { learning. }\end{array}$ & $\begin{array}{l}\text { Positive Psychology } \\
\text { (Seligman, 2002) } \\
\text { SCT (Palinscar, 1998; } \\
\text { Vygotsky, 1978) } \\
\text { EST (Bronfenbrenner, } \\
\text { 1979, 1989) } \\
\text { Self-determination } \\
\text { theory (Deci \& Ryan, } \\
\text { 2008) }\end{array}$ \\
\hline $\begin{array}{l}\text { Ogg, Fefer, } \\
\text { Sundman- }\end{array}$ & $\begin{array}{l}\text { DSM-IV (American Psychiatric Association, } \\
\text { 2000) core symptoms were adhered to in the }\end{array}$ & $\begin{array}{l}\text { Assessment of } \\
\text { children presenting }\end{array}$ & No & $\begin{array}{l}\text { Medical Model (Laing, } \\
\text { 1971) }\end{array}$ \\
\hline
\end{tabular}




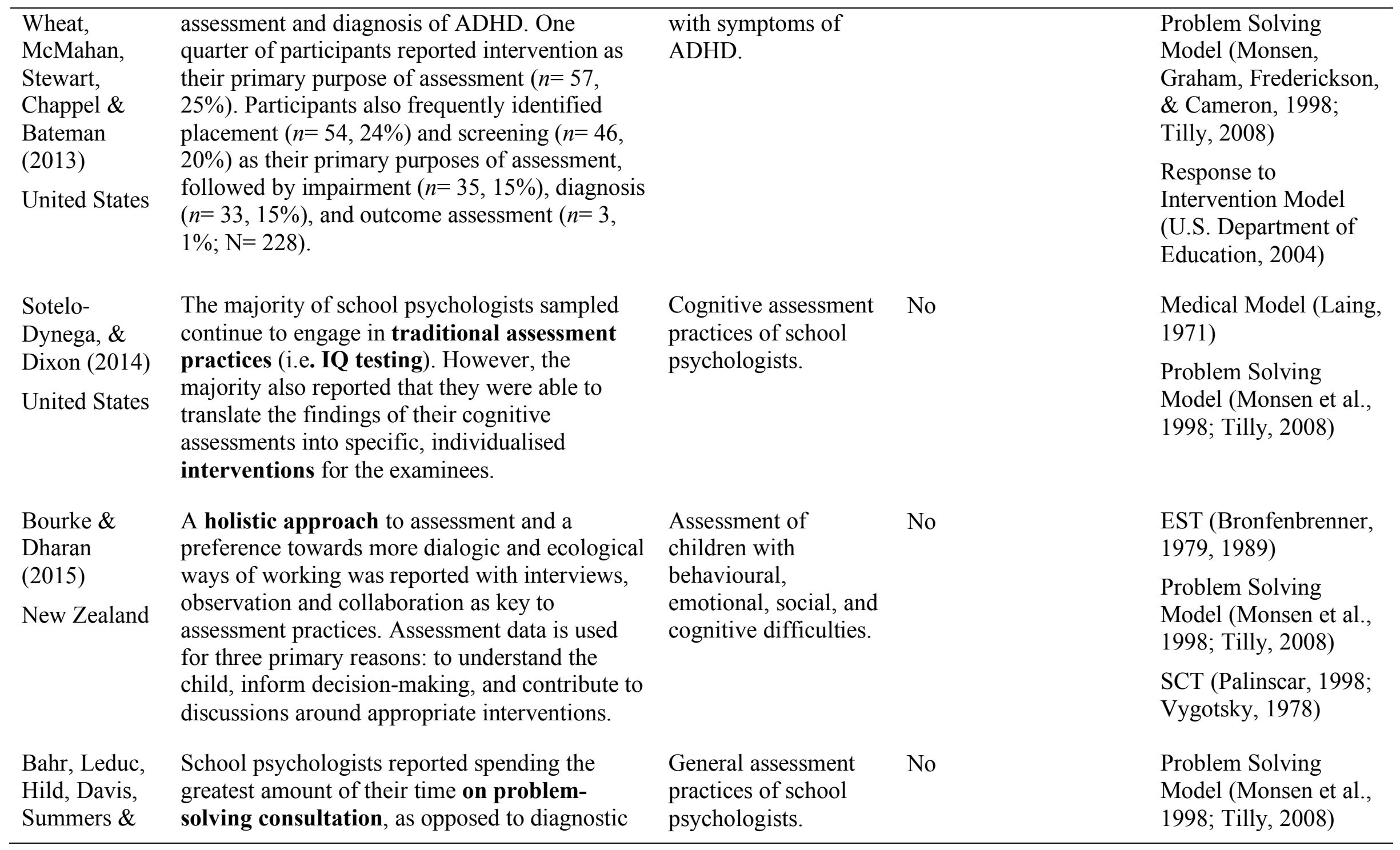




\begin{tabular}{|c|c|c|c|c|}
\hline $\begin{array}{l}\text { McNeal } \\
(2017) \\
\text { United States }\end{array}$ & $\begin{array}{l}\text { assessment. Most participants, } 112 \text { (or } 64 \% \text { ), } \\
\text { indicated problem-solving consultation as one of } \\
\text { their top five preferences. }\end{array}$ & & & \\
\hline $\begin{array}{l}\text { Hanchon \& } \\
\text { Allen (2013) } \\
\text { United States }\end{array}$ & $\begin{array}{l}\text { Inconsistent assessment practices are reported. } \\
\text { School psychologists report that they value a } \\
\text { multimethod, multisource assessment model } \\
\text { when ED is a classification consideration. } \\
\text { However, in many instances, their actual } \\
\text { assessments are missing commonly } \\
\text { recommended sources of data for making } \\
\text { eligibility decisions, including classroom } \\
\text { observations; parent, teacher, and student } \\
\text { interviews; and behaviour rating scales. }\end{array}$ & $\begin{array}{l}\text { Assessment of } \\
\text { children presenting } \\
\text { with symptoms of ED. }\end{array}$ & No & $\begin{array}{l}\text { Medical Model (Laing, } \\
\text { 1971) }\end{array}$ \\
\hline $\begin{array}{l}\text { Hill \& Turner } \\
(2016) \\
\text { United } \\
\text { Kingdom }\end{array}$ & $\begin{array}{l}\text { The data suggests that in current practice the } \\
\text { medical model dominates. Responses } \\
\text { highlighted how currently EPs are rarely } \\
\text { engaged in the assessment of ADHD. Where } \\
\text { Local Authorities have developed standardised } \\
\text { pathways or protocols governing the diagnostic } \\
\text { process, EPs are involved in the assessment } \\
\text { process and children are more likely to access } \\
\text { psychological interventions, and for contextual } \\
\text { factors to be considered. }\end{array}$ & $\begin{array}{l}\text { Assessment of } \\
\text { children presenting } \\
\text { with symptoms of } \\
\text { ADHD. }\end{array}$ & No & $\begin{array}{l}\text { Medical Model (Laing, } \\
\text { 1971) }\end{array}$ \\
\hline $\begin{array}{l}\text { Sansosti \& } \\
\text { Sansosti } \\
(2013)\end{array}$ & $\begin{array}{l}\text { Findings suggest that practitioners engage in } \\
\text { some of the best practice assessment methods } \\
\text { embraced by researchers and policy advocates } \\
\text { (National Autism Center), including use of }\end{array}$ & $\begin{array}{l}\text { Assessment of } \\
\text { children presenting } \\
\text { with symptoms of } \\
\text { ASD. }\end{array}$ & No & $\begin{array}{l}\text { Medical Model (Laing, } \\
\text { 1971) }\end{array}$ \\
\hline
\end{tabular}




\begin{tabular}{|c|c|c|c|c|}
\hline United States & $\begin{array}{l}\text { adaptive behaviour scales, autism } \\
\text { checklists/rating scales, direct observations, } \\
\text { developmental/health histories, interviews, and } \\
\text { standardised intelligence tests. 54\% of school } \\
\text { psychologists reported engaging either } \\
\text { frequently or very frequently in developing } \\
\text { interventions for students with ASD during the } \\
\text { prior school year. However, the researchers } \\
\text { conclude findings indicate that assessment may } \\
\text { be viewed more from the perspective of } \\
\text { eligibility determination rather than for the } \\
\text { development of interventions. }\end{array}$ & & & $\begin{array}{l}\text { Problem Solving } \\
\text { Model (Monsen et al., } \\
\text { 1998; Tilly, 2008) }\end{array}$ \\
\hline $\begin{array}{l}\text { Aiello, Ruble } \\
\text { \& Esler } \\
\text { (2016) } \\
\text { United States }\end{array}$ & $\begin{array}{l}402 \text { school psychologists were surveyed for } \\
\text { their knowledge of and training and experience } \\
\text { with ASD on assessment practices. The majority } \\
\text { of school psychologists reported that they did } \\
\text { not engage in comprehensive assessment of } \\
\text { ASD, which was defined as assessments that } \\
\text { consider all areas of development in addition to } \\
\text { the use of ASD-specific instruments. }\end{array}$ & $\begin{array}{l}\text { Assessment of } \\
\text { children presenting } \\
\text { with symptoms of } \\
\text { ASD. }\end{array}$ & No & $\begin{array}{l}\text { Medical Model (Laing, } \\
\text { 1971) }\end{array}$ \\
\hline $\begin{array}{l}\text { Vega, Lasser } \\
\text { \& Afifi } \\
\text { (2015) } \\
\text { United States }\end{array}$ & $\begin{array}{l}\text { Participants reported conducting } \\
\text { comprehensive assessments to determine } \\
\text { eligibility for special education among CLD } \\
\text { students. The majority of the participants ( } 75.5 \\
\% \text { ) reported assessing CLD students' cognitive, } \\
\text { achievement, and social-emotional functioning } \\
\text { when conducting an evaluation to determine } \\
\text { eligibility for special education services. Other }\end{array}$ & $\begin{array}{l}\text { Assessment of } \\
\text { culturally and } \\
\text { linguistically diverse } \\
\text { (CLD) students for } \\
\text { special education } \\
\text { eligibility. }\end{array}$ & No & $\begin{array}{l}\text { Medical Model (Laing, } \\
\text { 1971) }\end{array}$ \\
\hline
\end{tabular}


areas reportedly assessed included racial and ethnic identity development, acculturation, language proficiency, environmental impact, neuropsychological functioning, curriculumbased measurement, and motor functioning.

Cottrell \&

Barrett (2015)

United States

\section{The Ability-Achievement discrepancy method}

was the assessment method most used $(\mathrm{M}=$ 2.68, $\mathrm{SD}=1.19)$, followed by RTI $(\mathrm{M}=2.42$, $\mathrm{SD}=1.05)$ and pattern of strengths and weaknesses $(\mathrm{M}=1.86, \mathrm{SD}=1.03)$.

Filter, Ebsen \& Dibos

United States

The three most common activities reported were report writing $(\mathrm{M}=7.46$; $\mathrm{SD}=5.42)$, IQ testing $(\mathrm{M}=5.69 ; \mathrm{SD}=4.99)$, and staff consultation $(\mathrm{M}$ $=5.47 ; \mathrm{SD}=5.94)$. School psychologists reported spending 5.69 hours per week administering IQ tests but prefer to spend 1.83 hours per week less in this discrete practice. Furthermore, IQ tests traditionally comprised significant portions of reports written by school psychologists and the present sample reported wanting to spend 3.34 hours less per week writing reports.

Stothard,

Inconsistent assessment practices are reported.

Woods \& A narrow range of EP assessment and

Innoue (2018) intervention practice for Dyslexia was described, suggesting a need for professional clarification and development. Several EPs
Assessment of children presenting with symptoms of

SLD.

The general practice of school

psychologists including assessment, intervention, meetings, and continuing education
No

Medical Model (Laing, 1971)

Ability-Achievement Discrepancy Model (Franzen, 1920)

Medical Model (Laing, 1971)

Ability-Achievement Discrepancy Model (Franzen, 1920)
Assessment of children presenting with symptoms of

literacy

difficulties/Dyslexia. 


\begin{tabular}{|c|c|c|c|c|}
\hline $\begin{array}{l}\text { United } \\
\text { Kingdom }\end{array}$ & $\begin{array}{l}\text { reported using psychometric tests, some } \\
\text { discussed using unspecified assessments of } \\
\text { reading and/or spelling and some discussed } \\
\text { taking a strengths and needs approach. }\end{array}$ & & & $\begin{array}{l}\text { EST (Bronfenbrenner, } \\
1979,1989)\end{array}$ \\
\hline $\begin{array}{l}\text { Bozic, } \\
\text { Lawthom \& }\end{array}$ & \multirow{3}{*}{$\begin{array}{l}\text { A contextualised strength-based assessment } \\
\text { approach was applied. By engaging with the } \\
\text { 'Context Strength Finder' (CSF), all } \\
\text { children/young people identified situations or } \\
\text { contexts which they associated with the } \\
\text { presence of specific strengths. In some cases, } \\
\text { they highlighted aspects of a situation which } \\
\text { were hypothesised to have pedagogical value. }\end{array}$} & \multirow{3}{*}{$\begin{array}{l}\text { Assessments of } \\
\text { children with learning, } \\
\text { social and behaviour } \\
\text { difficulties. }\end{array}$} & \multirow[t]{3}{*}{ No } & $\begin{array}{l}\text { EST (Bronfenbrenner, } \\
1979,1989)\end{array}$ \\
\hline $\begin{array}{l}\text { Murray } \\
(2017)\end{array}$ & & & & $\begin{array}{l}\text { SCT (Palinscar, 1998; } \\
\text { Vygotsky, 1978) }\end{array}$ \\
\hline $\begin{array}{l}\text { United } \\
\text { Kingdom }\end{array}$ & & & & $\begin{array}{l}\text { Positive Psychology } \\
\text { (Seligman, 2002) }\end{array}$ \\
\hline $\begin{array}{l}\text { Harrison \& } \\
\text { McManus }\end{array}$ & \multirow{2}{*}{$\begin{array}{l}\text { This case highlighted a multimodal assessment } \\
\text { approach to inform diagnosis and intervention, } \\
\text { situated within a contemporary scientist- } \\
\text { practitioner framework. This approach involved } \\
\text { the following steps: (a) problem analysis and } \\
\text { development of hypotheses based on the } \\
\text { collection of background data (b) collection of } \\
\text { assessment data to test hypotheses; (c) synthesis } \\
\text { and interpretation of all assessment data in } \\
\text { relation to hypotheses; (d) intervention } \\
\text { development; and (e) intervention evaluation. }\end{array}$} & \multirow{2}{*}{$\begin{array}{l}\text { Assessment of child } \\
\text { presenting with } \\
\text { symptoms of Writing } \\
\text { Disorder. }\end{array}$} & \multirow[t]{2}{*}{ No } & $\begin{array}{l}\text { Medical Model (Laing, } \\
\text { 1971) }\end{array}$ \\
\hline $\begin{array}{l}\text { (2016) } \\
\text { Canada }\end{array}$ & & & & $\begin{array}{l}\text { Problem Solving } \\
\text { Model (Monsen et al., } \\
\text { 1998; Tilly, 2008) }\end{array}$ \\
\hline $\begin{array}{l}\text { McCrimmon } \\
\& \text { Yule } \\
(2016)\end{array}$ & \multirow{2}{*}{$\begin{array}{l}\text { Given the presenting issues and concerns, a } \\
\text { comprehensive assessment approach was } \\
\text { applied to examine the child's cognitive, } \\
\text { academic, behavioural, and attentional } \\
\text { functioning in addition to a specific assessment }\end{array}$} & \multirow[t]{2}{*}{$\begin{array}{l}\text { Assessment of child } \\
\text { presenting with } \\
\text { symptoms of ASD. }\end{array}$} & \multirow[t]{2}{*}{ No } & \multirow[t]{2}{*}{$\begin{array}{l}\text { Medical Model (Laing, } \\
\text { 1971) }\end{array}$} \\
\hline Canada & & & & \\
\hline
\end{tabular}


for symptoms of ASD. The practitioners'

Problem Solving

approach to assessment and case

Model (Monsen et al.,

conceptualisation was driven by advances in the

1998; Tilly, 2008)

clinical conceptualisation of diagnostic features

of childhood disorders and effective and

efficient approaches to obtain evidence about

the presence or absence of symptoms necessary

to yield a clinical diagnosis. The child's

intellectual, adaptive, academic, and behavioural

challenges were all important considerations in

determining the nature and context of potential interventions. 
Summary of findings table. Table 2.7 outlines the assessment approaches applied by EPs in the studies reviewed, and the context under which assessments were conducted. Twelve of the studies were consistent with the medical model of practice. Nine of the studies provided evidence of alternative forms of assessment and resonated with theories including EST (Bronfenbrenner, 1979, 1989), SCT (Palinscar, 1998; Vygotsky, 1978) and Positive Psychology (Seligman, 2002). The conceptual framework for the current research incorporates elements of EST (Bronfenbrenner, 1979) and SCT (Palinscar, 1998; Vygotsky, 1978) and a detailed account of these theories is provided in Sections 2.14 and 2.15. Six of the studies provided evidence of assessment approaches that informed appropriate interventions for clients. These findings are discussed in detail in the sections that follow.

\subsection{Review Question 1: What Assessment Approaches are Educational Psychologists Applying in Practice?}

\subsubsection{A Medical Model of Practice}

All studies included for review provided empirical evidence of assessment approaches that EPs are applying in practice. Overwhelmingly, assessment approaches were consistent with the medical model of practice, with classification and diagnosis of an SEN or disability reported in 12 of the studies reviewed. These studies utilised survey methods (9) interview methods (1) (Stothard et al., 2018) and case study designs (2) (Harrison \& McManus, 2016; McCrimmon \& Yule, 2016) and were conducted in the United States (8), Canada (2) and the United Kingdom (2). Most of the children referred for assessment in these studies were presenting with symptoms of a specified condition (e.g. ASD, ADHD, Dyslexia). The types of assessment approaches engaged in included diagnostic assessments for classification purposes, including comprehensive assessments and multimodal approaches (Aiello et al., 2016; Hanchon \& Allen, 2013; Harrison \& McManus, 2016; Hill \& Turner, 2016; McCrimmon \& Yule, 2016; Ogg et al., 2013; Sansosti \& Sansosti, 2013; Vega et al., 2015) and discrepancy methods and IQ testing (Cottrell \& Barrett, 2015; Filter et al., 2013; Sotelo-Dynega \& Dixon, 2014; Stothard et al., 2018). Some studies consistent with the medical model of practice also adhered to a problem solving approach to assessment, using assessment findings to contribute to discussions around interventions and intervention planning. However, the studies did not report empirical evidence of intervention utility in practice (Harrison \& McManus, 2016; McCrimmon \& Yule, 2016; Ogg et al., 2013; Sansosti \& Sansosti, 
2013; Sotelo-Dynega \& Dixon, 2014). Two of the studies adhering to the medical model referred to eligibility for special education as an outcome of their diagnostic assessment practices (Sansosti \& Sansosti, 2013; Vega et al., 2015). These studies were conducted in the United States and are considered consistent with current IDEIA legislation (NCSE, 2013; U.S. Department of Education, 2004).

\subsubsection{Inconsistencies in Assessment Practices}

There were several inconsistencies in reported assessment practices. With regards cognitive assessment practices of EPs, participants in Sotelo-Dynega and Dixon's (2014) survey study claimed their assessment practices were informed by Cattell Horn Carroll (CHC) theory, which endorses specific interpretation of cognitive processes and interpretation of a child's narrow abilities (Decker, Hale, \& Flanagan, 2013). However, the majority of participants reported engaging primarily in IQ testing and interpreting the student's global measure of ability. Similarly, discrepancies in assessment approaches were described by Hanchon and Allen (2013). Although participants indicated valuing a multimethod, multisource assessment when diagnosing ED, their actual assessment practices neglected recommended sources of data for diagnostic decision making, including classroom observations; parent, teacher, and student interviews; and use of behaviour rating scales (Hanchon \& Allen, 2013). Inconsistencies in assessment practices were also reported in interviews conducted by Stothard et al. (2018). While several EPs indicated using psychometric tests for assessment of Dyslexia, some discussed unspecified assessments of reading and/or spelling and others reported taking a strengths and needs-based approach. The researchers suggest the need for professional clarification and consistency in practice.

\subsubsection{Impact of Legislation on Assessment Approaches}

The prevalence of EP assessment approaches consistent with the medical model indicates assessment for diagnosis continues to dominate practice. However, eight of the 12 studies suggestive of the medical model were conducted in the United States and are in line with the IDEIA, requiring assessment and diagnosis of disability for access to special education resource provision (U.S. Department of Education, 2004). It should be noted that in these studies, presenting symptoms of a specified diagnosis (e.g. ASD, ADHD, Dyslexia) were stated as the reason for referral and therefore, comprehensive diagnostic assessments would be expected. Despite its prevalence, reports of preferred assessment practices indicate a shift away from the medical model. Filter et al. (2013) 
reported practices that school psychologists would prefer to engage in less, including report writing, IQ testing and special education eligibility meetings. Similarly, participants in Bahr et al. (2017) indicated problem-solving consultation as one of their top five preferred activities $(n=112)$, followed by mental health interventions $(n=90)$, participation on school-based teams or data teams $(n=79)$, interventions for academic problems $(n=64)$, and counseling $(n=54)$.

Ogg et al. (2013) demonstrated that in addition to adhering to the medical model for diagnosis of ADHD, school psychologists' practice was leaning towards an RTI model with one quarter of participants reporting intervention as the primary purpose of assessment. In case study research, Parker et al. (2016) described the application of a RTI approach with a child presenting with ADHD, oppositional behaviours, and explosive anger symptomology. Following a period of intervention after initial assessment, the child's behaviour and symptomology ratings were no longer in the clinically significant range. Respondents in Ogg et al. (2013) reported that progress monitoring, outcome evaluation and assessment of intervention integrity were conducted 'infrequently', despite these forms of assessment being rated as 'very important' to practice. Participants indicated that this was an area where they did not feel particularly well trained (Ogg et al., 2013). Although practice is reportedly moving towards an RTI approach in the United States, training and competency may need to be addressed, consistent with CPD training preferences reported by Jose et al. (2013) in Section 1.2.

Interestingly, studies demonstrating alternative assessment approaches in practice including strength-based, solution-focused, DA and AFI were conducted in the United Kingdom and Netherlands. Reasons for referral were described as learning, social, emotional and behaviour difficulties in contrast to symptoms associated with a particular diagnosis. With a shift away from evidence of diagnosis for resource provision, it could be inferred that legislative changes in the United Kingdom are beginning to be reflected in actual practice. However, the majority of these studies were case studies, having limited overall generalisability to current practice in the field. Further discussion on these assessment approaches is provided in the following section.

\subsubsection{Evidence of Alternative Assessment Approaches}

Assessment approaches consistent with the medical model of practice were evident in the findings of this systematic review. In addition, empirical findings indicate alternative assessment approaches are being applied in practice including strength-based 
and solution-focused approaches, DA, AFI and ecological and holistic approaches. Strength-based and solution-focused assessment approaches were applied in three case studies (Bozic, 2013; Bozic et al., 2017; Cane, 2016) with children presenting with learning, social-emotional and behaviour difficulties. Assessment measures used with the child or young person included the Sheffield Motivational Interviewing card-sort activity (Cane, 2016), the Child and Adolescent Strengths Assessment or Assets Interview (Bozic, 2013) and the Context Strength Finder (Bozic et al., 2017). The strengths of the child or young person were identified across contexts and then used to inform intervention planning. From a theoretical perspective, these approaches resonate with several theories including Positive Psychology, focusing on the child's strengths and how goals can be addressed using the skills and qualities they already possess (Seligman, 2002); EST, by identifying strengths across a range of ecological contexts (Bronfenbrenner, 1979, 1989) and SCT, by co-constructing knowledge and information with the child and relevant adults, to develop intervention plans and strategies (Palinscar, 1998, p. 353).

Two studies indicated a meaningful assessment process and useful recommendations as the aim of their assessment approach; DA (Lawrence \& Cahill, 2014) and AFI (Pameijer, 2017). The students in Pameijer (2017) presented with learning and/or behaviour difficulties and in Lawrence and Cahill (2014) were described as having special educational needs and referred for assessment of learning potential. DA is an assessment approach that aims to identify learning processes and cognitive functions, and the mediation strategies that advance the child's learning potential in a wide range of contexts (Lawrence \& Cahill, 2014). It is consistent with a social constructivist approach, where interaction between assessor and examinee and explicit assistance is encouraged during the assessment process. Dynamic assessment emanates from the Feuerstein et al. (1979) theory of 'Structural Cognitive Modifiability' and 'Mediated Learning Experience' where intelligence is viewed as malleable and open to change (Yeomans, 2008). Lawrence and Cahill (2014) outline a process whereby each child engaged in DA with the EP and arising intervention recommendations positively impacted upon the child's holistic needs, parenting practices and inclusive practices in the classroom. Similarly, Pameijer (2017) describes the implementation of the five stages of the AFI model with teachers, counsellors, parents and students. Survey responses indicate that the model provided structure to the assessment from beginning to end, all involved worked towards a shared goal and outcomes were meaningful to clients. The assessment approach outlined by Pameijer (2017) involved the 
collaborative sharing of information with clients and communication between systems (e.g. home and school), consistent with a social constructivist and ecological approach.

In a survey of EP assessment practices in New Zealand, Bourke and Dharan (2015) report a holistic approach to assessment and a preference towards dialogic and ecological forms of practice with interviews, observation and collaboration fundamental to their approach. In addition, participants in this study reported assessment data being used to contribute to discussions around appropriate interventions. Consistent with the current Irish context and issuance of Circular 0013/2017 (DES 2017b) and Circular $0014 / 2017$ (DES, 2017c), assessment data were used for three principal reasons; to understand the child, inform decision-making, and contribute to discussions around relevant interventions. Similarly, Tobias (2017) describes the use of a genogram as a social constructivist assessment approach for a child presenting with school refusal. The genogram is an assessment tool for identifying behavioural patterns and psychological aspects of family relationships, as well as being part of the therapeutic intervention itself (Tobias, 2017). As an assessment tool, the genogram gathered rich sources of information and its co-construction with the family facilitated meaningful discussion to address the referral issue. The researcher cautions applying the genogram as a standalone assessment and advises its use in conjunction with additional forms of assessment and in consideration of the impact of other ecological factors including school and social networks (Tobias, 2017). The findings relevant to review question 2 provide additional evidence of assessment approaches that informed appropriate interventions for clients, discussed in the next section.

\subsection{Review Question 2: Are the Assessment Approaches Informing Appropriate Interventions for Clients?}

Six studies included for review provided evidence of assessment approaches applied in practice that inform appropriate intervention recommendations for clients. Four of these studies adopted a case study design and each received a 'Medium' overall WoE D rating (Bozic, 2013; Cane, 2016; Parker et al., 2016; Tobias, 2017). A questionnaire evaluation study (Pameijer, 2017) and an interview and focus group study (Lawrence \& Cahill, 2014) were allocated a 'High' WoE D rating. The case studies received a lower WoE D rating due to limited generalisability to a sample population. The six studies were conducted in the United Kingdom (4), Netherlands (1) and United States (1) and reasons for referral included presentation of learning, social-emotional, behavioural and attentional difficulties. 
The assessment approaches that informed appropriate interventions for clients included the AFI model (Pameijer, 2017), DA (Lawrence \& Cahill, 2014), the genogram model (Tobias, 2017), strength-based assessment (Bozic, 2013), solutionfocused assessment (Cane, 2016) and a multi-modal assessment/RTI approach (Parker et al., 2016). Each of these studies outlined the purpose of assessment as informing intervention and working together with student, parents and/or teachers to address presenting concerns. From a theoretical perspective, these assessment and intervention practices reflect an ecological, social constructivist approach by collaborating with students, parents and/or teachers and working between systems (home and school) to effectuate change (Bronfenbrenner, 1979; Palinscar, 1998). Just one of the studies outlined symptomology of a diagnostic condition and possible eligibility for receipt of Exceptional Student Education (ESE) services as a consequence of the assessment (Parker et al., 2016). However, this study also demonstrated that following an RTI approach informed by a multi-modal assessment, the student no longer met the criteria for a diagnosis of ADHD. Participants reporting on whether the assessment approach informed appropriate interventions for clients included EPs (Bozic, 2013; Tobias, 2017), teachers, counsellors, parents, students and assessors (Pameijer, 2017), students, parents and teachers (Lawrence \& Cahill, 2014), parent and teachers (Parker et al., 2016) and student and school staff (Cane, 2016). The two studies that received a 'High' overall WoE D rating are discussed further in the following sections. Information on the remaining assessment approaches is provided in Appendix 7.

\subsubsection{Assessment for Intervention (Pameijer, 2017)}

Following engagement with the AFI model, the majority of teachers (70\%) reported that assessment provided recommendations and two out of three teachers indicated that they could implement the recommendations arising from assessment in their classroom (Pameijer, 2017). Conversely, only half of the parents (52\%) and even fewer assessors (43\%) reported that assessment provided recommendations for parents. Recommendations for future research suggest investigating ways that parents can support their child's success at school. Difficulty providing parents with targeted, individualised intervention recommendations is a pertinent issue in the field and may be due to recommendations focusing on the needs of the child rather than contextual issues in the home environment (Geffken, Keeley, Kellison, Storch, \& Rodrigue, 2006). The parent questionnaire included four questions exploring the child's experience of the Investigation stage. The majority of students (77\%) indicated that they enjoyed it some 
rated neutral $(23 \%)$ and none indicated angry or sad. Some of the students provided qualitative feedback to suggest the situation had improved "my teacher now understands me better", "she helps me more than before" and "now I get more attention from the teacher" (Pameijer, 2017, p. 76). The findings indicate that working collaboratively together throughout the assessment process resulted in meaningful intervention and change for students and teachers, reflective of an ecological, social constructivist approach (Bronfenbrenner, 1979; Palinscar, 1998).

\subsubsection{Dynamic Assessment (Lawrence \& Cahill, 2014)}

Lawrence and Cahill (2014) report DA impacted upon the child's holistic needs, parenting practices and the development of inclusive practice in the classroom context. Student and teacher responses during interview provided evidence of the utility of interventions in the classroom context. For example, one teacher commented "I think the process of DA makes the student feel more better and more confident ... the child's confidence has shot up ... I have seen huge amounts of progress" (Lawrence \& Cahill, 2014 , p. 201). Similarly, a student reported on strategies applied during classroom activities that benefited his learning "like in numeracy sometimes we have this ball we have to work out the problem like, so now I can think about how to slow down and chose the right shape and think it through like” (Lawrence \& Cahill, 2014, p. 200). Parent feedback indicated that DA resulted in attempts to manage their child's behaviour with an alternative, more positive approach. The findings of this study are consistent with Elliott, Resing, \& Beckmann's (2018) discussion paper, indicating the principal contribution of DA is in guiding intervention. The findings are also reflective of how social constructivist and mediated approaches applied during assessment, can be transferred to the classroom and home environment, for meaningful change.

\subsubsection{The Voice of the Child}

An additional finding across the studies reviewed was the inclusion or exclusion of the voice of the child during assessment and intervention processes. Students reported on the appropriateness of intervention recommendations in three studies (Cane, 2016; Lawrence \& Cahill, 2014; Pameijer, 2017). However, there was also an acknowledged absence of the voice of the child in studies. Hanchon and Allen (2013) state only $31 \%$ of respondents conducted diagnostic student interviews in their ED evaluations and participants in Hill and Turner (2016) reported few children being involved in decision making about their treatment for ADHD. In outlining implications 
for practice, Pameijer (2017) recommends actively including students in the assessment process as much as possible, regardless of their age, consistent with Article 12 of the International Convention on the Rights of the Child (Pameijer, 2017).

\subsection{Summary of the Systematic Review}

This systematic review sought to investigate the assessment approaches currently being applied by EPs in practice (review question 1) and the appropriateness of intervention recommendations arising from these assessments for clients (review question 2). Twenty-one studies were reviewed and limitations of findings and methodologies relative to the review questions were outlined and discussed. All studies provided empirical evidence of current assessment approaches used by EPs in practice and only six of these studies provided evidence to suggest the approach informed appropriate interventions for clients. This indicates a gap exists in EP practice, between the assessments being conducted and interventions that clients are receiving. This finding is consistent with the assertions of Resing et al. (2017) who outlined the challenge of linking assessment to intervention in EP practice and VanDerHeyden (2018) who described this task as the "next frontier for school psychology" (p.51) in Section 1.4. In the current systematic review, assessment approaches consistent with the medical model of practice were most prevalent in the United States. Although practice is reportedly moving towards an RTI approach, training and competency building may need to be addressed (Ogg et al., 2013), consistent with CPD training preferences outlined by Jose et al. (2013) in Section 1.2. Assessment approaches informing appropriate intervention for clients were found in studies conducted in Europe; AFI, DA, strength-based assessment, solution-focused assessment and the genogram, and in the United States; a multi-modal assessment/RTI approach. Two of these studies were allocated a 'High' overall WoE D rating and the aim of their assessment approach was for the process and resulting outcomes to be meaningful and useful for clients (Lawrence \& Cahill, 2014; Pameijer, 2017). The assessment approaches applied in these studies resonated with EST (Bronfenbrenner, 1979, 1989) and SCT (Palinscar, 1998; Vygotsky, 1978) and are considered most relevant to the stipulations of Circular 0013/2017 and Circular 0014/2017 regarding professional assessment; to provide understanding of a child's needs, the nature of difficulties, and inform appropriate interventions (DES, 2017b, p. 14; DES, 2017c, p.14). Limitations of the review and directions for future research are discussed in the following sections. 


\subsection{Limitations of the Systematic Review}

Surveys and interviews relied on participants' self-reporting of their practices and training, which may not accurately reflect actual practices and training. The validity of self-reported data is dependent upon the honesty and openness of the individual participant and this should be taken into account in the interpretation of findings (Mertens, 2015, p. 182). Social desirability and selection bias must also be considered when interpreting these findings as the participants who self-selected to take part may hold stronger views or have responded in a manner that represents them in a more favourable light (Mertens, 2015). Additionally, the case studies reviewed outline an assessment and intervention process applied in practice and findings are relevant to the specified context, as opposed to a population sample (Yin, 2009).

A broad review was conducted to generate a general picture of the assessment approaches currently being applied by EPs in practice, and whether these assessment approaches are informing appropriate interventions. Gough (2007) acknowledges that a systematic review may intend to include all research on a particular topic, no matter the research design employed and weighted judgements should be applied accordingly. Although published coding protocols and quality criteria checklists were applied for WoE A ratings, they were adapted for the purpose of this systematic review and WoE $\mathrm{B}, \mathrm{WoE} \mathrm{C}$ and WoE D criteria were devised by the current researcher, with reference to relevant literature and stipulated inclusion and exclusion criteria. For example, the acceptable response rate of $70 \%$ or higher, quoted by Johnson \& Christensen (2008, cited in Mertens, 2015 p. 191) and used as part of WoE B criteria, may be considered unrealistic by other researchers in the field. Krosnick (1999) states that "surveys with very low response rates can be more accurate than surveys with much higher response rates" (p. 540). Subjectivity of reviewer-designed quality checklists may be considered a limitation of the systematic review, having implications for overall ratings and weighing of evidence.

Inclusion criteria specified review of empirical data to investigate the review questions. The Pameijer (2017) study was conducted by an independent researcher (Alegra, 2013) and it was included for review because of its high quality methodology and strength of findings relevant to the review question. Finally, the proposed alignment of models and theories with the assessment approaches, identified in the systematic review, were discussed in the context of the researcher's own interpretation and alternative theoretical viewpoints are also possible. 


\subsection{Directions for Future Research}

The introduction of the new model for allocation of special education teaching resources in mainstream schools (DES, 2017a) has opened the forum for discussion on EP assessment approaches that are in line with Circular 0013/2017 and Circular $0014 / 2017$ assertions, in providing understanding of a child's needs, the nature of difficulties, and informing appropriate interventions (DES, 2017b, p. 14; DES, 2017c, p. 14). The findings of the systematic review suggest a gap exists between assessment and intervention in EP practice and offers two potential models that fit the criteria for professional assessment outlined in the circular, DA and AFI. Dynamic assessment aims to identify learning processes and cognitive functions, and the mediation strategies that advance the child's learning potential in a wide range of contexts (Lawrence \& Cahill, 2014). Research demonstrates its ability to inform intervention in practice (Lawrence \& Cahill, 2014) and a recent discussion paper outlines the need for research that systematically investigates the relationship between DA and intervention (Elliott et al., 2018). With regards proposals for the current research, DA is considered limited to measuring cognitive processes and Circular 0013/2017 (DES, 2017b) and Circular $0014 / 2017$ (DES, 2017c) specify the understanding and addressing of needs, that are not restricted to a particular domain. Furthermore, although the Lawrence and Cahill (2014) study received a 'High' overall WoE D rating in the systematic review, access to DA measures and CPD could have presented difficulties for the current researcher.

In contrast, AFI may be considered more consistent with Circular 0013/2017 and Circular 0014/2017 stipulations (DES, 2017b; DES, 2017c). AFI is a five-stage model of assessment and intervention underpinned by seven principles that outline the theoretical context and rationale of the model (Pameijer, 2017). Appendix 8 provides a detailed outline of the five stages (Intake; Strategy Development; Investigation; Integration; Recommendations, Appointments and Feedback). The overall aim of AFI is to bridge the gap between assessment and intervention in order to provide recommendations that are both scientifically sound and useful for the student, teacher and parent (Pameijer, 2017). This aim addresses the findings of the systematic review and assertions of Resing, Lauchlan, \& Elliott (2017) and VanDerHeyden (2018) in Section 1.4. Specifically, AFI works to offer a better understanding of the student and the support he/she needs, to generate recommendations for teachers and parents, and for teachers to be able to apply the recommendations in their classrooms (Pameijer, 2016). The Pameijer (2017) questionnaire evaluation study was allocated a 'High' overall WoE D rating. The findings revealed the stages of AFI structured the assessment process 
from beginning to end, all involved worked in cooperation towards a shared goal and outcomes were meaningful to clients (Pameijer, 2017). This process was considered consistent with EST (Bronfenbrenner, 1979) and SCT (Palinscar, 1998; Vygotsky, 1978). In the context of models of service delivery outlined in Section 1.3, the AFI process could also be considered a form of consultation as the EP works collaboratively with parents/guardians, school staff and the child to bring about meaningful change to the presenting referral situation.

The AFI model has been applied by EPs in the Netherlands and Belgium in primary and secondary schools for over twenty years and is described by the European Agency for Special Needs and Inclusive Education as a model of best practice (Pameijer, 2006, 2017). The model has been evaluated and adapted since it was first applied and the independent research study conducted by Alegra (2013) and reported in Pameijer (2017), is the first systematic evaluation study of the AFI model published in the English language. Rigorous, large scale research is needed to determine whether the model leads to more ecologically valid case formulations and effective interventions than 'assessment as usual'. However, practical evidence may continue to demonstrate that the model bridges the gap between assessment and intervention, making the assessment process more meaningful to teachers, parents and students (Pameijer, 2017). In consideration of the enactment of Circular 0013/2017 (DES, 2017b) and Circular $0014 / 2017$ (DES, 2017c), and the findings of the systematic review, the current researcher proposed exploring the application of AFI in an Irish Educational Psychological context. The theoretical context for conducting the research is discussed in the second phase of the literature review.

\subsection{Phase 2 of the Literature Review: A Theoretical Exploration of the Literature}

From a theoretical perspective, discussion of systematic review findings indicated alignment of the AFI model with EST (Bronfenbrenner, 1979) and SCT (Palinscar, 1998; Vygotsky, 1978). A second phase to the literature review was prompted to explore how these theories inform EP assessment practices generally, and more specifically, the AFI model (Pameijer, 2017). A conceptual framework reflects the researcher's understanding of how the research problem will be explored (Grant \& Osanloo, 2014). It was proposed that the findings of this phase would lead to the development of a conceptual framework and research question for the study, providing a methodological foundation for conducting the research. Two review questions were formulated: 
1. How does Ecological Systems Theory inform educational psychological assessment practices?

2. How does Social Constructivist Theory inform educational psychological assessment practices?

\subsection{Search Strategy/Literature Search}

An electronic database search of Academic Search Complete, PsycINFO and ERIC was conducted through EBSCO host in October, 2018. An initial search of the keyword phrases 'Assessment for Intervention' (Pameijer, 2016, 2017) and 'Needs Based Assessment' (Pameijer, 2006) was carried out. Keyword searches pertaining to review question 1 (Table 2.8) and review question 2 (Table 2.9) followed. A filter was applied so that only peer reviewed papers written in English would be included. A total search yield of 197 titles was generated. Titles and abstracts were screened for relevance to the review questions and eight papers were included for review. Papers included empirical research studies and review papers. An in-depth review of The Ecology of Human Development (Bronfenbrenner, 1979) and Mind in Society (Vygotsky, 1978) was also conducted and seminal papers were studied including “Ecological Systems Theory” (Bronfenbrenner, 1989), "Ecological Models of Human Development" (Bronfenbrenner, 1994) and "Social Constructivist Perspectives on Teaching and Learning” (Palinscar, 1998). "A Melange or a Mosaic of Theories?" (Ring, Sullivan, Ryan, \& Burke, 2018) was also referred to in the review process. The following sections discuss the application of EST and SCT to EP practice in detail.

Table 2.8

Database Search Items Review Question 1 (Search 1)

\begin{tabular}{|c|c|}
\hline Databases & Search Terms \\
\hline Academic Search, ERIC, PsycINFO & $\begin{array}{l}\text { "ecological systems theory" OR } \\
\text { "development in context" OR "human } \\
\text { ecology theory" AND "education* } \\
\text { psycholog*" OR "school psycholog*" } \\
\text { AND "assessment for intervention" OR } \\
\text { "needs based assessment" }\end{array}$ \\
\hline Academic Search, ERIC, PsycINFO & $\begin{array}{l}\text { "ecological systems theory" OR } \\
\text { "development in context" OR "human } \\
\text { ecology theory" AND "education* } \\
\text { psycholog*" OR "school psycholog*" } \\
\text { AND "assessment" }\end{array}$ \\
\hline Academic Search, ERIC, PsycINFO & \\
\hline
\end{tabular}


"ecological systems theory" OR "development in context" OR "human ecology theory" AND "education* psycholog*" OR "school psycholog*" AND "assessment practice" OR "assessment approach" OR "assessment model" OR "assessment framework"

Table 2.9

Database Search Items Review Question 2 (Search 2)

\begin{tabular}{|c|c|}
\hline Databases & Search Terms \\
\hline Academic Search, ERIC, PsycINFO & $\begin{array}{l}\text { "social constructivism" OR "social } \\
\text { constructivist" OR "social constructivist } \\
\text { theory" AND "education* psycholog*" } \\
\text { OR "school psycholog*" AND } \\
\text { "assessment for intervention" OR "needs } \\
\text { based assessment" }\end{array}$ \\
\hline Academic Search, ERIC, PsycINFO & $\begin{array}{l}\text { "social constructivism" OR "social } \\
\text { constructivist" OR "social constructivist } \\
\text { theory" AND "education* psycholog*" } \\
\text { OR "school psycholog*" AND } \\
\text { "assessment" }\end{array}$ \\
\hline Academic Search, ERIC, PsycINFO & $\begin{array}{l}\text { "social constructivism" OR "social } \\
\text { constructivist" OR "social constructivist } \\
\text { theory" AND "education* psycholog*" } \\
\text { OR "school psycholog*" AND } \\
\text { "assessment practice" OR "assessment } \\
\text { approach" OR "assessment model" OR } \\
\text { "assessment framework" }\end{array}$ \\
\hline
\end{tabular}

\subsection{Ecological Systems Theory: Definition and Model}

Bronfenbrenner (1989) states:

the ecology of human development involves the scientific study of the progressive, mutual accommodation, throughout the life course, between an active, growing human being and the changing properties of the immediate settings in which the developing person lives, as this process is affected by relations between these settings and by the larger contexts in which the settings are embedded. (p. 188)

From this ecological perspective, the developing person is viewed as an evolving, dynamic entity, reciprocally interacting and adapting to an ever-changing environment, which comprises of their immediate setting, interconnections between these settings and 
broader external influences of larger surroundings. According to EST, the ecological environment, in which the developing person is situated, is delineated topologically as layers of concentric structures referred to as the micro, meso, exo and macrosystems (Bronfenbrenner, 1979, p. 22). Bronfenbrenner and his colleagues have expanded upon the theory over the years to recognise the complex interaction between the child's biological disposition and environmental processes at eco-systemic levels, to form a 'bioecological' theory of child development (Odom et al., 2004; Ring et al., 2018).

Figure 2.2 delineates the theory and provides a definition of each of the system levels. Section 2.14.1 discusses key concepts. 


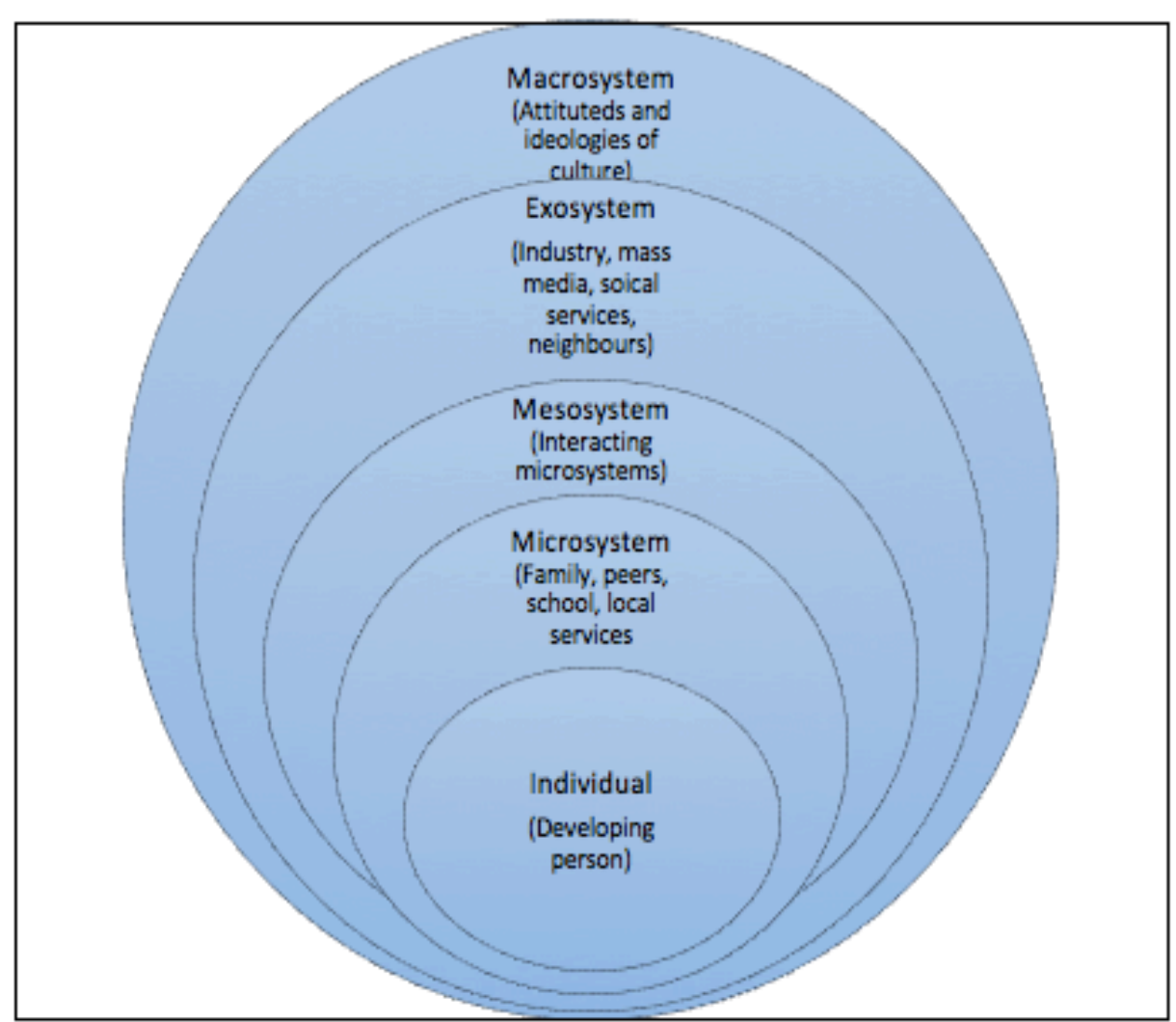

Microsystem: A microsystem is a pattern of activities, roles, and interpersonal relations experienced by the developing person in a given face-to-face setting with particular physical and material features, and containing other persons with distinctive characteristics of temperament, personality, and systems of belief.

Mesosystem: A mesosystem comprises the linkage and processes taking place between two or more settings containing the developing person (e.g. the relations between home and school, school and workplace, etc.). In other words, a mesosystem is a system of microsystems.

Exasystem: The exosystem, encompasses the linkage and processes taking place between two or more settings, at least one of which does not ordinarily contain the developing person, but in which events occur that influence processes within the immediate setting that does contain that person (c.g., for a child, the relation between the home and the parent's work place; for a parent, the relation between the school and the neighbourhood group).

Macrosystem: The macrosystem consists of the overarching pattern of micro-, meso-, and exosystems characteristic of a given culture, subculture, or other broader societal context, with particular reference to the developmentally-instigative belief systems, resources, hazards, life styles, opportunity structures, life course options, and patterns of social interchange that are embedded in each of these systems. The macrosystem may be thought of as a societal blueprint for a particular culture, subculture, or other broader social context.

Figure 2.2. Ecological Systems Theory (Bronfenbrenner, 1979)

\subsubsection{Ecological Systems Theory: Key Concepts}

There are several key elements and concepts of EST outlined in The Ecology of Human Development (Bronfenbrenner, 1979) including 'developmental potential', 'dyadic relations', 'molar activities' and 'experienced'. Each of these concepts are discussed in the paragraphs below, in the context of systematic review findings. 
Developmental potential and dyadic relations. At the mesosystem level, 'developmental potential' is strengthened to the extent that implicit linkages between settings facilitate the growth of mutual trust, positive orientation, goal consensus, and a 'balance of power' responsive to action on behalf of the developing person (Bronfenbrenner, 1979, p. 216). This supportive function for interconnections between settings is reflective of studies identified in the systematic review where communication between home, school and EP was encouraged (Bourke \& Dharan, 2015; Cane, 2016; Lawrence \& Cahill, 2014; Pameijer, 2017; Tobias, 2017). At the microsystem level, these supportive relations and interactions actively include the developing person and are termed 'dyadic relations'. According to Bronfenbrenner (1979), a dyad is formed whenever two individuals pay attention to or participate in one another's activities (Bronfenbrenner, 1979, p. 56). In any dyadic relation, and particularly during joint activity, the activity of A influences B and vice versa. Consequentially, one member (e.g. the parent, teacher or child) must coordinate his/her activities with those of the other. For a young child, this coordination can nurture the acquisition of interactive skills and fosters the growth of interdependence, essential for cognitive development. The extent to which A dominates B in a dyadic relationship, is referred to as a 'balance of power'. Joint activity dyads offer an opportunity for gradual transfer of power and as participants engage in dyadic interaction, they can start to develop more pronounced feelings toward one another (Bronfenbrenner, 1979, p. 58). Relevant to systematic review findings, the formation of dyadic relations between child and adult was evident in Lawrence and Cahill (2014) and (Pameijer, 2017), where children worked collaboratively with adults to generate meaningful interventions and their active participation was integral to the assessment process.

Molar activities and 'experienced'. Pertaining to dyadic relations, a 'molar activity' is an ongoing behaviour perceived as having meaning or intent by participants in a particular setting (Bronfenbrenner, 1979, p. 45). Molar activities function as indicators of the range and complexity of the perceived ecological setting and the potential of the child to alter and adapt to this environment, in accordance with his/her needs and desires. The extent to which a child develops a molar activity (e.g. building a tower of blocks, reading a book) depends upon the facilitation and activity of others within the setting, for example through joint participation and attracting attention (Bronfenbrenner, 1979, p. 45). This assistance is reflective of the mediation strategies applied during DA in the Lawrence and Cahill (2014) study, which were translated into intervention supports to address specific learning needs. Critical to the definition of 
microsystem presented in Figure 2.2 is the term 'experienced', which suggests the scientifically relevant properties of the environment include not only objective features but also the way in which these features are perceived by the developing person. Specifically, the aspects of the environment that are most influential in shaping psychological growth are those that have meaning to the person in a given situation (Bronfenbrenner, 1979, p. 22). This concept resonates with studies identified in the systematic review, where the voice of the child was gathered to determine the effectiveness of intervention recommendations, indicating consideration of their experience within the process (Cane, 2016; Lawrence \& Cahill, 2014; Pameijer, 2017) and contrasts with the acknowledged absence of the voice of the child in Hanchon and Allen (2013) and Hill and Turner (2016). The following section discusses EST in EP practice, in accordance with studies identified in the second phase of the literature review.

\subsubsection{Ecological Systems Theory in Educational Psychological Practice}

Burns (2013) suggests the unique contribution of EP practice is the application of an ecological perspective to school-based problems. Ecological Systems Theory can be applied broadly through a RTI approach, influencing positive change for all students and also more in-depth, to effectuate change for an individual student (Burns, 2013). An EP assessment conducted with a student is considered ecologically valid if the context of the assessment situation adequately represents reality, the assessment materials are relevant to daily activities, and the behaviour observed is natural and representative of the construct being assessed (Burns, 2013; Dean, Bums, Grialou, \& Varro, 2006). Therefore, EPs should identify the problem situation and devise a data collection plan that accurately represents the presenting context, for example, observing the student in the setting where the problem occurs, conducting a functional analysis to identify why the problem occurs, collecting data that represents multiple perspectives, assessing school climate, measuring the quality of relationships with teachers, parents, and peers, and reviewing samples of the student's work. Data can then be triangulated to develop a meaningful intervention plan that includes roles for the student, parents and school staff (Burns, 2013). This ecological approach to assessment resonates with the studies in the systematic review that informed appropriate interventions for clients (Bozic, 2013; Cane, 2016; Lawrence \& Cahill, 2014; Pameijer, 2017; Tobias, 2017). The assessment approaches applied required participants to work together and consider factors across 
contexts to develop interventions to address presenting concerns, consistent with the perspective outlined by Burns (2013).

Christenson and Anderson (2002) suggest assessment, in the context of EST, should be more than a process used to qualify a student for services; rather, a good assessment will lead to interventions that are personalised to each child. Assessment and intervention practices should be expanded to include broader contextual factors, alterable variables and student perspectives of these contexts and learning (e.g. belonging, motivation, relationships) (Christenson \& Anderson, 2002). Studies demonstrating how EST is applied to assessment and intervention in practice were identified in this second phase of the review. Frenzel, Goetz, Pekrun, and Watt (2010) examined adolescents' developmental trajectories of mathematics interest and investigated related effects of gender, family, and school context. Latent growth curve modeling was used to analyse longitudinal data of 53,193 students ( $51 \%$ female) from grades 5 to 9 from all three ability tracks of the German state school system. Results indicated that family values and classroom characteristics were positively related to within-person levels of interest over time and to average individual levels of interest, suggesting the impact of home and school factors on student motivation (Frenzel et al., 2010). Similarly, Aganza, Godinez, Smith, Gonzalez, and Robinson-Zañartu (2014) applied an EST approach to demonstrate how abilities of Latino students acquired at home could be reframed and applied to academic and behavioural challenges encountered in school. By bridging concepts and practices familiar to students and their parents into the school, positive outcomes were gained including improved relationships, communication and the potential for greater collaboration. Chun and Dickson (2011) investigated the effect of parental involvement, culturally responsive teaching and sense of school belonging on academic self-efficacy and academic performance amongst 478 (51.5\% female) Hispanic seventh graders in the United States-Mexico borderlands. Results demonstrated significant indirect effects of parental involvement, culturally responsive teaching, and sense of school belonging on academic performance (Chun \& Dickson, 2011). Collectively, these studies highlight the impact of ecological factors on student academic performance, wellbeing and motivation at school.

\subsubsection{Ecological Transitions in the Context of Ecological Systems Theory}

Throughout the life course of the developing person, ecological transitions are experienced, whereby positioning in the ecological environment alters due to changes in 
role, setting or both and continued development is dependent on the process of mutual accommodation (Bronfenbrenner, 1979, p. 26). Changes that trigger an ecological transition can occur at any of the four levels of the ecological environment (macro, exo, meso, micro), for example, the birth of a sibling, starting school, beginning a new job and getting married. In the current context, issuance of Circular 0013/2017 (DES, 2017b) and Circular 0014/2017 (DES, 2017c) at the macro level has directly impacted upon activities within the exosystem including assessment practices of school psychological services and resource allocation in schools (Figure 2.2). These changes may have effectuated change at the meso and microsystem levels, in terms of how schools identify and monitor needs of students with additional needs, how practices are communicated to parents and outside services and how support is being provided to children with identified needs. In accordance with EST, it is proposed that the issuance of Circular 0013/2017 (DES, 2017b) and Circular 0014/2017 (DES, 2017c) has instigated a period of ecological transition for the EP, school staff, parents and child with additional needs. Communication and interactions, occurring at the meso and microsystem levels between services, schools, home and student, need to be considered for the successful identification and support of student needs. Communication and interactions from a social constructivist perspective, specifically Vygotsky's (1978) socio-cultural theory, is discussed in the following sections.

\subsection{Social Constructivist Theory: Definition and Concepts}

Constructivism implies that "learners create their own learning" (Schunk, 2012, p. 230) and social constructivism acknowledges the influence of social factors in the construction of learning (Schunk, 2012). From a socio-cultural perspective, Vygotsky's (1978) view on constructivism implies the specific structures and processes demonstrated by individuals originate from their interactions with others (Palinscar, 1998, p. 351). Contrary to prevailing views of the time, where learning was considered an external process and development an internal process, Vygotsky (1978) proposed unity and interdependence of development and learning (Palinscar, 1998, p. 352). In her writings, Palinscar (1998) refers to the work of Wertsch (1991), who proposed major themes emerging from Vygotsky's theory that explicate the nature of this linkage between individual and social processes in learning and development. Two of these themes are discussed in the following paragraphs, in the context of systematic review findings. 
Theme 1: Genetic law of development. Vygotsky's (1978) "genetic law of development" states:

Every function in the child's cultural development appears twice on two levels. First on the social and later on the psychological level; first between people as an inter-psychological category and then inside the child as an intrapsychological category. This applies equally to voluntary attention, to logical memory and to the formation of concepts. The actual relations between human individuals underlie all the higher functions. (p. 128)

From this perspective, learners engage in a broad range of joint activities and internalise the effects of working together to develop novel strategies and knowledge of their world and culture (Palinscar, 1998). Vygotsky (1978) distinguished between two developmental levels: the actual and potential levels of development. The actual refers to those learning accomplishments a child can demonstrate alone or performs independently (Vygotsky, 1978, p. 85). The potential level of development is theorised through the Zone of Proximal Development (ZPD); what children can do with assistance (Vygotsky, 1978, p. 86). Vygotsky (1978) regarded the ZPD as a more accurate and dynamic indicator of cognitive development than what children have achieved alone and proposed productive interactions as those that facilitate learning through the ZPD (Palinscar, 1998, p. 353). This resembles the DA approach applied in Lawrence and Cahill (2014) in Section 2.8.2 and contrasts with the traditional, discrepancy assessment practices reported in several of the systematic review studies (Cottrell \& Barrett, 2015; Filter et al., 2013; Sotelo-Dynega \& Dixon, 2014; Stothard et al., 2018).

Theme 2: Tools and signs. Vygotsky's (1978) second theme posits that human activity, at the individual and social level, is mediated by tools and signs (Palinscar, 1998, p. 353). Tools are externally oriented, leading to change in the object of activity and signs are internally oriented for mastery of skill or behaviour (Vygotsky, 1978, p. 55). They can include "language; various systems of counting; mnemonic techniques; algebraic symbol systems; works of art; writing; schemes, diagrams, maps and mechanical drawings; all sorts of conventional signs and so on" (Vygotsky, 1981, as cited in Palinscar, 1998, p. 353). In relation to the preceding theme, tools and signs can be applied to assist the co-construction of knowledge and internalisation of problemsolving skills (Palinscar, 1998, p. 353). In the context of studies identified in the systematic review, language use was considered in the co-construction of knowledge between children and adults in the Pameijer (2017) study, on the 'goodness of fit' and 
'poorness of fit' of supports in place for the child (Table 2.10). In addition, student interview responses following DA in the Lawrence and Cahill (2014) study indicate internalisation of mediated strategies during the assessment process (Section 2.8.2). The application of SCT in EP practice is discussed in the following section.

\subsubsection{Social Constructivist Theory in Educational Psychological Practice}

In the context of EP practice, several elements of SCT can be considered (Green \& Gredler, 2002). An EP assessment consistent with SCT is dialogic and enables students to reflect as they take on increasingly independent roles in their own learning (Thomas \& Oldfather, 1997). During an assessment, observation of level of participation in discussion and evidence of verbal skill to participate is examined (Green \& Gredler, 2002). Observational data is invaluable in determining the learning potential of a student, for example, whether the student can complete a multistep task or assignments independently. In the classroom context, Ring et al. (2018) suggest teachers giving children clear guidance on how to use language to reason together can positively impact upon curriculum learning and intellectual development. In addition, investigation of whether the skills being assessed have been sufficiently taught to the student is necessary, to dispel within child assumptions and to emphasise learning as a reciprocal process (Green \& Gredler, 2002). Observing how the student solves problems and constructs meaning during classroom activities and individual assessment provides information necessary to formulate an accurate profile of students' strengths and needs and may inform suitable intervention recommendations (Green \& Gredler, 2002). Underlying this perspective is the significance of students' social constructions of their identities as learners and feelings of relatedness to teachers and peers in the learning environment, as well as to family and the larger community (Thomas \& Oldfather, 1997).

\subsection{Consistency of Theories with AFI Principles}

While SCT indicates that learning and development are dependent upon the direct interactions between the child and others within the environment, EST takes into account the influence of interacting external systems, beyond the immediate setting of the developing person (Bronfenbrenner, 1979, p. 18). Both theories are relevant to EP assessment and intervention practices, evident from the findings of the literature review. The theories emerged in the discussion of findings in Phase 1 and prompted an in-depth exploration and discussion on their application to EP assessment practices in Phase 2. 
There was a dearth in the literature on how EST and SCT inform the AFI model (Pameijer, 2016, 2017). Following a review of the (Pameijer, 2016, 2017) papers, EST and SCT concepts are proposed to resonate with several AFI principles. Table 2.10 overleaf outlines the proposed consistency. 
Consistency of AFI principles with EST and SCT

\begin{tabular}{ll}
\hline AFI Principle & EST/SCT Concepts \\
\hline $\begin{array}{l}\text { 1. AFI is goal-directed, aiming at } \\
\text { recommendations that are meaningful and } \\
\text { useful for clients and beneficial to the child. }\end{array}$ & \\
$\begin{array}{l}\text { 2. AFI applies a transactional perspective on } \\
\text { development and therefore not only focuses } \\
\text { on child factors, but also on teachers' }\end{array}$ & $\begin{array}{l}\text { Consistent with EST, the impact } \\
\text { of home and school environments } \\
\text { and interactions with others }\end{array}$ \\
$\begin{array}{l}\text { strategies and parental support. This principle } \\
\text { asserts that children develop through a }\end{array}$ & $\begin{array}{l}\text { influence the child's development. } \\
\text { continuous interaction with their school and } \\
\text { home environments. }\end{array}$ \\
$\begin{array}{l}\text { Development is dependent on } \\
\text { teachers' strategies and parental } \\
\text { support, which could include }\end{array}$ \\
strategies reflective of ZPD and \\
use of tools and signs.
\end{tabular}

3. AFI focuses on educational needs: what does this child need to achieve a specific goal? The EP discusses with the parents and teachers what they already offer the child ('goodness of fit') and what is missing ('poorness of fit').

4. As teachers and parents are essential in achieving educational goals, the model also focuses on their needs: what do they need to support this child's learning? The teacherstudent relationship is also focused upon.

5. AFI not only focuses on risk factors, but also on the protective factors of the child, school and parents/guardians.

6. The school psychologist works in a collaborative partnership with the teacher, child and parents. Parents, teachers and child are considered 'co-assessors' and 'hands on experts through experience'. The principle is to talk with the child, teacher and parents as much as possible, rather than about them.

7. The AFI-model follows a systematic and transparent stage-like process which increases the likelihood of objective decision making.
Focusing on the needs of the child through the lens of EST allows for identification of environmental factors that can be modified or implemented, to achieve a goal. Consistent with SCT, ZPD and tools and signs can be applied to identify what the child needs to achieve a specific goal.

Focusing on needs of teachers and parents offers an eco-systemic and inter-psychological perspective on supporting the child's learning, consistent with EST and SCT.

Focusing on the eco-systemic factors that may be contributing to risk and protective factors, including interactions with others, resonates with SCT and EST.

Parents/guardians, teachers, child and EP interact and communicate inter-psychologically, as a group and in dyadic relations, consistent with SCT and EST. 


\subsubsection{Conceptual Framework Development}

A conceptual framework offers a logical structure of connected concepts that help provide a picture or visual display of how ideas in a study are associated to one another (Grant \& Osanloo, 2014). The current research sought to explore the utility of the AFI model in bridging the gap between assessment and intervention, through the conceptual lens of EST and SCT. The seven AFI principles describe the theoretical context and rationale that underlie the five stages of the AFI-model (Pameijer, 2017). The research endeavoured to explore the applicability of EST and SCT to the AFI model, to further strengthen its theoretical underpinnings and the combination of theories informed the analysis of findings. In accordance with EST (Bronfenbrenner, 1979, p. 26), it is proposed that the issuance of Circular 0013/2017 (DES, 2017b) and Circular 0014/2017 (DES, 2017c) at the macro level has instigated a period of ecological transition for the EP, school staff, parents and child with additional needs. The five stages of the AFI model were applied to case referrals at the meso and microsystem levels, with parents, school staff and child. Table 2.11 presents the development of the conceptual framework for the current study, outlining key concepts of EST, SCT and AFI principles. This is followed by a diagram of the conceptual framework outlining concepts and principles and the proposed ecological transition at the macro, exo, meso and microsystem levels (Figure 2.3). The diagram is accompanied by a theoretical statement for the current study and the research question.

Table 2.11

Conceptual Framework

\begin{tabular}{ll}
\hline Theories/Models & Conceptual Framework \\
\hline $\begin{array}{l}\text { Assessment for Intervention } \\
\text { model (Pameijer, 2016; 2017) }\end{array}$ & $\begin{array}{l}\text { Core principles of AFI: goal directed, } \\
\text { transactional perspective, needs of child, parents } \\
\text { and teachers, risk and protective factors, } \\
\text { collaborative partnership, systematic and } \\
\text { transparent process }\end{array}$ \\
$\begin{array}{l}\text { Ecological Systems Theory } \\
\text { (Bronfenbrenner, 1979) }\end{array}$ & $\begin{array}{l}\text { Key EST concepts: micro, meso, exo and } \\
\text { macro-systems, 'experienced', developmental } \\
\text { potential, molar activities, dyadic relations, } \\
\text { balance of power }\end{array}$ \\
$\begin{array}{l}\text { Social Constructivist Theory } \\
\text { (Palinscar, 1998; Vygotsky, }\end{array}$ & $\begin{array}{l}\text { Key SCT concepts: “zone of proximal } \\
\text { development", tools and signs }\end{array}$ \\
\hline
\end{tabular}




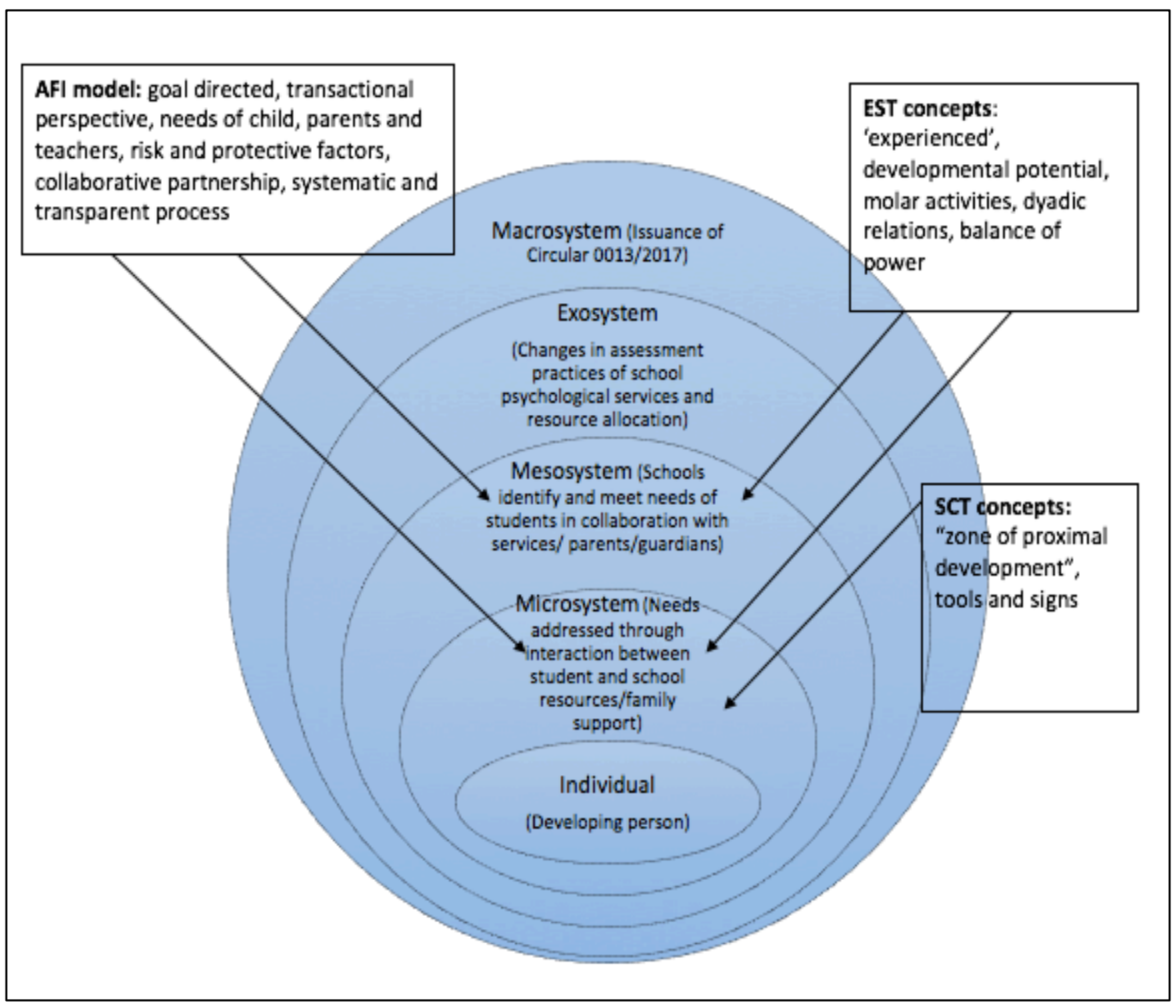

Figure 2.3. Diagram of Conceptual Framework

\subsubsection{Theoretical Statement and Research Question}

The conceptual framework was refined into a theoretical statement and proposition for the current research project:

During a time of ecological transition with the issuance of Circular 0013/2017 (DES, 2017b) and Circular 0014/2017 (DES, 2017c), the EP applies the five stages of the AFI model to a case referral. By interacting with the child, parents/guardians and teachers in accordance with AFI principles at the meso and microsystem levels, the assessment process leads to intervention recommendations that are meaningful to teachers, parents/ guardians and child. Engaging together in the five-stage process exposes EST and SCT concepts.

Subsequently, the research question outlined below evolved from this theoretical statement and literature review findings:

'Can the Assessment for Intervention model bridge the gap between assessment and intervention, through activity and interaction ${ }^{2}$ at the meso and microsystem levels?' 


\subsection{Chapter Conclusion}

This chapter reviewed the literature pertaining to EP assessment and intervention approaches and has set the context and rationale for the current study. A systematic review of the assessment approaches currently being applied by EPs in practice was conducted. Approaches informing appropriate interventions for clients were discussed. The second phase of the literature review presented the conceptual framework for the study. Chapter 3 outlines the research methodology, followed by a presentation of findings (Chapter 4), discussion of findings (Chapter 5) and conclusions and implications for practice (Chapter 6). 


\section{Chapter 3 Methodology}

\subsection{Overview of Chapter}

This chapter outlines the design for the research project, 'An exploration of the Assessment for Intervention model in an Irish Educational Psychological context'. The current research sought to explore the utility of the AFI model in bridging the gap between assessment and intervention, through the conceptual lens of EST and SCT. The constructivist paradigm was considered consistent with the researcher's conceptual framework and influenced the selection of an exploratory case study design and qualitative measures. Two case studies were conducted in parallel. Participants included parents, teachers and students in two different mainstream primary schools $(n=7)$. A pilot case study was conducted initially to inform overall design and measures $(n=3)$. The design and components of the case study; the research question, propositions, unit of analysis, logic for linking the data to the propositions and criteria for interpreting findings are outlined and discussed. The measures applied included Likert scale statements, semi-structured interviews and a researcher diary and journal. Data collection procedures and methods of analysis are outlined and ethical considerations for conducting the research are presented.

\subsection{Paradigm and Assumptions}

When examining the analysis and interpretation of findings in qualitative research, it is essential to outline the researcher's own paradigmatic position (Cresswell \& Poth, 2017). The conceptual framework outlined in Section 2.16.1 implies consideration of eco-systemic factors and collaboration and communication during the five stages of the AFI process. This assumption resonates most with the constructivist paradigm. From an ontological and epistemological perspective, reality and knowledge are socially constructed and the researcher and participants engage in an interactive process throughout to uncover meaningful information pertaining to presenting issues (Mertens, 2015). The researcher's ontological and epistemological values are applied in practice through interaction and dialogue with students, parents/guardians and teachers, to reveal the reality of a situation and to construct meaning and knowledge around referral issues. In psychological practice, Strong (2005) asserts that from a constructivist viewpoint, individuals put together their experience in somewhat unique ways due to the various mediating processes and resources (mostly linguistic and symbolic) that they are engaged in. When conducting research within the constructivist paradigm, the 
researcher strives to understand the complex world of lived experience from the point of view of those who live it (Schwandt, 2000). This approach to the research process requires a more personal, interactive mode of data collection and validity of findings is supported by the multiple methods used to collect data (Mertens, 2015). Such constructivist perspectives were considered in the design of data collection measures for the current study (Section 3.7).

\subsection{Design}

Qualitative methods utilising an exploratory 'two case' case study were employed to explore the application of the AFI model in an Irish Educational Psychological context. Case study designs are most suited to research questions that seek to provide explanation to a present circumstance and require an extensive, thorough description of a particular phenomenon, explaining "how" or "why" a phenomenon works (Yin, 2009, p. 4). When conducting a case study, the goal is to expand and generalise theories (analytic generalisation) rather than enumerate frequencies (statistical generalisation) (Yin, 2009). Articulating theory about what is being researched assists in operationalising case study designs and makes them more explicit (Yin, 2009). With regards the current study, the conceptual framework devised served as the vehicle for generalising to other cases and in the generation of case propositions (Section 3.5.2). Within the current context of an existing gap between assessment and intervention in EP practice, presented in the findings of the systematic review, a case study design was considered appropriate for an in-depth exploration into the utility of the AFI model in 'bridging the gap' (Pameijer, 2017). Applying the case study method allowed for EST and SCT concepts to emerge while working through the AFI model.

\subsubsection{An Exploratory Case Study Design}

When there is limited existing knowledge on a particular topic, empirical research in the area may take the form of an 'exploratory case study' (Yin, 2009, p. 37). As there has been no published research on the AFI model in an Irish Educational Psychological context to date, the current case study design was considered exploratory by nature. The 'two case' case study design was employed to feasibly replicate the fivestage AFI process within a limited timeframe (Yin, 2009, p. 61). A pilot study was carried out before conducting the two case studies (Section 3.8). External validity of the methodological procedure was established by replicating the process across two cases 
and the researcher's conceptual framework served as the vehicle to generalise findings case by case (Horner et al., 2005; Yin, 2009). When applying a case study design, a 'case' can refer to an individual, group, situation or organisation (Robson, 2011). In the current study, 'case' referred to a group (the student, the student's parents and teachers) in two separate primary schools.

\subsection{Participants}

The number of participants taking part in this study was subject to case presentation. The cases were referrals to the school psychological service and formed part of the researcher's caseload, while completing a school psychology placement. Case 1 included the student, the student's class teacher, SET, and one parent $(n=4)$. Case 2 included the student, the student's class teacher and one parent $(n=3)$. Participant sampling was purposive. Table 3.1 below presents the pseudonyms applied to each participant. See Section 3.8 for information related to the pilot study.

Table 3.1

Participant Pseudonyms

\begin{tabular}{lll}
\hline Case & Participant/Role & Pseudonym \\
\hline Pilot Case & Class teacher & Grace \\
& Parent & Louise \\
& Student & John \\
& Class teacher & Michael \\
Case 1 & SET & Sarah \\
& Parent & Tina \\
& Student & Jim \\
& Class teacher & \\
Case 2 & Parent & Anne \\
& Student & Mary \\
& & Michelle \\
\hline
\end{tabular}

Case referral forms were reviewed with the researcher's placement supervisor and a case selection protocol with specified criteria was adhered to in selecting referrals to approach for interest in taking part in the study (Appendix 9). The criteria for selection included:

- A student in a senior class in primary school $\left(4^{\text {th }}, 5^{\text {th }}\right.$ or $\left.6^{\text {th }}\right)$ referred to the school psychological service to address behaviour, social-emotional and/or learning needs. Students in senior classes were sought so as to maximise the voice of the child in the research project, in line with Article 12 of the International 
Convention on the Rights of the Child (2013) and recommendations for practice outlined in Pameijer (2017).

- The referral form preferably outlined a complex case in terms of learning, behaviour and/or social-emotional needs, which would be suited to the stages of the AFI model. The researcher's placement supervisor assisted in determining the level of complexity.

- The parents/guardians and teachers preferably showed interest in the AFI model and five-stage process, and motivation to take part in the study.

The Principals in Case 1 and Case 2 were initially approached to gauge interest in taking part in the research project. An information sheet outlining the aims of the research project and an informed consent form were provided for signature of the Board of Management (BOM). Following consent of the BOM, an information sheet was provided and discussed with the class teachers, SET and parents. A child friendly information sheet was also provided and explained to the students. Participants were informed that participation was voluntary, and they would receive a service from the school psychological service regardless of their participation in the research project. Upon confirmation of interest in taking part in the research project, a consent form was signed by the class teachers, SET and parents. A child friendly assent form was signed by the students. Participants completed a series of Likert scale statements before and after engagement with the AFI model (Section 3.7.1). In addition to completing the Likert scale statements, participants also engaged in a semi-structured interview following engagement with the AFI model (Section 3.7.2).

\subsection{Components of a Case Study}

According to (Yin, 2009, p. 27) there are five essential components to the design of a case study:

1. The study's questions

2. The propositions

3. The unit(s) of analysis

4. The logic linking the data to the propositions

5. The criteria for interpreting the findings

In addition, a case study protocol is essential to carrying out a multiple case study to guide the researcher in the data collection process and to enhance the reliability of the research (Yin, 2009). Each of these components was considered in the context of the current study and are discussed in the following sections. 


\subsubsection{Research question}

The research question was formed from the literature review and conceptual framework and sought to explore:

'Can the Assessment for Intervention model bridge the gap between assessment and intervention, through activity and interaction ${ }^{3}$ at the meso and microsystem levels?'

The case propositions outlined below, arose from the research question.

\subsubsection{Case propositions}

Case study propositions direct attention to something that should be examined within the scope of the study (Yin, 2009, p. 28). It is when a researcher is obliged to state some propositions that he/she begins to move in the right direction (Yin, 2009). Case study propositions were used to guide data collection and relevant analytic strategies (Yin, 2009, p. 130). The propositions outlined below arose from the conceptual framework, research question and the aims of AFI (Pameijer, 2016, 2017). Following engagement with the five stages of the AFI model:

1. Parents, teachers and TEP will have a better understanding of the child's situation, as a result of interactions and activities that have occurred at the meso and microsystem levels.

2. Parents, teachers and TEP will know what intervention supports the child needs at home and at school, as a result of interactions and activities that have occurred at the meso and microsystem levels.

3. Teachers and parents will have greater perceived competence in supporting the child's needs at home and at school, as a result of interactions and activities that have occurred at the meso and microsystem levels.

4. The child will have a better understanding of their strengths and needs and what to do to improve their needs, as a result of interactions and activities that have occurred at the meso and microsystem levels.

5. At a time of ecological transition, teachers will feel competent in their ability to work according to the aims of Circular 0013/2017 , in meeting and monitoring the needs of the child.

\footnotetext{
${ }^{3}$ Activity and interaction are those activities and interactions that resonate with EST and SCT concepts, including molar activities, dyadic relations, ZPD and tools and signs

${ }^{4}$ As the research was conducted with participants in mainstream primary schools, the aims and findings of the research project are discussed within the context of Circular 0013/2017 (DES, 2017b)
} 


\subsubsection{Unit of Analysis}

The 'case' or unit of analysis can be an individual, event or entity and its definition depends upon the specifications of the research question (Yin, 2009, p. 30). The unit of analysis in the current study was the AFI model. The utility of the model in bridging the gap between assessment and intervention was explored with Likert scale statements (Section 3.7.1) and semi-structured interviews (Section 3.7.2) with participants. Researcher reflections were also included to determine the model's utility in practice (Section 3.7.3).

\subsubsection{Linking Data to Propositions}

In accordance with Yin (2009, p. 130), case propositions shaped the data collection plan and informed relevant analytic strategies. The propositions and research question were explored through Likert scale statements, semi-structured interviews and researcher reflections. These measures were considered appropriate for gathering and linking data to the propositions for the reasons outlined below:

- A previous evaluation study on AFI had also applied Likert scale ratings (from 1 to 10) to explore participant experience (Pameijer, 2017).

- Semi-structured interviews are flexible to allow for a rich development of discussion with participants including children (Robson, 2011).

- Diary methods allow an individual to document their experiences for a specified period of time (Iida, Shrout, Laurenceau, \& Bolger, 2012).

\subsubsection{Criteria for Interpreting the Findings}

Table 3.2 outlines the criteria for interpreting the findings from the Likert scale statements, semi-structured interviews and entries in the researcher diary, in accordance with the five propositions. Changes in Likert scale statement ratings before and after implementation of the five stages of the AFI model were analysed according to corresponding propositions. Pattern-matching was applied to comments accompanying participants' Likert scale ratings and interview responses. Pattern-matching was considered an appropriate analytic technique because it compares an empirically-based pattern with a predicted one (Trochim, 1989 cited in Yin, 2009) and the case study's internal validity is strengthened if the patterns coincide (Yin, 2009). Thematic analysis was also applied to interview data as a method for identifying, analysing and reporting patterns related to the study's propositions (Braun \& Clarke, 2006). Excerpts from the researcher's diary and reflective journal demonstrated interpretation and reflections on 
the application of the AFI model, in the context of the propositions (Mertens, 2015). Section 4.1 provides signposting to relevant appendices for examples of analyses conducted. 
Table 3.2

Criteria for Interpreting the Findings

\begin{tabular}{|c|c|c|c|c|}
\hline Proposition & $\begin{array}{l}\text { Likert Scale } \\
\text { Statements (See } \\
\text { Section 3.7.1) } \\
\end{array}$ & $\begin{array}{l}\text { Likert Scale Statements } \\
\text { (Comments Section) }\end{array}$ & $\begin{array}{l}\text { Semi-structured Interviews } \\
\text { (See Section 3.7.2) }\end{array}$ & $\begin{array}{l}\text { Researcher Diary } \\
\text { (See Section 3.7.3) }\end{array}$ \\
\hline $\begin{array}{l}\text { Proposition 1: } \\
\text { 'Parents, teachers and } \\
\text { TEP will have a better } \\
\text { understanding of the } \\
\text { child's situation, as a } \\
\text { result of interactions } \\
\text { and activities that have } \\
\text { occurred at the meso } \\
\text { and microsystem } \\
\text { levels' }\end{array}$ & $\begin{array}{l}\text { A change in rating on } \\
\text { associated Likert scale } \\
\text { statements indicates } \\
\text { change in } \\
\text { understanding of the } \\
\text { child's situation } \\
\text { following engagement } \\
\text { with the AFI model and } \\
\text { as a result of } \\
\text { interactions and } \\
\text { activities that have } \\
\text { occurred at the meso } \\
\text { and microsystem levels. }\end{array}$ & $\begin{array}{l}\text { Pattern-matching: } \\
\text { Comments that link } \\
\text { understanding of the } \\
\text { child's situation, } \\
\text { engagement with the AFI } \\
\text { model and interactions } \\
\text { and activities that have } \\
\text { occurred at the meso and } \\
\text { microsystem levels and } \\
\text { vice versa. }\end{array}$ & $\begin{array}{l}\text { Pattern-matching: Interview } \\
\text { responses that link understanding of } \\
\text { the child's situation, engagement } \\
\text { with the AFI model and interactions } \\
\text { and activities that have occurred at } \\
\text { the meso and microsystem levels and } \\
\text { vice versa. } \\
\text { Thematic analysis: Themes and } \\
\text { patterns within the data set that refer } \\
\text { to understanding of the child's } \\
\text { situation and interactions and } \\
\text { activities that have occurred at the } \\
\text { meso and microsystem levels were } \\
\text { included in the analysis of the AFI } \\
\text { model. }\end{array}$ & $\begin{array}{l}\text { Excerpts from the } \\
\text { researcher diary that } \\
\text { referred to } \\
\text { understanding of the } \\
\text { child's situation and } \\
\text { interactions and } \\
\text { activities that have } \\
\text { occurred at the meso } \\
\text { and microsystem } \\
\text { levels were included in } \\
\text { the analysis of the AFI } \\
\text { model. }\end{array}$ \\
\hline $\begin{array}{l}\text { Proposition 2: } \\
\text { 'Parents, teachers and } \\
\text { TEP will know what } \\
\text { intervention supports } \\
\text { the child needs at } \\
\text { home and at school, as } \\
\text { a result of interactions }\end{array}$ & $\begin{array}{l}\text { A change in rating on } \\
\text { associated Likert scale } \\
\text { statements indicates } \\
\text { change in knowledge of } \\
\text { what intervention } \\
\text { supports the child needs } \\
\text { following engagement }\end{array}$ & $\begin{array}{l}\text { Pattern-matching: } \\
\text { Comments that link } \\
\text { knowledge of } \\
\text { intervention supports the } \\
\text { child needs, engagement } \\
\text { with the AFI model and } \\
\text { interactions and activities }\end{array}$ & $\begin{array}{l}\text { Pattern-matching: Interview } \\
\text { responses that link knowledge of } \\
\text { intervention supports the child needs, } \\
\text { engagement with the AFI model and } \\
\text { interactions and activities that have } \\
\text { occurred at the meso and } \\
\text { microsystem levels and vice versa. }\end{array}$ & $\begin{array}{l}\text { Excerpts from the } \\
\text { researcher diary that } \\
\text { referred to knowledge } \\
\text { of intervention } \\
\text { supports the child } \\
\text { needs and interactions } \\
\text { and activities that have }\end{array}$ \\
\hline
\end{tabular}


and activities that have with the AFI model and occurred at the meso and microsystem levels'

as a result of interactions and activities that have occurred at the meso and microsystem levels.

\section{Proposition 3:}

'Teachers and parents will have greater perceived competence in supporting the child's needs at home and at school, as a result of interactions and activities that have occurred at the meso and microsystem levels'

\section{Proposition 4:}

'The child will have a better understanding of their strengths and needs and what to do

A change in rating on associated Likert scale statements indicates change in perceived competence in supporting the child's needs following engagement with the AFI model and as a result of interactions and activities that have occurred at the meso and microsystem levels.

A change in rating on the associated Likert scale statements indicates change in the child's understanding of
Pattern-matching:

Comments that link the child's understanding of their strengths and needs and what to do to that have occurred at the meso and microsystem levels and vice versa.

Thematic analysis: Themes and patterns within the data set that refer to knowledge of intervention supports the child needs and interactions and activities that have occurred at the meso and microsystem levels were included in the analysis of the AFI model.

\section{Pattern-matching:} Comments that link competence in supporting the child's needs, engagement with the AFI model and interactions and activities that have occurred at the meso and microsystem levels and vice versa.

\section{Pattern-matching: Interview} responses that link competence in supporting the child's needs, engagement with the AFI model and interactions and activities that have occurred at the meso and

microsystem levels and vice versa.

Thematic analysis: Themes and patterns within the data set that refer to competence in supporting the
N/A child's needs and interactions and activities that have occurred at the meso and microsystem levels were included in the analysis of the AFI model.

occurred at the meso

and microsystem

levels were included in the analysis of the AFI model.

Pattern-matching: Interview responses that link the child's understanding of their strengths and needs and what to do to improve their needs, engagement with the 


to improve their needs,
as a result of
interactions and
activities that have
occurred at the meso
and microsystem
levels'

levels' their strengths and needs and what to do to improve their needs, following engagement with the AFI model and as a result of

interactions and activities that have occurred at the meso and microsystem levels improve their needs, engagement with the AFI model and interactions and activities that have occurred at the meso and microsystem levels and vice versa.

AFI model and interactions and activities that have occurred at the meso and microsystem levels and vice versa.

Thematic analysis: Themes and patterns within the data set that refer to understanding of strengths and needs and what to do to improve their needs, and interactions and activities that have occurred at the meso and microsystem levels were included in the analysis of the AFI model.

\section{Proposition 5:}

N/A

N/A

ecological transition, teachers will feel competent in their ability to work according to the aims of Circular $0013 / 2017$, in meeting and monitoring the needs of the child'

\section{Pattern-matching: Interview} responses that link competence in in meeting and monitoring the needs of the child, engagement with the AFI model and interactions and activities that have occurred at the meso and microsystem levels and vice versa. Thematic analysis: Themes and patterns within the data set that refer to meeting and monitoring the needs of the child, and interactions and activities that have occurred at the meso and microsystem levels were included in the analysis of the AFI model. 


\subsubsection{Case Study Protocol}

Devising a case study protocol increased the study's reliability and guided the data collection process (Yin, 2009). It served to keep the researcher focused on the case study topic and to foresee any potential issues in data collection, analysis and report writing (Yin, 2009, p. 80). See Appendix 10 for an outline of the case study protocol.

\subsection{Measures}

Qualitative measures were employed to address the research question and case propositions. Measures included Likert scale statements, semi-structured interviews and a researcher diary and reflective journal.

\subsubsection{Likert Scale Statements}

Likert scales are commonly applied in psychological research to measure participants' judgements (Barker, Pistrang \& Elliott, 2002). The Pameijer (2017) study used Likert scale ratings (from 1 to 10) to explore student, parent, teacher, counsellor and assessor experience and views on AFI. In the researcher's systematic review, Cane (2016) also applied Likert scale ratings to monitor student experience of an intervention arising from a solution-focused assessment. Scaling questions are considered an effective method when working with children to elicit information regarding their learning and feelings (Wagner, 2008). As the voice of the child is central to the AFI model and the aims of the current study, this measure was deemed appropriate. The Likert scale statements were devised to measure the case study propositions and were reviewed by an expert in the field, Noelle Pameijer, in April 2018, enhancing the measure's construct validity. Parents and teachers were asked to rate three Likert scale statements before and after engagement with the AFI model. Space was provided to write comments underneath each statement. The statements were rated on a scale of 110, consistent with previous AFI research (Pameijer, 2017), where 1 was 'Strongly Disagree', 5 was 'Neither Agree or Disagree' and 10 was 'Strongly Agree'. The student was also asked to rate three Likert scale statements with smiley faces, in accordance with previous AFI research (Pamejier, 2017) to represent 'Yes', 'Not Sure' and 'No'. Revisions to the parent and teacher Likert statements were made following feedback provided by the parent and teacher in the pilot study. A space for writing the referral issue or presenting concern at the top of the page was included in an effort to focus statements and participant responses. See Appendix 11 for the pilot and revised Parent Likert Statements and Appendix 12 for the pilot and revised Teacher Likert Statements. 
The student's feedback was also sought, and Student Likert Statements did not undergo revisions following the pilot study (Appendix 13).

\subsubsection{Semi-Structured Interviews}

Semi-structured interviews are flexible to allow for a rich development of discussion and allowed for key questions relevant to the research question and case propositions to be explored (Robson, 2011). The flexibility of this data collection technique was considered appropriate for use with children under the age of 18 and interview style and questions for this age group were adapted accordingly. For example, the child in each case was informed they could use drawings to represent their response to a question.

All participants were asked to engage in a semi-structured interview following engagement with the model, to explore their views on the utility of the AFI model in bridging the gap between assessment and intervention (research question) and to address the case propositions. The interview questions in the current study were devised according to the case propositions, literature on AFI (Pameijer, 2017) and cognisance of a changing context and attitude towards assessment and intervention with the issuance of Circular 0013/2017 (DES, 2017b). The interview questions were revised following the pilot study and were reviewed by a practicing educational psychologist (school psychology placement supervisor) to enhance the measure's construct validity. The researcher adhered to an interview guide when conducting interviews to increase the reliability of the measure. See Appendix 14 for the pilot and revised Parent Interview Schedules, Appendix 15 for the pilot and revised Teacher Interview Schedules, Appendix 16 for the pilot and revised Student Interview Schedules and Appendix 17 for the pilot and revised Interview Guides.

\subsubsection{Researcher Diary and Journal}

The researcher aimed to be reflexive while engaging in the research process. In the current study, the implementation of the five stages of the AFI model was documented in a researcher diary (AFI templates) and the researcher's views on the process were recorded in a reflective journal. Diary methods in psychological research require an individual to provide information on their experiences for a specified period of time (Iida et al., 2012). Traditional diary designs can be categorised into two general categories: time-based and event-based protocols. Event-based design necessitates researchers to give a clear definition of the event in which they are interested and data 
collection is prompted by its occurrence (Iida et al., 2012). With regards the current study, the 'event' was each stage of the AFI model and AFI templates represented the diary for documenting the process. AFI templates function as a guide for EPs implementing the five-stage process with space for remarks, questions and examples (Pameijer, 2016). There are four templates pertaining to the stages and the researcher recorded actions accordingly. The researcher journal was used to elucidate actions recorded and to reflect upon the process.

\subsection{Pilot Study}

A pilot study was conducted initially, to inform and refine the research design, measures and data collection procedures (Mertens, 2015; Yin, 2009). The Pilot Case was carried out with a case referral on the researcher's school psychology placement. The case selection and case study protocol were adhered to during the pilot study. The student, the student's class teacher and one of his parents participated $(n=3)$ and findings were used to inform and revise measures as documented in Section 3.7. The pilot study also informed the development of an in-depth procedure for conducting the proceeding cases. To enhance reliability, a procedural template was devised and was based on the case study protocol, AFI templates and instructional guidance provided in Pameijer (2016). Appendix 18 provides an outline of the revisions made to measures following the pilot study and Appendix 19 outlines the detailed procedural template. The parent and student in Case 2 did not complete the Likert statements and semistructured interview following engagement with the AFI model. Due to an incomplete data set for Case 2, the pilot study data were analysed and presented as a case in Chapter 4.

\subsection{Data Collection Procedures}

Case 1 and Case 2 were conducted in parallel due to time constraints. Upon receipt of informed consent and assent from participants, procedural phases for data collection were implemented as outlined below:

1. Parents, teachers and students rated Likert scale statements before engagement with the AFI model. Space for comments was provided. Instruction and clarification was provided as needed. The researcher documented the process and reflections in the researcher diary (AFI templates) and reflective journal.

2. The researcher applied the five-stage AFI model to the case referral. The researcher documented the process and reflections to demonstrate transparency 
and reliability. See Table 3.3 below for a summary of the procedure followed for conducting the cases, in accordance with Pameijer $(2016,2017)$ guidelines. Appendix 19 provides a more in-depth account of the procedure applied.

3. Parents, teachers and student rated the same Likert scale statements from Phase 1 , following engagement with the stages of the AFI model. Space for comments was provided. The researcher also conducted semi-structured interviews with parents and teachers to explore their views on the utility of the AFI model in bridging the gap between assessment and intervention (research question) and to address the case propositions. Interviews were also conducted with the students to explore their views on the process and to address case propositions. Interviews were recorded on a recording device borrowed from the Mary Immaculate College library. The researcher documented reflections in the reflective journal. While participants completed the Likert scale statements and engaged in semi-structured interviews, the researcher made every effort to ensure they felt safe and at ease by:

a. Ensuring the activity was carried out in a quiet and appropriate environment.

b. Engaging in some initial rapport building to promote a contained and safe environment.

c. Clarifying that they do not have to answer any questions that they do not wish to.

d. Clarifying that they could withdraw from the study at any time.

e. Offering a debrief afterwards.

Following data collection, a participant database was created. The anonymity of participants was protected throughout the research process. Alpha-numeric codes were assigned to protect anonymity and to link participant Likert scale data to semistructured interview data. Pseudonyms were applied in the transcription of interview data and the write up of the results and discussion sections. No identifiable details were reported, including names of participants, schools and regional locations. Sensitive assessment information gathered during Phase 2 of the data collection procedure was kept confidential. To enhance reliability, a case study database was created, to compile all anonymised data from each case study, including the researcher's diary (AFI templates) and interview transcripts (Yin, 2009). 
Table 3.3

The Five Stages of the AFI Model (Pameijer, 2016, 2017)

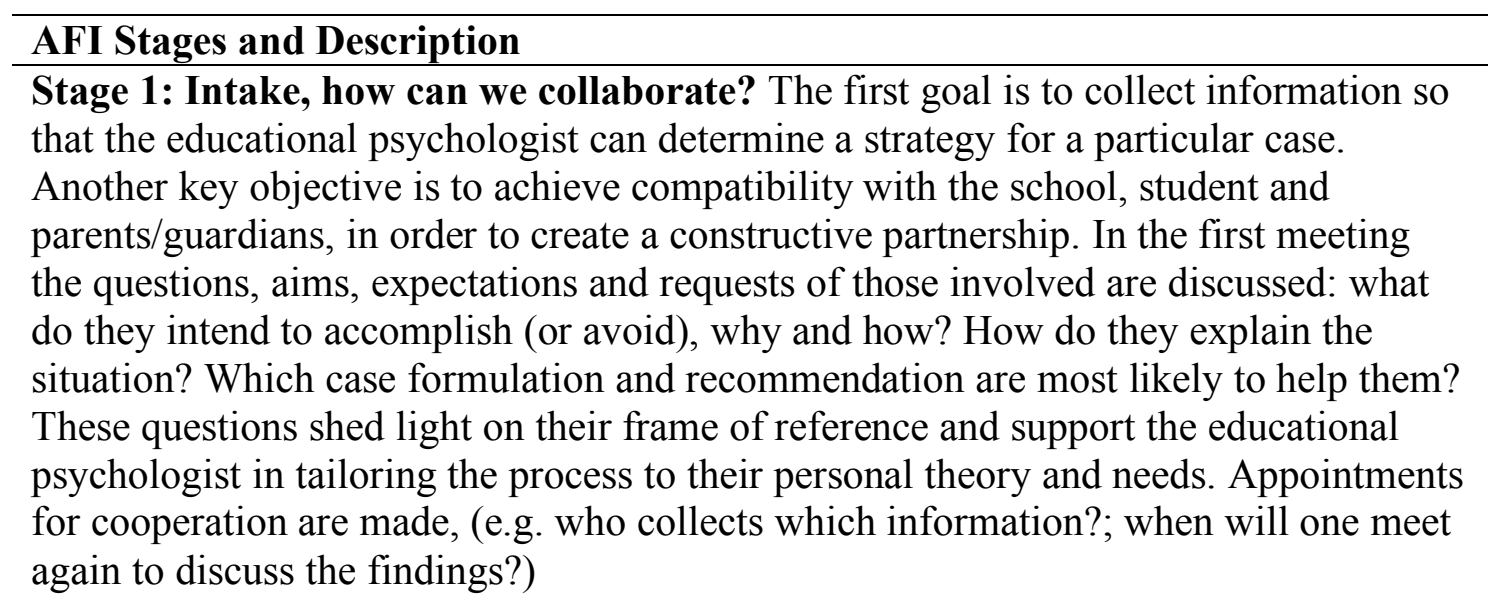

Stage 2: Strategy, how to proceed in this particular case? The input of this stage is the information collected in the intake and the output is the strategy that best fits a specific situation. First, the relevant information is organised in four sections: student, instructional environment, parental support of learning and relevant history. Then the educational psychologist decides what more needs to be known to answer the clients' and her questions. Is the investigation stage (Stage 3) necessary? This is the case when more information is needed in order to formulate recommendations. Or can she already move on to stage 4 (Integration stage)? The bottom line is: no investigation will be conducted unless its outcomes will influence the choice of the intervention. If stage 3 is required, alternate hypotheses from a transactional frame of reference are formulated, relevant hypotheses are selected, based on their impact on the choice of an intervention and these hypotheses are translated into questions for investigation.

Stage 3: Investigation, answering the selected answers: This stage involves a goaldirected rather than a routine collection of data. The selected hypotheses determine the information to be gathered. The content of this stage thus varies in each case, ranging from using one instrument to several different tools.

Stage 4: Integration, goals and needs: The information is integrated into a specific case formulation: how can the situation be understood? This summary is translated into goals for the student, teaching strategies and parental support, educational needs of the student and support needs of his teacher and/or parents/guardians. As several interventions focus on the same target, choices have to be made. The AFI-model prefers interventions that have been proven to be effective.

Stage 5: Recommendations, appointments and feedback: In this stage the clients are informed about the outcomes of the assessment. By providing them with clear and meaningful information, related to their personal theory, hopes and worries, they can choose for themselves which option is both desirable and achievable. An important aim is to arrive at a feasible intervention, supported by all parties. The educational psychologist therefore asks if those involved are willing and able to 'start tomorrow'. If the answer is affirmative, the child, teacher and parent/guardian are encouraged to change their behaviour. If this is not yet the case, the assessment process continues with further consultation. 


\subsection{Validity}

In addition to applying procedures and measures to address case study validity (Sections $3.3 .1 ; 3.5 .4 ; 3.5 .5 ; 3.7 .1 ; 3.7 .2$ ), ecological validity and developmental validity were addressed in accordance with the researcher's conceptual framework. Ecological validity is defined as "the extent to which the environment experienced by the subjects in a scientific investigation has the properties it is supposed or assumed to have by the investigator" (Bronfenbrenner, 1979, p. 29). When conducting ecological research, factors of the person and environment, including the structural aspects of environmental settings, and the processes taking place within and between them must be considered interdependent and analysed in systems terms. All phases of the data collection process were conducted in the school setting, which allowed for observations, consultations and one to one work with the child. To demonstrate developmental validity in ecological research, Bronfenbrenner $(1979$, p. 29) states it is necessary to establish that a change produced in the person's conceptions and/or activities is transferable to other settings and other times. The interview question 'Do you think you would apply anything that you have learned from working through the five stages of the AFI model to a different situation?' was devised to address developmental validity in the current research project.

\subsection{Data Analysis}

The following section describes the researcher's approach to the analysis of data gathered and is followed by an outline of the presentation of findings in Chapter 4.

\subsubsection{Analysis of Likert Scale Ratings}

For each case report, participant ratings on the Likert scale statements, before and after engagement with the AFI model were presented in tabular format, according to their corresponding proposition. Ratings were presented to outline any changes in understanding of the student's situation, knowledge of supports needed and perceived competency to provide these supports following engagement with the AFI process. The student's ratings were presented in tabular format to outline any changes in understanding of their strengths, needs and how to address identified needs. Average ratings were reported in the cross-case discussion, to analyse changes in ratings according to participant group (teachers, parents, students), before and after engagement with the AFI model, consistent with the Pameijer (2017) evaluation study. 


\subsubsection{Pattern-Matching}

Pattern-matching compares an empirically-based pattern with a predicted one (Trochim, 1989 cited in Yin, 2009). Pattern-matching logic was applied to each participant's interview transcript and comments accompanying participants' Likert scale statement ratings, in accordance with guidelines in Yin (2009). The comments accompanying the participants' Likert statements were often left blank or with limited information, therefore pattern matching was focused on interview comments. See Section 4.1 for signposting to the analysis of this data. Pattern-matching was reported case by case and used to determine level of consistency or inconsistency between the case propositions and actual participant experiences in terms of:

1. Understanding of the child's situation following engagement with the AFI model and interactions and activities that occurred at the meso and microsystem levels.

2. Knowledge of interventions to support the child needs, following engagement with the AFI model and interactions and activities that occurred at the meso and microsystem levels.

3. Competence in supporting the child's needs following engagement with the AFI model and interactions and activities that occurred at the meso and microsystem levels.

4. Understanding of strengths and needs and what to do to address needs, following engagement with the AFI model and interactions and activities that occurred at the meso and microsystem levels (Child only).

5. Competence in meeting and monitoring the needs of the child, following engagement with the AFI model and interactions and activities that occurred at the meso and microsystem levels (Teachers only).

\subsubsection{Thematic Analysis}

Interviews were listened to and stored as encrypted files on the researcher's laptop. The eight interviews were transcribed by the current researcher and thematic analysis was also applied to the data. Braun and Clarke (2006) describe thematic analysis as a flexible, qualitative analytic method that is compatible with the constructivist paradigm. Through its theoretical freedom, thematic analysis offered a useful research tool to provide a rich, yet complex explanation of the data in the current study (Braun and Clarke, 2006). Before conducting the analysis, the theoretical position of the thematic analysis was considered (Braun \& Clarke, 2006). As the research was 
conducted through the conceptual lens of EST and SCT, the analysis was driven by the researcher's theoretical interest and a deductive approach to thematic analysis was adopted (Braun \& Clarke, 2006).

Thematic analysis was applied to interview data as a method for identifying, analysing and reporting patterns related to the study's propositions and to address the research question. The researcher adhered to the six phases of thematic analysis outlined in Braun and Clarke (2006); familiarising yourself with your data (Phase 1); generating initial codes (Phase 2); searching for themes (Phase 3); reviewing themes (Phase 4); defining and naming themes (Phase 5) and producing the report (Phase 6). Appendix 20 provides a detailed outline of the six phases. The prevalence of codes across the data set was observed and a process of categorising codes was engaged in to form themes and subthemes (Braun and Clarke, 2006). Section 4.1 provides signposting to the analysis of this data. Themes that related to case propositions were documented under the corresponding proposition in each case report. The Braun and Clarke (2006) checklist criteria for good thematic analysis was applied to ensure high quality analysis (Appendix 21). To establish dependability and credibility of findings, the researcher documented each step of the analysis process to produce a 'chain of evidence' and recruited an objective peer to review examples of coded interview data (Yin, 2009).

\subsubsection{Analysis of the Researcher Diary}

The researcher aimed to be reflexive during the research process, in accordance with the constructivist paradigm (Mertens, 2015). The implementation of the five stages of the AFI model was documented in a researcher diary (AFI templates) and the views of the researcher on the process were recorded in a reflective journal. Excerpts from the reflective journal and diary elucidated findings presented and were analysed in the context of the conceptual framework.

\subsubsection{Outline of Case Reports}

The following is an outline of the structure of the presentation of findings and case study reports in Chapter 4:

1. Results under each proposition (Pilot Case)

a. Participant ratings on the Likert scale statements, before and after implementation of the AFI model presented in tabular format

b. Pattern-matching of interview responses

c. Thematic analysis of interview responses 
d. Researcher diary and reflective journal

2. Summary of findings addressing the research question (Pilot Case)

3. Discussion of findings under related themes in accordance with the literature and conceptual framework (Pilot Case)

4. Repeat steps 1-3 for Case 1 and 2

5. Cross-case analysis and discussion

6. Conclusions and directions for future research

\subsection{Ethical Considerations}

Ethical approval was obtained from Mary Immaculate College in May 2018 and approval to conduct the research project was granted by the school psychological service in June 2018. Transparency and credibility of researcher actions and intentions were made clear to the participants at all times. The BOM, teachers and parents were briefed on the aims and goals of the research project and were provided with an information sheet. The BOM, teachers and parents also signed an informed consent form with explicit information indicating that they could withdraw at any time and for any reason. See Appendix 22 and 23 for a sample parent information and consent sheet. Parents were also informed of their right to withdraw their child from the study at any time. The students participating were considered vulnerable as they were under the age of 18 and referred for behaviour, social-emotional and/or learning needs. The researcher took careful steps to ensure the students fully understood the aims of the project, that their participation was voluntary and of their right to withdraw at any time. A child friendly information sheet and assent form was presented and signed by the students. See Appendix 24 and 25 for the student information sheet and assent form. Procedures were devised for situations where it was considered the child may be at risk. Although such issues did not arise during the research process, it was considered important to outline what would have been done in such situations (Appendix 26).

\subsection{Chapter Conclusion}

This chapter presented the methodological framework which underpinned the current research design. The research design and data analysis procedures were described in detail. Ethical considerations for undertaking the research were also outlined. Chapter 4 presents the research findings, which emerged from analysis of the data. Findings are presented in case reports, followed by a discussion of findings in Chapter 5. 


\section{Chapter 4 Findings}

\subsection{Overview of Chapter}

This chapter reports the findings of the current research project. Case reports for the Pilot Case, Case 1 and Case 2 are presented. Each report provides a rich case description and findings to explicate the five case propositions. Summary sections demonstrate how the findings address the research question 'Can the Assessment for Intervention Model bridge the gap between assessment and intervention, through activity and interaction at the meso and microsystem levels?'. For each case proposition, Likert scale ratings are presented in tabular format and pattern-matching logic applied to interview responses expands upon these ratings. Analysis of data was informed by the researcher's conceptual framework. Figures are presented for each case proposition, delineating how proposition findings resonate with AFI principles (Pameijer, 2017), EST concepts (Bronfenbrenner, 1979) and SCT concepts (Palinscar, 1998; Vygotsky, 1978). Appendices 27-32 (Pilot Case) 33-38 (Case 1) and 39-44 (Case 2) provide detailed accounts of the analysis of data addressing each of the case propositions, in accordance with the criteria for interpreting findings outlined in Section 3.5.5, including the coding procedure applied for pattern-matching of interview responses. Four themes and their associated subthemes were generated through thematic analysis. The themes included 'A team approach to assessment', 'Perspectives on barriers to parental engagement', 'Factors bridging the gap between assessment and intervention' and 'Views and experiences of AFI'. Proposition findings are discussed under each of these themes, in accordance with the literature and conceptual framework in Chapter 5. Sections 4 and 5 of Appendices 27-44 detail how these themes resonate with proposition findings, for each case proposition. Appendix 45 presents the thematic maps and Appendix 46 outlines the initial, revised and final category clusters and codes applied to interview responses. Appendix 47 provides a sample of the coding applied to a participant's interview transcript and Appendix 48 demonstrates the main themes, subthemes, codes and relevant statements. Entries in the researcher diary (AFI templates) and reflective journal are presented in the analysis of findings, in accordance with the criteria for interpreting findings (See Section 3.5.5 in Chapter 3 and Section 2 of Appendices 27-44). Appendices 49-53 present sample sections of AFI templates that addressed relevant propositions, which are also included in the discussion of findings in Chapter 5. 


\subsection{Pilot Case: Case Description}

John is a ten-year old boy, attending $4^{\text {th }}$ class in a mainstream primary school. He has a diagnosis of ASD and presents with a fixated, restricted interest. John was referred to the school psychological service to provide insight into how to address his engagement in the restricted interest and to broaden his interests. At the initial intake stage, questions of the parent (Louise), teacher (Grace) and child (John) were gathered. These questions, along with background information guided the development of questions for investigation. The case log of actions presented in Table 4.1 outlines the stages and tasks applied to address these questions, in addition to the timescale for the assessment process. 
Table 4.1

Case Log of Actions Pilot Case

\begin{tabular}{ll}
\hline Stage & Actions \\
\hline $\begin{array}{l}\text { 1. Intake: } \\
\left(\text { October } 17^{\text {th }}\right)\end{array}$ & -Initial consultation with John, Louise and Grace \\
$\begin{array}{l}\text { 2.Strategy: } \\
\left(\text { October } 17^{\text {th }}\right)\end{array}$ & $\begin{array}{l}\text {-TEP devised a strategy for moving forward, based on } \\
\text { information gathered at intake }\end{array}$ \\
$\begin{array}{l}\text { 3. Investigation: } \\
(\text { October 23 }\end{array}$ & $\begin{array}{l}\text {-Functional Assessment Interview (FAI) to identify triggers } \\
\text { and maintaining factors associated with restricted interest } \\
\text { (TEP, Louise and Grace) }\end{array}$ \\
& $\begin{array}{l}\text {-Interest Inventory and pie chart task to demonstrate time } \\
\text { spent engaging in the restricted interest and to explore } \\
\text { alternative interests (TEP and John) } \\
\text {-Classroom observation to identify triggers, interactions } \\
\text { working well between John and his teacher and interactions } \\
\text { that may need to be modified (TEP) }\end{array}$
\end{tabular}

3. Investigation: (October $23^{\text {rd }}$ November $7^{\text {th }}$ )

-Completion of behaviour observation charts to identify triggers, maintaining factors, interactions working well, interactions that may need to be modified and John's response to behaviour specific praise (Louise and Grace)

-Discussion with John to identify the aspects of his subjects that are difficult (TEP and John) -Co-investigator activity to record alternative interests that provide a sense of calm (John with support of Louise and Grace)
4. Integration of assessment information: -Meeting with Louise and Grace to discuss assessment information -Meeting with John to discuss co-investigator activity (November $7^{\text {th }}$ ) -Integration of assessment information (TEP)
5. Feedback on report, goals and recommendations: -Meeting with Louise and Grace to discuss needs, goals and intervention recommendations -Meeting with John, Louise and Grace to discuss feedback on (November $14^{\text {th }}$ ) child friendly report, goals and intervention recommendations -John, Louise and Grace chose recommendations to implement.

The proceeding sections present the findings for Propositions 1, 2, 3, 4 and 5 in the pilot study. This is followed by a summary, outlining how the proposition findings address the research question. 
4.3 Proposition 1: 'Parents, teachers and TEP will have a better understanding of the child's situation, as a result of interactions and activities that have occurred at the meso and microsystem levels'

\subsubsection{Likert Scale Ratings}

Likert scale ratings, provided by the class teacher, indicate greater understanding of John's situation after engagement with the AFI model. Ratings, provided by the parent, prove understanding remained unchanged, however, pattern-matching logic applied to interview responses suggest a change in understanding occurred.

Table 4.2

Pilot Case Proposition 1 Ratings

\begin{tabular}{llcc}
\hline Likert Statement & Participant & Before AFI Model & After AFI Model \\
\hline $\begin{array}{l}\text { I understand the } \\
\text { student's situation }\end{array}$ & $\begin{array}{l}\text { Class teacher } \\
\text { (Grace) }\end{array}$ & 7 & 8 \\
$\begin{array}{l}\text { I understand my } \\
\text { child's situation }\end{array}$ & Parent (Louise) & 8 & 8 \\
\hline
\end{tabular}

\subsubsection{Pattern-Matching Logic}

Pattern-matching logic was applied to responses to questions exploring change in understanding of the student's situation and experience of the AFI model. Table 4.3 outlines each participant's key quote, for which pattern-matching logic was applied. Appendix 28 presents the coding procedure and a thorough presentation of the analysis. Patterns within parent and teacher interview responses connected understanding of John's situation to interactions and activities that occurred during the AFI process, at the meso and microsystem level (See Section 2.14 for meso and microsystem level definitions). Louise (parent) noted that she felt "a bit more restrictive about feeding into it [the restricted interest]" following "information taking' and the "team approach to identifying what the issues was". Grace (teacher) commented that "hearing" the parent's voice and "hearing from John's own perspective" helped her to better understand his situation. These responses resonate with the specific activities and interactions that participants engaged in during the assessment process at the micro and mesosystem levels, outlined in Table 4.1, which included dialogue between team members, coinvestigator observation tasks, joint activity tasks and an FAI. Activities and interactions such as "information taking", a "team approach" to identifying issues and 
"hearing" the voices of the parent and child are considered consistent with the AFI process. In accordance with AFI Principle 6, participants actively engaged in these activities and were included in the process as 'co-assessors' (Pameijer, 2017). In addition, obtaining the perspective of the parent, teacher and child is consistent with AFI Principle 2, in applying a 'transactional perspective' to the presenting situation (Pameijer, 2017).

Table 4.3

Pilot Case Key Quotes Addressing Proposition 1

\begin{tabular}{|c|c|}
\hline Participant & Key Quote \\
\hline $\begin{array}{l}\text { Louise } \\
\text { (parent) }\end{array}$ & $\begin{array}{l}\text { When describing how her understanding of John's situation had } \\
\text { changed and her experience of the AFI model, Louise commented: } \\
\text { Yeah, definitely [understanding] because I probably feel a bit more } \\
\text { restrictive about feeding into it [restrictive interest] (line 41)...it } \\
\text { [AFI model] identified and kind of had a team approach to it where } \\
\text { it involved the teacher and information taking (lines 4-5) }\end{array}$ \\
\hline $\begin{array}{l}\text { Grace (class } \\
\text { teacher) }\end{array}$ & $\begin{array}{l}\text { Grace described how her understanding of John's situation had } \\
\text { changed following engagement in the AFI process: I definitely do } \\
\text { (line 70)...it was lovely to actually just to meet her [parent] in this } \\
\text { situation and to be able to hear her voice am...I think to understand } \\
\text { that was a big part of it that there was this kind of opening up of } \\
\text { communication between home and school and then I suppose } \\
\text { hearing from John's own perspective then from chatting to you } \\
\text { (lines 74-77)...am, so I really think...I think I do understand the } \\
\text { situation a bit more (line } 83 \text { ) }\end{array}$ \\
\hline
\end{tabular}

\subsubsection{Researcher Diary and Thematic Analysis}

Consistent with AFI principle 7 (Pameijer, 2017), the researcher adhered to the systematic and transparent assessment process of AFI and progressing understanding of John's situation was evidenced in the AFI templates (researcher diary) and reflective journal (See Appendix 28 and Figure 4.1 below). The templates documented the assessment process and guided hypothesis formation, needs, goals and recommendations throughout the five stages (Appendix 49). Themes within the data set that referred to understanding of John's situation included 'A team approach to assessment', and 'Views and Experiences of AFI'. Proposition 1 findings are discussed under each of these themes, in accordance with the literature and conceptual framework in Sections 5.3 and 5.4 of Chapter 5. Figure 4.1 below delineates how Proposition 1 findings resonate with AFI principles (Pameijer, 2017), EST concepts (Bronfenbrenner, 1979) and SCT concepts (Palinscar, 1998; Vygotsky, 1978). See Chapter 2 for a 
Proposition 1: 'Parents, teachers and TEP will have a better understanding of the child's situation, as a result of interactions and activities that have occurred at the meso and microsystem levels'

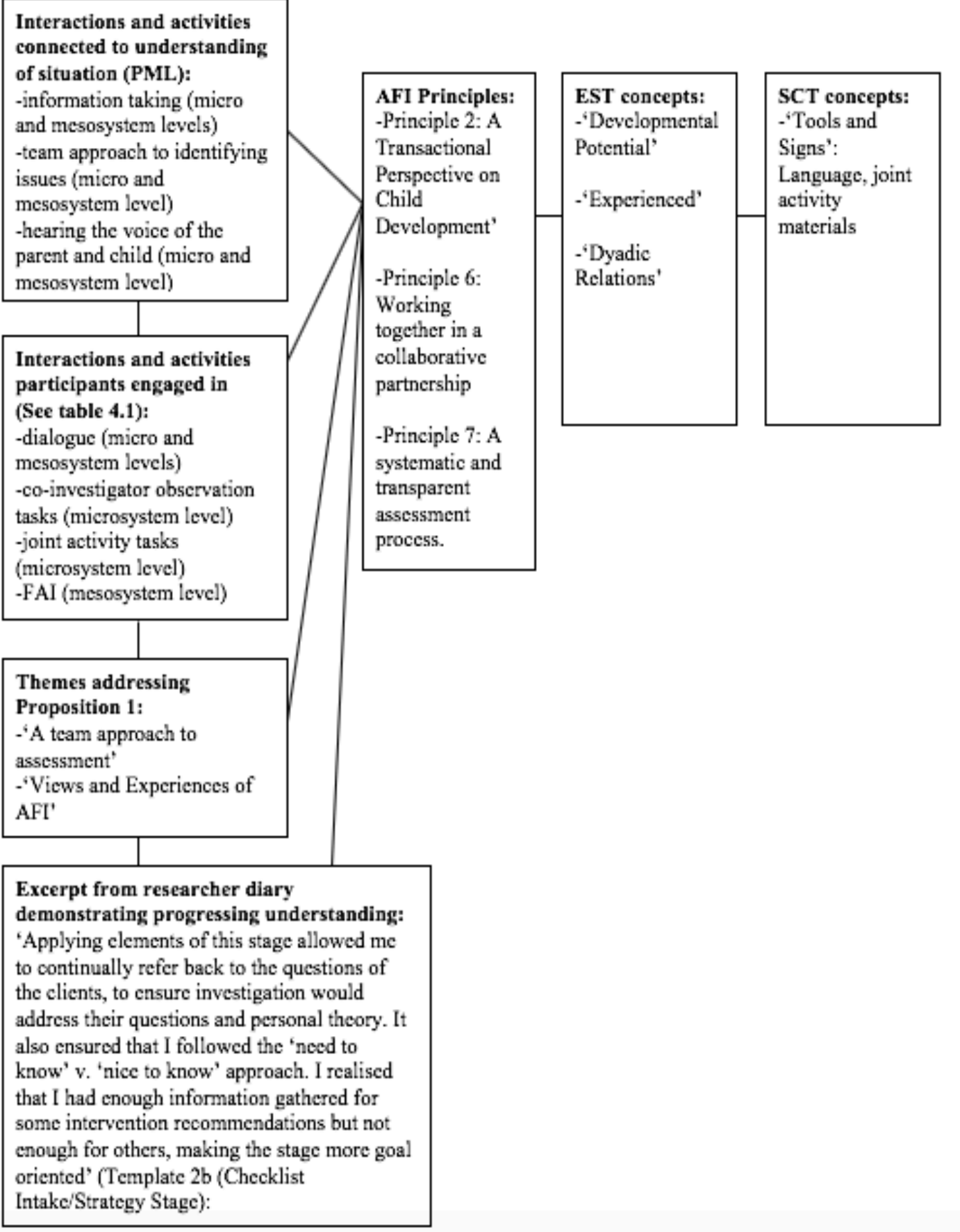

Figure 4.1. Pilot Case Proposition 1 Findings 
4.4 Proposition 2: 'Parents, teachers and TEP will know what intervention supports the child needs at home and at school, as a result of interactions and activities that have occurred at the meso and microsystem levels'

\subsubsection{Likert Scale Ratings}

Higher teacher and parent Likert scale ratings, following engagement with the AFI model, indicates greater knowledge of intervention supports the child needs. Pattern-matching logic applied to interview responses elucidates this finding.

Table 4.4

Pilot Case Proposition 2 Ratings

\begin{tabular}{llcc}
\hline Likert Statement & Participant & Before AFI Model & After AFI Model \\
\hline $\begin{array}{l}\text { I know what support } \\
\text { the student needs at } \\
\text { school }\end{array}$ & $\begin{array}{l}\text { Class teacher } \\
\text { (Grace) }\end{array}$ & 6 & 8 \\
$\begin{array}{l}\text { I know what support } \\
\text { my child needs at } \\
\text { home }\end{array}$ & Parent (Louise) & 2 & 8 \\
\hline
\end{tabular}

\subsubsection{Pattern-Matching Logic}

Pattern-matching logic was applied to responses to questions on intervention recommendations and supporting John's needs (See Table 4.5 below for key quotes and Appendix 29 for a thorough presentation of the analysis). Patterns identified indicate interactions and activities that occurred during the AFI process, at the meso and microsystem levels, led to knowledge of intervention supports that could be applied at home and at school. Grace (class teacher) observed John's (child) positive response to "the close proximity" and "behaviour specific praise" in the classroom during the Investigation stage and Louise (parent) commented that she would "explore other avenues that you might get him interested in", including "having a chat", as this was something that John expressed he would like during a joint activity task with the TEP. The intervention strategies described by Grace and Louise arose from interactions and activities at the meso and microsystem levels, including dialogue between team members, co-investigator observation tasks, joint activity tasks and an FAI (Table 4.1) The activities and interactions resonate with the AFI process as John, Louise and Grace were co-assessors during the Investigation stage and co-constructed knowledge around intervention supports with the TEP (Pameijer, 2017). Furthermore, developing 
intervention strategies to effectively address John's needs is in accordance with AFI

Principles 3 and 4, in identifying teacher and parent approaches that the child needs to achieve a certain goal (Pameijer, 2017).

Table 4.5

Pilot Case Key Quotes Addressing Proposition 2

\begin{tabular}{ll}
\hline Participant & Key Quote \\
\hline $\begin{array}{l}\text { Louise } \\
\text { parent) }\end{array}$ & $\begin{array}{l}\text { Louise referred to her knowledge of intervention strategies, when } \\
\text { describing how she intends to support John's needs: Well I suppose } \\
\text { you're trying to explore other avenues that you might get him } \\
\text { interested in (line 65)...I suppose like maybe just a simple thing that } \\
\text { he identified that he likes having a chat, so it's to sit down with him } \\
\text { and actually say to him am... Do you know why we had to put other } \\
\text { little activities on his agenda (lines 68-70) }\end{array}$ \\
$\begin{array}{l}\text { Grace (class } \\
\text { teacher) }\end{array}$ & $\begin{array}{l}\text { Grace also referred to her knowledge of intervention strategies, } \\
\text { when commenting on how she intends to support John's } \\
\text { needs...And having the other strategies like the close proximity, } \\
\text { the behaviour specific praise, like they're all things I think are... } \\
\text { they're really useful and are useful for any other child in the class as } \\
\text { well but for him especially because he does seem to be responding } \\
\text { to them (lines 142-144) }\end{array}$ \\
\hline
\end{tabular}

\subsubsection{Researcher Diary and Thematic Analysis}

The researcher's knowledge of intervention supports was demonstrated in the AFI templates (researcher diary) and reflective journal (See Appendix 50 and Figure 4.2 below) and the researcher followed a systematic and transparent assessment process, in accordance with AFI Principle 7 (Pameijer, 2017). The theme 'Factors bridging the gap between assessment and intervention' referred to knowledge of intervention supports and Proposition 2 findings are discussed under this theme, in conjunction with the literature and conceptual framework in Section 5.6 (Chapter 5). Figure 4.2 below presents how Proposition 2 findings are consistent with AFI principles (Pameijer, 2017), EST concepts (Bronfenbrenner, 1979) and SCT concepts (Palinscar, 1998; Vygotsky, 1978). See Appendix 29, Section 5 for this analysis. 
Proposition 2: 'Parents, teachers and TEP will know what intervention supports the child needs at home and at school, as a result of interactions and activities that have occurred at the meso and microsystem levels'

Interactions and activities connected to knowledge of intervention supports (PML):

-Intervention recommendations observed and discussed during the AFI process including 'close proximity' and 'bchaviour specific praise' and exploring 'other avenues that you might get him interested in' (micro and mesosystem levels)

Interactions and activities participants engaged in

(See Table 4.1):

-dialogue (micro and mesosystem levels) -co-investigator observation tasks (microsystem level) -joint activity tasks (microsystem level) -FAI (mesosystem level)

\section{Themes addressing}

Proposition 2:

-'Factors bridging the gap between assessment and intervention'

Excerpt from reflective journal demonstrating knowledge of intervention supports:

'Helpful meeting in the sense that information was communicated, John's voice was heard on his suggested replacement interests' (Reflective Journal November $7^{\text {th }}$, Integration stage).

Figure 4.2. Pilot Case Proposition 2 Findings 
4.5 Proposition 3: 'Teachers and parents will have greater perceived competence in supporting the child's needs at home and at school, as a result of interactions and activities that have occurred at the meso and microsystem levels'

\subsubsection{Likert Scale Ratings}

Higher Likert scale ratings indicate greater perceived competence of Grace (class teacher) and Louise (parent) in supporting John's needs after engaging with the AFI model. Pattern-matching logic of interview responses expands upon this finding.

Table 4.6

Pilot Case Proposition 3 Ratings

\begin{tabular}{llcc}
\hline Likert Statement & Participant & Before AFI Model & After AFI Model \\
\hline $\begin{array}{l}\text { I am able to support } \\
\text { the student's needs at } \\
\text { school }\end{array}$ & Class teacher & 6 & 8 \\
$\begin{array}{l}\text { I am able to support } \\
\text { my child's needs at } \\
\text { home }\end{array}$ & Parent & 3 & 8 \\
\hline
\end{tabular}

\subsubsection{Pattern-Matching Logic}

Pattern-matching logic was applied to parent and teacher responses to an interview question exploring their ability to support John's needs (See Table 4.7 below for key quotes and Appendix 30 for the analysis). Patterns within their responses connected activities and interactions that occurred during the AFI process, at the meso and microsystem levels, to perceived competence to support John's needs. Louise's response associated competence with knowledge of alternative interest activities such as "Zumba class and Boy Scouts", suggested by Grace during a meeting at the Strategy Stage. Grace's response connected competence to awareness of "triggers" and understanding "the situation he [John] is coming from". Activities and interactions that led to the identification of triggers and understanding of John's situation are listed in Table 4.1. These activities and interactions are considered consistent with the AFI process for reasons outlined in the following paragraph.

In accordance with AFI Principle 6, all participants were co-assessors during the AFI process and suggestions of alternative interest activities, for example Zumba and Boy Scouts were considered and valued during meetings (Pameijer, 2017). This knowledge of alternative interest activities strengthened Louise's perceived competence 
to support John's needs, as evidenced in Table 4.7 below. Identifying parent and teacher needs to support John moving forward is consistent with AFI Principle 4 and also supported feasible intervention planning, in accordance with AFI Principle 1, 'Goal directed and functional assessment' (Pameijer, 2017). In adherence to this principle, the TEP worked towards developing feasible interventions for participants during the AFI process (Appendix 50). Patterns within Louise's response also connected competence to support John's needs to strategies she already provides in the home environment, "continuing to bring him out for a cycle or a walk in the woods". Consistent with AFI Principle 3, parent and teacher approaches effectively addressing John's needs were encouraged by the TEP during discussions, in adherence with the concept of 'goodness of fit' (Pameijer, 2017). Regarding competence, Grace commented there were still areas of need that she did not feel confident supporting, including helping John to discern between reality and fantasy. She considered that such support warranted outside services and that this issue was "beyond us [school]". These findings are discussed further in Section 5.24 of Chapter 5.

Table 4.7

Pilot Case Key Quotes Addressing Proposition 3

\begin{tabular}{|c|c|}
\hline Participant & Key Quote \\
\hline $\begin{array}{l}\text { Louise } \\
\text { (parent) }\end{array}$ & $\begin{array}{l}\text { When commenting on whether she felt she could support John's } \\
\text { needs at home, Louise stated: Well I suppose you're trying to } \\
\text { explore other avenues that you might get him interested in, i.e. } \\
\text { maybe Zumba class or the Boy Scouts or.....am...maybe look, } \\
\text { continuing with bringing him out for a cycle or a walk in the woods } \\
\text { am (lines 79-81) }\end{array}$ \\
\hline $\begin{array}{l}\text { Grace (class } \\
\text { teacher) }\end{array}$ & $\begin{array}{l}\text { Grace described how she felt about supporting John's needs: I do } \\
\text { feel more confident and because I understand the situation he is } \\
\text { coming from and from maybe little triggers that have been setting it } \\
\text { up, I actually have an understanding now well if the [trigger for } \\
\text { restricted interest occurs] he can [engage in appropriate activities } \\
\text { related to restricted interest] but if it gets to that I can go down and } \\
\text { give a gentle reminder (lines } 144-148 \text { )...but no definitely the reality } \\
\text { and fantasy is a huge thing but that's a much bigger issue than } \\
\text { school like, that's beyond us now so, but that would be the one thing } \\
\text { I wouldn't feel confident in supporting (lines } 164-166 \text { ) }\end{array}$ \\
\hline
\end{tabular}

\subsubsection{Thematic Analysis}

Themes within the data set that referred to competence in supporting John's needs included 'A team approach to assessment', 'Perspectives on barriers to parental 
engagement' and 'Factors bridging the gap between assessment and intervention'. Proposition 3 findings are discussed under these themes, in accordance with the literature and conceptual framework in Sections 5.4, 5.5 and 5.6. Figure 4.3 below presents how Proposition 3 findings are consistent with AFI principles (Pameijer, 2017) and EST concepts (Bronfenbrenner, 1979). See Section 5 in Appendix 30 for this analysis.

Proposition 3: 'Teachers and parents will have greater perceived competence in supporting the child's needs at home and at school, as a result of interactions and activities that have occurred at the meso and microsystem levels'

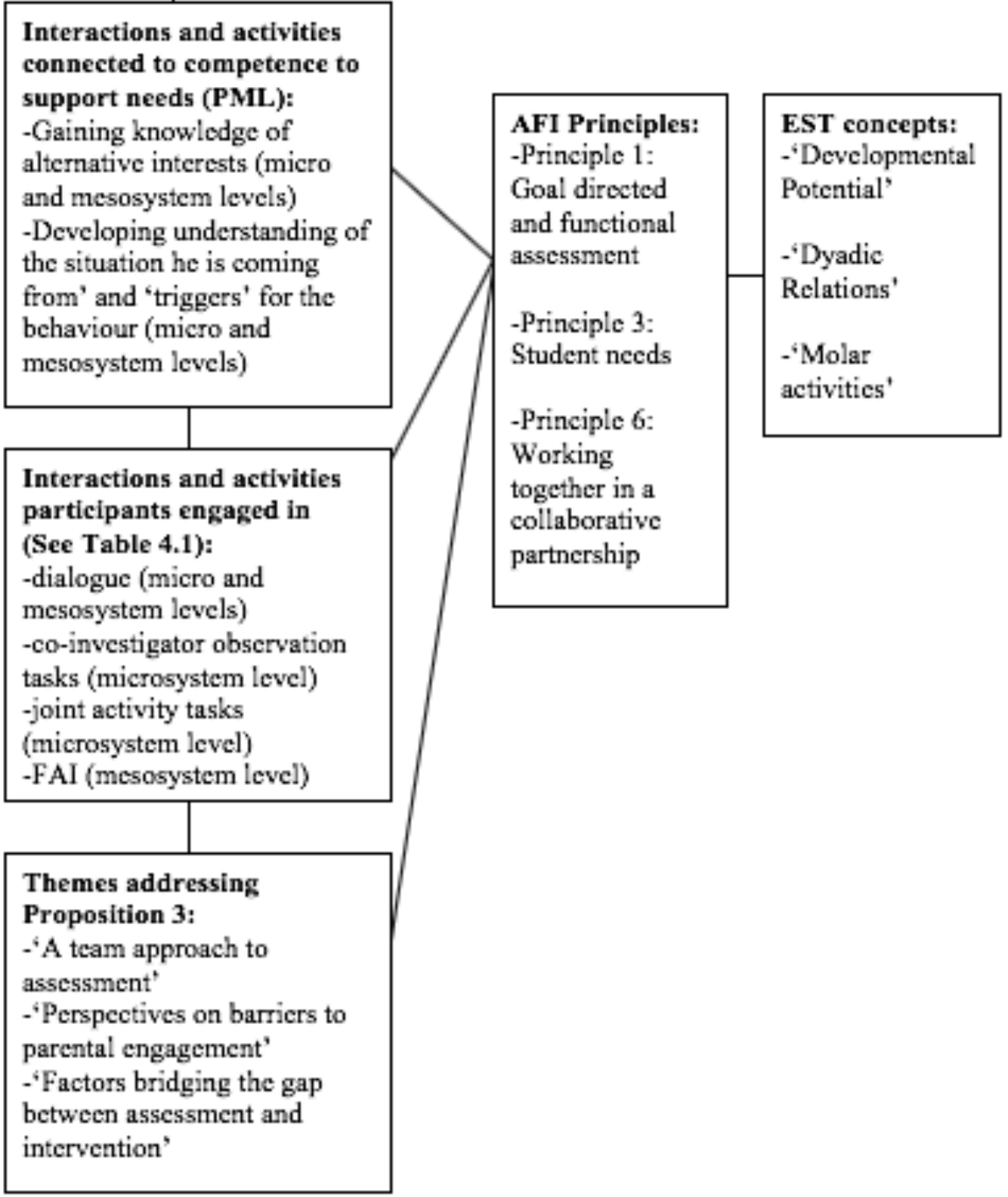

Figure 4.3. Pilot Case Proposition 3 Findings 
4.6 Proposition 4: 'The child will have a better understanding of their strengths and needs and what to do to improve their needs, as a result of interactions and activities that have occurred at the meso and microsystem levels'

\subsubsection{Likert Scale Ratings}

Likert scale ratings provided by John indicate that he was "not sure" of his strengths following engagement with the AFI model. His ratings show that he now knows what he needs help with at school and still does not know what he can do to improve on his needs at school. Pattern-matching logic of interview responses expands upon these ratings.

Table 4.8

Pilot Case Proposition 4 Ratings

\begin{tabular}{llcc}
\hline Likert Statement & Participant & Before AFI Model & After AFI Model \\
\hline $\begin{array}{l}\text { I know what my } \\
\text { strengths are at school }\end{array}$ & Student & Yes & Not sure \\
$\begin{array}{l}\text { I know what I need } \\
\text { help with at school }\end{array}$ & Student & Not sure & Yes \\
$\begin{array}{l}\text { I know what I can do } \\
\text { to get better at what I } \\
\text { need help with at } \\
\text { school }\end{array}$ & Student & No & No \\
\hline
\end{tabular}

\subsubsection{Pattern-Matching Logic}

Pattern-matching logic was applied to responses to interview questions exploring John's thoughts on his strengths, his needs, and what he needs to do to address needs. He was also asked a question on his experience of working together with his mother, teacher and the TEP (See Table 4.9 below for key quotes and Appendix 31 for the analysis). Patterns within John's responses connected understanding of strengths and needs to interactions and activities that occurred during the AFI process, at the microsystem level. Working together with the TEP, parent and teacher was associated with a "kind of" understanding of strengths and needs. Activities and interactions where John worked with the TEP, parent and teacher included dialogue throughout the assessment process, co-investigator observation tasks and joint activity tasks to identify alternative interests and activities that provide him with a sense of calm (Table 4.1). These activities and interactions are considered consistent with AFI Principle 6 
(Pameijer, 2017) as John acted as a co-assessor when completing co-investigator tasks and his opinions and feedback contributed to intervention planning (Appendix 50). Patterns within John's response indicate that it was more difficult to find out about his strengths and needs with his mother and teacher, than with the TEP and that the assessment process was a challenging experience with making "hard decisions". Making decisions around engaging in alternative interests may have been a difficult experience for John as the restricted interest provides him with a sense of calm and security. With regards addressing needs moving forward, patterns within John's response were associated with interactions and activities that occurred during the AFI process, at the meso and microsystem level. John mentioned to "study my best at school" and engaging in "new activities", which were identified and discussed during joint activity tasks at the Investigation stage.

Table 4.9

Pilot Case Key Quotes Addressing Proposition 4

\begin{tabular}{|c|c|}
\hline Participant & Key Quote \\
\hline \multirow[t]{4}{*}{ John (child) } & $\begin{array}{l}\text { When asked if working together with his mother, teachers and TEP } \\
\text { helped in finding out about his strengths, John responded: Kind of } \\
\text { (line 22)... at least I know a little bit about working with you but it's } \\
\text { hard to do with the teachers and mom and yeah (lines } 24-25)\end{array}$ \\
\hline & $\begin{array}{l}\text { When asked if working together with his mother, teachers and TEP } \\
\text { helped in finding out about what he needs help with, John } \\
\text { commented: Kind of (line } 30) . . . \text { I think for that question I wouldn't } \\
\text { really want to answer (line } 32 \text { ) }\end{array}$ \\
\hline & $\begin{array}{l}\text { When asked if he knew what to do to address these areas of need, } \\
\text { John replied: Kind of yeah (line } 39) \text {... Study my best in school } \\
\text { (line } 41) . . \text { [Interviewer: What about your new activities]...Yeah that } \\
\text { would be one (line } 43 \text { ) }\end{array}$ \\
\hline & $\begin{array}{l}\text { When describing his experience of working with the TEP, teacher } \\
\text { and parent, John commented: Part of the time it would be hard, part } \\
\text { of the time it could be easy but most of the time its hard (line 7)... } \\
\text { [Interviewer: Tell me a little bit more about that]...Just doing hard } \\
\text { decisions (line 9) }\end{array}$ \\
\hline
\end{tabular}

\subsubsection{Thematic Analysis}

The theme 'A team approach to assessment' referred to John's understanding of strengths and needs and what to do to address needs. Proposition 4 findings are discussed under this theme, in conjunction with the literature and conceptual framework 
in Section 5.4. Figure 4.4 below outlines how Proposition 4 findings are in agreement with AFI principles (Pameijer, 2017), EST concepts (Bronfenbrenner, 1979) and SCT concepts (Palinscar, 1998; Vygotsky, 1978). Section 5 in Appendix 31 presents this analysis in detail.

Proposition 4: 'The child will have a better understanding of their strengths and needs and what to do to improve their needs, as a result of interactions and activities that have occurred at the meso and microsystem levels'

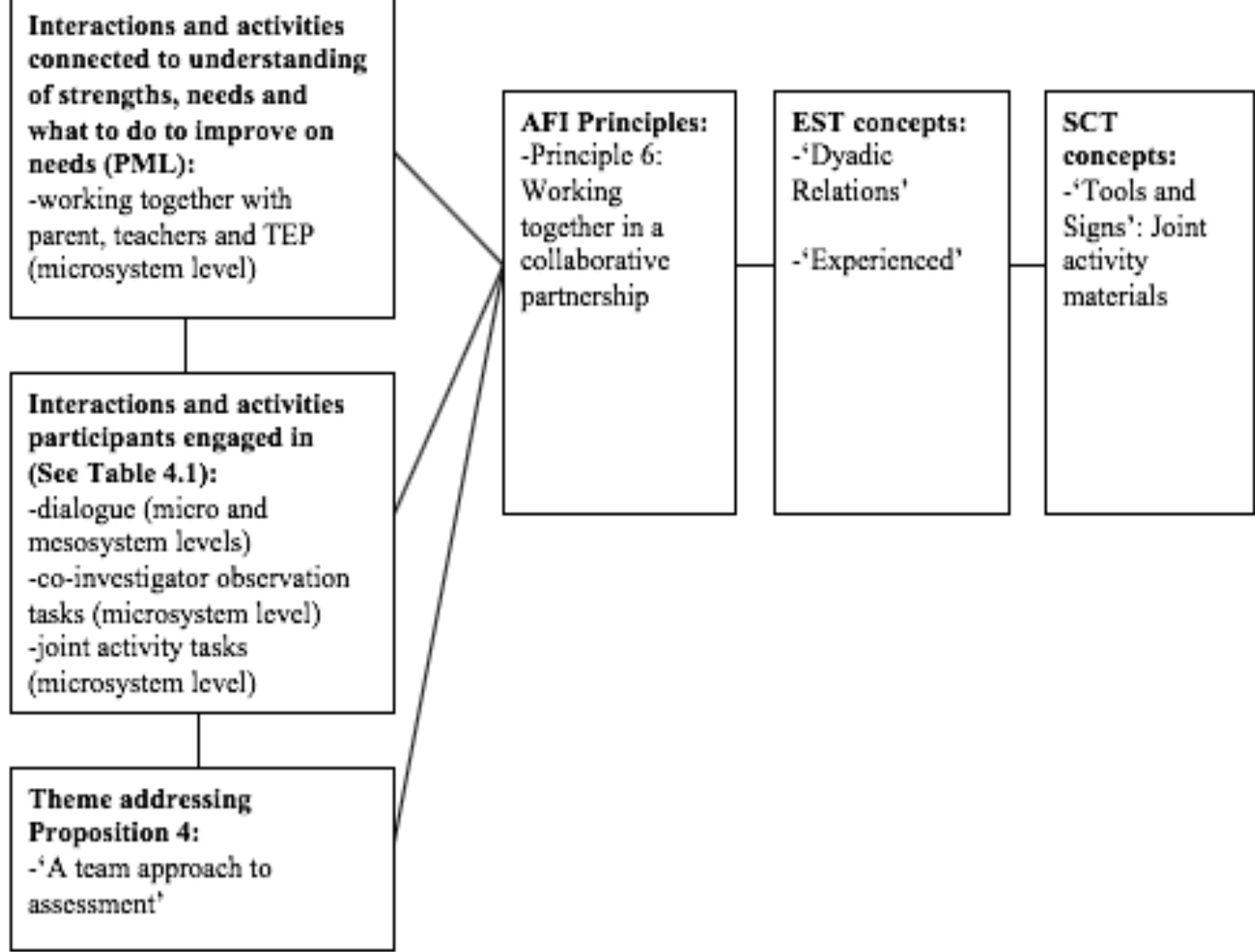

Figure 4.4. Pilot Case Proposition 4 Findings 
4.7 Proposition 5: 'At a time of ecological transition, teachers will feel competent in their ability to work according to the aims of Circular 0013/2017, in meeting and monitoring the needs of the child'

\subsubsection{Pattern-Matching Logic}

Pattern-matching logic was applied to Grace's (teacher) response to an interview question exploring her perceived levels of competence to monitor John's needs moving forward (See Table 4.10 below for key quotes and Appendix 32 for the analysis). Patterns within her response connected competence to meet and monitor John's needs to "being aware of the situation". Awareness of the situation resulted from interactions and activities that occurred during the AFI process, at the meso and microsystem level, listed in Table 4.1. These activities and interactions are consistent with AFI Principle 6 as participants were co-assessors during the Investigation stage and their opinions and feedback were valued as 'hands-on experts through experience' throughout the process (Pameijer, 2017). Increased awareness of the situation also resonates with AFI Principle 3, with greater awareness and insight into John's needs. Patterns within Grace's response also connected competence to meet and monitor John's needs to her general teaching skills, as this is something that she would "do with everyone anyway". In accordance with AFI Principle 3 and the concept of goodness of fit, effective monitoring approaches were encouraged by the TEP during discussions (Pameijer, 2017).

Table 4.10

Pilot Case Key Quotes Addressing Proposition 5

\begin{tabular}{ll}
\hline Participant & Key Quote \\
\hline Grace (class & When asked if she felt she could monitor John's needs moving \\
teacher) & forward, Grace commented: Yeah I think that's something that I \\
& will be able to do I mean it's something that I do with everyone \\
& anyway...but especially because I think I'm aware of the situation \\
& and because the behaviours they were, I think it's something that I'd \\
& be extra tuned into anyway, am, but I couldn't see any issue in \\
& monitoring his progress especially because even over the last few \\
& weeks I have seen an improvement so if it only continues on, I'd \\
& definitely be able to compare (lines 221-225)
\end{tabular}

\subsubsection{Thematic Analysis}

The theme 'Factors bridging the gap between assessment and intervention' referred to meeting and monitoring of needs. Proposition 5 findings are discussed under 
this theme, in conjunction with the literature and conceptual framework in Section 5.6. Figure 4.5 below delineates how Proposition 5 findings resonate with AFI principles (Pameijer, 2017) and EST concepts (Bronfenbrenner, 1979). This analysis is presented in Section 5 in Appendix 32.

Proposition 5: 'At a time of ecological transition, teachers will feel competent in their ability to work according to the aims of Circular 0013/2017, in meeting and monitoring the needs of the child'

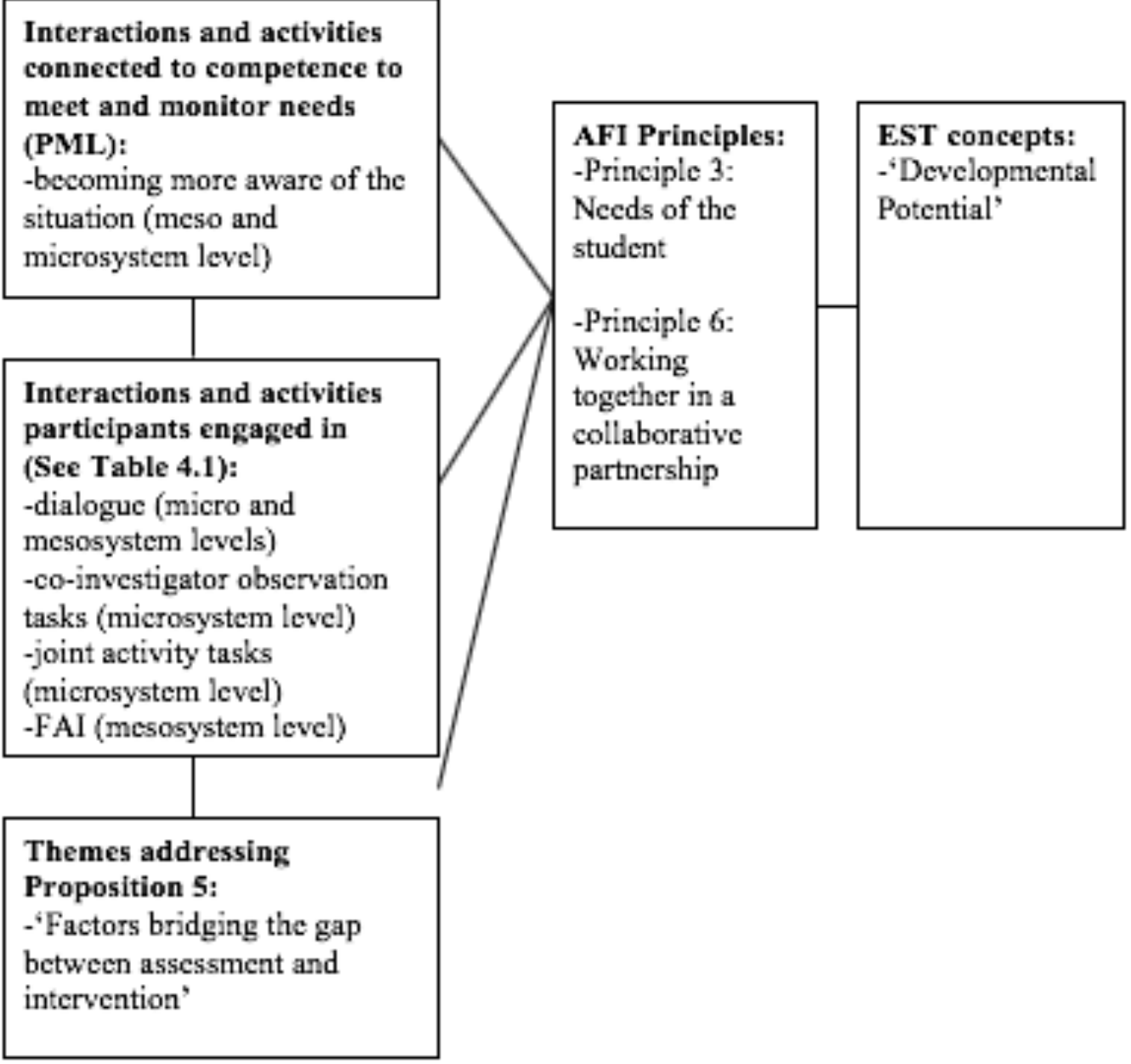

Figure 4.5. Pilot Case Proposition 5 Findings 


\subsection{Summary of Pilot Case Proposition Findings}

Overall, the findings of the Pilot Case suggest the AFI model can bridge the gap between assessment and intervention through activity and interaction at the meso and microsystem levels. Supporting evidence was presented for Proposition 1, 2, 3 and 5. Likert scale ratings and pattern-matching of parent and teacher interview responses indicated greater understanding of John's situation, knowledge of intervention supports and greater perceived competence in supporting and monitoring John's needs following engagement with the AFI model. Activities undertaken at the micro level included dialogue between team members, co-investigator observation tasks, joint activity tasks and an FAI. Activities undertaken at the mesosystem level included meetings and dialogue between the TEP, teacher and parent (Table 4.1). Evidence was presented to support Proposition 4 in the Pilot Case. John responded "kind of" to questions exploring if working together helped him to find out about his strengths and needs. His comments during the interview indicate his experience of the team assessment may have been challenging with "doing hard decisions". Figures 4.1, 4.2, 4.3, 4.4 and 4.5 outlined how proposition findings resonate with AFI principles (Pameijer, 2017), EST concepts (Bronfenbrenner, 1979) and SCT concepts (Palinscar, 1998; Vygotsky, 1978). Findings are discussed in greater depth under related themes in Chapter 5, in accordance with the literature and conceptual framework. Applicability of the AFI model to other situations is also discussed. Case 1 findings are presented overleaf. 


\subsection{Case 1: Case description}

$\mathrm{Jim}$ is a ten-year old boy, attending $4^{\text {th }}$ class in a mainstream primary school. He was referred to the school psychological service to provide insight into difficulties in literacy and numeracy and information on specific supports and strategies to target these areas. At the Intake stage, questions of the parent (Tina), class teacher (Michael), Special Education Teacher (Sarah) and child (Jim) were gathered. These questions, along with background information guided the development of questions for investigation. The case $\log$ of actions presented in Table 4.11 outlines the stages and tasks applied to address these questions, in addition to the timescale for the assessment process. 
Table 4.11

Case Log of Actions Case 1

\begin{tabular}{|c|c|}
\hline Stage & Actions \\
\hline $\begin{array}{l}\text { 1. Intake } \\
\left(\text { November } 22^{\text {nd }} \text { ) }\right.\end{array}$ & -Initial consultation with Jim, his mother and teachers \\
\hline $\begin{array}{l}\text { 2. Strategy } \\
\text { (November } 22^{\text {nd }} \text { ) }\end{array}$ & $\begin{array}{l}\text {-TEP devised a strategy for moving forward, based on } \\
\text { information gathered at intake }\end{array}$ \\
\hline $\begin{array}{l}\text { 3. Investigation } \\
\text { (November } 28^{\text {th }} / \\
\left.29^{\text {th }}\right)\end{array}$ & $\begin{array}{l}-1: 1 \text { attainment and informal testing in areas of literacy (word } \\
\text { reading, pseudoword decoding, spelling) and numeracy/problem } \\
\text { solving skills (The Wechsler Individual Achievement Test } \\
\text { (WIAT-III }{ }^{\text {UK }} \text { ) } \\
-1: 1 \text { assessment of cognitive strengths and weaknesses (The } \\
\text { Wechsler Scale of Intelligence for Children-Fifth UK Edition- } \\
\text { WISC-V }{ }^{\mathrm{UK}} \text { ) } \\
\text {-Dynamic assessment during cognitive and attainment testing } \\
\text {-Running record of reading and oral comprehension of a reading } \\
\text { passage and analysis of a written passage (TEP) } \\
\text {-Classroom ecological observation to observe Jim's learning in } \\
\text { context, task expectations and response to strategies and } \\
\text { methodologies (TEP) }\end{array}$ \\
\hline
\end{tabular}

3. Investigation (November 29 ${ }^{\text {th }}$ December $5^{\text {th }}$ )

-Completion of observation sheets to observe Jim's learning in context, task expectations and response to strategies and methodologies (Tina, Michael and Sarah) -Co-investigator activity to identify the strategies that help Jim learn (Jim, with the support of Tina, Michael and Sarah)

4. Integration of assessment information -Meeting with Jim's teachers to discuss assessment information -Phone call with Tina to discuss assessment information (December $6^{\text {th }}$ ) -Meeting with Jim to discuss co-investigator activity -Integration of assessment information (TEP)

5. Feedback on report, goals and recommendations (December $12^{\text {th }}$ )

-Meeting with Tina, Michael and Sarah to discuss needs, goals and intervention recommendations

-Meeting with Jim, his mother and teachers to discuss feedback on child friendly report and to choose goals and intervention recommendations.

The proceeding sections present the findings for Propositions 1, 2, 3, 4 and 5 in Case 1. This is followed by a summary, outlining how the proposition findings address the research question. 
4.10 Proposition 1: 'Parents, teachers and TEP will have a better understanding of the child's situation, as a result of interactions and activities that have occurred at the meso and microsystem levels'

\subsubsection{Likert Scale Ratings}

Likert scale ratings indicate greater understanding of the child's situation after engaging with the AFI model. Pattern-matching logic applied to interview responses elaborates upon this finding in the section below.

Table 4.12

Case 1 Proposition 1 Ratings

\begin{tabular}{llcc}
\hline Likert Statement & Participant & Before AFI Model & After AFI Model \\
\hline $\begin{array}{l}\text { I understand the } \\
\text { student's situation }\end{array}$ & $\begin{array}{l}\text { Class teacher } \\
\text { (Michael) }\end{array}$ & 4 & 9 \\
$\begin{array}{l}\text { I understand the } \\
\text { student's situation }\end{array}$ & SET (Sarah) & 3 & 10 \\
$\begin{array}{l}\text { I understand my } \\
\text { child's situation }\end{array}$ & Parent (Tina) & 4 & 5 \\
\hline
\end{tabular}

\subsubsection{Pattern-Matching Logic}

Pattern-matching logic was applied to responses to interview questions exploring change in understanding of the student's situation, and experience of the AFI model (See Table 4.13 below for key quotes and Appendix 34 for the analysis). Patterns within parent and teacher interview responses connected greater understanding of the situation to interactions and activities that occurred during the AFI process, at the micro and mesosystem levels, including dialogue between team members, co-investigator observation tasks and 1:1 assessments (Table 4.11). Michael (class teacher) commented "getting the voice of the pupil, our voice [teachers] and the parent was really good" and helped to provide "a better understanding of the specific needs". Sarah (SET) responded, "Yes 100\%, 100\%", when asked if her understanding of Jim's situation had changed and reflected that she "never realised there was any working memory problem". Working memory was established as an area of cognitive weakness following 1:1 assessments with the TEP at the microsystem level and completion of a teacher task expectation observation sheet. Tina (parent) commented "I understand more about Jim" as a result of "discussing things" with Jim's teachers and the TEP during meetings at 
the mesosystem level (Table 4.11). Activities and interactions connected to Proposition 1 are considered unique to the AFI process as consistent with AFI Principle 6, participants actively engaged in activities and were included in the process as coassessors (Pameijer, 2017).

Table 4.13

Case 1 Key Quotes Addressing Proposition 1

\begin{tabular}{ll}
\hline Participant & Key Quote \\
\hline Tina (parent) & $\begin{array}{l}\text { When describing her experience of the AFI model, Tina commented: } \\
\text { Yeah it [AFI model] was good like, it was interesting as well you } \\
\text { know and I understand more about Jim (line 3)... discussing things } \\
\text { like you know, about Jim at home and his work and I know more } \\
\text { about Jim about his work and his homework and stuff (lines 5-6) }\end{array}$ \\
& $\begin{array}{l}\text { When describing his experience of the AFI model, Michael noted: I } \\
\text { Michael } \\
\text { (class } \\
\text { teacher) }\end{array}$ \\
& $\begin{array}{l}\text { really good and am, just helps us get a better understanding of the } \\
\text { specific needs and then the various stages of it made it very clear } \\
\text { (lines 3-5) }\end{array}$ \\
& $\begin{array}{l}\text { Sarah described how her understanding of Jim's situation had } \\
\text { changed following engagement in the AFI process: Yes 100\%, } \\
\text { Sarah (SET) } \\
\text { 100\% [understanding] (line 78)...I never realised there was any } \\
\text { working memory problem whatsoever which is huge in my opinion, } \\
\text { like it's immense like if that was gone unnoticed... it would have } \\
\text { been a disaster for him (lines 83-85) }\end{array}$ \\
\hline
\end{tabular}

\subsubsection{Researcher Diary and Thematic Analysis}

In accordance with AFI principle 7, the researcher followed a systematic and transparent assessment process and understanding of Jim's situation was evidenced in the AFI templates (researcher diary) and reflective journal (See Appendix 51 and Figure 4.6 below). Themes within the data set that referred to understanding of Jim's situation included 'A team approach to assessment', 'Perspectives on barriers to parental engagement' and 'Views and Experiences of AFI'. Proposition 1 findings are discussed under each of these themes, in accordance with the literature and conceptual framework in Sections 5.9, 5.10 and 5.11 of Chapter 5. Figure 4.6 below delineates how Proposition 1 findings are consistent with AFI principles (Pameijer, 2017), EST concepts (Bronfenbrenner, 1979) and SCT concepts (Palinscar, 1998; Vygotsky, 1978). This analysis is outlined in Appendix 34 (Section 5). 
Proposition 1: 'Parents, teachers and TEP will have a better understanding of the child's situation, as a result of interactions and activities that have occurred at the meso and microsystem levels'

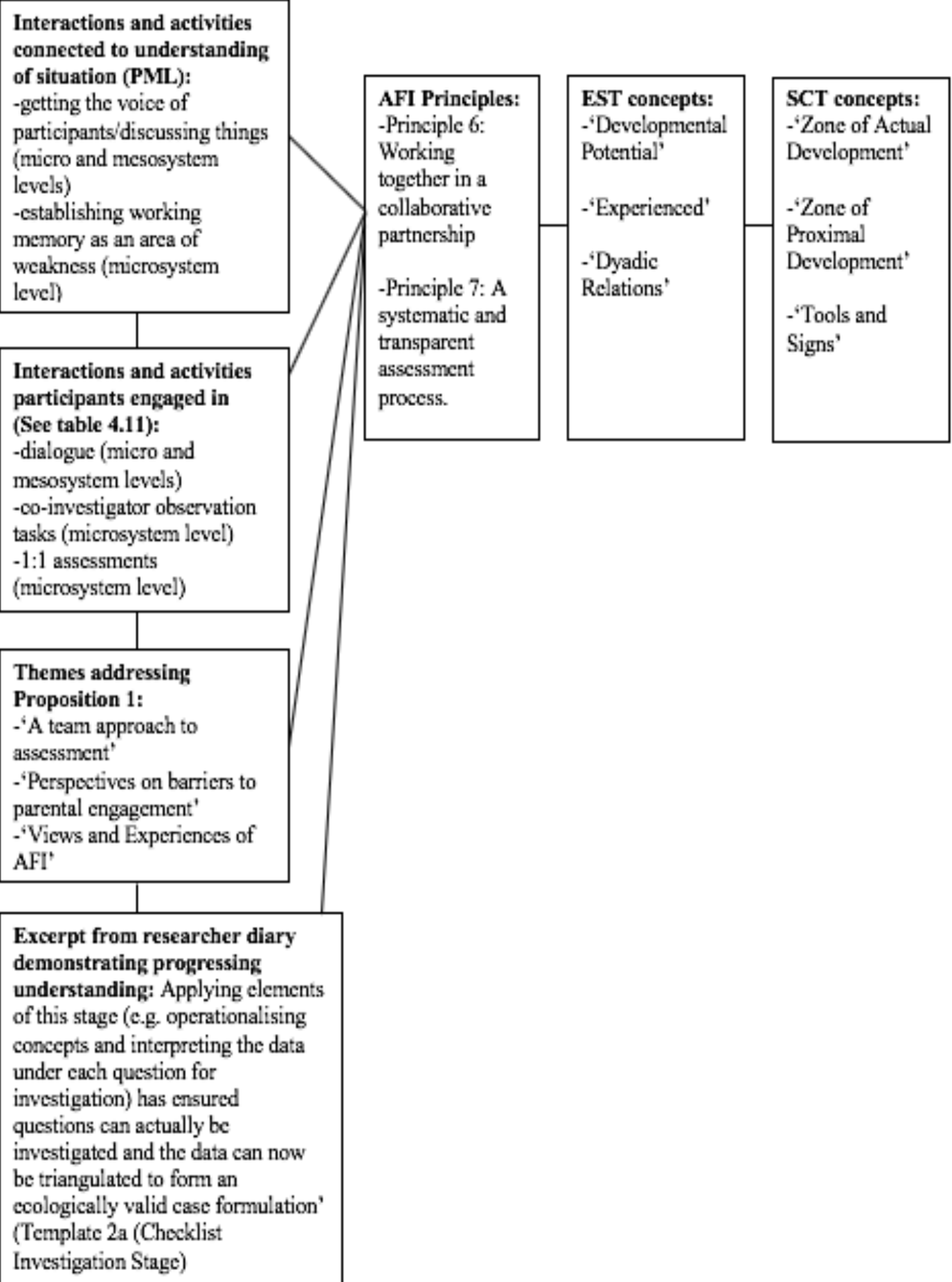

Figure 4.6. Case 1 Proposition 1 Findings 
4.11 Proposition 2: 'Parents, teachers and TEP will know what intervention supports the child needs at home and at school, as a result of interactions and activities that have occurred at the meso and microsystem levels'

\subsubsection{Likert Scale Ratings}

Higher ratings were provided on knowledge of supports that the student needs, after engaging with the AFI model. Pattern-matching logic applied to interview responses expands upon this finding.

Table 4.14

Case 1 Proposition 2 Ratings

\begin{tabular}{llcc}
\hline Likert Statement & Participant & Before AFI Model & After AFI Model \\
\hline $\begin{array}{l}\text { I know what support } \\
\text { the student needs at } \\
\text { school }\end{array}$ & Class teacher & 5 & 9 \\
$\begin{array}{l}\text { I know what support } \\
\text { the student needs at } \\
\text { school }\end{array}$ & SET & 5 & 10 \\
$\begin{array}{l}\text { I know what support } \\
\text { my child needs at } \\
\text { home }\end{array}$ & Parent & 5 & 10 \\
\hline
\end{tabular}

\subsubsection{Pattern-Matching Logic}

Pattern-matching logic was applied to responses to questions on intervention recommendations and supporting Jim's needs (See Table 4.15 below for key quotes and Appendix 35 for the analysis). Patterns within parent and teacher interview responses connected knowledge of intervention supports to interactions and activities that occurred during the AFI process, at the meso and microsystem levels. Michael referred to intervention supports discussed at the Feedback stage, including "appraisal" and "ideas for working on his oral language and his memory and auditory skills". These intervention strategies developed from interactions and activities at the meso and microsystem levels, including dialogue between team members, co-investigator observation tasks and 1:1 assessments (Table 4.11). Similarly, Sarah and Tina referred to their knowledge of the "acronym", a mnemonic intervention for spelling, which arose during 1:1 dynamic assessment with Jim and became a strategy that he later shared with team members as helping him to learn from his co-investigator activity. These activities 
and interactions resonate with AFI Principle 6, as participants were co-assessors during the Investigation stage and co-constructed knowledge around intervention with the TEP (Pameijer, 2017). In addition, intervention strategies that effectively addressed Jim's needs are in accordance with AFI Principles 3 and 4, identifying teacher and parent approaches that the child needs to achieve a certain goal (Pameijer, 2017).

Table 4.15

Case 1 Key Quotes Addressing Proposition 2

\begin{tabular}{ll}
\hline Participant & Key Quote \\
\hline Tina (parent) & $\begin{array}{l}\text { Tina referred to her knowledge of the spelling mnemonic aid, } \\
\text { when commenting on intervention recommendations: Yeah } \\
\text { because at home with the spellings and stuff...like if I told him to } \\
\text { do spellings at home like, if I was doing it like you were doing } \\
\text { [with mnemonic aid], he would do it after me, he would do it like } \\
\text { (lines 129-130) }\end{array}$ \\
& $\begin{array}{l}\text { When describing how intervention recommendations addressed } \\
\text { Michael } \\
\text { (class }\end{array}$ \\
teacher) & $\begin{array}{l}\text { his questions at the Intake stage, Michael noted: Oh the } \\
\text { recommendations they did for sure, I suppose definitely the }\end{array}$ \\
& $\begin{array}{l}\text { working on his oral language and his memory and auditory skills } \\
\text { (lines 69-71) }\end{array}$ \\
Sarah (SET) & $\begin{array}{l}\text { Sarah also referred to her knowledge of the spelling mnemonic } \\
\text { aid, when describing how she intends to support Jim's needs: We } \\
\text { did notice from the offset that he wasn't overly strong in spelling, } \\
\text { but now from the recommendations and even Jim, I suppose it } \\
\text { stands out more so in my head because he's so keen to use this } \\
\text { recommendation which is brilliant, it's the acronym for a word } \\
\text { (lines 118-121) }\end{array}$
\end{tabular}

\subsubsection{Researcher Diary and Thematic Analysis}

Consistent with AFI principle 7 (Pameijer, 2017), the researcher applied a systematic and transparent assessment process and knowledge of intervention supports was demonstrated in the AFI templates and reflective journal (See Appendix 52 and Figure 4.7 below). The theme 'Factors bridging the gap between assessment and intervention' referred to knowledge of intervention supports and Proposition 2 findings are discussed in greater depth under this theme, in conjunction with the literature and conceptual framework in Section 5.12. Figure 4.7 below presents how Proposition 2 findings are consistent with AFI principles (Pameijer, 2017), EST concepts (Bronfenbrenner, 1979) and SCT concepts (Palinscar, 1998; Vygotsky, 1978). See Appendix 35, Section 5 for this analysis. 
Proposition 2: 'Parents, teachers and TEP will know what intervention supports the child needs at home and at school, as a result of interactions and activities that have occurred at the meso and microsystem levels'

Interactions and activities connected to knowledge of intervention supports (PML):

-Intervention recommendations discussed during the AFI process including appraisal, spelling acronym and ideas to address memory and auditory skills (micro and mesosystem levels)

\section{Interactions and activities} participants engaged in (See Table 4.11): -dialogue (micro and mesosystem levels) -co-investigator observation tasks (microsystem level) $-1: 1$ assessments

\section{Themes addressing}

Proposition 2:

-'Factors bridging the gap between assessment and intervention'

\section{Excerpt from reflective} journal demonstrating knowledge of intervention supports: 'Teachers provided feodback on their observations, the SET reflected on her expectations and teaching strategies working well/what could be modified. I shared some strategies that I found worked well during testing"

(Reflective Journal

November $7^{\hbar}$, Integration stage). 
4.12 Proposition 3: 'Teachers and parents will have greater perceived competence in supporting the child's needs at home and at school, as a result of interactions and activities that have occurred at the meso and microsystem levels'

\subsubsection{Likert Scale Ratings}

Likert scale ratings indicate greater perceived competence in supporting Jim's needs at home and at school following engagement with the AFI model. Patternmatching logic of interview responses expands upon this finding.

Table 4.16

Case 1 Proposition 3 Ratings

\begin{tabular}{llcc}
\hline Likert Statement & Participant & Before AFI Model & After AFI Model \\
\hline $\begin{array}{l}\text { I am able to support } \\
\text { the student's needs at } \\
\text { school }\end{array}$ & Class teacher & 3 & 9 \\
$\begin{array}{l}\text { I am able to support } \\
\text { the student's needs at } \\
\text { school }\end{array}$ & SET & 4 & 10 \\
$\begin{array}{l}\text { I am able to support } \\
\text { my child's needs at } \\
\text { home }\end{array}$ & Parent & 6 & 10 \\
\hline
\end{tabular}

\subsubsection{Pattern-Matching Logic}

Pattern-matching logic applied to responses to an interview question exploring ability to support Jim's needs, indicates interactions and activities that occurred during the AFI process, at the micro and mesosystem levels contributed to greater perceived competence to support his needs (See Table 4.17 below for key quotes and Appendix 36 for the analysis). Michael commented that he feels "more prepared and equipped to support them [needs] now" and attributes this to knowing "what activities and what areas would help Jim", the "recommendations" that will address his needs and his "strong relationship" with the SET and parent. Similarly, Sarah commented that "100\%" she feels better able to support Jim's needs because she now knows "exactly what his needs are" and feels they can "work on those recommendations". Tina also commented that she feels she can support Jim because she knows "what to do now". Interactions and activities at the meso and microsystem levels that led to the identification of Jim's needs and the formation of recommendations to address these 
needs are outlined in Table 4.11. Consistent with AFI Principle 6 (Pameijer, 2017), all participants were co-assessors during the Investigation stage and their active participation led to the identification of needs and subsequently, intervention recommendations. Competence to support needs is also consistent with AFI Principle 1, 'Goal directed and functional assessment', with the TEP aiming at feasible intervention recommendations for participants (Pameijer, 2017).

Table 4.17

Key Quotes Addressing Proposition 3

\begin{tabular}{ll}
\hline Participant & Key Quote \\
\hline Tina (parent) & $\begin{array}{l}\text { When commenting on whether she felt she could support Jim 's } \\
\text { needs, Tina stated: Yeah, I can now, cause I know what to do now } \\
\text { (line 163) }\end{array}$ \\
$\begin{array}{l}\text { Michael } \\
\text { (class }\end{array}$ & $\begin{array}{l}\text { Michael described how he felt about supporting Jim 's needs: I } \\
\text { definitely feel more prepared and equipped to support them [needs] } \\
\text { now, so am I think yeah for sure having a very strong relationship } \\
\text { with the learning support teacher and with the parents and obviously } \\
\text { the recommendations here, I think yeah like, investing time and } \\
\text { prioritising, you know his needs, which I'm definitely more aware of } \\
\text { now, I think we can support them better (lines 74-77) }\end{array}$ \\
& $\begin{array}{l}\text { When commenting on whether she felt she could support Jim's } \\
\text { needs, Sarah stated: Yes 100\% now, did I feel at the beginning, no I } \\
\text { didn't feel that I could, I wasn't sure of his needs. Now I know } \\
\text { exactly what his needs are, I know what his needs are in literacy and } \\
\text { in numeracy and with regard to working memory, I know his needs } \\
\text { and I know that the recommendations that you have given us, I } \\
\text { know that we can work on those recommendations (lines 109-112) }\end{array}$ \\
\hline
\end{tabular}

\subsubsection{Thematic Analysis}

Themes within the data set that referred to competence in supporting Jim's needs included 'A team approach to assessment', 'Perspectives on barriers to parental engagement' and 'Factors bridging the gap between assessment and intervention'. Proposition 3 findings are discussed under each of these themes, in accordance with the literature and conceptual framework in Sections 5.10, 5.11 and 5.12. Figure 4.8 below outlines how Proposition 3 findings are in accordance with AFI principles (Pameijer, 2017) and EST concepts (Bronfenbrenner, 1979). See Appendix 36, Section 5 for this analysis. 
Proposition 3: 'Teachers and parents will have greater perceived competence in supporting the child's needs at home and at school, as a result of interactions and activities that have occurred at the meso and microsystem levels'

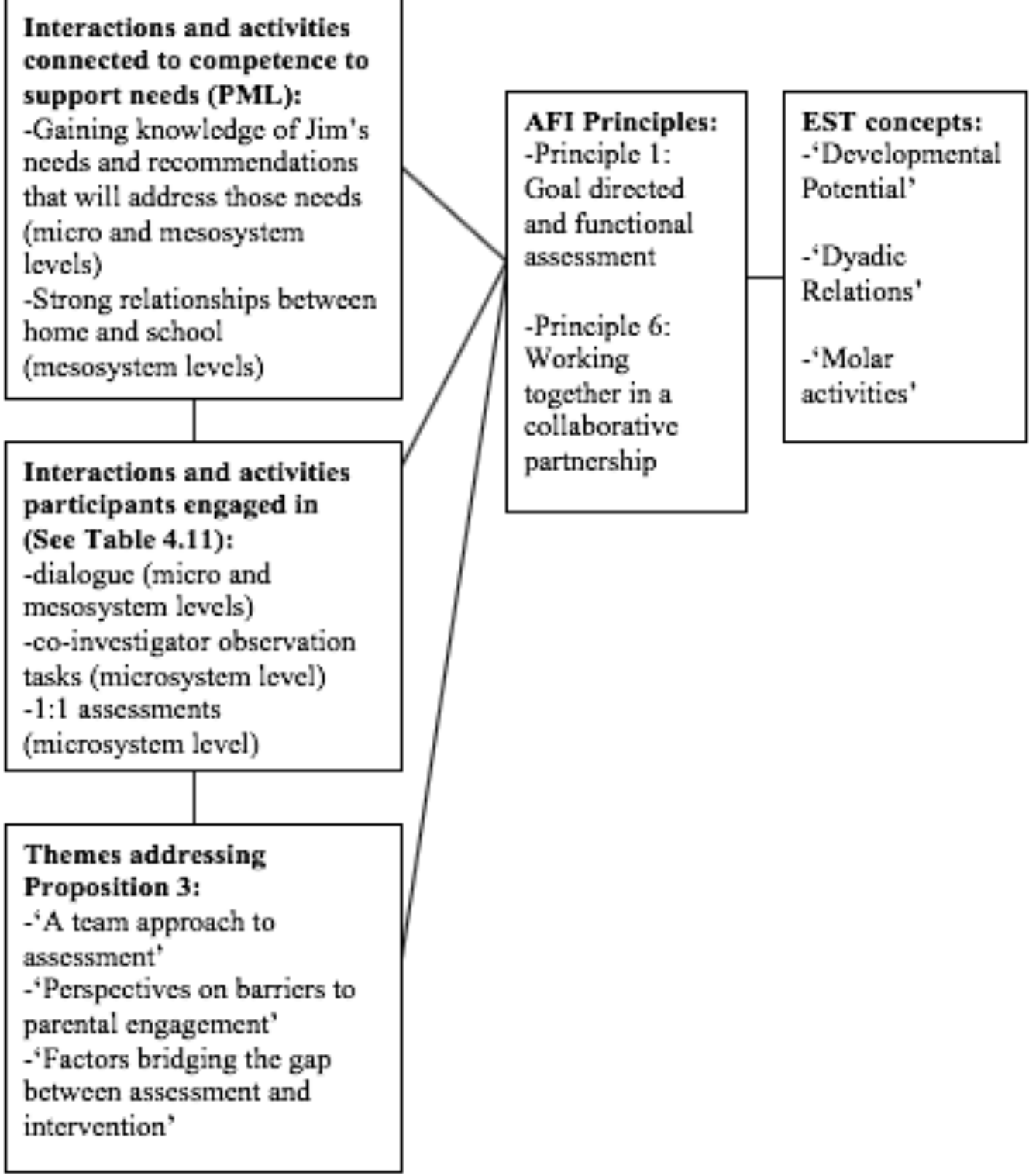

Figure 4.8. Case 1 Proposition 3 Findings 
4.13 Proposition 4: 'The child will have a better understanding of their strengths and needs and what to do to improve their needs, as a result of interactions and activities that have occurred at the meso and microsystem levels'

\subsubsection{Likert Scale Ratings}

Likert scale ratings provided by Jim indicate he knew what his strengths and needs were before and after engaging with the AFI model. Jim's rating changed from not knowing what he can do to improve on needs at school to being "not sure" following engagement with the AFI model. Pattern-matching logic of interview responses expands upon these ratings in the section below.

Table 4.18

Case 1 Proposition 4 Ratings

\begin{tabular}{llcc}
\hline Likert Statement & Participant & Before AFI Model & After AFI Model \\
\hline $\begin{array}{l}\text { I know what my } \\
\text { strengths are at school }\end{array}$ & Student & Yes & Yes \\
$\begin{array}{l}\text { I know what I need } \\
\text { help with at school }\end{array}$ & Student & Yes & Yes \\
$\begin{array}{l}\text { I know what I can do } \\
\text { to get better at what I } \\
\text { need help with at } \\
\text { school }\end{array}$ & Student & No & Not sure \\
\hline
\end{tabular}

\subsubsection{Pattern-Matching Logic}

Pattern-matching logic was applied to responses to interview questions exploring Jim's thoughts on his strengths and needs and what to do to address those needs. He was also asked a question regarding his experience of working together with his mother, his teachers and with the TEP. Patterns within Jim's responses connected understanding of strengths and needs and what to do to address needs, to interactions and activities that occurred during the AFI process, at the microsystem level (See Table 4.19 below for key quotes and Appendix 37 for the analysis). Jim responded "yes" to questions exploring if working together helped him to find out about his strengths and needs. Jim identified his strengths as "drawing, reading and a little bit of maths and history". He identified his needs as "maths and spelling". Activities and interactions that led to the identification of areas of strength and need included dialogue between team members, co-investigator tasks (observations) and 1:1 assessments at the Investigation 
Stage (Table 4.11). A child friendly report compiled for Jim at the Feedback stage, listed specific areas of strength and need in reading, maths and spelling, which were discussed with Jim, his class teacher and mother. When asked how he could improve at maths and spelling, Jim mentioned strategies that had been practiced during 1:1 dynamic assessment including "silly sentences" for spelling and the use of "counters" for maths. Consistent with AFI Principle 6, Jim acted as a co-assessor during the Investigation stage. His opinions and feedback were valued during the process and contributed to overall intervention planning (Pameijer, 2017).

Table 4.19

Case 1 Key Quotes Addressing Proposition 4

\begin{tabular}{|c|c|}
\hline Participant & Key Quote \\
\hline \multirow[t]{3}{*}{$\operatorname{Jim}$ (child) } & $\begin{array}{l}\text { When asked if working together with his mother, teachers and TEP } \\
\text { helped in finding out about his strengths, Jim responded: Yeah (line } \\
66) \text {... am, drawing and reading and a little bit of maths... and history } \\
\text { (line } 68 \text { ) }\end{array}$ \\
\hline & $\begin{array}{l}\text { When asked if working together with his mother, teachers and TEP } \\
\text { helped in finding out about what he needs help with, Jim } \\
\text { commented: Yes (line 86)...maths and spelling (line 89) }\end{array}$ \\
\hline & $\begin{array}{l}\text { When asked if he knew what to do to address these areas of need, } \\
\text { Jim replied: Yeah (line 91)...spellings like...just make up silly } \\
\text { sentences like and then for maths, like use counters and you'll get } \\
\text { better (lines 93-94) }\end{array}$ \\
\hline
\end{tabular}

\subsubsection{Thematic Analysis}

The theme 'A team approach to assessment' referred to Jim's understanding of strengths and needs and what to do to address needs. Proposition 4 findings are discussed under this theme, in accordance with the literature and conceptual framework in Section 5.10. Figure 4.9 below outlines how Proposition 4 findings resonate with AFI principles (Pameijer, 2017), EST concepts (Bronfenbrenner, 1979) and SCT concepts (Palinscar, 1998; Vygotsky, 1978). See Appendix 37, Section 5 for this analysis. 
Proposition 4: 'The child will have a better understanding of their strengths and needs and what to do to improve their needs, as a result of interactions and activities that have occurred at the meso and microsystem levels'

\section{Interactions and activities} connected to understanding of strengths, needs and what to do to improve on needs (PML):

-working together with parent, teachers and TEP (microsystem level)

Interactions and activities participants engaged in (See Table 4.11): -dialogue (micro and mesosystem levels) -co-investigator observation tasks (microsystem level)

$-1: 1$ assessments (microsystem level)

Theme addressing Proposition 4:

-'A team approach to assessment'

Figure 4.9. Case 1 Proposition 4 Findings 
4.14 Proposition 5: "At a time of ecological transition, teachers will feel competent in their ability to work according to the aims of Circular 0013/2017, in meeting and monitoring the needs of the child'

\subsubsection{Pattern-Matching Logic}

Pattern-matching logic was applied to responses to interview questions exploring Michael and Sarah's perceived competence to monitor Jim's needs moving forward (See Table 4.20 below for key quotes and Appendix 38 for the analysis). Patterns within Michael's response connected competence to his "strengthened relationship with Jim" as a result of the "whole process" and "having a good relationship with the learning support teacher". Sarah attributed competence to knowing "what the needs [Jim's needs] are". Activities and interactions during the AFI process that resulted in identification of Jim's needs are listed in Table 4.11. Increased awareness of Jim's needs reflects the aims of AFI Principle 3, focusing on the educational needs of the child and strengthening relationships between class teacher, SET and student resonates with the collaborative partnerships promoted by AFI Principle 6 (Pameijer, 2017).

Table 4.20

Case 1 Key Quotes Addressing Proposition 5

\begin{tabular}{ll}
\hline Participant & Key Quote \\
\hline Michael & When asked if he felt he could monitor Jim 's needs moving forward, \\
teacher) & $\begin{array}{l}\text { Michael commented: I do feel like I can monitor them [needs] yeah, } \\
\text { am I think also the relationship I've...this whole process has } \\
\text { strengthened my relationship with Jim and you know, he's aware of } \\
\text { you know, areas where he needs support and just to talk to him and } \\
\text { ask him the questions like what worked well or you know, where he } \\
\text { is struggling, so I think monitoring for sure and between having a } \\
\text { good relationship with the learning support teacher, that we can } \\
\text { monitor his progress (lines 99-103) } \\
\text { When asked if she felt she could monitor Jim's needs moving } \\
\text { forward, Sarah commented: Yeah 100\%, yes I can, now that I know } \\
\text { what the needs are, I can monitor them. We can assess, and again } \\
\text { him being involved, seeing from, we know where he's at at the } \\
\text { moment, with those recommendations in place, building on them } \\
\text { and monitoring and making sure that they're working do you know, } \\
\text { to the best that they can for him so it's no problem to monitor (lines } \\
\text { 151-154) }\end{array}$ \\
\hline
\end{tabular}




\subsubsection{Thematic Analysis}

The theme 'Factors bridging the gap between assessment and intervention' referred to meeting and monitoring of needs. Proposition 5 findings are discussed in greater depth under this theme, in conjunction with the literature and conceptual framework in Section 5.12. Figure 4.10 below delineates how Proposition 5 findings are consistent with AFI principles (Pameijer, 2017), EST concepts (Bronfenbrenner, 1979) and SCT concepts (Palinscar, 1998; Vygotsky, 1978). This analysis is outlined in Section 5 in Appendix 38.

Proposition 5: 'At a time of ccological transition, teachers will feel competent in their ability to work according to the aims of Circular 0013/2017, in meeting and monitoring the needs of the child'

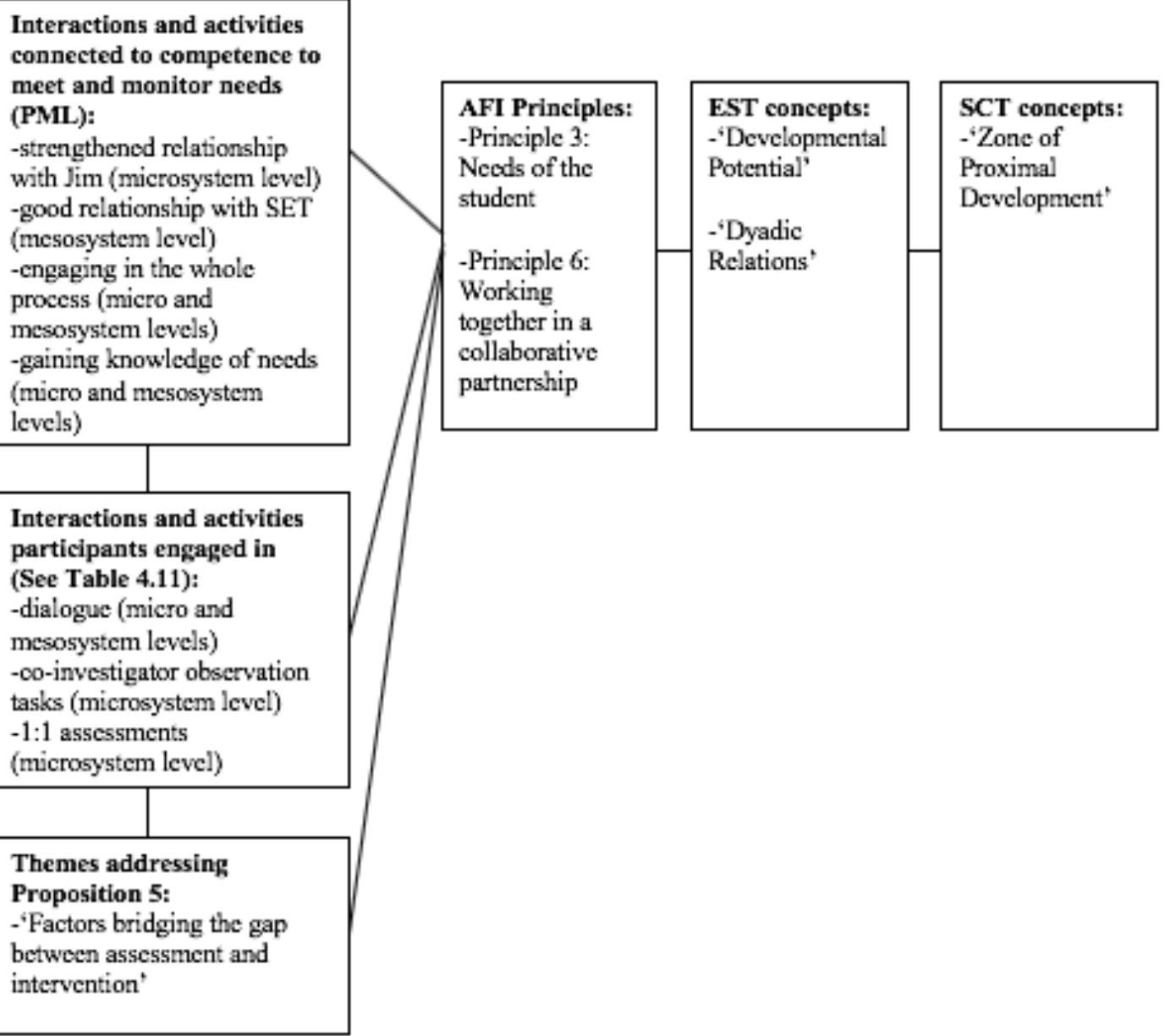

Figure 4.10. Case 1 Proposition 5 Findings 


\subsection{Summary of Case 1 Proposition Findings}

The findings presented for each proposition in Case 1 suggest the AFI model can bridge the gap between assessment and intervention through activity and interaction at the meso and microsystem levels. Likert scale ratings and pattern-matching of parent and teacher interview responses indicate greater understanding of Jim's situation, knowledge of intervention supports and greater perceived competence in supporting and monitoring Jim's needs, following engagement with the AFI model. Activities undertaken at the micro level included dialogue, observation tasks completed by Jim, his mother and teachers and 1:1 assessments conducted by the TEP, including attainment and cognitive testing and dynamic assessment of skills and potential. Activities that occurred at the mesosystem level included meetings between TEP, parents and teachers (Table 4.11). Pattern-matching logic applied to Jim's interview responses linked knowledge of strengths and needs to working with the TEP, parent and teachers and what to do to improve on needs to activities that were practiced during $1: 1$ dynamic assessment at the microsystem level. Figures 4.6, 4.7, 4.8, 4.9 and 4.10 delineated how proposition findings are consistent with AFI principles (Pameijer, 2017), EST concepts (Bronfenbrenner, 1979) and SCT concepts (Palinscar, 1998; Vygotsky, 1978). Findings are discussed in greater depth under related themes in

Chapter 5 , in accordance with the literature and conceptual framework. Applicability of the AFI model to other situations is also discussed. Case 2 findings are presented overleaf. 


\subsection{Case 2: Case description}

Michelle is a twelve-year old girl, attending $6^{\text {th }}$ class in a mainstream primary school. Michelle's mother and school requested the involvement of the school psychological service to provide insight into learning difficulties in English and Maths and support around social-emotional and communication skills. At the Intake stage, questions of the parent (Mary) class teacher (Anne) and child (Michelle) were gathered. These questions, along with background information guided the development of questions for investigation. The case log of actions presented in Table 4.21 outlines the stages and tasks applied to address these questions, in addition to the timescale for the assessment process. 
Table 4.21

Case Log of Actions Case 2

\begin{tabular}{|c|c|}
\hline Stage & Actions \\
\hline $\begin{array}{l}\text { 1. Intake } \\
\left(\text { November } 23^{\text {rd }}\right) \\
\text { 2. Strategy } \\
\left(\text { November } 23^{\text {rd }}\right)\end{array}$ & $\begin{array}{l}\text {-Initial consultation with Michelle, her mother and class teacher } \\
\text {-TEP devised a strategy for moving forward, based on } \\
\text { information gathered at intake }\end{array}$ \\
\hline $\begin{array}{l}\text { 3. Investigation } \\
\left(\text { November } 29^{\text {th }}\right)\end{array}$ & $\begin{array}{l}-1: 1 \text { attainment and informal testing in areas of literacy (word } \\
\text { reading, pseudoword decoding, spelling) and } \\
\text { numeracy/problem solving skills (The Wechsler Individual } \\
\text { Achievement Test (WIAT-III }{ }^{\mathrm{UK}} \text { ) } \\
-1: 1 \text { assessment of cognitive strengths and weaknesses The } \\
\text { Wechsler Scale of Intelligence for Children-Fifth UK Edition } \\
\text { (WISC-V }{ }^{\mathrm{UK}} \text { ) } \\
\text {-Dynamic assessment during cognitive and attainment testing } \\
\text {-Running record of reading and oral comprehension of a } \\
\text { reading passage } \\
\text {-Observing the effectiveness of a graphic organiser activity for } \\
\text { assisting the development of metacognitive skills (TEP and } \\
\text { Michelle) } \\
\text {-Classroom and yard observation to observe Michelle's learning } \\
\text { and social interactions in context (TEP) } \\
\text {-Informal discussion with Michelle on her social relationships } \\
\text { (TEP and Michelle) }\end{array}$ \\
\hline $\begin{array}{l}\text { 3. Investigation } \\
\left(\text { November } 29^{\text {th }}-\right. \\
\left.\text { December } 12^{\text {th }}\right)\end{array}$ & $\begin{array}{l}\text { Teaching of 'I Statements' as a communication, problem } \\
\text { solving tool (Anne) } \\
\text {-Observing the effectiveness of a self-monitoring checklist for } \\
\text { maths to facilitate independence and responsibility in } \\
\text { completing tasks and assignments (Anne and Michelle) } \\
\text {-Completion of observation activities to investigate teaching } \\
\text { supports and strategies that Michelle responds well to (Mary } \\
\text { and Anne) } \\
\text {-Co-investigator activity to investigate what helps Michelle to } \\
\text { learn (Michelle with the support of Mary and Anne) } \\
\text {-Adaptive Behaviour Assessment System-Third Edition } \\
\text { (Teacher Form) }\end{array}$ \\
\hline $\begin{array}{l}\text { 4. Integration of } \\
\text { assessment } \\
\text { information } \\
\left(\text { December } 13^{\text {th }}\right)\end{array}$ & $\begin{array}{l}\text {-Meeting with Michelle's teacher to discuss assessment } \\
\text { information } \\
\text {-Phone call with Mary to discuss assessment information } \\
\text {-Meeting with Michelle to discuss co-investigator activity } \\
\text {-Integration of assessment information (TEP) }\end{array}$ \\
\hline $\begin{array}{l}\text { 5. Feedback on } \\
\text { report, goals and } \\
\text { recommendation } \\
\left(\text { December } 20^{\text {th }}\right)\end{array}$ & $\begin{array}{l}\text {-Meeting with Michelle's class teacher to discuss feedback on } \\
\text { report, goals and intervention recommendations. }\end{array}$ \\
\hline
\end{tabular}


The proceeding sections present the findings for Propositions 1, 2, 3, 4 and 5 in Case 2. This is followed by a summary, outlining how the proposition findings address the research question.

4.17 Proposition 1: 'Parents, teachers and TEP will have a better understanding of the child's situation, as a result of interactions and activities that have occurred at the meso and microsystem levels'

\subsubsection{Likert Scale Ratings}

Likert scale ratings provided by the class teacher indicate understanding of the child's situation remained unchanged, however pattern-matching logic applied to interview responses suggests a change in understanding occurred.

Table 4.22

Case 2 Proposition 1 Ratings

\begin{tabular}{llcc}
\hline Likert Statement & Participant & Before AFI Model & After AFI Model \\
\hline $\begin{array}{l}\text { I understand the } \\
\text { student's situation }\end{array}$ & Class teacher & 8 & 8 \\
$\begin{array}{l}\text { I understand my } \\
\text { child's situation }\end{array}$ & Parent & 7 & \\
\hline
\end{tabular}

\subsubsection{Pattern-Matching Logic}

Pattern-matching logic was applied to responses to questions exploring change in understanding of the student's situation and experience of the AFI model (See Table 4.23 below for key quotes and Appendix 40 for the analysis). Patterns within Anne's (class teacher) interview responses connected understanding of the situation to interactions and activities that occurred during the five stages of the AFI process, at the meso and microsystem levels. These activities and interactions included "talking through everything" with team members and having to "analyse" the "situation". Interactions and activities engaged in by participants are outlined in Table 4.21 and included dialogue between team members, co-investigator observation tasks and 1:1 assessment of attainment and cognitive functioning and dynamic assessment of learning potential. In accordance with AFI Principle 6, participants were active in their engagement in assessment tasks and included in the process as co-assessors (Pameijer, 2017). 
Table 4.23

Case 2 Key Quotes Addressing Proposition 1

\begin{tabular}{ll}
\hline Participant & Key Quote \\
\hline Anne & Anne described how her understanding of Michelle's situation had \\
(class & changed following engagement in the AFI process: Yeah I definitely \\
teacher) & think I understand everything a lot more (line 55)...I felt that just \\
& through talking through everything and just being way more aware \\
& of the situation as well (lines 57-58)...just having to hone in on one \\
& particular child and really analyse that situation and then put \\
& information or put words on it (lines 60-61)
\end{tabular}

\subsubsection{Researcher Diary and Thematic Analysis}

Consistent with AFI principle 7 (Pameijer, 2017), the researcher adhered to the systematic and transparent assessment process and progressing understanding of Michelle's situation was evidenced through the AFI templates (researcher diary) and reflective journal (See Appendix 53 and Figure 4.11 below). Themes within the data set that referred to understanding of Michelle's situation included 'A team approach to assessment', 'Perspectives on barriers to parental engagement' and 'Views and Experiences of AFI'. Proposition 1 findings are discussed in greater detail under each of these themes, in conjunction with the literature and conceptual framework in Sections 5.15, 5.16 and 5.17 of Chapter 5. Figure 4.11 below delineates how Proposition 1 findings are consistent with AFI principles (Pameijer, 2017), EST concepts (Bronfenbrenner, 1979) and SCT concepts (Palinscar, 1998; Vygotsky, 1978). This analysis is outlined in Appendix 40, Section 5. 
Proposition 1: 'Parents, teachers and TEP will have a better understanding of the child's situation, as a result of interactions and activities that have occurred at the meso and microsystem levels'

\section{Interactions and activities connected to understanding of situation (PML): \\ -talking through everything (micro and mesosystem levels) \\ -analysing the situation (micro and mesosystem level)}

\section{Interactions and activities} participants engaged in (See table 4.21) : -dialogue (micro and mesosystem levels) -co-investigator observation tasks (microsystem level)

$-1: 1$ assessments (microsystem level)

\section{Themes addressing}

Proposition 1:

-A team approach to assessment'

-'Perspectives on barriers to parental engagement'

-Views and Experiences of AFI'

\section{Excerpt from researcher diary} demonstrating progressing understanding: 'Applying elements of this stage ensured that I understood the situation and interactions between the risk and protective factors for Michelle, her school and home environment. Consideration of these factors allowed me to present a valid, transactional case formulation and devise feasible goals, needs and intervention recommendations' (Template 2b: Checklist Integration/Feedback Stage)

\begin{tabular}{|c|c|c|}
\hline $\begin{array}{l}\text { AFI Principles: } \\
\text {-Principle 6: } \\
\text { Working } \\
\text { together in a } \\
\text { collaborative } \\
\text { partnership } \\
\text {-Principle 7: A } \\
\text { systematic and } \\
\text { transparent } \\
\text { assessment } \\
\text { process. }\end{array}$ & $\begin{array}{l}\text { EST concepts: } \\
\text {-'Developmental } \\
\text { Potential' } \\
\text {-'Dyadic } \\
\text { Relations' }\end{array}$ & $\begin{array}{l}\text { SCT concepts: } \\
\text {-'Zone of Actual } \\
\text { Development' } \\
\text {-'Zone of } \\
\text { Proximal } \\
\text { Development' } \\
\text {-'Tools and } \\
\text { Signs' }\end{array}$ \\
\hline
\end{tabular}

AFI Principles:

-Principle 6 :

collaborative

partnership

-Principle 7: A

systematic and

assessment

process.

Figure 4.11. Case 2 Proposition 1 Findings 
4.18 Proposition 2: 'Parents, teachers and TEP will know what intervention supports the child needs at home and at school, as a result of interactions and activities that have occurred at the meso and microsystem levels'

\subsubsection{Likert Scale Ratings}

A higher teacher Likert scale rating following engagement with the AFI model indicates greater knowledge of intervention supports the child needed. Pattern-matching logic applied to interview responses elucidates this finding.

Table 4.24

Case 2 Proposition 2 Ratings

\begin{tabular}{llcc}
\hline Likert Statement & Participant & Before AFI Model & After AFI Model \\
\hline $\begin{array}{l}\text { I know what support } \\
\text { the student needs at }\end{array}$ & Class teacher & 6 & 8 \\
school & & & \\
$\begin{array}{l}\text { I know what support } \\
\text { my child needs at } \\
\text { home }\end{array}$ & Parent & 5 & \\
\hline
\end{tabular}

\subsubsection{Pattern-Matching Logic}

Pattern-matching logic was applied to responses to questions on intervention recommendations and supporting Michelle's needs (See Table 4.25 below for key quotes and Appendix 41 for analysis). Patterns within Anne's interview responses associated knowledge of intervention supports with interactions and activities that occurred during the stages of the AFI model, at the meso and microsystem level. At the microsystem level, Anne observed the effectiveness of a graphic organiser for "mapping out things ahead of a lesson" and a self-checklist for maths, making things "a little bit easier for her [Michelle] at the start of a lesson". At the mesosystem level, dialogue between TEP, parent and teacher at the Intake and Feedback stage led to knowledge of evidence-based social-emotional intervention resources including "Talkabout" and "cognitive behaviour therapy". These activities and interactions are considered consistent with the AFI process. In accordance with AFI Principle 6, parent, teacher and child were co-assessors during the Investigation stage and provided feedback on intervention strategies designed to address needs (Pameijer, 2017). Their opinions and feedback were valued during the process and contributed to intervention planning. Furthermore, identifying intervention strategies that effectively addressed Michelle's 
needs is consistent with AFI Principles 3 and 4 (Pameijer, 2017). Patterns within Anne's response also connected knowledge of intervention supports to differentiation activities she already applies in her general practice as a teacher (See Table 4.25 below). Consistent with AFI Principle 3 and the concept of goodness of fit, the effective approaches addressing Michelle's needs were encouraged by the TEP (Pameijer, 2017).

Table 4.25

Case 2 Key Quotes Addressing Proposition 2

\begin{tabular}{|c|c|}
\hline Participant & Key Quote \\
\hline \multirow[t]{2}{*}{$\begin{array}{l}\text { Anne (class } \\
\text { teacher) }\end{array}$} & $\begin{array}{l}\text { When describing how intervention recommendations addressed her } \\
\text { questions at the Intake stage, Anne commented: I think that's really } \\
\text { the practical tips and the 'Talkabout' and am, the cognitive- } \\
\text { behavioral therapy, all of that like that's what works, it's practical, } \\
\text { it's very doable, it's very user-friendly (lines } 85-86 \text { ) }\end{array}$ \\
\hline & $\begin{array}{l}\text { When commenting on how she intended to support Michelle's needs } \\
\text { moving forward, Anne referred to intervention strategies she had } \\
\text { observed to be effective at the Investigation stage: ...obviously put } \\
\text { in place these extra you know ideas, especially say along the lines } \\
\text { for English and mapping out things ahead of a lesson and again with } \\
\text { the maths and checklists (lines 101-103)... again we would be doing } \\
\text { it anyway with brainstorming but sometimes just if a template looks } \\
\text { different or is different it can also help, you know which is great } \\
\text { (lines 124-125) }\end{array}$ \\
\hline
\end{tabular}

\subsubsection{Researcher Diary and Thematic Analysis}

In accordance with AFI principle 7 (Pameijer, 2017), the researcher followed a systematic and transparent assessment process and knowledge of intervention supports was demonstrated in the AFI templates (researcher diary) and reflective journal (See Appendix 53 and Figure 4.12 below). The theme 'Factors bridging the gap between assessment and intervention' referred to knowledge of intervention supports and Proposition 2 findings are discussed in greater depth under this theme, in conjunction with the literature and conceptual framework in Section 5.18. Figure 4.12 below presents how Proposition 2 findings are consistent with AFI principles (Pameijer, 2017), EST concepts (Bronfenbrenner, 1979) and SCT concepts (Palinscar, 1998; Vygotsky, 1978). See Appendix 41, Section 5 for this analysis. 
Proposition 2: 'Parents, teachers and TEP will know what intervention supports the child needs at home and at school, as a result of interactions and activities that have occurred at the meso and microsystem levels'

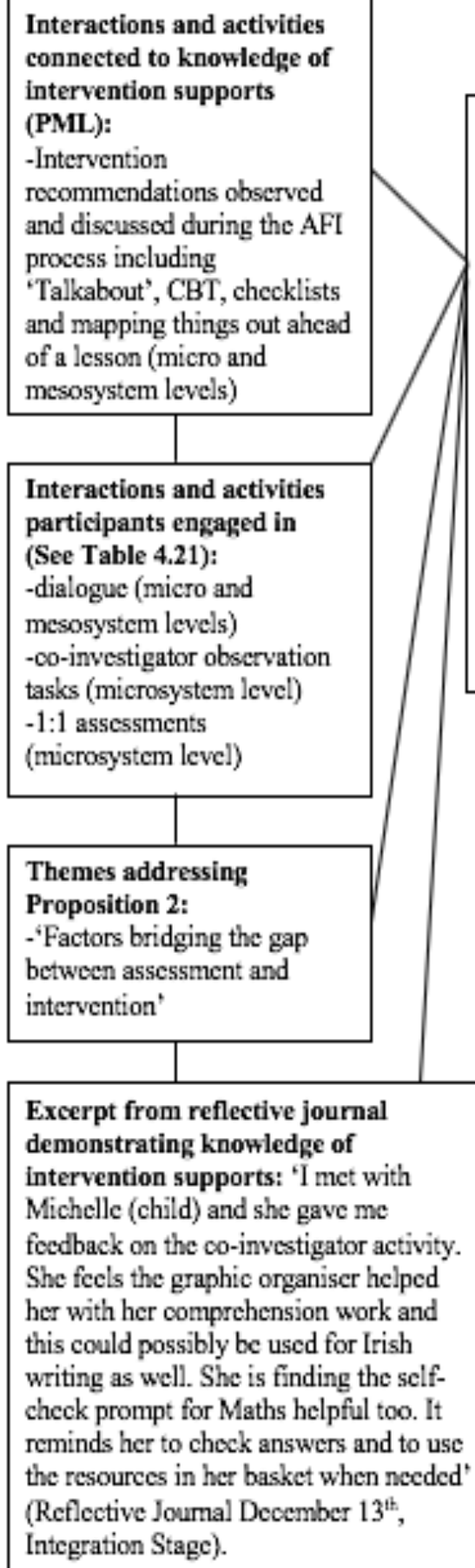

Figure 4.12. Case 2 Proposition 2 Findings 
4.19 Proposition 3: 'Teachers and parents will have greater perceived competence in supporting the child's needs at home and at school, as a result of interactions and activities that have occurred at the meso and microsystem levels'

\subsubsection{Likert Scale Ratings}

Class teacher Likert scale ratings indicate greater perceived competence in supporting Michelle's needs, after engaging with the AFI model. Pattern-matching logic applied to interview responses expands upon this finding.

Table 4.26

Case 2 Proposition 3 Ratings

\begin{tabular}{llcc}
\hline Likert Statement & Participant & Before AFI Model & After AFI Model \\
\hline $\begin{array}{l}\text { I am able to support } \\
\text { the student's needs at } \\
\text { school }\end{array}$ & Class teacher & 6 & 9 \\
$\begin{array}{l}\text { I am able to support } \\
\text { my child's needs at } \\
\text { home }\end{array}$ & Parent & & \\
\hline
\end{tabular}

\subsubsection{Pattern-Matching Logic}

Pattern-matching logic was applied to Anne's response to an interview question exploring her ability to support Michelle's needs (See Table 4.27 below for key quotes and Appendix 42 for analysis). Her perceived competence to support Michelle's needs was connected to "the help of all these...extra interventions". These "extra interventions" resulted from activities and interactions that occurred during the stages of the AFI model, at the meso and microsystem levels listed in Table 4.21. Consistent with AFI Principle 6, all participants were co-assessors during the Investigation stage and their active participation led to the identification of needs and subsequently, intervention recommendations (Pameijer, 2017). Teacher competence to implement these intervention recommendations is in accordance with AFI Principle 1, to devise feasible intervention recommendations for participants (Pameijer, 2017). Patterns within Anne's response also associated competence to support Michelle's needs to "what I [Anne] would be doing anyway". In adherence with AFI Principle 3 and the concept of goodness of fit, the effective approaches addressing Michelle's needs were promoted by the TEP during discussions (Pameijer, 2017). 
Table 4.27

Case 2 Key Quotes Addressing Proposition 3

\begin{tabular}{|c|c|}
\hline Participant & Key Quote \\
\hline $\begin{array}{l}\text { Anne (class } \\
\text { teacher) }\end{array}$ & $\begin{array}{l}\text { When commenting on whether she felt she could support } \\
\text { Michelle's needs, Anne stated: I think so yeah, absolutely, } \\
\text { am... and again with the help of all these again, extra interventions } \\
\text { yeah absolutely (lines 96-97)...I suppose obviously what I would } \\
\text { be doing anyway, all the extra sort of resources and over the weeks } \\
\text { when you get to know how a child learns and you adapt things and } \\
\text { sort of differentiate to suit their needs am I'll continue to do that } \\
\text { (lines 99-101) }\end{array}$ \\
\hline
\end{tabular}

\subsubsection{Thematic Analysis}

The theme 'Factors bridging the gap between assessment and intervention' referred to competence in supporting Michelle's needs and Proposition 3 findings are discussed under this theme, in accordance with the literature and conceptual framework in Section 5.18. Figure 4.13 outlines how Proposition 3 findings resonate with AFI principles (Pameijer, 2017) and EST concepts (Bronfenbrenner, 1979). See Appendix 42, Section 5 for this analysis. 
Proposition 3: 'Teachers and parents will have greater perceived competence in supporting the child's needs at home and at school, as a result of interactions and activities that have occurred at the meso and microsystem levels'

\section{Interactions and activities connected to competence to support needs (PML): \\ -Gaining knowledge around 'extra interventions' (micro and mesosystem levels) -Strategies Anne 'would be doing anyway' (mesosystem levels)}

\section{Interactions and activities} participants engaged in (See Table 4.21): -dialogue (micro and mesosystem levels) -co-investigator observation tasks (microsystem level) $-1: 1$ assessments (microsystem level)

\section{Themes addressing}

Proposition 3:

-'Factors bridging the gap between assessment and intervention'

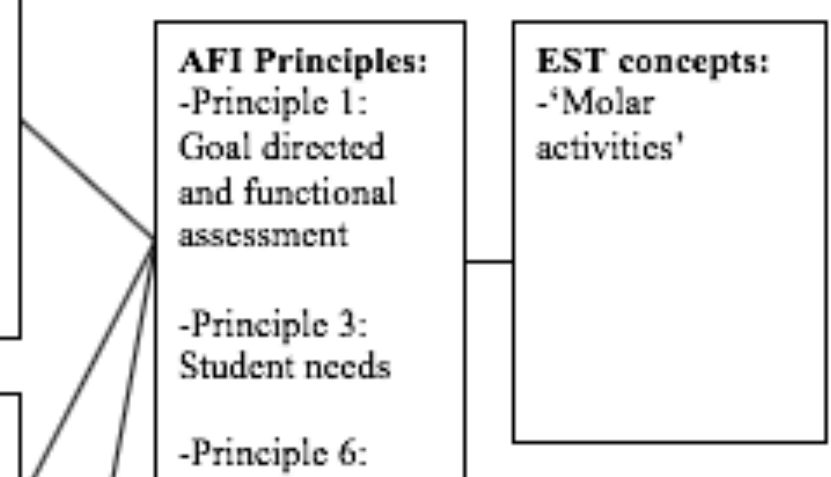

Working together in a collaborative partnership

Figure 4.13. Case 2 Proposition 3 Findings 
4.20 Proposition 4: 'The child will have a better understanding of their strengths and needs and what to do to improve their needs, as a result of interactions and activities that have occurred at the meso and microsystem levels'

\subsubsection{Likert Scale Ratings}

Likert scale ratings provided by Michelle (child) indicate that she knew what her strengths and needs were before engaging with the AFI model. Ratings also suggest that she knew what she could do to improve on her needs at school. Data were not collected from Mary (parent) and Michelle following engagement with the AFI model and there was limited evidence that could be drawn upon for analysis. Researcher reflections and thematic analysis provide an interpretation of Michelle's experience of the assessment process.

Table 4.28

Case 2 Proposition 4 Ratings

\begin{tabular}{llcc}
\hline Likert Statement & Participant & Before AFI Model & After AFI Model \\
\hline $\begin{array}{l}\text { I know what my } \\
\text { strengths are at school }\end{array}$ & Student & Yes & \\
$\begin{array}{l}\text { I know what I need } \\
\text { help with at school }\end{array}$ & Student & Yes & \\
$\begin{array}{l}\text { I know what I can do } \\
\text { to get better at what I } \\
\text { need help with at } \\
\text { school }\end{array}$ & Student & Yes & \\
\hline
\end{tabular}

\subsubsection{Researcher Diary and Thematic Analysis}

The theme 'A team approach to assessment' referred to Michelle's understanding of strengths and needs and what to do to address needs. Researcher reflections also suggest Michelle was an active participant, sharing information at the Intake stage, engaging in assessment and co-investigator tasks and also providing feedback on what was helping her to learn. See Appendix 43 for a presentation of these findings, which are discussed under the theme 'A team approach to assessment' and related to the literature and conceptual framework in Section 5.16. 
4.21 Proposition 5: 'At a time of ecological transition, teachers will feel competent in their ability to work according to the aims of Circular 0013/2017, in meeting and monitoring the needs of the child'

\subsubsection{Pattern -Matching Logic}

Pattern-matching logic was applied to Anne's response to a question exploring her perceived competence to monitor Michelle's needs moving forward (See Table 4.29 below for key quotes and Appendix 44 for analysis). Patterns within her response linked competence to being "more mindful now" and being able to "pick up on things", following all the "analysis" completed during the assessment process. This "analysis" consisted of activities and interactions that occurred during the stages of the AFI model, at the meso and microsystem levels, listed in Table 4.21. These activities and interactions are consistent with AFI Principle 6 as participants were co-assessors during the Investigation stage and their opinions and feedback were valued as hands-on experts through experience during the process (Pameijer, 2017). Being more "mindful" of, and monitoring Michelle's needs moving forward also resonates with AFI Principle 3, focusing on the educational needs of the child. Patterns within Anne's response connected competence to meet and monitor Michelle's needs to the "observations" that she conducts "all day every day". In agreement with AFI Principle 3, effective monitoring approaches were encouraged during meetings and discussions (Pameijer, 2017).

Table 4.29

Case 2 Key Quotes Addressing Proposition 5

\begin{tabular}{|c|c|}
\hline Participant & Key Quote \\
\hline $\begin{array}{l}\text { Anne (class } \\
\text { teacher) }\end{array}$ & $\begin{array}{l}\text { When asked if she felt she could monitor Michelle's needs moving } \\
\text { forward, Anne commented: Yes, am... yeah I think so I mean } \\
\text { obviously I think just in general just using... sorry now I'm just } \\
\text { trying to think. In general just in terms of observations and we're } \\
\text { doing that all day every day, and I suppose maybe I'd be doing it } \\
\text { anyway but I would feel I would be a lot more mindful now and I'd } \\
\text { probably pick up on things, we've just done so much analysis of her } \\
\text { maybe that now I feel I'd be more clued in, so yeah moving forward } \\
\text { absolutely (lines 136-140) }\end{array}$ \\
\hline
\end{tabular}




\subsubsection{Thematic Analysis}

The theme 'Factors bridging the gap between assessment and intervention' referred to meeting and monitoring of needs. Proposition 5 findings are discussed under this theme, in accordance with the literature and conceptual framework in Section 5.18. Figure 4.14 below delineates how Proposition 5 findings resonate with AFI principles (Pameijer, 2017) and EST concepts (Bronfenbrenner, 1979). See Appendix 44, Section 5 for this analysis.

Proposition 5: 'At a time of ecological transition, teachers will feel competent in their ability to work according to the aims of Circular 0013/2017, in meeting and monitoring the needs of the child'

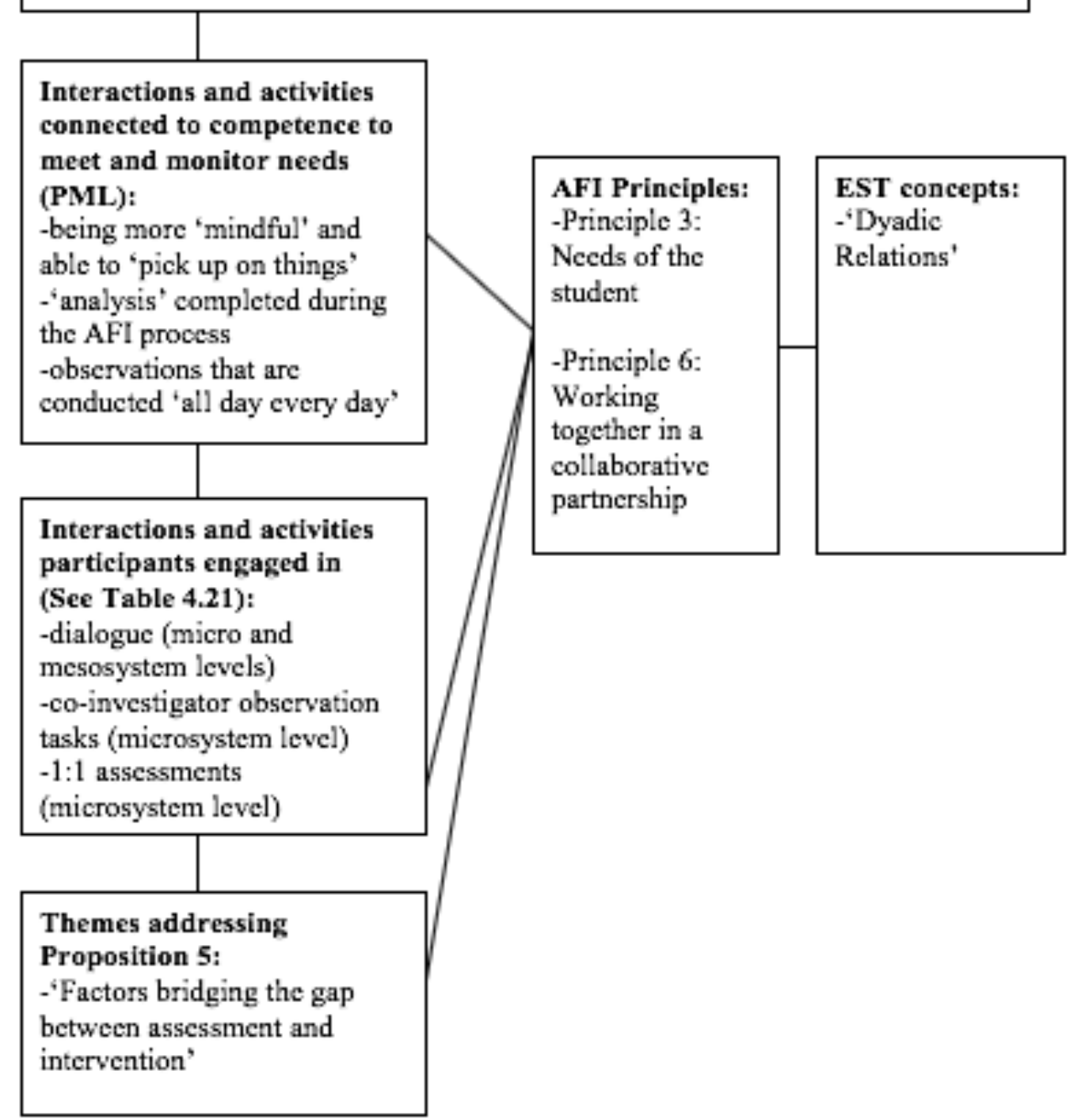

Figure 4.14. Case 2 Proposition 5 Findings 


\subsection{Summary of Case 2 Proposition Findings}

Overall, the findings of Case 2 provide evidence to suggest the AFI model can bridge the gap between assessment and intervention through activity and interaction at the meso and microsystem levels. As data were collected from the teacher only following engagement with the AFI model, there was limited evidence that could be drawn upon for analysis in this case. Supporting evidence was presented for Proposition 1, 2, 3 and 5. Likert scale ratings and pattern-matching of teacher interview responses indicated greater understanding of Michelle's situation, knowledge of intervention supports and greater perceived competence in supporting and monitoring Michelle's needs following activity and interaction at the meso and microsystem levels. Activities undertaken at the microsystem level included dialogue, investigation and observation tasks completed by Michelle, Anne and the TEP and 1:1 assessments including attainment and cognitive testing and dynamic assessment of skills and potential. Activities that occurred at the mesosystem level included meetings and dialogue between TEP, parent and teacher (Table 4.21). With regards Proposition 4, data were not collected from Michelle (child) following engagement with the AFI model, however excerpts from the researcher diary and thematic analysis indicate a positive experience of the process and active involvement and strengthening relationships, discussed further in Section 5.16. Figures 4.11, 4.12, 4.13 and 4.14 outlined how proposition findings are consistent with AFI principles (Pameijer, 2017), EST concepts (Bronfenbrenner, 1979) and SCT concepts (Palinscar, 1998; Vygotsky, 1978). Findings are discussed in greater depth under related themes in Chapter 5, in accordance with the literature and conceptual framework. Applicability of the AFI model to other situations is also discussed.

\subsection{Chapter Conclusion}

This chapter outlined the findings of the current research project. Each case report provided a rich case description and the data presented addressed the case propositions and research question. Analysis was informed by researcher's conceptual framework. In Chapter 5, proposition findings are discussed under related themes, in conjunction with the literature and conceptual framework. This is followed by a crosscase analysis discussion. 


\section{Chapter 5 Discussion}

\subsection{Overview of Chapter}

This chapter discusses the findings of the research project. The findings of the Pilot Case, Case 1 and Case 2 are discussed separately, followed by a cross-case analysis discussion. Proposition findings are discussed under themes, in the context of the literature and conceptual framework.

\subsection{Discussion of Findings: Pilot Case}

Collectively, the Pilot Case findings suggest the AFI model can bridge the gap between assessment and intervention through activity and interaction at the meso and microsystem levels. Pilot Case findings are discussed in the sections that follow.

\subsection{Views and Experiences of the AFI Model}

Thematic analysis of participant interview responses depicts AFI as a framework for inclusive team assessment. Louise (parent) described the "team approach" facilitated by AFI, an opinion shared by Grace (teacher) who referred to the model's "clear process" and the "channel of communication" supported between home and school (See Appendix 28, Section 3 for key quotes). This framework enabled participants to work together as a team, to engage in activities and interactions that built a better understanding of John's situation, as evidenced in the findings presented for Proposition 1 (Section 4.3). At the micro and mesosystem levels, active participation in activities and communication during the AFI process between child, home, school and school psychological service served to strengthen the developmental potential of these settings to identify and understand John's needs (Bronfenbrenner, 1979, p. 216), consistent with the aims of Circular 0013/2017 (DES, 2017b). Activities, including information taking from all participants and hearing each other's perspectives on the presenting situation (Section 4.3), reflect the ecological assessment approach outlined by Burns (2013), through collection of data that represents multiple perspectives (Section 2.14.3). Furthermore, engagement in activities that required dialogue and interaction (Section 4.1, Table 4.1), is consistent with the social constructivist assessment process described by Thomas and Oldfather (1997) (Section 2.15.1). These findings resonate with outcomes of the Pameijer (2017) evaluation study, where clients and assessors reported the stages of AFI structured the assessment process from beginning to end and all involved worked in cooperation towards shared goals (Pameijer, 2017). 
Grace compared her experience of the AFI model to previous assessment and intervention experiences:

Like my only prior experience with these kind of interventions was purely me filling in the report and then getting a report back...or maybe seeing a note that the parents had sent in but not actually getting to speak to them, so I think having that interaction and then hearing my voice and seeing what I've been experiencing and then vice versa me seeing them and hearing their experiences I think that was a huge benefit of it (Grace, lines 250-254)

This reflection is perhaps indicative of Grace's experiences with traditional assessment practices, with less emphasis placed on the contextual, transactional perspective on child development promoted by the AFI model (Pameijer, 2017) and EST (Bronfenbrenner, 1979). The findings of the systematic review identified a prevalence of assessment approaches consistent with the medical model of practice and within the Irish context, the traditional resource allocation system prevailed until 2017 (DES, 2017b). Grace's reflection suggests preference for the communication and insight into perspectives and experiences of others facilitated by AFI, consistent with an ecological assessment approach (Bronfenbrenner, 1979). This presents an interesting finding for EP practice and policy. Communication and interaction were pivotal to the assessment process and were proposed as essential factors to achieve the aims of Circular 0013/2017 and Circular 0014/2017 in the researcher's theoretical statement, in understanding and addressing the needs of students (DES, 2017b, p. 14; DES, 2017c, p. 14) (Section 2.16.2).

The AFI model was discussed as having practical applicability to other situations, reflective of the framework's developmental validity (Bronfenbrenner, 1979, p. 29) (Section 3.10). Consistent with an ecological perspective, Grace commented that she would now be more perceptive to the home situation, if a child was having difficulties at school:

so I think in other situations that maybe if there's a child who isn't getting their homework done that maybe there is something else happening at home, so being aware of home situations is definitely a huge advantage (Grace, lines 235-238)

It could be inferred that Grace's perceptiveness to the home situation in future problem situations is an adjunct to the transactional, contextual processes she experienced during her engagement with the AFI model (Pameijer, 2017). Grace acknowledged that there was a time commitment involved in the AFI process and that she lost out on class 
teaching time, however the cost-benefit was balanced by the information gathered and strengthened communication between home and school:

I lost out on some class teaching time with it (line 266)...but in the long run, it's going to be beneficial because we have so much information and we have so much line of discussion between home and school (Grace, lines 270-271)

This reflects findings from the Pameijer (2017) study, where, $80 \%$ of teachers acknowledged that time and energy invested in the assessment process provided time saving in the long run and greater knowledge of the student and how to respond to his needs. Overall, parent and teacher views on the AFI model in the Pilot Case provide noteworthy findings for practice and policy, discussed further in the cross-case discussion and Chapter 6.

\subsection{A Team Approach to Assessment}

The theme 'A team approach to assessment' had three associated subthemes; 'Active roles and communication', 'Insight into strengths and needs' and 'Strengthening relationships'. Proposition findings are discussed under associated subthemes in the sections below.

\subsubsection{Active Roles and Communication}

Thematic analysis of participants' interview responses suggests active participation and communication between parent, teacher, child and TEP during the assessment process and the TEP as a "facilitator", reflective of a team assessment approach (See Appendix 28, Section 3 (Proposition 1) and Appendix 31, Section 3 (Proposition 4) for key quotes). Participants' active inclusion as co-assessors (Pameijer, 2017) with the TEP during the assessment process supported understanding of John's situation, as evidenced in Proposition 1 (Section 4.3) and Proposition 4 (Section 4.6) findings. Tools, including the interest inventory and pie chart (Section 4.1, Table 4.1) facilitated John's active engagement in joint activity tasks with the TEP, and coconstruction of knowledge around alternative interest activities (Palinscar, 1998, p. 353; Vygotsky, 1978, p. 55). The active roles and communication experienced by participants in the Pilot Case is consistent with findings in Pameijer (2017). Teachers, counsellors and parents reported a positive collaborative relationship with the assessor, analysing the problematic situation together and co-operating to form specific goals and needs (Pameijer, 2017). These findings are also in accordance with ecological, social 
constructivist assessment approaches identified in the literature review. For example, Bourke and Dharan (2015) described a holistic assessment approach applied by EPs, where dialogue and collaboration with teachers and parents were fundamental to identifying and meeting the needs of children. Similarly, Tobias (2017) discussed the application of the genogram, where the EP, family members and child engaged together in meaningful discussion to address the referral issue. Collectively, these findings suggest active engagement and communication between child, home, school and school psychological services, at the micro and mesosystem levels (Bronfenbrenner, 1979) are important factors to consider in developing an understanding of a child's situation.

\subsubsection{Insight into Strengths, Needs and Perspectives}

Louise and Grace referred to greater insight into the perspectives of home and school settings and John's needs, including understanding around his need to engage in the restricted interest, following team engagement in the AFI process (See Appendix 28, Section 3 (Proposition 1) for key quotes). Sharing information on the situation at home and at school is consistent with the transactional perspective on child development promoted by AFI principles (Pameijer, 2017) and provided insight into how events may be experienced by John in these environments (Bronfenbrenner, 1979, p. 22). These findings provided support for Proposition 1 (Section 4.3) and are consistent with outcomes in the Pameijer (2017) study, where assessment provided most teachers and counsellors, and some parents with greater insight into the child's situation. Evidence of greater insight into needs also satisfies the stipulations for professional assessment outlined in Circular 0013/2017 (DES, 2017b, p. 14), in terms of providing understanding of student needs and the nature of difficulties.

\subsubsection{Strengthening Relationships}

Consistent with Proposition 3 findings in the Pilot Case, teachers in the Pameijer (2017) study felt more capable to teach the child following engagement in the AFI process. Grace and Louise commented on how relationships had been strengthened following team engagement in the AFI process and this may have contributed to greater perceived competence to support John's needs at home and at school (See Appendix 30, Section 3 (Proposition 3) for key quotes). For example, Grace reflected on "an opening up of communication between home and school" and observed John to be "much more responsive" to her. Improved communication and relationships at the meso and microsystem levels signifies strengthened developmental potential of home and school 
settings in working towards supporting John's needs and the possible development of affective dyadic relations between student and teacher (Bronfenbrenner, 1979, p. 56). This finding is in agreement with the Pameijer (2017) study, where engaging in the AFI process provided the school and parents with a perspective on how to further collaborate and come to an agreement in the best interests of the child. Similarly, Aganza et al. (2014) (Section 2.14.2) reported improved relationships between home and school and potential for greater collaboration, as a positive outcome of the ecological assessment approach applied. Relevant to Circular 0013/2017 (DES, 2017b), these findings suggest that strengthened relationships between home and school at the mesosystem level is a factor for consideration, in supporting and meeting the needs of students.

Strengthening relationships: Considering the child's perspective. With regards Proposition 4, John's responses, during the interview, indicate that working as part of a team and engaging in the assessment process led to understanding of strengths, needs and what to do to address needs (Section 4.6). Findings also suggest this may have been a challenging experience for him, with "hard decisions" and difficulty finding out about strengths and needs with his mother and teacher. From the perspective of Bronfenbrenner (1979, p. 22), continued consideration of John's views and experience is required to support psychological growth and communication and co-operation between parents, teachers and child is fundamental to this process. Considering the child's experience during an assessment and including his thoughts and feelings around the process, is in accordance with AFI principles where talking with the student is encouraged as much as possible, rather than talking about them (Pameijer, 2017). The findings of the systematic review indicated an overall absence of the voice of the child. Hanchon and Allen (2013) reported only 31\% of respondents conducted diagnostic student interviews in their ED evaluations and participants in Hill and Turner (2016) reported few children being involved in decision making about their treatment for ADHD. Consideration of the child's experience and perspective during an assessment presents as an implication for EP practice in Chapter 6.

\subsection{Perspectives on Barriers to Parental Engagement}

Comments made by Grace indicate that team engagement in the AFI process may have addressed a communication "barrier" that existed previously between home and school, reflective perhaps of inherent communication practices in the school (See Appendix 30, Section 3 (Proposition 3) for key quotes). Limited communication between home and school prior to the AFI model may have hindered the developmental 
potential of these settings to identify, meet and support John's needs (Bronfenbrenner, 1979, p. 216; DES, 2017a). Barriers in communication between home and school contradict the inclusive assessment practices for school promoted by the European Agency for Special Needs and Inclusive Education, where assessment is considered an ongoing communicative process of teachers, parents and students developing an understanding of the student's learning needs (Watkins, 2007). Enhanced communication practices between home and school settings at the mesosystem level is a factor for consideration as we work towards meeting the aims of Circular 0013/2017 (DES, 2017b), discussed further in Chapter 6.

\subsection{Factors Bridging the Gap between Assessment and Intervention}

Findings presented in the Pilot Case provide supportive evidence for Proposition 2 and parent, teacher and TEP indicated knowledge of appropriate intervention supports following engagement in the AFI process (Section 4.4). This finding is in accordance with the Pameijer (2017) study, where the majority of teachers and half of parents indicated assessment provided meaningful recommendations. It is also consistent with the findings of the five additional studies identified in the systematic review, that provided evidence of assessment approaches informing appropriate interventions for clients (Bozic, 2013; Cane, 2016; Lawrence \& Cahill, 2014; Parker et al., 2016; Tobias, 2017) (Section 2.8). In the current study, the theme 'Factors bridging the gap between assessment and intervention' was comprised of four subthemes; 'Experiencing or observing intervention effectiveness', 'Practical interventions', 'Shared awareness of strengths, needs and perspectives' and 'A collaborative approach to implementing and monitoring interventions'. These subthemes are expanded upon in the sections below. Collectively, it is proposed that these factors address the gap in practice identified in the systematic review, between assessments being conducted and the interventions that clients are receiving, having implications for EP practice, discussed further in Chapter 6.

\subsubsection{Experiencing Intervention Effectiveness and Practical Interventions}

Thematic analysis suggests factors involved in bridging the gap between assessment and intervention in the Pilot Case included experiencing and observing intervention effectiveness during the five-stage AFI process and the recommendation of practical interventions that were beneficial to John and other children in the class, for example behaviour specific praise and "little steps" that could be taken in the home 
environment (See Appendix 29, Section 3 (Proposition 2) and Appendix 30, Section 3 (Proposition 3) for key quotes). Consistent with AFI principles, parent, teacher and child were co-assessors and engaged in tasks and activities to investigate the effectiveness of intervention strategies that supported John's need to engage in alternative activities (Pameijer, 2017). Engaging in alternative interest activities was considered a molar activity, a behaviour perceived as having meaning or intent by participants in a particular setting (Bronfenbrenner, 1979, p. 45). Implementing practical interventions to support John's need to engage in alternative activities supported the development of this molar activity (Bronfenbrenner, 1979). Entries in the researcher's diary indicated Grace and Louise were able to identify an intervention recommendation that they were willing to start 'tomorrow', in line with the aims of AFI (Pameijer, 2017). Participant satisfaction with intervention recommendations is reflective of findings presented in the Lawrence and Cahill (2014) study, where student and teacher interview responses suggested the utility of interventions in the classroom context, and parent feedback indicated intervention effectiveness in the home setting (Section 2.8.2).

\subsubsection{Shared Awareness of Strengths, Needs and Goals}

Shared awareness of needs and goals identified also facilitated meaningful intervention planning and knowledge of effective supports (See Appendix 29, Section 3 (Proposition 2) for key quotes). John's awareness of information being communicated between child, parents, teacher and TEP and having his voice heard in meetings is indicative of his central role during the assessment process (Pameijer, 2017). It also suggests a shifting balance of power towards him as the developing person and may have encouraged the development of dyadic relations between parent, teacher, TEP and child (Bronfenbrenner, 1979, p. 56). Additionally, for information to be shared and understood by child, parent and TEP during the AFI process, appropriate language use was considered before, during and after interactions (Palinscar, 1998, p. 353). Appendix 19 provides a sample account of the questions and language applied during the AFI process, in accordance with procedural guidelines (Pameijer, 2016). This reflects the approach of Tobias (2017), who co-constructed knowledge and information between child, family and EP with a genogram assessment tool. The process directly informed meaningful intervention planning and the family's attention was drawn to repeated patterns of behaviour and unresolved or unvoiced issues. 


\subsubsection{A Collaborative Approach to Implementing Interventions}

With regard to Proposition 5, Grace felt competent to monitor John's needs moving forward and accounted her ability to "being aware of the situation" and her general teaching skills (Section 4.7). This is a positive finding in consideration of stipulations of the new allocation model requiring school staff to meet and monitor the needs of students (DES, 2017a, p. 3). Thematic analysis suggests the inclusion of John's parents in the monitoring of goals and interventions moving forward (See Appendix 32, Section 3 (Proposition 5) for key quotes). From an ecological systems perspective, this reflects the strengthened developmental potential of home and school settings at the mesosystem level to meet and monitor John's needs (Bronfenbrenner, 1979, p. 216). This collaboration between home and school settings also resonates with findings presented by Parker et al. (2016), where teachers and parents worked together to monitor the effectiveness of a CBT and behaviour intervention programme (Section 2.7).

\subsection{Pilot Case Summary}

In summary, discussion of Pilot Case findings suggests the AFI model can bridge the gap between assessment and intervention through activity and interaction at the meso and microsystem levels. Key findings were expanded upon in the context of the literature and conceptual framework and further analysis and discussion is presented in the cross-case analysis discussion in Section 5.20. Case 1 findings are discussed in the following section. 


\subsection{Discussion of Findings: Case 1}

The findings presented for Case 1 propositions demonstrate that the AFI model can bridge the gap between assessment and intervention through activity and interaction at the meso and microsystem levels. These findings are discussed under themes in the sections below, with reference to the literature and conceptual framework.

\subsection{Views and Experiences of AFI}

Participant experience of engaging with the AFI model was positive. Thematic analysis of participants' interview responses suggest the AFI model facilitated a clear and structured process and a framework to engage in a team assessment (See Appendix 34, Section 3 (Proposition 1) for key quotes). Relevant to Proposition 1, this framework supported team engagement in activities and interactions and resulted in a better understanding of Jim's situation (Section 4.10). In accordance with Circular 0013/2017 stipulations (DES, 2017b) and at the micro and mesosystem levels, active participation and communication between child, home, school and school psychological service served to strengthen the developmental potential of these settings to identify and understand Jim's needs (Bronfenbrenner, 1979, p. 216). In agreement with AFI principles, the researcher adhered to a systematic and transparent assessment process with the implementation of the five stages. Findings are comparable to the Pameijer (2017) study, where assessment was structured from beginning to end and those important to teaching the child worked in co-operation towards shared goals. Michael (class teacher) compared his experience of the AFI process to a more formalised assessment approach and commented on how AFI empowered him to feel more involved:

but I think the whole concept and the model I think it's much better than you know, a one on one, very formalised setting, getting your feedback, this you feel like...it is a real process and you're involved in it and there's much more consultation and communication which I think was key moving forward (Michael, lines 151-154)

Consistent with feedback provided by teachers and parents in the Pameijer (2017) study, who expressed their appreciation for the AFI model, Michael's comment implies preference for the interactive, collaborative process promoted by AFI (Pameijer, 2017). This communicative, interactive approach is reflective of the ecological, dialogical assessment approaches described by Burns (2013) and Thomas and Oldfather (1997) in Sections 2.14.2 and 2.15.1 and could be applied in practice to satisfy the aims 
of Circular 0013/2017 (DES, 2017b). Tina (parent) noted that the AFI model helped her "to see more" and Sarah described how AFI "was a tool for him [Jim] to use, to express his opinion on how his learning might be improved" (See Appendix 34, Section 3 (Proposition 1) for key quotes). This finding addresses recommendations for practice, outlined in the Pameijer (2017) study, encouraging the active inclusion of students in the assessment process in as much as possible. It also contrasts with studies identified in the systematic review that indicated an absence of the voice of the child in the assessment process (Hanchon \& Allen, 2013; Hill \& Turner, 2016).

The AFI model was discussed as having practical applicability to other situations, reflecting its developmental validity (Bronfenbrenner, 1979, p. 29). Sarah (SET) commented on how she could apply the model to assess and meet the needs of other children in her class:

I found the model...the process hugely beneficial, not only to the child but to me as a teacher, in my learning and in the things that I have learned from the process, about how to address the needs, how to assess the needs of the children in the first place and then how I'm going to, as the teacher address those needs, and to ensure that that child can achieve to the best that they can (Sarah, lines 711)

This is a significant finding for EP and educational practice. Sarah reflected on how she could apply the stages of the AFI model to her own teaching and in accordance with Circular 0013/2017 (DES, 2017b, p. 17), how she could identify and meet the needs of children in her class. Michael noted that AFI could be applied as a framework for communication between home and school; "I think like parental attitude and for anything even for like behaviour contracts or anything because... it's basically like, giving a voice and hearing different people's perspectives", demonstrating its adaptability and interactive, ecological approach (Bronfenbrenner, 1979). Overall, parent and teacher views on the AFI model in Case 1 offer noteworthy findings for practice and policy, deliberated further in the cross-case discussion and Chapter 6.

\subsection{A Team Approach to Assessment}

Proposition findings are discussed within the context of the subthemes of "A team approach to assessment' in the sections that follow. 


\subsubsection{Active Roles and Communication}

Active participation and communication were described by Michael, Sarah and Tina, with engagement in discussion, voicing "concerns", conducting "observations" and "going through the different steps" (See Appendix 34, Section 3 (Proposition 1) and Appendix 37, Section 3 (Proposition 4) for key quotes).The TEP's role was described as a "facilitator" and it was acknowledged that Jim had "a very central role" in the process, reflecting a shifting balance of power towards him as the developing person (Bronfenbrenner, 1979, p. 58). This assessment approach is in agreement with specifications for an ecological assessment, described by Christenson and Anderson (2002) in Section 2.14.2, where practice considers contextual factors, alterable variables and student perspectives of these contexts and learning. Team engagement in assessment tasks as co-assessors (Pameijer, 2017) during the assessment process generated understanding of Jim's situation, as evidenced in Proposition 1 (Section 4.10) and Proposition 4 (Section 4.13) findings. Tools and signs, including co-investigator observation sheets, documented supports that help Jim to learn (Section 4.9, Table 4.11) and facilitated his active engagement in the co-construction of knowledge during the AFI process (Palinscar, 1998, p. 353; Vygotsky, 1978, p. 55). These findings are consistent with the Pameijer (2017) study, where teachers, parents and counsellors described a positive working relationship with the EP, and active co-operation in working to identify specific needs and goals. Active participation and communication between teachers, parents, family members and student was also portrayed in the EP assessment approaches outlined by Bourke and Dharan (2015) and Tobias (2017) in Section 2.7.4. The active participation and communication of teachers, parents and student during the AFI process in the current study, and the EP as "facilitator" of the process, appears to be in contrast with the traditional, medical model assessment practices outlined in twelve of the studies identified in the systematic review, where determination of a diagnosis or special education provision was the primary purpose of the assessment (Section 2.7.1). EPs in two of these studies reported a preference for moving away from assessment practices driven by the medical model (Bahr et al., 2017; Filter et al., 2013), further suggestive of the potential relevance of the AFI model (Pameijer, 2017) in current EP practice.

\subsubsection{Insight into Strengths, Needs and Perspectives}

In support of Proposition 1 and 4, working together as a team provided insight into Jim's strengths and needs and perspective on the situation in the home and school 
environment. For example, dialogue between team members generated understanding of what was working well at home that could be applied in school, and perspective on Jim's experience in these environments (See Appendix 34, Section 3 (Proposition 1) and Appendix 37, Section 3 (Proposition 4) for key quotes). This is consistent with findings presented in Pameijer (2017), where teachers and counsellors reported greater insight into the child's situation. Jim's understanding of strengths and needs following team engagement in the assessment process is also reflective of findings outlined in Bozic (2013); Bozic et al. (2017) and Cane (2016), where students worked with the EP to identify strengths across a range of contexts to inform an intervention plan. In the context of the current study, insight into Jim's actual and potential levels of development arose from conducting assessment tasks, including observations and 1:1 cognitive and attainment testing, theorised through ZAD, what Jim could do unassisted and ZPD, what Jim could do with the assistance of tools and signs, such as counters and mnemonic aids (Palinscar, 1998, p. 353; Vygotsky, 1978, pp. 85-86). The insight into strengths, needs and perspectives demonstrated in Case 1 addresses the aims of Circular $0013 / 2017$ (DES, 2017b, p. 14), in providing understanding of student needs and the nature of difficulties.

\subsubsection{Strengthening Relationships}

Consistent with Proposition 3 findings in Case 1, teachers in the Pameijer (2017) study felt more capable to teach the child following engagement in the AFI process (Section 4.12). Strengthening relationships emerged as a positive outcome of the team assessment approach in the current study, which may have contributed to perceived competence in supporting Jim's needs (See Appendix 36, Section 3 (Proposition 3) for key quotes). For example, Tina commented that she is now "doing more" with Jim and that she and Jim can "talk to the teachers more" if they have a problem. These factors may have also served to strengthen the developmental potential of home and school settings to identify and meet Jim's needs at the mesosystem level, and the formation of dyadic relations at the microsystem level (Bronfenbrenner, 1979, pp. 56, 216). This finding is consistent with the results of Aganza et al. (2014), who demonstrated improved relationships between home and school and the potential for greater collaboration between these settings as an outcome of their ecological assessment approach (Section 2.14.2). Similarly, Chun and Dickson (2011) outlined the significant indirect effects of parental involvement and culturally responsive teaching on students' sense of belonging and academic performance. Flexibility in working as part of a team 
was required in Case 1, which is further discussed under the theme 'Perspectives on barriers to parental engagement'. Researcher reflections report that although there were difficulties at times in coordinating a team assessment approach in Case 1, the process was very much worthwhile in terms of positive outcomes:

Overall, I really feel the model was very beneficial in this case. Although at times difficult to co-ordinate meetings where all participants could be involved, I feel participants were as active and included as possible, given individual situations and I feel confident leaving this school that positive change will occur for the child, parent and teachers (Reflective Journal, December $17^{\text {th }}$, reflection following interview)

In accordance with the researcher's reflection, AFI acknowledges that every case is unique and requires a tailored approach accordingly (Pameijer, 2017).

\subsection{Perspectives on Barriers to Parental Engagement}

Thematic analysis of participants' interview responses generated perspectives on barriers to parental engagement (See Appendix 34, Section 3 (Proposition 1) and Appendix 36, Section 3 (Proposition 3) for key quotes). Tina could not always be present for meetings scheduled due to work commitments and the assessment process was flexible and pragmatic to adapt to this situation, for example by communicating and gathering information over the phone. Latent analysis of Sarah's comments during the interview indicated disappointment that Tina could not be more present throughout the process, perhaps reflective of her own values and belief systems as a parent and teacher (See Appendix 34, Section 3 (Proposition 1) for key quotes). Unaddressed belief systems and values could potentially hinder the development of authentic supportive links between home and school, and the developmental potential of these settings to identify, understand and support student needs, in accordance with Proposition 1 (Section 4.10) and Proposition 3 (Section 4.12) (Bronfenbrenner, 1979, p. 216; Pameijer, 2017). The European Agency for Special Needs and Inclusive Education posits that the attitudes a mainstream class teacher holds in relation to inclusion and assessment are crucial (Watkins, 2007). In the context of the current study, values and belief systems were not directly addressed during interview, which is further discussed in Chapter 6.

In addition, although relationships appear to have strengthened following engagement in the AFI process in Case 1, communication between home and school presented a challenge for Tina in the previous school year and interview comments 
indicate she was unaware of the extent of Jim's learning needs at school (See Appendix 36, Section 3 (Proposition 3) for key quotes). Latent analysis of Tina's comments during interview suggested past experience and language used by a previous teacher, describing Jim as a "fairy", may have caused feelings of apprehension, with regard to engaging with the current team. Sarah reflected on how she valued home school communication but acknowledged this is not characteristic of typical practice; "Even like the communication with the parents like...I find that hugely important, but do we ever try to make that time to do it no". Barriers to communication between home and school challenge the inclusive assessment practices for school promoted by the European Agency for Special Needs and Inclusive Education (Watkins, 2007), where assessment is deemed an ongoing communicative process of teachers, parents and students developing an understanding of the student's learning needs (Section 1.2). Communication practices between home and school settings at the mesosystem level should be reflected upon as we work towards meeting the aims of Circular 0013/2017 (DES, 2017b). Furthermore, and consistent with social constructivist perspectives (Palinscar, 1998, p. 353), the impact of language on the co-construction of knowledge and communicative relations between home and school is an important factor for consideration.

\subsection{Factors Bridging the Gap between Assessment and Intervention}

Findings presented in Case 1 provide support for Proposition 2 and parent and teachers demonstrated knowledge of appropriate intervention supports following engagement in the AFI process (Section 4.11). This is consistent with findings in the Pameijer (2017) study where the majority of teachers and half of parents reported assessment provided them with recommendations. It is also congruent with the findings of studies identified in the systematic review that provided evidence of assessment informing appropriate interventions for clients (Bozic, 2013; Cane, 2016; Lawrence \& Cahill, 2014; Parker et al., 2016; Tobias, 2017) (Section 2.8). There were several factors identified as bridging the gap between assessment and intervention in Case 1, which are discussed in the sections below in accordance with proposition findings. Collectively, it is proposed that these factors address the gap identified in the systematic review, between assessments being conducted and the interventions that clients are receiving, having implications for EP practice discussed in Chapter 6. 


\subsubsection{Experiencing Intervention Effectiveness and Practical Interventions}

Thematic analysis of interview responses indicates experiencing and observing the effectiveness of intervention recommendations during the Investigation stage of the AFI process contributed to bridging the gap between assessment and intervention (See Appendix 35, Section 3 (Proposition 2) and Appendix 36, Section 3 (Proposition 3) for key quotes). Participants were co-assessors and Sarah, Tina and Jim commented on the effectiveness of the mnemonic aid for remembering tricky spelling words during the Investigation stage (Pameijer, 2017). For example, Tina (parent) commented "I feel it [mnemonic aid] working”. Spelling was considered a molar activity in Case 1, a behaviour perceived as having meaning or intent and thematic analysis suggests practical interventions such as the mnemonic aid supported the development of this skill (Bronfenbrenner, 1979, p. 45). As a sign, it functioned as a mediation strategy to assist Jim's internalisation of learning during 1:1 assessment, and was observed to be effective by parent, teachers and student in the home and classroom context (Palinscar, 1998, p. 353; Vygotsky, 1978, p. 55). Team engagement in these observation tasks during the Investigation stage reflects strengthening dyadic relations between parent and child and teacher and student, through participation in one another's activities (Bronfenbrenner, 1979, p. 56). Providing feedback on intervention effectiveness is also consistent with findings presented by Lawrence and Cahill (2014), where student and teacher interview responses indicated the utility of interventions in the classroom context, and parent comments suggested effectiveness in the home setting (Section 2.8.2). Congruent with the aims of AFI (Pameijer, 2017), researcher reflections documented that parent and teachers were able to choose recommendations they would be willing to start tomorrow, including the mnemonic aid for spelling at home and specific strategies for targeting working memory at school.

\subsubsection{Shared Awareness of Strengths, Needs and Goals}

Findings presented for Proposition 2 and 5 provide evidence to suggest greater knowledge of intervention supports and competency to monitor Jim's needs moving forward, following the AFI process (Section 4.11 and 4.14). Thematic analysis of interview responses indicates shared awareness of strengths, needs and goals identified during the five-stage process further contributed to knowledge of intervention strategies and competency to monitor Jim's needs (See Appendix 35, Section 3 (Proposition 2) for key quotes and Appendix 38, Section 3 (Proposition 5) for key quotes). For example, Sarah commented on Jim's awareness of strategies that he could implement to achieve 
goals, following engagement in the AFI process; "He's [Jim] aware of strategies that he can do and that he can put in place as well for him to achieve, he knows what he needs to achieve in". Shared awareness of Jim's learning potential, achieved through observations conducted by team members at home and in the classroom and engaging in 1:1 dynamic assessment with the TEP, helped to form realistic and achievable goals and intervention recommendations, in accordance with Vygotsky's ZPD (Vygotsky, 1978, p. 86) and AFI principles (Pameijer, 2017). This is consistent with social constructivist assessment approaches outlined by Green and Gredler (2002) and Thomas and Oldfather (1997) in Section 2.15.1, where learning is considered a reciprocal process as the student takes on an increasingly independent role and observations of how a student solves problems informs intervention planning.

\subsubsection{A Collaborative Approach to Implementing Interventions}

Findings presented for Proposition 5 demonstrate that Michael and Sarah felt competent to monitor Jim's needs, following engagement in the AFI process (Section 4.14). Thematic analysis of interview responses portrays a collaborative, team approach to monitoring needs, where Jim was central to the process (See Appendix 38, Section 3 (Proposition 5) for key quotes). Michael commented that he planned to consult with Jim and would also rely on his good relationship with the SET when monitoring intervention recommendations. Jim noted that he would need his parents and teachers to help him "complete" his goals, reflective of his inclusion in the process. Jim's active involvement in the monitoring of intervention recommendations is in adherence with AFI principles (Pameijer, 2017) and reflects a shifting balance of power towards him as the developing person (Bronfenbrenner, 1979, p. 58). This finding is consistent with the aims of Circular 0013/2017 (DES, 2017b) and findings presented by Cane (2016) in the systematic review, where student and school staff input was sought during assessment, to directly inform a solution-focused intervention. Student and staff were involved in the monitoring of the intervention and scaling and qualitative feedback indicated improvements in the student's learning and behaviour.

\subsection{Case 1 Summary}

In summary, the discussion presented for Case 1 indicates the AFI model can bridge the gap between assessment and intervention through activity and interaction at the meso and microsystem levels. Key findings were discussed under themes in conjunction with the literature and conceptual framework. A cross-case analysis 
discussion presented in Section 5.20 integrates these findings with the Pilot Case and Case 2 findings. Case 2 findings are discussed overleaf.

\subsection{Discussion of Findings: Case 2}

Overall, the findings presented for Case 2 propositions suggest the AFI model can bridge the gap between assessment and intervention through activity and interaction at the meso and microsystem levels. However, it is noted that as data were collected from the teacher only post engagement in the AFI process, there was limited evidence that could be drawn upon for analysis in this case. Findings are discussed under themes in the sections below, in accordance with the literature and conceptual framework.

\subsection{Views and Experiences of AFI}

Anne's (teacher) views on the AFI model were positive. She described AFI as a structured process that was very "user-friendly" and involved everyone "sort of working together" (See Appendix 40, Section 3 (Proposition 1) for key quotes). Researcher reflections suggest Mary (parent) may have also appreciated the framework that AFI provided for coming together and voicing concerns:

Mary found the process [Intake] very helpful, and said she felt very happy with how it went and she hopes the questions can be answered. I think she really valued having someone to listen and acknowledge her concerns (Reflective Journal, November $23^{\text {rd }}$, Intake stage)

With regard to Proposition 1, the framework for team assessment facilitated by AFI delivered a better understanding of Michelle's situation (Section 4.17). This reflects findings outlined in Pameijer (2017), where teachers, parents and counsellors reported the assessment was structured from beginning to end and those involved in teaching the child worked collaboratively towards shared goals. In the current study, working together during the AFI process at the micro and mesosystem levels also functioned to strengthen the developmental potential of the home, school and school psychological service to identify and understand Michelle's needs (Bronfenbrenner, 1979, p. 216). The AFI model was discussed as having practical applicability to other situations, reflecting its developmental validity (Bronfenbrenner, 1979, p. 29). Anne described how she could see the process being applied "across the board" and also how information gathered and interventions could be used with other children: 
Yeah, absolutely I think even though you're dealing with one specific child and their needs, it [AFI process] does crossover into so many other things, so yeah, no I definitely think I would and also in terms of the resources and in terms of the information that's come from all of this, I could see how it would work across the board, you know am, with Michelle obviously but also there are definitely things there that would work with other children and just has given me greater insight into everything (Anne, lines 159-164)

Anne's comment indicates that the AFI process provided her with greater insight and knowledge of how to meet needs, consistent with the three-step process outlined for schools in Guidelines for Primary Schools: Supporting Pupils with Special Educational Needs in Mainstream Schools (DES, 2017a, p. 3). Most noteworthy to EP and educational practice is the indication that Anne may apply what she learned from her experience with the AFI process to other students. These findings are discussed further in the cross-case discussion and Chapter 6.

\subsection{A Team Approach to Assessment}

Anne's description of participant roles reflects an active team assessment approach, with the TEP "facilitating the whole process" and teacher, parent and student sharing information and working together during the assessment process (See Appendix 40, Section 3 (Proposition 1) and Appendix 43, Section 3 (Proposition 4) for key quotes). Engaging in tasks and sharing of information provided insight into strengths, needs and the home and school environment, supporting Proposition 1 (Section 4.17) and Proposition 4 (Section 4.20). For example, Anne commented that information offered during meetings was based on her "everyday observations" and Mary had also provided "information that we [school and school psychological service] wouldn't be aware of". Researcher reflections indicate Michelle was an active participant, sharing information at the Intake stage, engaging in assessment and co-investigator tasks and also providing feedback on what was helping her to learn (See Appendix 43, Section 2 (Proposition 4) for key quotes). Relevant to Proposition 1 and 4, sharing information between home, school, school psychological service and child at the meso and microsystem levels served to strengthen the developmental potential of these settings to identify and understand Michelle's needs (Bronfenbrenner, 1979, p. 216). Furthermore, Michelle's active participation and enabling her participation during the process reflects a shifting balance of power towards her, as the developing person (Bronfenbrenner, 1979, p. 58). Tools, including checklists and a graphic organiser helped to facilitate 
Michelle's active involvement during the Investigation stage and task completion in the classroom (Palinscar, 1998, p. 353; Vygotsky, 1978, p. 55).

These findings are in agreement with the Pameijer (2017) study, where teachers, parents and counsellors described a positive working relationship with the EP, active co-operation in working to form specific needs and goals and greater insight into the child's situation. The active engagement and communication between all team members in Case 2 contrasts with the traditional, medical model assessment practices outlined in twelve of the studies presented in the systematic review, where determination of a diagnosis or special education provision was the primary objective of the assessment (Section 2.7.1). The team assessment approach is more reflective of the ecological, dialogical assessment approaches applied by Bourke and Dharan (2015) and Tobias (2017) in Section 2.7.4 and the ecological assessment methods described by Burns (2013) and Christenson and Anderson (2002) in Section 2.14.2.

\subsubsection{Strengthening Relationships}

In agreement with Proposition 3 findings in Case 2, teachers in the Pameijer (2017) study reported feeling more capable to teach the child after working through the AFI process (Section 4.19). Comments made by Anne (teacher) during the interview indicate a strengthened relationship between the TEP and Michelle following engagement with the AFI model, and researcher reflections suggest activities and interactions between the TEP and Michelle may have served to balance the power (Bronfenbrenner, 1979, p. 58) (See Appendix 43, Section 2 and 3 (Proposition 4) for key quotes). For example, Anne noted that Michelle was enthusiastic to see the TEP again and was "calling you [TEP] by your name". This finding addresses points of improvement for assessors outlined in the Pameijer (2017) evaluation study, advocating the active inclusion of students during the assessment process as co-assessors. In addition, researcher reflections, following the interview with Anne, suggest strengthened relationships between TEP and teacher, as an outcome of working collaboratively throughout the AFI process:

After working through the model, I really felt like I was speaking to a colleague, we had collaborated so much throughout the assessment process and I really feel we learned from each other (December $20^{\text {th }}$, reflections following the interview)

Relevant to Circular 0013/2017 (DES, 2017b), these collective findings suggest strengthened relationships between school psychological service, school, home and 
student at the meso and microsystem levels is a factor to consider in supporting and meeting the needs of students. Collaborative, strengthened partnerships between these settings and the child is integral to the AFI process (Pameijer, 2017) and further indicates the model's relevance in the context of current findings and Circular 0013/2017 (DES, 2017b).

Although findings indicate strengthened relationships at the meso and microsystem levels, Michelle was absent from school for a number of days during the assessment process and Mary was not always available to attend meetings at school due to illness and other commitments. The AFI model was flexibly applied to ensure Mary was included and informed throughout, with phone calls and updates during the process. Researcher reflections suggest the flexibility of the AFI model in practice is key to enabling meaningful change:

While Mary and Michelle were not as present [physically], they were part of the process as much as they could, given the situation. I feel the model has to be flexibly applied in practice and every case is going to present with its own challenges, it's how you adapt accordingly to the situation and show understanding and respect, is how change can still be effectuated (December $20^{\text {th }}$, Reflections following interview with class teacher)

Consistent with this reflective commentary, AFI acknowledges that every case is unique and requires a tailored approach accordingly (Pameijer, 2017). The theme 'Perspectives on barriers to parental involvement' provides further interpretation on this situation.

\subsection{Perspectives on Barriers to Parental Involvement}

Thematic analysis of participants' interview responses presented perspectives on barriers to parental engagement (See Appendix 40, Section 3 (Proposition 1) for key quotes). Latent analysis of Anne's comments regarding Michelle's absence during the AFI process indicated feelings of disappointment as she felt Michelle could have benefited more from the process had she been present, perhaps reflective of her own belief systems as a teacher and the value she had placed on the AFI process; "it's just really unfortunate because it could have been something that she could have got much more out of". Anne also felt that Mary (parent) could have "put herself forward" more during the assessment process and that "there's only so much you [school and school psychological service] can do". She felt that Mary may have been apprehensive about engaging with the school and the school psychological services, perhaps attributable to a "fear of the unknown". Although Mary was involved in the team assessment 
approach, Anne felt that making small changes in the home environment may have been too demanding a task for her:

I do honestly believe that she genuinely wants that help, but I think as time goes on if there is any input that she has to put in, it could be something that's just a little bit, a stretch too far for her, even small little things like making changes, the small changes that she has to make she might be thinking of them as much bigger and it's just finding it hard to break it down, so I think that's her own issues and her own abilities really (Anne, lines 198-202)

It is interesting that Anne felt these were Mary's "issues" and "her own abilities", a comment interpreted as in line with a medical, within-person perspective and reflective of studies identified in the systematic review, where assessment practices adhered to the medical model of practice (Section 2.7.1). In the context of the aims of Circular 0013/2017 (DES, 2017b), unaddressed belief systems, values and judgements could potentially hinder the development of genuine supportive links between home and school and consequently the developmental potential of these settings to meet Michelle's needs (Bronfenbrenner, 1979, p. 216). The European Agency for Special Needs and Inclusive Education asserts the attitudes a mainstream class teacher holds in relation to inclusion and assessment are crucial (Watkins, 2007). In relation to the current study, values and belief systems were not openly challenged during interview which is further discussed in Chapter 6.

In order to facilitate an inclusive assessment process, the TEP was mindful to apply language during meetings that would permit all participants to access and coconstruct knowledge (Palinscar, 1998, p. 353). Appendix 19 provides a sample account of the questions and language utilised during the AFI process, in compliance with procedural guidelines (Pameijer, 2016). Despite this form of planning, the TEP was possibly not perceptive enough to the implicit thoughts or feelings that the parent may have had around engaging in the process, and although the parent confirmed the assessment tasks were manageable, for example to conduct observations in the home environment, this may not have been the reality. This finding presents an implication for EP practice and could be addressed with further consideration on empowering parents to support their child's needs at school, discussed further in Section 6.5 of Chapter 6.

\subsection{Factors Bridging the Gap between Assessment and Intervention}

Case 2 findings provide support for Proposition 2 and Anne (teacher) indicated knowledge of appropriate intervention supports following engagement in the AFI 
process (Section 4.18). This is in agreement with outcomes of the Pameijer (2017) study, and studies identified in the systematic review that provided evidence of assessment informing appropriate interventions for clients (Bozic, 2013; Cane, 2016; Lawrence \& Cahill, 2014; Parker et al., 2016; Tobias, 2017). There were several factors identified as bridging the gap between assessment and intervention in Case 2, discussed under associated subthemes in the sections below, with implications for EP practice discussed in Chapter 6.

\subsubsection{Experiencing Intervention Effectiveness and Practical Interventions}

Thematic analysis of interview responses and researcher reflections indicate Anne and Michelle observed or experienced the effectiveness of interventions during the Investigation stage (See Appendix 41, Section 3 (Proposition 2), Appendix 42, Section 3 (Proposition 3) and Appendix 44, Section 3 (Proposition 5) for key quotes). Interventions observed to be effective by Anne included the self-monitoring checklist and graphic organiser, which were subsequently translated into intervention recommendations at the Feedback stage and these tools supported Michelle to complete tasks and assignments (Palinscar, 1998, p. 353; Vygotsky, 1978, p. 55). Researcher reflections indicate intervention planning was supported by Michelle (child) sharing her feedback on co-investigator activities:

I met with Michelle [child] and she gave me feedback on the co-investigator activity. She feels the graphic organiser helped her with her comprehension work and this could possibly be used for Irish writing as well. She is finding the self-check prompt for Maths helpful too. It reminds her to check answers and to use the resources in her basket when needed (Reflective Journal December $13^{\text {th }}$, Integration Stage)

Becoming more independent and responsible in completing tasks was considered a molar activity in Case 2, a behaviour perceived as having meaning or intent (Bronfenbrenner, 1979, p. 45) and thematic analysis suggests practical interventions including checklists and the graphic organiser supported its development. This finding is in agreement with the outcomes of the Lawrence and Cahill (2014) study, where parents, students and teachers provided feedback on the utility of interventions in the home and school setting (Section 2.8.2). Furthermore, and in line with AFI principles, Anne commented that the intervention recommendations arising from the assessment would be "beneficial for everybody [in the class]", indicating their universal applicability (Pameijer, 2017). 


\subsubsection{Shared Awareness of Strengths, Needs and Goals}

Findings presented for Proposition 2 and 5 provided evidence to suggest improved teacher knowledge of intervention supports and competency to monitor Michelle's needs following the AFI process (Section 4.18 and 4.21). Thematic analysis of interview responses indicates shared awareness of strengths, needs and goals identified during the AFI process, enhanced knowledge of intervention strategies and ability to monitor needs moving forward (See Appendix 41, Section 3 (Proposition 2) for key quotes and Appendix 44, Section 3 (Proposition 5) for key quotes). In agreement with the AFI concept of goodness of fit, sharing of assessment information helped Anne to realise she was "working towards the right way of doing it [supporting Michelle's needs]" (Pameijer, 2017). Furthermore, observations conducted by Michelle and Anne in the classroom and engaging in 1:1 standardised and dynamic assessment resulted in shared awareness of Michelle's actual and learning potential (Vygotsky, 1978, pp. 85-86). Sharing of information revealed some of the mediation strategies applied during assessment were similar to approaches that Anne was using in the classroom, for example, use of concrete materials for explaining concepts and rephrasing of questions to ensure understanding. These approaches to assessment are in accordance with the social constructivist assessment methods outlined by Green and Gredler (2002) and Thomas and Oldfather (1997) in Section 2.15.1, where learning is deemed reciprocal and the student progressively becomes more independent in the process.

\subsubsection{A Collaborative Approach to Implementing Interventions}

Proposition 5 findings demonstrate that Anne felt competent to monitor Michelle's needs following the AFI process (Section 4.21). Thematic analysis of interview responses suggests awareness of specific needs and also knowledge of intervention approaches that were "going to work for her (Michelle)", contributed to perceived ability to monitor needs moving forward (See Appendix 44, Section 3 (Proposition 5) for key quotes). This finding is relevant within the context of Circular 0013/2017 (DES, 2017b), as school staff are expected to monitor student needs under the allocation model of special education teaching resources (DES, 2017a, p. 3). Anne did not however refer to Michelle or Mary's role in the process of monitoring needs and interventions, which contradicts the aims of inclusive assessment outlined by the European Agency for Special Needs and Inclusive Education (Watkins, 2007). 


\subsection{Case 2 Summary}

In summary, the findings and discussion presented for Case 2 indicate the AFI model can bridge the gap between assessment and intervention through activity and interaction at the meso and microsystem levels. Key findings were expanded upon under themes in the discussion, in the context of the literature and conceptual framework. A cross-case analysis discussion is presented overleaf. 


\subsection{Cross-Case Analysis Discussion}

This section of the Discussion Chapter provides a cross-case analysis discussion of the Pilot Case, Case 1 and Case 2 findings. The first section outlines a table of parent and teacher Average Likert scale rating scores and findings are discussed in the context of the Pameijer (2017) evaluation study. Pattern-matching applied to interview responses to address case propositions are discussed in the context of resonating AFI principles (Pameijer, 2017). This is followed by a cross-case discussion of integrated findings under themes, in the context of the literature and conceptual framework.

\subsection{Likert Scale Ratings}

Average parent and teacher Likert scale ratings indicate greater understanding of the situation, knowledge of intervention supports and greater perceived competence in supporting the child's needs following engagement with the AFI model. Averages were rounded to the nearest decimal point, consistent with the Pameijer (2017) evaluation study and are reported in Table 5.1, for participants who completed the Likert scale statements before and after engaging with the AFI model. The Average ratings reflect percentages presented in Pameijer (2017), where $90 \%$ of teachers and $65 \%$ of parents indicated the assessment offered a better understanding of the student, $70 \%$ of teachers and $52 \%$ of parents reported the assessment offered them recommendations and $65 \%$ of teachers felt they could apply the recommendations in their class. Due to the small sample size of the current exploratory case study, reported Average ratings are not statistically generalisable but provide supportive evidence for Propositions 1, 2 and 3 along with pattern-matching logic of interview responses. With regards Proposition 4, Likert scale ratings provided by John in the Pilot Study indicate that he was "not sure" of his strengths following engagement with the AFI model. His ratings indicate that he now knows what he needs help with at school and still does not know what he can do to address needs at school. Likert scale ratings provided by Jim in Case 1 indicate he knew what his strengths and needs were before and after engaging with the AFI model. Jim's rating changed from not knowing what he can do to improve on needs at school to being "not sure" following engagement with the AFI model. These findings are supported by pattern-matching logic applied to interview responses, presented in the following section. 
Table 5.1

Participant Average Likert Scale Ratings

\begin{tabular}{llcc}
\hline Likert Statement & Participant & Before AFI Model & After AFI Model \\
\hline $\begin{array}{l}\text { I understand the } \\
\text { student's situation }\end{array}$ & Teacher $(n=4)$ & 5.5 & 8.9 \\
$\begin{array}{l}\text { I understand my } \\
\text { child's situation }\end{array}$ & Parent $(n=2)$ & 6 & 6.5 \\
$\begin{array}{l}\text { I know what } \\
\text { support the student } \\
\text { needs at school }\end{array}$ & Teacher $(n=4)$ & 5.5 & 8.9 \\
$\begin{array}{l}\text { I know what } \\
\text { support my child } \\
\text { needs at home }\end{array}$ & Parent $(n=2)$ & 3.5 & 9 \\
$\begin{array}{l}\text { I am able to the } \\
\text { student's needs at } \\
\text { school }\end{array}$ & Teacher $(n=4)$ & & \\
$\begin{array}{l}\text { I am able to } \\
\text { support my child's } \\
\text { needs at home }\end{array}$ & Parent $(n=2)$ & 4.8 & 9 \\
\hline
\end{tabular}

\subsection{Pattern-Matching Logic}

Pattern-matching logic was applied to the responses of participants who engaged in interview following engagement in the AFI model in each of the three cases. Responses are discussed in the context of resonating AFI principles (Pameijer, 2017). Proposition findings are further discussed under associated themes in Sections 5.23, $5.24,5.25$ and 5.26, in the context of the conceptual framework and literature.

\subsubsection{Proposition 1}

Pattern-matching logic was applied to parent and teacher responses to questions exploring change in understanding and experience of the AFI model. Responses across all three studies suggest interactions and activities that occurred at the meso and microsystem levels during the five stages were connected to greater understanding of the situation (Sections 4.3, 4.10, 4.17). Activities and interactions included information taking, a team approach to identifying the issues and hearing each other's voices (Pilot Case); getting the voice of the student, parent and teachers and establishing working memory as an area of cognitive weakness (Case 1) and talking through everything and analysing the situation (Case 2). These activities and interactions resonated with several 
AFI principles as outlined in the aforementioned sections, including applying a transactional perspective to the presenting situation (Principle 2), engaging in tasks as co-assessors (Principle 6) and adherence to a systematic and transparent assessment process (Principle 7) (Pameijer, 2017).

\subsubsection{Proposition 2}

To address Proposition 2, parents and teachers were questioned on intervention recommendations and supporting the child's needs. Patterns across participant responses indicate interactions and activities that occurred at the meso and microsystem levels supported knowledge of intervention supports the child needed (Sections 4.4, 4.11, 4.18). These included intervention recommendations observed and discussed during the AFI process such as close proximity praise, behaviour specific praise and exploring alternative interests (Pilot Case); appraisal, spelling acronym, ideas to address memory and auditory skills (Case 1); social communication and CBT interventions, checklists and graphic organisers (Case 2). Consistent with Proposition 1, these activities and interactions were in accordance with AFI Principles 6 and 7, and in addition Principles 3 and 4, identifying teacher and parent approaches to address the child's needs (Pameijer, 2017).

\subsubsection{Proposition 3}

Proposition 3 was addressed with a question exploring ability to support the child's needs. Patterns within parent and teacher interview responses suggest interactions and activities that occurred at the meso and microsystem levels during the AFI process were connected to greater perceived competence to support the child's needs (Sections 4.5, 4.12, 4.19). These activities and interactions included gaining knowledge of alternative interests and developing understanding of the situation and triggers for behaviour (Pilot Case); gaining knowledge of student's needs, recommendations to address needs and strengthening relationships between home and school (Case 1) and gaining knowledge around extra interventions and continuing with strategies that are working well (Case 2). Akin to Proposition 2, these activities and interactions were consistent with AFI Principles 3 and 6 and additionally, Principle 1, participating in a goal directed and functional assessment, with the development of feasible intervention recommendations (Pameijer, 2017). 


\subsubsection{Proposition 4}

To address Proposition 4, John (child in Pilot Study) and Jim (child in Case 1) were asked interview questions exploring their thoughts on their strengths, needs and what they need to do to address needs. They were also asked a question on how they found the process of working together with their parent, teachers and the TEP. Patterns identified within their responses connected interactions and activities that occurred at the microsystem levels during the AFI process, including dialogue between team members, co-investigator observation tasks and 1:1 assessments/joint activity tasks to knowledge of strengths, needs and what to do to address needs (Sections 4.6, 4.13, 4.20). John responded "kind of" to questions exploring if working together helped him to find out about his strengths and needs and that it was more difficult to find out about his strengths and needs with his mother and teacher, than with the TEP. His responses indicated that the assessment process was a challenging experience in "making hard decisions". As regards addressing needs, John commented that he needs to "study my best in school". When asked about making time for new activities and interests, John commented "yeah that would be one". In contrast, Jim responded "yes" to questions exploring if working together helped him to find out about his strengths and needs. When asked what he needs to do to improve on needs, Jim mentioned strategies that had been practiced during 1:1 dynamic assessment. Activities and interactions that Jim and John engaged in at the microsystem level are considered consistent with AFI Principle 6 (Pameijer, 2017) as they were both considered co-assessors when completing coinvestigator tasks and their opinions and feedback contributed to intervention planning. Proposition 4 findings are discussed further under related themes in Section 5.24, in conjunction with the conceptual framework and literature.

\subsubsection{Proposition 5}

With regards Proposition 5, teachers were asked an interview question exploring their perceived levels of competence to monitor the student's needs moving forward. Patterns within their responses associated ability to monitor needs to interactions and activities that occurred at the microsystem levels during the AFI process (Sections 4.7, 4.14, 4.21). In Case 1, patterns within Michael's response associated competence with his strengthened relationship with Jim and having a good relationship with the learning support teacher. Sarah attributed competency to her awareness of Jim's learning needs following engagement with the AFI process. Similarly, in Case 2 patterns within Anne's response related competence to greater awareness and insight into needs following the 
AFI process. Patterns within Anne and Grace's response also connected competence to monitor needs to their general teaching skills. These activities and interactions were considered consistent with AFI Principle 3, focusing on the educational needs of the child and AFI Principle 6, engaging in tasks as co-assessors. The following sections examine proposition findings under relevant themes, in accordance with the conceptual framework and literature.

\subsection{Views and Experiences of AFI}

Parent and teacher experience of engaging with the AFI model was positive in all three cases. The AFI model was described as a clear, structured process that facilitated engagement in a team assessment to better understand the students' situation, as evidenced by Proposition 1 findings and consistent with outcomes presented in the Pameijer (2017) evaluation study. Teachers in Case 1 and the Pilot Study compared their experience of AFI to traditional, formalised assessment approaches and commented on their preference for AFI (Pameijer, 2017). Both Michael (Case 1) and Grace (Pilot Case) commented on the interaction and communication that AFI facilitated, consistent with ecological and social constructivist assessment approaches outlined by Burns (2013) and Thomas and Oldfather (1997) in Sections 2.14.2 and 2.15.1. The theoretical statement presented in Section 2.16.2 proposed that communication and interaction at the meso and micro system levels would be necessary to successfully fulfill the aims of Circular 0013/2017 (DES, 2017b) and is supported by Grace and Michael's interview comments. Relevant to EST, active participation in activities and communication during the AFI process at the micro and mesosystem levels, between child, home, school and school psychological service served to strengthen the developmental potential of these settings to identify and understand student needs (Bronfenbrenner, 1979, p. 216). Consistent with the aims of AFI (Pameijer, 2017) and inclusive assessment approaches promoted by the European Agency for Special Needs and Inclusive Education (Watkins, 2007), the students were included in the team assessment facilitated by AFI. For example, in Case 1, Sarah described how AFI "was a tool for him [Jim] to use, to express his opinion on how his learning might be improved". This finding contrasts with studies identified in the systematic review that indicated an absence of the voice of the child in the assessment process (Hanchon \& Allen, 2013; Hill \& Turner, 2016). 


\subsubsection{Applicability to Other Situations}

The AFI model was discussed as having practical applicability to other situations by the teachers in each of the case studies, reflecting the model's developmental validity (Bronfenbrenner, 1979, p. 29). In Case 1, Sarah commented on how she could apply the model to assess and meet the needs of other children in her class and Michael reflected on how it could be applied to any school situation that required communication. Grace commented that she would now be more perceptive to the home situation if a child was having difficulties at school, reflective of an ecological perspective (Pilot Case). She also acknowledged that there was a time commitment involved in the AFI process and that she lost out on class teaching time, however the cost-benefit was balanced by the information gathered and strengthened communication between home and school, reflective of Pameijer (2017) findings. Anne (Case 2) discussed how she could see the process being applied "across the board" and also how information gathered, and interventions could be used with other children. Overall, collective views on the AFI model provide noteworthy findings for practice and policy, particularly on how the model could be applied to address the aims of Circular 0013/2017 (DES, 2017b), discussed as an implication for practice in Chapter 6.

\subsection{A Team Approach to Assessment}

Active participation and communication, suggestive of a team assessment approach was described by parents and teachers in the Pilot Case and Case 1, and by the teacher in Case 2. They acknowledged the involvement of all team players, including the child and the TEP as a "facilitator" of the process. Relevant to Proposition 1 and 4, active participation and communication between home, school, school psychological service and child at the meso and microsystem levels served to empower the developmental potential of these settings to identify and understand the child's needs (Bronfenbrenner, 1979, p. 216). This is reflective of the assessment approaches applied by Bourke and Dharan (2015), Pameijer (2017) and Tobias (2017) in the systematic review and the ecological assessment approaches described by Burns (2013) and Christenson and Anderson (2002) in Section 2.14.2. The active engagement of team members appears to be in contrast with the traditional, medical model assessment practices outlined in twelve of the studies presented in the systematic review, where determination of a diagnosis or special education provision was the main objective of the assessment. Working together as a team provided insight into strengths and needs and the home and school environment in each of the case studies, consistent with 
findings presented in Pameijer (2017) and the stipulations for professional assessment outlined by Circular 0013/2017 (DES, 2017b, p. 14), to provide understanding of student needs and the nature of difficulties. Relevant to Proposition 3, strengthening relationships was interpreted as an outcome of engagement in team assessment in each of the case studies and may have contributed to perceived competence in supporting the child's needs moving forward, consistent with findings presented in the literature review (Aganza et al., 2014; Chun \& Dickson, 2011; Pameijer, 2017).

Consideration of the child's experience during the assessment was presented as a factor for consideration in a team assessment in the Pilot Study and is in accordance with the concept of 'experienced' proposed by Bronfenbrenner (1979, p. 22). In line with AFI (Pameijer, 2017), inclusion of the voice of the child was encouraged during the AFI process in each of the three cases. Relevant to Circular 0013/2017 (DES, 2017b), strengthened relationships between school psychological service, home and school at the mesosystem level and between child and adults at the microsystem level is a factor for consideration in supporting and meeting the needs of students moving forward. In addition, Grace referred to the role of additional outside services at the mesosystem level, for providing optimal levels of support to meet John's needs, requiring effective co-ordination and communication across systems (Bronfenbrenner, 1979). Flexibility in working as a team was discussed in Case 1 and Case 2 and researcher reflections documented that although there were difficulties at times coordinating a team assessment approach, the process was very much worthwhile in terms of positive outcomes.

\subsection{Perspectives on Barriers to Parental Engagement}

Comments made by the teacher in the Pilot Case and the parent and SET in Case 1 suggest there were barriers to communication between home and school before engaging with the AFI model, which may reflect inherent communication practices of the school. Tina in Case 1 also referred to the language used by a previous class teacher, to describe her son which had a lasting, negative impact. In the context of the conceptual framework, poor communication between home and school could hinder the developmental potential of these settings to identify, meet and monitor the needs of students (DES, 2017a) and contrasts with the inclusive assessment practices for schools promoted by the European Agency for Special Needs and Inclusive Education (Watkins, 2007). Belief systems, values and judgements of teachers was also discussed as a factor that could potentially hinder the developmental potential of home and school 
settings to meet students' needs (Bronfenbrenner, 1979, p. 216). During the assessment process, the parents in Case 1 and Case 2 could not be present for all meetings scheduled due to work commitments and illness and the model was flexibly applied to gather and communicate information over the phone (Sections 5.11 and 5.16.1). Teachers in Case 1 and Case 2 (Sarah and Anne), expressed their disappointment in interview that the parents were not as present during the assessment process, reflective perhaps of their own belief systems, values and habitus as teachers and/or parents (Bourdieu, 1977). In the context of the current study, these values and belief systems were not directly addressed during interview, discussed further in Section 6.4 of Chapter 6. In addition, Anne expressed that having to make small changes in the home environment may have been too demanding a task for Mary (parent). Although Mary was involved during the assessment process, the TEP may not have been fully perceptive to the implicit thoughts or feelings that she had around engaging in the process. Despite confirming that assessment tasks were manageable, including observations in the home environment, this may not have been the reality. Such findings present implications for EP practice and could be addressed with further consideration of how to empower parents to support their child's needs at school, discussed in Section 6.5 .

\subsection{Factors Bridging the Gap Between Assessment and Intervention}

Relevant to Proposition 2, the findings presented in each case study indicate that assessment informed appropriate interventions for participants. These finding are consistent with the studies identified in the systematic review that also provided evidence of assessment informing appropriate interventions for clients (Bozic, 2013; Cane, 2016; Pameijer, 2017; Parker et al., 2016; Tobias, 2017). There were several factors identified as bridging the gap between assessment and intervention including shared awareness of strengths and needs identified, experiencing or observing intervention effectiveness, practical intervention recommendations and a collaborative approach to implementing and monitoring interventions. In Case 1 and Case 2, shared awareness of students' learning potential, achieved through observations conducted by team members at home and in the classroom as co-assessors (Pameijer, 2017), and engaging in 1:1 assessment of actual and learning potential with the TEP, helped to form realistic and achievable goals and intervention recommendations, consistent with Vygotsky's ZAD and ZPD (Vygotsky, 1978, pp. 85-86). 
Experiencing or observing intervention effectiveness occurred during the Investigation stage in each of the three cases and these interventions were later translated into recommendations. For example in Case 1, Sarah, Tina and Jim commented on how they tried the mnemonic aid for remembering tricky spelling words during the Investigation stage and observed it to be effective. In Case 2, Anne and Michelle observed or experienced the effectiveness of interventions during the Investigation stage, including the self-monitoring checklist and graphic organiser, which became intervention recommendations at the Feedback stage. This is in agreement with findings presented in Lawrence and Cahill (2014), where student and teacher interview responses provided evidence of the utility of interventions in the classroom context, and parent comments indicated intervention effectiveness in the home setting.

Pertinent to Proposition 3 and competency to support needs, practical intervention recommendations were provided and were described by teachers and parents as being feasible to implement and beneficial to other children in the class or "little steps" that could be taken in the home environment. Consistent with AFI (Pameijer, 2017), researcher reflections indicated that parents and teachers in the Pilot Case and Case 1 were able to identify an intervention recommendation that they were willing to start 'tomorrow'. These finding are consistent with the aims of Circular 0013/2017 (DES, 2017b) and findings presented by Cane (2016) in the systematic review, where student and school staff input was sought during assessment to directly inform a solution-focused intervention. Relevant to Proposition 5 and monitoring of needs, Grace's responses in the Pilot Case indicated parental inclusion in the monitoring of goals and interventions and Michael commented that he planned to consult with Jim in this process in Case 1. Jim also commented that he would need his parents and teachers to help him "complete" his goals, reflective of his inclusion in the process (Pameijer, 2017). Collectively, these factors have implications for EP practice and address the gap between assessments being conducted and the interventions that clients receive identified in the current systematic review.

\subsection{Chapter Conclusion}

This chapter discussed the findings of the current research project in the context of the literature and conceptual framework. Implications for practice are discussed in Chapter 6 along with directions for future research and limitations of the study. 


\section{Chapter 6 Conclusions}

\subsection{Overview of Chapter}

This concluding chapter provides a summary of the research and the unique contribution of the work. Implications and recommendations for EP practice and the broader educational context are outlined, including the evolving role of the EP in a progressing needs-based assessment context with the issuance of Circular 0013/2017 (DES, 2017b) and Circular 0014/2017 (DES, 2017c). Limitations of the research are discussed and the scholarly significance of the research is critiqued in accordance with Yin (2009) stipulations. Directions for future research are outlined along with proposals for the dissemination of findings.

\subsection{Summary of Research Findings}

Within the Irish Educational Psychological context, the model for allocation of special education teaching resources in mainstream schools stipulates resource allocation based on identified needs rather than diagnosis of SEN (DES, 2017a). Relevant to its implementation, assertions for professional assessment outlined in Circular 0013/2017 (DES, 2017b) and Circular 0014/2017 (DES, 2017c) pertaining to mainstream primary and post-primary schools, specify a shift in focus from assessment for diagnosis towards a needs-based approach that informs appropriate interventions, having direct implications for EP practice. Linking assessment to intervention is discussed as a presenting challenge in practice in academic discourse (Resing et al., 2017; VanDerHeyden, 2018). In the context of the issuance of Circular 0013/2017 (DES, 2017b) and Circular 0014/2017 (DES, 2017c), a systematic review was conducted to explore assessment approaches that EPs are currently applying in practice and to determine if they are informing appropriate interventions for clients. Twenty-one studies provided empirical evidence of assessment approaches used by EPs in practice and only six of these studies provided evidence to suggest the approach informed appropriate interventions for clients, indicating a gap in EP practice. Of all the assessment approaches reviewed, the AFI model (Pameijer, 2017) was considered most consistent with the aims of Circular 0013/2017 (DES, 2017b) and Circular 0014/2017 (DES, 2017c), as it aims to provide a better understanding of the child and the support he/she needs. The current research sought to explore the utility of the AFI model in bridging the gap between assessment and intervention, through the conceptual lens of EST and SCT with participants in mainstream primary schools. It was proposed that 
conducting the research through this conceptual framework would further strengthen the theoretical underpinnings of the AFI model.

The presented cases were analysed to derive authentic cross-case conclusions, in the context of the conceptual framework and literature, and validity of findings were demonstrated by maintaining a chain of evidence (Appendices 27-53). In summary, the collective findings of the case studies suggest the AFI model can bridge the gap between assessment and intervention through activity and interaction at the meso and microsystem levels (Bronfenbrenner, 1979). Average parent and teacher Likert scale ratings indicated greater understanding of the situation, knowledge of intervention supports and greater perceived competence in supporting the child's needs following engagement with the AFI model, consistent with findings presented in Pameijer (2017). The current research extended these findings with the application of pattern-matching and thematic analysis to participant interview responses. Pattern-matching logic provided supportive evidence for Propositions 1, 2, 3, 4 and 5 in each of the three cases ${ }^{5}$ and indicated activities and interactions that occurred at the meso and micro system levels, including dialogue between team members, co-investigator observation tasks and 1:1 assessments/joint activity tasks, were connected to:

1. Greater understanding of the child's situation (Proposition 1)

2. Knowledge of intervention supports the child needed (Proposition 2)

3. Greater perceived competence in supporting the child's needs (Proposition 3)

4. Greater understanding of strengths, needs and what to do to address needs (Proposition 4-child only)

5. Greater perceived competence to meet and monitor needs moving forward (Proposition 5-teachers only)

An in-depth analysis of findings demonstrated that these activities and interactions were in accordance with AFI principles, EST and SCT concepts (Appendices 27-44). In addition, discussion of these findings under related themes generated through thematic analysis and in the context of the conceptual framework and literature, demonstrated consistency of the assessment process with ecological, socialconstructivist assessment approaches (Burns, 2013; Christenson \& Anderson, 2002),

\footnotetext{
${ }^{5}$ Data were collected from the teacher only post engagement in the AFI process in Case 2, therefore there was limited evidence that could be drawn upon for analysis in this case.
} 
further strengthening the theoretical underpinnings of the AFI model. These approaches contrast with the traditional, medical model assessment practices outlined in twelve of the studies presented in the systematic review, where determination of a diagnosis or special education provision was the primary objective of the assessment (Section 2.7.1). It is proposed that the collective findings of this exploratory research project, expanded upon in the sections below, address the gap identified in the current systematic review, between assessments being conducted and the interventions that clients receive in EP practice. The findings satisfy the stipulations for professional assessment outlined in Circular 0013/2017 by providing understanding of the child's needs, the nature of difficulties, and informing appropriate interventions (DES, 2017b, p. 14). The implications of research findings are presented in the following section.

\subsection{Implications of Research Findings}

The research presents significant implications for the discipline of educational psychology in the context of Circular 0013/2017 (DES, 2017b). The first key implication for EP practice is the presentation of the AFI model (Pameijer, 2017) as an assessment framework consistent with ecological and social constructivist perspectives, that can satisfy the aims of Circular 0013/2017 (DES, 2017b). A second implication is the consideration of factors that bridged the gap between assessment and intervention in the current research project, in EP practice. These implications are expanded upon in the sections below.

\subsubsection{Implications for Policy}

The cross-case analysis discussion indicates parents, teachers and TEP found AFI (Pameijer, 2017) to be a clear, structured process that facilitated engagement in a team assessment approach. The assessment process was considered consistent with ecological and social constructivist assessment approaches outlined by Burns (2013) and Thomas and Oldfather (1997) in Sections 2.14.2 and 2.15.1. Findings suggest parents and teachers had a greater understanding of the child's situation, knowledge of intervention supports and greater perceived competence in supporting the child's needs following engagement with the AFI model, consistent with the aims of Circular 0013/2017 (DES, 2017b). Teachers in Case 1 and the Pilot Case compared their experience of AFI to traditional, formalised assessment approaches and commented on their preference for AFI (Pameijer, 2017). The theoretical statement outlined in Section 2.16.2 of the literature review proposed that communication and interaction at the meso 
and micro system levels would be necessary to successfully meet the aims of Circular 0013/2017 (DES, 2017b, p. 14) in providing understanding of needs and informing meaningful interventions, supported by the findings presented. AFI provides a structured framework for communication and interaction at the meso and microsystem levels, between school psychological service, school, home and student and strengthens the developmental potential (Bronfenbrenner, 1979, p. 216) of these settings to work together to meet the aims of Circular 0013/2017 (DES, 2017b). Furthermore, the students' voice and their active participation was facilitated and encouraged throughout the process, shifting the balance of power towards them as the developing person (Bronfenbrenner, 1979, p. 58) and contrasting with studies in the systematic review that acknowledged the absence of the voice of the child during the assessment process (Hanchon \& Allen, 2013; Hill \& Turner, 2016). AFI provides a framework for the inclusion of the voice of the child, which is consistent with the inclusive assessment approaches promoted by the European Agency for Special Needs and Inclusive Education (Watkins, 2007). The AFI model could be applied in EP practice to facilitate the child's active involvement in working towards meeting the aims of Circular 0013/2017 (DES, 2017b).

\subsubsection{Implications for EP Practice}

The researcher's systematic review identified a gap between assessment being conducted by EPs and the interventions that clients receive, consistent with assertions by Resing et al. (2017) and VanDerHeyden (2018) in Section 1.4. Stipulations for professional assessment in Circular 0013/2017 (2017b, p. 14) outline the role of the EP in providing understanding of a child's needs, the nature of difficulties, and informing appropriate interventions. The overall aim of the AFI model is to bridge the gap between assessment and intervention in order to provide recommendations that are both scientifically sound and useful for the student, teacher and parent (Pameijer, 2017). Factors identified as bridging the gap in the current research project included shared awareness of strengths and needs identified between child, parents, teachers and TEP; experiencing or observing intervention effectiveness during the Investigation stage; practical intervention recommendations and a collaborative approach to implementing and monitoring interventions. These factors could be considered by EPs in practice as they work in accordance to the aims of Circular 0013/2017 (DES, 2017b) in mainstream primary schools. 


\subsubsection{Implications for Educational Contexts}

The AFI model was discussed as having practical applicability to educational settings by teachers in each of the case studies, reflecting the model's developmental validity (Bronfenbrenner, 1979, p. 29). Teachers indicated the model could be applied to consider the home situation when a child is having difficulties at school (Pilot Case), to assess and meet the needs of other children in the class and to provide a framework for communication between home and school (Case 1) and its applicability "across the board" as information gathered and interventions were useful to other children (Case 2). In terms of monitoring needs, teachers in two of the cases discussed involving the parent (Pilot Case) and student (Case 1) in monitoring the student's needs moving forward. The child in Case 1 also stated that he would need his parents and teachers to help him "complete" his goals, reflective of his inclusion in the process. This feedback on the applicability of the AFI model in educational practice suggests its relevance to this field, in consideration of Circular 0013/2017 (DES, 2017b) and specific recommendations for practice are outlined in the following section.

\subsubsection{Summary of Recommendations for Practice}

In summary, it is recommended that training and input on the AFI model (Pameijer, 2017) be considered on a national level by school psychological services and professional training bodies as the unique role of the EP shifts away from a medical model of practice, towards an ecological, interactionist approach. Additionally, in consideration of the aims of Circular 0013/2017 (DES, 2017b) and the endorsement of inclusive assessment by the European Agency for Special Needs and Inclusive Education (Watkins, 2007), training and input on the AFI model (Pameijer, 2017) could be considered for teachers and school staff, in line with The Teaching Council's (2017) national framework for teacher learning 'Cosán'. Working together at a systems level to apply the aims and principles of AFI would support the developmental potential of home, school and school psychological services in identifying, meeting and monitoring the needs of students (Bronfenbrenner, 1979, p. 216). Furthermore, factors identified by the current research project as bridging the gap between assessment and intervention could be considered by EPs in practice as they work in accordance to the aims of Circular 0013/2017 (DES, 2017b). Proposals for dissemination of these research findings are outlined in Section 6.7 and limitations of the research and a critique of the AFI model is provided in the following section. 


\subsection{Limitations of the Research}

While the findings of this study can be used to inform future practice, the exploratory case study design would suggest generalisability of findings is limited to theory, similar settings, population and age group (Yin, 2009). The researcher adhered to a case selection protocol with specified selection criteria and a larger, more varied group of participants may have provided greater variation and depth of findings. Recommendations to address these limitations are outlined in Section 6.6, including replicating the research project with participants in post-primary schools, within the context of Circular 0014/2017 (DES, 2017c). Due to the referral of participants through the school psychological service, participant and researcher bias could have influenced responses on the Likert scale statements, interviews and researcher diary and journal (Mertens, 2015). Although the constructivist paradigm promotes the co-construction of knowledge between researcher and participants (Mertens, 2015), the potential for social desirability bias in responses should be considered as a possible limitation of findings. With regard to interviews, teachers in Case 1 and Case 2 expressed their disappointment that the parents were not as present during the assessment process, reflective perhaps of the value they had placed on the AFI process and their own belief systems and habitus as parents and teachers (Bourdieu, 1977). In the context of the current study, belief systems and values were not directly addressed during interview, which may have provided for a richer data set. An additional limitation is that data were collected from the teacher only in Case 2 following engagement with the AFI model and therefore there was limited evidence in this case that could be drawn upon for analysis. The time of year in which the research was conducted (October-December) presented a challenge as data were collected right up until the Christmas holidays, which is a very busy time for schools and parents. Finally, although the researcher had received training on the AFI model, level of experience and competency as a TEP applying the model in practice should be considered in the interpretation of findings.

Critique of the AFI model. The AFI model offers a systematic, decisionmaking process for EPs to apply in practice and the five stages provide a framework to assessment and intervention activities (Pameijer, 2017). It has been successfully applied in practice, as demonstrated in the findings of the current research project and previous evaluation studies (Pameijer, 2017). Despite its strengths, there are challenges associated with the AFI model, including difficulty assessing what is only strictly necessary and the time-consuming nature of applying a tailored approach to casework (Pameijer, 2017). In the current research, the class teacher in the Pilot Case discussed 
the time commitment involved in engaging in the AFI process, but also acknowledged the cost-benefit in terms of strength of information gathered and improved communication links between home and school (Section 5.23.1). In terms of its application in EP practice, it should be acknowledged that the AFI model is just one of many frameworks in existence. Kelly, Woolfson, and Boyle (2008) outline a number of frameworks for EP practice including the 'Problem Solving Model' (Monsen, Graham, Frederickson, \& Cameron, 1998), 'Integrated Framework'(Woolfson, Whaling, Stewart, \& Monsen, 2003) and the 'Constructionist Model of Informed and Reasoned Action (COMOIRA) (Gameson \& Rhydderch, 2008). Similar to the AFI model, these frameworks offer a series of systematic steps, to ensure that theory is clearly and appropriately applied throughout the assessment process and enable the EP to explicate their reasoning and justify their choice of interventions (Kelly et al., 2008). The AFI model is considered unique however, as it provides practical guidelines on how to gather and integrate relevant information, in close collaboration with teachers, parents and students (Pameijer, 2017, p. 80). The AFI checklists support this process and guide the EP through the five stages and provide opportunity for continuous feedback and reflection. In addition, the AFI model explicitly promotes the active participation of all relevant personnel during the assessment and intervention process (Pameijer, 2017). Despite limitations presented in this section, the current research may be the starting point for a more extended exploration of the AFI model in an Irish Educational Psychological context, discussed further in Section 6.6. The noteworthy implications of the research findings outlined in Section 6.3 above suggests the scholarly significance of the current research project, discussed in the following section.

\subsection{Scholarly Significance of the Research}

The findings of the current research project demonstrate scholarly significance as they are deemed nationally important, in theoretical and policy terms and are considered to be of general public interest (Yin, 2009). In accordance with EST, the theoretical statement in Section 2.16.2 proposed the issuance of Circular 0013/2017 (DES, 2017b) at the macro level instigated a period of ecological transition for the EP, school staff, parents and child with additional needs, in moving from a traditional resource allocation model, reflective of the medical model of practice towards a needsbased resource allocation model (DES, 2017a). In the context of Circular 0013/2017, the research findings demonstrate the AFI model can bridge the gap between assessment and intervention, by providing understanding of the child's situation and 
informing appropriate interventions for parents, teachers and students (DES, 2017b, p. 14). Activity and interaction between the TEP, child, parents and teachers during the AFI process, at the meso and microsystem levels was fundamental to this success, as evidenced in the findings outlined in Sections 4.2, 4.9 and 4.16. These activities and interactions were demonstrated to be consistent with AFI principles (Pameijer, 2017), EST and SCT concepts (Bronfenbrenner, 1979; Palinscar, 1998; Vygotsky, 1978), further strengthening the theoretical underpinnings of the AFI model. The findings presented are considered scholarly significant as to date, there has been no published research on the application of the AFI model in an Irish Educational Psychological context. It is suggested that outcomes of the research could be used to further inform policy and practice, in accordance with Circular 0013/2017 assertions (DES, 2017b). Consistent with Yin (2009) criteria for an exemplary case study, considerations for conducting the research through an alternative theoretical lens may also be worthy.

The current research question was explored through the conceptual lens of EST (Bronfenbrenner, 1979) and SCT (Palinscar, 1998; Vygotsky, 1978), as these theories were interpreted from the findings of the systematic review. Alternative theoretical frameworks could have been applied to conduct the research, including Positive Psychology (Seligman, 2002). Positive Psychology proposes the client has the potential and intrinsic motivation for growth and development (Seligman, 2002). Applying Positive Psychology in an assessment context allows an EP to modify aspects of the scientific method to the issues presented and focuses on strengths and resilience building (Seligman, 2002). Positive Psychology (Seligman, 2002) is consistent with elements of AFI principles (Pameijer, 2017), including focusing on educational needs and protective factors as well as risk factors to form an ecologically valid case formulation. In practice, Positive Psychology (Seligman, 2002) identifies the strengths of the parent/guardian, teacher and child and their capacity for resilience. In the context of AFI (Pameijer, 2017), awareness of these concepts could facilitate discussion on how goals could be addressed at home and at school, using the skills and qualities clients already possess and in exploring positive exceptions and strengths. With regards Case 2 in the current study, consideration of the parent's capacity to effectuate change in the home environment, through the theoretical lens of Positive Psychology (Seligman, 2002), may have empowered her to follow through on observation tasks and express any hesitations that she may have been experiencing (Section 5.17). Future research may explore the application of the AFI model through the theoretical lens of Positive 
Psychology (Seligman, 2002). Additional recommendations for future research are outlined in the following section.

\subsection{Recommendations for Future Research}

There are a number of opportunities for further research to consolidate and enhance the findings of the current research. The study could be replicated for a more extended exploration of the AFI model in an Irish Educational Psychological context and also to evaluate the effectiveness of intervention recommendations following a specified period of implementation. The current research was conducted with participants in mainstream primary schools and could be replicated with participants in post-primary schools, to explore the utility of the model in addressing the aims for professional assessment outlined in Circular 0014/2017 (DES, 2017c). For a richer data set, the research could include the views and perspectives of additional stakeholders, including School Principals and Inclusion Support Assistants and also consider the impact of habitus on views held, as outlined in Section 6.4. In addition, the research question could be explored though an alternative theoretical lens, such as Positive Psychology, presented in Section 6.5. In accordance with recommendations for future research discussed in Pameijer (2017), further research is warranted to investigate if AFI produces more ecologically valid case formulations and effective interventions than “assessment as usual" (p. 80).

\subsection{Dissemination of Research}

It is the intention that the findings of the current research project will be disseminated in relevant journal articles and conference presentations (Yin, 2009). An empirical paper has been written for submission to the journal 'Educational Psychology in Practice' (Appendix 54) and the researcher presented on the study at a PSI national conference in 2018.

\subsection{Conclusion}

To conclude, the current research project provided original insight into the application of the AFI model in EP practice, through the conceptual lens of EST (Bronfenbrenner, 1979) and SCT (Palinscar, 1998; Vygotsky, 1978). The presented findings suggest the AFI model can bridge the gap between assessment and intervention, through activity and interaction at the meso and microsystem levels. The findings address assertions for professional assessment outlined in Circular 0013/2017 
(DES, 2017b), indicating the scholarly significance of this doctoral research with practical implications for EP and educational practice (Yin, 2009). Limitations include the exploratory case study design and limited generalisability of findings to theory, similar settings, population and age group (Yin, 2009). However, this study may be the foundation for a more extended exploration of the AFI model in Irish Educational Psychological practice, as detailed in the recommendations for future research. 


\section{References}

Aganza, J. S., Godinez, A., Smith, D., Gonzalez, L. G., \& Robinson-Zañartu, C. (2014). Using cultural assets to enhance assessment of Latino students. Contemporary School Psychology, 19(1), 30-45. doi:10.1007/s40688-014-0041-7

Aiello, R., Ruble, L., \& Esler, A. (2016). National study of school psychologists' use of evidence-based assessment in autism spectrum disorder. Journal of Applied School Psychology, 33(1), 67-88. doi:10.1080/15377903.2016.1236307

Alegra, M. (2013). Assessment for intervention: Second evaluation of a pilot in Unita, a centre for inclusive education. Rotterdam, NL: CED-groep.

American Psychiatric Association. (2000). Diagnostic and statistical manual of mental disorders (4th ed.). Washington, DC: Author.

Bahr, M. W., Leduc, J. D., Hild, M. A., Davis, S. E., Summers, J. K., \& McNeal, B. (2017). Evidence for the Expanding Role of Consultation in the Practice of School Psychologists. Psychology in the Schools, 54(6), 581-595. doi:10.1002/pits. 22020

Bourke, R., \& Dharan, V. (2015). Assessment practices of educational psychologists in Aotearoa/New Zealand: from diagnostic to dialogic ways of working. Educational Psychology in Practice, 31(4), 369-381. doi:10.1080/02667363.2015.1070709

Bowles, T., Scull, J., Clinton, J. H. a. J., Larkins, G., Cicconi, V., Kumar, D., \& Arnup, J. L. (2016). Conducting psychological assessments in schools: Adapting for converging skills and expanding knowledge. Issues in Educational Reserach, 26(1), 10-28.

Bourdieu, P. (1977). Outline of a theory of practice. Cambridge: University Press.

Bozic, N. (2013). Developing a strength-based approach to educational psychology practice: A multiple case study. Educational \& Child Psychology, 30(4), 18-29.

Bozic, N., Lawthom, R., \& Murray, J. (2017). Exploring the context of strengths - a new approach to strength-based assessment. Educational Psychology in Practice, 34(1), 26-40. doi:10.1080/02667363.2017.1367917

Braun, V., \& Clarke, V. (2006). Using thematic analysis in psychology. Qualitative Research in Psychology, 3(2), 77-101. doi:10.1191/1478088706qp063oa

Bronfenbrenner, U. (1979). The ecology of human development: Experiments by nature and design. Boston, MA: Harvard College.

Bronfenbrenner, U. (1989). Ecological systems theory. Annals of Child Development, 6, 187-249. 
Bronfenbrenner, U. (1994). Ecological models of human development International encyclopedia of education (2 ed., Vol. 3). Oxford: Elsevier.

Burns, M. K. (2013). Contextualizing school psychology practice: Introducing featured research commentaries. School Psychology Review, 42(3), 334-342.

Cane, F. (2016). Everyone's solution: A case study of a systemic and solution-focused approach to therapeutic intervention in a secondary school. Educational \& Child Psychology, 33(4), 66-79.

Christenson, S. L., \& Anderson, A. R. (2002). Commentary: The centrality of the learning context for students' academic enabler skills. School Psychology Review, 31(3), 378-393.

Chun, H., \& Dickson, G. (2011). A psychoecological model of academic performance among Hispanic adolescents. Journal of Youth Adolescence, 40(12), 1581-1594. doi:10.1007/s10964-011-9640-z

Clarke, S., \& Jenner, S. (2006). The psychology of delivering a psychological service: Self-organised learning as a model for consultation. Educational Psychology in Practice, 22(3), 185-197. doi:10.1080/02667360600845703

Cottrell, J. M., \& Barrett, C. A. (2015). Job satisfaction among practicing school psychologists: The impact of SLD identification. Contemporary School Psychology, 20(1), 21-30. doi:10.1007/s40688-015-0076-4

Cresswell, J. W., \& Poth, C. N. (2017). Qualitative inquiry research design: Choosing among ve approaches (4th ed.). London: Sage Publications.

Davis, J. M., \& Deponio, P. (2013). Analysing conflicting approaches to dyslexia on a European project: moving to a more strategic, participatory, strength-based and integrated approach. International Journal of Inclusive Education, 18(5), 515534. doi:10.1080/13603116.2013.802023

Dean, V. J., Bums, M. K., Grialou, T., \& Varro, P. (2006). Comparison of ecological validity of leaming disabilities diagnostic models. Psychology in the Schools, $43,157-168$.

Deci, E. L., \& Ryan, R. M. (2008). Self-determination theory: A macrotheory of human motivation, development and health. Canadian Psychology, 49(3), 182-185.

Decker, S. L., Hale, J. B., \& Flanagan, D. P. (2013). Professional practice issues in the assessment of cognitive functioning for educational applications. Psychology in the Schools, 50(3), 300-313. doi:10.1002/pits.2167510.1002/pits

Department of Children and Youth Affairs. (2016). Access \& Inclusion Model: A new model for supporting access to early childhood care and education (ECCE) programme for children with a disability. Retrieved from http://aim.gov.ie/wpcontent/uploads/2016/06/AIM-Policy.pdf. 
Department of Education and Skills. (2017). Guidelines for primary schools supporting pupils with special educational needs in mainstream schools. Athlone:

Department of Education and Skills.

Department of Education and Skills. (2017). Special education teaching allocation circular 0013/2017. Retrieved from http://www.sess.ie/sites/default/files/inlinefiles/c10013_2017.pdf.

Department of Education and Skills. (2017). Special education teaching allocation circular 0014/2017. Retrieved from https://www.education.ie/en/Circulars-andForms/Active-Circulars/c10014_2017.pdf.

Desforges, M., \& Lindsay, G. (2010). Procedures used to diagnose a disability and to assess special educational needs: An international review (5). Retrieved from http://ncse.ie/wp-content/uploads/2014/10/5_NCSE_Diag_Ass.pdf

Elliott, J. G., Resing, W. C. M., \& Beckmann, J. F. (2018). Dynamic assessment a case of unfulfilled potential. Educational Review, 70(1), 7-17.

doi:10.1080/00131911.2018.1396806

Farrell, P. (2010). School psychology: Learning lessons from history and moving forward. School Psychology International, 31(6), 581-598.

Feuerstein, R., Rand, Y., \& Hoffman, M. (1979). The DA of retarded performers: The learning potential assessment device. Baltimore, MD: University Park Press.

Filter, K. J., Ebsen, S., \& Dibos, R. (2013). School psychology crossroads in America: Discrepancies between actual and preferred discrete practices and barriers to preferred practice. International Journal of Special Education, 28(1), 88-100.

Franzen, R. (1920). The accomplishment quotient: A school mark in terms of individual capacity. Teachers College Record, 21, 432-440.

Frenzel, A. C., Goetz, T., Pekrun, R., \& Watt, H. M. G. (2010). Development of mathematics interest in adolescence: Influences of gender, family, and school context. Journal of Research on Adolescence, 20(2), 507-537.

doi:10.1111/j.1532-7795.2010.00645.x

Gameson, J., \& Rhydderch, G. (2008). The constructionist model of informed and reasoned action (COMOIRA). In B. Kelly, L. Woolfson, \& J. Boyle (Eds.), Frameworks for Practice in Educational Psychology: A textbook for trainees and practitioners. London: Jessica Kingsley.

Geffken, G. R., Keeley, M. L., Kellison, I., Storch, E. A., \& Rodrigue, J. R. (2006). Parental adherence to child psychologists' recommendations from psychological testing. Professional Psychology: Research and Practice, 37(5), 499-505. doi:10.1037/0735-7028.37.5.499

Gough, D. (2007). Weight of Evidence: a framework for the appraisal 
of the quality and relevance of evidence. Research Papers in Education, 22(2), 213-228. doi:10.1080/02671520701296189

Gough, D., Oliver, S., \& Thomas, J. (2013). Learning from research: Systematic reviews for informing policy decisions. University of London: EPPI-Centre, Social Science Reserach Unit, Institute of Education.

Grant, C., \& Osanloo, A. (2014). Understanding, selecting, and integrating a theoretical framework in dissertation research: Creating the blueprint for your "House". Administrative Issues Journal Education Practice and Research. doi:10.5929/2014.4.2.9

Green, S. K., \& Gredler, M. E. (2002). A review and analysis of constructivism for school-based practice. School Psychology Review, 31(1), 53-70.

Guest, G., Bunce, A., \& Johnson, L. (2016). How many interviews are enough? Field Methods, 18(1), 59-82. doi:10.1177/1525822x05279903

Hanchon, T. A., \& Allen, R. A. (2013). Identifying students with emotional disturbance: School psychologists' practices and perceptions. Psychology in the Schools, 50(2), 193-208. doi:10.1002/pits. 21668

Harrison, G. L., \& McManus, K. L. (2016). Clinical reasoning in the assessment and intervention planning for writing disorder. Canadian Journal of School Psychology, 32(1), 73-86. doi:10.1177/0829573516658997

Hill, V., \& Turner, H. (2016). Educational psychologists' perspectives on the medicalisation of childhood behaviour: A focus on Attention Deficit Hyperactive Disorder (ADHD). Educational \& Child Psychology, 33(2), 12-29.

Horner, R. H., Carr, E. G., Halle, J., McGee, G., Odom, S., \& Wolery, M. (2005). The use of single-subject research to identify evidence-based practice in special education. Exceptional Children, 71(2), 165-179.

Iida, M., Shrout, P. E., Laurenceau, J.-P., \& Bolger, N. (2012). Using diary methods in psychological research. In H. Cooper (Ed.), APA Handbook of Research Methods in Psychology: Vol. 1. Foundations, Planning, Measures, and Psychometrics, (pp. 277-305): American Psychological Association.

Jose, M. C., Michael, J. C., Chappel, A., \& Cunningham, J. (2013). School psychologists' continuing professional development preferences and practices. Psychology in the Schools, 50(4), 415-432. doi:10.1002/pits.2168410.1002/pits

Kelly, B., Woolfson, L., \& Boyle, J. (2008). Frameworks for practice in educational psychology: A textbook for trainees and practitioners. London: Jessica Kingsley Publishers. 
Kennedy, E. K., Frederickson, N., \& Monsen, J. (2008). Do educational psychologists "walk the talk" when consulting? Educational Psychology in Practice, 24(3), 169-187. doi:10.1080/02667360802256733

Krosnick, J., A. (1999). Survey research. Annual Review of Psychology, 50, 537-567.

Laing, R. D. (1971). The politics of the family and other essays. London: Tavistock.

Lawrence, N., \& Cahill, S. (2014). The impact of dynamic assessment: an exploration of the views of children, parents and teachers. British Journal of Special Education, 41(2), 191-211. doi:10.1111/1467-8578.12060

McCrimmon, A. W., \& Yule, A. E. (2016). Clinical reasoning in the assessment and planning for intervention for autism spectrum disorder. Canadian Journal of School Psychology, 32(1), 59-72. doi:10.1177/0829573516658564

Mertens, D. M. (2015). Research and evaluation in education and psychology. London: Sage.

Monsen, J., Graham, B., Frederickson, N., \& Cameron, R. J. (1998). An accountable model of practice. Educational Psychology in Practice, 13(4), 234-249. doi:10.1080/0266736980130405

National Council for Special Education. (2013). Supporting students with special educational needs in schools. Trim: National Council for Special Education.

National Council for Special Education. (2014). Delivery for students with special educational needs: A better and more equitable way. Retrieved from http://ncse.ie/wpcontent/uploads/2014/09/Allocating_resources_1_5_14_Web_a ccessible_version_FINAL.pdf.

National Educational Psychological Service. (2003). Working together to make a difference for children: The NEPS model of service. Dublin: NEPS.

National Educational Psychological Service. (2007). Special educational needs: A continuum of support. Dublin: The Stationary Office.

National Educational Psychological Service. (2010). Behavioural, emotional and social difficulties: A continuum of support. Dublin: The Stationary Office.

Odom, S. L., Vitztum, J., Wolery, R., Lieber, J., Sandall, S., Hanson, M. J., . . Horn, E. (2004). Preschool inclusion in the United States: A review of research from an ecological systems perspective. Journal of Research in Special Educational Needs, 4(1), 17-49. doi:10.1111/J.1471-3802.2004.00016.x

Ogg, J., Fefer, S., Sundman-Wheat, A., McMahan, M., Stewart, T., Chappel, A., \& Bateman, L. (2013). School-based assessment of ADHD: Purpose, alignment 
with best practice guidelines, and training. Journal of Applied School Psychology, 29(4), 305-327. doi:10.1080/15377903.2013.836775

Palinscar, A. S. (1998). Social constructivist perspectives on teaching and learning. Annual Review of Psychology, 49, 345-375.

Pameijer, N. (2006). Towards needs-based assessment: Bridging the gap between assessment and practice. Educational \& Child Psychology, 23(3), 12-24.

Pameijer, N. (2016). Assessment for intervention: A practice-based model.

Pameijer, N. (2017). Assessment and intervention: Bridging the gap with a practicebased model. Educational \& Child Psychology, 34(1), 66-82.

Parker, J., Zaboski, B., \& Joyce-Beaulieu, D. (2016). School-based cognitive-behavioral therapy for an adolescent presenting with ADHD and explosive anger: A case study. Contemporary School Psychology, 20(4), 356-369. doi:10.1007/s40688016-0093-y

Passenger, T. (2013). Introduction to educational psychology ractice. In A. J. Holliman (Ed.), The Routledge International Companion to Educational Psychology. London: Routledge. Retrieved from https://www.routledgehandbooks.com/doi/10.4324/9780203809402.ch3. doi:10.4324/9780203809402.ch3

Resing, W., Lauchlan, F., \& Elliott, J. (2017). Guest editorial: Bridging the gap between psychological assessment and educational instruction. Educational \& Child Psychology, 34(1), 6-8.

Ring, E., Sullivan, L. O., Ryan, M., \& Burke, P. (2018). A melange or a mosaic of theories?: How theoretical perspectives on children's learning and development can inform a responsive pedagogy in a redeveloped primary school curriculum. Limerick: Mary Immaculate College.

Robson, C. (2011). Real world research 3e (3rd ed.). Chichester: John Wiley \& Sons.

Sansosti, F. J., \& Sansosti, J. M. (2013). Effective school-based service delivery for students with autism spectrum disorders: Where we are and where we need to go. Psychology in the Schools, 50(3), 229-244. doi:10.1002/pits.21669

Schunk, D. H. (2012). Learning theories an educational perspective (6th ed.). London: Pearson.

Schwandt, T. A. (2000). Three epistemological stances for qualitative inquiry: Interpretivism, hermeneutics, and social constructionism. In N. K. Denzin \& Y. S. Lincoln (Eds.), Handbook of qualitative reserach (Vol. 2, pp. 189-214). Thousand Oaks: CA: Sage. 
Seligman, M. E. P. (2002). Positive psychology, positive prevention, and positive therapy. In C. R. Snyder \& S. J. Lopez (Eds.), Handbook of positive psychology. Oxford: University Press.

Sotelo-Dynega, M., \& Dixon, S. G. (2014). Cognitive assessment practices: A survey of school psychologists. Psychology in the Schools, 51(10), 1031-1045. doi:10.1002/pits.21802

Stothard, J., Woods, K., \& Innoue, A. (2018). An exploration of practitioner educational psychologists' understandings and practice in relation to dyslexia. Educational \& Child Psychology, 35(1), 13-26.

Strong, T. O. M. (2005). Constructivist ethics? let's talk about them: an introduction to the special issue on ethics and constructivist psychology. Journal of Constructivist Psychology, 18(2), 89-102. doi:10.1080/10720530590914752

Thomas, S., \& Oldfather, P. (1997). Intrinsic motivations, literacy, and assessment practices: "That's my grade. That's me.". Educational Psychologist, 32(2), 107123.

The Teaching Council. (2017). Cosán: Framework for Teacher's Learning Development Process (2016-2020. Retrieved from https://www.teachingcouncil.ie/en/Publications/Teacher-Education/CosanDevelopment-Process.pdf.

Tilly, W. D. (2008). The Evolution of School Psychology to Science-Based Practice: Problem Solving and the Three-Tiered Model. In A. Thomas \& J. Grimes (Eds.), Best Practices in School Psychology (Vol. 5). Bethesda: NASP.

Tobias, A. (2017). The use of genograms in educational psychology practice. Educational Psychology in Practice, 34(1), 89-104. doi:10.1080/02667363.2017.1411787

U.S. Department of Education. (2004). Individuals with Disabilities Education Improvement Act (IDEIA). Retrieved from https://sites.ed.gov/idea/.

VanDerHeyden, A. M. (2018). Why do school psychologists cling to ineffective practices? Let's do what works. School Psychology Forum: Research in Practice, 12(1), 44-52.

Vega, D., Lasser, J., \& Afifi, A. F. M. (2015). School psychologists and the assessment of culturally and linguistically diverse students. Contemporary School Psychology, 20(3), 218-229. doi:10.1007/s40688-015-0075-5

Vygotsky, L. (1978). Mind in society: The development of higher psychological processes. Cambridge: Harvard University Press. 
Wagner, P. (2008). Consultation as a framework for practice. In B. Kelly, L. Woolfson, \& J. Boyle (Eds.), Frameworks for practice in eduactional psychology. London: Jessica Kingsley Publishers.

Watkins, A. (2007). Assessment in Inclusive Settings: Key Issues for Policy and Practice. Odense, Denmark: European Agency for Development in Special Needs Education.

Wertsch, J. (1991). Voices of the mind: A socio-cultural approach to mediated action. Cambridge: Harvard University Press.

Woolfson, L., Whaling, R., Stewart, A., \& Monsen, J. (2003). An integrated framework to guide educational psychologist practice. Educational Psychology in Practice, 19(4), 283-304. doi:10.1080/0266736032000138535

Yeomans, J. (2008). Dynamic Assessment practice: some suggestions for ensuring follow up. Educational Psychology in Practice, 24(2), 105-114.

doi:10.1080/02667360802076107

Yin, R. K. (2009). Case study research: Design and methods (4th ed.). London: Sage Publications Inc.

Yin, R. K. (2018). Case study research and applications: Design and methods (6th ed.). London: SAGE Publications Ltd. 


\section{Included Studies}

Aiello, R., Ruble, L., \& Esler, A. (2016). National study of school psychologists' use of evidence-based assessment in autism spectrum disorder. Journal of Applied School Psychology, 33(1), 67-88. doi:10.1080/15377903.2016.1236307

Bahr, M. W., Leduc, J. D., Hild, M. A., Davis, S. E., Summers, J. K., \& McNeal, B. (2017). Evidence for the Expanding Role of Consultation in the Practice of School Psychologists. Psychology in the Schools, 54(6), 581-595. doi:10.1002/pits.22020

Bourke, R., \& Dharan, V. (2015). Assessment practices of educational psychologists in Aotearoa/New Zealand: from diagnostic to dialogic ways of working. Educational Psychology in Practice, 31(4), 369-381. doi:10.1080/02667363.2015.1070709

Bozic, N. (2013). Developing a strength-based approach to educational psychology practice: A multiple case study. Educational \& Child Psychology, 30(4), 18-29.

Bozic, N., Lawthom, R., \& Murray, J. (2017). Exploring the context of strengths - a new approach to strength-based assessment. Educational Psychology in Practice, 34(1), 26-40. doi:10.1080/02667363.2017.1367917

Cane, F. (2016). Everyone's solution: A case study of a systemic and solution-focused approach to therapeutic intervention in a secondary school. Educational \& Child Psychology, 33(4), 66-79.

Cottrell, J. M., \& Barrett, C. A. (2015). Job satisfaction among practicing school psychologists: The impact of SLD identification. Contemporary School Psychology, 20(1), 21-30. doi:10.1007/s40688-015-0076-4

Filter, K. J., Ebsen, S., \& Dibos, R. (2013). School psychology crossroads in America: Discrepancies between actual and preferred discrete practices and barriers to preferred practice. International Journal of Special Education, 28(1), 88-100.

Hanchon, T. A., \& Allen, R. A. (2013). Identifying students with emotional disturbance: School psychologists' practices and perceptions. Psychology in the Schools, 50(2), 193-208. doi:10.1002/pits.21668

Harrison, G. L., \& McManus, K. L. (2016). Clinical reasoning in the assessment and intervention planning for writing disorder. Canadian Journal of School Psychology, 32(1), 73-86. doi:10.1177/0829573516658997

Hill, V., \& Turner, H. (2016). Educational psychologists' perspectives on the medicalisation of childhood behaviour: A focus on Attention Deficit Hyperactive Disorder (ADHD). Educational \& Child Psychology, 33(2), 12-29.

Lawrence, N., \& Cahill, S. (2014). The impact of dynamic assessment: an exploration of the views of children, parents and teachers. British Journal of Special Education, 41(2), 191-211. doi:10.1111/1467-8578.12060 
McCrimmon, A. W., \& Yule, A. E. (2016). Clinical reasoning in the assessment and planning for intervention for autism spectrum disorder. Canadian Journal of School Psychology, 32(1), 59-72. doi:10.1177/0829573516658564

Ogg, J., Fefer, S., Sundman-Wheat, A., McMahan, M., Stewart, T., Chappel, A., \& Bateman, L. (2013). School-based assessment of ADHD: Purpose, alignment with best practice guidelines, and training. Journal of Applied School Psychology, 29(4), 305-327. doi:10.1080/15377903.2013.836775

Pameijer, N. (2017). Assessment and intervention: Bridging the gap with a practicebased model. Educational \& Child Psychology, 34(1), 66-82.

Parker, J., Zaboski, B., \& Joyce-Beaulieu, D. (2016). School-based cognitivebehavioral therapy for an adolescent presenting with ADHD and explosive anger: A case study. Contemporary School Psychology, 20(4), 356-369. doi:10.1007/s40688016-0093-y

Sansosti, F. J., \& Sansosti, J. M. (2013). Effective school-based service delivery for students with autism spectrum disorders: Where we are and where we need to go. Psychology in the Schools, 50(3), 229-244. doi:10.1002/pits.21669

Sotelo-Dynega, M., \& Dixon, S. G. (2014). Cognitive assessment practices: A survey of school psychologists. Psychology in the Schools, 51(10), 1031-1045. doi:10.1002/pits.21802

Stothard, J., Woods, K., \& Innoue, A. (2018). An exploration of practitioner educational psychologists' understandings and practice in relation to dyslexia. Educational \& Child Psychology, 35(1), 13-26.

Tobias, A. (2017). The use of genograms in educational psychology practice. Educational Psychology in Practice, 34(1), 89-104. doi:10.1080/02667363.2017.1411787

Vega, D., Lasser, J., \& Afifi, A. F. M. (2015). School psychologists and the assessment of culturally and linguistically diverse students. Contemporary School Psychology, 20(3), 218-229. doi:10.1007/s40688-015-0075-5 
Appendix 2 Excluded Articles with Rationale and Full References (full text)

\begin{tabular}{l}
\hline Excluded Study \\
\hline Aljunied, M., \& Frederickson, N. (2014). \\
Utility of the International Classification \\
of Functioning, Disability and Health \\
(ICF) for educational psychologists' \\
work. Educational Psychology in \\
Practice, 30(4), 380-392.
\end{tabular}

Allen, R. A., \& Hanchon, T. A. (2013). What can we learn from school-based emotional disturbance assessment practices? Implications for practice and preparation in school psychology. Psychology in the Schools, 50(3), 290299.

Amod, Z., Heafield, D., \& Seabi, J. (2017). Assessing a remedial intervention programme in developing the planning skills of grade 4 and 5 learners. International Journal of Disability, Development and Education, 65(4), 428-441.

Baas, D., Castelijns, J., Vermeulen, M., Martens, R., \& Segers, M. (2015). The relation between Assessment for Learning and elementary students' cognitive and metacognitive strategy use. British Journal of Educational Psychology, 85(1), 33-46. Barker-Collo, S. L., \& Fernando, K. (2015). A survey of New Zealand psychologists' practices with respect to the assessment of performance validity. New Zealand Journal of Psychology, 44(2), 35-42.

Bell, H. D., \& McKenzie, V. (2013). Perceptions and realities: The role of school psychologists in Melbourne, Australia. The Australian Educational

\section{Rationale for Exclusion}

(3) The objective of this study was to make an initial appraisal of the utility of the ICF evaluation system for use by EPs with children with ASD. This study evaluates the utility of the ICF framework, it does not investigate or provide data on its use by EPs in practice.

(5) This study used the same data set as a study that has already been included in the present literature review, by Hanchon \& Allen (2013)

(3) The aim of this study was to investigate the effectiveness of a planning intervention programme based on Feuerstein's criteria for Mediated Learning Experience (MLE) with a group of Grade 4 and 5 remedial school learners who presented with a range of barriers to learning. The study provides experimental data on the programmes effectiveness. It does not describe an assessment model used by EPs in practice that informs an appropriate intervention.

(3) This study aimed to enhance students' motivation and self-regulated learning by means of Assessment for Learning. The study is situated in an educational context and measures were administered by teachers.

(6) This study collected data from psychologists practicing in various domains including counselling and clinical psychologists.

(3) The study investigated how psychologists assess symptom validity.

(3) This study investigated the role of school psychologists and did not provide evidence of an assessment approach used in practice. 
and Developmental Psychologist,

30(01), 54-73.

Clark, G. C., \& Parker, D. C. (2016).

Comparing assessment approaches for use with brief experimental analysis.

School Psychology Forum: Research in

Practice, 10(1), 93-105.

(3) This study was a pilot comparison of two curriculum-based assessment approaches with respect to measuring growth during the context of evidencebased interventions. The study compares curriculum-based assessment approaches and is situated in an educational context.

Grossen, M., Florez, D., \& Lauvergeon, S. (2014). Dealing with clients' diversity in test situations: Client categorisations in psychologists' accounts of their practices. International Journal of Educational Research, 63, 15-25.

Kleinert, W. L., Silva, M. R., Codding, R. S., Feinberg, A. B., \& James, P. S. S. (2017). Enhancing classroom management using the classroom checkup consultation model with in-vivo coaching and goal setting components. School Psychology Forum: Research in Practice, 11(1), 5-19.

Lebeer, J., Partanen, P., Candeias, A., Gracio, M. L., Bohacs, K., Sonnesyn, G., ... Dawson, L. The need for a more dynamic and ecological assessment of children experiencing barriers to learning to move towards inclusive education: A summary of results of the daffodil project. Transylvanian Journal of Psychology, 175-205.

Massé, L., Couture, C., Levesque, V., \& Bégin, J.-Y. (2013). Impact of a school consulting programme aimed at helping teachers integrate students with behavioural difficulties into secondary school: actors' points of view. Emotional and Behavioural Difficulties, 18(3), 327343.

Miciak, J., Williams, J. L., Taylor, W. P., Cirino, P. T., Fletcher, J. M., \& Vaughn, S. (2016). Do processing patterns of strengths and weaknesses predict differential treatment response? Journal of Educational Psychology, 108(6), 898909.

(6) This study collected data from counselling psychologists and school psychologists.

(3) This study investigated the effectiveness of The Classroom Check-Up (CCU) structured consultation model in improving teachers' classroom management strategies.

(5) This is a descriptive article and does not provide empirical data to address the review questions.

(3) This study provides data on the utility of an individual consultation and smallgroup consultation offered to secondary school teachers, not an EP assessment approach.

(3) This study investigated whether the identification decisions of the concordance/discordance model and cross-battery assessment were consistent and whether they predicted intervention response beyond that accounted for by pretest performance on measures of 
reading. This study did not provide evidence of assessment and intervention approaches used by educational psychologists in practice.

Newman, D. S., Barrett, C. A., \& Hazel, C. E. (2015). School consultation practices in the early career: Does training matter? Consulting Psychology Journal: Practice and Research, 67(4), 326-347.

O’Neill, R. E., Bundock, K., Kladis, K., \& Hawken, L. S. (2015). Acceptability of functional behavioral assessment procedures to special educators and school psychologists. Behavioral Disorders, 41(1), 51-66.

Pat-El, R. J., Tillema, H., Segers, M., \& Vedder, P. (2013). Validation of assessment for learning questionnaires for teachers and students. British Journal of Educational Psychology, 83(Pt 1), 98113.

Pat-El, R. J., Tillema, H., Segers, M., \& Vedder, P. (2014). Multilevel predictors of differing perceptions of Assessment for Learning practices between teachers and students. Assessment in Education: Principles, Policy \& Practice, 22(2), 282-298.

Rahill, S. A. (2018). Parent and teacher satisfaction with school-based psychological reports. Psychology in the Schools, 55(6), 693-706.

Shenoy, S. (2014). Assessment tools to differentiate between language differences and disorders in English language learners. Berkeley Review of Education, 5(1), 33-52.

Strohmeier, C. W., Murphy, A., \& O'Connor, J. T. (2017). Parent-informed
(3) This study investigated the link between school psychologists' consultation training at the preservice level, and the enactment of the consultant role in schools during the early career. The focus of the study is investigating training in consultation and consultative practice.

(3) This survey study assessed the acceptability of functional behavioural assessment (FBA) procedures, it does not explore its use in EP practice.

(3) This study provided data to validate Assessment for Learning Questionnaires for Teachers (TAFL-Q) and for students (SAFL- Q, developed by the authors.

(3) In this study, perceptions regarding Assessment for Learning practices in their classroom were collected from both teachers and students. The study is situated in an educational context.

(3) This study examined parent and teacher perceptions regarding the quality, understandability, and usefulness of school-based psychological reports. It does not provide data on assessment approaches that inform interventions in EP practice.

(6) This study collected data from health professionals, educational psychologists and educators. 
test-control functional analysis and treatment of problem behavior related to combined establishing operations.

Developmental Neurorehabilitation, 20(4), 247-252.

Waters, A. M., Groth, T. A., Sanders, M., O'Brien, R., \& Zimmer-Gembeck, M. J. (2015). Developing partnerships in the provision of youth mental health services and clinical education: A school-based cognitive behavioral intervention targeting anxiety symptoms in children. Behavior Therapy, 46(6), 844-855.

Wright, C. V., Beattie, S. G., Galper, D. I., Church, A. S., Bufka, L. F., Brabender, V. M., \& Smith, B. L. (2017). Assessment practices of professional psychologists: Results of a national survey. Professional Psychology: Research and Practice, 48(2), 73-78. clinic and delivered by a clinician, not an EP.

(3) This CBT intervention was administered by student psychologists and registered psychologists.

(6) This study collected data from psychologists practicing in various domains (e.g. forensic and outpatient mental health settings), not school psychologists 
WoE A measures the study's general quality of design and methodology. To ensure a reliable critique of the studies in the current review, published coding protocols and quality criteria checklists were applied. The Brantlinger, Jimenez, Klingner, Pugach, and Richardson (2005) coding protocol was applied to studies with a qualitative design. An adapted version of the Hyett, Kenny, and Dickson-Swift (2014) quality checklist was used to appraise case study designs. A checklist for critically analysing survey research in Mertens (2015, p. 214) was adapted for use as a coding protocol for survey studies. The tables below outline how coding criteria was converted into WoE A ratings. An example of each of the coding protocols applied is also included in this appendix.

WoE A Criteria and Rationale

\begin{tabular}{|c|c|c|}
\hline $\begin{array}{l}\text { WoE A } \\
\text { Score }\end{array}$ & Criteria & Rationale \\
\hline 3 (High) & $\begin{array}{l}\text { Average score of } 0.67-1 \text { across the } \\
\text { judgement areas }\end{array}$ & $\begin{array}{l}\text { Possible scores range } \\
\text { from 0-9 (Mertens, 2015, p. } \\
\text { 214), 0-11(Brantlinger, }\end{array}$ \\
\hline 2 (Medium) & $\begin{array}{l}\text { Average score of } 0.34-0.66 \text { across } \\
\text { the judgement areas }\end{array}$ & $\begin{array}{l}\text { Jimenez, Klingner, Pugach, } \\
\text { and Richardson, 2005) and } \\
0-13 \text { (Hyett, Kenny, and }\end{array}$ \\
\hline 1 (Low) & $\begin{array}{l}\text { Average score of } 0-0.33 \text { across } \\
\text { the judgement areas }\end{array}$ & $\begin{array}{l}\text { Dickson-Swift, 2014). This } \\
\text { criteria converts them into } \\
\text { scores between } 1-3 \text {. }\end{array}$ \\
\hline
\end{tabular}

WoE A Scoring

\begin{tabular}{|c|c|c|c|}
\hline Study & High & Medium & Low \\
\hline $\begin{array}{l}\text { Bahr, Leduc, Hild, Davis, Summers \& } \\
\text { McNeal (2017) }\end{array}$ & $\mathrm{X}$ & & \\
\hline Stothard, Woods \& Innoue (2018) & $\mathrm{X}$ & & \\
\hline Sotelo-Dynega, \& Dixon (2014) & $\mathrm{X}$ & & \\
\hline Ogg et al. (2013) & $\mathrm{X}$ & & \\
\hline Hill \& Turner (2016) & $\mathrm{X}$ & & \\
\hline Sansosti \& Sansosti (2013) & $X$ & & \\
\hline
\end{tabular}




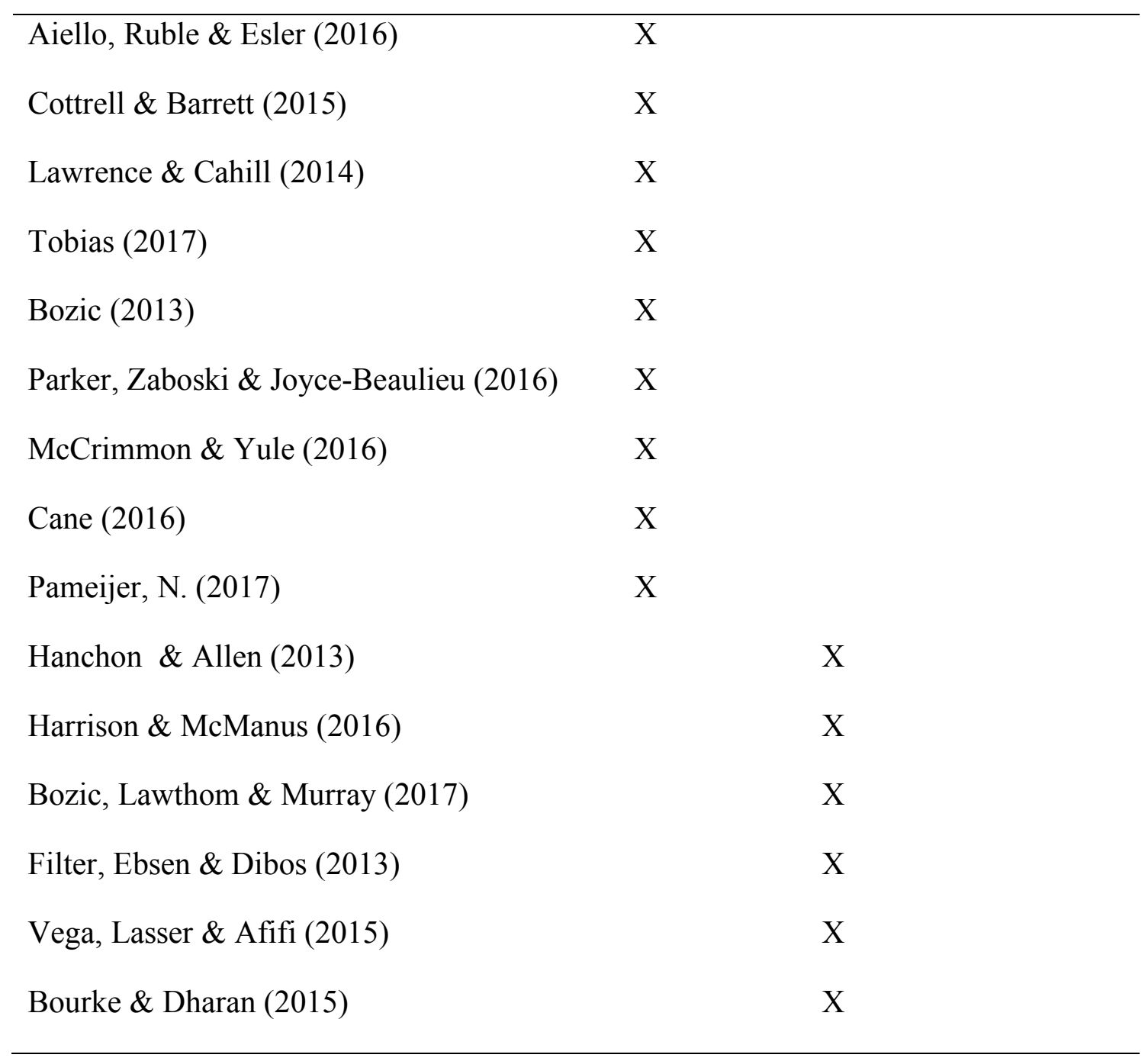


Quality Indicators Within Survey Research (Adapted from Mertens, 2015, p. 214)

Article Reference: Aiello, R., Ruble, L., \& Esler, A. (2016). National study of school psychologists' use of evidence-based assessment in autism spectrum disorder. Journal of Applied School Psychology, 33(1), 67-88.

Wording of survey questions account for bias and are not considered leading.

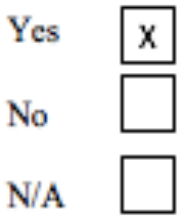

Unknown/Unable to Code

Response pattern biases are not evident (c.g. question order effects, no opinion filter effects).

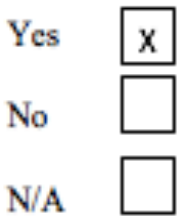

Unknown/Unable to Code

Participant response rate is accounted for.

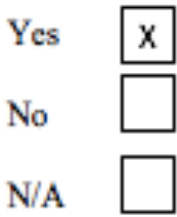

Unknown/Unable to Code

The person who experienced the phenomenon in question answered the questions.

$\begin{array}{ll}\text { Yes } & \mathrm{X} \\ \text { No } & \square \\ \text { N/A } & \square\end{array}$


Unknown/Unable to Code

Researchers accounted for differences between themselves and respondents in terms of gender, race or ethnicity, socioeconomic status or disability.

Yes

No

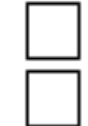

N/A $\mathrm{X}$

Unknown/Unable to Code

Researcher/Interviewer effects were accounted for.

Yes

No $\mathrm{x}$

N/A

Unknown/Unable to Code

The translation process is accounted for in surveys that were translated into another language.

Yes

No

N/A $\mathrm{X}$

Unknown/Unable to Code

The sampling strategy is described.

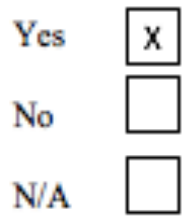

Unknown/Unable to Code 
The impact of the survey design (descriptive, cross-sectional, longitudinal) on the interpretation of the results is discussed.

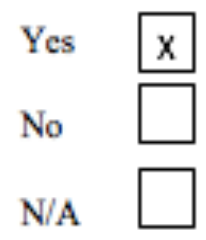

Unknown/Unable to Code

Average WoE A across the judgement areas:

Sum of $\mathrm{X} / \mathrm{N}=6 / 7$

$\mathrm{X}=$ total quality rating across judgement areas

$\mathrm{N}=$ number of judgement areas

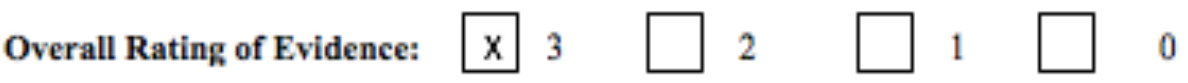


Quality Indicators Within Qualitative Research (Adapted from Brantlinger et al., 2005).

Article Reference: Lawrence, N., \& Cahill, S. (2014). The impact of dynamic assessment: an exploration of the views of children, parents and teachers. British Joumal of Special Education, 4I(2), 191-211. doi:10.1111/1467-8578.12060

\section{Interview Studies (or Interview Components of Comprehensive Studies)}

Appropriate participants are selected (purposefully identified, effectively recruited, adequate number, representative of population of interest).

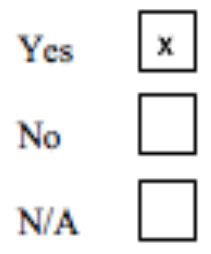

Unknown/Unable to Code

Interview questions are reasonable (clearly worded, not leading, appropriate and sufficient for exploring domains of interest).

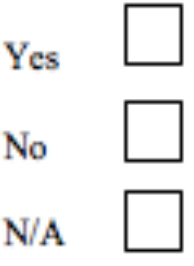

Unknown/Unable to Code $\mathrm{X}$

Adequate mechanisms are used to record and transcribe interviews.

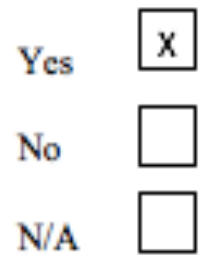

Unknown/Unable to Code

Participants are represented sensitively and fairly in the report.
Yes $\mathrm{X}$
No 
N/A

Unknown/Unable to Code

Sound measures are used to ensure confidentiality

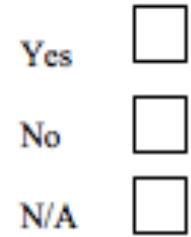

Unknown/Unable to Code $\mathrm{X}$

Observation Studies (or Observation Components of Comprehensive Studies)

Appropriate setting (s) and/or people are selected for observation

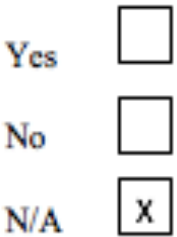

Unknown/Unable to Code

Sufficient time is spent in the field (number and duration of observations, study time span)

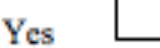

No $\square$

N/A $\mathrm{X}$

Unknown/Unable to Code

Researcher fits into the site (accepted, respected, unobtrusive)

Yes

No 
$\mathrm{N} / \mathrm{A} \quad \mathrm{X}$

Unknown/Unable to Code

Research has minimal impact on setting (except for action research, which is purposely designed to have an impact)

Yes

No

$\mathrm{N} / \mathrm{A} \quad \mathrm{X}$

Unknown/Unable to Code

Field notes systematically collected (videotaped, audiotaped, written during or soon after observations)

Yes $\square$

No $\square$

$\mathrm{N} / \mathrm{A} \mathrm{X}$

Unknown/Unable to Code

Sound measures are used to ensure confidentiality of participants and settings

Yes

No

$\mathrm{N} / \mathrm{A} \quad \mathrm{X}$

Unknown/Unable to Code

Overall Rating of Evidence: $\square$ 3 $\square 2 \begin{array}{lll}\square & \square\end{array}$ 


\section{Document Analysis}

Meaningful documents (texts, artifacts, objects, pictures) are found and their relevance is established

Yes $\square$

No $\square$

N/A $\mathrm{x}$

Unknown/Unable to Code

Documents are obtained and stored in a careful manner

Yes $\square$

No $\square$

N/A $\mathrm{X}$

Unknown/Unable to Code

Documents are sufficiently described and cited

Yes $\square$

No $\square$

N/A $\mathrm{X}$

Unknown/Unable to Code

Sound measures are used to ensure confidentiality of private documents

Yes $\square$

No $\square$

N/A $\mathrm{X}$

Unknown/Unable to Code 
Overall Rating of Evidence:

3

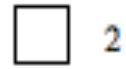

2

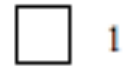

$\square 0$

\section{Data Analysis}

Results are sorted and coded in a systematic and meaningful way

Yes $\mathrm{X}$

No $\square$

N/A

Unknown/Unable to Code

Sufficient rationale is provided for what was (or was not) included in the report

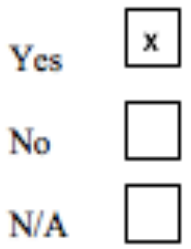

Unknown/Unable to Code

Documentation of methods used to establish trustworthiness and credibility are clear

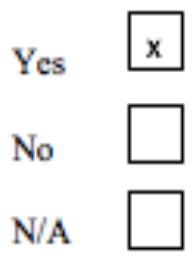

Unknown/Unable to Code

Reflection about researchers' personal position/perspectives are provided
Yes $\mathrm{x}$
No
N/A 
Unknown/Unable to Code

Conclusions are substantiated by sufficient quotations from participants, field notes of observations and evidence of documentation inspection

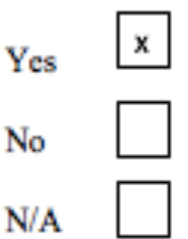

Unknown/Unable to Code

Connections are made with related research

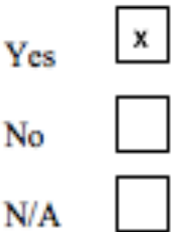

Unknown/Unable to Code

\section{Average $\mathrm{WoE}_{\mathrm{A}} \mathrm{A}$ across the 7 judgement areas:}

Sum of $\mathrm{X} / \mathrm{N}=9 / 11$

$\mathrm{X}=$ individual quality rating for each judgement area

$\mathrm{N}=$ number of judgement areas

Overall Rating of Evidence: $\mathrm{x}$

\section{3}

$\square 2$

$\square 1$<smiles>[O]C1CCC1</smiles> 
Quality Indicators Within Case Study Research (Adapted from Hyett, Kenny, \& Dickson-Swift, 2014)

Article Reference: Parker, J., Zaboski, B., \& Joyce-Beaulieu, D. (2016). School-based cognitive-behavioral therapy for an adolescent presenting with ADHD and explosive anger: A case study. Contemporary School Psychology, 20(4), 356-369.

The case study report demonstrates conceptual structure (i.e., themes or issues).

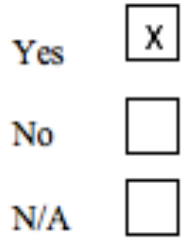

Unknown/Unable to Code

Issues are developed in a series and scholarly way.

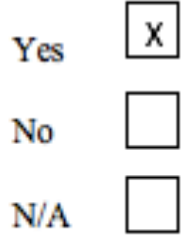

Unknown/Unable to Code

The case has been adequately defined.

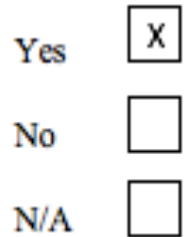

Unknown/Unable to Code

The nature of the intended audience is apparent.

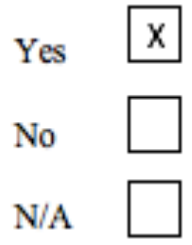


Headings, figures, artefacts, appendices, indexes have been effectively used.

Yes $\mathrm{X}$

No $\square$

N/A

Unknown/Unable to Code

Sufficient raw data is presented.

Yes $\mathrm{X}$

No $\square$

N/A

Unknown/Unable to Code

Quotations have been used effectively.

Yes

No $\mathrm{x}$

N/A

Unknown/Unable to Code

Data sources were well-chosen and in sufficient number.

Yes $\mathrm{X}$

No

N/A

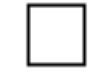

Unknown/Unable to Code 
Adequate attention has been paid to various contexts.

Yes $\mathrm{X}$

No $\square$

N/A

Unknown/Unable to Code

It does not appear that individuals were put at risk.

Yes $\mathrm{X}$

No

N/A $\square$

Unknown/Unable to Code

Observations and interpretations appear to have been triangulated.

Yes $\mathrm{X}$

No

N/A

Unknown/Unable to Code

The writer has made sound assertions, neither over- or under-interpreting.

Yes $\mathrm{X}$

No

N/A

Unknown/Unable to Code 
The role and point of view of the researcher is apparent.

Yes $\mathrm{X}$

No

N/A $\square$

Unknown/Unable to Code

Average WoE A across the judgement areas:

Sum of $\mathrm{X} / \mathrm{N}=12 / 13$

$\mathrm{X}=$ individual quality rating for each judgement area

$\mathrm{N}=$ number of judgement areas

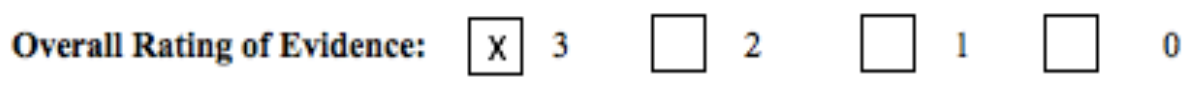




\section{Appendix 4 WoE B Criteria and Scoring}

Weight of Evidence B measures whether the design of the study was relevant to addressing the specified review questions (Gough, 2007). The criteria for WoE B weighting was devised by the reviewer in accordance with the quality criteria used for WoE $A$ and inclusion and exclusion criteria. The current review sought to gather empirical data to investigate the assessment approaches currently being applied by EPs in practice (review question 1) and the appropriateness of intervention recommendations arising from these assessments (review question 2). Study designs that used methods for gathering participant views (i.e. survey and interview studies) were considered most appropriate to addressing the review questions and were allocated a higher WoE B rating. Survey studies facilitate collection of data from a larger population sample than is generally possible with experimental or quasi-experimental designs (Mertens, 2015, p. 182) and are a method for understanding the workings of the human mind and dynamics of social interaction (Krosnick, 1999). Similarly, qualitative interviews allow the researcher to get an in-depth account of a topic and allow for respondents' perspectives or stories to be told (Barker, Pistrang, \& Elliott, 2002). In survey research, two criteria for determining quality and sample representativeness include the reported response rate and procedure for follow-up of non- respondents (Mertens, 2015, p. 215). With regards interview methods, saturation has become the gold standard by which purposive sample sizes are determined (Guest et al., 2016). This information was taken into account in devising the WoE B criteria for survey and interview studies. A 'High' WoE B rating was allocated to survey studies with a high response rate (70\% or higher) (Johnson \& Christensen, 2008, cited in Mertens, 2015 p. 191) or interview studies with evidence of data saturation (12 interviews) (Guest et al., 2016).

Case studies were allocated a 'Low' WoE B rating because of limited generalisability to a sample population. In case study research, the case does not represent a 'sample' and the goal is to expand and generalise theories, rather than enumerate frequencies (statistical generalisation) (Yin, 2009, p. 15). Case studies were still considered appropriate to addressing the review question as they outline an assessment process applied in practice and provide empirical evidence relevant to the specified context. In devising WoE B criteria for case studies, the reviewer referred to Yin (2009). In outlining elements of an 'exemplary' case study, Yin (2009) makes reference to case significance. Significant case(s) are unusual and of general public 
interest and the underlying issues are nationally important, either in theoretical terms or in policy or practical terms. The current review question is of general public interest (investigating assessment approaches that are informing appropriate interventions for clients) and any potential issues explored are relevant to policy terms (Circular 0013/2017). To meet WoE B criteria, case studies reviewed must be significant and describe the process of an assessment approach used by an EP in practice. The tables below outline WoE B criteria and scoring.

WoE B Criteria and Rationale

\begin{tabular}{|c|c|}
\hline WoE B Weighting & Description \\
\hline 3 (High) & $\begin{array}{l}\text { Survey or interview methods are } \\
\text { employed in the study to address the } \\
\text { review questions. There is evidence of a } \\
\text { high response rate ( } 70 \% \text { or higher) for } \\
\text { survey studies (Johnson \& Christensen, } \\
2008 \text {, cited in Mertens, } 2015 \text { p. 191) or in } \\
\text { the case of interview studies, the study } \\
\text { reaches data saturation (12 interviews) } \\
\text { (Guest et al., 2016). }\end{array}$ \\
\hline 2 (Medium) & $\begin{array}{l}\text { Survey or interview methods are } \\
\text { employed in the study to address the } \\
\text { review questions. There is evidence of a } \\
\text { low response rate ( } 69 \% \text { or less) in survey } \\
\text { studies or in the case of interview studies, } \\
\text { less than } 12 \text { interviews are conducted } \\
\text { (Guest et al., 2016). }\end{array}$ \\
\hline & $\begin{array}{l}\text { A case study design is employed in the } \\
\text { study. The case study is significant and } \\
\text { describes the process of an assessment } \\
\text { approach used by an EP in practice. }\end{array}$ \\
\hline
\end{tabular}

WoE B Scoring

\begin{tabular}{|c|c|c|c|}
\hline Study & High & Medium & Low \\
\hline Lawrence \& Cahill (2014) & $\mathrm{X}$ & & \\
\hline Ogg et al. (2013) & & $X$ & \\
\hline Stothard, Woods \& Innoue (2018) & & $X$ & \\
\hline Sotelo-Dynega, \& Dixon (2014) & & $X$ & \\
\hline Bourke \& Dharan (2015) & & $\mathrm{X}$ & \\
\hline
\end{tabular}


Bahr, Leduc, Hild, Davis, Summers \& $\mathrm{X}$

McNeal (2017)

Hanchon \& Allen (2013)

X

Hill \& Turner (2016)

$\mathrm{X}$

Sansosti \& Sansosti (2013)

X

Aiello, Ruble \& Esler (2016)

X

Vega, Lasser \& Afifi (2015)

X

Cottrell \& Barrett (2015)

X

Filter, Ebsen \& Dibos (2013)

X

Pameijer, N. (2017)

X

Tobias (2017) X

Bozic, Lawthom \& Murray (2017) X

Bozic (2013)

Harrison \& McManus (2016) X

Parker, Zaboski \& Joyce-Beaulieu (2016) X

McCrimmon \& Yule (2016) X X

Cane (2016) X X 


\section{Appendix 5 WoE C Criteria and Scoring}

WoE $\mathrm{C}$ is also review specific, measuring the extent to which the study and its findings are relevant to answering the review question (Gough, 2007). The WoE C criteria was devised by the reviewer, with reference to inclusion and exclusion criteria stipulated. For a 'High' WoE C rating, the study must provide empirical evidence of an assessment approach used by EPs in practice and evidence on whether this approach informs appropriate interventions for clients. The tables below outline $\mathrm{WoE} \mathrm{C}$ criteria and scoring.

WoE C Criteria and Rationale

\begin{tabular}{ll}
\hline WoE C Weighting & Description \\
\hline 3 (High) & The study provides empirical evidence for \\
& an assessment approach used by EPs in \\
& practice and evidence on whether this \\
& approach informs appropriate \\
& interventions for clients. Findings are \\
& considered relevant to the review \\
& questions (i.e. Assessment practices are \\
& not limited to a specified domain, such as \\
& cognitive assessment practices and are \\
& applied with more than one referral \\
& type/population). \\
& \\
& The study provides empirical evidence for \\
an assessment approach used by EPs in \\
practice and evidence on whether this \\
approach informs appropriate \\
interventions for clients. Findings are \\
considered less relevant to the review \\
questions (i.e. Assessment practices are \\
limited to a specified domain and are \\
applied with one referral \\
type/population). \\
The study provides empirical evidence for \\
an assessment approach used by EPs in \\
practice. Findings are relevant to the \\
review questions (i.e. Assessment \\
practices are not limited to a specified \\
domain, such as cognitive assessment \\
practices and are applied with more than \\
one referral type/population). \\
The study provides empirical evidence for \\
an assessment approach used by EPs in \\
practice. Findings are considered less \\
\hline \\
\end{tabular}


relevant to the review questions (i.e. Assessment practices are limited to a specified domain and are applied with one referral type/population).

WoE C Scoring

\begin{tabular}{lc}
\hline Study & H \\
\hline Lawrence \& Cahill (2014) & X \\
Bozic (2013) & X \\
Pameijer, N. (2017) & X
\end{tabular}

Tobias (2017)

Bahr, Leduc, Hild, Davis, Summers \&

McNeal (2017)

Bourke \& Dharan (2015)

Filter, Ebsen \& Dibos (2013)

Bozic, Lawthom \& Murray (2017)

X

Parker, Zaboski \& Joyce-Beaulieu (2016)

X

Cane (2016)

$\mathrm{X}$

Ogg et al. (2013)

Stothard, Woods \& Innoue (2018)

Sotelo-Dynega, \& Dixon (2014)

Hanchon \& Allen (2013)

Hill \& Turner (2016)

Sansosti \& Sansosti (2013)

Aiello, Ruble \& Esler (2016)

$\mathrm{X}$

Vega, Lasser \& Afifi (2015)

Cottrell \& Barrett (2015)

Harrison \& McManus (2016)

X

McCrimmon \& Yule (2016) 
WoE D is a general overall weighting of the study, calculated by averaging the scores for WoE A, WoE B and WoE C (Gough, 2007). For the purpose of this systematic review, a common scoring system for weighting the evidence was implemented across categories (See table below). Ratings of 1-1.6 are considered 'Low', 1.7-2.3 'Medium', and ratings of 2.4-3 are considered 'High'.

WoE Common Scoring System

\begin{tabular}{ll}
\hline Weighting of Evidence & Score \\
\hline High (3) & $2.4------3$ \\
Medium (2) & $1.7----2.3$ \\
Low (1) & $1------1.6$ \\
\hline
\end{tabular}


Appendix 7 Assessment Approaches Informing Appropriate Interventions

\section{Strength-Based Assessment (Bozic, 2013)}

Strengths arising from this assessment approach were incorporated into intervention planning for six cases (Bozic, 2013). For five cases, pre-intervention levels and targets were plotted on a 10-point Target, Monitoring and Evaluation (TME) Scale. For one case, the initial Child and Adolescent Strengths Assessment (CASA) was converted into a score, which could be re-administered post-intervention. Where an intervention plan was agreed, repeated measures were taken (five cases). Positive change was found in four of these cases ( 80 per cent), through positive movement on TME rating scales (three cases) or an increase in repeated measures on the CASA (one case).

\section{Multi-modal Assessment/RTI Approach (Parker et al., 2016)}

Assessment data gathered from the multi-modal assessment approach applied by Parker et al. (2016) indicates the student struggled to manage his attention and hyperactivity, use healthy coping strategies when angry or frustrated, view himself in a positive manner and maintain pro-social relationships with peers and adults. It was decided by the school's 'Student Success Team' that Tier III intervention of the RTI framework was warranted. The intervention plan consisted of intensive counseling, which took place weekly over the course of 6 months within the school campus, a daily behaviour report card strategy implemented over a nine week period, and regular home/school collaboration on behavioural progress. Measures used to track progress included The Behavior Assessment System for Children, Second Edition (BASC-2), the number of office discipline referrals and behaviour plan data. Outcome data indicated a decrease in office discipline referrals, lower levels of behaviour symptoms, and an increase in prosocial classroom behaviours. Improvement was maintained into the following school year.

\section{Genogram Model (Tobias, 2017)}

The genogram was used both as an assessment tool and as the initial intervention in a piece of casework with an 11-year old girl who was presenting with school refusal. The genogram was drawn up on a large piece of flip chart paper in the middle of the family's living room. Themes that emerged through application of the genogram included attachments, loyalty and exclusion, anxiety, health concerns and 
school attendance within the wider family, mutism, strength and gender. The EP reports the genogram was helpful as part of an initial intervention, because it directed the family's attention to ongoing patterns of behaviour and unanswered, or unspoken, issues. It helped family members to view events and relationships from different perspectives and to reflect on how other members might be feeling within the family system (Tobias, 2017).

\section{Solution-Focused Approach (Cane, 2016)}

A solution-focused approach was applied in the assessment of a child presenting with externalising behavioural difficulties (Cane, 2016). Intervention goals arising from assessment included; being on time to school and to lessons, getting work done, asking for help, and being more mature and independent. The use of scaling illustrated how, over the course of the intervention, the student rated himself as progressing from 3.5 to 8 on his scale and at seven-week follow-up, he demonstrated maintenance of positive changes by rating himself as remaining at eight on his scale. Qualitative feedback from school staff described the student as "loads better" and more mature and sensible, including reduced incidences of shouting out and pushing other students, and an improved ability to wait calmly (e.g. at lunchtime) (Cane, 2016, p. 77). Staff commented that he was learning more and seemed proud of his work, as well as giving more articulate responses to class discussions with a reduced need for adult prompts (Cane, 2016). 
(Pameijer, 2016, 2017)

\begin{tabular}{|c|c|}
\hline Stage & Description \\
\hline $\begin{array}{l}\text { 1. Intake, how can we } \\
\text { collaborate? }\end{array}$ & $\begin{array}{l}\text { The first goal is to collect information so that the school } \\
\text { psychologist can determine a strategy for a particular case. } \\
\text { Another key objective is to achieve compatibility with the } \\
\text { school, student and parents/guardians, in order to create a } \\
\text { constructive partnership. In the first meeting the questions, } \\
\text { aims, expectations and requests of those involved are } \\
\text { discussed: what do they intend to accomplish (or avoid), } \\
\text { why and how? How do they explain the situation? Which } \\
\text { case formulation and recommendation are most likely to } \\
\text { help them? These questions shed light on their frame of } \\
\text { reference and support the school psychologist in tailoring the } \\
\text { process to their personal theory and needs. Appointments for } \\
\text { cooperation are made, e.g. who collects which information?; } \\
\text { when will one meet again to discuss the findings? }\end{array}$ \\
\hline $\begin{array}{l}\text { 2. Strategy, how to } \\
\text { proceed in this } \\
\text { particular case? }\end{array}$ & $\begin{array}{l}\text { The input of this stage is the information collected in the } \\
\text { intake and the output is the strategy that best fits a specific } \\
\text { situation. First, the relevant information is organised in four } \\
\text { sections: student, instructional environment, parental support } \\
\text { of learning and relevant history. Then the school } \\
\text { psychologist decides what more needs to be known to } \\
\text { answer the clients' and his questions. Is the investigation } \\
\text { stage (Stage 3) necessary? This is the case when more } \\
\text { information is needed in order to formulate } \\
\text { recommendations. Or can he already move on to stage } 4 \\
\text { (Integration stage)? The bottom line is: no investigation will } \\
\text { be conducted unless its outcomes will influence the choice } \\
\text { of the intervention. Each question is justified with the 'if- } \\
\text { then-rationale': if we know ..., then we can recommend... } \\
\text { However, if we don't know..., we then cannot recommend ... } \\
\text { This way, collecting data is goal-directed and directly linked } \\
\text { to intervention. If stage } 3 \text { is required, alternate hypotheses } \\
\text { from a transactional frame of reference are formulated, } \\
\text { relevant hypotheses are selected, based on their impact on } \\
\text { the choice of an intervention and these hypotheses are } \\
\text { translated into questions for investigation. }\end{array}$ \\
\hline $\begin{array}{l}\text { 3: Investigation, } \\
\text { answering the selected } \\
\text { answers }\end{array}$ & $\begin{array}{l}\text { This stage involves a goal-directed rather than a routine } \\
\text { collection of data. The selected hypotheses determine the } \\
\text { information to be gathered. The content of this stage thus } \\
\text { varies in each case, ranging from using one instrument to } \\
\text { several different tools. }\end{array}$ \\
\hline $\begin{array}{l}\text { 4: Integration, goals } \\
\text { and needs }\end{array}$ & $\begin{array}{l}\text { The information is integrated into a specific case } \\
\text { formulation: how can the situation be understood? This } \\
\text { summary is translated into goals for the student, teaching } \\
\text { strategies and parental support, educational needs of the }\end{array}$ \\
\hline
\end{tabular}


student and support needs of his teacher and/or parents/guardians. As several interventions focus on the same target, choices have to be made. The AFI-model prefers interventions that have been proven to be effective. The school psychologist can benefit from several metaanalyses when deciding in this.

5: Recommendations, In this stage the clients are informed about the outcomes of appointments the assessment. By providing them with clear and and feedback meaningful information, related to their personal theory, hopes and worries, they can choose for themselves which option is both desirable and achievable. An important aim is to arrive at a feasible intervention, supported by all parties. The school psychologist therefore asks if those involved are willing and able to 'start tomorrow'. If the answer is affirmative, the child, teacher and parent/guardian are encouraged to change their behaviour. If this is not yet the case, the assessment process continues with further consultation. 


\section{Criteria 1}

The initial criteria for participant selection will be a student in a senior class in primary school $\left(4^{\text {th }}, 5^{\text {th }}\right.$ or $\left.6^{\text {th }}\right)$ referred to the school psychology service to address behaviour, socialemotional and/or learning needs. Students in senior classes are being sought so as to maximise the voice of the child in the research project, in line with article 12 of the International Convention on the Rights of the Child (2013) (Pameijer, 2017).

\section{Criteria 2}

The referral form will preferably outline a complex case in terms of learning, behaviour and/or social-emotional needs, that will be suited to the stages of the AFI model. The researcher's placement supervisor will help decide on the level of complexity.

\section{Criteria 3}

The parents/guardians and school staff (teachers and SNA) will preferably show interest in the AFI model and five-stage process, and motivation to take part in the study. 
Appendix 10 Case Study Protocol (Devised in accordance with Yin, 2009, p. 80 guidelines) and Yin (2018)

A. Introduction to the Case Study and Purpose of Protocol

1. Case study questions, hypotheses and propositions

a. Research question: 'Can the Assessment for Intervention model bridge the gap between assessment and intervention, through activity and interaction* at the meso and microsystem levels?'

*Activity and interaction are those activities and interactions that resonate with EST and SCT concepts, including but not limited to molar activities, dyadic relations, "law of genetic development", ZPD and tools and semiotics.

b. Case study propositions: Five propositions arising from from the conceptual framework and theoretical statement, research question and the aims of AFI (Pameijer, 2016, 2017).

Following application of the five stages of the AFI model:

1. Parents/guardians, school staff and TEP will have a better understanding of the child's situation, as a result of interactions and activities that have occurred at the meso and microsystem levels.

2. Parents/guardians, school staff and TEP will know what intervention supports the child needs at home and at school, as a result of interactions and activities that have occurred at the meso and microsystem levels.

3. School staff and parents/guardians will have greater perceived competence in supporting the child's needs at home and at school, as a result of interactions and activities that have occurred at the meso and microsystem levels.

4. The child will have a better understanding of their strengths and needs and what to do to improve their needs, as a result of interactions and activities that have occurred at the meso and microsystem levels. 
5. At a time of ecological transition, school staff will feel competent in their ability to work according to the aims of Circular 0013/2017, in meeting and monitoring the needs of the child.

2. Conceptual framework for the case study: The current research is conducted through the theoretical lens of EST and SCT, because these were the theories that emerged in the discussion of findings in the literature review. To design a conceptual framework for the current study, the concepts within these theories were analysed in accordance with AFI principles. The conceptual framework was refined into a theoretical statement, or proposition for the current study:

'During a time of ecological transition with the issuance of Circular 0013/2017, the EP applies the five stages of the AFI model to a case referral. By interacting with the child, parents/guardians and teachers in accordance with AFI principles at the meso and microsystem levels, the assessment process leads to intervention recommendations that are meaningful to teachers, parents/guardians and child. Engaging together in the five-stage process exposes EST and SCT concepts'.

3. Role of protocol in guiding the case study investigator: The protocol guides the researcher in the data collection process and enhance the reliability of the research. It is considered a standardised agenda for the researcher's line of inquiry (Yin, 2009).

\section{B. Data Collection Procedures}

1. Names of sites to be visited, including contact persons: Schools 1 and 2 and their respective SENCOs/principals

2. Data Collection Plan:

a. October/November 2018: Complete pilot study. Revise interview questions/consent forms/printing etc. following pilot study. ${ }^{*}$ See separate document outlining revisions made.

b. October 2018: Choose suitable case referrals with placement supervisor 
c. October 2018: Distribute information sheets to schools, parents/guardians and student. Informed consent/ assent of school staff, parents/guardians and student.

d. November 2018: Set up database of participants (School 1 and School 2)

e. November 2018: Cases are conducted in parallel due to time constraints. Participants in School 1 and 2 complete Likert scale statements before application of AFI model and researcher records reflections in researcher diary.

f. November/December 2018: Apply the five-stage AFI model to the case referral in School 1 and 2 and record researcher reflections in the researcher diary*.

g. December 2018: Participants complete Likert scale statements and semistructured interviews after application of AFI model and researcher records reflections in researcher diary

*See detailed outline of data collection procedures and researcher actions at each stage of the AFI model in a separate appendix.

3. Expected preparation prior to school visits: Print information and consent/assent sheets, distribute and collect signed informed consent/assent sheets, print researcher diary (AFI templates), Likert scale questionnaires, interview questions and interview protocol. Borrow voice recording device from MIC library.

\section{Protocol Questions}

The main purpose of the protocol's questions is to keep the researcher on track as data collection proceeds and serves as the researcher's line of inquiry (Yin, 2018 p. 99).

1. Does the AFI model link assessment to meaningful intervention for clients? If so, how? What activities and interactions lead to meaningful intervention recommendations for clients?

- Source of evidence: Researcher reflections, interviews with clients, Likert scale statements

2. Do clients feel competent in meeting and monitoring the needs of the child identified?

- Source of evidence: Researcher reflections, interviews with clients, Likert scale statements 
3. To what extent are all clients actively involved in the five-stage process?

- Source of evidence: Researcher reflections, interviews with clients

4. What factors promote and hinder client involvement in the five-stage process?

- Source of evidence: Researcher reflections, interviews with clients

5. Can the model be feasibly applied by EPs in practice?

- Source of evidence: Researcher reflections

6. What are the benefits and limitations of the AFI model?

- Source of evidence: Researcher reflections, interviews with clients,

Likert scale statements

D. Outline of Case Study Report

7. Results under each proposition (Pilot Case)

a. Participant ratings on the Likert scale statements, before and after implementation of the AFI model presented in tabular format

b. Pattern-matching of interview responses

c. Thematic analysis of interview responses

d. Researcher diary and reflective journal

8. Summary of findings addressing the research question (Pilot Case)

9. Discussion of findings under related themes in accordance with the literature and conceptual framework (Pilot Case)

10. Repeat steps 1-3 for Case 1 and 2

11. Cross-case analysis and discussion

12. Conclusions and directions for future research 
Appendix 11 Pilot and Revised Parent Likert Scale Statements (before and after engagement with the AFI model)

Please rate the following items on a scale of 1-10, where 1 is strongly disagree, 5 is neither agree or disagree and 10 is strongly agree. Please use the spaces below for any additional comments.

1. I understand my child's situation

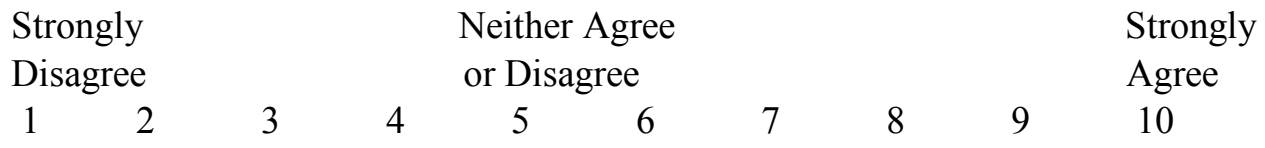

2. I know what support my child needs at home

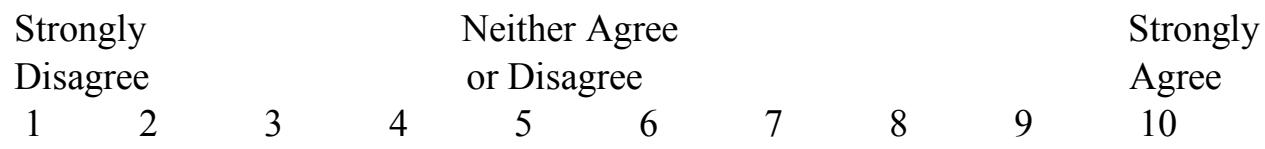

3. I am able to support my child's needs at home

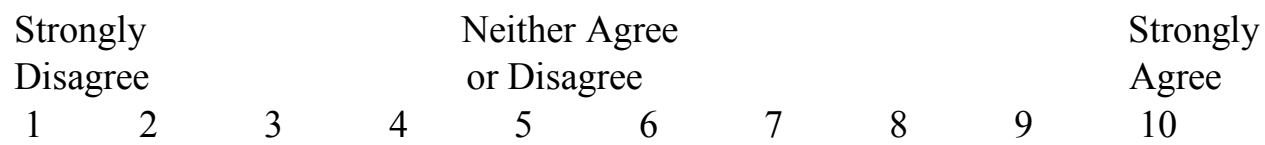


Revised Parent Likert Scale Statements (before and after engagement with the AFI model)

\section{My concern}

is:

Please rate the following items on a scale of 1-10, where 1 is strongly disagree, 5 is neither agree or disagree and $\mathbf{1 0}$ is strongly agree. Please use the spaces below for any additional comments.

1. I understand my child's situation

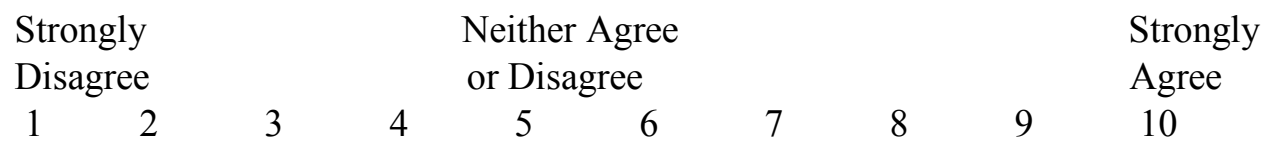

2. I know what support my child needs at home

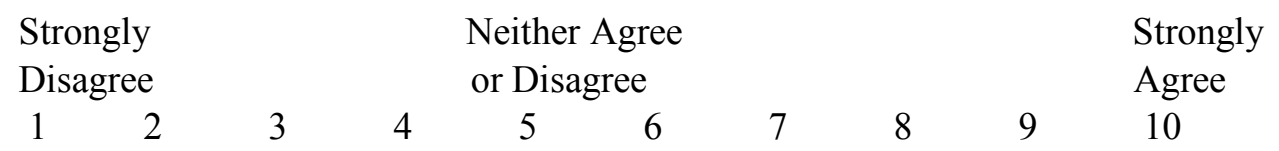

3. I am able to support my child's needs at home

\begin{tabular}{ccccccccc}
$\begin{array}{c}\text { Strongly } \\
\text { Disagree } \\
1\end{array}$ & \multicolumn{9}{c}{$\begin{array}{c}\text { Neither Agree } \\
\text { or Disagree }\end{array}$} & 3 & 4 & 5 & 6 & 7 & 8 & 9 & $\begin{array}{c}\text { Strongly } \\
\text { Agree }\end{array}$
\end{tabular}


Appendix 12 Pilot and Revised Teacher Likert Scale Statements (before and after engagement with the AFI model)

Please rate the following items on a scale of 1-10, where 1 is strongly disagree, 5 is neither agree or disagree and 10 is strongly agree. Please use the spaces below for any additional comments.

1. I understand the student's situation

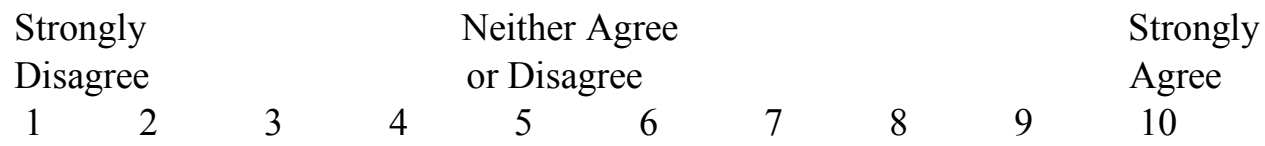

2. I know what support the student needs at school

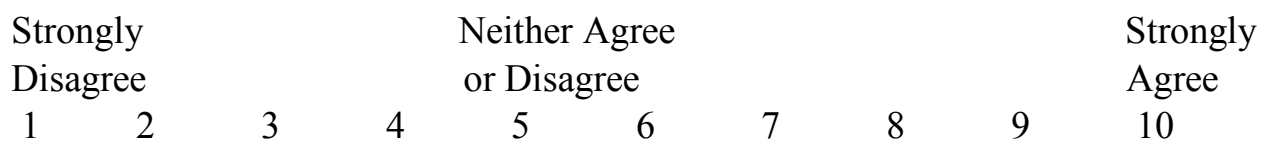

3. I am able to support the student's needs at school

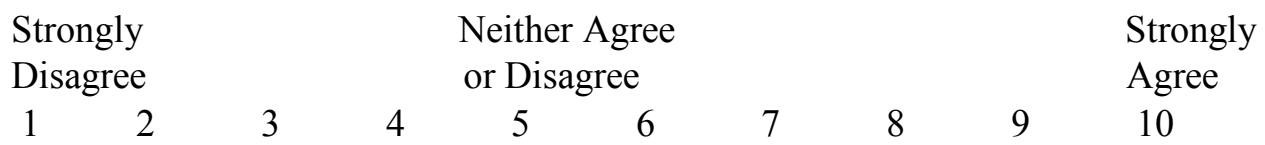


Revised Teacher Likert scale Statements (before and after engagement with the AFI model)

\section{My concern}

is:

Please rate the following items on a scale of 1-10, where 1 is strongly disagree, 5 is neither agree or disagree and $\mathbf{1 0}$ is strongly agree. Please use the spaces below for any additional comments.

1. I understand the student's situation

\begin{tabular}{|c|c|c|c|c|}
\hline $\begin{array}{l}\text { Strongly } \\
\text { Disagree }\end{array}$ & & & $\begin{array}{l}\text { Jeith } \\
\text { or Di }\end{array}$ & $\begin{array}{l}\text { Strongly } \\
\text { Agree }\end{array}$ \\
\hline 2 & 3 & 4 & 5 & 10 \\
\hline
\end{tabular}

2. I know what support the student needs at school

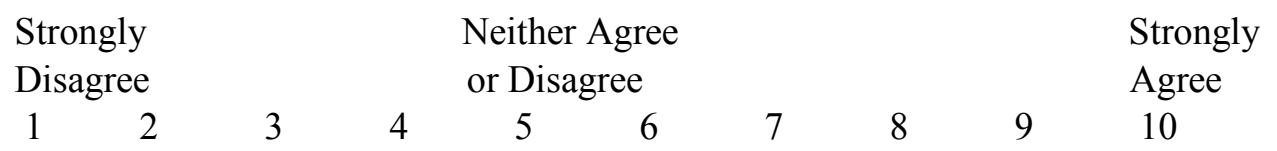

3. I am able to support the student's needs at school

\begin{tabular}{ccccccccc}
$\begin{array}{c}\text { Strongly } \\
\text { Disagree } \\
1\end{array}$ & \multicolumn{9}{c}{$\begin{array}{c}\text { Neither Agree } \\
\text { or Disagree }\end{array}$} & 3 & 4 & 5 & 6 & 7 & 8 & 9 & $\begin{array}{c}\text { Strongly } \\
\text { Agree }\end{array}$
\end{tabular}


Appendix 13 Student Likert Scale Statements (before and after engagement with the AFI model)

Please circle the smileys below and use the lines to write anything you would like to say.

1. I know what my strengths are at school

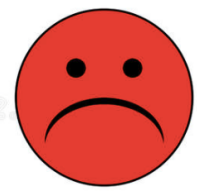

No

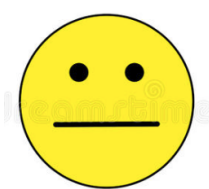

Not sure

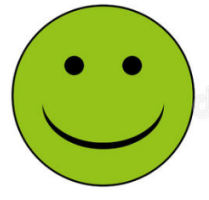

Yes

2. I know what I need help with at school

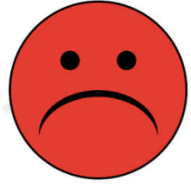

No

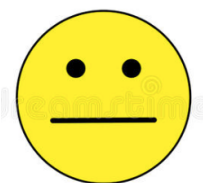

Not sure

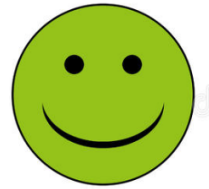

Yes

3. I know what I can do to get better at what I need help with at school

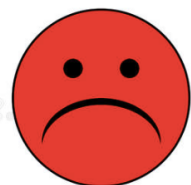

No

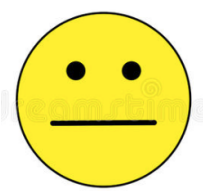

Not sure

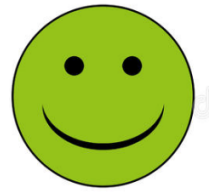

Yes 
Appendix 14 Pilot and Revised Parent Interview Schedule

Research Question: 'Can the Assessment for Intervention model bridge the gap between assessment and intervention, through activity and interaction at the meso and microsystem levels?'

1. Can you describe your experience of working with the Assessment for Intervention model?

2. What were the roles of the EP, parent(s)/guardian(s), teacher(s), SNA and child throughout the process?

3. From your perspective, what was your child's experience of the process?

4. Do you feel your understanding of your child's situation has changed? If so, how?

5. Did the intervention recommendations address the needs identified? If so, how?

6. Do you feel you can support your child's needs at home? If so, how?

7. In your opinion, were the intervention recommendations useful, in what way?

8. From your perspective, what were the benefits of the Assessment for Intervention model, if any?

9. What were some of the limitations of the Assessment for Intervention model, if any?

Note: The questions may be revised following completion of the pilot phase of the study 


\section{Revised Parent Interview Schedule}

Research Question: 'Can the Assessment for Intervention model bridge the gap between assessment and intervention, through activity and interaction at the meso and microsystem levels?'

1. Can you describe your experience of working with the Assessment for Intervention model?

2. From your perspective, what was my role* throughout the Assessment for Intervention process?

$>$ From your perspective, what was your role, throughout the process?

$>$ From your perspective, what was the teacher (s) role, throughout the process?

$>$ From your perspective, what was your child's role, throughout the process?

*Note: Clarification of my title 'Trainee Educational Psychologist' will be provided if needed. The word 'role' will be explained and elaborated upon as needed (e.g. job).

3. From your perspective, what was your child's experience of the process?

4. Do you feel your understanding of your child's situation has changed after working through the five stages of the AFI model?

$>$ Prompt question: If yes:

○ Why do you feel your understanding has changed?

Prompt question: If no:

○ Why do you not feel your understanding has changed?

*Note: 'the child's situation will be clarified as the presenting concerns or reason for referral at the intake stage.

5. Did the intervention recommendations address your questions at the intake stage?

$>$ Prompt question: If yes:

- How did the intervention recommendations address your questions?

Prompt question: If no:

○ Why do you think the intervention recommendations did not address your questions?

○ What recommendations would you have preferred to receive?

6. Do you feel you can support your child's needs at home?

$>$ Prompt question: If yes: 
○ How will you support your child's needs at home?

○ Had you supported your child in this way before working through the five stages of the AFI model?

Prompt question: If no:

○ Can you explain why you feel you can't support your child's needs at home?

○ Did you feel the same way before working through the five stages of the AFI model?

*Note: 'the child's needs' will be clarified as the needs that were identified at stage five of the model, at the recommendations, appointments and feedback stage.

7. Do you feel you can monitor* your child's needs at home?

$>$ Prompt question: If yes:

○ How will you monitor your child's needs at home?

- Had you monitored your child's needs in this way before working through the five stages of the AFI model?

Prompt question: If no:

○ Why do you feel you can't monitor your child's needs at home?

○ Did you feel the same way before working through the five stages of the AFI model?

*Note: the word 'monitor' will be explained and elaborated upon if needed (e.g. keep an eye on).

8. In your opinion, were the intervention* recommendations useful?

$>$ Prompt question: If yes:

○ In what way were the intervention recommendations useful?

Prompt question: If no:

- Are there any other intervention recommendations that you feel would have been useful?

*Note: the word 'intervention' will be explained and elaborated upon if needed (e.g. ideas for helping

9. Do you think you would apply anything that you have learned from working through the five stages of the AFI model to a different situation?

10. From your perspective, were there benefits to the Assessment for Intervention model? 
11. From your perspective, were there limitations to the Assessment for Intervention model?

*Note: The questions will be rephrased and clarified further for understanding, as necessary 
Appendix 15 Pilot and Revised Teacher Interview Schedule

Research Question: 'Can the Assessment for Intervention model bridge the gap between assessment and intervention, through activity and interaction at the meso and microsystem levels?'

1. Can you describe your experience of working with the Assessment for Intervention model?

2. What were the roles of the EP, parent(s)/guardian(s), teacher(s), SNA and child throughout the process?

3. From your perspective, what was the child's experience of the process?

4. Do you feel your understanding of the child's situation has changed? If so, how?

5. Did the intervention recommendations address the needs identified? If so, how?

6. Do you feel you can support the child's needs at school? If so, how?

7. In your opinion, were the intervention recommendations useful, in what way?

8. Do you feel you are able to meet and monitor the needs of the child, in line with the aims of Circular 0013/2014?

9. From your perspective, what were the benefits of the Assessment for Intervention model, if any?

10. What were some of the limitations of the Assessment for Intervention model, if any?

Note: The questions may be revised following completion of the pilot phase of the study 


\section{Revised Teacher Interview Schedule}

Research Question: 'Can the Assessment for Intervention model bridge the gap between assessment and intervention, through activity and interaction at the meso and microsystem levels?'

1. Can you describe your experience of working with the Assessment for Intervention model?

2. From your perspective, what was my role* throughout the Assessment for Intervention process?

$>$ From your perspective, what was the parent's role, throughout the process?

$>$ From your perspective, what was your role, throughout the process?

$>$ From your perspective, what was the child's role, throughout the process?

*Note: Clarification of my title 'Trainee Educational Psychologist' will be provided if needed

3. From your perspective, what was the child's experience of the process?

4. Do you feel your understanding of the child's situation has changed after working through the five stages of the AFI model?

Prompt question: If yes:

○ Why do you feel your understanding has changed?

Prompt question: If no:

○ Why do you not feel your understanding has changed?

*Note: 'the child's situation will be clarified as the presenting concerns or reason for referral at the intake stage.

5. Did the intervention recommendations address your questions at the intake stage?

$>$ Prompt question: If yes:

○ How did the intervention recommendations address your questions?

Prompt question: If no:

$\circ$ Why do you think the intervention recommendations did not address your questions?

○ What recommendations would you have preferred to receive?

6. Do you feel you can support the child's needs at school?

$>$ Prompt question: If yes:

- How will you support the child's needs at school? 
○ Had you supported the child in this way before working through the five stages of the AFI model?

Prompt question: If no:

○ Can you explain why you feel you can't support the child's needs at school?

○ Did you feel the same way before working through the five stages of the AFI model?

*Note: 'the child's needs' will be clarified as the needs that were identified at stage five of the model, at the recommendations, appointments and feedback stage.

7. Do you feel you can monitor* the child's needs at school?

Prompt question: If yes:

○ How will you monitor the child's needs at school?

○ Had you monitored the child's needs in this way before working through the five stages of the AFI model?

Prompt question: If no:

○ Why do you feel you can't monitor the child's needs at school?

○ Did you feel the same way before working through the five stages of the AFI model?

*Note: the word 'monitor' will be explained and elaborated upon if needed

8. In your opinion, were the intervention* recommendations useful?

$>$ Prompt question: If yes:

○ In what way were the intervention recommendations useful?

Prompt question: If no:

- Are there any other intervention recommendations that you feel would have been useful?

*Note: the word 'intervention' will be explained and elaborated upon if needed

9. Do you think you would apply anything that you have learned from working through the five stages of the AFI model to a different situation?

10. From your perspective, were there benefits to the Assessment for Intervention model?

11. From your perspective, were there limitations to the Assessment for Intervention model? 
Appendix 16 Pilot and Revised Student Interview Schedule

Warm up question: What are some of the things you like about school?

1. What did you like about working with me (researcher), your parent(s)/guardian(s), teacher(s)?

2. Was there anything you didn't like?

3. Did working with me (researcher), your parent(s)/guardian(s) and teacher(s) help you to find out about your strengths and needs? What are your strengths and needs?

4. Do you know what you need to do to improve on your needs?

5. Did working with me (researcher), your parent(s)/guardian(s) and teacher(s) help you to make goal(s)*? How?

6. What can we do to help you achieve your goal(s)?

Note: The student will be told that they can draw a picture to represent their response, if they wish. The questions may be revised following completion of the pilot phase of the study.

*In stage four of the AFI model, the information gathered in previous stages is put together to make goals for the student and intervention recommendations that support goals and needs are created. The goals created will depend on the reason for referral (behaviour, social-emotional and/or learning needs) and this question will be phrased accordingly (e.g. Did working with me (the researcher), your parent(s)/guardian(s) and teacher(s) help you to make goal(s) for copying homework from the board? How?) 


\section{Revised Student Interview Schedule}

Warm up question: What are some of the things you like about school?

1. What did you like about working with me, your mother and teacher?

2. Was there anything you didn't like?

3. Did working with me, your mother and teacher help you to find out about your strengths*?

Prompt question: What are your strengths?

*Note: Strengths will be clarified as 'the things you are good at'

4. Did working with me, your mother and teacher help you to find out about what you need help with?

Prompt question: What do you need help with?

5. Do you know what you need to do to get better at (insert needs identified)

6. Did working with me, your mother and teacher help you to make goal(s)* for___ (insert needs identified)? How?

7. Have you thought about how we might help you achieve your goal(s)?

8. Is there anything that you have learned working with me, your mother and teacher that you can use in a situation in the future?

Note: The student will be told that they can draw a picture to represent their response, if they wish. The questions may be revised following completion of the pilot phase of the study.

*In stage four of the AFI model, the information gathered in previous stages is put together to make goals for the student and intervention recommendations that support goals and needs are created. The goals created will depend on the reason for referral (behaviour, social-emotional and/or learning needs) and this question will be phrased accordingly (e.g. Did working with me (the researcher), your parent(s)/guardian(s) and teacher(s) help you to make goal(s) for copying homework from the board? How?) 
1. Set up environment appropriately i.e. suitable seating arrangements and some light refreshments, sound check of audio equipment.

2. Thank participant to agreeing to be involved and use rapport and attunement skills to promote a relaxed and safe environment.

3. Clarification and reminders provided to participants:

- Reminder of what I will be doing with the audio data and why

- The information sheet has been read and consent forms signed

- Assurance of anonymity and confidentiality

- Re-emphasise, I am interested in their thoughts on the topic and there are no right or wrong answers

- Re-emphasise participants right to not answer a question or to withdraw from study at any point

- Check if there are any questions before we get started.

4. Carrying out interview according to schedule.

5. At the end of the interview thank participants again for being involved and enquire as to their experience of being involved. Check as to whether anything needs to be followed up. 


\section{Revised Interview Guide}

1. Set up environment appropriately i.e. suitable seating arrangements and some light refreshments, sound check of audio equipment.

2. Thank participant for agreeing to be involved and use rapport and attunement skills to promote a relaxed and safe environment.

3. Clarification and reminders provided to participants:

- Reminder of what I will be doing with the audio data and why

- This is a semi-structured interview to gather information on your experience of the model and to explore your views on how useful the Assessment for Intervention model is in linking assessment to intervention.

- I will be transcribing these interviews and anonymized quotes will be used in the write up of the thesis. Pseudonyms will be applied throughout. You are welcome to read over the transcript to ensure anonymity is maintained.

- The information sheet has been read and consent forms signed

- Assurance of anonymity and confidentiality

- Re-emphasise, I am interested in their thoughts on the topic and there are no right or wrong answers. Please give your most honest feedback

- Re-emphasise participants right to not answer a question or to withdraw from study at any point

- Interview will take approx. 20-30 mins

- Check if there are any questions before we get started.

4. Carrying out interview according to schedule (take some notes).

5. At the end of the interview thank participants again for being involved and enquire as to their experience of being involved. Check as to whether anything needs to be followed up.

\section{Interview Guide (Student)}

- Set up environment appropriately i.e. suitable seating arrangements and some light refreshments, sound check of audio equipment.

- Show recording device

- Carry out interview

- Let child listen to some of the recording after

At the end of the interview thank participants again for being involved and enquire as to their experience of being involved. Check as to whether anything needs to be followed up. 
Appendix 18 Documentation of Revisions Following Pilot Study

\section{Changes after pilot}

- Parent and teacher Likert statements, included the statement at top of page My concern is , for clarity, structure and following feedback

from parent and teacher in pilot case.

- Changes to interview questions (teacher):

○ Question 5: Did the intervention recommendations address the needs identified? If so, how?

○ Did the intervention recommendations address your questions at the intake stage? If so, how?

$\circ \quad$ New question (question 7): Do you feel you can monitor the child's needs at school? If so, how? (addressing aims of the new model)

○ New question (question 9): Do you think you could apply anything that you have learned to a different situation? (to address developmental validity)

- Changes to interview questions (parent):

○ Question 5: Did the intervention recommendations address the needs identified? If so, how?

○ Did the intervention recommendations address your questions at the intake stage? If so, how?

○ New question (question 7): Do you feel you can monitor your child's needs at home? If so, how? (addressing aims of the new model)

○ New question (question 9): Do you think you could apply anything that you have learned to a different situation? (to address developmental validity)

- Changes to interview questions (child):

○ Question 3: Did working with me, your parents/guardians and teachers help you to find out about your strengths and needs? What are your strengths and needs?

- Did working with me, your mother and teacher help you to find out about your strengths? Did working with me, your mother and teacher help you to find out about what you need help with? What are your strengths? What do you need help with?

○ Question 4: Do you know what you need to do to improve on your needs? 
○ Do you know what you need to do to improve on (insert what you need help with at school?)

$\circ \quad$ New question (question 7): Is there anything that you have learned working with me, your parents and teacher that you can use in a different situation?

- Detailed procedure for conducting cases, based on case study protocol and stages of the pilot study 


\section{Informed consent (parent/teacher)}

- Talk through information sheet/informed consent

- Explain context of research and aim

- Explain AFI model (go through each of the five stages, make sure participant knows which stage we are at now)

○ Leave to let participant read information/informed consent sheets

- Ensure participant knows what is involved before signing

○ Show parents the child information sheet and informed assent sheet.

\section{Informed assent (child)}

1. Talk through information sheet/informed consent My name is I'm a trainee educational psychologist. I work with schools to help children do the best they can in school. Will I show you some of the areas we can help? You can tick off if I have done a good job explaining and you understand

- Behaviour: How we follow directions at home and at school, like when you are playing a game of football and the teacher explains the rules/tell you what you do and you do it.

- Feelings: we all have different feelings, happy, sad, worried. We can help boys and girls that have a hard time talking about or managing their feelings.

- Sensations: sensations is how parts of our body react to things going on around us. Like for some boys and girls their head or ears can hurt if the classroom gets too noisy.

- Images: Sometimes pictures and schedules help boys and girls manage their day at school. We can help children learn better and manage their school day with pictures and schedules.

- Learning: Sometimes boys and girls are finding a subject hard like reading or maths, or don't know how to do some of the instructions teachers give, or forget the instructions. We can help with this.

- Body: Some boys and girls have a hard time moving, walking or running. We can help make some changes to make things better.

- Interpersonal relationships: How we get on with other boys and girls in our class, teachers in our school, how we make friends.

\section{Informed consent/assent}

- If you would like, I can work with you, your teacher(s) and parent(s)/guardian(s) to find out about what you are good at (strengths) and what you might need 
some help with. Will we read through this information and assent sheet together?

- Ensure child understands the nature of the study before signing.

*Researcher documents actions/procedures in researcher notebook and fills out template 1a of researcher diary

\section{Likert Scale Statements (Parent and teacher)}

- Explain Likert scale statements (script):

○ So before getting started with the intake stage, I want you to think about the presenting concern and reason for referral. Write your concern at the top of the page and rate these 3 statements from 1-10 where 1 is strongly disagree, 5 is neither agree or disagree and 10 is strongly agree.

- Leave the room and give parent/teacher five minutes to fill out

- Return and give the option to talk through the Likert statements

- Explain we will do this again after application of the five stages and complete an interview as well to explore your experience of the process.

\section{Likert Scale Statements (Child)}

- Read instructions and question for understanding before asking child to proceed with rating statements.

- Stay in room but move away to another area in the room, to give child space to rate the statements.

- Return and give the option to talk through the Likert statements

- Explain we will do this again in a few weeks, after we have done some activities/tasks together and complete a short interview as well to find out about your experience of the process.

*Researcher documents actions/procedures in researcher notebook

\section{Stage 1: Intake (General procedure)}

- Meet with teacher (go through intake questions)

- Meet with child (go through assent and intake questions)

- Meet with parent (go through intake questions)

- Meet with parent and teacher (share questions)

- Meet with parent, teacher and child (discuss everyone's questions/requests) 


\section{Stage 1: Intake (Teacher) \\ Show AFI model, explain at stage 1 \\ Intro and intake questions:}

The goal for me for today's meeting is to gather some information about your concerns, and the problem situation. But before we do this, I'm going to ask a few questions about your hopes and aims and also what you might fear or want to avoid in terms of my involvement and us working together.

\section{Reasons for assessment, questions, aims, expectations and requests}

1. So what are your questions? What do you want to know and why? (come back to this again if too hard at the start)

- The reason I ask this is because these are the questions that I will keep referring back to in any assessment or consultation work that we do, and these questions will guide any recommendations or intervention planning. Why those questions?

So in terms of your questions for my involvement (repeat)

2. What do you want to achieve and what would you like to avoid?

3. When you think of the outcomes of any assessment or of us working together, what do you hope for and what do you fear?

4. What information or supports do you feel you need to address the problem situation?

5. Is there anything in particular that you want me to observe (in classroom)

\section{Overview}

6. Tell me a little about and what is going on at home/school

- For how long has it been like this? (Clarify the issue)

- What does do that contributes to the prob situation?

- What aspects of the home environment contribute to the prob situation?

- What aspects of the school environment contribute to the prob situation

- What are 's positives traits?

- What are the positive aspects about the home environment?

- What are the positive aspects of the school environment? What is going well?

\section{Relevant past history}

7. Anamnestic information (do with parent/possible to view previous reports? On waitlist for further assessment? School files?)

8. In terms of the problem situation, what has previously been tried? what worked or did not work and why not? 


\section{Attributions, goals and solutions}

9. What do you think explains the situation? What do you think is causing the problem? (find the similarities, analyse the differences)

10 . What do you see as the solution to the problem situation?

\section{Conclusion}

11. Repeat questions of the clients and school psychologist

12. Have we collected relevant information and was the cooperation constructive?

13. Appointments for cooperation (who collects which information, why, how and when? When will we meet again to discuss our findings?)

14. Best way/time to contact?

15. Feedback now-can you tell me one thing I did well and one thing I can improve on?

Show AFI model-explain that I will now take the information gathered to come up with a strategy

\section{AFI Notes/reminders}

- $\quad$ sincere compliments

- need to know vs. nice to know

- high realistic expectations

- Feedback (what am I already doing well, keep doing this)

- Feed forward (where is there room for improvement? Something to try)

- metacommunication: I am going to ask you a question about ..., because ...;

- metacommunication: I am going to talk about ..., so that ...;

\section{Stage 1: Intake (Child)}

So, now I have some questions here to ask you to try to get to know you a little better (Thoughts about school checklist)
$\circ \quad$ What I am good at (explain as strengths)
- I need help with (use picture sheet as prompt if there is difficulty).
Which one of these things do you need help with most?

On a scale of $1-10$, where one is really bad and 10 is really good, where would you say you are in relation to ?

How could we get you to one step higher?

How can I/your teachers/your parents help you with this?

What questions do you have for me and your parents/teachers? What would you like us to be able to help you with? Can you draw it? 
I will be speaking to your parents and teachers, what would you like me to tell them? (compliment/something they can do a bit better)

I'll talk to parents and teachers and be back, I might need your help as co-investigator!!

\section{Stage 1: Intake (Parent) \\ Show AFI model, explain at stage 1 \\ Intro and intake questions:}

The goal for me for today's meeting is to gather some information about your concerns, and the problem situation. But before we do this, I'm going to ask a few questions about your hopes and aims and also what you might fear or want to avoid in terms of my involvement and us working together.

\section{Reasons for assessment, questions, aims, expectations and requests}

1. So what are your questions? What do you want to know and why? (come back to this again if too hard at the start)

- The reason I ask this is because these are the questions that I will keep referring back to in any assessment or consultation work that we do, and these questions will guide any recommendations or intervention planning. Why those questions?

So in terms of your questions for my involvement (repeat)

2. What do you want to achieve and what would you like to avoid?

3 . When you think of the outcomes of any assessment or of us working together, what do you hope for and what do you fear?

4. What information or supports do you feel you need to address the problem situation?

5. Is there anything in particular that you want me to observe (in classroom)

\section{Overview}

6. Tell me a little about and what is going on at home/school

- For how long has it been like this? (Clarify the issue)

- What does do that contributes to the prob situation?

- What aspects of the home environment contribute to the prob situation?

- What aspects of the school environment contribute to the prob situation

- What are 's positives traits?

- What are the positive aspects about the home environment?

- What are the positive aspects of the school environment? What is going well? 


\section{Relevant past history}

7. Anamnestic information (do with parent/possible to view previous reports? On waitlist for further assessment? School files?)

8. In terms of the problem situation, what has previously been tried? what worked or did not work and why not?

\section{Attributions, goals and solutions}

9. What do you think explains the situation? What do you think is causing the problem? (find the similarities, analyse the differences)

10 . What do you see as the solution to the problem situation?

\section{Conclusion}

11. Repeat questions of the clients and school psychologist

12. Have we collected relevant information and was the cooperation constructive?

13. Appointments for cooperation (who collects which information, why, how and when? When will we meet again to discuss our findings?)

14. Best way/time to contact?

15. Feedback now-can you tell me one thing I did well and one thing I can improve on?

\section{Show AFI model-explain that I will now take the information gathered to come up} with a strategy

\section{AFI Notes/reminders}

- sincere compliments

- need to know vs. nice to know

- high realistic expectations

- Feedback (what am I already doing well, keep doing this)

- Feed forward (where is there room for improvement? Something to try)

- metacommunication: I am going to ask you a question about ..., because ...;

- metacommunication: I am going to talk about ..., so that ...;

\section{Stage 1: Intake (Parent and teacher)}

- Discuss questions of parent and teacher together

- Inform parent and teacher of child's questions

- Offer parent and teacher opportunity to ask each other questions about their questions (degree of similarity and difference)

\section{Stage 1: Intake (Parent, teacher and child)}

- Clarify and discuss everyone's questions together

- Offer parent, teacher and child opportunity to ask each other questions about their questions (degree of similarity and difference) 
*Researcher documents actions/procedures in researcher notebook, records notes/reflections in accordance with protocol questions and fills out researcher diary (template 1a)

\section{Stage 2: Strategy}

- Researcher completes researcher diary (template $1 \mathrm{~b}$ )

- Researcher complies a feedback on strategy and justification for conducting further investigation document for clients:

○ What we know

- Where we need more information (and rationale for this)

- Questions for investigation and methods of investigation

\section{Stage 3: Investigation}

- Go through feedback on strategy and questions for investigation with parent and teacher (and child)

- Conduct assessments/investigations

- Researcher completes researcher diary (template 2a)

- Compile feedback on investigation stage template

- Go through feedback on investigation stage template with parent and teacher (share assessment information gathered)

- Go through feedback on investigations with child

- Researcher completes researcher diary (template 2a)

*Researcher documents actions/procedures in researcher notebook, records notes/reflections in accordance with protocol questions and fills out researcher diary (template 2a-checklist investigation stage)

\section{Stage 4: Integration}

- Researcher completes researcher diary (template $2 b$ )

- Researcher writes assessment for intervention report and child friendly report

*Researcher documents actions/procedures in researcher notebook, records notes/reflections in accordance with protocol questions and fills out researcher diary (template 2b)

\section{Stage 5: Feedback and Recommendations}

- Meet parent first (read as far as goal)

- Teacher (read as far as goals)

- Meet parent and teacher and follow steps 1-7 below:

- Meet with child, discuss report and goals and how we can achieve goals with intervention recommendations 
1. Discussion of the case formulation:

- Does this summary make sense?

- To what extent do you agree or see the situation differently?

2. Discussion of the goals, needs and recommendations, supported by arguments.

- Do you have additional ideas, solutions or arguments?

- How useful do you find it?

- Are you confident of its success?

- Can you apply the desired approach in daily practice?

- If we achieved this goal, what would we see, what would we hear? (makes it concrete)

3. Clients choose one (or more) of the suggested recommendations. This workable option then becomes the intervention.

4. Conclusions, evaluations and appointments.

- Have we achieved our goals?

- Are questions answered?

- Was the assessment functional?

- Has our insight into the problematic situation increased?

- Do the child, teacher, counsellor and parents have more perspective?

5. Show child report-what do you think?

6. Feedback

- What did I do professionally well (compliments for ...)

- And what could he do better in a similar case in the future (suggestions for the next case ...)?

*Researcher documents actions/procedures in researcher notebook, records notes/reflections in accordance with protocol questions and fills out researcher diary (template $2 b$ )

\section{Likert scale statements and interviews (see data collection post application of AFI model)}

*Researcher documents actions/procedures in researcher notebook, records notes/reflections in accordance with protocol questions 
Appendix 20 Phases of Thematic Analysis (Braun \& Clarke, 2006)

\begin{tabular}{ll}
\hline Phase & $\begin{array}{l}\text { Description of the Process } \\
\text { 1. Foumiliarising }\end{array}$ \\
$\begin{array}{l}\text { 2. Generating initial } \\
\text { codes }\end{array}$ & $\begin{array}{l}\text { Transcribing data (if necessary), reading and re-reading the } \\
\text { data, noting down initial ideas. } \\
\text { Coding interesting features of the data in a systematic } \\
\text { fashion across the entire data set, collating data relevant to } \\
\text { each code. }\end{array}$ \\
$\begin{array}{l}\text { 3. Searching for } \\
\text { themes }\end{array}$ & $\begin{array}{l}\text { Collating codes into potential themes, gathering all data } \\
\text { relevant to each potential theme. }\end{array}$ \\
$\begin{array}{l}\text { 4. Reviewing themes } \\
\text { Checking if the themes work in relation to the coded } \\
\text { extracts (Level 1) and the entire data set (Level 2), } \\
\text { generating a thematic 'map' of the analysis. }\end{array}$ \\
$\begin{array}{l}\text { 5. Defining and } \\
\text { naming themes }\end{array}$ \\
$\begin{array}{l}\text { Ongoing analysis to refine the specifics of each theme, and } \\
\text { the overall story the analysis tells, generating clear } \\
\text { definitions and names for each theme. }\end{array}$ \\
$\begin{array}{l}\text { The final opportunity for analysis. Selection of vivid, } \\
\text { compelling extract examples, final analysis of selected } \\
\text { extracts, relating back of the analysis to the research } \\
\text { question and literature, producing a scholarly report of the } \\
\text { analysis. }\end{array}$
\end{tabular}


Appendix 21 15-Point Checklist of Criteria for Good Thematic Analysis (Braun \&

Clarke, 2006)

\begin{tabular}{|c|c|c|}
\hline Process & No. & Criteria \\
\hline Transcription & 1 & $\begin{array}{l}\text { The data have been transcribed to an appropriate level of } \\
\text { detail, and the transcripts have been checked against the } \\
\text { tapes for 'accuracy'. }\end{array}$ \\
\hline \multirow[t]{5}{*}{ Coding } & 2 & $\begin{array}{l}\text { Each data item has been given equal attention in the coding } \\
\text { process. }\end{array}$ \\
\hline & 3 & $\begin{array}{l}\text { Themes have not been generated from a few vivid examples } \\
\text { (an anecdotal approach), but instead the coding process has } \\
\text { been thorough, inclusive and comprehensive. }\end{array}$ \\
\hline & 4 & All relevant extracts for all each theme have been collated. \\
\hline & 5 & $\begin{array}{l}\text { Themes have been checked against each other and back to } \\
\text { the original data set. }\end{array}$ \\
\hline & 6 & Themes are internally coherent, consistent, and distinctive. \\
\hline \multirow[t]{4}{*}{ Analysis } & 7 & $\begin{array}{l}\text { Data have been analysed / interpreted, made sense of / rather } \\
\text { than just paraphrased or described. }\end{array}$ \\
\hline & 8 & $\begin{array}{l}\text { Analysis and data match each other / the extracts illustrate } \\
\text { the analytic claims. }\end{array}$ \\
\hline & 9 & $\begin{array}{l}\text { Analysis tells a convincing and well-organized story about } \\
\text { the data and topic. }\end{array}$ \\
\hline & 10 & $\begin{array}{l}\text { A good balance between analytic narrative and illustrative } \\
\text { extracts is provided. }\end{array}$ \\
\hline Overall & 11 & $\begin{array}{l}\text { Enough time has been allocated to complete all phases of the } \\
\text { analysis adequately, without rushing a phase or giving it a } \\
\text { once-over-lightly. }\end{array}$ \\
\hline \multirow[t]{4}{*}{ Written report } & 12 & $\begin{array}{l}\text { The assumptions about, and specific approach to, thematic } \\
\text { analysis are clearly explicated. }\end{array}$ \\
\hline & 13 & $\begin{array}{l}\text { There is a good fit between what you claim you do, and what } \\
\text { you show you have done / i.e., described method and } \\
\text { reported analysis are consistent. }\end{array}$ \\
\hline & 14 & $\begin{array}{l}\text { The language and concepts used in the report are consistent } \\
\text { with the epistemological position of the analysis. }\end{array}$ \\
\hline & 15 & $\begin{array}{l}\text { The researcher is positioned as active in the research } \\
\text { process; themes do not just 'emerge'. }\end{array}$ \\
\hline
\end{tabular}




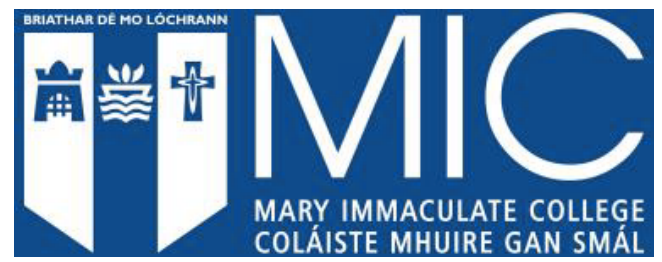

'An exploration of the Assessment for Intervention model in an Irish Educational Psychological context'

\section{Parent/Guardian Information Sheet}

What is the project about?

In 2017, the new model for allocation of special education teaching resources in mainstream schools was launched. Under the new model, schools follow a three step process to support children with special educational needs by asking:

1. How can we identify needs?

2. How can we meet needs?

3. How can we monitor and report on progress?

In supporting this process, the focus of an educational psychological assessment will be on providing understanding of a child's needs, the nature of difficulties, and informing appropriate interventions. 'Assessment for Intervention' is a model that can be applied to educational psychological practice and aims to bridge the gap between assessment and intervention to give rise to meaningful interventions for parents/guardians, teachers, Special Needs Assistants (SNAs) and students. The aim of this research is to explore primary school teacher, SNA, parent/guardian, student and Trainee Educational Psychologist (TEP)/ researcher views on how useful the Assessment for Intervention model is in linking assessment to intervention.

Who is undertaking it?

My name is Carol Slattery and I am currently a second year student studying for a Doctorate in Educational and Child Psychology at Mary Immaculate College, Limerick, under the supervision of Dr. Margaret Egan. I am inviting you to take part in my research 'An exploration of the Assessment for Intervention model in an Irish Educational Psychological context' which will form part of my thesis.

\section{Why is it being undertaken?}

This study is being undertaken to explore the views of teachers, SNAs, parents/guardians, students and educational psychologists (EP) on how useful the Assessment for Intervention model is in identifying a child's needs through assessment and making recommendations that are meaningful to teachers, SNAs, parents/guardians and students.

Exactly what is involved for the participant? (time, location, etc.)

Parents/guardians, teachers, SNA and students will be asked to rate Likert scale questions (see sample below) and write additional comments underneath before the assessment and intervention process. Following this, teacher(s), SNA, parent(s)/guardian(s), student and TEP will engage in the five stages of the Assessment for Intervention model which may include classroom observations, consultations and 
individual assessment if required. A separate information sheet on the Assessment Intervention model is attached. The Assessment for Intervention model will be similar to the [school psychological service] model of service delivery (identifying the concern, information gathering and assessment, planning and intervention and review). The time frame for assessment and intervention will depend on the reason for referral. Following the five stage process, parent(s)/guardian(s), teacher(s), SNA and student will rate the same Likert scale questions and can write additional comments in the spaces underneath the questions. Teacher(s), SNA, parent(s)/guardian(s) and student will also be invited to take part in semi-structured interview to explore their views on how useful the Assessment for Intervention model is in linking assessment to intervention. It is expected that the interview will take approximately 30 minutes. All research activities will take place in the school.

What are the benefits of this research?

It is hoped that this study will (a) provide evidence of the application of the AFI model in EP practice in Ireland and, (b) provide findings on how useful the AFI model is in linking assessment to intervention.

Right to withdraw/not answer questions

Your anonymity is assured and you are free to not answer questions or withdraw from the research at any time without giving a reason and without consequence.

\section{How will the information be used/disseminated?}

The scaling questions and interviews will be coded and anonymised so that any individual participants will not be identifiable. The researcher's supervisor, the research coordinator and data analysis supports in Mary Immaculate College may view this data to support the researcher in analysing the data. Anonymised quotes from individual participants may be used in the researcher's thesis or publications arising from the research. Assessment information gathered will not be reported. Descriptor information (e.g. age, sex, nationality and reason for referral) will be reported but details of the case will be kept confidential. The focus of the research is on the researcher reflections and responses of the participants gathered from the scaling questions and semi-structured interviews.

How will confidentiality be ensured?

The anonymity of participants will be protected throughout the research process. No identifiable details will be used, including names of participants, schools and regional locations. Pseudonyms will be applied throughout. Participants are welcome to read over the transcript to ensure anonymity is maintained.

All hard copies (e.g. copies of transcripts, scaling questions, consent forms, researcher's diary) will be kept in a locked filing cabinet in the researcher's home. Soft-data, including anonymised transcriptions will be stored in an encrypted file on the researcher's password protected laptop. Assessment information gathered will be stored and handled according to [the school psychological service] guidelines.

What will happen to the data after research has been completed? All research data will be stored for the duration of the project plus three years. Anonymised research data may be held indefinitely or as required by the researcher. 


\section{Contact details}

If at any time you have any queries / issues with regard to this study, my contact details are as follows:

Carol Slattery (Researcher)

E-mail:00656003@micstudent.mic.ul.ie

Phone: 0871642443

If you have concerns about this study you may contact:

Dr Margaret Egan (Research Supervisor)

Mary Immaculate College

South Circular Road

Limerick

Email: margaret.egan@mic.ul.ie

Phone:061204337

Dr Therese Brophy (DECPsy Research Co-ordinator)

Mary Immaculate College

South Circular Road

Limerick

Email: therese.brophy@mic.ul.ie

Phone: 061774767

Thank you for taking the time to read this information letter. 
The Five stages of the AFI-model (Adapted from Pameijer, 2016, 2017)

\begin{tabular}{|c|c|}
\hline Stage & Description \\
\hline $\begin{array}{l}\text { 1. Intake, how can } \\
\text { we collaborate? }\end{array}$ & $\begin{array}{l}\text { The first goal is to develop a relationship with the teacher(s), } \\
\text { student and parent(s)/guardian(s) and collect information for } \\
\text { making a plan. The educational psychologist will meet with the } \\
\text { student and then parent(s)/guardian(s) and teacher(s). She will } \\
\text { then meet with parent(s)/guardian(s), teacher(s) and student. In } \\
\text { these meetings the questions, aims, expectations and requests of } \\
\text { everyone involved are discussed. This information allows the } \\
\text { educational psychologist to create a strategy that reflects } \\
\text { everyone's personal theory and needs. }\end{array}$ \\
\hline $\begin{array}{l}\text { 2. Strategy, how to } \\
\text { proceed in this } \\
\text { particular case? }\end{array}$ & $\begin{array}{l}\text { The information gathered in stage } 1 \text { is used to create a strategy } \\
\text { that best fits the specific situation. If there is enough } \\
\text { information gathered, the process moves to stage } 4 \text {. If more } \\
\text { information is needed, the educational psychologist engages in } \\
\text { further investigation (stage } 3 \text { ). No investigation will be carried } \\
\text { out unless its outcomes will influence the choice of the } \\
\text { intervention recommendation. This way, collecting data is goal- } \\
\text { directed and directly linked to intervention. If stage } 3 \text { is needed, } \\
\text { questions for investigation are created by the educational } \\
\text { psychologist. }\end{array}$ \\
\hline $\begin{array}{l}\text { 3: Investigation, } \\
\text { answering the } \\
\text { selected answers }\end{array}$ & $\begin{array}{l}\text { This stage involves a goal-directed rather than a routine } \\
\text { collection of data. The questions created in stage } 2 \text { determine } \\
\text { the information to be gathered. This stage can vary in each case, } \\
\text { ranging from using one assessment tool to several different } \\
\text { tools. }\end{array}$ \\
\hline $\begin{array}{l}\text { 4: Integration, } \\
\text { goals and needs }\end{array}$ & $\begin{array}{l}\text { The information gathered in stage } 1,2 \text { and } 3 \text { is put together and } \\
\text { is translated into goals for the student, teaching strategies and } \\
\text { parental support, educational needs of the student and support } \\
\text { needs of his teacher(s) and/or parent(s)/guardian(s). Intervention } \\
\text { recommendations that support goals and needs are created. }\end{array}$ \\
\hline $\begin{array}{l}\text { 5: } \\
\text { Recommendations, } \\
\text { appointments } \\
\text { and feedback }\end{array}$ & $\begin{array}{l}\text { In this stage the parent(s)/guardian(s), teacher(s) and student are } \\
\text { informed about the outcomes of the assessment. By providing } \\
\text { them with clear and meaningful information, they can choose } \\
\text { for themselves which intervention option is both desirable and } \\
\text { achievable. The educational psychologist therefore asks if those } \\
\text { involved are willing and able to 'start tomorrow'. If the answer } \\
\text { is yes, the student, teacher(s) and parent(s)/guardian(s) are } \\
\text { encouraged to start the intervention. If this is not yet the case, } \\
\text { the assessment process continues with further consultation. }\end{array}$ \\
\hline
\end{tabular}


Examples of Likert Scale Questions

Please rate the following items on a scale of 1-10, where 1 is strongly disagree, 5 is neither agree or disagree and 10 is strongly agree. Please use the lines below for any additional comments.

1. I understand my child's situation

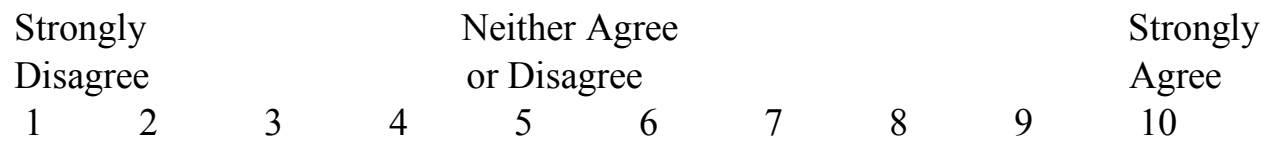

2. I know what support my child needs at home

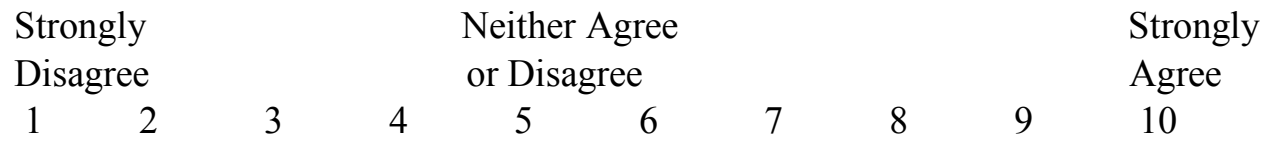




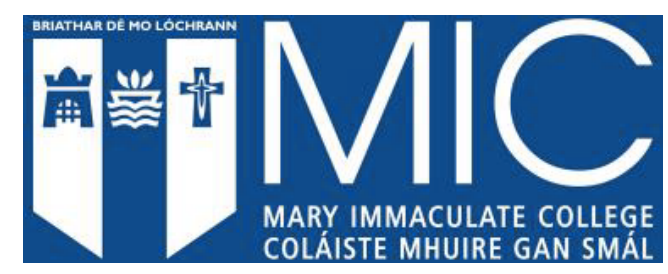

'An exploration of the Assessment for Intervention model in an Irish Educational Psychological context'

\section{Parent/Guardian Informed Consent Form}

\section{Dear Parent(s)/Guardian(s),}

As outlined in the parent/guardian information sheet, the current study will explore primary school teacher, SNA, parent/guardian, student and Trainee Educational Psychologist (TEP)/researcher views on how useful the Assessment for Intervention model is in linking assessment to intervention.

The information sheet outlines what will be involved in this research project. This should be read fully and carefully before consenting to take part in the research project.

Your anonymity is assured and you are free to withdraw from the research at any time. All information gathered will remain confidential and will not be released to any third party. The researcher's supervisor, the research coordinator and data analysis supports in Mary Immaculate College will have access to this data. Anonymised quotes from individual participants may be used in the researcher's thesis or publications arising from the research. In accordance with the MIC Record Retention Schedule all participant data will be stored for the duration of the project plus three years at which time it will be destroyed. Anonymised research data may be held indefinitely or as required by the researcher.

Please read the following statements before signing the consent form.

- I have read and understood the participant information sheet.

- I understand what the project is about, and what the findings will be used for.

- I am fully aware of all of the procedures involving myself and my child, and of any risks and benefits associated with the study.

- I know that my participation and my child's participation is voluntary and that I/he/she can withdraw from the project at any stage without giving any reason.

- I am aware that assessment information will be kept confidential and responses to scaling questions and interviews will be anonymised. 
- I am aware that anonymised responses may be used in the researcher's thesis or publications arising from the research.

Name of Parent/Guardian 1 (Printed):

Name of Parent/Guardian 1 (Signature):

Date:

Name of Parent/Guardian 2 (Printed):

Name of Parent/Guardian 2 (Signature):

Date: 


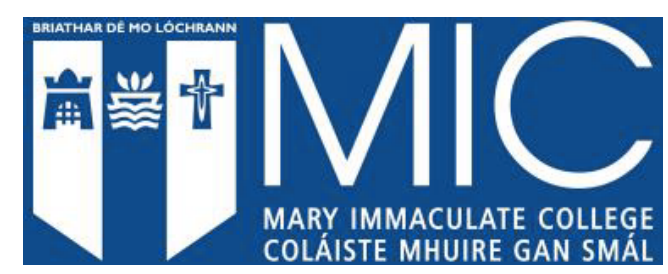

'Assessment for Intervention'

Student Information Sheet

My name is Carol Slattery. I am a Trainee Educational Psychologist in Mary Immaculate College in Limerick. Educational Psychologists work with teachers, parents/guardians and children to try to support children's strengths and needs. The pictures below show the areas where we can help.

As part of my college work, I am working with parents/guardians, teachers and children in finding out about children's strengths and needs and making helpful recommendations. If you would like, I can work with you, your teacher(s) and parent(s)/guardian(s) to find out about your strengths and needs. First, I will ask you to answer some questions with smiley faces. Then we will talk, do some tasks and make goals for an area where you need help. A goal is something that you want to do or get better at. I will also observe for a little while in your classroom. After we have come up with some helpful recommendations for achieving your goals, I will ask you to answer the same questions at the start with smiley faces. I will also ask you some other questions about what you liked and didn't like about working together and if we were able to help you make useful goals. I will be using a voice recorder to record your answers to these questions and I can show you how this works before we start. There are no right or wrong answers to these questions and if you don't want to answer a question, or want to stop at any time, that is 
okay. You can draw pictures for your answers too if you like. My research supervisors and people who work at the college to help college students with their work will look at the answers but they will not know your name. I will use the answers to write about how educational psychologists support children's strengths and needs at school in my 'thesis' (like a really long essay) for people to read about. 


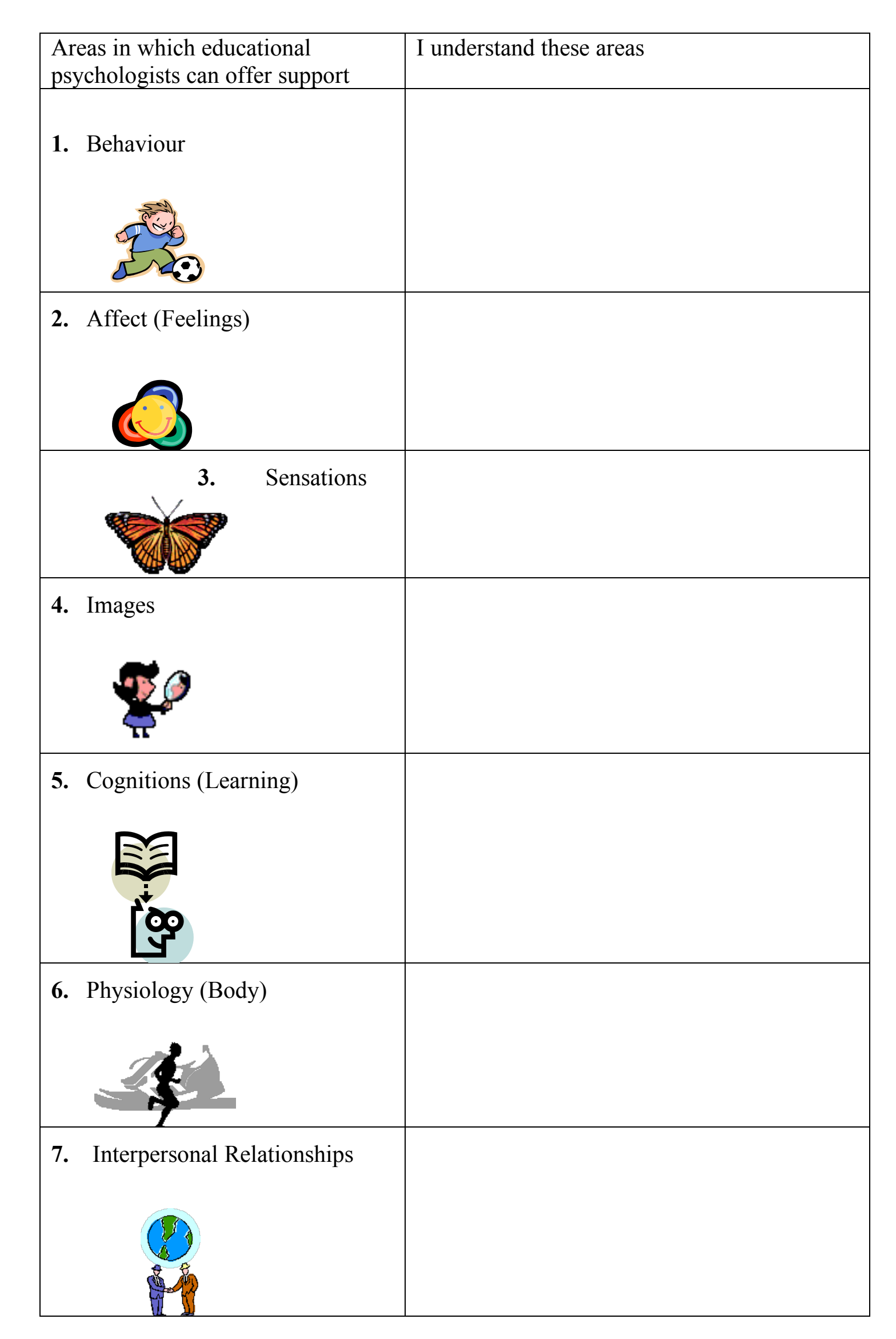




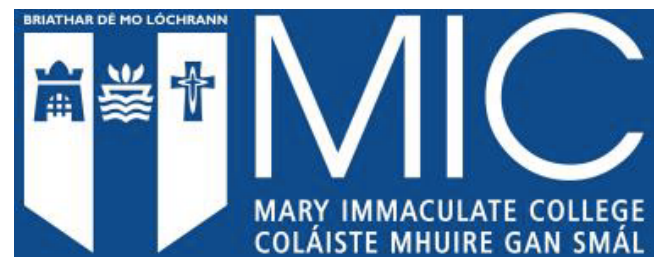

'Assessment for Intervention'

Student Informed Assent Form

My name is

I am going to work with Carol, my parents(s)/guardian(s) and teacher(s) to find out about my strengths and needs. I may have to complete some tasks and answer some questions.

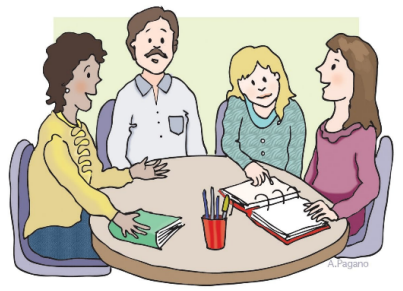

I know that I can draw pictures to answer questions

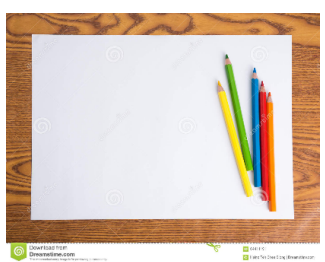

and don't have to answer questions if I don't want to.

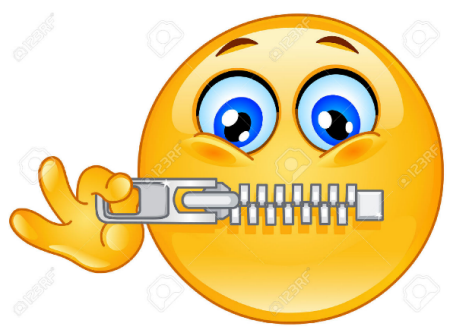


I know that whenever I feel like stopping that's okay, I won't get in trouble and I don't have to say why I feel like stopping.

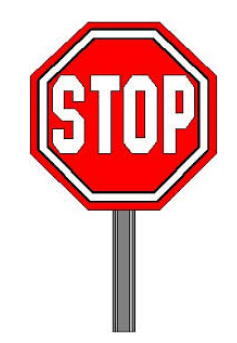

I know that my answers to questions might be used in Carol's thesis for people to read about, but my name will not be used.

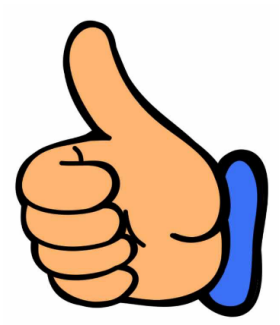

Name of Student (Printed):

Name of Student (Signature):

Date: 
Appendix 26 Procedures for Ethical Issues During the Research Process

In the situation where a student got upset, the researcher would adhere to the following procedure:

- Provide reassurance that they do not have to answer any question that they do not wish to and that it is absolutely fine to stop at any time.

- Exercise professional judgement to discontinue a line of questioning or stop the interview if a child appeared distressed.

- Inform parents and teacher and offer a debrief afterwards.

In the situation where the researcher found out the child required additional/alternative support, the researcher would adhere to the new Children First guidelines (DCYA, 2011) and the following procedure:

- The researcher would discuss the concern with her placement supervisor and determine the appropriate supports to be offered.

- The researcher would discuss the additional/alternative supports with the child's parents, with the support of the TEP's placement supervisor if necessary.

- Any referral forms or contact with other services would be completed by the researcher, under the supervision of her placement supervisor and with the consent of the child's parents.

- The researcher would follow up on any referrals or contact with other services, to ensure continuity of care for the child.

In the situation where concerns presented around Child Protection, the researcher would adhere to the following procedure:

- The researcher would discuss the concern with her placement supervisor.

- The researcher/ placement supervisor would contact the local TUSLA social work duty service in the area where the child lives to discuss the query in general and to decide whether a formal report of the concern to Tusla is appropriate at this stage.

- If the concern was below the threshold for reporting, the researcher would follow Tusla's advice in terms of keeping an eye on the child and exploring other services that may be suitable to meeting the needs of the child and/or family. 
This section outlines the analysis of findings for each case proposition in the Pilot Case Study. The analysis for each proposition is divided into four sections. Section 1 provides a table outlining the criteria for interpreting the findings, also documented in Section 3.5.5 in Chapter 3. Section 2 provides the analysis of findings for each participant in accordance with the proposed criteria, in tabular format. The Changes in ratings on Likert scale statements before and after engaging with the AFI model are outlined. Pattern-matching logic is applied to responses to interview questions addressing each of the Propositions. The interview questions and codes applied to responses are outlined in Table 1 below. Themes and subthemes relevant to each proposition are also outlined and Section 3 provides a table of key quotes for each participant Appendix 45 presents the thematic maps. Appendix 46 outlines the initial and final category clusters and codes applied to interview responses and Appendix 47 provides a sample of the coding applied to a participant's interview transcript. Appendix 48 outlines the process of devising all themes and subthemes. Section 4 outlines a summary of findings for each proposition and Section 5 provides a conceptual analysis, in accordance with AFI principles, EST and SCT concepts. 
Table 1 Pattern-Matching Codes Applied to Interview Responses

\begin{tabular}{|c|c|c|}
\hline Proposition & Interview Questions Addressing Proposition & Pattern-Matching Codes Applied \\
\hline $\begin{array}{l}\text { 1: 'Parents, teachers and TEP will have a better } \\
\text { understanding of the child's situation, as a } \\
\text { result of interactions and activities that have } \\
\text { occurred at the meso and microsystem levels' }\end{array}$ & $\begin{array}{l}\text { 1. Do you feel your understanding of John's } \\
\text { situation has changed? } \\
\text { 2. Can you describe your experience of working } \\
\text { with the Assessment for Intervention model? }\end{array}$ & $\begin{array}{l}\text { 'understanding' } \\
\text { 'interactions and activities during the AFI } \\
\text { process' }\end{array}$ \\
\hline $\begin{array}{l}\text { 2: 'Parents, teachers and TEP will know what } \\
\text { intervention supports the child needs at home } \\
\text { and at school, as a result of interactions and } \\
\text { activities that have occurred at the meso and } \\
\text { microsystem levels' }\end{array}$ & $\begin{array}{l}\text { 1. Do you feel you can support John's needs at } \\
\text { home/school? } \\
\text { 2. Did the intervention recommendations address } \\
\text { your questions at the Intake stage? }\end{array}$ & $\begin{array}{l}\text { 'knowledge of intervention' } \\
\text { 'interactions and activities during the AFI } \\
\text { process' }\end{array}$ \\
\hline $\begin{array}{l}\text { 3: 'Teachers and parents will have greater } \\
\text { perceived competence in supporting the child's } \\
\text { needs at home and at school, as a result of } \\
\text { interactions and activities that have occurred at } \\
\text { the meso and microsystem levels' }\end{array}$ & $\begin{array}{l}\text { 1. Do you feel you can support John's needs at } \\
\text { home/school? }\end{array}$ & $\begin{array}{l}\text { 'competence' } \\
\text { 'interactions and activities during the AFI } \\
\text { process' }\end{array}$ \\
\hline $\begin{array}{l}\text { 4: 'The child will have a better understanding } \\
\text { of their strengths and needs and what to do to } \\
\text { improve their needs, as a result of interactions } \\
\text { and activities that have occurred at the meso } \\
\text { and microsystem levels' }\end{array}$ & $\begin{array}{l}\text { 1. What did you like about working with me, } \\
\text { your teacher and mom? } \\
\text { 2. Did working together help you to find out } \\
\text { about your strengths? } \\
\text { 3. Did working together help you to find out } \\
\text { about what you need help with? } \\
\text { 4. Do you know what you need to do to get } \\
\text { better at what you need help with? }\end{array}$ & $\begin{array}{l}\text { 'understanding strengths' } \\
\text { 'understanding needs' } \\
\text { 'improve on needs' } \\
\text { 'interactions and activities during the AFI } \\
\text { process' }\end{array}$ \\
\hline $\begin{array}{l}\text { 5: 'At a time of ecological transition, teachers } \\
\text { will feel competent in their ability to work } \\
\text { according to the aims of Circular } 0013 / 2017 \text {, in } \\
\text { meeting and monitoring the needs of the child' }\end{array}$ & $\begin{array}{l}\text { 1. Do you feel you will be able to meet and } \\
\text { monitor John's needs moving forward? }\end{array}$ & $\begin{array}{l}\text { 'competence to meet/monitor needs' } \\
\text { 'interactions and activities during the AFI } \\
\text { process' }\end{array}$ \\
\hline
\end{tabular}


Appendix 28 Pilot Case Analysis of Findings: Proposition 1

The proceeding sections provide a comprehensive analysis of Proposition 1 findings for the Pilot Case. Section 1 below outlines the criteria for interpreting the findings, also presented in in Section 3.5.5 in Chapter 3. Section 2 presents the analysis of findings. Section 3 outlines the themes relevant to Proposition 1 and Section 4 provides a summary of findings. Section 5 provides a conceptual analysis of Proposition 1 findings.

Section 1: Criteria for Interpreting Proposition 1 Findings (Pilot Case)

\begin{tabular}{|c|c|c|c|c|}
\hline $\begin{array}{l}\text { Proposition: } \\
\text { Following } \\
\text { Engagement } \\
\text { with the AFI } \\
\text { Model: }\end{array}$ & $\begin{array}{l}\text { Likert Scale } \\
\text { Statements }\end{array}$ & $\begin{array}{l}\text { Likert Scale } \\
\text { Statements } \\
\text { (Comments } \\
\text { Section) }\end{array}$ & $\begin{array}{l}\text { Semi-structured } \\
\text { Interviews }\end{array}$ & $\begin{array}{l}\text { Researcher } \\
\text { Diary }\end{array}$ \\
\hline $\begin{array}{l}\text { Proposition } \\
\text { 1: 'Parents, } \\
\text { teachers and } \\
\text { TEP will } \\
\text { have a better } \\
\text { understanding } \\
\text { of the child's } \\
\text { situation, as a } \\
\text { result of } \\
\text { interactions } \\
\text { and activities } \\
\text { that have } \\
\text { occurred at } \\
\text { the meso and } \\
\text { microsystem } \\
\text { levels' }\end{array}$ & $\begin{array}{l}\text { A change in } \\
\text { rating on } \\
\text { associated } \\
\text { Likert scale } \\
\text { statements } \\
\text { indicates } \\
\text { change in } \\
\text { understanding } \\
\text { of the child's } \\
\text { situation } \\
\text { following } \\
\text { engagement } \\
\text { with the AFI } \\
\text { model and as } \\
\text { a result of } \\
\text { interactions } \\
\text { and activities } \\
\text { that have } \\
\text { occurred at } \\
\text { the meso and } \\
\text { microsystem } \\
\text { levels. }\end{array}$ & $\begin{array}{l}\text { Pattern- } \\
\text { matching: } \\
\text { Comments } \\
\text { that link } \\
\text { understanding } \\
\text { of the child's } \\
\text { situation, } \\
\text { engagement } \\
\text { with the AFI } \\
\text { model and } \\
\text { interactions } \\
\text { and activities } \\
\text { that have } \\
\text { occurred at } \\
\text { the meso and } \\
\text { microsystem } \\
\text { levels and } \\
\text { vice versa. }\end{array}$ & $\begin{array}{l}\text { Pattern- } \\
\text { matching: } \\
\text { Interview } \\
\text { responses that link } \\
\text { understanding of } \\
\text { the child's } \\
\text { situation, } \\
\text { engagement with } \\
\text { the AFI model and } \\
\text { interactions and } \\
\text { activities that have } \\
\text { occurred at the } \\
\text { meso and } \\
\text { microsystem levels } \\
\text { and vice versa. } \\
\text { Thematic } \\
\text { analysis: Themes } \\
\text { and patterns within } \\
\text { the data set that } \\
\text { refer to } \\
\text { understanding of } \\
\text { the child's } \\
\text { situation and } \\
\text { interactions and } \\
\text { activities that have } \\
\text { occurred at the } \\
\text { meso and } \\
\text { microsystem levels } \\
\text { were included in } \\
\text { the analysis of the } \\
\text { AFI model. }\end{array}$ & $\begin{array}{l}\text { Excerpts from } \\
\text { the researcher } \\
\text { diary that } \\
\text { referred to } \\
\text { understanding } \\
\text { of the child's } \\
\text { situation and } \\
\text { interactions } \\
\text { and activities } \\
\text { that have } \\
\text { occurred at the } \\
\text { meso and } \\
\text { microsystem } \\
\text { levels were } \\
\text { included in the } \\
\text { analysis of the } \\
\text { AFI model. }\end{array}$ \\
\hline
\end{tabular}


Section 2: Analysis of Proposition 1 Findings (Pilot Case)

\begin{tabular}{|c|c|c|c|c|}
\hline $\begin{array}{l}\text { Proposition1 } \\
\text { Evidence } \\
\text { (Pilot Case) }\end{array}$ & $\begin{array}{l}\text { Likert } \\
\text { Scale } \\
\text { Statements }\end{array}$ & $\begin{array}{l}\text { Likert Scale } \\
\text { Statements } \\
\text { (Comments) }\end{array}$ & Semi-structured Interviews & Researcher Diary \\
\hline $\begin{array}{l}\text { Parent } \\
\text { (Louise) }\end{array}$ & $\begin{array}{l}\text { I } \\
\text { understand } \\
\text { my child's } \\
\text { situation: } \\
\text { Before } \\
\text { AFI: } 8 \\
\text { After AFI: } \\
8\end{array}$ & $\begin{array}{l}\text { N/A (See } \\
\text { section } \\
3.11 .2)\end{array}$ & $\begin{array}{l}\text { Pattern-matching: Do you feel your understanding of John's } \\
\text { situation has changed? Yeah, definitely because I probably } \\
\text { feel a bit more restrictive about feeding into it } \\
\text { (understanding) (line 41) } \\
\text { Can you describe your experience of working with the } \\
\text { Assessment for Intervention model? ...it identified and kind } \\
\text { of had a team approach to it where it involved the teacher and } \\
\text { information taking (interactions and activities during the } \\
\text { AFI process) (lines 4-5) } \\
\text { Thematic analysis (See Section } 3 \text { below for key quotes of } \\
\text { themes and subthemes relevant to Proposition 1): } \\
\text { A team approach to assessment } \\
\text {-Active roles and communication } \\
\text {-Insight into strengths, needs and perspectives } \\
\text { Views and experiences of AFI } \\
\text {-A clear and structured process } \\
\text {-A framework to facilitate teamwork and positive outcomes }\end{array}$ & $\begin{array}{l}\text { Researcher diary (AFI templates) } \\
\text { documented specific activities and } \\
\text { interactions that took place during the } \\
\text { five-stage AFI process (See Section } \\
\text { 4.2, Table 4.1) }\end{array}$ \\
\hline $\begin{array}{l}\text { Class teacher } \\
\text { (Grace) }\end{array}$ & $\begin{array}{l}\text { I } \\
\text { understand } \\
\text { the } \\
\text { student's } \\
\text { situation }\end{array}$ & $\begin{array}{l}\text { N/A (See } \\
\text { section } \\
3.11 .2)\end{array}$ & $\begin{array}{l}\text { Pattern-matching: Do you feel your understanding of John's } \\
\text { situation has changed? I definitely do (understanding) (line } \\
\text { 70). The only information that I had before was that I had } \\
\text { only met mum once, am before the intervention and... I didn't } \\
\text { get a full picture of what it was like at home, there definitely } \\
\text { was a barrier (lines } 70-72 \text { ).... and it was lovely to actually just }\end{array}$ & $\begin{array}{l}\text { Researcher diary (AFI templates) } \\
\text { documented specific activities and } \\
\text { interactions that took place during the } \\
\text { five-stage AFI process (See Section } \\
\text { 4.2, Table 4.1) }\end{array}$ \\
\hline
\end{tabular}


Before

AFI: 7

After AFI:

8

to meet her in this situation and to be able to hear her voice

am (interactions and activities during the AFI process)...I

think to understand that was a big part of it that there was this kind of opening up of communication between home and school (interactions and activities during the AFI process) and then I suppose hearing from John's own perspective then from chatting to you (interactions and activities during the AFI process) (lines 74-77)...Am so I really think, I think I do understand the situation a bit more (understanding) (line 83)

Can you describe your experience of working with the Assessment for Intervention model? Yeah am, I found it quite a positive experience. Am, I think having the parents and children involved really actually... gave another dimension to the whole intervention process that it's not just me talking about school (interactions and activities during the AFI process, that I actually saw what was happening at home and that kind of reassured that behaviours that I had noticed were actually happening at home as well (interactions and activities during the AFI process), that it wasn't just schoolbased (lines 3-7)

Thematic analysis (See Section 3 below for key quotes of themes and subthemes relevant to Proposition 1):

A team approach to assessment

-Active roles and communication

-Insight into strengths, needs and perspectives

Views and experiences of AFI

-A clear and structured process

-A framework to facilitate teamwork and positive outcomes 


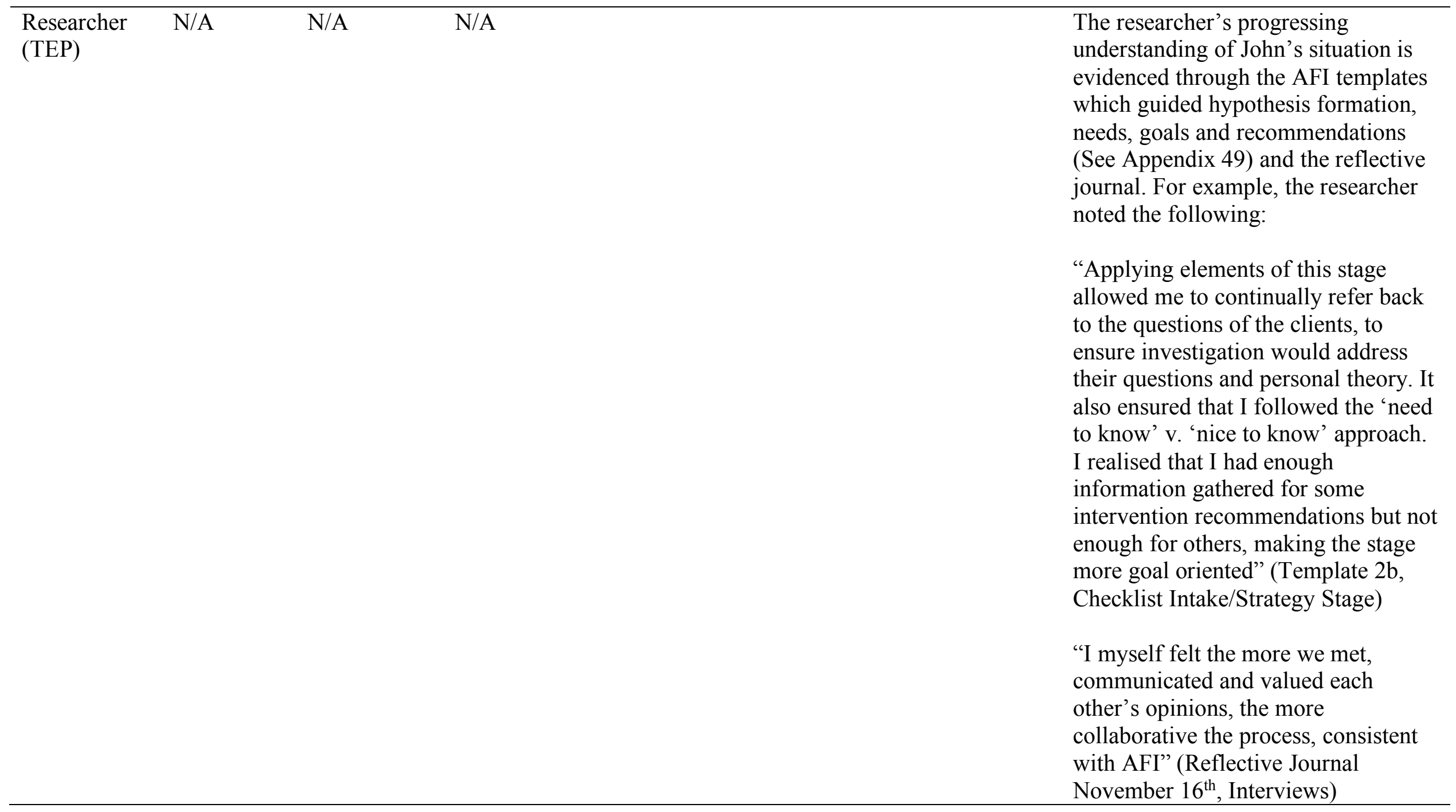




\begin{tabular}{ll}
\hline Theme 1: A & Team Approach to Assessment \\
\hline Subtheme & Key Quotes \\
Active roles & $\begin{array}{l}\text { When describing the roles of participants, Grace (class teacher) } \\
\text { and }\end{array}$ \\
$\begin{array}{ll}\text { commented: Am, I saw kind of your role as facilitator, that the } \\
\text { ation }\end{array}$ & $\begin{array}{l}\text { questions you asked kind of guided us in a way that got us thinking } \\
\text { about things (lines 26-27) }\end{array}$
\end{tabular}

In describing her experience of the AFI process, Grace stated: I think having the parents and children involved... gave another dimension to the whole intervention process that it's not just me talking about school (lines 3-5)

When describing the roles of participants, Louise (parent) noted: I suppose your role [TEP] fundamentally was kind of, teaching us the model of assessment, how to implement it in the home environment and in the school environment (lines 15-17). With regard to the roles of participant Louise also commented: You needed collectively engagement from the parents, the teachers and from the child (lines 1718)

Insight into When describing her understanding of John's situation, Louise strengths, (parent) commented: Like you have understanding and insight into his needs and need for the obsessional interest that he has but I think, I suppose you perspectives have...greater understanding as to why he does participate in these activities (lines 48-50)

Grace (class teacher) also commented on her understanding: I can see his side, I can see the parent's side and I think they see my side of it as well... that we're all just, I think we're just more aware, I think would be the word I would use (lines 84-85)

Louise described the benefits of the AFI model: Sometimes you might feel there's certain problems in the home environment and they might be different to the problems that are going on inside the school (lines 108-109)

\begin{tabular}{ll}
\hline Theme 4: Views and Experiences of AFI \\
\hline $\begin{array}{l}\text { Subtheme } \\
\text { A clear and } \\
\text { structured } \\
\text { process }\end{array}$ & $\begin{array}{l}\text { Key Quotes } \\
\text { the five steps were very beneficial and it was a very clear process to } \\
\text { follow (line 267) }\end{array}$ \\
& $\begin{array}{l}\text { Louise described how the process addressed her questions at the intake } \\
\text { stage: I think it would have been very difficult not to be able to } \\
\text { identify what the problem was because most of the questions were kind } \\
\text { of, I won't say bullet points but they were kind of descriptive type } \\
\text { questions, identifying what the problems was (lines 61-63) }\end{array}$ \\
$\begin{array}{l}\text { Framework } \\
\text { to facilitate } \\
\text { teamwork }\end{array}$ & $\begin{array}{l}\text { Grace commented on what she had learned from the AFI process: Like } \\
\text { parent teacher meeting and beyond that it's maybe a phone call or the } \\
\text { odd meeting so I think having that channel of communication is }\end{array}$ \\
\hline
\end{tabular}


and positive definitely important....and that's a huge part of the model as well, outcomes having that communication so I think that was really beneficial (lines 243-246)

When discussing the benefits of the AFI model, Louise stated: Am, I suppose the benefits was it [AFI model] was kind of a team approach (line 107)

\section{Section 4: Summary of Proposition 1 Findings (Pilot Case)}

Likert scale ratings, provided by the class teacher indicate greater understanding of John's situation after engaging with the AFI model. Ratings, provided by the parent proof understanding remained unchanged, however pattern-matching logic applied to interview responses suggest a change occurred. Patterns within parent and teacher interview responses connected understanding of the situation to interactions and activities that occurred during the AFI process, at the meso and microsystem level (e.g. engaging in a team assessment approach, participating in meetings and hearing each other's voices throughout the process) (See Section 2 above). These activities and interactions are considered consistent with the AFI process. In accordance with AFI Principle 6, participants were active in their engagement and included in the process as 'co-assessors'. Additionally and consistent with AFI principle 7, the researcher adhered to the systematic and transparent assessment process of AFI and the researcher's progressing understanding of John's situation was evidenced through the AFI templates. The templates guided hypothesis formation, needs, goals and recommendations during the five stages (See Appendix 49). Reflections in the researcher's journal also documented a communicative, collaborative process facilitated by AFI (See Section 2 above). Themes within the data set that referred to understanding of John's situation and interactions and activities that occurred at the meso and microsystem levels included 'A team approach to assessment' and 'Views and Experiences of AFI' (See Section 3 above). Section 5 below provides a conceptual analysis of the findings and themes, in accordance with AFI principles, EST and SCT concepts. 


\section{Theme/ AFI Principles (See section 2.16 for description of AFI} Subtheme Principles)

Active roles Patterns within parent and teacher interview responses linked and communic- occurred during the AFI process, at the meso and microsystem understanding of the situation to interactions and activities that ation level (e.g. engaging in a team assessment approach, participating in meetings and hearing each other's voices throughout the process). Reflections in the researcher's journal also documented a communicative, collaborative process facilitated by AFI. Thematic analysis of interview responses in the data set indicates the subtheme 'Active roles and communication' was a factor that contributed to understanding of the situation (see key quotes in Section 3). Engaging in a team approach to understand John's situation, as evidenced through pattern-matching, thematic analysis and researcher reflections, resonates with AFI Principle 6: Working together in a collaborative partnership to search for explanations to the situation.
EST Concepts (See section SCT Concepts (See 2.14 for concept definitions) section 2.15 for concept definitions)

At the mesosystem level, active participation and communication between home, school and school psychological service may have served to strengthen the developmental potential of these settings to identify and understand John's needs. At the microsystem level, inclusion of John in the process may have encouraged strengthening of dyadic relations (Bronfenbrenner, 1979).
Thematic analysis evidenced that the language used, including the descriptive type AFI questions, assisted the coconstruction of knowledge around the situation

between the team (Vygotsky, 1981). See Section 3 above. Tools and signs, including the interest inventory and pie chart (See Section 4.2, Table 4.1) facilitated John's active engagement in the process, and helped to develop understanding of the situation (Palinscar, 1998; Vygotsky, 1978)
Insight into Patterns within parent and teacher interview responses linked strengths, needs and perspectives occurred during the AFI process, at the meso and microsystem level. Thematic analysis of interview responses in the data set understanding of the situation to interactions and activities that indicates the subtheme 'Insight into strengths, needs and perspectives' was a factor that contributed to understanding of
Sharing information on the situation at home and at school provided additional insight into how events may be 'experienced' by John in 
the situation (See key quotes in Section 3 This subtheme

resonates with AFI Principle 2: 'Applying a Transactional Perspective to Child Development' as the parent and teacher gained insight into the impact of home and school environments on the presenting situation.

Views and experiences of AFI theme in the dataset and provides further explanation of how participants' understanding of Jim's situation improved
Thematic analysis identified 'Views and experiences of AFI' as a following engagement in the AFI process. Grace (teacher) and Louise (parent) considered AFI a framework to facilitate teamwork, consistent with AFI Principle 6. For example Grace commented that "communication" was a "huge part of the model" and Louise referred to the model's "team approach". Grace commented on the AFI model's "very clear process" and Louise noted that the "descriptive type questions", obtained from AFI procedural guidelines (Pameijer, 2016), helped to identify "what the problem was". A clear and structured assessment process resonates with AFI Principle 7: A systematic and transparent stage-like process. Comments made by the parent and teacher (See Section 3 above) indicate their awareness of working through the stages of the AFI process and the researcher adhered to the systematic and transparent assessment process with the use of the AFI templates. these environments

(Bronfenbrenner, 1979).

Thematic analysis suggests the AFI model provided a framework to facilitate teamwork and positive outcomes with (See Section 3 above). At the mesosystem level, active participation and communication between home, school and school psychological service may have served to strengthen the developmental potential of these settings to identify and understand John's needs. At the microsystem level, inclusion of John in the process may have encouraged strengthening of dyadic relations (Bronfenbrenner, 1979) 
Appendix 29 Pilot Case Analysis of Findings: Proposition 2

The proceeding sections provide a comprehensive analysis of Proposition 2 findings for the Pilot Case. Section 1 below outlines the criteria for interpreting the findings, also presented in in Section 3.5.5 in Chapter 3. Section 2 presents the analysis of findings. Section 3 outlines the themes relevant to Proposition 2 and Section 4 provides a summary of findings. Section 5 provides a conceptual analysis of Proposition 2 findings.

Section 1: Criteria for Interpreting Proposition 2 Findings (Pilot Case)

\begin{tabular}{|c|c|c|c|c|}
\hline $\begin{array}{l}\text { Proposition: } \\
\text { Following } \\
\text { Engagement } \\
\text { with the } \\
\text { AFI Model: }\end{array}$ & $\begin{array}{l}\text { Likert Scale } \\
\text { Statements }\end{array}$ & $\begin{array}{l}\text { Likert Scale } \\
\text { Statements } \\
\text { (Comments } \\
\text { Section) }\end{array}$ & $\begin{array}{l}\text { Semi-structured } \\
\text { Interviews }\end{array}$ & $\begin{array}{l}\text { Researcher } \\
\text { Diary }\end{array}$ \\
\hline $\begin{array}{l}\text { Proposition } \\
\text { 2: 'Parents, } \\
\text { teachers and } \\
\text { TEP will } \\
\text { know what } \\
\text { intervention } \\
\text { supports the } \\
\text { child needs } \\
\text { at home and } \\
\text { at school, as } \\
\text { a result of } \\
\text { interactions } \\
\text { and activities } \\
\text { that have } \\
\text { occurred at } \\
\text { the meso and } \\
\text { microsystem } \\
\text { levels' }\end{array}$ & $\begin{array}{l}\text { A change in } \\
\text { rating on } \\
\text { associated } \\
\text { Likert scale } \\
\text { statements } \\
\text { indicates } \\
\text { change in } \\
\text { knowledge of } \\
\text { what } \\
\text { intervention } \\
\text { supports the } \\
\text { child needs } \\
\text { following } \\
\text { engagement } \\
\text { with the AFI } \\
\text { model and as } \\
\text { a result of } \\
\text { interactions } \\
\text { and activities } \\
\text { that have } \\
\text { occurred at } \\
\text { the meso and } \\
\text { microsystem } \\
\text { levels. }\end{array}$ & $\begin{array}{l}\text { Pattern- } \\
\text { matching: } \\
\text { Comments } \\
\text { that link } \\
\text { knowledge } \\
\text { of } \\
\text { intervention } \\
\text { supports the } \\
\text { child needs, } \\
\text { engagement } \\
\text { with the AFI } \\
\text { model and } \\
\text { interactions } \\
\text { and activities } \\
\text { that have } \\
\text { occurred at } \\
\text { the meso and } \\
\text { microsystem } \\
\text { levels and } \\
\text { vice versa. }\end{array}$ & $\begin{array}{l}\text { Pattern-matching: } \\
\text { Interview responses } \\
\text { that link knowledge } \\
\text { of intervention } \\
\text { supports the child } \\
\text { needs, engagement } \\
\text { with the AFI model } \\
\text { and interactions and } \\
\text { activities that have } \\
\text { occurred at the } \\
\text { meso and } \\
\text { microsystem levels } \\
\text { and vice versa. } \\
\text { Thematic analysis: } \\
\text { Themes and } \\
\text { patterns within the } \\
\text { data set that refer to } \\
\text { knowledge of } \\
\text { intervention } \\
\text { supports the child } \\
\text { needs and } \\
\text { interactions and } \\
\text { activities that have } \\
\text { occurred at the } \\
\text { meso and } \\
\text { microsystem levels } \\
\text { were included in the } \\
\text { analysis of the AFI } \\
\text { model. }\end{array}$ & $\begin{array}{l}\text { Excerpts from } \\
\text { the researcher } \\
\text { diary that } \\
\text { referred to } \\
\text { knowledge of } \\
\text { intervention } \\
\text { supports the } \\
\text { child needs } \\
\text { and } \\
\text { interactions } \\
\text { and activities } \\
\text { that have } \\
\text { occurred at the } \\
\text { meso and } \\
\text { microsystem } \\
\text { levels were } \\
\text { included in the } \\
\text { analysis of the } \\
\text { AFI model. }\end{array}$ \\
\hline
\end{tabular}


Section 2: Analysis of Proposition 2 Findings (Pilot Case)

\begin{tabular}{|c|c|c|c|c|}
\hline $\begin{array}{l}\text { Evidence for } \\
\text { Proposition2 } \\
\text { (Pilot Case) } \\
\end{array}$ & $\begin{array}{l}\text { Likert } \\
\text { Scale } \\
\text { Statements } \\
\end{array}$ & $\begin{array}{l}\text { Likert Scale } \\
\text { Statements } \\
\text { (Comments) }\end{array}$ & Semi-structured Interviews & Researcher Diary \\
\hline $\begin{array}{l}\text { Parent } \\
\text { (Louise) }\end{array}$ & $\begin{array}{l}\text { I know } \\
\text { what } \\
\text { supports } \\
\text { my child } \\
\text { needs at } \\
\text { home: } \\
\text { Before AFI: } \\
2 \\
\text { After AFI: } \\
8\end{array}$ & $\begin{array}{l}\text { N/A (See } \\
\text { section } \\
3.11 .2)\end{array}$ & $\begin{array}{l}\text { Pattern-matching: Do you feel you can support John's } \\
\text { needs at home? Well I suppose you're trying to explore other } \\
\text { avenues that you might get him interested in (knowledge of } \\
\text { intervention) (line 65)... I suppose like maybe just a simple } \\
\text { thing that he identified that he likes having a chat } \\
\text { (interactions and activities during the AFI process) so it's } \\
\text { to sit down with him and actually say to him am...Do you } \\
\text { know why we had to put other little activities on his agenda } \\
\text { (knowledge of intervention) (lines 68-70) } \\
\text { Did the intervention recommendations address your } \\
\text { questions at the Intake stage? Am, I do really because they } \\
\text { were broad and kind of collectively tried to outline am... what } \\
\text { the problem was, do you know kind of what the aims and } \\
\text { goals was, what the outcomes, what work needed to be done } \\
\text { (interactions and activities during the AFI process)...I } \\
\text { thought they did yeah I did think... and it was broad-based } \\
\text { you know that sort of way, so I think there wasn't probably } \\
\text { an avenue that... you couldn't have had engaged and } \\
\text { am...engaged and ... outlined what the problem was the way } \\
\text { they were worded, do you know, I think it would have been } \\
\text { very difficult not to be able to identify what the problem was } \\
\text { because most of the questions were kind of, I won't say bullet } \\
\text { points but they were kind of descriptive type questions, } \\
\text { identifying what the problems was (interactions and }\end{array}$ & $\begin{array}{l}\text { Researcher diary (AFI templates) } \\
\text { documented specific activities } \\
\text { and interactions that took place } \\
\text { during the five-stage AFI process } \\
\text { (See Section 4.2, Table 4.1) }\end{array}$ \\
\hline
\end{tabular}


activities during the AFI process) so I do really yeah (lines 56-63)

Thematic analysis (See Section 3 for key quotes of themes and subthemes relevant to Proposition 2):

Factors bridging the gap between assessment and intervention -Shared awareness of strengths, needs and goals identified

-Experiencing or observing intervention effectiveness

$\begin{array}{lll}\text { Class teacher } & \text { I know } & \text { N/A (See } \\ \text { (Grace) } & \text { what } & \text { section } \\ & \text { supports the } & 3.11 .2 \text { ) } \\ & \text { student } & \\ & \text { needs at } \\ & \text { school: } \\ & \text { Before AFI: } \\ & 6 \\ & \text { After AFI: } 8\end{array}$

Pattern-matching: Do you feel you can support John's needs at school?...And having the other strategies like the close proximity, the behaviour specific praise (knowledge of intervention) like they're all things I think are... they're really useful and are useful for any other child in the class as well but for him especially because he does seem to be responding to them (interactions and activities during the AFI process) (lines 142-144)

Did the intervention recommendations address your questions at the Intake stage? Yeah they did am, I know one of mine was how could I support him in school and definitely there's a lot of strategies there like the checklist am...for himself to kinda self-assess am, coming up with a bank of different interests for him to try out throughout the day (knowledge of interventions/interactions and activities during the AFI process), they're all very simple ideas that I probably wouldn't have thought of myself (laughs)

(interactions and activities during the AFI process), but they'll be a huge support in class especially when you work with 26 other kids, that there are things that I can actually implement that won't detract from the others and that can be

Researcher diary (AFI templates) documented specific activities and interactions that took place during the five-stage AFI process (See Section 4.2, Table 4.1) 
easily implemented and that they could all actually even try

(lines 89-95)

Thematic analysis (See Section 3 below for key quotes of themes and subthemes relevant to Proposition 2):

Factors bridging the gap between assessment and intervention

-Shared awareness of strengths, needs and goals identified

-Experiencing or observing intervention effectiveness

Researcher N/A N/A N/A

(TEP)

/A

The researcher's knowledge of intervention supports is evidenced through the AFI templates (researcher diary) which guided hypothesis formation, needs, goals and recommendations (See Appendix 50).

Researcher reflections indicate intervention planning was supported by John sharing his thoughts on engaging in alternative interests with his mother and teacher:

"Helpful meeting in the sense that information was communicated, John's voice was heard on his suggested replacement interests" (Reflective Journal November $7^{\text {th }}$, Integration stage). 


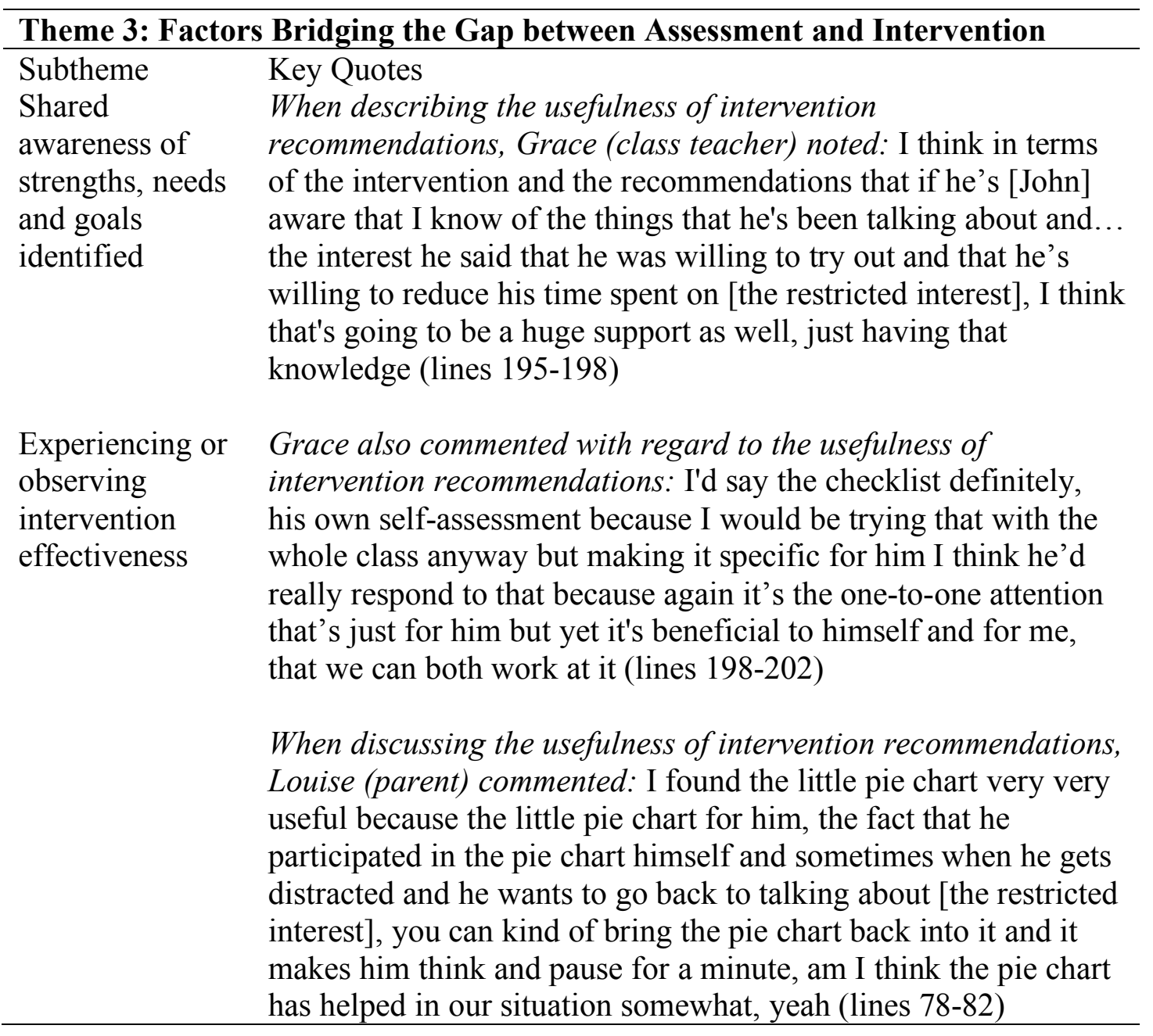

\section{Section 4: Summary of Proposition 2 Findings (Pilot Case)}

Higher Likert scale ratings, following engagement with the AFI model, indicate greater knowledge of intervention supports. Patterns within parent and teacher interview responses linked knowledge of intervention supports to interactions and activities that occurred during the AFI process, at the meso and microsystem levels. Grace (class teacher) observed John's positive response to "the close proximity" and "behaviour specific praise" in the classroom during the Investigation stage and Louise commented that she would "explore other avenues that you might get him interested in", including "having a chat", as this was something that he identified he would like during a joint activity task with the TEP. See Section 4.2, Table 4.1 for a list of the activities and interactions that participants engaged in. The activities and interactions are considered consistent with the AFI process as John, Louise and Grace were 'co-assessors' during the Investigation stage and co-constructed knowledge around intervention supports in this regard (See Section 5 below). Additionally and in accordance with AFI principle 7, the researcher's knowledge of intervention supports was evidenced through the AFI 
templates (researcher diary). The templates guided hypothesis formation, needs, goals and recommendations throughout the AFI process. Researcher reflections indicate intervention planning was supported by John sharing his thoughts on engaging in alternative interests with his mother and teacher, consistent with a collaborative assessment process advocated by AFI (Pameijer, 2017). The theme 'Factors bridging the gap between assessment and intervention' referred to knowledge of intervention supports and interactions and activities that occurred at the meso and microsystem levels (See Section 3 above). Section 5 below provides a conceptual analysis of the findings and themes, in accordance with AFI principles, EST and SCT concepts. 
Section 5: Conceptual Analysis of Proposition 2 Findings (Pilot Case)

\begin{tabular}{|c|c|c|c|}
\hline $\begin{array}{l}\text { Theme/ } \\
\text { Subtheme }\end{array}$ & AFI Principles (See section 2.16 for description of AFI Principles) & $\begin{array}{l}\text { EST Concepts (See } \\
\text { section 2.14) }\end{array}$ & $\begin{array}{l}\text { SCT Concepts } \\
\text { (See section 2.15) }\end{array}$ \\
\hline $\begin{array}{l}\text { Shared } \\
\text { awareness of } \\
\text { strengths, } \\
\text { needs and } \\
\text { goals } \\
\text { identified }\end{array}$ & $\begin{array}{l}\text { Knowledge of intervention supports resulted from sharing information during } \\
\text { the AFI process, as evidenced through pattern-matching applied to interview } \\
\text { responses. For example, Louise's knowledge to 'explore other avenues that you } \\
\text { might get him (John) interested in", resulted from the TEP sharing information } \\
\text { on activities that John had expressed interest in during a joint activity at the } \\
\text { Investigation stage. Thematic analysis (See Section 3) and researcher reflections } \\
\text { (See Section 2) indicate intervention planning was supported by shared } \\
\text { awareness of needs and goals. This is consistent with AFI Principle 6: 'Working } \\
\text { together in a collaborative partnership', as insights and solutions of the parent, } \\
\text { teacher and child were considered just as important as those of the TEP and } \\
\text { information was discussed 'with' team members rather than 'about' or 'to' them } \\
\text { (Pameijer, 2017). }\end{array}$ & $\begin{array}{l}\text { John's awareness of } \\
\text { information being } \\
\text { communicated and } \\
\text { having his voice heard } \\
\text { in meetings suggests a } \\
\text { shifting balance of } \\
\text { power towards him as } \\
\text { the developing person } \\
\text { and encouraged dyadic } \\
\text { relations between } \\
\text { parent, teacher, TEP } \\
\text { and child. }\end{array}$ & $\begin{array}{l}\text { In order for } \\
\text { information to be } \\
\text { shared with John, } \\
\text { Louise and Grace, } \\
\text { appropriate } \\
\text { language use was } \\
\text { considered before, } \\
\text { during and after } \\
\text { interactions, } \\
\text { consistent with } \\
\text { SCT. }\end{array}$ \\
\hline $\begin{array}{l}\text { Experiencing } \\
\text { or observing } \\
\text { intervention } \\
\text { effectiveness }\end{array}$ & $\begin{array}{l}\text { Knowledge of intervention supports resulted from experiencing or observing } \\
\text { intervention effectiveness, as evidenced through pattern-matching. For example, } \\
\text { Grace's knowledge of intervention supports was linked to observing John's } \\
\text { positive response to "close proximity" and "behaviour specific praise" in the } \\
\text { classroom during the Investigation stage. Thematic analysis of interview } \\
\text { responses in the data set indicates experiencing or observing intervention } \\
\text { effectiveness during the Investigation stage was a factor in bridging the gap } \\
\text { between assessment and intervention (See Section 3). This resonates with AFI } \\
\text { Principle } 6 \text { as Grace, Louise and John were considered 'co-assessors' and } \\
\text { engaged in tasks and activities to investigate the effectiveness of intervention } \\
\text { strategies during the Investigation stage. The close proximity and behaviour } \\
\text { specific praise observed to be effective by Grace is also consistent with AFI } \\
\text { Principle } 3 \text { and 4; Focusing on the needs of the teacher, parent and student to } \\
\text { discover what the ideal approach for John might be and to strengthen the } \\
\text { teacher-student relationship (Pameijer, 2017). }\end{array}$ & $\begin{array}{l}\text { Dyadic relations may } \\
\text { be beginning to develop } \\
\text { between Grace (class } \\
\text { teacher) and John } \\
\text { (student) as Grace } \\
\text { started to direct more } \\
\text { focused, positive } \\
\text { attention to John's } \\
\text { activities and } \\
\text { behaviours during the } \\
\text { Investigation stage } \\
\text { (Bronfenbrenner, } \\
\text { 1979). }\end{array}$ & $\begin{array}{l}\text { Tools and signs } \\
\text { applied, including } \\
\text { the pie chart were } \\
\text { observed to be } \\
\text { effective in } \\
\text { supporting John to } \\
\text { consider alternative } \\
\text { interests during the } \\
\text { Investigation stage, } \\
\text { consistent with } \\
\text { SCT (Palinscar, } \\
\text { 1998; Vygotsky, } \\
\text { 1978). }\end{array}$ \\
\hline
\end{tabular}


The proceeding sections provide a comprehensive analysis of Proposition 3 findings for the Pilot Case. Section 1 below outlines the criteria for interpreting the findings, also presented in in Section 3.5.5 in Chapter 3. Section 2 presents the analysis of findings. Section 3 outlines the themes relevant to Proposition 3 and Section 4 provides a summary of findings. Section 5 provides a conceptual analysis of Proposition 3 findings.

\section{Section 1: Criteria for Interpreting Proposition 3 Findings (Pilot Case)}

\begin{tabular}{|c|c|c|c|c|}
\hline $\begin{array}{l}\text { Proposition: } \\
\text { Following } \\
\text { Engagement } \\
\text { with the AFI } \\
\text { Model: }\end{array}$ & $\begin{array}{l}\text { Likert Scale } \\
\text { Statements }\end{array}$ & $\begin{array}{l}\text { Likert Scale } \\
\text { Statements } \\
\text { (Comments } \\
\text { Section) }\end{array}$ & $\begin{array}{l}\text { Semi-structured } \\
\text { Interviews }\end{array}$ & $\begin{array}{l}\text { Researcher } \\
\text { Diary }\end{array}$ \\
\hline $\begin{array}{l}\text { Proposition 3: } \\
\text { 'Teachers and } \\
\text { parents will } \\
\text { have greater } \\
\text { perceived } \\
\text { competence in } \\
\text { supporting the } \\
\text { child's needs } \\
\text { at home and at } \\
\text { school, as a } \\
\text { result of } \\
\text { interactions } \\
\text { and activities } \\
\text { that have } \\
\text { occurred at the } \\
\text { meso and } \\
\text { microsystem } \\
\text { levels' }\end{array}$ & $\begin{array}{l}\text { A change in } \\
\text { rating on } \\
\text { associated } \\
\text { Likert scale } \\
\text { statements } \\
\text { indicates } \\
\text { change in } \\
\text { perceived } \\
\text { competence in } \\
\text { supporting the } \\
\text { child's needs } \\
\text { following } \\
\text { engagement } \\
\text { with the AFI } \\
\text { model and as a } \\
\text { result of } \\
\text { interactions } \\
\text { and activities } \\
\text { that have } \\
\text { occurred at the } \\
\text { meso and } \\
\text { microsystem } \\
\text { levels. }\end{array}$ & $\begin{array}{l}\text { Pattern- } \\
\text { matching: } \\
\text { Comments } \\
\text { that link } \\
\text { competence } \\
\text { in supporting } \\
\text { the child's } \\
\text { needs, } \\
\text { engagement } \\
\text { with the AFI } \\
\text { model and } \\
\text { interactions } \\
\text { and activities } \\
\text { that have } \\
\text { occurred at } \\
\text { the meso and } \\
\text { microsystem } \\
\text { levels and } \\
\text { vice versa. }\end{array}$ & $\begin{array}{l}\text { Pattern-matching: } \\
\text { Interview } \\
\text { responses that link } \\
\text { competence in } \\
\text { supporting the } \\
\text { child's needs, } \\
\text { engagement with } \\
\text { the AFI model and } \\
\text { interactions and } \\
\text { activities that have } \\
\text { occurred at the } \\
\text { meso and } \\
\text { microsystem levels } \\
\text { and vice versa. } \\
\text { Thematic } \\
\text { analysis: Themes } \\
\text { and patterns within } \\
\text { the data set that } \\
\text { refer to competence } \\
\text { in supporting the } \\
\text { child's needs and } \\
\text { interactions and } \\
\text { activities that have } \\
\text { occurred at the } \\
\text { meso and } \\
\text { microsystem levels } \\
\text { were included in } \\
\text { the analysis of the } \\
\text { AFI model. }\end{array}$ & N/A \\
\hline
\end{tabular}


Section 2: Analysis of Proposition 3 Findings (Pilot Case)

\begin{tabular}{|c|c|c|c|c|}
\hline $\begin{array}{l}\text { Evidence } \\
\text { for } \\
\text { Proposition } \\
3 \text { (Pilot } \\
\text { Case) }\end{array}$ & $\begin{array}{l}\text { Likert Scale } \\
\text { Statements }\end{array}$ & $\begin{array}{l}\text { Likert } \\
\text { Scale } \\
\text { Statements } \\
\text { (Comments } \\
\text { Section) }\end{array}$ & Semi-structured Interviews & Researcher Diary \\
\hline $\begin{array}{l}\text { Parent } \\
\text { (Louise) }\end{array}$ & $\begin{array}{l}\text { I am able to } \\
\text { support my } \\
\text { child's needs } \\
\text { at home } \\
\text { Before AFI: } 3 \\
\text { After AFI: } 8\end{array}$ & $\begin{array}{l}\text { N/A (See } \\
\text { section } \\
3.11 .2)\end{array}$ & $\begin{array}{l}\text { Pattern-matching: Do you feel you can support John's needs } \\
\text { at home? Well I suppose you're trying to explore other avenues } \\
\text { (competence) that you might get him interested in, i.e. maybe } \\
\text { Zumba class or the Boy Scouts (interaction and activities } \\
\text { during the AFI process) or..... am...maybe look, continuing } \\
\text { with bringing him out for a cycle or a walk in the woods am } \\
\text { (interaction and activities during the AFI process), and then } \\
\text { am...basically just trying to distract his attention away from the } \\
\text { obsessional interest (lines 79-82) } \\
\text { Thematic analysis (See Section } 3 \text { below for key quotes of } \\
\text { themes and subthemes relevant to Proposition 3): } \\
\text { A team approach to assessment } \\
\text {-Strengthening relationships } \\
\text { Perspectives on barriers to parental engagement } \\
\text {-Judgements, belief systems, practices and values } \\
\text { Factors bridging the gap between assessment and intervention } \\
\text {-Practical interventions }\end{array}$ & $\begin{array}{l}\text { Researcher diary (AFI } \\
\text { templates) documented } \\
\text { specific activities and } \\
\text { interactions that took place } \\
\text { during the five-stage AFI } \\
\text { process (See Section } 4.2 \text {, Table } \\
4.1 \text { ) }\end{array}$ \\
\hline
\end{tabular}




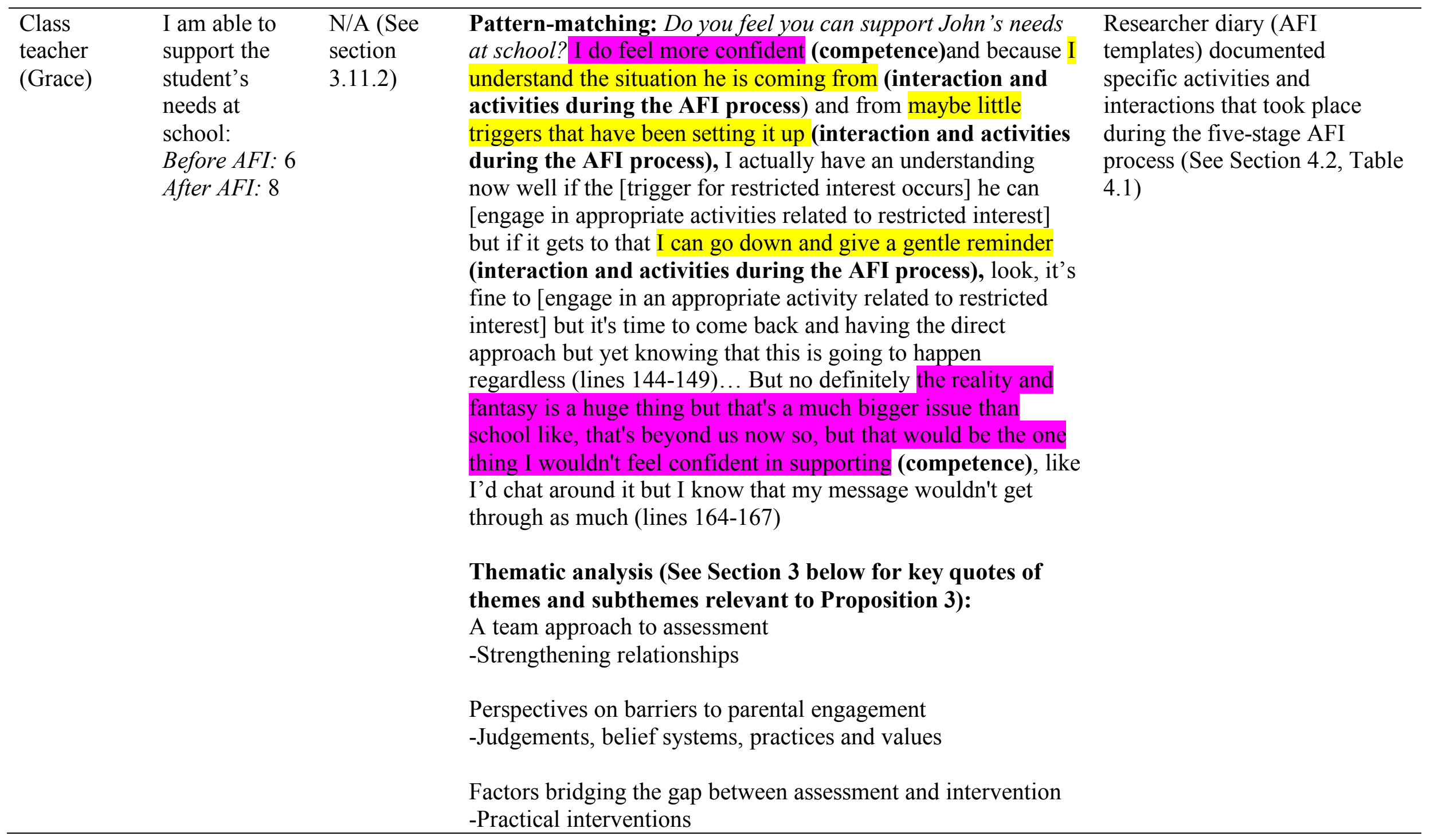




\begin{tabular}{|c|c|}
\hline \multicolumn{2}{|c|}{ Theme 1: A Team Approach to Assessment } \\
\hline Subthemes & Key Quotes \\
\hline \multirow{11}{*}{$\begin{array}{l}\text { Strengthening } \\
\text { relationships }\end{array}$} & When describing how she would support John's needs at school, \\
\hline & $\begin{array}{l}\text { Grace (class teacher) commented: As I said to you even in the } \\
\text { couple of weeks I've seen a change in his behaviour even so far } \\
\text { that he is more responsive, and kind of more positive towards } \\
\text { me...(lines 150-151) }\end{array}$ \\
\hline & $\begin{array}{l}\text { When discussing her understanding of John's situation, Grace } \\
\text { stated: }\end{array}$ \\
\hline & I think to understand that was a big part of it that there was this \\
\hline & $\begin{array}{l}\text { kind of opening up of communication between home and school } \\
\text { (lines 75-76) }\end{array}$ \\
\hline & When describing how she would support John's needs at home, \\
\hline & Louise (parent) commented: I suppose like maybe just a simple \\
\hline & thing that he identified that he likes having a chat so it's to sit \\
\hline & down with him and actually say to him am... Do you know why \\
\hline & we had to put other little activities on his agenda and the reason \\
\hline & for it is $\mathrm{X}, \mathrm{Y}$ and $\mathrm{Z}$ (lines $68-71)$ \\
\hline \multicolumn{2}{|c|}{ Theme 2: Perspectives on Barriers to Parental Engagement } \\
\hline \multirow{10}{*}{$\begin{array}{l}\text { Subthemes } \\
\text { Judgements, } \\
\text { belief systems, } \\
\text { practices and } \\
\text { values }\end{array}$} & Key Quotes \\
\hline & When describing her role in the AFI process, Grace commented: \\
\hline & Maybe they [parents] weren't being told absolutely everything \\
\hline & that was happening in the school but that there was a build-up of, \\
\hline & like the note things and those kind of things, that there was these \\
\hline & behaviours that were actually kind of worrying (lines 47-50) \\
\hline & Grace noted when describing what she had learned during the \\
\hline & AFI process: Like we only get to meet them [parents] maybe \\
\hline & once a year with parent teacher meetings and beyond that it's \\
\hline & maybe a phone call or the odd meeting (lines $243-244)$ \\
\hline \multicolumn{2}{|c|}{ Theme 3: Factors Bridging the Gap between Assessment and Intervention } \\
\hline Subthemes & Key Quotes \\
\hline \multirow[t]{5}{*}{$\begin{array}{l}\text { Practical } \\
\text { interventions }\end{array}$} & $\begin{array}{l}\text { With regard to intervention recommendations, Grace } \\
\text { commented: }\end{array}$ \\
\hline & $\begin{array}{l}\text { They'll [recommendations] be a huge support in class especially } \\
\text { when you work with } 26 \text { other kids, that there are things that I can } \\
\text { actually implement that won't detract from the others and that can } \\
\text { be easily implemented and that they could all actually even try } \\
\text { (lines 92-95) }\end{array}$ \\
\hline & With regard to intervention recommendations, Louise \\
\hline & commented: \\
\hline & $\begin{array}{l}\text { Little steps that you can take in the home environment as a } \\
\text { mother and just little steps just about listening, because you } \\
\text { identified that he likes a little chat (lines } 76-78 \text { ) }\end{array}$ \\
\hline
\end{tabular}




\section{Section 4: Summary of Proposition 3 Findings (Pilot Case)}

Higher Likert scale ratings indicate greater perceived competence of Grace (class teacher) and Louise (parent) in supporting John's needs after engaging with the AFI model. Patterns within parent and teacher interview responses connected activities and interactions that occurred during the AFI process, at the meso and microsystem levels, to perceived competence to support John's needs. Louise's response connected competence to knowledge of alternative interest activities such as "Zumba class and Boy Scouts", which were suggested by Grace during a meeting at the Investigation Stage. Grace's response linked competence to awareness of "triggers" and understanding "the situation he [John] is coming from". Activities and interactions that led to the identification of triggers and understanding of John's situation included dialogue between team members, observation tasks and an FAI at the Investigation stage. See Section 4.2, Table 4.1 for a list of the activities and interactions that participants engaged in. These activities and interactions are considered consistent with the AFI process for several reasons. In accordance with AFI Principle 6, all participants were 'co-assessors' during the Investigation stage and suggestions of alternative interest activities, for example Zumba and Boy Scouts suggested by Grace during meetings, were considered and valued. This knowledge of alternative interest activities strengthened Louise's perceived competence to support John's needs, as evidenced in Section 2 above. Understanding John's situation and his triggers for engaging in the restricted interest enhanced Grace's perceived competence to support his needs, as evidenced in Section 2 above. This understanding resulted from the activities and interactions (e.g. FAI, dialogue, observations) engaged in as a 'co-assessor' during the AFI process. In addition, identifying parent and teacher needs to support John moving forward is consistent with AFI Principle 4, focusing on teacher and parent needs. Identifying these needs also supported feasible intervention planning, consistent with AFI Principle 1 (Pameijer, 2017).

Patterns within Louise's response also connected competence to support John's needs to supports that she already provides in the home environment, "continuing to bring him out for a cycle or a walk in the woods". In accordance with AFI Principle 3, effective approaches addressing John's needs were encouraged by the TEP during meetings and discussions ('goodness of fit') (Pameijer, 2017). Grace also commented there were still areas of need that she did not feel confident supporting, including helping John to discern between reality and fantasy, she considered that such support warranted outside services and that this issue was "beyond us [school]". Themes within 
the data set that referred to competence in supporting John's needs and interactions and activities that occurred at the meso and microsystem levels included 'A team approach to assessment', 'Perspectives on barriers to parental engagement approach' and 'Factors bridging the gap between assessment and intervention' (See Section 3 above). Section 5 below provides a conceptual analysis of the findings and themes, in accordance with AFI principles, EST and SCT concepts. 


\section{Subtheme AFI Principles (See section 2.16 for description of AFI Principles) \\ Strengthening Pattern-matching of interview responses indicated perceived competence to relationships \\ support John's needs at home and at school was linked to activities and interactions that occurred at the meso and microsystem levels, including dialogue between team members and engagement in assessment tasks (e.g. FAI) as co- assessors. Thematic analysis of interview responses suggests strengthened relationships as a result of engaging in a team assessment approach, may further account for perceived competence in meeting John's needs (See key quotes in Section 3). This subtheme resonates with AFI Principle 6, where assessment is built upon collaborative partnerships between TEP, parents, teachers and child.}

Judgements,

belief

systems,

practices and

values

Practical

interventions

Pattern-matching of interview responses indicated perceived competence to support John's needs at home and at school was linked to dialogue between team members and engagement in assessment tasks as co-assessors. Communication and co-operation between team members was required during the AFI process in accordance with AFI Principle 6. Collaborative partnerships between home and school during the AFI process is in direct contrast to the previous lack of communication and barrier between home and school described by Grace during interview (See key quotes in Section 3).

Pattern-matching of interview responses indicated perceived competence to support John's needs at home and at school was linked to dialogue between team members and engagement in assessment tasks as co-assessors. Thematic analysis of interview responses suggests practical intervention recommendations may further account for perceived competence in meeting John's needs as interventions were considered practical and easy to implement by Grace and Louise (See key quotes in Section 3). This subtheme resonates with AFI Principle 1 'Goal directed and functional assessment', as the EP aims at feasible intervention recommendations from the beginning of the assessment process.

\section{EST Concepts (See section 2.14)}

Opening of communication between home and school and John's increased responsiveness to his teacher reflects strengthened developmental potential of home and school settings working towards supporting John's needs and the possible development of affective dyadic relations between John (child) and Grace (teacher) (Bronfenbrenner, 1979).

Lack of communication between home and school prior to the AFI model may have hindered the developmental potential of these settings to work together to support John's needs (Bronfenbrenner, 1979).

Implementing practical interventions to support John's need to engage in alternative activities supports the development of this molar activity (Bronfenbrenner, 1979). Molar activities are behaviours perceived as having meaning or intent by participants in a particular setting (Bronfenbrenner, 1979). 


\section{Appendix 31 Pilot Case Analysis of Findings: Proposition 4}

The proceeding sections provide a comprehensive analysis of Proposition 4 findings for the Pilot Case. Section 1 below outlines the criteria for interpreting the findings, also presented in in Section 3.5.5 in Chapter 3. Section 2 presents the analysis of findings. Section 3 outlines the themes relevant to Proposition 4 and Section 4 provides a summary of findings. Section 5 provides a conceptual analysis of Proposition 4 findings.

Section 1: Criteria for Interpreting Proposition 4 Findings (Pilot Case)

\begin{tabular}{|c|c|c|c|c|}
\hline $\begin{array}{l}\text { Proposition: } \\
\text { Following } \\
\text { Engagement } \\
\text { with the AFI } \\
\text { Model: }\end{array}$ & $\begin{array}{l}\text { Likert Scale } \\
\text { Statements }\end{array}$ & $\begin{array}{l}\text { Likert Scale } \\
\text { Statements } \\
\text { (Comments } \\
\text { Section) }\end{array}$ & $\begin{array}{l}\text { Semi-structured } \\
\text { Interviews }\end{array}$ & $\begin{array}{l}\text { Researcher } \\
\text { Diary }\end{array}$ \\
\hline $\begin{array}{l}\text { Proposition } \\
\text { 4: 'The child } \\
\text { will have a } \\
\text { better } \\
\text { understanding } \\
\text { of their } \\
\text { strengths and } \\
\text { needs and } \\
\text { what to do to } \\
\text { improve their } \\
\text { needs, as a } \\
\text { result of } \\
\text { interactions } \\
\text { and activities } \\
\text { that have } \\
\text { occurred at } \\
\text { the meso and } \\
\text { microsystem } \\
\text { levels' }\end{array}$ & $\begin{array}{l}\text { A change in } \\
\text { rating on the } \\
\text { associated } \\
\text { Likert scale } \\
\text { statements } \\
\text { indicates } \\
\text { change in the } \\
\text { child's } \\
\text { understanding } \\
\text { of their } \\
\text { strengths and } \\
\text { needs and } \\
\text { what to do to } \\
\text { improve their } \\
\text { needs, } \\
\text { following } \\
\text { engagement } \\
\text { with the AFI } \\
\text { model and as } \\
\text { a result of } \\
\text { interactions } \\
\text { and activities } \\
\text { that have } \\
\text { occurred at } \\
\text { the meso and } \\
\text { microsystem } \\
\text { levels }\end{array}$ & $\begin{array}{l}\text { Pattern- } \\
\text { matching: } \\
\text { Comments } \\
\text { that link the } \\
\text { child's } \\
\text { understanding } \\
\text { of their } \\
\text { strengths and } \\
\text { needs and } \\
\text { what to do to } \\
\text { improve their } \\
\text { needs, } \\
\text { engagement } \\
\text { with the AFI } \\
\text { model and } \\
\text { interactions } \\
\text { and activities } \\
\text { that have } \\
\text { occurred at } \\
\text { the meso and } \\
\text { microsystem } \\
\text { levels and } \\
\text { vice versa. }\end{array}$ & $\begin{array}{l}\text { Pattern-matching: } \\
\text { Interview responses } \\
\text { that link the child's } \\
\text { understanding of } \\
\text { their strengths and } \\
\text { needs and what to do } \\
\text { to improve their } \\
\text { needs, engagement } \\
\text { with the AFI model } \\
\text { and interactions and } \\
\text { activities that have } \\
\text { occurred at the meso } \\
\text { and microsystem } \\
\text { levels and vice } \\
\text { versa. } \\
\text { Thematic analysis: } \\
\text { Themes and patterns } \\
\text { within the data set } \\
\text { that refer to } \\
\text { understanding of } \\
\text { strengths and needs } \\
\text { and what to do to } \\
\text { improve their needs, } \\
\text { and interactions and } \\
\text { activities that have } \\
\text { occurred at the meso } \\
\text { and microsystem } \\
\text { levels were included } \\
\text { in the analysis of the } \\
\text { AFI model. }\end{array}$ & $\mathrm{N} / \mathrm{A}$ \\
\hline
\end{tabular}


Section 2: Analysis of Proposition 4 Findings (Pilot Case)

\begin{tabular}{|c|c|c|c|c|}
\hline $\begin{array}{l}\text { Proposition } \\
4 \text { Evidence } \\
\text { (Pilot Case) }\end{array}$ & $\begin{array}{l}\text { Likert Scale } \\
\text { Statements }\end{array}$ & $\begin{array}{l}\text { Likert Scale } \\
\text { Statements } \\
\text { (Comments) }\end{array}$ & Semi-structured Interviews & $\begin{array}{l}\text { Researcher } \\
\text { Diary }\end{array}$ \\
\hline $\begin{array}{l}\text { John } \\
\text { (Student) }\end{array}$ & $\begin{array}{l}\text { I know what } \\
\text { my strengths } \\
\text { are at school } \\
\text { Before AFI: } \\
\text { Yes } \\
\text { After AFI: Not } \\
\text { sure } \\
\text { I know what I } \\
\text { need help with } \\
\text { at school } \\
\text { Before AFI: } \\
\text { Not sure } \\
\text { After AFI: Yes } \\
\text { I know what I } \\
\text { can do to get } \\
\text { better at what I } \\
\text { need help with } \\
\text { at school } \\
\text { Before AFI: } \\
\text { No } \\
\text { After AFI: No }\end{array}$ & $\begin{array}{l}\text { N/A (See } \\
\text { section } \\
3.11 .2)\end{array}$ & $\begin{array}{l}\text { Pattern-matching: What did you like about working with me, your teacher and } \\
\text { mom? Part of the time it would be hard, part of the time it could be easy but most } \\
\text { of the time its hard (interactions and activities during the AFI process) (line } \\
\text { 7)...(Interviewer: Tell me a little bit more about that)...Just doing hard decisions } \\
\text { (interactions and activities during the AFI process) (line 9) } \\
\text { Did working together (interactions and activities during the AFI process) help } \\
\text { you to find out about your strengths? Kind of (understanding strengths) (line } \\
\text { 22)... at least I know a little bit about working with you but it's hard to do with the } \\
\text { teachers and mom (interactions and activities during the AFI process) and yeah } \\
\text { (lines } 24-25 \text { ) } \\
\text { Did working together (interactions and activities during the AFI process) help } \\
\text { you to find out about what you need help with? Kind of (understanding needs) } \\
\text { (line 30)...I think for that question I wouldn't really want to answer (line 32) } \\
\text { Do you know what you need to do to get better at what you need help with? Kind } \\
\text { of yeah...(line 39) Study my best in school (improve on needs) (line } \\
41 \text { )...(Interviewer: What about your new activities) (improve on needs)... Yeah } \\
\text { that would be one (improve on needs/ interactions and activities during the AFI } \\
\text { process) (line } 43 \text { ) } \\
\text { Thematic analysis (See Section } 3 \text { below for key quotes of themes and } \\
\text { subthemes relevant to Proposition 4): } \\
\text { A team approach to assessment }\end{array}$ & $\begin{array}{l}\text { Researcher } \\
\text { diary (AFI } \\
\text { templates) } \\
\text { documented } \\
\text { specific } \\
\text { activities } \\
\text { and } \\
\text { interactions } \\
\text { that took } \\
\text { place } \\
\text { during the } \\
\text { five-stage } \\
\text { AFI process } \\
\text { (See } \\
\text { Section 4.2, } \\
\text { Table 4.1) }\end{array}$ \\
\hline
\end{tabular}




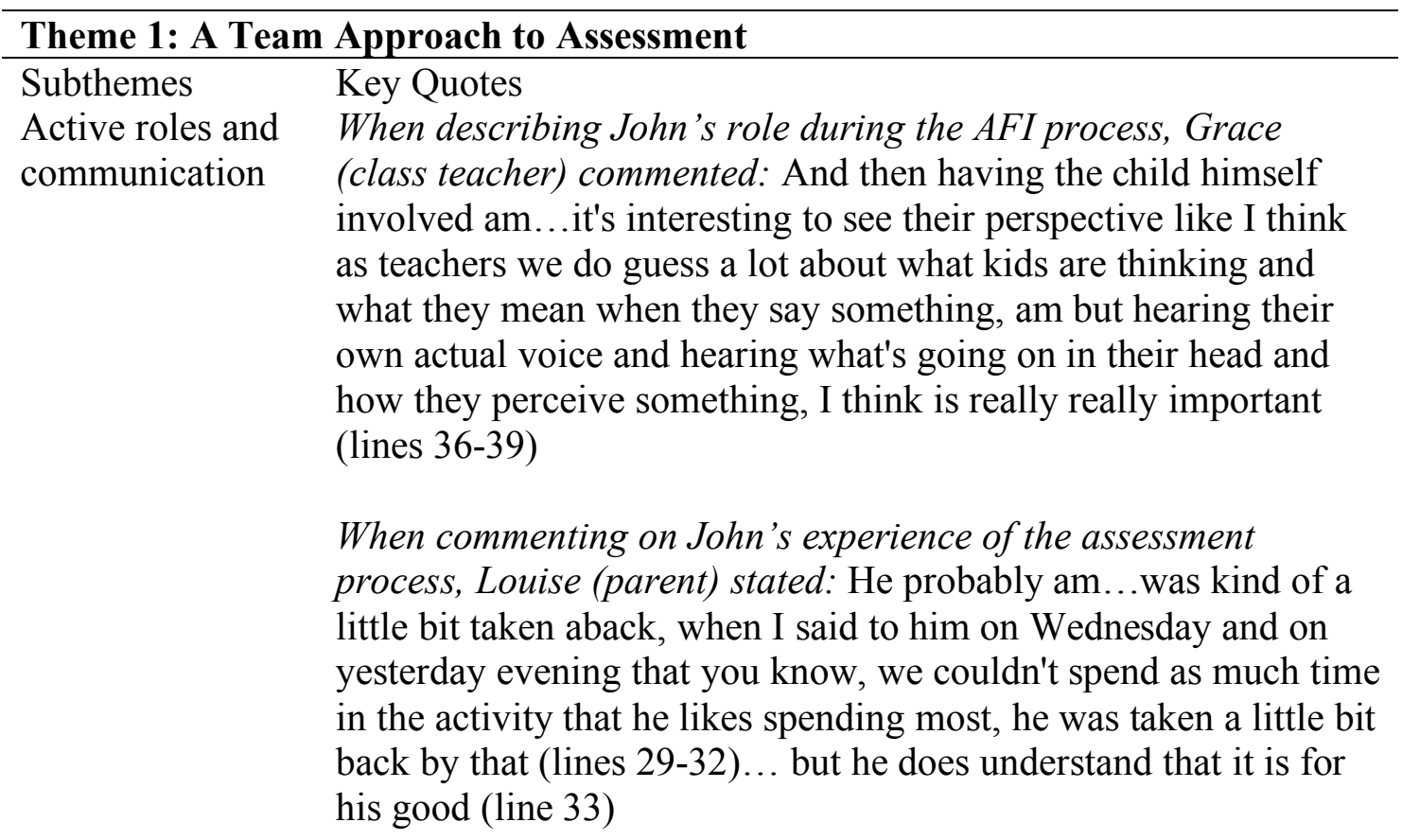

Strengthening relationships

When commenting on John's responsiveness to intervention strategies applied during the Investigation stage, Grace noted: So but I think that even he does seem to be much more responsive now to things we've been saying (lines 116-117)

When describing how she would support John's needs at school, Grace commented: As I said to you even in the couple of weeks I've seen a change in his behaviour even so far that he is more responsive, and kind of more positive towards me...(lines 150151)

Insight into When describing John's responsiveness to intervention strategies strengths, needs applied during the Investigation stage, Grace noted: So hopefully and perspectives even if we can get him to see it slightly, that maybe it will start to click (lines 129-130)

When describing how she would support John's needs at home, Louise commented: And I suppose the other thing is, to try and do is to kind of get him to reason so that he might participate in the idea of spending less time [engaging in restricted interest], and so look if he understands, he might buy into it more readily (lines 71-73)

\section{Section 4: Summary of Proposition 4 Findings (Pilot Case)}

Likert scale ratings provided by John indicate that he was "not sure" of his strengths following engagement with the AFI model. His ratings indicate that he now knows what he needs help with at school and still does not know what he can do to improve on needs at school. Patterns within John's responses to interview questions 
addressing Proposition 4 linked understanding of strengths and needs to interactions and activities that occurred during the AFI process, at the meso and microsystem levels. Working together with the TEP, parent and teacher was associated with a "kind of" understanding of strengths and needs. Activities and interactions where John worked together with the TEP, parent and teacher included dialogue throughout the assessment process, co-investigator observation tasks and joint activity tasks to identify alternative interests and activities that provide him with a sense of calm (See Section 4.2, Table 4.1). These activities and interactions are considered unique to the AFI process as consistent with AFI Principle 6, John, was considered a 'co-assessor' during the Investigation stage and co-constructed knowledge around intervention supports in this regard (See Section 5 below).

Patterns within John's responses indicate that it was more difficult to find out about his strengths and needs with his mother and teacher, than with the TEP and that the assessment process was a challenging experience with making "hard decisions". Making decisions around engaging in alternative interests may have been a difficult experience for John as the restricted interest provides him with a sense of calm and security. Appropriate engagement in this interest and alternative activities that offer a sense of calm were considered during intervention planning and difficulty adapting to changes in routine can be experienced by children with ASD. Patterns within John's responses linked understanding of what to do to address needs to interactions and activities that occurred during the AFI process, at the meso and microsystem level. John identified "study my best at school" and engaging in "new activities" and interests, which were discussed and identified during joint activity tasks at the Investigation stage (See Section 4.2, Table 4.1). The theme 'A team approach to assessment' referred to understanding of strengths and needs and what to do to address needs, and interactions and activities that occurred at the meso and microsystem levels (See Section 3 above). Section 5 below provides a conceptual analysis of the findings and themes, in accordance with AFI principles, EST and SCT concepts. 
Section 5: Conceptual Analysis of Proposition 4 Findings (Pilot Case)

\begin{tabular}{|c|c|c|c|}
\hline $\begin{array}{l}\text { Theme/ } \\
\text { Subtheme }\end{array}$ & $\begin{array}{l}\text { AFI Principles (See section } 2.16 \text { for description of AFI } \\
\text { Principles) }\end{array}$ & $\begin{array}{l}\text { EST Concepts (See } \\
\text { section } 2.14 \text { for } \\
\text { definitions of EST } \\
\text { concepts) }\end{array}$ & $\begin{array}{l}\text { SCT Concepts (See } \\
\text { section } 2.15 \text { for } \\
\text { definitions of SCT } \\
\text { concepts) }\end{array}$ \\
\hline $\begin{array}{l}\text { Active roles } \\
\text { and } \\
\text { communication }\end{array}$ & $\begin{array}{l}\text { Patterns within John's responses to interview questions linked } \\
\text { understanding of strengths and needs and what to do to address } \\
\text { needs, to interactions and activities that occurred during the AFI } \\
\text { process at the meso and microsystem level. Activities and } \\
\text { interactions where John worked together with the TEP, parent and } \\
\text { teacher included dialogue throughout the assessment process, co- } \\
\text { investigator observation tasks and joint activity tasks. Thematic } \\
\text { analysis of interview responses suggests John's active role in the } \\
\text { team assessment approach provided the opportunity to voice his } \\
\text { opinions (See key quotes above), however this process may have } \\
\text { been difficult in making 'hard decisions' and in implementing the } \\
\text { intervention recommendations discussed. John's active involvement } \\
\text { as a 'co-assessor' in the team assessment approach is consistent } \\
\text { with AFI Principle 6: Working together in a collaborative }\end{array}$ & $\begin{array}{l}\text { At the microsystem level, } \\
\text { John's active } \\
\text { engagement and } \\
\text { inclusion in the AFI } \\
\text { process may have } \\
\text { strengthened dyadic } \\
\text { relations between John, } \\
\text { his teacher, parent and } \\
\text { TEP in working together } \\
\text { to understand and meet } \\
\text { his needs } \\
\text { (Bronfenbrenner, 1979). }\end{array}$ & $\begin{array}{l}\text { Tools and signs, } \\
\text { including the pie chart } \\
\text { and interest inventory } \\
\text { helped to facilitate } \\
\text { John's active } \\
\text { involvement during the } \\
\text { Investigation stage to } \\
\text { co-construct knowledge } \\
\text { with the TEP, parent } \\
\text { and teacher and } \\
\text { supported consideration } \\
\text { of alternative interests } \\
\text { (Palinscar, 1998; } \\
\text { Vygotsky, 1978). }\end{array}$ \\
\hline
\end{tabular}

Strengthening Patterns within John's responses to interview questions linked relationships understanding of strengths and needs and what to do to address needs, to engagement with the AFI model and interactions and activities that occurred at the meso and microsystem level. Thematic analysis of interview responses suggests strengthened relationships as a result of engaging in a team assessment approach, may have contributed to John's understanding of strengths and needs. Grace had started to notice John being "more positive towards" her following engagement with the AFI model (See key

John's increased responsiveness to his teacher following engagement in the AFI process suggests the possible development of affective dyadic relations between child (John) and 
quotes in Section 3). This subtheme resonates with AFI Principle 6, where assessment is built upon collaborative partnerships between TEP, parents, teachers and child.

Insight into strengths, needs and perspectives

Patterns within John's responses to interview questions linked understanding of strengths and needs and what to do to address needs, to interactions and activities that occurred during the AFI process, at the meso and microsystem levels. Thematic analysis of parent and teacher interview responses suggests continued reasoning and perspective taking with John may be needed in order to achieve his goals (See key quotes in Section 3 above). This resonates with AFI Principle 6 as parent, teacher and child will need to continue to co-operate and collaborate to meet on needs.

Working together to meet needs requires continued consideration of AFI Principle 3 and 4, John's needs and Grace and Louise's needs to support his needs. teacher (Grace)

(Bronfenbrenner, 1979).

Bronfenbrenner (1979) states the aspects of the environment that are most powerful in influencing psychological growth are those that have meaning to the person in a given situation. Continued consideration of John's perspective and experience is necessary as team members work towards addressing needs identified during the AFI process. 
Appendix 32 Pilot Case Analysis of Findings: Proposition 5

The proceeding sections provide a comprehensive analysis of Proposition 5 findings for the Pilot Case. Section 1 below outlines the criteria for interpreting the findings, also presented in in Section 3.5.5 in Chapter 3. Section 2 presents the analysis of findings. Section 3 outlines the themes relevant to Proposition 5 and Section 4 provides a summary of findings. Section 5 provides a conceptual analysis of Proposition 5 findings.

Section 1: Criteria for Interpreting Proposition 5 Findings (Pilot Case)

\begin{tabular}{|c|c|c|c|c|}
\hline $\begin{array}{l}\text { Proposition: } \\
\text { Following } \\
\text { Engagement } \\
\text { with the AFI } \\
\text { Model: }\end{array}$ & $\begin{array}{l}\text { Likert Scale } \\
\text { Statements }\end{array}$ & $\begin{array}{l}\text { Likert Scale } \\
\text { Statements } \\
\text { (Comments } \\
\text { Section) }\end{array}$ & $\begin{array}{l}\text { Semi-structured } \\
\text { Interviews }\end{array}$ & $\begin{array}{l}\text { Researcher } \\
\text { Diary }\end{array}$ \\
\hline $\begin{array}{l}\text { Proposition 5: } \\
\text { 'At a time of } \\
\text { ecological } \\
\text { transition, } \\
\text { teachers will } \\
\text { feel competent } \\
\text { in their ability } \\
\text { to work } \\
\text { according to the } \\
\text { aims of Circular } \\
\text { 0013/2017, in } \\
\text { meeting and } \\
\text { monitoring the } \\
\text { needs of the } \\
\text { child' }\end{array}$ & $\mathrm{N} / \mathrm{A}$ & $\mathrm{N} / \mathrm{A}$ & $\begin{array}{l}\text { Pattern-matching: } \\
\text { Interview responses } \\
\text { that link } \\
\text { competence in in } \\
\text { meeting and } \\
\text { monitoring the } \\
\text { needs of the child, } \\
\text { engagement with } \\
\text { the AFI model and } \\
\text { interactions and } \\
\text { activities that have } \\
\text { occurred at the } \\
\text { meso and } \\
\text { microsystem levels } \\
\text { and vice versa. } \\
\text { Thematic } \\
\text { analysis: Themes } \\
\text { and patterns within } \\
\text { the data set that } \\
\text { refer to meeting } \\
\text { and monitoring the } \\
\text { needs of the child, } \\
\text { and interactions } \\
\text { and activities that } \\
\text { have occurred at } \\
\text { the meso and } \\
\text { microsystem levels } \\
\text { were included in } \\
\text { the analysis of the } \\
\text { AFI model. }\end{array}$ & N/A \\
\hline
\end{tabular}


Section 2: Analysis of Proposition 5 Findings (Pilot Case)

\begin{tabular}{|c|c|c|c|c|}
\hline $\begin{array}{l}\text { Evidence } \\
\text { for } \\
\text { Proposition } \\
5 \text { (Pilot } \\
\text { Case) }\end{array}$ & $\begin{array}{l}\text { Likert Scale } \\
\text { Statements }\end{array}$ & $\begin{array}{l}\text { Likert } \\
\text { Scale } \\
\text { Statements } \\
\text { (Comments } \\
\text { Section) }\end{array}$ & Semi-structured Interviews & Researcher Diary \\
\hline $\begin{array}{l}\text { Class } \\
\text { teacher } \\
\text { (Grace) }\end{array}$ & $\mathrm{N} / \mathrm{A}$ & $\mathrm{N} / \mathrm{A}$ & $\begin{array}{l}\text { Pattern-matching: Do you feel you will be able to meet and } \\
\text { monitor John's needs moving forward? Yeah I think that's } \\
\text { something that I will be able to do (competence to } \\
\text { meet/monitor needs) I mean it's something that I do with } \\
\text { everyone anyway (competence to meet/monitor needs) } \\
\text {...but especially because I think I'm aware of the situation } \\
\text { (interactions and activities during the AFI process) and } \\
\text { because the behaviours they were, I think it's something that } \\
\text { I'd be extra tuned into anyway, am, but I couldn't see any } \\
\text { issue in monitoring his progress especially because even } \\
\text { over the last few weeks I have seen an improvement } \\
\text { (interactions and activities during the AFI process) so if } \\
\text { it only continues on, I'd definitely be able to compare (lines } \\
\text { 221-225) } \\
\text { Thematic analysis (See Section } 3 \text { below for key quotes of } \\
\text { themes and subthemes relevant to Proposition 5): } \\
\text {-Factors bridging the gap between assessment and } \\
\text { intervention }\end{array}$ & $\begin{array}{l}\text { Researcher diary (AFI } \\
\text { templates) documented } \\
\text { specific activities and } \\
\text { interactions that took place } \\
\text { during the five-stage AFI } \\
\text { process (See Section 4.2, Table } \\
4.1 \text { ) }\end{array}$ \\
\hline
\end{tabular}




\begin{tabular}{ll}
\hline \multicolumn{2}{l}{ Theme 4: Factors Bridging the Gap between Assessment and Intervention } \\
\hline $\begin{array}{ll}\text { Subthemes } & \text { Key Quotes } \\
\text { A collaborative } & \text { When describing how she would monitor John's needs, Grace } \\
\text { approach to } & \text { (class teacher) referred to the involvement of his parents: And for } \\
\text { implementing } & \text { the parents to see that they have changed as well it would be } \\
\text { and monitoring } & \text { fantastic for them to see as well because it's proof that there is } \\
\text { interventions } & \text { something happening (lines 229-230) }\end{array}$ \\
\hline
\end{tabular}

\section{Section 4: Summary of Proposition 5 Findings (Pilot Case)}

Patterns within Grace's interview response connected competence to meet and monitor John's needs moving forward to "being aware of the situation". Awareness of the situation resulted from interactions and activities that occurred during the AFI process, at the meso and microsystem level, including dialogue between team members and co-assessor observation tasks. These activities and interactions are consistent with AFI Principle 6, as participants were 'co-assessors' during the Investigation stage and their opinions and feedback were valued as 'hands-on experts through experience' throughout the process (Pameijer, 2017). See Section 4.2, Table 4.1 for a list of the activities and interactions that participants engaged in. Increased awareness of the situation resonates with AFI Principle 3, with greater awareness and insight into John's needs (Pameijer, 2017). Patterns within Grace's response also connected competence to meet and monitor John's needs to her general teaching skills, as this is something that she would "do with everyone anyway". Consistent with AFI Principle 3, effective monitoring approaches were encouraged to be continued during meetings and discussions ('goodness of fit') (Pameijer, 2017). The theme 'Factors bridging the gap between assessment and intervention' referred to meeting and monitoring of needs and interactions and activities that occurred at the meso and microsystem levels (See Section 3 above). Section 5 below provides a conceptual analysis of the findings and themes, in accordance with AFI principles, EST and SCT concepts. 
Section 5: Conceptual Analysis of Proposition 5 Findings (Pilot Case)

\begin{tabular}{|c|c|c|c|}
\hline $\begin{array}{l}\text { Theme/ } \\
\text { Subtheme }\end{array}$ & $\begin{array}{l}\text { AFI Principles (See } \\
\text { section } 2.16 \text { for } \\
\text { description of AFI } \\
\text { Principles) }\end{array}$ & $\begin{array}{l}\text { EST Concepts (See } \\
\text { section } 2.14 \text { for } \\
\text { definitions of EST } \\
\text { concepts) }\end{array}$ & $\begin{array}{l}\text { SCT } \\
\text { Concepts } \\
\text { (See section } \\
2.15 \text { for } \\
\text { definitions } \\
\text { of concepts) }\end{array}$ \\
\hline $\begin{array}{l}\text { A } \\
\text { collaborative } \\
\text { approach to } \\
\text { implementing } \\
\text { and } \\
\text { monitoring } \\
\text { interventions }\end{array}$ & $\begin{array}{l}\text { Patterns within Grace's } \\
\text { interview response } \\
\text { connected competence to } \\
\text { meet and monitor John's } \\
\text { needs moving forward to } \\
\text { her increased awareness of } \\
\text { the situation, which } \\
\text { resulted from interactions } \\
\text { and activities that occurred } \\
\text { during the AFI process, at } \\
\text { the meso and microsystem } \\
\text { levels. Thematic analysis of } \\
\text { interview responses } \\
\text { suggests the subtheme 'A } \\
\text { collaborative approach to } \\
\text { implementing and } \\
\text { monitoring interventions' } \\
\text { may further account for } \\
\text { competence to meet and } \\
\text { monitor John's needs } \\
\text { moving forward (See } \\
\text { Section } 3 \text { for key quotes). } \\
\text { Grace referred to parental } \\
\text { involvement in the } \\
\text { monitoring of intervention } \\
\text { recommendations, in terms } \\
\text { of observing changes in } \\
\text { behaviour and progress. } \\
\text { This is reflective of AFI } \\
\text { Principle } 6 \text { as home and } \\
\text { school work together to } \\
\text { meet and monitor John's } \\
\text { needs (Pameijer, } 2017 \text { ). }\end{array}$ & $\begin{array}{l}\text { Including the parents } \\
\text { in monitoring } \\
\text { effectiveness of } \\
\text { interventions may be } \\
\text { reflective of the } \\
\text { strengthened } \\
\text { developmental } \\
\text { potential of home and } \\
\text { school settings in } \\
\text { working together } \\
\text { towards monitoring } \\
\text { John's needs and } \\
\text { goals } \\
\text { (Bronfenbrenner, } \\
\text { 1979). }\end{array}$ & \\
\hline
\end{tabular}


This section outlines the analysis of findings for each case proposition in Case 1. The analysis for each proposition is divided into four sections. Section 1 provides a table outlining the criteria for interpreting the findings, also documented in Section 3.5.5 in Chapter 3. Section 2 provides the analysis of findings for each participant in accordance with the proposed criteria, in tabular format. Changes in ratings on Likert scale statements before and after engaging with the AFI model are outlined. Pattern-matching logic is applied to responses to interview questions addressing each of the propositions. The interview questions and codes applied to responses are outlined in Table 1 below. Themes and subthemes relevant to each proposition are also outlined and Section 3 provides a table of key quotes for each participant Appendix 45 presents the thematic maps. Appendix 46 outlines the initial and final category clusters and codes applied to interview responses and Appendix 47 provides a sample of the coding applied to a participant's interview transcript. Appendix 48 outlines the process of devising all themes and subthemes. Section 4 provides a summary of findings for each proposition and Section 5 provides a conceptual analysis, in accordance with AFI principles, EST and SCT concepts. 
Table 1 Pattern-Matching Codes Applied to Interview Responses

\begin{tabular}{|c|c|c|}
\hline Proposition & Interview Questions Addressing Proposition & Pattern-matching Codes Applied \\
\hline $\begin{array}{l}\text { 1: 'Parents, teachers and TEP will have a better } \\
\text { understanding of the child's situation, as a } \\
\text { result of interactions and activities that have } \\
\text { occurred at the meso and microsystem levels' }\end{array}$ & $\begin{array}{l}\text { 1. Do you feel your understanding of Jim's situation } \\
\text { has changed after working through the five stages } \\
\text { of the AFI model? } \\
\text { 2. Can you describe your experience of working } \\
\text { with the Assessment for Intervention model? }\end{array}$ & $\begin{array}{l}\text { 'understanding' } \\
\text { 'interactions and activities during the } \\
\text { AFI process' }\end{array}$ \\
\hline $\begin{array}{l}\text { 2: 'Parents, teachers and TEP will know what } \\
\text { intervention supports the child needs at home } \\
\text { and at school, as a result of interactions and } \\
\text { activities that have occurred at the meso and } \\
\text { microsystem levels' }\end{array}$ & $\begin{array}{l}\text { 1. Did the intervention recommendations address } \\
\text { your questions at the intake stage? } \\
\text { 2. Do you feel you can support Jim's needs at } \\
\text { home/school? }\end{array}$ & $\begin{array}{l}\text { 'knowledge of intervention' } \\
\text { 'interactions and activities during the } \\
\text { AFI process' }\end{array}$ \\
\hline $\begin{array}{l}\text { 3: 'Teachers and parents will have greater } \\
\text { perceived competence in supporting the child's } \\
\text { needs at home and at school, as a result of } \\
\text { interactions and activities that have occurred at } \\
\text { the meso and microsystem levels' }\end{array}$ & $\begin{array}{l}\text { 1. Do you feel you can support Jim's needs at } \\
\text { home/school? }\end{array}$ & $\begin{array}{l}\text { 'competence' } \\
\text { 'interactions and activities during the } \\
\text { AFI process' }\end{array}$ \\
\hline $\begin{array}{l}\text { 4: 'The child will have a better understanding } \\
\text { of their strengths and needs and what to do to } \\
\text { improve their needs, as a result of interactions } \\
\text { and activities that have occurred at the meso } \\
\text { and microsystem levels' }\end{array}$ & $\begin{array}{l}\text { 1. Did working with me, your mother and teacher } \\
\text { help you to find out about your strengths? } \\
\text { 2. Did working with me, your mother and teacher } \\
\text { help you to find out about what you need help with? } \\
\text { 3. Do you know what you need to do to get better } \\
\text { at__ (insert needs identified) }\end{array}$ & $\begin{array}{l}\text { 'understanding strengths' } \\
\text { 'understanding needs' } \\
\text { 'improve on needs' } \\
\text { 'interactions and activities during the } \\
\text { AFI process' }\end{array}$ \\
\hline $\begin{array}{l}\text { 5: 'At a time of ecological transition, teachers } \\
\text { will feel competent in their ability to work } \\
\text { according to the aims of Circular } 0013 / 2017 \text {, in } \\
\text { meeting and monitoring the needs of the child' }\end{array}$ & $\begin{array}{l}\text { 1. Do you feel you can monitor Jim's needs at } \\
\text { school? }\end{array}$ & $\begin{array}{l}\text { 'competence to meet/monitor needs' } \\
\text { 'interactions and activities during the } \\
\text { AFI process' }\end{array}$ \\
\hline
\end{tabular}


Appendix 34 Case 1 Analysis of Findings: Proposition 1

The proceeding sections provide a comprehensive analysis of Proposition 1 findings for Case 1. Section 1 below outlines the criteria for interpreting the findings, also presented in in Section 3.5.5 in Chapter 3. Section 2 presents the analysis of findings. Section 3 outlines the themes relevant to Proposition 1 and Section 4 provides a summary of findings. Section 5 provides a conceptual analysis of Proposition 1 findings.

Section 1: Criteria for Interpreting Proposition 1 Findings (Pilot Case)

\begin{tabular}{|c|c|c|c|c|}
\hline $\begin{array}{l}\text { Proposition: } \\
\text { Following } \\
\text { Engagement } \\
\text { with the AFI } \\
\text { Model: }\end{array}$ & $\begin{array}{l}\text { Likert Scale } \\
\text { Statements }\end{array}$ & $\begin{array}{l}\text { Likert Scale } \\
\text { Statements } \\
\text { (Comments } \\
\text { Section) }\end{array}$ & $\begin{array}{l}\text { Semi-structured } \\
\text { Interviews }\end{array}$ & $\begin{array}{l}\text { Researcher } \\
\text { Diary }\end{array}$ \\
\hline $\begin{array}{l}\text { Proposition } \\
\text { 1: 'Parents, } \\
\text { teachers and } \\
\text { TEP will } \\
\text { have a better } \\
\text { understanding } \\
\text { of the child's } \\
\text { situation, as a } \\
\text { result of } \\
\text { interactions } \\
\text { and activities } \\
\text { that have } \\
\text { occurred at } \\
\text { the meso and } \\
\text { microsystem } \\
\text { levels' }\end{array}$ & $\begin{array}{l}\text { A change in } \\
\text { rating on } \\
\text { associated } \\
\text { Likert scale } \\
\text { statements } \\
\text { indicates } \\
\text { change in } \\
\text { understanding } \\
\text { of the child's } \\
\text { situation } \\
\text { following } \\
\text { engagement } \\
\text { with the AFI } \\
\text { model and as } \\
\text { a result of } \\
\text { interactions } \\
\text { and activities } \\
\text { that have } \\
\text { occurred at } \\
\text { the meso and } \\
\text { microsystem } \\
\text { levels. }\end{array}$ & $\begin{array}{l}\text { Pattern- } \\
\text { matching: } \\
\text { Comments } \\
\text { that link } \\
\text { understanding } \\
\text { of the child's } \\
\text { situation, } \\
\text { engagement } \\
\text { with the AFI } \\
\text { model and } \\
\text { interactions } \\
\text { and activities } \\
\text { that have } \\
\text { occurred at } \\
\text { the meso and } \\
\text { microsystem } \\
\text { levels and } \\
\text { vice versa. }\end{array}$ & $\begin{array}{l}\text { Pattern- } \\
\text { matching: } \\
\text { Interview } \\
\text { responses that link } \\
\text { understanding of } \\
\text { the child's } \\
\text { situation, } \\
\text { engagement with } \\
\text { the AFI model and } \\
\text { interactions and } \\
\text { activities that have } \\
\text { occurred at the } \\
\text { meso and } \\
\text { microsystem levels } \\
\text { and vice versa. } \\
\text { Thematic } \\
\text { analysis: Themes } \\
\text { and patterns within } \\
\text { the data set that } \\
\text { refer to } \\
\text { understanding of } \\
\text { the child's } \\
\text { situation and } \\
\text { interactions and } \\
\text { activities that have } \\
\text { occurred at the } \\
\text { meso and } \\
\text { microsystem levels } \\
\text { were included in } \\
\text { the analysis of the } \\
\text { AFI model. }\end{array}$ & $\begin{array}{l}\text { Excerpts from } \\
\text { the researcher } \\
\text { diary that } \\
\text { referred to } \\
\text { understanding } \\
\text { of the child's } \\
\text { situation and } \\
\text { interactions } \\
\text { and activities } \\
\text { that have } \\
\text { occurred at the } \\
\text { meso and } \\
\text { microsystem } \\
\text { levels were } \\
\text { included in the } \\
\text { analysis of the } \\
\text { AFI model. }\end{array}$ \\
\hline
\end{tabular}


Section 2: Analysis of Proposition 1 Findings (Case 1)

\begin{tabular}{|c|c|c|c|c|}
\hline $\begin{array}{l}\text { Evidence for } \\
\text { Proposition1 } \\
\text { (Case 1) }\end{array}$ & $\begin{array}{l}\text { Likert } \\
\text { Scale } \\
\text { Statements }\end{array}$ & $\begin{array}{l}\text { Likert } \\
\text { Scale } \\
\text { Statements } \\
\text { (Comments } \\
\text { Section) }\end{array}$ & Semi-structured Interviews & Researcher Diary \\
\hline Parent & $\begin{array}{l}\text { I } \\
\text { understand } \\
\text { my child's } \\
\text { situation: } \\
\text { Before } \\
\text { AFI: } 4 \\
\text { After AFI: } \\
5\end{array}$ & $\begin{array}{l}\text { N/A (See } \\
\text { section } \\
3.11 .2 \text { ) }\end{array}$ & $\begin{array}{l}\text { Pattern-matching: Can you describe your experience of } \\
\text { working with the Assessment for Intervention model? Yeah it } \\
\text { was good like, it was interesting as well you know and I } \\
\text { understand more about Jim (understanding) (line 3)... } \\
\text { Discussing things (interactions and activities during the } \\
\text { AFI process) like you know, about Jim at home and his } \\
\text { work and I know more about Jim (understanding), about } \\
\text { his work and his homework and stuff (lines 5-6) } \\
\text { Do you feel your understanding of Jim's situation has } \\
\text { changed after working through the five stages of the AFI } \\
\text { model? Yeah (understanding) cause I didn't know anything } \\
\text { about Jim's learning (interactions and activities during the } \\
\text { AFI process) (line 102) } \\
\text { Thematic analysis (See Section } 3 \text { below for key quotes of } \\
\text { themes and subthemes relevant to Proposition 1): } \\
\text { A team approach to assessment } \\
\text {-Active roles and communication } \\
\text {-Insight into strengths, needs and perspectives } \\
\text { Perspectives on barriers to parental engagement } \\
\text { - Judgements, belief systems, practices and values }\end{array}$ & $\begin{array}{l}\text { Researcher diary (AFI templates) } \\
\text { documented specific activities and } \\
\text { interactions that took place during the } \\
\text { five-stage AFI process (See Section } \\
\text { 4.9, Table 4.11) }\end{array}$ \\
\hline
\end{tabular}


Views and experiences of AFI

-A clear and structured process

-A framework to facilitate teamwork and positive outcomes

Class teacher I (Michael)

understand

the

student's

situation

Before

AFI: 4

After AFI:

9
N/A (See

section

$3.11 .2)$
Pattern-matching: Can you describe your experience of working with the Assessment for Intervention model? I found it a very worthwhile experience. I think getting the voice of the pupil, our voice and the parent (interactions and activities during the AFI process) was really good and am, just helps us get a better understanding of the specific needs (understanding) and then the various stages of it made it very clear (interactions and activities during the AFI process) and we saw the progress then and it was really beneficial (lines 3-6)

Do you feel your understanding of Jim's situation has changed after working through the five stages of the AFI model? Yeah I definitely think it has (understanding), am I suppose I would have had concerns about his numeracy and oral language skills but now I can see that he has very good like visual skills and it's just possibly the difficulty in, maybe the difficulty and the weakness in his working memory (interactions and activities during the AFI process) and trying to implement, you know, skills into processing and you know, processing information, so I think that's the biggest thing and even ideas to work with him and things that will help train the muscles and improve possibly his working memory will be good(interactions and activities during the AFI process), so I think I do have a better understanding, definitely (understanding) (lines 50-56)
Researcher diary (AFI templates) documented specific activities and interactions that took place during the five-stage AFI process (See Section 4.9, Table 4.11) 
Thematic analysis (See Section 3 below for key quotes of themes and subthemes relevant to Proposition 1):

A team approach to assessment

-Active roles and communication

-Insight into strengths, needs and perspectives

Perspectives on barriers to parental engagement

- Judgements, belief systems, practices and values

Views and experiences of AFI

-A clear and structured process

-A framework to facilitate teamwork and positive outcomes

SET (Sarah) I

understand

the

student's

situation

Before

AFI: 3

After AFI:

10
N/A (See

section

3.11.2)

ern-matching: Do you feel your understanding of Jim 's situation has changed after working through the five stages of the AFI model? Yes 100\%, 100\% (understanding).

Am... before the model, yes we knew that there were problems, that he was having difficulties, but it was trying to pinpoint, yeah he was struggling a bit in maths, he was struggling a bit with English... but there was nothing concrete (lines 78-80)... Am and then just a big thing I suppose for me, I never realised there was any working memory problem whatsoever (interactions and activities during the AFI process) which is huge in my opinion, like it's immense like if that was gone unnoticed... it would have been a disaster for him, like we would have kept on trying whatever interventions we had, and overloading him constantly (interactions and activities during the AFI

process) ...oh just with the working memory, just with that we probably got so much (lines 83-87)
Researcher diary (AFI templates) documented specific activities and interactions that took place during the five-stage AFI process (See Section 4.9, Table 4.11) 
Can you describe your experience of working with the

Assessment for Intervention model? Yeah my experience I suppose when you introduced it to us in the first place,

am...well I had no experience of it in the first place until you came to the school and you spoke about it and you explained it in detail, all the five steps that was involved in the model, am... and before that I didn't have any experience with regards to the model at all, but overall from the start of the beginning, I found the model...the process hugely

beneficial, not only to the child but to me as a teacher, in my learning and in the things that I have learned from the process, about how to address the needs, how to assess the needs of the children in the first place and then how I'm going to, as the teacher address those needs (interactions and activities during the AFI process), and to ensure that that child can achieve to the best that they can (lines 3-11)

Thematic analysis (See Section 3 below for key quotes of themes and subthemes relevant to Proposition 1):

A team approach to assessment

-Active roles and communication

-Insight into strengths, needs and perspectives

Perspectives on barriers to parental engagement

- Judgements, belief systems, practices and values

Views and experiences of AFI

-A clear and structured process

-A framework to facilitate teamwork and positive outcomes 


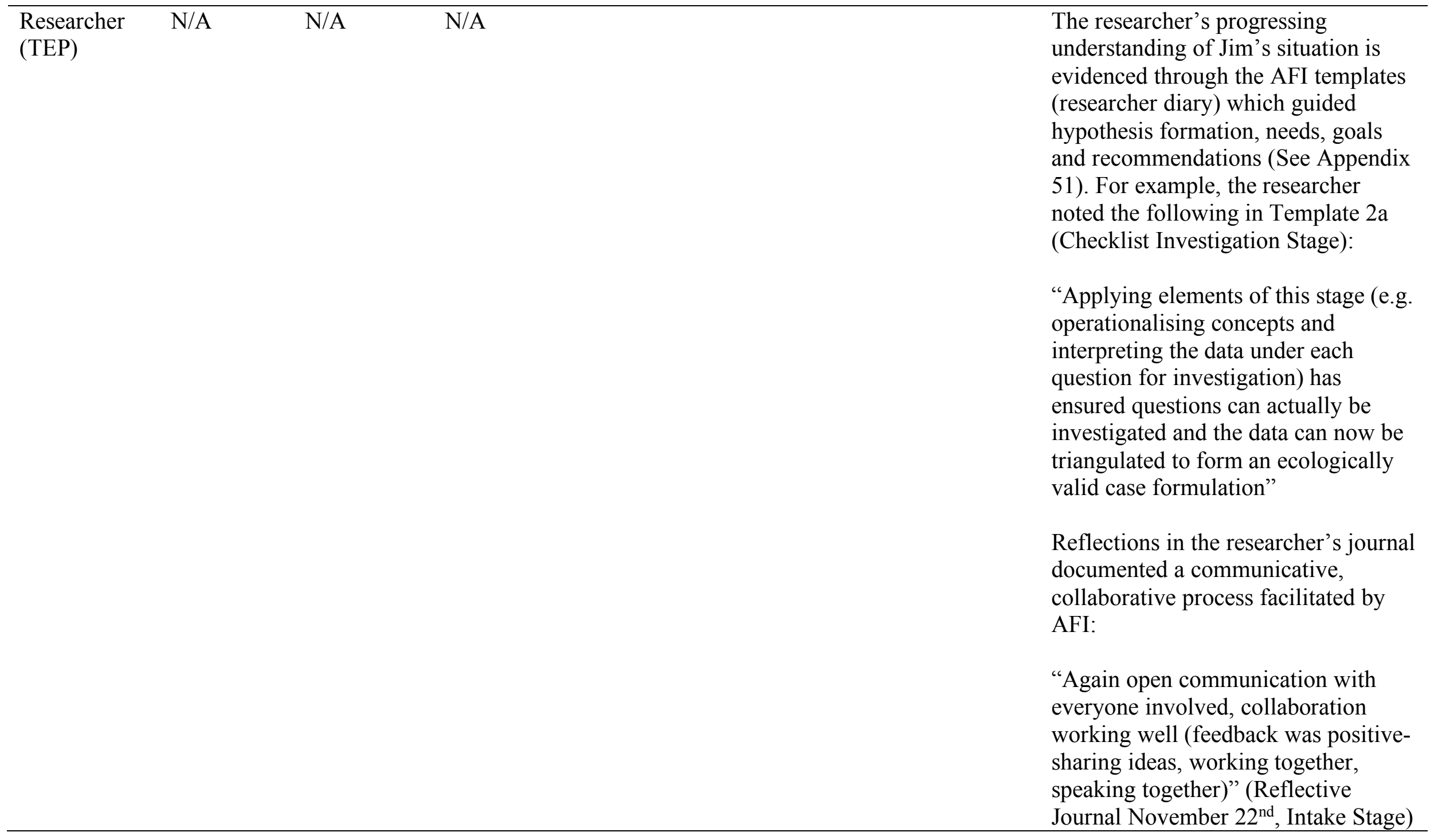




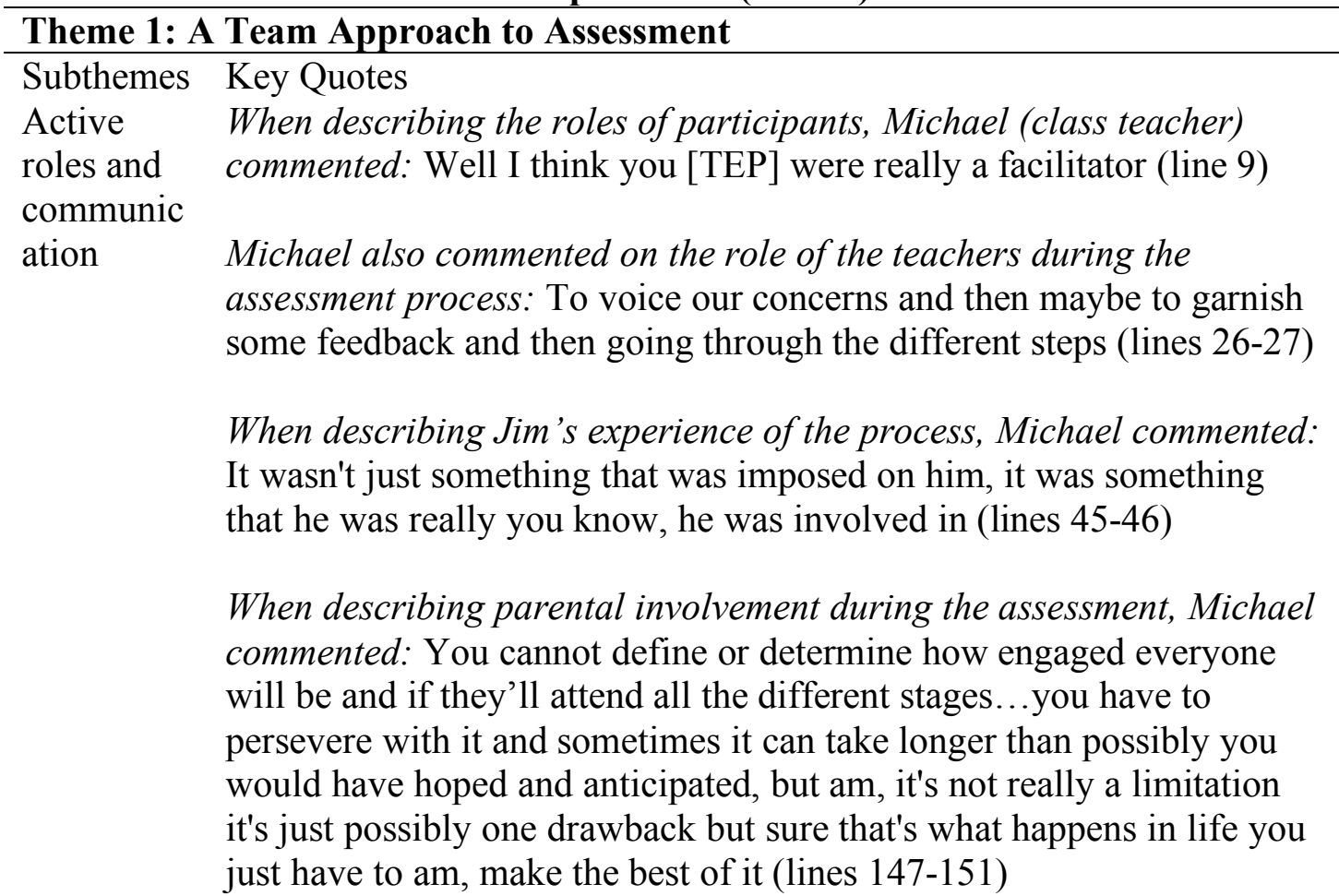

When describing the role of the teachers during the assessment process, Sarah (SET) commented: ...from the beginning, trying to discuss to see where we felt that he may have problems... where his needs might be and then just having our observations in class also (lines 44-46)

When describing Jim's role during the assessment process, Sarah noted: He was able to discuss it even with us in class, do you know his role...he felt more willing to let us know, oh that worked, or that didn't work (lines 58-60)

When describing the TEP's role during the assessment process, Tina (parent) commented: Helping Jim, yeah and helping to find out what his strengths were, you know what he's capable of (line 11)

Tina also described her active involvement during the assessment process: Discussing things like you know, about Jim at home and his work (line 5)

Insight When describing his understanding of Jim's situation, Michael (class into teacher) commented: I can see that he has very good like visual skills strengths, and it's just possibly the difficulty in, maybe the difficulty and the needs and weakness in his working memory (lines 51-52)

perspectiv es

When describing Tina's role during the process, Michael referred to how she provided greater insight into the home situation: Her role was to give us a sort of broader picture of Jim and his home life and her particular concerns and needs and things that work well, what he responds well to at home (lines 17-18) 
Sarah (SET) also referred to how Tina provided greater insight into the home situation: ... anything that might be happening at home that we could take on board in school or things the school could do that she might find helpful (lines 33-34)

Sarah described her insight into Jim's specific needs, as a result of the assessment process: Now I know exactly what his needs are, I know what his needs are in literacy and in numeracy and with regard to working memory (lines 110-111)

When describing her experience of the AFI model, Sarah referred to Jim's growing awareness of his own needs: And it was good for them (Jim) to recognise, any needs that they might have (lines 16-17)

When describing her experience of the AFI model, Tina (parent) referred to her understanding of Jim's situation: I understand more about Jim (line 3)...I know more about Jim, about his work and his homework and stuff (lines 5-6)

\section{Theme 2: Perspectives on Barriers to Parental Engagement}

Subtheme Key Quotes

Judgement When describing parental involvement during the assessment, Sarah $\mathrm{s}$, belief (SET) commented: I suppose from a parent's perspective as well and systems, also from a teacher's perspective, but it's such a pity that...yeah do you practices know when these [assessment] are available for you and your child, and values that you don't avail of them (lines 264-267)

Sarah also commented: I feel that I could access it [assessment process] no problem, again when the child was in school again something that he could do no problem as well, he was happy to do it and then the parents... it's a pity (lines 271-273)

\section{Theme 4: Views and Experiences of AFI}

\section{Subtheme Key Quotes}

A clear Michael commented on what he had learned from the AFI process: It's and just like gradual, step-by-step, so it's like sometimes when we have a structured difficult situation and we try and jump right into it, so I think going process with the gradual approach and like getting positive feedback so I think behaviour, any sort of initiative where you want to support learning, it would be a really good process model to follow (lines 123-126)

When describing her experience of the assessment process, Sarah commented: It was lovely to see it broken down into the steps and to be so clear and so precise of what we had to do at each step, what our involvement was, what the parent's involvement was, what the child's involvement was (lines 12-14)
A
When describing the benefits of the AFI model, Michael stated: I think
framework
to the whole concept and the model I think it's much better than you facilitate know, a one on one, very formalised setting, getting your feedback, teamwork this you feel like...it is a real process and you're involved in it and and there's much more consultation and communication which I think was key moving forward (lines 151-154) 
positive Sarah commented on how the AFI model benefited Jim: It was a tool

outcomes for him to use, to express his opinion on how his learning might be improved (lines 74-75)

When describing the benefits of the AFI model, Tina stated: It helped me a lot now it did, it did now it helped me to see more and stuff you know (lines 238-239)

\section{Section 4: Summary of Proposition 1 Findings (Case 1)}

Parent and teacher Likert scale ratings indicate greater understanding of Jim's situation after engaging with the AFI model. Patterns within parent and teacher interview responses connected greater understanding of the situation to interactions and activities that occurred during the AFI process, at the meso and microsystem level (e.g. engaging in discussion, hearing the voice of each team member and assessment tasks including observations and 1:1 assessment). For example, pattern-matching logic applied to Michael's response linked greater understanding of Jim's situation to engaging in the "clear" process facilitated by AFI, consistent with AFI Principle 7 and interactions and activities at the meso and micro system levels, including "getting the voice" of each team member (See Section 2). Pattern-matching logic applied to Sarah's response connected greater understanding of Jim's situation to interactions and activities during the AFI process that highlighted area of need for his working memory. These activities included a teacher task expectation observation sheet and 1:1 testing of attainment and cognitive functioning and dynamic assessment of learning potential with the TEP. Activities and interactions connected to Proposition 1 are considered unique to the AFI process as consistent with AFI Principle 6, participants were active in their engagement and included in the process as 'co-assessors'. Additionally and in accordance with AFI principle 7, the researcher adhered to the systematic and transparent assessment process and the researcher's progressing understanding of Jim's situation was evidenced in the AFI templates. The templates guided hypothesis formation, needs, goals and recommendations during the five stages (See Appendix 51). Reflections in the researcher's reflective journal documented a communicative, collaborative process facilitated by AFI (See Section 2). Themes within the data set that referred to understanding of Jim's situation and interactions and activities that occurred at the meso and microsystem levels included 'A team approach to assessment', 'Perspectives on barriers to parental engagement' and 'Views and Experiences of AFI' (See Section 3). Section 5 below provides a conceptual analysis of the findings and themes, in accordance with AFI principles, EST and SCT concepts. 
Section 5: Conceptual Analysis of Proposition 1 Findings (Case 1)

\begin{tabular}{|c|c|c|c|}
\hline $\begin{array}{l}\text { Theme/ } \\
\text { Subtheme }\end{array}$ & $\begin{array}{l}\text { AFI Principles (See section } 2.16 \text { for description of AFI } \\
\text { Principles) }\end{array}$ & $\begin{array}{l}\text { EST Concepts (See section } 2.14 \\
\text { for definitions of EST concepts) }\end{array}$ & $\begin{array}{l}\text { SCT Concepts (See section } \\
\text { 2.15) }\end{array}$ \\
\hline $\begin{array}{l}\text { A team } \\
\text { approach to } \\
\text { assessment }\end{array}$ & $\begin{array}{l}\text { Patterns within parent and teacher interview responses } \\
\text { linked understanding of the situation to engagement with } \\
\text { the AFI model and interactions and activities that occurred } \\
\text { at the meso and microsystem level (e.g. engaging in } \\
\text { discussion, hearing the voice of each team member and } \\
\text { team assessment tasks including observations and } 1: 1 \\
\text { assessment). Reflections in the researcher's journal } \\
\text { documented a communicative, collaborative assessment } \\
\text { process. Thematic analysis of interview responses in the } \\
\text { data set indicates a team approach to assessment was a } \\
\text { factor that contributed to understanding of the situation } \\
\text { (See key quotes in Section } 3 \text { ). This factor resonates with } \\
\text { AFI Principle } 6 \text { : Working together in a collaborative } \\
\text { partnership to search for explanations to the situation. }\end{array}$ & $\begin{array}{l}\text { At the mesosystem level, active } \\
\text { participation and communication } \\
\text { between home, school and school } \\
\text { psychological service served to } \\
\text { strengthen supportive links and } \\
\text { the developmental potential of } \\
\text { these settings to identify and } \\
\text { understand needs } \\
\text { (Bronfenbrenner, 1979). Engaging } \\
\text { in discussion also provided insight } \\
\text { into how features and events may } \\
\text { be 'experienced' by Jim in these } \\
\text { settings. Furthermore, at the } \\
\text { microsystem level, Jim's active } \\
\text { involvement during the process } \\
\text { indicates a shifting balance of } \\
\text { power towards him as the } \\
\text { developing person } \\
\text { (Bronfenbrenner, 1979). }\end{array}$ & $\begin{array}{l}\text { From a SCT perspective, } \\
\text { insight into Jim's actual and } \\
\text { potential levels of } \\
\text { development was conducted } \\
\text { through assessment tasks, } \\
\text { including observations and } \\
\text { 1:1 cognitive and attainment } \\
\text { testing and was theorised } \\
\text { through ZAD: what Jim could } \\
\text { do unassisted and ZPD; what } \\
\text { Jim could do with tools and } \\
\text { signs (e.g. counters and } \\
\text { mnemonic aids) and } \\
\text { assistance (Palinscar, 1998; } \\
\text { Vygotsky, 1978). Interactions } \\
\text { that facilitated learning were } \\
\text { later translated into } \\
\text { intervention } \\
\text { recommendations. }\end{array}$ \\
\hline $\begin{array}{l}\text { Perspectives } \\
\text { on barriers } \\
\text { to parental } \\
\text { engagement }\end{array}$ & $\begin{array}{l}\text { Pattern-matching of interview responses connected } \\
\text { understanding of Jim's situation to interactions and } \\
\text { activities during the AFI process, including engaging in } \\
\text { discussion, hearing the voice of each team member and } \\
\text { team assessment tasks. Active involvement of participants } \\
\text { varied during the process. Tina (parent) was not always } \\
\text { present at meetings and researcher reflections document }\end{array}$ & $\begin{array}{l}\text { Unaddressed teacher belief } \\
\text { systems and values could hinder } \\
\text { the developmental potential of } \\
\text { home and school settings to } \\
\text { identify and understand Jim's } \\
\text { needs (Bronfenbrenner, 1979). }\end{array}$ & \\
\hline
\end{tabular}


pragmatism was applied in proceeding through the five stages of AFI (See Appendices 51 and 52). Thematic analysis of participant interview responses identified 'Perspectives on barriers to parental engagement' as a theme in the data set, presenting viewpoints on parental engagement during the assessment process. For example, Sarah noted it was "such a pity" Tina could not be present at all scheduled meetings, which could be reflective of her own belief systems and values as a teacher and parent (See Section 3). Judgements and belief systems could hinder the development of collaborative partnerships between home, school, school psychological service and child promoted by AFI Principle 6.

Views and Thematic analysis identified 'Views and experiences of experiences AFI' as a theme in the dataset and provides further of AFI explanation of how participants' understanding of Jim's situation improved following engagement in the AFI process. Michael and Sarah considered AFI a framework to facilitate teamwork, consistent with AFI Principle 6. Michael commented "it is a real process and you're involved in it" and Sarah described AFI as "a tool for him (Jim) to use, to express his opinion on how his learning might be improved". Michael reflected on the "gradual, step-by-step" approach and Sarah referred to the process as "so clear and so precise of what we had to do". A clear and structured assessment process, resonates with AFI Principle 7: A systematic and transparent assessment process. The researcher adhered to the systematic and transparent assessment process with the use of the AFI templates.
Thematic analysis suggest the AFI model provided a framework to facilitate teamwork and positive outcomes with (See Section 3). At the mesosystem level, active participation and communication between home, school and school psychological service may have served to strengthen the developmental potential of these settings to identify and understand Jim's needs. At the microsystem level, inclusion of Jim in the process may have encouraged strengthening of dyadic relations (Bronfenbrenner, 1979) 
Appendix 35 Case 1 Analysis of Findings: Proposition 2

The proceeding sections provide a comprehensive analysis of Proposition 2 findings for Case 1 . Section 1 below outlines the criteria for interpreting the findings, also presented in in Section 3.5.5 in Chapter 3. Section 2 presents the analysis of findings. Section 3 outlines the themes relevant to Proposition 2 and Section 4 provides a summary of findings. Section 5 provides a conceptual analysis of Proposition 2 findings.

Section 1: Criteria for Interpreting Proposition 2 Findings (Case 1)

\begin{tabular}{|c|c|c|c|c|}
\hline $\begin{array}{l}\text { Proposition: } \\
\text { Following } \\
\text { Engagement } \\
\text { with the } \\
\text { AFI Model: }\end{array}$ & $\begin{array}{l}\text { Likert Scale } \\
\text { Statements }\end{array}$ & $\begin{array}{l}\text { Likert Scale } \\
\text { Statements } \\
\text { (Comments } \\
\text { Section) }\end{array}$ & $\begin{array}{l}\text { Semi-structured } \\
\text { Interviews }\end{array}$ & $\begin{array}{l}\text { Researcher } \\
\text { Diary }\end{array}$ \\
\hline $\begin{array}{l}\text { Proposition } \\
\text { 2: 'Parents, } \\
\text { teachers and } \\
\text { TEP will } \\
\text { know what } \\
\text { intervention } \\
\text { supports the } \\
\text { child needs } \\
\text { at home and } \\
\text { at school, as } \\
\text { a result of } \\
\text { interactions } \\
\text { and activities } \\
\text { that have } \\
\text { occurred at } \\
\text { the meso and } \\
\text { microsystem } \\
\text { levels' }\end{array}$ & $\begin{array}{l}\text { A change in } \\
\text { rating on } \\
\text { associated } \\
\text { Likert scale } \\
\text { statements } \\
\text { indicates } \\
\text { change in } \\
\text { knowledge of } \\
\text { what } \\
\text { intervention } \\
\text { supports the } \\
\text { child needs } \\
\text { following } \\
\text { engagement } \\
\text { with the AFI } \\
\text { model and as } \\
\text { a result of } \\
\text { interactions } \\
\text { and activities } \\
\text { that have } \\
\text { occurred at } \\
\text { the meso and } \\
\text { microsystem } \\
\text { levels. }\end{array}$ & $\begin{array}{l}\text { Pattern- } \\
\text { matching: } \\
\text { Comments } \\
\text { that link } \\
\text { knowledge } \\
\text { of } \\
\text { intervention } \\
\text { supports the } \\
\text { child needs, } \\
\text { engagement } \\
\text { with the AFI } \\
\text { model and } \\
\text { interactions } \\
\text { and activities } \\
\text { that have } \\
\text { occurred at } \\
\text { the meso and } \\
\text { microsystem } \\
\text { levels and } \\
\text { vice versa. }\end{array}$ & $\begin{array}{l}\text { Pattern-matching: } \\
\text { Interview responses } \\
\text { that link knowledge } \\
\text { of intervention } \\
\text { supports the child } \\
\text { needs, engagement } \\
\text { with the AFI model } \\
\text { and interactions and } \\
\text { activities that have } \\
\text { occurred at the meso } \\
\text { and microsystem } \\
\text { levels and vice } \\
\text { versa. } \\
\text { Thematic analysis: } \\
\text { Themes and patterns } \\
\text { within the data set } \\
\text { that refer to } \\
\text { knowledge of } \\
\text { intervention } \\
\text { supports the child } \\
\text { needs and } \\
\text { interactions and } \\
\text { activities that have } \\
\text { occurred at the meso } \\
\text { and microsystem } \\
\text { levels were included } \\
\text { in the analysis of the } \\
\text { AFI model. }\end{array}$ & $\begin{array}{l}\text { Excerpts } \\
\text { from the } \\
\text { researcher } \\
\text { diary that } \\
\text { referred to } \\
\text { knowledge } \\
\text { of } \\
\text { intervention } \\
\text { supports the } \\
\text { child needs } \\
\text { and } \\
\text { interactions } \\
\text { and } \\
\text { activities } \\
\text { that have } \\
\text { occurred at } \\
\text { the meso } \\
\text { and } \\
\text { microsystem } \\
\text { levels were } \\
\text { included in } \\
\text { the analysis } \\
\text { of the AFI } \\
\text { model. }\end{array}$ \\
\hline
\end{tabular}


Section 2: Analysis of Proposition 2 Findings (Case 1)

\begin{tabular}{|c|c|c|c|c|}
\hline $\begin{array}{l}\text { Evidence } \\
\text { for } \\
\text { Proposition } \\
2 \text { (Case 1) }\end{array}$ & $\begin{array}{l}\text { Likert Scale } \\
\text { Statements }\end{array}$ & $\begin{array}{l}\text { Likert } \\
\text { Scale } \\
\text { Statements } \\
\text { (Comments } \\
\text { Section) } \\
\end{array}$ & Semi-structured Interviews & Researcher Diary \\
\hline $\begin{array}{l}\text { Parent } \\
\text { (Tina) }\end{array}$ & $\begin{array}{l}\text { I know what } \\
\text { supports my } \\
\text { child needs at } \\
\text { home: } \\
\text { Before AFI: } 5 \\
\text { After AFI: } 10\end{array}$ & $\begin{array}{l}\text { N/A (See } \\
\text { section } \\
3.11 .2)\end{array}$ & $\begin{array}{l}\text { Pattern-matching: Did the intervention recommendations } \\
\text { address your questions at the intake stage? Yeah because at } \\
\text { home with the spellings and stuff (knowledge of } \\
\text { interventions)...like if I told him to do spellings at home like, } \\
\text { if I was doing it like you were doing, he would do it after me, } \\
\text { he would do it like (interactions and activities during the } \\
\text { AFI process) (lines 129-130) } \\
\text { Do you feel you can support Jim's needs at home? Yeah, I can } \\
\text { now, cause I know what to do now. I know what to do } \\
\text { and...(line 163) I get him books and stuff do you know, and } \\
\text { getting him to do it (knowledge of interventions/interactions } \\
\text { and activities during the AFI process), and yeah, he's } \\
\text { enjoying it now more...yeah he's enjoying it now more (line } \\
\text { 165-166) } \\
\text { Thematic analysis (See Section } 3 \text { below for key quotes of } \\
\text { themes and subthemes relevant to Proposition 2): } \\
\text { Factors bridging the gap between assessment and intervention } \\
\text {-Shared awareness of strengths, needs and goals identified } \\
\text {-Experiencing or observing intervention effectiveness }\end{array}$ & $\begin{array}{l}\text { Researcher diary (AFI } \\
\text { templates) documented } \\
\text { specific activities and } \\
\text { interactions that took place } \\
\text { during the five-stage AFI } \\
\text { process (See Section 4.9, Table } \\
\text { 4.11) }\end{array}$ \\
\hline
\end{tabular}




\begin{tabular}{lll}
\hline Class & I know what & N/A (See \\
teacher & supports the & section \\
(Michael) & student needs & $3.11 .2)$ \\
& at school: & \\
& Before AFI: 5 \\
& After AFI: 9
\end{tabular}

Pattern-matching: Did the intervention recommendations address your questions at the intake stage? Oh the recommendations they did for sure, I suppose definitely the recommendations, you know appraisal and also just ideas for working on his oral language and his memory and auditory skills (knowledge of interventions/interactions and activities during the AFI process), so definitely recommendations that have addressed and I feel moving forward they will be a very good tool to help support Jim's needs (lines 69-72)

Do you feel you can support Jim's needs at school?

$\mathrm{Hmm}$, am I definitely feel more prepared and equipped to support them now, so am I think yeah for sure having a very strong relationship with the learning support teacher and with the parents and obviously the recommendations here ,I think yeah like, investing time and prioritising, you know his needs, which I'm definitely more aware of now, I think we can support them better to definitely help you know, maybe am...to strengthen his working memory in particular and oral language, so good ideas like with the resources, definitely worthwhile investing in them and we can use them to really strengthen and... working memory in particular(knowledge of interventions/interactions and activities during the AFI process), I think that's the biggest one I think, so...(lines 7482)

Thematic analysis (See Section 3 below for key quotes of themes and subthemes relevant to Proposition 2):

Factors bridging the gap between assessment and intervention -Shared awareness of strengths, needs and goals identified
Researcher diary (AFI

templates) documented specific activities and interactions that took place during the five-stage AFI process (See Section 4.9, Table 4.11) 
SET (Sarah) I know what supports the Before AFI: 5 After AFI: 10

Researcher

N/A

(TEP) student needs at school:

N/A (See section
Pattern-matching: Did the intervention recommendations address your questions at the intake stage? 100\% we know now (knowledge of interventions), because it's so clear it's so specific now, like I said we were unsure as to what to do, totally unaware on one aspect but unsure also as to our questions at the beginning (lines 98-99)

Do you feel you can support Jim's needs at school? ...I suppose one of the big ones was in the literacy and the spelling (knowledge of interventions), we did notice from the offset that he wasn't overly strong in spelling, but now from the recommendations and even Jim, I suppose it stands out more so in my head because he's so keen to use this recommendation which is brilliant (interactions and activities during the AFI process), it's the acronym for a word like for arguments sake (knowledge of interventions/(interactions and activities during the AFI process), his one that he produced in class on Friday, he said for the word 'said'(118-122)

Thematic analysis (See Section 3 below for key quotes of themes and subthemes relevant to Proposition 2):

Factors bridging the gap between assessment and intervention -Shared awareness of strengths, needs and goals identified -Experiencing or observing intervention effectiveness
Researcher diary (AFI templates) documented specific activities and interactions that took place during the five-stage AFI process (See Section 4.9, Table 4.11)
The researcher's knowledge of intervention supports is evidenced through the AFI 
templates (researcher diary)

which guided hypothesis

formation, needs, goals and

recommendations (See

Appendix 52).

Researcher reflections indicate intervention planning was

supported by participants

sharing their feedback on co-

investigator activities. For

example:

"Teachers provided feedback on their observations, the SET reflected on her expectations and teaching strategies working well/what could be modified. I shared some strategies that I found worked well during testing" (Reflective Journal November $7^{\text {th }}$, Integration stage). 


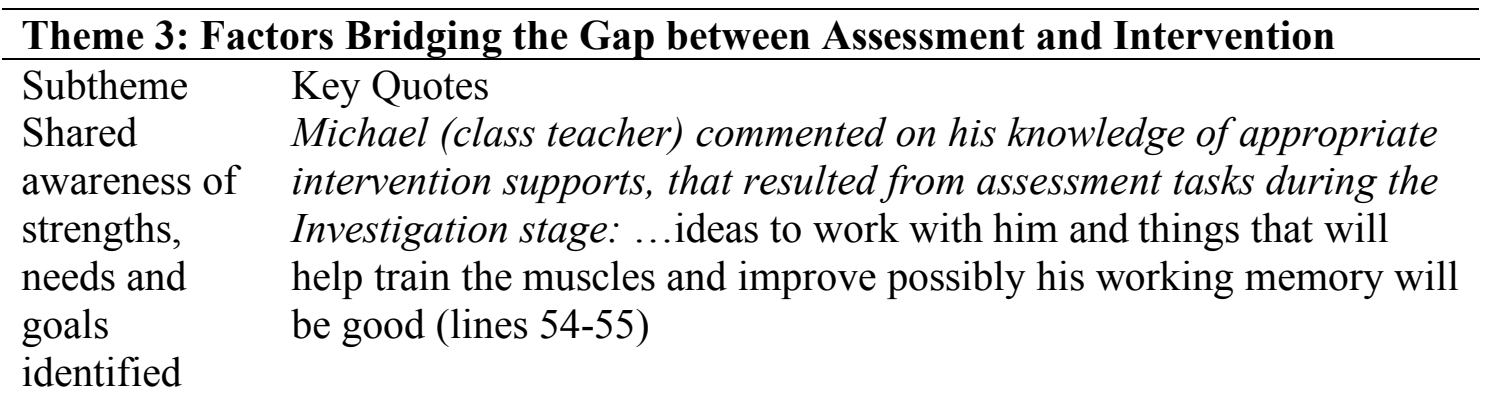

When describing the benefits of the AFI model, Michael remarked on how sharing information between home and school led to the development of intervention supports: ... how you could bring those strengths and his personal interests into the school setting to give an opportunity to, maybe work on his oral language skills (lines 137139)

When commenting on her role during the AFI process, Sarah (SET) referred to the identification of Jim's strengths and weaknesses and using strengths to address areas of weakness, which was discussed between team member during the process:...trying to work his strengths on his weaknesses also, do you know, trying to see if they helped in developing his weaknesses (lines 53-54)

When describing how the intervention recommendations addressed her questions at the Intake stage, Sarah commented: But now we know what the situation is, we know, we have recommendations to address those problems, and how to constantly to keep on improving, step-by-step how we can build and develop Jim to the child that he can possibly be (lines 100-102)

When describing how she would support Jim's needs at home, Tina (mother) referred to providing support during homework, which was discussed at meetings during the AFI process: I get him books and stuff do you know, and getting him to do it and yeah (line 165)

Tina also commented: I know now to get him to do a bit more, do more with him (lines 172-173)

Experiencing When describing his experience of the AFI model, Michael (class or observing intervention effectiveness teacher) commented: we saw the progress [in reference to Jim's progress in areas of need] (line 5)

When commenting on the mnemonic intervention strategy for remembering spelling words, Sarah (SET) commented: it's great because it works (line 135)

When commenting on the mnemonic intervention strategy for remembering spelling words, Tina (mother) also commented: I feel it working (lines 133-134) 


\section{Section 4: Summary of Proposition 2 Findings (Case 1)}

Higher parent and teacher Likert scale ratings, following engagement with the AFI model, indicate greater knowledge of intervention supports. Patterns within parent and teacher interview responses linked knowledge of intervention supports to engagement with the AFI model and interactions and activities that occurred at the meso and microsystem level during the Investigation Stage. Michael referred to intervention supports that arose from assessment activities at the microsystem level during the Investigation stage, including "appraisal" and "ideas for working on his oral language and his memory and auditory skills". Similarly, Sarah and Tina referred to their knowledge of the "acronym", a mnemonic intervention for spelling, which arose during 1:1 dynamic assessment with Jim and became a strategy that he later referred to in his co-investigator activity. See Section 4.9, Table 4.11 for a list of the activities and interactions that participants engaged in. These activities and interactions are considered consistent with the AFI process. In accordance with AFI Principle 6, Michael, Sarah, Tina and Jim were considered 'co-assessors' during the Investigation stage and coconstructed knowledge around intervention supports in this regard (See Section 5 above). Additionally and consistent with AFI principle 7, the researcher's knowledge of intervention supports was evidenced through the AFI templates (researcher diary) (Pameijer, 2017). The templates guided hypothesis formation, needs, goals and recommendations throughout the AFI process. Researcher reflections indicate intervention planning was supported by participants sharing their feedback on coinvestigator activities, consistent with the collaborative partnerships promoted in AFI Principle 6. The theme 'Factors bridging the gap between assessment and intervention' referred to knowledge of intervention supports and interactions and activities that occurred at the meso and microsystem levels (See Section 3). Section 5 below provides a conceptual analysis of the findings and themes, in accordance with AFI principles, EST and SCT concepts. 
Section 5: Conceptual Analysis of Proposition 2 Findings (Case 1)

\begin{tabular}{|c|c|c|c|}
\hline $\begin{array}{l}\text { Theme/ } \\
\text { Subtheme }\end{array}$ & AFI Principles (See section 2.16 for description of AFI Principles) & $\begin{array}{l}\text { EST Concepts (See } \\
\text { section 2.14) }\end{array}$ & $\begin{array}{l}\text { SCT Concepts (See } \\
\text { section 2.15) }\end{array}$ \\
\hline $\begin{array}{l}\text { Shared } \\
\text { awareness of } \\
\text { strengths, } \\
\text { needs and } \\
\text { goals } \\
\text { identified }\end{array}$ & $\begin{array}{l}\text { Knowledge of intervention supports resulted from sharing of } \\
\text { information gathered during the Investigation Stage, as evidenced } \\
\text { through pattern-matching logic applied to interview responses. } \\
\text { Researcher reflections indicate intervention planning was supported } \\
\text { by participants sharing their feedback on co-investigator activities } \\
\text { (See Section } 2 \text { above). Thematic analysis of interview responses in the } \\
\text { data set indicates shared awareness of needs and goals was a factor in } \\
\text { bridging the gap between assessment and intervention (See key quotes } \\
\text { in Section 3). This is consistent with AFI Principle } 6 \text { as insights and } \\
\text { solutions of the parent, teacher and child were considered just as } \\
\text { important as those of the TEP and information was discussed 'with' } \\
\text { team members rather than 'about' or 'to' them (Pameijer, 2017). }\end{array}$ & $\begin{array}{l}\text { Shared awareness of } \\
\text { strengths, needs and } \\
\text { goals identified between } \\
\text { home, school, child and } \\
\text { school psychological } \\
\text { service is reflective of } \\
\text { strengthening links } \\
\text { between these settings } \\
\text { and their developmental } \\
\text { potential to meet Jim's } \\
\text { needs (Bronfenbrenner, } \\
\text { 1979). }\end{array}$ & $\begin{array}{l}\text { For information to be } \\
\text { shared with } \\
\text { participants, appropriate } \\
\text { language use was } \\
\text { considered by the TEP } \\
\text { before, during and after } \\
\text { interactions. Shared } \\
\text { information on Jim's } \\
\text { learning potential and } \\
\text { strategies observed to } \\
\text { assist his learning } \\
\text { demonstrates ZPD. }\end{array}$ \\
\hline $\begin{array}{l}\text { Experiencing } \\
\text { or observing } \\
\text { intervention } \\
\text { effectiveness }\end{array}$ & $\begin{array}{l}\text { Knowledge of intervention supports was linked to activities and } \\
\text { interactions that occurred during the Investigation stage, including co- } \\
\text { investigator tasks (See Section 4.9, Table } 4.11 \text { ). Thematic analysis of } \\
\text { interview responses in the data set indicates experiencing or observing } \\
\text { intervention effectiveness during the Investigation stage was a factor } \\
\text { in bridging the gap between assessment and intervention (See key } \\
\text { quotes in Section 3). This resonates with AFI Principle } 6 \text { as } \\
\text { participants were considered 'co-assessors' and engaged in tasks and } \\
\text { activities to investigate the effectiveness of intervention strategies at } \\
\text { the Investigation stage. See Section } 4.9 \text {, Table } 4.11 \text { for list of specific } \\
\text { activities that participants engaged in. Observing the effectiveness of } \\
\text { intervention strategies is also consistent with AFI Principle } 4 \text { and 5; } \\
\text { Focusing on the needs of the teacher, parent and student to discover } \\
\text { what the ideal approach for Jim might be (Pameijer, 2017). }\end{array}$ & $\begin{array}{l}\text { Parent and teacher } \\
\text { observations of the } \\
\text { effectiveness of } \\
\text { intervention strategies } \\
\text { during reflects } \\
\text { strengthening dyadic } \\
\text { relations between parent } \\
\text { and child and teacher } \\
\text { and student, through } \\
\text { participation in one } \\
\text { another's activities } \\
\text { (Bronfenbrenner, 1979), } \\
\text { e.g. joint participation } \\
\text { in the mnemonic } \\
\text { spelling strategy. }\end{array}$ & $\begin{array}{l}\text { Signs, including the } \\
\text { spelling mnemonic aid } \\
\text { were observed to be } \\
\text { effective in supporting } \\
\text { Jim's learning during } \\
\text { the Investigation stage } \\
\text { consistent with SCT } \\
\text { (Palinscar, 1998; } \\
\text { Vygotsky, 1978). }\end{array}$ \\
\hline
\end{tabular}


Appendix 36 Case 1 Analysis of Findings: Proposition 3

The proceeding sections provide a comprehensive analysis of Proposition 3 findings for Case 1 . Section 1 below outlines the criteria for interpreting the findings, also presented in in Section 3.5.5 in Chapter 3. Section 2 presents the analysis of findings. Section 3 outlines the themes relevant to Proposition 3 and Section 4 provides a summary of findings. Section 5 provides a conceptual analysis of Proposition 3 findings.

Section 1: Criteria for Interpreting Proposition 3 Findings (Case 1)

\begin{tabular}{|c|c|c|c|c|}
\hline $\begin{array}{l}\text { Proposition: } \\
\text { Following } \\
\text { Engagement } \\
\text { with the AFI } \\
\text { Model: }\end{array}$ & $\begin{array}{l}\text { Likert Scale } \\
\text { Statements }\end{array}$ & $\begin{array}{l}\text { Likert Scale } \\
\text { Statements } \\
\text { (Comments } \\
\text { Section) }\end{array}$ & $\begin{array}{l}\text { Semi-structured } \\
\text { Interviews }\end{array}$ & $\begin{array}{l}\text { Researcher } \\
\text { Diary }\end{array}$ \\
\hline $\begin{array}{l}\text { Proposition 3: } \\
\text { 'Teachers and } \\
\text { parents will } \\
\text { have greater } \\
\text { perceived } \\
\text { competence in } \\
\text { supporting the } \\
\text { child's needs } \\
\text { at home and at } \\
\text { school, as a } \\
\text { result of } \\
\text { interactions } \\
\text { and activities } \\
\text { that have } \\
\text { occurred at the } \\
\text { meso and } \\
\text { microsystem } \\
\text { levels' }\end{array}$ & $\begin{array}{l}\text { A change in } \\
\text { rating on } \\
\text { associated } \\
\text { Likert scale } \\
\text { statements } \\
\text { indicates } \\
\text { change in } \\
\text { perceived } \\
\text { competence in } \\
\text { supporting the } \\
\text { child's needs } \\
\text { following } \\
\text { engagement } \\
\text { with the AFI } \\
\text { model and as a } \\
\text { result of } \\
\text { interactions } \\
\text { and activities } \\
\text { that have } \\
\text { occurred at the } \\
\text { meso and } \\
\text { microsystem } \\
\text { levels. }\end{array}$ & $\begin{array}{l}\text { Pattern- } \\
\text { matching: } \\
\text { Comments } \\
\text { that link } \\
\text { competence } \\
\text { in } \\
\text { supporting } \\
\text { the child's } \\
\text { needs, } \\
\text { engagement } \\
\text { with the AFI } \\
\text { model and } \\
\text { interactions } \\
\text { and } \\
\text { activities } \\
\text { that have } \\
\text { occurred at } \\
\text { the meso } \\
\text { and } \\
\text { microsystem } \\
\text { levels and } \\
\text { vice versa. }\end{array}$ & $\begin{array}{l}\text { Pattern-matching: } \\
\text { Interview responses } \\
\text { that link competence } \\
\text { in supporting the } \\
\text { child's needs, } \\
\text { engagement with the } \\
\text { AFI model and } \\
\text { interactions and } \\
\text { activities that have } \\
\text { occurred at the meso } \\
\text { and microsystem } \\
\text { levels and vice } \\
\text { versa. } \\
\text { Thematic analysis: } \\
\text { Themes and patterns } \\
\text { within the data set } \\
\text { that refer to } \\
\text { competence in } \\
\text { supporting the } \\
\text { child's needs and } \\
\text { interactions and } \\
\text { activities that have } \\
\text { occurred at the meso } \\
\text { and microsystem } \\
\text { levels were included } \\
\text { in the analysis of the } \\
\text { AFI model. }\end{array}$ & N/A \\
\hline
\end{tabular}


Section 2: Analysis of Proposition 3 Findings (Case 1)

\begin{tabular}{|c|c|c|c|c|}
\hline $\begin{array}{l}\text { Evidence } \\
\text { for } \\
\text { Proposition } \\
3 \text { (Case 1) }\end{array}$ & $\begin{array}{l}\text { Likert } \\
\text { Scale } \\
\text { Statements }\end{array}$ & $\begin{array}{l}\text { Likert Scale } \\
\text { Statements } \\
\text { (Comments) }\end{array}$ & Semi & $\begin{array}{l}\text { Researcher } \\
\text { Diary }\end{array}$ \\
\hline $\begin{array}{l}\text { Parent } \\
\text { (Tina) }\end{array}$ & $\begin{array}{l}\text { I am able to } \\
\text { support my } \\
\text { child's } \\
\text { needs at } \\
\text { home } \\
\text { Before AFI: } \\
6 \\
\text { After AFI: } \\
10\end{array}$ & $\begin{array}{l}\text { N/A (See } \\
\text { section } \\
3.11 .2)\end{array}$ & $\begin{array}{l}\text { Pattern-matching: Do you feel you can support Jim's needs at home? Yeah, I can now } \\
\text { (competence), cause I know what to do now (interactions and activities during the } \\
\text { AFI process) (line 163) } \\
\text { Thematic analysis (See Section } 3 \text { below for key quotes of themes and subthemes } \\
\text { relevant to Proposition 3): } \\
\text { A team approach to assessment } \\
\text {-Strengthening relationships } \\
\text { Perspectives on barriers to parental engagement } \\
\text {-Judgements, belief systems, practices and values } \\
\text {-Apprehension of the assessment process and outcomes } \\
\text { Factors bridging the gap between assessment and intervention } \\
\text {-Practical interventions }\end{array}$ & $\begin{array}{l}\text { Researcher } \\
\text { diary (AFI } \\
\text { templates) } \\
\text { documented } \\
\text { specific } \\
\text { activities } \\
\text { and } \\
\text { interactions } \\
\text { that took } \\
\text { place during } \\
\text { the five- } \\
\text { stage AFI } \\
\text { process } \\
\text { (See Section } \\
4.9, \text { Table } \\
4.11)\end{array}$ \\
\hline
\end{tabular}


Thematic analysis (See Section 3 below for key quotes of themes and subthemes relevant to Proposition 3):

A team approach to assessment

-Strengthening relationships

Perspectives on barriers to parental engagement

-Judgements, belief systems, practices and values

-Apprehension of the assessment process and outcomes

Factors bridging the gap between assessment and intervention

-Practical interventions

$\begin{array}{lll}\text { SET } & \text { I am able to } & \text { N/A (See } \\ \text { Sarah) } & \text { support the } & \text { section } \\ & \text { student's } & 3.11 .2) \\ & \text { needs at } \\ & \text { school: } \\ & \text { Before AFI: } \\ & 4 \\ & \text { After AFI: }\end{array}$

10
Pattern-matching: Do you feel you can support Jim's needs at school? Yes 100\% now (competence), did I feel at the beginning, no I didn't feel that I could, I wasn't sure of his needs. Now I know exactly what his needs are (interactions and activities during the AFI process), I know what his needs are in literacy and in numeracy and with regard to working memory (interactions and activities during the AFI process), I know his needs and I know that the recommendations that you have given us (interactions and activities during the AFI process) I know that we can work on those recommendation (competence) (lines 109-112)

Thematic analysis (See Section 3 below for key quotes of themes and subthemes relevant to Proposition 3):

A team approach to assessment

-Strengthening relationships

Perspectives on barriers to parental engagement

-Judgements, belief systems, practices and values

-Apprehension of the assessment process and outcomes

Factors bridging the gap between assessment and intervention

-Practical interventions place during

the five-

stage AFI

process

(See Section

4.9, Table

4.11)

Researcher diary (AFI templates)

documented specific activities

and

interactions that took place during the fivestage AFI process (See Section 4.9,

Table 4.11) 


\section{Section 3: Themes Relevant to Proposition 3 (Case 1)}

\section{Theme 1: A Team Approach to Assessment \\ Subthemes Key Quotes \\ Strengthen- $\quad$ When commenting on how he would monitor Jim's needs moving \\ ing forward, Michael (class teacher) referred to his strengthened \\ relationships relationship with Jim: This whole process has strengthened my relationship with Jim (lines 99-100) \\ When describing her role in the AFI process, Tina (parent) referred to her increased involvement in Jim's activities: I kind of got more involved with Jim do you know, he let me do more with him, with his homework and stuff (lines 26-27) \\ Tina described strengthening relationships between home and school and child and teachers following engagement with the AFI model: I can talk to the teachers more and if I've a problem I can go to them now and Jim can as well (line 246)}

Tina described strengthening relationships between child and TEP: He was happy coming in here and doing work with you (line 96)

\section{Theme 2: Perspectives on Barriers to Parental Engagement}

\section{Subthemes Judgements, belief systems, practices and values}

\section{Key Quotes}

When describing how she would support Jim's needs, Tina commented on poor communication between home and school the previous school year and she had not been aware of Jim's level of need before the AFI process: Yeah cause I didn't know he was...he needed that much help, no I didn't think he needed it (line 138)

Tina also commented that she felt she wasted time last year: You know I kinda feel like I've wasted a year of his life, that's what I feel...of learning, not now, last year all last year... (lines 107-108)
Apprehension of the assessment process and outcomes
Tina referred to interactions with a previous class teacher, which may have caused her to feel apprehensive about engaging with the current team: he's a bit of a fairy I didn't like that, that's still in my brain, he doesn't want to work... but like he's not going to if you're not going to help him like (lines 149-150)

\begin{tabular}{ll}
\hline Theme 3: Factors Bridging the Gap between Assessment and Intervention \\
\hline $\begin{array}{l}\text { Subthemes } \\
\text { Practical } \\
\text { interventions Quotes }\end{array}$ & $\begin{array}{l}\text { When describing how she would support Jim's needs, Sarah (SET) } \\
\text { referred to how intervention recommendations were useful to all the } \\
\text { class: ...that all of the class wanted to take on (mnemonic spelling } \\
\text { aid), which was great for Jim because it gave him ownership of this } \\
\text { new game and now all the class, they ask 'Miss can we all have one } \\
\text { each night? (lines 128-130) }\end{array}$ \\
& $\begin{array}{l}\text { Sarah commented on the utility of intervention recommendations: } \\
\text { Very clear, easy, specific recommendations, simple like again you } \\
\text { don't have to buy a shop to implement them...(lines 232-233) }\end{array}$
\end{tabular}




\section{Section 4: Summary of Proposition 3 Findings (Case 1)}

Higher Likert scale ratings indicate greater perceived competence by parent and teachers in supporting Jim's needs after engaging with the AFI model. Patterns within parent and teacher interview responses connected activities and interactions that occurred at the meso and microsystem levels throughout the AFI process, including dialogue during meetings, co-investigator tasks (e.g. observations) and 1:1 assessment to perceived competence to support Jim's needs. See Section 4.9, Table 4.11 for a list of the activities and interactions that participants engaged in. For example, Michael commented that he feels "more prepared and equipped to support them (needs) now' and attributes this to knowing what Jim's needs are, the recommendations that will address his needs and his strong relationship with the SET and parent. Consistent with AFI Principle 6, all participants were 'co-assessors' during the Investigation stage and their active participation led to the identification of needs and subsequently, intervention recommendations (Pameijer, 2017). It is possible that working together as co-assessors also strengthened relationships between home and school. These factors appear to have strengthened Michael's perceived competence to support Jim's needs, as evidenced through pattern-matching logic in Section 2 above. Similarly, awareness of Jim's needs and what to do to support his needs following engagement in the AFI process strengthened Sarah and Tina's competence to support Jim's needs. Arriving at feasible intervention recommendations that can be implemented by parents and teachers is consistent with AFI Principle 1 'Goal directed and functional assessment', with the EP aiming at feasible intervention recommendations from the outset of the assessment (Pameijer, 2017).Themes within the data set that referred to competence in supporting Jim's needs and interactions and activities that occurred at the meso and microsystem levels included 'A team approach to assessment', 'Perspectives on barriers to parental engagement approach' and 'Factors bridging the gap between assessment and intervention' (See Section 3). Section 5 below provides a conceptual analysis of the findings and themes, in accordance with AFI principles, EST and SCT concepts. 
Section 5: Conceptual Analysis of Proposition 3 Findings (Case 1)

\begin{tabular}{|c|c|c|}
\hline $\begin{array}{l}\text { Theme/ } \\
\text { Subtheme }\end{array}$ & AFI Principles (See section 2.16 for description of AFI Principles) & $\begin{array}{l}\text { EST Concepts (See section } 2.14 \text { for } \\
\text { definitions) }\end{array}$ \\
\hline $\begin{array}{l}\text { Strength- } \\
\text { ening } \\
\text { relation- } \\
\text { ships }\end{array}$ & $\begin{array}{l}\text { Patterns within parent and teacher interview responses connected activities and } \\
\text { interactions that occurred at the meso and microsystem levels throughout the AFI } \\
\text { process, including dialogue during meetings, co-investigator tasks and } 1: 1 \text { assessment to } \\
\text { perceived competence to support Jim's needs. Thematic analysis of interview responses } \\
\text { suggests strengthened relationships as a result of engaging in a team assessment } \\
\text { approach, may further account for perceived competence in meeting Jim's needs (See } \\
\text { key quotes in Section 3). This subtheme resonates with AFI Principle } 6 \text {, where } \\
\text { assessment is built upon collaborative partnerships between TEP, parents, teachers, child }\end{array}$ & $\begin{array}{l}\text { Strengthening relationships at the } \\
\text { mesosystem level reflects the } \\
\text { strengthened developmental potential of } \\
\text { home and school settings, working } \\
\text { towards meeting Jim's needs and at the } \\
\text { microsystem level, suggests strengthened } \\
\text { dyadic relations (Bronfenbrenner, 1979) }\end{array}$ \\
\hline $\begin{array}{l}\text { Perspectives } \\
\text { on barriers } \\
\text { to parental } \\
\text { engagement }\end{array}$ & $\begin{array}{l}\text { Patterns within parent and teacher interview responses connected activities and } \\
\text { interactions that occurred at the meso and microsystem levels throughout the AFI } \\
\text { process to perceived competence to support Jim's needs. Communication and co- } \\
\text { operation between team members was required in accordance with AFI Principle } 6 \\
\text { (Pameijer, 2017). Thematic analysis suggests the level of communication and } \\
\text { collaboration during the AFI process contrasted with the poor communication between } \\
\text { home and school felt by Tina in the previous school year and may have conjured up } \\
\text { feelings of guilt as she stated she "wasted a year of his life". Additionally, language used } \\
\text { by a previous teacher, describing Jim as a "fairy" may have resulted in Tina feeling } \\
\text { apprehensive about engaging with the current team. Poor communication between home } \\
\text { and school contradicts the collaborative partnerships promoted by AFI Principle } 6 \text { and } \\
\text { hinders these settings in working together to support Jim's needs. }\end{array}$ & $\begin{array}{l}\text { Poor communication between home and } \\
\text { school in the previous school year may } \\
\text { have hindered the developmental } \\
\text { potential of these settings in working } \\
\text { together to support Jim's needs } \\
\text { (Bronfenbrenner, 1979). }\end{array}$ \\
\hline $\begin{array}{l}\text { Practical } \\
\text { intervent- } \\
\text { ions }\end{array}$ & $\begin{array}{l}\text { Patterns within parent and teacher interview responses connected activities and } \\
\text { interactions that occurred at the meso and microsystem levels to perceived competence } \\
\text { to support Jim's needs. Thematic analysis of interview responses suggests practical } \\
\text { intervention recommendations may further account for perceived competence in meeting } \\
\text { Jim's needs as they were considered easy to implement (See key quotes in Section 3). } \\
\text { This subtheme resonates with AFI Principle } 1 \text { 'Goal directed and functional assessment', } \\
\text { with the EP aiming at feasible intervention recommendations (Pameijer, 2017). }\end{array}$ & $\begin{array}{l}\text { Providing Jim with opportunities to } \\
\text { develop his spelling skills at home and at } \\
\text { school facilitated the development of this } \\
\text { molar activity. Molar activities are } \\
\text { behaviours perceived as having meaning } \\
\text { or intent by participants in a particular } \\
\text { setting (Bronfenbrenner, 1979). }\end{array}$ \\
\hline
\end{tabular}


Appendix 37 Case 1 Analysis of Findings: Proposition 4

The proceeding sections provide a comprehensive analysis of Proposition 4 findings for Case 1 . Section 1 below outlines the criteria for interpreting the findings, also presented in in Section 3.5.5 in Chapter 3. Section 2 presents the analysis of findings. Section 3 outlines the themes relevant to Proposition 4 and Section 4 provides a summary of findings. Section 5 provides a conceptual analysis of Proposition 4 findings.

Section 1: Criteria for Interpreting Proposition 3 Findings (Case 1)

\begin{tabular}{|c|c|c|c|c|}
\hline $\begin{array}{l}\text { Proposition: } \\
\text { Following } \\
\text { Engagement } \\
\text { with the AFI } \\
\text { Model: }\end{array}$ & $\begin{array}{l}\text { Likert Scale } \\
\text { Statements }\end{array}$ & $\begin{array}{l}\text { Likert Scale } \\
\text { Statements } \\
\text { (Comments } \\
\text { Section) }\end{array}$ & $\begin{array}{l}\text { Semi-structured } \\
\text { Interviews }\end{array}$ & $\begin{array}{l}\text { Researcher } \\
\text { Diary }\end{array}$ \\
\hline $\begin{array}{l}\text { Proposition 4: } \\
\text { 'The child will } \\
\text { have a better } \\
\text { understanding } \\
\text { of their } \\
\text { strengths and } \\
\text { needs and what } \\
\text { to do to } \\
\text { improve their } \\
\text { needs, as a } \\
\text { result of } \\
\text { interactions and } \\
\text { activities that } \\
\text { have occurred } \\
\text { at the meso and } \\
\text { microsystem } \\
\text { levels' }\end{array}$ & $\begin{array}{l}\text { A change in } \\
\text { rating on the } \\
\text { associated } \\
\text { Likert scale } \\
\text { statements } \\
\text { indicates } \\
\text { change in the } \\
\text { child's } \\
\text { understanding } \\
\text { of their } \\
\text { strengths and } \\
\text { needs and } \\
\text { what to do to } \\
\text { improve their } \\
\text { needs, } \\
\text { following } \\
\text { engagement } \\
\text { with the AFI } \\
\text { model and as } \\
\text { a result of } \\
\text { interactions } \\
\text { and activities } \\
\text { that have } \\
\text { occurred at } \\
\text { the meso and } \\
\text { microsystem } \\
\text { levels }\end{array}$ & $\begin{array}{l}\text { Pattern- } \\
\text { matching: } \\
\text { Comments } \\
\text { that link the } \\
\text { child's } \\
\text { understanding } \\
\text { of their } \\
\text { strengths and } \\
\text { needs and } \\
\text { what to do to } \\
\text { improve their } \\
\text { needs, } \\
\text { engagement } \\
\text { with the AFI } \\
\text { model and } \\
\text { interactions } \\
\text { and activities } \\
\text { that have } \\
\text { occurred at } \\
\text { the meso and } \\
\text { microsystem } \\
\text { levels and } \\
\text { vice versa. }\end{array}$ & $\begin{array}{l}\text { Pattern-matching: } \\
\text { Interview responses } \\
\text { that link the child's } \\
\text { understanding of } \\
\text { their strengths and } \\
\text { needs and what to } \\
\text { do to improve their } \\
\text { needs, engagement } \\
\text { with the AFI model } \\
\text { and interactions and } \\
\text { activities that have } \\
\text { occurred at the } \\
\text { meso and } \\
\text { microsystem levels } \\
\text { and vice versa. } \\
\text { Thematic analysis: } \\
\text { Themes and } \\
\text { patterns within the } \\
\text { data set that refer to } \\
\text { understanding of } \\
\text { strengths and needs } \\
\text { and what to do to } \\
\text { improve their } \\
\text { needs, and } \\
\text { interactions and } \\
\text { activities that have } \\
\text { occurred at the } \\
\text { meso and } \\
\text { microsystem levels } \\
\text { were included in } \\
\text { the analysis of the } \\
\text { AFI model. }\end{array}$ & N/A \\
\hline
\end{tabular}


Section 2: Analysis of Proposition 4 Findings (Case 1)

\begin{tabular}{|c|c|c|c|c|}
\hline $\begin{array}{l}\text { Evidence } \\
\text { for } \\
\text { Proposition } \\
4 \text { (Case 1) }\end{array}$ & $\begin{array}{l}\text { Likert Scale } \\
\text { Statements }\end{array}$ & $\begin{array}{l}\text { Likert Scale } \\
\text { Statements } \\
\text { (Comments } \\
\text { Section) }\end{array}$ & Semi-structured Interviews & Researcher Diary \\
\hline \multirow[t]{4}{*}{$\begin{array}{l}\text { Jim } \\
\text { (Student) }\end{array}$} & $\begin{array}{l}\text { I know what } \\
\text { my strengths } \\
\text { are at school } \\
\text { Before AFI: } \\
\text { Yes } \\
\text { After AFI: Yes }\end{array}$ & \multirow[t]{4}{*}{$\begin{array}{l}\text { N/A (See } \\
\text { section 3.11.2) }\end{array}$} & $\begin{array}{l}\text { Pattern-matching: Did working with me, your mother and } \\
\text { teacher (interactions and activities during AFI process) help } \\
\text { you to find out about your strengths? Yeah (understanding } \\
\text { strengths) (line 66)... Am, drawing and reading and a little } \\
\text { bit of maths... and history (interactions and activities during } \\
\text { the AFI process) (line 68) }\end{array}$ & \multirow[t]{4}{*}{$\begin{array}{l}\text { Researcher diary (AFI } \\
\text { templates) documented } \\
\text { specific activities and } \\
\text { interactions that took place } \\
\text { during the five-stage AFI } \\
\text { process (See Section 4.9, } \\
\text { Table 4.11) }\end{array}$} \\
\hline & $\begin{array}{l}\text { I know what I } \\
\text { need help with } \\
\text { at school } \\
\text { Before AFI: } \\
\text { Yes } \\
\text { After AFI: Yes }\end{array}$ & & $\begin{array}{l}\text { Did working with me, your mother and teacher (interactions } \\
\text { and activities during the AFI process) help you to find out } \\
\text { about what you need help with? Yes (understanding needs) } \\
\text { (line } 86 \text { )...Maths and spelling (interactions and activities } \\
\text { during the AFI process) (line 89) }\end{array}$ & \\
\hline & $\begin{array}{l}\text { I know what I } \\
\text { can do to get } \\
\text { better at what I } \\
\text { need help with } \\
\text { at school }\end{array}$ & & $\begin{array}{l}\text { Do you know what you need to do to get better at Maths and } \\
\text { Spelling? Yeah (improve on needs) (line 91)...Spellings } \\
\text { like...just make up silly sentences like and then for maths, } \\
\text { like use counters and you'll get better (interactions and } \\
\text { activities during the AFI process) (lines 93-94) }\end{array}$ & \\
\hline & $\begin{array}{l}\text { Before AFI: } \\
\text { No } \\
\text { After AFI: Not } \\
\text { sure }\end{array}$ & & $\begin{array}{l}\text { Thematic analysis (See Section } 3 \text { below for key quotes of } \\
\text { themes and subthemes relevant to Proposition 4): } \\
\text { A team approach to assessment } \\
\text { - Active roles and communication } \\
\text { - Strengthening relationships }\end{array}$ & \\
\hline
\end{tabular}




\section{Section 3: Themes Relevant to Proposition 4 (Case 1)}

\section{Subthemes \\ Active roles and communication}

Theme 1: A Team Approach to Assessment

\section{Key Quotes}

When describing Jim's role during the AFI process, Michael (class teacher) commented: He was honest in his feedback and he was telling us what works well and what doesn't and what he finds difficult so I think it was really important, like his voice was heard and I think he really appreciated that (line 38-40)

Michael also commented: He [Jim] had a very central role (line 38)

When describing Jim's role during the AFI process, Sarah (SET) commented: In Maths like I said even he was more willing now to tell us 'Miss that didn't work right' or 'I like when you say it that was Miss', which was great (lines 139-140)

When describing the benefits of the AFI model, Tina (parent) commented: So he's [Jim] more open, more putting his hand up (line 212)

Strengthening When describing how he planned to monitor Jim's needs, relationships
Michael (class teacher) referred to his strengthened relationship with Jim as a result of the AFI process: This whole process has strengthened my relationship with Jim (lines 99-100)

When describing her role during the AFI process, Tina commented: Doing more with Jim, I kind of got more involved with Jim do you know, he let me do more with him, with his homework and stuff (lines 26-27)

Tina elaborated further on Jim's reaction to her trying to be more involved in his activities : Like letting me sit beside him and do the homework, do you know what I mean (line 29)

\section{Section 4: Summary of Proposition 4 Findings (Case 1)}

Likert scale ratings provided by Jim indicate he knew what his strengths and needs were before and after engaging with the AFI model. Jim's rating changed from not knowing what he can do to improve on needs at school to being "not sure" following engagement with the AFI model. Patterns within Jim's responses to interview questions addressing Proposition 4 linked understanding of strengths and needs to interactions and activities that occurred during the AFI process, at the microsystem level. Working together with the TEP, parent and teacher was connected to his understanding of strengths and needs. Jim responded "yes" to questions exploring if working together helped him to find out about his strengths and needs. Jim identified his strengths as "drawing, reading and a little bit of maths and history". Activities and 
interactions that led to the identification of reading and maths as strengths included dialogue and 1:1 assessment at the Investigation Stage (See Section 4.9, Table 4.11). A child friendly report complied for Jim at the Feedback stage listed specific areas of reading and maths where Jim showed particular strength and this was discussed with Jim, his class teacher and mother. Jim identified his needs as "maths and spelling". Activities and interactions that led to the identification of reading and maths as strengths included dialogue and 1:1 assessment at the Investigation Stage. The child friendly report also outlined specific needs in the areas of maths and spellings. When asked what he needs to do to get better at maths and spelling, Jim mentioned strategies that had been practiced during 1:1 dynamic assessment at the Investigation stage of the AFI process (making up silly sentences and using counters). The activities and interactions outlined here are considered unique to the AFI process. Consistent with AFI Principle 6, Jim, was considered a 'co-assessor' during the Investigation stage and co-constructed knowledge and planning around intervention supports in this regard (See Section 5 below). The theme 'A team approach to assessment' referred to understanding of strengths and needs and what to do to address needs, and interactions and activities that occurred at the meso and microsystem levels (See Section 3). Section 5 below provides a conceptual analysis of the findings and themes, in accordance with AFI principles, EST and SCT concepts. 
Section 5: Conceptual Analysis of Proposition 4 Findings (Case 1)

\begin{tabular}{|c|c|c|c|}
\hline $\begin{array}{l}\text { Theme/ } \\
\text { Subtheme }\end{array}$ & $\begin{array}{l}\text { AFI Principles (See section } 2.16 \text { for description of AFI } \\
\text { Principles) }\end{array}$ & $\begin{array}{l}\text { EST Concepts (See } \\
\text { section } 2.14 \text { for } \\
\text { concept definitions) }\end{array}$ & $\begin{array}{l}\text { SCT Concepts (See section } 2.15 \text { for } \\
\text { concept definitions) }\end{array}$ \\
\hline $\begin{array}{l}\text { Active roles } \\
\text { and } \\
\text { communication }\end{array}$ & $\begin{array}{l}\text { Patterns within John's responses to interview questions linked } \\
\text { understanding of strengths and needs and what to do to } \\
\text { address needs, to engagement with the AFI model and } \\
\text { interactions and activities that occurred at the meso and } \\
\text { microsystem level. Activities and interactions where Jim } \\
\text { worked together with the TEP, parent and teachers included } \\
\text { dialogue, co-investigator observation tasks and 1:1 } \\
\text { assessments. Thematic analysis of interview responses } \\
\text { suggests Jim's active role in the team assessment approach } \\
\text { provided him the opportunity to give feedback on strategies } \\
\text { that were helping him to learn and he was considered central } \\
\text { to the assessment process. Jim's active involvement as a 'co- } \\
\text { assessor' in the team assessment approach is consistent with } \\
\text { AFI Principle 6: Working together in a collaborative } \\
\text { partnership. }\end{array}$ & $\begin{array}{l}\text { At the microsystem } \\
\text { level, Jim's active } \\
\text { participation reflects } \\
\text { a shifting balance of } \\
\text { power towards him, } \\
\text { as the developing } \\
\text { person } \\
\text { (Bronfenbrenner, } \\
\text { 1979). }\end{array}$ & $\begin{array}{l}\text { From a SCT perspective, insight into } \\
\text { Jim's actual and potential levels of } \\
\text { development was conducted through } \\
\text { assessment tasks, including } \\
\text { observations and 1:1 cognitive and } \\
\text { attainment testing and was theorised } \\
\text { through ZAD: what Jim could do } \\
\text { unassisted and ZPD; what Jim could } \\
\text { do with tools and signs (e.g. counters } \\
\text { and mnemonic aids) and assistance } \\
\text { (Palinscar, 1998; Vygotsky, 1978). } \\
\text { Interactions that facilitated learning } \\
\text { were later translated into intervention } \\
\text { recommendations. }\end{array}$ \\
\hline $\begin{array}{l}\text { Strengthening } \\
\text { relationships }\end{array}$ & $\begin{array}{l}\text { Thematic analysis of interview responses suggests } \\
\text { strengthened relationships as a result of engaging in a team } \\
\text { assessment approach, may further account for understanding } \\
\text { of strengths and needs. Michael and Tina described how their } \\
\text { relationship with Jim had strengthened during the assessment } \\
\text { process and could be explained by increased interaction and } \\
\text { attention towards each other's activities (See key quotes in } \\
\text { Section 3). This subtheme resonates with AFI Principle 6, } \\
\text { where assessment is built upon collaborative partnerships } \\
\text { between TEP, parents, teachers and child. }\end{array}$ & $\begin{array}{l}\text { Strengthening } \\
\text { relationships } \\
\text { between parent and } \\
\text { child and teacher and } \\
\text { student is suggestive } \\
\text { of strengthened } \\
\text { dyadic relations at } \\
\text { the microsystem } \\
\text { level } \\
\text { (Bronfenbrenner, } \\
\text { 1979). }\end{array}$ & \\
\hline
\end{tabular}


Appendix 38 Case 1 Analysis of Findings: Proposition 5

The proceeding sections provide a comprehensive analysis of Proposition 5 findings for Case 1. Section 1 below outlines the criteria for interpreting the findings, also presented in in Section 3.5.5 in Chapter 3. Section 2 presents the analysis of findings. Section 3 outlines the themes relevant to Proposition 5 and Section 4 provides a summary of findings. Section 5 provides a conceptual analysis of Proposition 3 findings.

Section 1: Criteria for Interpreting Proposition 5 Findings (Case 1)

\begin{tabular}{|c|c|c|c|c|}
\hline $\begin{array}{l}\text { Proposition: } \\
\text { Following } \\
\text { Engagement } \\
\text { with the AFI } \\
\text { Model: }\end{array}$ & $\begin{array}{l}\text { Likert Scale } \\
\text { Statements }\end{array}$ & $\begin{array}{l}\text { Likert Scale } \\
\text { Statements } \\
\text { (Comments } \\
\text { Section) }\end{array}$ & $\begin{array}{l}\text { Semi-structured } \\
\text { Interviews }\end{array}$ & $\begin{array}{l}\text { Researcher } \\
\text { Diary }\end{array}$ \\
\hline $\begin{array}{l}\text { Proposition 5: } \\
\text { 'At a time of } \\
\text { ecological } \\
\text { transition, } \\
\text { teachers will } \\
\text { feel competent } \\
\text { in their ability } \\
\text { to work } \\
\text { according to the } \\
\text { aims of Circular } \\
\text { 0013/2017, in } \\
\text { meeting and } \\
\text { monitoring the } \\
\text { needs of the } \\
\text { child' }\end{array}$ & N/A & $\mathrm{N} / \mathrm{A}$ & $\begin{array}{l}\text { Pattern-matching: } \\
\text { Interview responses } \\
\text { that link } \\
\text { competence in in } \\
\text { meeting and } \\
\text { monitoring the } \\
\text { needs of the child, } \\
\text { engagement with } \\
\text { the AFI model and } \\
\text { interactions and } \\
\text { activities that have } \\
\text { occurred at the } \\
\text { meso and } \\
\text { microsystem levels } \\
\text { and vice versa. }\end{array}$ & N/A \\
\hline & & & $\begin{array}{l}\text { Thematic } \\
\text { analysis: Themes } \\
\text { and patterns within } \\
\text { the data set that } \\
\text { refer to meeting } \\
\text { and monitoring the } \\
\text { needs of the child, } \\
\text { and interactions } \\
\text { and activities that } \\
\text { have occurred at } \\
\text { the meso and } \\
\text { microsystem levels } \\
\text { were included in } \\
\text { the analysis of the } \\
\text { AFI model. }\end{array}$ & \\
\hline
\end{tabular}


Section 2: Analysis of Proposition 5 Findings (Case 1)

\begin{tabular}{|c|c|c|c|c|}
\hline $\begin{array}{l}\text { Evidence } \\
\text { for } \\
\text { Proposition } \\
5 \text { (Case 1) }\end{array}$ & $\begin{array}{l}\text { Likert } \\
\text { Scale } \\
\text { Statements }\end{array}$ & $\begin{array}{l}\text { Likert Scale } \\
\text { Statements } \\
\text { (Comments) }\end{array}$ & Semi-structured Interviews & Researcher Diary \\
\hline $\begin{array}{l}\text { Class } \\
\text { teacher } \\
\text { (Michael) }\end{array}$ & $\mathrm{N} / \mathrm{A}$ & $\mathrm{N} / \mathrm{A}$ & $\begin{array}{l}\text { Pattern-matching: Do you feel you can monitor Jim's needs at school? } \\
\text { I do feel like I can monitor them yeah (competence to meet/monitor } \\
\text { needs), am I think also the relationship I've...this whole process has } \\
\text { strengthened my relationship with Jim (interactions and activities } \\
\text { during AFI process) and you know, he's aware of you know, areas } \\
\text { where he needs support and just to talk to him and ask him the questions } \\
\text { like what worked well or you know, where he is struggling, so I think } \\
\text { monitoring for sure and between having a good relationship with the } \\
\text { learning support teacher, that we can monitor his progress (competence } \\
\text { to meet/monitor needs) (lines 99-103) } \\
\text { Thematic analysis (See Section } \mathbf{3} \text { below for key quotes of themes } \\
\text { and subthemes relevant to Proposition 5): } \\
\text { Factors bridging the gap between assessment and intervention } \\
\text { - Shared awareness of strengths, needs and goals identified } \\
\text { - A collaborative approach to implementing and monitoring } \\
\text { interventions }\end{array}$ & $\begin{array}{l}\text { Researcher diary (AFI } \\
\text { templates) documented } \\
\text { specific activities and } \\
\text { interactions that took place } \\
\text { during the five-stage AFI } \\
\text { process See Section 4.9, } \\
\text { Table 4.11) }\end{array}$ \\
\hline $\begin{array}{l}\text { SET } \\
\text { (Sarah) }\end{array}$ & $\mathrm{N} / \mathrm{A}$ & $\mathrm{N} / \mathrm{A}$ & $\begin{array}{l}\text { Pattern-matching: Do you feel you can monitor Jim's needs at school? } \\
\text { Yeah } 100 \% \text {, yes I can (competence to meet/monitor needs) now that I } \\
\text { know what the needs are (interactions and activities during the AFI } \\
\text { process), I can monitor them (competence to meet/monitor needs). We } \\
\text { can assess, and again him being involved, seeing from, we know where } \\
\text { he's at at the moment, with those recommendations in place }\end{array}$ & $\begin{array}{l}\text { Researcher diary (AFI } \\
\text { templates) documented } \\
\text { specific activities and } \\
\text { interactions that took place } \\
\text { during the five-stage AFI }\end{array}$ \\
\hline
\end{tabular}


(interactions and activities during the AFI process), building on them and monitoring and making sure that they're working do you know, to

process See Section 4.9, the best that they can for him so it's no problem to monitor (competence

to meet/monitor needs) (lines 151-154)

Thematic analysis (See Section 3 below for key quotes of themes and subthemes relevant to Proposition 5):

Factors bridging the gap between assessment and intervention

- Shared awareness of strengths, needs and goals identified

- A collaborative approach to implementing and monitoring interventions 


\section{Theme 3: Factors Bridging the Gap between Assessment and Intervention \\ Subthemes Key Quotes \\ Shared awareness Sarah (SET) connected her ability to monitor Jim's needs of strengths, needs and goals identified moving forward to her awareness of Jim's needs, gained through the process of AFI: I can now that I know what the needs are (line 151)}

When discussing how she would support Jim's needs, Sarah referred to his awareness of goals and strategies: He's aware of strategies that he can do and that he can put in place as well for him to achieve, he knows what he needs to achieve in (lines 144145)

When describing how she would monitor Jim's needs, Sarah referred to monitoring of strategies that were shared between the team and implemented during the Investigation stage of the AFI model: And even like that I suppose, when we're talking about spellings, like starting, the class would get 20 spellings, which was totally out of the Jim's ability, so really going starting at 10 and again with his spelling trick to remember his words, how to spell his words, even if needs be we'll start at 8 and then we'll go up one every week depending on how he's achieving and not overloading and then again seeing if that trick works, it will help him, even if he's only doing half of the words with the trick and then the other half learning them rotely, am but again another way of monitoring to see if the amount of spellings that he's able to do has increased (lines 172-178)

A collaborative Michael (class teacher) described a team approach to approach to monitoring Jim's needs moving forward: Just to talk to him and implementing and monitoring ask him the questions like what worked well or you know, where he is struggling, so I think monitoring for sure and between interventions having a good relationship with the learning support teacher, that we can monitor his progress (lines 101-103)

When describing how she would monitor Jim's needs, Sarah referred to a team approach which included the class teacher and student: I can monitor them. We can assess, and again him being involved, seeing from, we know where he's at at the moment, with those recommendations in place, building on them and monitoring and making sure that they're working do you know, to the best that they can for him (lines 151-154)

Jim commented during interview that he would need the support of his parents and teachers to complete his goals moving forward, reflective of a team approach: To complete them (line 125) 


\section{Section 4: Summary of Proposition 5 Findings (Case 1)}

Pattern-matching logic applied to Michael and Sarah's interview responses connected competence to meet and monitor Jim's needs moving forward to activities and interactions during the AFI process that occurred at the meso and microsystem levels. Patterns within Michael's response linked competence to his "strengthened relationship with Jim" as a result of the "whole process" and "having a good relationship with the learning support teacher". Sarah attributed ability to meet and monitor Jim's needs to her improved awareness of his learning needs, following engagement with the AFI process. Activities and interactions during the AFI process that resulted in identification of Jim's learning needs included dialogue during meetings, observation tasks completed by Jim, his mother and teachers and 1:1 assessments conducted by the TEP, including attainment and cognitive testing and dynamic assessment of skills and potential. Increased awareness of Jim's needs resonates with AFI Principle 3, focusing on the educational needs of the child (Pameijer, 2017). Strengthening relationships between class teacher, SET and student resonates with the collaborative partnerships promoted by AFI Principle 6 . The theme 'Factors bridging the gap between assessment and intervention' referred to meeting and monitoring needs and interactions and activities that occurred at the meso and microsystem levels (See Section 3). Section 5 below provides a conceptual analysis of the findings and themes, in accordance with AFI principles, EST and SCT concepts. 
Section 5: Conceptual Analysis of Proposition 5 Findings (Case 1)

\begin{tabular}{|c|c|c|c|}
\hline $\begin{array}{l}\text { Theme/ } \\
\text { Subtheme }\end{array}$ & $\begin{array}{l}\text { AFI Principles (See section } \\
2.16 \text { for description of AFI } \\
\text { Principles) }\end{array}$ & $\begin{array}{l}\text { EST Concepts } \\
\text { (See section } 2.14 \\
\text { for definitions of } \\
\text { EST concepts) }\end{array}$ & $\begin{array}{l}\text { SCT Concepts } \\
\text { (See section } 2.15 \\
\text { for definitions of } \\
\text { SCT concepts) }\end{array}$ \\
\hline $\begin{array}{l}\text { Factors } \\
\text { bridging } \\
\text { the gap } \\
\text { between } \\
\text { assessment } \\
\text { and } \\
\text { intervention }\end{array}$ & $\begin{array}{l}\text { Pattern-matching logic } \\
\text { applied to Michael and } \\
\text { Sarah's interview responses } \\
\text { connected competence to } \\
\text { meet and monitor Jim's needs } \\
\text { moving forward to activities } \\
\text { and interactions that occurred } \\
\text { at the meso and microsystem } \\
\text { level and engagement with the } \\
\text { AFI model. Thematic analysis } \\
\text { of interview responses } \\
\text { suggests the theme 'Factors } \\
\text { bridging the gap between } \\
\text { assessment and intervention' } \\
\text { may provide further } \\
\text { explanation for competence to } \\
\text { meet and monitor John's } \\
\text { needs moving forward. } \\
\text { Specifically, the subthemes } \\
\text { 'Shared awareness of needs } \\
\text { and goals identified' and 'A } \\
\text { collaborative approach to } \\
\text { implementing and monitoring } \\
\text { interventions' are factors that } \\
\text { provide further explanation. } \\
\text { For example, Sarah referred to } \\
\text { the benefit of Jim being aware } \\
\text { of strategies that he can } \\
\text { implement to achieve goals } \\
\text { and participants also } \\
\text { commented on a team } \\
\text { approach to monitoring his } \\
\text { needs moving forward (See } \\
\text { Section } 3 \text { ). Increased } \\
\text { awareness of Jim's needs } \\
\text { resonates with AFI Principle } \\
3 \text {, focusing on the educational } \\
\text { needs of the child and } \\
\text { working together with Jim to } \\
\text { monitor needs resonates with } \\
\text { the collaborative partnerships } \\
\text { promoted by AFI Principle } 6 .\end{array}$ & $\begin{array}{l}\text { Jim's active } \\
\text { involvement in } \\
\text { the monitoring of } \\
\text { intervention } \\
\text { recommendations } \\
\text { reflects a shifting } \\
\text { balance of power } \\
\text { towards him as } \\
\text { the developing } \\
\text { person } \\
\text { (Bronfenbrenner, } \\
\text { 1979). At the } \\
\text { mesosystem level, } \\
\text { including the } \\
\text { parents in } \\
\text { monitoring } \\
\text { effectiveness of } \\
\text { interventions may } \\
\text { be reflective of } \\
\text { the strengthened } \\
\text { developmental } \\
\text { potential of home } \\
\text { and school } \\
\text { settings in } \\
\text { working together } \\
\text { towards } \\
\text { monitoring needs } \\
\text { and goals } \\
\text { (Bronfenbrenner, } \\
\text { 1979). }\end{array}$ & $\begin{array}{l}\text { From a social } \\
\text { constructivist } \\
\text { perspective, } \\
\text { Sarah alluded to } \\
\text { applying ZPD to } \\
\text { monitor Jim's } \\
\text { progress in } \\
\text { spelling } \\
\text { (Vygotsky, 1978) } \\
\text { (See Section } 3 \\
\text { above). }\end{array}$ \\
\hline
\end{tabular}


This section outlines the analysis of findings for each case proposition in Case 2. The analysis for each proposition is divided into four sections. Section 1 provides a table outlining the criteria for interpreting the findings, also documented in Section 3.5.5 in Chapter 3. Section 2 provides the analysis of findings for each participant in accordance with the proposed criteria, in tabular format. The Changes in ratings on Likert scale statements before and after engaging with the AFI model are outlined. Pattern-matching logic is applied to responses to interview questions addressing each of the Propositions. The interview questions and codes applied to responses are outlined in Table 1 below. Themes and subthemes relevant to each proposition are also outlined and Section 3 provides a table of key quotes for each participant Appendix 45 presents the thematic maps. Appendix 46 outlines the initial and final category clusters and codes applied to interview responses and Appendix 47 provides a sample of the coding applied to a participant's interview transcript. Appendix 48 outlines the process of devising all themes and subthemes. Section 4 provides a summary of findings for each proposition and Section 5 provides a conceptual analysis, in accordance with AFI principles, EST and SCT concepts. 
Table 1 Pattern-Matching Codes Applied to Interview Responses

\begin{tabular}{|c|c|c|}
\hline Proposition & Interview Questions Addressing Proposition & Pattern-Matching Codes Applied \\
\hline $\begin{array}{l}\text { 1: 'Parents, teachers and TEP will have a better } \\
\text { understanding of the child's situation, as a } \\
\text { result of interactions and activities that have } \\
\text { occurred at the meso and microsystem levels' }\end{array}$ & $\begin{array}{l}\text { 1. Do you feel your understanding of Michelle's } \\
\text { situation has changed after working through the five } \\
\text { stages of the AFI model? } \\
\text { 2. Can you describe your experience of working } \\
\text { with the Assessment for Intervention model? }\end{array}$ & $\begin{array}{l}\text { 'understanding' } \\
\text { 'interactions and activities during the } \\
\text { AFI process' }\end{array}$ \\
\hline $\begin{array}{l}\text { 2: 'Parents, teachers and TEP will know what } \\
\text { intervention supports the child needs at home } \\
\text { and at school, as a result of interactions and } \\
\text { activities that have occurred at the meso and } \\
\text { microsystem levels' }\end{array}$ & $\begin{array}{l}\text { 1. Did the intervention recommendations address } \\
\text { your questions at the intake stage? } \\
\text { 2. Do you feel you can support Michelle's needs at } \\
\text { home/school? }\end{array}$ & $\begin{array}{l}\text { 'knowledge of intervention' } \\
\text { 'interactions and activities during the } \\
\text { AFI process' }\end{array}$ \\
\hline $\begin{array}{l}\text { 3: 'Teachers and parents will have greater } \\
\text { perceived competence in supporting the child's } \\
\text { needs at home and at school, as a result of } \\
\text { interactions and activities that have occurred at } \\
\text { the meso and microsystem levels' }\end{array}$ & $\begin{array}{l}\text { 1. Do you feel you can support Michelle's needs at } \\
\text { home/school? }\end{array}$ & $\begin{array}{l}\text { 'competence' } \\
\text { 'interactions and activities during the } \\
\text { AFI process' }\end{array}$ \\
\hline $\begin{array}{l}\text { 4: 'The child will have a better understanding } \\
\text { of their strengths and needs and what to do to } \\
\text { improve their needs, as a result of interactions } \\
\text { and activities that have occurred at the meso } \\
\text { and microsystem levels' }\end{array}$ & $\begin{array}{l}\text { 1. Did working with me, your mother and teacher } \\
\text { help you to find out about your strengths? } \\
\text { 2. Did working with me, your mother and teacher } \\
\text { help you to find out about what you need help with? } \\
\text { 3. Do you know what you need to do to get better } \\
\text { at_ (insert needs identified) }\end{array}$ & $\begin{array}{l}\text { 'understanding strengths' } \\
\text { 'understanding needs' } \\
\text { 'improve on needs' } \\
\text { 'interactions and activities during the } \\
\text { AFI process' }\end{array}$ \\
\hline $\begin{array}{l}\text { 5: 'At a time of ecological transition, teachers } \\
\text { will feel competent in their ability to work } \\
\text { according to the aims of Circular } 0013 / 2017 \text {, in } \\
\text { meeting and monitoring the needs of the child' }\end{array}$ & $\begin{array}{l}\text { 1. Do you feel you can monitor Michelle's needs at } \\
\text { school? }\end{array}$ & $\begin{array}{l}\text { 'competence to meet/monitor needs' } \\
\text { 'interactions and activities during the } \\
\text { AFI process' }\end{array}$ \\
\hline
\end{tabular}


The proceeding sections provide a comprehensive analysis of Proposition 1 findings for Case 2. Section 1 below outlines the criteria for interpreting the findings, also presented in in Section 3.5.5 in Chapter 3. Section 2 presents the analysis of findings. Section 3 outlines the themes relevant to Proposition 1 and Section 4 provides a summary of findings. Section 5 provides a conceptual analysis of Proposition 1 findings.

Section 1: Criteria for Interpreting Proposition 1 Findings (Case 2)

\begin{tabular}{|c|c|c|c|c|}
\hline $\begin{array}{l}\text { Proposition: } \\
\text { Following } \\
\text { Engagement } \\
\text { with the AFI } \\
\text { Model: } \\
\end{array}$ & $\begin{array}{l}\text { Likert Scale } \\
\text { Statements }\end{array}$ & $\begin{array}{l}\text { Likert Scale } \\
\text { Statements } \\
\text { (Comments } \\
\text { Section) }\end{array}$ & $\begin{array}{l}\text { Semi-structured } \\
\text { Interviews }\end{array}$ & $\begin{array}{l}\text { Researcher } \\
\text { Diary }\end{array}$ \\
\hline $\begin{array}{l}\text { Proposition } \\
\text { 1: 'Parents, } \\
\text { teachers and } \\
\text { TEP will } \\
\text { have a better } \\
\text { understanding } \\
\text { of the child's } \\
\text { situation, as a } \\
\text { result of } \\
\text { interactions } \\
\text { and activities } \\
\text { that have } \\
\text { occurred at } \\
\text { the meso and } \\
\text { microsystem } \\
\text { levels' }\end{array}$ & $\begin{array}{l}\text { A change in } \\
\text { rating on } \\
\text { associated } \\
\text { Likert scale } \\
\text { statements } \\
\text { indicates } \\
\text { change in } \\
\text { understanding } \\
\text { of the child's } \\
\text { situation } \\
\text { following } \\
\text { engagement } \\
\text { with the AFI } \\
\text { model and as } \\
\text { a result of } \\
\text { interactions } \\
\text { and activities } \\
\text { that have } \\
\text { occurred at } \\
\text { the meso and } \\
\text { microsystem } \\
\text { levels. }\end{array}$ & $\begin{array}{l}\text { Pattern- } \\
\text { matching: } \\
\text { Comments } \\
\text { that link } \\
\text { understanding } \\
\text { of the child's } \\
\text { situation, } \\
\text { engagement } \\
\text { with the AFI } \\
\text { model and } \\
\text { interactions } \\
\text { and activities } \\
\text { that have } \\
\text { occurred at } \\
\text { the meso and } \\
\text { microsystem } \\
\text { levels and } \\
\text { vice versa. }\end{array}$ & $\begin{array}{l}\text { Pattern-matching: } \\
\text { Interview } \\
\text { responses that link } \\
\text { understanding of } \\
\text { the child's } \\
\text { situation, } \\
\text { engagement with } \\
\text { the AFI model and } \\
\text { interactions and } \\
\text { activities that have } \\
\text { occurred at the } \\
\text { meso and } \\
\text { microsystem levels } \\
\text { and vice versa. } \\
\text { Thematic } \\
\text { analysis: Themes } \\
\text { and patterns within } \\
\text { the data set that } \\
\text { refer to } \\
\text { understanding of } \\
\text { the child's situation } \\
\text { and interactions } \\
\text { and activities that } \\
\text { have occurred at } \\
\text { the meso and } \\
\text { microsystem levels } \\
\text { were included in } \\
\text { the analysis of the } \\
\text { AFI model. }\end{array}$ & $\begin{array}{l}\text { Excerpts } \\
\text { from the } \\
\text { researcher } \\
\text { diary that } \\
\text { referred to } \\
\text { understanding } \\
\text { of the child's } \\
\text { situation and } \\
\text { interactions } \\
\text { and activities } \\
\text { that have } \\
\text { occurred at } \\
\text { the meso and } \\
\text { microsystem } \\
\text { levels were } \\
\text { included in } \\
\text { the analysis } \\
\text { of the AFI } \\
\text { model. }\end{array}$ \\
\hline
\end{tabular}


Section 2: Analysis of Proposition 1 Findings (Case 2)

\begin{tabular}{|c|c|c|c|c|}
\hline $\begin{array}{l}\text { Evidence for } \\
\text { Proposition1 } \\
\text { (Case 2) } \\
\end{array}$ & $\begin{array}{l}\text { Likert } \\
\text { Scale } \\
\text { Statements } \\
\end{array}$ & $\begin{array}{l}\text { Likert Scale } \\
\text { Statements } \\
\text { (Comments) }\end{array}$ & Semi-structured Interviews & Researcher Diary \\
\hline $\begin{array}{l}\text { Class teacher } \\
\text { (Anne) }\end{array}$ & $\begin{array}{l}\text { I } \\
\text { understand } \\
\text { the } \\
\text { student's } \\
\text { situation } \\
\text { Before AFI: } \\
8 \\
\text { After AFI: } 8\end{array}$ & $\begin{array}{l}\text { N/A (See } \\
\text { section } \\
3.11 .2)\end{array}$ & $\begin{array}{l}\text { Pattern-matching: Do you feel your understanding } \\
\text { of Michelle's situation has changed after working } \\
\text { through the five stages of the AFI model? Yeah I } \\
\text { definitely think I understand everything a lot more } \\
\text { (understanding), it's just even you know the } \\
\text { information was there and I knew a certain amount, } \\
\text { obviously I've only taught her for a couple of months, } \\
\text { a few months, well since September obviously so } \\
\text { what's that } 4 \text { months, am but I felt that just through } \\
\text { talking through everything and just being way more } \\
\text { aware of the situation as well } \\
\text { (understanding/interactions and activities during } \\
\text { the AFI process) and those observations are there all } \\
\text { the time, you're just doing that as a teacher anyway, } \\
\text { you're always looking but just having to hone in on } \\
\text { one particular child and really analyse that situation } \\
\text { and then put information or put words on it } \\
\text { (interactions and activities during the AFI } \\
\text { process) (lines 55-61) } \\
\text { Can you describe your experience of working with } \\
\text { the Assessment for Intervention model? Ok, am...I } \\
\text { would say that it's been a very positive experience } \\
\text { am, in terms of the structure of everything and the } \\
\text { whole process and, am very sort of like user-friendly } \\
\text { (interactions and activities during the AFI }\end{array}$ & $\begin{array}{l}\text { Researcher diary (AFI templates) } \\
\text { documented specific activities and } \\
\text { interactions that took place during the five- } \\
\text { stage AFI process (See Section } 4.16 \text {, Table } \\
4.21 \text { ) }\end{array}$ \\
\hline
\end{tabular}


process), in terms of the way everything was

explained and just being able to go through

everything very comprehensively with practical

results (interactions and activities during the AFI

process), every step of the way, does that make

sense? (lines 3-6)

Thematic analysis (See Section 3 below for key

quotes of themes and subthemes relevant to

Proposition 1):

A team approach to assessment

-Active roles and communication

-Insight into strengths, needs and perspectives

Perspectives on barriers to parental engagement - Judgements, belief systems, practices and values -Apprehension of the assessment process and outcomes

Views and experiences of AFI

-A clear and structured process

-A framework to facilitate teamwork and positive

outcomes

Researcher N/A N/A N/A

The researcher's progressing understanding of Michelle's situation is evidenced through the AFI templates (researcher diary) which guided hypothesis formation, needs, goals and recommendations (See Appendix 53). For example, the researcher noted the 
following in Template $2 \mathrm{~b}$ (Checklist

Integration/Feedback Stage):

"Applying elements of this stage ensured that I understood the situation and interactions between the risk and protective factors for Michelle, her school and home environment. Consideration of these factors allowed me to present a valid, transactional case formulation and devise feasible goals, needs and intervention recommendations".

Reflections in the researcher's journal documented a communicative, collaborative process facilitated by AFI. Following meetings at the Intake stage, the researcher reflected:

"We went through the information, "what we know' and 'questions for investigation', teacher and parent agreed that Michelle may benefit from developing positive self-talk and CBT strategies. There was great discussion around this between teacher and parent and myself. I was very aware and conscious of trying to adapt and pitch the language used in the meeting, for equal participation and understanding" (Reflective Journal November $29^{\text {th }}$, Intake Stage). 


\section{Section 3: Themes Relevant to Proposition 1 (Case 2)}

Theme 1: A Team Approach to Assessment
$\begin{aligned} & \text { Subtheme } \\ & \begin{array}{l}\text { Active roles and } \\ \text { communication }\end{array}\end{aligned}$
$\begin{aligned} & \text { When describing the roles of participants, Anne (class teacher) } \\ & \text { commented: Just facilitating the whole process and am, guiding } \\ & \text { us through it all, all the different people that were involved (lines } \\ & 9-11)\end{aligned}$
$\begin{aligned} & \text { When describing the benefits of the AFI model, Anne stated: } \\ & \text { There's only so much thinking you can do about an individual } \\ & \text { child on your own, whereas like when you have such great } \\ & \text { support and you can talk about it and sort of tease out ideas and I } \\ & \text { think it's really good (lines 174-176) } \\ & \text { Anne commented on the reality of the situation when Michelle } \\ & \text { was absent from school during the assessment process: Missing } \\ & \text { a lot of time [Michelle] and then you know when I had planned } \\ & \text { to do lessons or planned to implement some of the little tips and } \\ & \text { things like that, not being here was obviously do you know, a big } \\ & \text { problem (lines 27-29) }\end{aligned}$

Anne refereed to making the best of the presenting situation (Michelle's absences from school): Yes so I think obviously we did the very best that we could with the situation we had (line 188)

Insight into When describing the role of the parent during the assessment strengths, needs $\quad$ process, Anne commented: The parent had to provide and perspectives information that we wouldn't be aware of, am so to add that other dimension to things (lines 13-14)

Anne commented on her perception of her own role during the process: Yeah again I suppose providing the information that I could provide based on what I know about the child and my everyday observations, am so providing all of that information and then just kind of working together to try to come up with the best plan moving forward, really (lines 17-19)

Anne commented also on Michelle's role during the assessment process: Again kind of information but that can be a little bit challenging because they might be reluctant to give you that information (lines 21-22)

When describing her changing in understanding of Michelle's situation, Anne referred to greater awareness of the situation: Just being way more aware of the situation (line 58)

\begin{tabular}{ll}
\hline \multicolumn{2}{l}{ Theme 2: Perspectives on Barriers to Parental Engagement } \\
\hline $\begin{array}{l}\text { Subtheme } \\
\text { Judgements, belief }\end{array}$ & $\begin{array}{l}\text { Key Quotes } \\
\text { systems, practices } \\
\text { and values }\end{array}$ \\
$\begin{array}{l}\text { assessment process, Anne commented: It's just really unfortunate } \\
\text { because it could have been something that she could have got } \\
\text { much more out of (lines 44-45) }\end{array}$ \\
\hline
\end{tabular}


When describing the parent's involvement, Anne commented: There's only so much you can do and then she [parent] has to come forward and put herself forward so it's just unfortunate that she just couldn't...couldn't do that (lines 207-208)

Apprehension of the assessment outcomes process and

With regards to the parent's involvement, Anne commented:

I do honestly believe that she genuinely wants that help, but I think as time goes on if there is any input that she has to put in, it could be something that's just a little bit, a stretch too far for her, even small little things like making changes, the small changes that she has to make she might be thinking of them as much bigger and it's just finding it hard to break it down, so I think that's her own issues and her own abilities really (lines 198-202)

Anne commented further on the parent's involvement: And am, then there could be a slight fear of the unknown as well, sometimes in these situations [assessments] (lines 202-203)

\section{Theme 4: Views and Experiences of AFI}

Subtheme Key Quotes

A clear and When describing her experience of the AFI process, Anne structured process commented: In terms of the structure of everything and the whole process and, am very sort of like user-friendly (lines 3-4)

Anne also referred to how she could understand the process: I really understood the whole process, am...there was no kind of, you know the way sometimes things can get a little bit wordy or too much information (lines 72-73)

A framework to When describing the benefits of the AFI model, Anne commented: facilitate teamwork and positive outcomes
So I think that's really beneficial, to have that opportunity you know what I mean, to deal with somebody and am...to come together and come up with a plan together, you know when everyone is sort of working together, it just really makes it a lot easier (lines 179-181)

\section{Section 4: Summary of Proposition 1 Findings (Case 2)}

Ratings, provided by the teacher indicate understanding remained unchanged, however pattern-matching logic and thematic analysis suggest a change occurred. Patterns within the teacher's interview responses linked understanding of the situation to interactions and activities that occurred during the five stages of the AFI process, at the meso and microsystem levels. These activities and interactions included "talking through everything" with team members and having to "analyse that situation" with the various assessment tasks. Assessment tasks are outlined in Section 4.16, Table 4.21 and included observation tasks, 1:1 assessment of attainment and cognitive functioning and dynamic assessment of learning potential. The activities and interactions connected to 
Proposition 1 are considered consistent with the AFI process. In accordance with AFI Principle 6, participants were active in their engagement in assessment tasks and included in the process as 'co-assessors' (Pameijer, 2017). Additionally and consistent with AFI principle 7, the researcher adhered to the systematic and transparent assessment process and the researcher's progressing understanding of Michelle's situation was evidenced through the AFI templates. The templates guided hypothesis formation, needs, goals and recommendations during the five stages (See Appendix 53). Reflections in the researcher's journal documented a communicative, collaborative process facilitated by AFI (See Section 2 above). Themes within the data set that referred to understanding of Michelle's situation and interactions and activities that occurred at the meso and microsystem levels included 'A team approach to assessment', 'Perspectives on barriers to parental engagement' and 'Views and Experiences of AFI' (See Section 3 above). Section 5 below provides a conceptual analysis of the findings and themes, in accordance with AFI principles, EST and SCT concepts. 
Section 5: Conceptual Analysis of Proposition 1 Findings (Case 2)

\section{Theme/ AFI Principles (See section 2.16 for description of AFI Subtheme Principles)}

\section{A team}

approach to

assessment

\section{Patterns within the teacher's interview responses linked}

understanding of the situation to interactions and activities that

occurred at the meso and microsystem levels during the five

stages of the AFI model, including "talking through everything" with team members and having to "analyse that situation", with the various assessment tasks. Researcher reflections documented a communicative, collaborative assessment process (See Section 2). Thematic analysis of interview responses in the data set indicates a team approach to assessment was a factor that contributed to understanding of the situation (See key quotes in Section 3). This factor resonates with AFI Principle 6: Working together in a collaborative partnership to search for explanations to the situation.

\section{EST Concepts (See \\ section 2.14 for \\ definitions of EST concepts)}

At the mesosystem level, active participation and communication between home, school and school psychological service may have served to strengthen the developmental potential of these settings to identify and understand Michelle's needs. At the microsystem level, inclusion of Michelle in the process may have encouraged strengthening of dyadic relations between adults and child (Bronfenbrenner, 1979).
SCT Concepts (See section 2.15 for definitions of SCT concepts)

\section{From a SCT perspective,} insight into Michelle's actual and potential levels of development was conducted through assessment tasks, including observations and 1:1 cognitive and attainment testing and was theorised through ZAD: what Michelle could do unassisted and ZPD; what Michelle could do with tools and signs (e.g. graphic organiser) and assistance (Palinscar, 1998; Vygotsky, 1978). Interactions that facilitated learning were later translated into intervention recommendations.

Appropriate use of language applied during the assessment process may have assisted the co-construction of knowledge and understanding between participants, in accordance 
Perspectives on barriers to parental engagement
Patterns within the teacher's interview responses linked understanding of the situation to interactions and activities that occurred at the meso and microsystem levels during the five stages of the AFI model, including "talking through everything" with team members and having to "analyse that situation" with the various assessment tasks. Active involvement of participants varied during the process. Mary (parent) was not always present at meetings and Michelle (child) was absent for a number of days during the assessment process. Researcher reflections document that pragmatism was applied in proceeding through the five stages of AFI. Thematic analysis of participant interview responses identified 'Perspectives on barriers to parental engagement' as a theme in the data set, presenting viewpoints on parental engagement during the assessment process. For example, Anne commented that "we did the very best that we could with the situation we had" and that there may have been a "fear of the unknown" for Mary and although wanting help, "small changes" may have been "a stretch too far for her". These thoughts are perhaps reflective of Anne's own judgements, belief systems and values (See Section 3 above). Judgements and belief systems could hinder the development of collaborative partnerships between home, school, school psychological service and child promoted by AFI Principle 6 (Pameijer, 2017).
Unaddressed teacher belief systems and values could hinder the developmental potential of home and school settings to identify and understand Michelle's needs (Bronfenbrenner, 1979). 


\begin{tabular}{|c|c|c|}
\hline $\begin{array}{l}\text { Views and } \\
\text { experiences } \\
\text { of AFI }\end{array}$ & $\begin{array}{l}\text { Thematic analysis identified 'Views and experiences of AFI' as a } \\
\text { theme in the dataset and provides further explanation of how } \\
\text { understanding of Michelle's situation improved following } \\
\text { engagement with the AFI process. Anne commented on the } \\
\text { opportunity AFI provided "to come together and come up with a } \\
\text { plan together", that she "really understood the whole process" } \\
\text { and that it was "user-friendly" (See Section 3). A clear and } \\
\text { structured assessment process, resonates with AFI Principle 7: A } \\
\text { systematic and transparent stage-like process (Pameijer, 2017). } \\
\text { The researcher adhered to the systematic and transparent } \\
\text { assessment process with the AFI templates. AFI was also } \\
\text { considered a framework to facilitate teamwork, providing the } \\
\text { opportunity to "to come together and come up with a plan } \\
\text { together", consistent with AFI Principle } 6 \text {. }\end{array}$ & $\begin{array}{l}\text { Thematic analysis suggests } \\
\text { the AFI model provided a } \\
\text { framework to facilitate } \\
\text { teamwork and positive } \\
\text { outcomes with everyone } \\
\text { "sort of working together" } \\
\text { (See Section 3). At the } \\
\text { mesosystem level, active } \\
\text { participation and } \\
\text { communication between } \\
\text { home, school and school } \\
\text { psychological service may } \\
\text { have served to strengthen } \\
\text { the developmental } \\
\text { potential of these settings } \\
\text { to identify and understand } \\
\text { Michelle's needs. At the } \\
\text { microsystem level, } \\
\text { inclusion of Michelle in } \\
\text { the process may have } \\
\text { encouraged strengthening } \\
\text { of dyadic relations } \\
\text { (Bronfenbrenner, 1979) }\end{array}$ \\
\hline
\end{tabular}


Appendix 41 Case 2 Analysis of Findings: Proposition 2

The proceeding sections provide a comprehensive analysis of Proposition 2 findings for Case 2. Section 1 below outlines the criteria for interpreting the findings, also presented in in Section 3.5.5 in Chapter 3. Section 2 presents the analysis of findings. Section 3 outlines the themes relevant to Proposition 2 and Section 4 provides a summary of findings. Section 5 provides a conceptual analysis of Proposition 2 findings.

Section 1: Criteria for Interpreting Proposition 2 Findings (Case 2)

\begin{tabular}{|c|c|c|c|c|}
\hline $\begin{array}{l}\text { Proposition: } \\
\text { Following } \\
\text { Engagement } \\
\text { with the } \\
\text { AFI Model: }\end{array}$ & $\begin{array}{l}\text { Likert Scale } \\
\text { Statements }\end{array}$ & $\begin{array}{l}\text { Likert Scale } \\
\text { Statements } \\
\text { (Comments } \\
\text { Section) }\end{array}$ & $\begin{array}{l}\text { Semi-structured } \\
\text { Interviews }\end{array}$ & $\begin{array}{l}\text { Researcher } \\
\text { Diary }\end{array}$ \\
\hline $\begin{array}{l}\text { Proposition } \\
\text { 2: 'Parents, } \\
\text { teachers and } \\
\text { TEP will } \\
\text { know what } \\
\text { intervention } \\
\text { supports the } \\
\text { child needs } \\
\text { at home and } \\
\text { at school, as } \\
\text { a result of } \\
\text { interactions } \\
\text { and activities } \\
\text { that have } \\
\text { occurred at } \\
\text { the meso and } \\
\text { microsystem } \\
\text { levels' }\end{array}$ & $\begin{array}{l}\text { A change in } \\
\text { rating on } \\
\text { associated } \\
\text { Likert scale } \\
\text { statements } \\
\text { indicates } \\
\text { change in } \\
\text { knowledge of } \\
\text { what } \\
\text { intervention } \\
\text { supports the } \\
\text { child needs } \\
\text { following } \\
\text { engagement } \\
\text { with the AFI } \\
\text { model and as } \\
\text { a result of } \\
\text { interactions } \\
\text { and activities } \\
\text { that have } \\
\text { occurred at } \\
\text { the meso and } \\
\text { microsystem } \\
\text { levels. }\end{array}$ & $\begin{array}{l}\text { Pattern- } \\
\text { matching: } \\
\text { Comments } \\
\text { that link } \\
\text { knowledge } \\
\text { of } \\
\text { intervention } \\
\text { supports the } \\
\text { child needs, } \\
\text { engagement } \\
\text { with the AFI } \\
\text { model and } \\
\text { interactions } \\
\text { and activities } \\
\text { that have } \\
\text { occurred at } \\
\text { the meso and } \\
\text { microsystem } \\
\text { levels and } \\
\text { vice versa. }\end{array}$ & $\begin{array}{l}\text { Pattern-matching: } \\
\text { Interview responses } \\
\text { that link knowledge } \\
\text { of intervention } \\
\text { supports the child } \\
\text { needs, engagement } \\
\text { with the AFI model } \\
\text { and interactions and } \\
\text { activities that have } \\
\text { occurred at the meso } \\
\text { and microsystem } \\
\text { levels and vice } \\
\text { versa. } \\
\text { Thematic analysis: } \\
\text { Themes and patterns } \\
\text { within the data set } \\
\text { that refer to } \\
\text { knowledge of } \\
\text { intervention } \\
\text { supports the child } \\
\text { needs and } \\
\text { interactions and } \\
\text { activities that have } \\
\text { occurred at the meso } \\
\text { and microsystem } \\
\text { levels were included } \\
\text { in the analysis of the } \\
\text { AFI model. }\end{array}$ & $\begin{array}{l}\text { Excerpts } \\
\text { from the } \\
\text { researcher } \\
\text { diary that } \\
\text { referred to } \\
\text { knowledge of } \\
\text { intervention } \\
\text { supports the } \\
\text { child needs } \\
\text { and } \\
\text { interactions } \\
\text { and activities } \\
\text { that have } \\
\text { occurred at } \\
\text { the meso and } \\
\text { microsystem } \\
\text { levels were } \\
\text { included in } \\
\text { the analysis } \\
\text { of the AFI } \\
\text { model. }\end{array}$ \\
\hline
\end{tabular}


Section 2: Analysis of Proposition 2 Findings (Case 2)

\begin{tabular}{|c|c|c|c|c|}
\hline $\begin{array}{l}\text { Evidence } \\
\text { for } \\
\text { Proposition } \\
2 \text { (Case 2) }\end{array}$ & $\begin{array}{l}\text { Likert } \\
\text { Scale } \\
\text { Statements }\end{array}$ & $\begin{array}{l}\text { Likert Scale } \\
\text { Statements } \\
\text { (Comments) }\end{array}$ & Semi-structured Interviews & Researcher Diary \\
\hline
\end{tabular}


little bit easier for her at the start of the lesson (lines 101-

104)... Like say for example with regards to maths and things like that if I was doing a lesson it was differentiated for her and that maybe she might have examples to start with and then she can continue on and sort of knows what she's doing until there's a chance for me to get there and work with her one to one maybe (interactions and activities during the AFI process) and then we have the little checklist now that she puts into her pencil case (knowledge of interventions/AFI /interactions and activities during the AFI process), do you know what I mean, they're similar but just additional and anything extra is a help, you know any extra ideas and same with the English and just sort of writing out the words and doing, again we would be doing it anyway with brainstorming (interactions and activities during the AFI process) but sometimes just if a template looks different or is different it can also help, you know which is great (lines 118-125)

Thematic analysis (See Section 3 below for key quotes of themes and subthemes relevant to Proposition 2):

Factors bridging the gap between assessment and intervention

-Shared awareness of strengths, needs and goals identified

-Experiencing or observing intervention effectiveness

Researcher N/A N/A N
(TEP)

The researcher's knowledge of intervention supports is evidenced through the AFI templates (researcher diary) which guided hypothesis formation, needs, goals and recommendations (See 
Appendix 53). Researcher

reflections indicate intervention

planning was supported by

participants sharing their feedback

on co-investigator activities. For

example:

"I met with Michelle (child) and she gave me feedback on the co-

investigator activity. She feels the graphic organiser helped her with

her comprehension work and this

could possibly be used for Irish

writing as well. She is finding the

self-check prompt for Maths helpful too. It reminds her to check answers and to use the resources in her basket when needed" (Reflective Journal December $13^{\text {th }}$, Integration Stage).

"Anne was able to teach how to use an 'I Statement' at an opportune moment and Michelle was able to understand the concept. Anne feels she will be able to draw on this again at another stage" (Reflective Journal December $13^{\text {th }}$, Integration Stage). 


\begin{tabular}{|c|c|}
\hline \multicolumn{2}{|c|}{ Theme 3: Factors Bridging the Gap between Assessment and Intervention } \\
\hline Subtheme & Key Quotes \\
\hline $\begin{array}{l}\text { Shared awareness } \\
\text { of strengths, } \\
\text { needs and goals } \\
\text { identified }\end{array}$ & $\begin{array}{l}\text { When commenting on how she would support Michelle's needs } \\
\text { moving forward, Anne (class teacher) described the benefit of } \\
\text { sharing assessment information gathered: And sometimes as } \\
\text { well it's good even things that you have been doing in the past } \\
\text { but just to sort of know that we had similar ideas on that, so that } \\
\text { you know I'm working towards the right way of doing it, do you } \\
\text { know what I mean? (lines } 114-116 \text { ) }\end{array}$ \\
\hline & $\begin{array}{l}\text { When describing the benefits of the AFI model, Anne referred to } \\
\text { the value of shared awareness of how best to work towards } \\
\text { meeting Michelle's needs: Most of the time you're just trying to } \\
\text { do this on your own and you're just trying to find a way of } \\
\text { coming up with ideas and sometimes you'll be wondering are } \\
\text { you doing the right thing or is this going to work and I found } \\
\text { that [sharing of assessment information] really beneficial (lines } \\
\text { 181-184) }\end{array}$ \\
\hline
\end{tabular}

Experiencing or When describing how she would support Michelle's needs observing moving forward, Anne referred to applying intervention intervention strategies that were observed to be effective during the effectiveness Investigation Stage: Obviously put in place these extra you know ideas, especially say along the lines for English and mapping out things ahead of a lesson and again with the maths and checklists and just I suppose making things a little bit easier for her at the start of the lesson and then obviously then when I get a chance I can do a little bit more one to one with her but she is encouraged to get started independently (lines 101-105)

Anne also commented on how she plans to use the self-
monitoring checklist, which was also observed to be effective
during the Investigation Stage: We have the little checklist now
that she puts into her pencil case, do you know what I mean,
they're similar but just additional and anything extra is a help,
you know any extra ideas and same with the English and just
sort of writing out the words and doing, again we would be
doing it anyway with brainstorming but sometimes just if a
template looks different or is different it can also help, you
know which is great (lines 121-125)

\section{Section 4: Summary of Proposition 2 Findings (Case 2)}

Higher teacher Likert scale ratings, following engagement with the AFI model, indicates greater knowledge of intervention supports. Patterns within the teacher's interview responses linked knowledge of intervention supports to interactions and activities that occurred during the stages of the AFI model, at the meso and microsystem 
level. At the microsystem level, Anne observed the effectiveness of a graphic organiser for "mapping out things ahead of a lesson" and a self-checklist for maths, making things "a little bit easier for her [Michelle] at the start of a lesson". At the mesosystem level, dialogue between TEP, parent and teacher at the Intake and Feedback stage led to knowledge of evidence-based social-emotional intervention resources including "Talkabout" and "cognitive behaviour therapy". The AFI templates documented these meetings and discussions. These activities and interactions are considered consistent with the AFI process. In accordance with AFI Principle 6, parent, teacher and child were considered 'co-assessors' during the Investigation stage and provided feedback on intervention strategies designed to address needs (Pameijer, 2017). Their opinions and feedback were valued during the process and contributed to intervention planning (See Section 2 above). Patterns within the teacher's interview responses also connected knowledge of intervention supports to differentiation activities that she already applies in her general practice as a teacher (e.g. brainstorming of words in an English lesson). Consistent with AFI Principle 3, the effective approaches addressing Michelle's needs were encouraged by the TEP ('goodness of fit'). In accordance with AFI principle 7, the researcher's knowledge of intervention supports was evidenced in the AFI templates (researcher diary) (Pameijer, 2017). The templates guided the formation of hypotheses, needs, goals and recommendations throughout the AFI process. The theme 'Factors bridging the gap between assessment and intervention' referred to knowledge of intervention supports and interactions and activities that occurred at the meso and microsystem levels (See Section 3 above). Section 5 below provides a conceptual analysis of the findings and themes, in accordance with AFI principles, EST and SCT concepts. 
Section 5: Conceptual Analysis of Proposition 2 Findings (Case 2)

\begin{tabular}{|c|c|c|c|}
\hline $\begin{array}{l}\text { Theme/ } \\
\text { Subtheme }\end{array}$ & $\begin{array}{l}\text { AFI Principles (See section } 2.16 \text { for description of AFI } \\
\text { Principles) }\end{array}$ & $\begin{array}{l}\text { EST Concepts (See section } 2.14 \\
\text { for definitions of EST concepts) }\end{array}$ & $\begin{array}{l}\text { SCT Concepts (See } \\
\text { section 2.15) }\end{array}$ \\
\hline $\begin{array}{l}\text { Experiencing } \\
\text { or observing } \\
\text { intervention } \\
\text { effectiveness }\end{array}$ & $\begin{array}{l}\text { Thematic analysis of interview responses in the data set indicates } \\
\text { experiencing or observing intervention effectiveness during the } \\
\text { Investigation stage was another factor in bridging the gap between } \\
\text { assessment and intervention (see key quotes in Section } 3 \text { ). This } \\
\text { resonates with AFI Principle } 6 \text { as participants were considered 'co- } \\
\text { assessors' and engaged in tasks and activities to investigate the } \\
\text { effectiveness of intervention strategies at the Investigation stage. } \\
\text { See Section 4.16, Table } 4.21 \text { for list of specific activities that } \\
\text { participants engaged in. Observing the effectiveness of intervention } \\
\text { strategies resonates with AFI Principle } 4 \text { and 5; Focusing on the } \\
\text { needs of the teacher, parent and student to discover what the ideal } \\
\text { approach for Michelle might be (Pameijer, 2017). }\end{array}$ & $\begin{array}{l}\text { Teacher observations of the } \\
\text { effectiveness of intervention } \\
\text { strategies during the Investigation } \\
\text { stage reflects strengthening dyadic } \\
\text { relations between teacher and } \\
\text { student, by paying increased } \\
\text { attention to Michelle's learning } \\
\text { and her activities } \\
\text { (Bronfenbrenner, 1979). For } \\
\text { example, Anne (class teacher) } \\
\text { providing Michelle (student) with } \\
\text { tools (checklists and graphic } \\
\text { organiser) to assist her learning. }\end{array}$ & $\begin{array}{l}\text { Tools and signs, } \\
\text { including checklists and } \\
\text { a graphic organiser } \\
\text { were observed to be } \\
\text { effective in supporting } \\
\text { Michelle's involvement } \\
\text { in the co-construction } \\
\text { of knowledge and } \\
\text { learning, consistent } \\
\text { with SCT (Palinscar, } \\
\text { 1998; Vygotsky, 1978). }\end{array}$ \\
\hline
\end{tabular}


Appendix 42 Case 2 Analysis of Findings: Proposition 3

The proceeding sections provide a comprehensive analysis of Proposition 3 findings for Case 2. Section 1 below outlines the criteria for interpreting the findings, also presented in in Section 3.5.5 in Chapter 2. Section 2 presents the analysis of findings. Section 3 outlines the themes relevant to Proposition 3 and Section 4 provides a summary of findings. Section 5 provides a conceptual analysis of Proposition 3 findings.

Section 1: Criteria for Interpreting Proposition 3 Findings (Case 2)

\begin{tabular}{|c|c|c|c|c|}
\hline $\begin{array}{l}\text { Proposition: } \\
\text { Following } \\
\text { Engagement } \\
\text { with the AFI } \\
\text { Model: }\end{array}$ & $\begin{array}{l}\text { Likert Scale } \\
\text { Statements }\end{array}$ & $\begin{array}{l}\text { Likert Scale } \\
\text { Statements } \\
\text { (Comments } \\
\text { Section) }\end{array}$ & $\begin{array}{l}\text { Semi-structured } \\
\text { Interviews }\end{array}$ & $\begin{array}{l}\text { Researcher } \\
\text { Diary }\end{array}$ \\
\hline $\begin{array}{l}\text { Proposition } \\
\text { 3: 'Teachers } \\
\text { and parents } \\
\text { will have } \\
\text { greater } \\
\text { perceived } \\
\text { competence in } \\
\text { supporting the } \\
\text { child's needs } \\
\text { at home and at } \\
\text { school, as a } \\
\text { result of } \\
\text { interactions } \\
\text { and activities } \\
\text { that have } \\
\text { occurred at the } \\
\text { meso and } \\
\text { microsystem } \\
\text { levels' }\end{array}$ & $\begin{array}{l}\text { A change in } \\
\text { rating on } \\
\text { associated } \\
\text { Likert scale } \\
\text { statements } \\
\text { indicates } \\
\text { change in } \\
\text { perceived } \\
\text { competence } \\
\text { in supporting } \\
\text { the child's } \\
\text { needs } \\
\text { following } \\
\text { engagement } \\
\text { with the AFI } \\
\text { model and as } \\
\text { a result of } \\
\text { interactions } \\
\text { and activities } \\
\text { that have } \\
\text { occurred at } \\
\text { the meso and } \\
\text { microsystem } \\
\text { levels. }\end{array}$ & $\begin{array}{l}\text { Pattern- } \\
\text { matching: } \\
\text { Comments } \\
\text { that link } \\
\text { competence in } \\
\text { supporting the } \\
\text { child's needs, } \\
\text { engagement } \\
\text { with the AFI } \\
\text { model and } \\
\text { interactions } \\
\text { and activities } \\
\text { that have } \\
\text { occurred at the } \\
\text { meso and } \\
\text { microsystem } \\
\text { levels and vice } \\
\text { versa. }\end{array}$ & $\begin{array}{l}\text { Pattern- } \\
\text { matching: } \\
\text { Interview } \\
\text { responses that link } \\
\text { competence in } \\
\text { supporting the } \\
\text { child's needs, } \\
\text { engagement with } \\
\text { the AFI model and } \\
\text { interactions and } \\
\text { activities that have } \\
\text { occurred at the } \\
\text { meso and } \\
\text { microsystem levels } \\
\text { and vice versa. } \\
\text { Thematic } \\
\text { analysis: Themes } \\
\text { and patterns within } \\
\text { the data set that } \\
\text { refer to } \\
\text { competence in } \\
\text { supporting the } \\
\text { child's needs and } \\
\text { interactions and } \\
\text { activities that have } \\
\text { occurred at the } \\
\text { meso and } \\
\text { microsystem levels } \\
\text { were included in } \\
\text { the analysis of the } \\
\text { AFI model. }\end{array}$ & $\mathrm{N} / \mathrm{A}$ \\
\hline
\end{tabular}


Section 2: Analysis of Proposition 3 Findings (Case 2)

\begin{tabular}{|c|c|c|c|c|}
\hline $\begin{array}{l}\text { Evidence } \\
\text { for } \\
\text { Proposition } \\
3 \text { (Case 2) }\end{array}$ & $\begin{array}{l}\text { Likert Scale } \\
\text { Statements }\end{array}$ & $\begin{array}{l}\text { Likert } \\
\text { Scale } \\
\text { Statements } \\
\text { (Comments } \\
\text { Section) }\end{array}$ & Semi-structured Interviews & Researcher Diary \\
\hline $\begin{array}{l}\text { Class } \\
\text { teacher } \\
\text { (Anne) }\end{array}$ & $\begin{array}{l}\text { I am able to } \\
\text { support the } \\
\text { student's } \\
\text { needs at } \\
\text { school: } \\
\text { Before AFI: } 6 \\
\text { After AFI: } 9\end{array}$ & $\begin{array}{l}\text { N/A (See } \\
\text { section } \\
3.11 .2)\end{array}$ & $\begin{array}{l}\text { Pattern-matching: Do you feel you can support Michelle's } \\
\text { needs at school? I think so yeah, absolutely (competence), } \\
\text { am... and again with the help of all these again, extra } \\
\text { interventions yeah absolutely (interactions and activities } \\
\text { during the AFI process) (lines 96-97)...I suppose } \\
\text { obviously what I would be doing anyway (competence), all } \\
\text { the extra sort of resources and over the weeks when you get } \\
\text { to know how a child learns and you adapt things and sort of } \\
\text { differentiate to suit their needs am I'll continue to do that } \\
\text { (competence) and then obviously put in place these extra } \\
\text { you know ideas (lines 99-102) } \\
\text { Thematic analysis (See Section } 3 \text { below for key quotes of } \\
\text { themes and subthemes relevant to Proposition 3): } \\
\text { Factors bridging the gap between assessment and } \\
\text { intervention } \\
\text {-Practical interventions }\end{array}$ & $\begin{array}{l}\text { Researcher diary (AFI } \\
\text { templates) documented } \\
\text { specific activities and } \\
\text { interactions that took place } \\
\text { during the five-stage AFI } \\
\text { process (See Section } 4.16 \text {, } \\
\text { Table } 4.21 \text { ) }\end{array}$ \\
\hline
\end{tabular}




\section{Section 3: Themes Relevant to Proposition 3 (Case 2)}

\section{Theme 3: Factors Bridging the Gap between Assessment and Intervention}

$\begin{array}{ll}\text { Subthemes } & \text { Key Quotes } \\ \text { Practical } & \text { When commenting on how the intervention recommendations }\end{array}$

interventions addressed her Intake questions, Anne referred to the usefulness

of intervention recommendations and how they would be

beneficial to others in the class: All of those tips are brilliant and can be used for everybody, which is great as well and again that's again getting back to the practicalities of things, a class of 25 it is very hard to get the one to one time or even sort of small groups or whatever so when you have something that you can use with everybody but that's going to be beneficial for everybody, that's really important you know (lines 89-93)

When commenting on whether the intervention recommendations were useful, Anne noted: As I said probably repeating myself again here but practical [intervention recommendations] and that's what it's all about just getting it done (lines 155-156)

\section{Section 4: Summary of Proposition 3 Findings (Case 2)}

Class teacher Likert scale ratings indicate greater perceived competence in supporting Michelle's needs, after engaging with the AFI model. Pattern-matching logic applied to Anne's interview response connected her perceived competence to support Michelle's needs to "the help of all these...extra interventions". These "extra interventions" resulted from activities and interactions that occurred during the stages of the AFI model, at the meso and micro system levels. Activities and interactions are listed in Section 4.16, Table 4.21 and at the microsystem level included dialogue between adults and child, investigation and observation tasks, 1:1 assessments and at the mesosystem level, meetings and dialogue between TEP, parent and teacher. Consistent with AFI Principle 6, all participants were 'co-assessors' during the Investigation stage and their active participation led to the identification of needs and subsequently, intervention recommendations (Pameijer, 2017). Patterns within Anne's response also connected competence to support Michelle's needs to "what I [she] would be doing anyway". In accordance with AFI Principle 3, the effective approaches addressing Michelle's needs were encouraged by the TEP during meetings and discussions ('goodness of fit'). The theme 'Factors bridging the gap between assessment and intervention' referred to competence in supporting Michelle's needs and interactions and activities that occurred at the meso and microsystem levels (See Section 3). Section 5 below provides a conceptual analysis of the findings and themes, in accordance with AFI principles, EST and SCT concepts. 
Section 5: Conceptual Analysis of Proposition 3 Findings (Case 2)

\begin{tabular}{|c|c|c|c|}
\hline $\begin{array}{l}\text { Theme/ } \\
\text { Subtheme }\end{array}$ & $\begin{array}{l}\text { AFI Principles (See section } 2.16 \text { for description of AFI } \\
\text { Principles) }\end{array}$ & $\begin{array}{l}\text { EST Concepts (See } \\
\text { section } 2.14 \text { for } \\
\text { definitions of EST } \\
\text { concepts) }\end{array}$ & $\begin{array}{l}\text { SCT Concepts (See section } \\
2.15 \text { for definitions of SCT } \\
\text { concepts) }\end{array}$ \\
\hline
\end{tabular}


Appendix 43 Case 2 Analysis of Findings: Proposition 4

The proceeding sections provide a comprehensive analysis of Proposition 4 findings for Case 2. Section 1 below outlines the criteria for interpreting the findings, also presented in in Section 3.5.5 in Chapter 3. Section 2 presents the analysis of findings. Section 3 outlines the themes relevant to Proposition 4 and Section 4 provides a summary of findings. Section 5 provides a conceptual analysis of Proposition 4 findings.

Section 1: Criteria for Interpreting Proposition 4 Findings (Case 2)

\begin{tabular}{|c|c|c|c|c|}
\hline $\begin{array}{l}\text { Proposition: } \\
\text { Following } \\
\text { Engagement } \\
\text { with the AFI } \\
\text { Model: }\end{array}$ & $\begin{array}{l}\text { Likert Scale } \\
\text { Statements }\end{array}$ & $\begin{array}{l}\text { Likert Scale } \\
\text { Statements } \\
\text { (Comments } \\
\text { Section) }\end{array}$ & $\begin{array}{l}\text { Semi-structured } \\
\text { Interviews }\end{array}$ & $\begin{array}{l}\text { Researcher } \\
\text { Diary }\end{array}$ \\
\hline $\begin{array}{l}\text { Proposition } \\
\text { 4: 'The child } \\
\text { will have a } \\
\text { better } \\
\text { understanding } \\
\text { of their } \\
\text { strengths and } \\
\text { needs and } \\
\text { what to do to } \\
\text { improve their } \\
\text { needs, as a } \\
\text { result of } \\
\text { interactions } \\
\text { and activities } \\
\text { that have } \\
\text { occurred at } \\
\text { the meso and } \\
\text { microsystem } \\
\text { levels' }\end{array}$ & $\begin{array}{l}\text { A change in } \\
\text { rating on the } \\
\text { associated } \\
\text { Likert scale } \\
\text { statements } \\
\text { indicates } \\
\text { change in the } \\
\text { child's } \\
\text { understandin } \\
\text { g of their } \\
\text { strengths and } \\
\text { needs and } \\
\text { what to do to } \\
\text { improve their } \\
\text { needs, } \\
\text { following } \\
\text { engagement } \\
\text { with the AFI } \\
\text { model and as } \\
\text { a result of } \\
\text { interactions } \\
\text { and activities } \\
\text { that have } \\
\text { occurred at } \\
\text { the meso and } \\
\text { microsystem } \\
\text { levels }\end{array}$ & $\begin{array}{l}\text { Pattern- } \\
\text { matching: } \\
\text { Comments that } \\
\text { link the child's } \\
\text { understanding } \\
\text { of their } \\
\text { strengths and } \\
\text { needs and what } \\
\text { to do to } \\
\text { improve their } \\
\text { needs, } \\
\text { engagement } \\
\text { with the AFI } \\
\text { model and } \\
\text { interactions } \\
\text { and activities } \\
\text { that have } \\
\text { occurred at the } \\
\text { meso and } \\
\text { microsystem } \\
\text { levels and vice } \\
\text { versa. }\end{array}$ & $\begin{array}{l}\text { Pattern-matching: } \\
\text { Interview responses } \\
\text { that link the child's } \\
\text { understanding of } \\
\text { their strengths and } \\
\text { needs and what to do } \\
\text { to improve their } \\
\text { needs, engagement } \\
\text { with the AFI model } \\
\text { and interactions and } \\
\text { activities that have } \\
\text { occurred at the meso } \\
\text { and microsystem } \\
\text { levels and vice } \\
\text { versa. } \\
\text { Thematic analysis: } \\
\text { Themes and patterns } \\
\text { within the data set } \\
\text { that refer to } \\
\text { understanding of } \\
\text { strengths and needs } \\
\text { and what to do to } \\
\text { improve their needs, } \\
\text { and interactions and } \\
\text { activities that have } \\
\text { occurred at the meso } \\
\text { and microsystem } \\
\text { levels were included } \\
\text { in the analysis of the } \\
\text { AFI model. }\end{array}$ & $\mathrm{N} / \mathrm{A}$ \\
\hline
\end{tabular}


Section 2: Analysis of Proposition 4 Findings (Case 2)

\begin{tabular}{|c|c|c|c|c|}
\hline $\begin{array}{l}\text { Evidence } \\
\text { for } \\
\text { Proposition } \\
4 \text { (Case 2) } \\
\end{array}$ & $\begin{array}{l}\text { Likert Scale } \\
\text { Statements }\end{array}$ & $\begin{array}{l}\text { Likert Scale } \\
\text { Statements } \\
\text { (Comments } \\
\text { Section) }\end{array}$ & $\begin{array}{l}\text { Semi- } \\
\text { structured } \\
\text { Interviews }\end{array}$ & Researcher Diary \\
\hline $\begin{array}{l}\text { Michelle } \\
\text { (Student) }\end{array}$ & $\begin{array}{l}\text { I know what } \\
\text { my strengths } \\
\text { are at school } \\
\text { Before AFI: } \\
\text { Yes } \\
\text { After AFI: - } \\
\text { I know what I } \\
\text { need help with } \\
\text { at school } \\
\text { Before AFI: } \\
\text { Yes } \\
\text { After AFI: - } \\
\text { I know what I } \\
\text { can do to get } \\
\text { better at what I } \\
\text { need help with } \\
\text { at school } \\
\text { Before AFI: } \\
\text { Yes } \\
\text { After AFI: }\end{array}$ & $\begin{array}{l}\text { N/A (See section } \\
3.11 .2 \text { ) }\end{array}$ & $\begin{array}{l}\text { Data was not } \\
\text { collected from } \\
\text { Michelle } \\
\text { following } \\
\text { engagement } \\
\text { with the AFI } \\
\text { model. }\end{array}$ & $\begin{array}{l}\text { Researcher diary (AFI templates) documented specific activities and } \\
\text { interactions that took place during the five-stage AFI process (See } \\
\text { Section 4.16, Table 4.21). } \\
\text { Researcher reflections suggest Michelle was an active participant, } \\
\text { sharing information at the Intake stage, engaging in assessment and } \\
\text { co-investigator tasks and also providing feedback on what was } \\
\text { helping her to learn: } \\
\text { "I met with Michelle [child] and she gave me feedback on the co- } \\
\text { investigator activity. She feels the graphic organiser helped her with } \\
\text { her comprehension work and this could possibly be used for Irish } \\
\text { writing as well. She is finding the self-check prompt for Maths } \\
\text { helpful too. It reminds her to check answers and to use the resources } \\
\text { in her basket when needed" (Reflective Journal December } 13^{\text {th }} \text {, } \\
\text { Integration Stage). } \\
\text { Researcher reflections recorded activities that served to balance the } \\
\text { power which may have strengthened the relationship between TEP } \\
\text { and child: } \\
\text { "I met with Michelle after [meeting with parent and teacher] and } \\
\text { explained the role of the TEP and asked Michelle to tick off what she } \\
\text { understood and if I did a good job explaining, this served to balance } \\
\text { the power" (Reflective Journal November } 23^{\text {rd }} \text {, Intake stage). }\end{array}$ \\
\hline
\end{tabular}




\section{Section 3: Themes Relevant to Proposition 4 (Case 2)}

\begin{tabular}{|c|c|}
\hline Subthemes & Key Ouotes \\
\hline $\begin{array}{l}\text { Active roles and } \\
\text { communication }\end{array}$ & $\begin{array}{l}\text { Anne described how tools such as the self-monitoring checklist } \\
\text { and graphic organiser helped Michelle to feel more involved } \\
\text { in the process and encouraged confidence and independence } \\
\text { in her work: I think, like when a child like Michelle can even } \\
\text { just start writing, straight away they just feel a little bit more } \\
\text { involved and a little bit more confident about things so again } \\
\text { just encouraging that bit of independence as well (lines 132- } \\
\text { 134) }\end{array}$ \\
\hline $\begin{array}{l}\text { Strengthening } \\
\text { relationships }\end{array}$ & $\begin{array}{l}\text { When describing Michelle's experience of the AFI process, } \\
\text { Anne referred to an interaction with Michelle that could be } \\
\text { indicative of a strengthening relationship between TEP and } \\
\text { child: You know, she would be saying at the start anyway, 'do } \\
\text { I go to see that lady today?' You know and then she obviously } \\
\text { was calling you by your name and that so she loved that I } \\
\text { suppose...(lines } 36-38 \text { ) }\end{array}$ \\
\hline
\end{tabular}

\section{Section 4: Summary of Proposition 4 Findings (Case 2)}

Likert scale ratings provided by Michelle indicate that she knew what her strengths and needs were before engaging with the AFI model. Ratings also indicate that she knew what she could do to improve on needs at school. Data were not collected from Mary (parent) and Michelle (child) following engagement with the AFI model and there was limited evidence that could be drawn upon for analysis in this case. The theme 'A team approach to assessment' referred to understanding of strengths and needs and what to do to address needs and interactions and activities that occurred at the meso and microsystem levels (See Section 3). Researcher reflections also suggest Michelle was an active participant, sharing information at the Intake stage, engaging in assessment and co-investigator tasks and also providing feedback on what was helping her to learn. Consistent with AFI Principle 6, Michelle was considered a 'co-assessor' during the Investigation stage and her feedback and opinions were valued during the AFI process (Pameijer, 2017). Activities and interactions that Michelle engaged in are listed in Section 4.16, Table 4.21. Section 5 below provides a conceptual analysis of the findings and themes, in accordance with AFI principles, EST and SCT concepts. 
Section 5: Conceptual Analysis of Proposition 4 Findings (Case 2)

\begin{tabular}{|c|c|c|c|}
\hline $\begin{array}{l}\text { Theme/ } \\
\text { Subtheme }\end{array}$ & $\begin{array}{l}\text { AFI Principles (See section } 2.16 \text { for description of } \\
\text { AFI Principles) }\end{array}$ & $\begin{array}{l}\text { EST Concepts (See section } 2.14 \\
\text { for definitions of EST concepts) }\end{array}$ & $\begin{array}{l}\text { SCT Concepts (See } \\
\text { section } 2.15 \text { for } \\
\text { definitions of SCT } \\
\text { concepts) }\end{array}$ \\
\hline $\begin{array}{l}\text { Active roles } \\
\text { and } \\
\text { communication }\end{array}$ & $\begin{array}{l}\text { Researcher reflections and thematic analysis of } \\
\text { interview responses suggests Michelle's active role in } \\
\text { the team assessment approach provided her the } \\
\text { opportunity to be more involved in the process and } \\
\text { encouraged confidence and independence in her work. } \\
\text { Anne commented that providing Michelle with tools, } \\
\text { such as the self-monitoring checklist helped her to "feel } \\
\text { a little bit more involved" and encouraged } \\
\text { "independence". Michelle's active involvement as a }\end{array}$ & $\begin{array}{l}\text { Michelle's active participation and } \\
\text { enabling her participation during } \\
\text { the process reflects a shifting } \\
\text { balance of power towards her, as } \\
\text { the developing person } \\
\text { (Bronfenbrenner, 1979). }\end{array}$ & $\begin{array}{l}\text { Tools and signs, including } \\
\text { checklists and a graphic } \\
\text { organiser helped to } \\
\text { facilitate Michelle's active } \\
\text { involvement during the } \\
\text { Investigation stage and her } \\
\text { learning during the process } \\
\text { (Palinscar, 1998; } \\
\text { Vygotsky, 1978) }\end{array}$ \\
\hline
\end{tabular}

Strengthening Researcher reflections and thematic analysis of relationships interview responses suggests a strengthening relationship between the child and TEP. Anne noted that Michelle felt comfortable working with the TEP and "was calling you (TEP) by your name". Researcher reflections recorded activities that served to balance the power which may have subsequently strengthened the relationship. Strengthening relationships resonates with AFI Principle 6, where assessment is built upon collaborative partnerships between TEP, parents, teachers and child (Pameijer, 2017).

Strengthening relationships between the TEP and student is suggestive of strengthened dyadic relations at the microsystem level (Bronfenbrenner, 1979). 
Appendix 44 Case 2 Analysis of Findings: Proposition 5

The proceeding sections provide a comprehensive analysis of Proposition 5 findings for Case 2. Section 1 below outlines the criteria for interpreting the findings, also presented in in Section 3.5.5 in Chapter 3. Section 2 presents the analysis of findings. Section 3 outlines the themes relevant to Proposition 5 and Section 4 provides a summary of findings. Section 5 provides a conceptual analysis of Proposition 3 findings.

Section 1: Criteria for Interpreting Proposition 5 Findings (Case 2)

\begin{tabular}{|c|c|c|c|c|}
\hline $\begin{array}{l}\text { Proposition: } \\
\text { Following } \\
\text { Engagement } \\
\text { with the AFI } \\
\text { Model: }\end{array}$ & $\begin{array}{l}\text { Likert Scale } \\
\text { Statements }\end{array}$ & $\begin{array}{l}\text { Likert Scale } \\
\text { Statements } \\
\text { (Comments } \\
\text { Section) }\end{array}$ & $\begin{array}{l}\text { Semi-structured } \\
\text { Interviews }\end{array}$ & $\begin{array}{l}\text { Researcher } \\
\text { Diary }\end{array}$ \\
\hline $\begin{array}{l}\text { Proposition 5: } \\
\text { 'At a time of } \\
\text { ecological } \\
\text { transition, } \\
\text { teachers will } \\
\text { feel competent } \\
\text { in their ability } \\
\text { to work } \\
\text { according to the } \\
\text { aims of Circular } \\
0013 / 2017 \text {, in } \\
\text { meeting and } \\
\text { monitoring the } \\
\text { needs of the } \\
\text { child' }\end{array}$ & N/A & N/A & $\begin{array}{l}\text { Pattern-matching: } \\
\text { Interview responses } \\
\text { that link } \\
\text { competence in in } \\
\text { meeting and } \\
\text { monitoring the } \\
\text { needs of the child, } \\
\text { engagement with } \\
\text { the AFI model and } \\
\text { interactions and } \\
\text { activities that have } \\
\text { occurred at the } \\
\text { meso and } \\
\text { microsystem levels } \\
\text { and vice versa. } \\
\text { Thematic } \\
\text { analysis: Themes } \\
\text { and patterns within } \\
\text { the data set that } \\
\text { refer to meeting } \\
\text { and monitoring the } \\
\text { needs of the child, } \\
\text { and interactions } \\
\text { and activities that } \\
\text { have occurred at } \\
\text { the meso and } \\
\text { microsystem levels } \\
\text { were included in } \\
\text { the analysis of the } \\
\text { AFI model. }\end{array}$ & N/A \\
\hline
\end{tabular}


Section 2: Analysis of Proposition 5 Findings (Case 2)

\begin{tabular}{|c|c|c|c|c|}
\hline $\begin{array}{l}\text { Evidence } \\
\text { for } \\
\text { Proposition } \\
5 \text { (Case 2) }\end{array}$ & $\begin{array}{l}\text { Likert Scale } \\
\text { Statements }\end{array}$ & $\begin{array}{l}\text { Likert } \\
\text { Scale } \\
\text { Statements } \\
\text { (Comments } \\
\text { Section) } \\
\end{array}$ & Semi-structured Interviews & Researcher Diary \\
\hline $\begin{array}{l}\text { Class } \\
\text { teacher } \\
\text { (Anne) }\end{array}$ & N/A & N/A & $\begin{array}{l}\text { Pattern-matching: Do you feel you can monitor Michelle's } \\
\text { needs at school? Yes, am...yeah I think so (competence to } \\
\text { meet/monitor needs), I mean obviously I think just in } \\
\text { general just using... sorry now I'm just trying to think. In } \\
\text { general just in terms of observations and we're doing that all } \\
\text { day every day (competence to meet/monitor needs), and I } \\
\text { suppose maybe I'd be doing it anyway but I would feel I } \\
\text { would be a lot more mindful now and I'd probably pick up } \\
\text { on things, we've just done so much analysis of her maybe } \\
\text { that now I feel I'd be more clued in (interactions and } \\
\text { activities during the AFI process), so yeah moving forward } \\
\text { absolutely (competence to meet/monitor needs) (lines 136- } \\
\text { 140) } \\
\text { Thematic analysis (See Section } 3 \text { below for key quotes of } \\
\text { themes and subthemes relevant to Proposition 5): } \\
\text { Factors bridging the gap between assessment and } \\
\text { intervention } \\
\text { - Shared awareness of strengths, needs and goals identified } \\
\text { - Experiencing or observing intervention effectiveness }\end{array}$ & $\begin{array}{l}\text { Researcher diary (AFI } \\
\text { templates) documented } \\
\text { specific activities and } \\
\text { interactions that took place } \\
\text { during the five-stage AFI } \\
\text { process See Section 4.16, Table } \\
\text { 4.21) }\end{array}$ \\
\hline
\end{tabular}




\section{Section 3: Themes Relevant to Proposition 5 (Case 2)}

\begin{tabular}{ll}
\hline Theme 3: Factors Bridging the Gap between Assessment and Intervention \\
\hline $\begin{array}{l}\text { Subthemes } \\
\begin{array}{l}\text { Shared awareness of } \\
\text { strengths, needs and } \\
\text { goals identified }\end{array}\end{array}$ & $\begin{array}{l}\text { When describing how she would monitor Michelle's } \\
\text { (child's) needs, Anne (class teacher) commented on how she } \\
\text { felt more tuned into her specific needs, following } \\
\text { engagement in the AFI process: More clued into the } \\
\text { specific but specifically what she needs... (line 142) }\end{array}$ \\
$\begin{array}{l}\text { Experiencing or } \\
\text { observing } \\
\text { intervention } \\
\text { effectiveness }\end{array}$ & $\begin{array}{l}\text { Anne also referred to how she is more aware of what } \\
\text { strategies are going to work for Michelle, following } \\
\text { engagement in the AFI process, which would make } \\
\text { monitoring of needs easier moving forward: ...Just a lot } \\
\text { more aware of what's going to work for her, you know so it } \\
\text { might make it [monitoring] a bit easier (lines 142-143) }\end{array}$ \\
\hline
\end{tabular}

\section{Section 4: Summary of Proposition 5 Findings (Case 2)}

Pattern-matching logic applied to Anne's interview response connected competence to meet and monitor Michelle's needs moving forward to activities and interactions that occurred during the AFI process, at the meso and microsystem levels. Patterns within her response linked competence to being "more mindful now" and being able to "pick up on things", following all the "analysis" completed during the assessment process. This "analysis" consisted of activities and interactions that occurred during the stages of the AFI model, at the meso and micro system levels, including dialogue, investigation and observation tasks completed by all participants, 1:1 assessments and at the mesosystem level, meetings and dialogue between TEP, parent and teacher. These activities and interactions are consistent with AFI Principle 6 as participants were 'co-assessors' during the Investigation stage and their opinions and feedback were valued as 'hands-on experts through experience' throughout the process (Pameijer, 2017). See Section 4.16, Table 4.21 for a list of activities and interactions that participants engaged in. Being more "mindful" of, and monitoring Michelle's needs moving forward also resonates with AFI Principle 3, focusing on the educational needs of the child. Patterns within Anne's response also connected competence to meet and monitor Michelle's needs to the "observations" that she conducts "all day every day". Consistent with AFI Principle 3, effective monitoring approaches were encouraged to be continued during meetings and discussions ('goodness of fit') (Pameijer, 2017). The theme 'Factors bridging the gap between assessment and intervention' referred to meeting and monitoring needs and interactions and activities that occurred at the meso 
and microsystem levels (See Section 3). Section 5 below provides a conceptual analysis of the findings and themes, in accordance with AFI principles, EST and SCT concepts. 
Section 5: Conceptual Analysis of Proposition 5 Findings (Case 2)

\begin{tabular}{|c|c|c|c|}
\hline $\begin{array}{l}\text { Theme/ } \\
\text { Subtheme }\end{array}$ & $\begin{array}{l}\text { AFI Principles (See section } 2.16 \\
\text { for description of AFI } \\
\text { Principles) }\end{array}$ & $\begin{array}{l}\text { EST Concepts } \\
\text { (See section } 2.14 \\
\text { for definitions of } \\
\text { EST concepts) }\end{array}$ & $\begin{array}{l}\text { SCT } \\
\text { Concepts } \\
\text { (See } \\
\text { section } \\
2.15 \text { for } \\
\text { definitions) }\end{array}$ \\
\hline $\begin{array}{l}\text { Factors } \\
\text { bridging the } \\
\text { gap between } \\
\text { assessment } \\
\text { and } \\
\text { intervention }\end{array}$ & $\begin{array}{l}\text { Pattern-matching logic applied to } \\
\text { Anne's interview response } \\
\text { connected competence to meet } \\
\text { and monitor Michelle's needs } \\
\text { moving forward to activities and } \\
\text { interactions that occurred at the } \\
\text { meso and microsystem level, } \\
\text { during the AFI process. Patterns } \\
\text { within her response linked } \\
\text { competence to being "more } \\
\text { mindful now" and being able to } \\
\text { "pick up on things", following all } \\
\text { the "analysis" completed during } \\
\text { the assessment process. Thematic } \\
\text { analysis of interview responses } \\
\text { suggests the theme 'Factors } \\
\text { bridging the gap between } \\
\text { assessment and intervention' may } \\
\text { provide further explanation for } \\
\text { competence to meet and monitor } \\
\text { Michelle's needs moving } \\
\text { forward. 'Shared awareness of } \\
\text { needs and goals identified' and } \\
\text { 'Experiencing or observing } \\
\text { intervention effectiveness' are } \\
\text { factors that provide further } \\
\text { explanation. For example, Anne } \\
\text { commented that having worked } \\
\text { through the AFI process, she } \\
\text { feels more aware of Michelle's } \\
\text { needs and the intervention } \\
\text { approaches that she responds well } \\
\text { to, which will make monitoring } \\
\text { of needs easier moving forward } \\
\text { (See Section } 3 \text { ). }\end{array}$ & $\begin{array}{l}\text { Teacher } \\
\text { monitoring of } \\
\text { student needs } \\
\text { reflects } \\
\text { strengthening } \\
\text { dyadic relations } \\
\text { between the } \\
\text { teacher and } \\
\text { student at the } \\
\text { microsystem } \\
\text { level, by paying } \\
\text { increased } \\
\text { attention to } \\
\text { Michelle's needs } \\
\text { and progress } \\
\text { (Bronfenbrenner, } \\
\text { 1979). }\end{array}$ & \\
\hline
\end{tabular}


Appendix 45 Thematic Maps (Braun and Clarke, 2006)

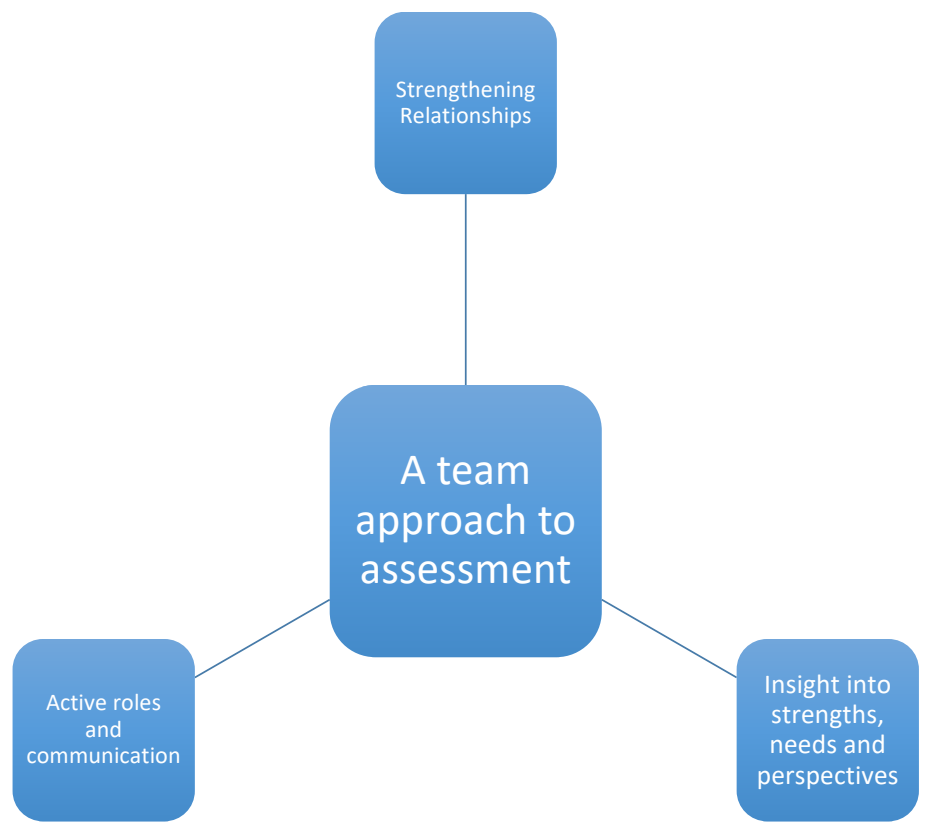

A team approach to assessment (Theme 1)

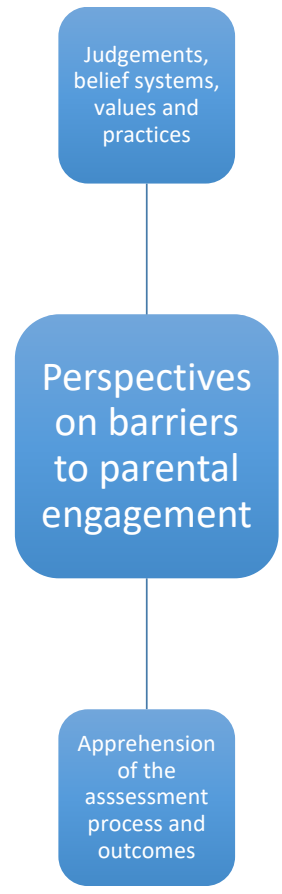

Perspectives on barriers to parental engagement (Theme 2) 


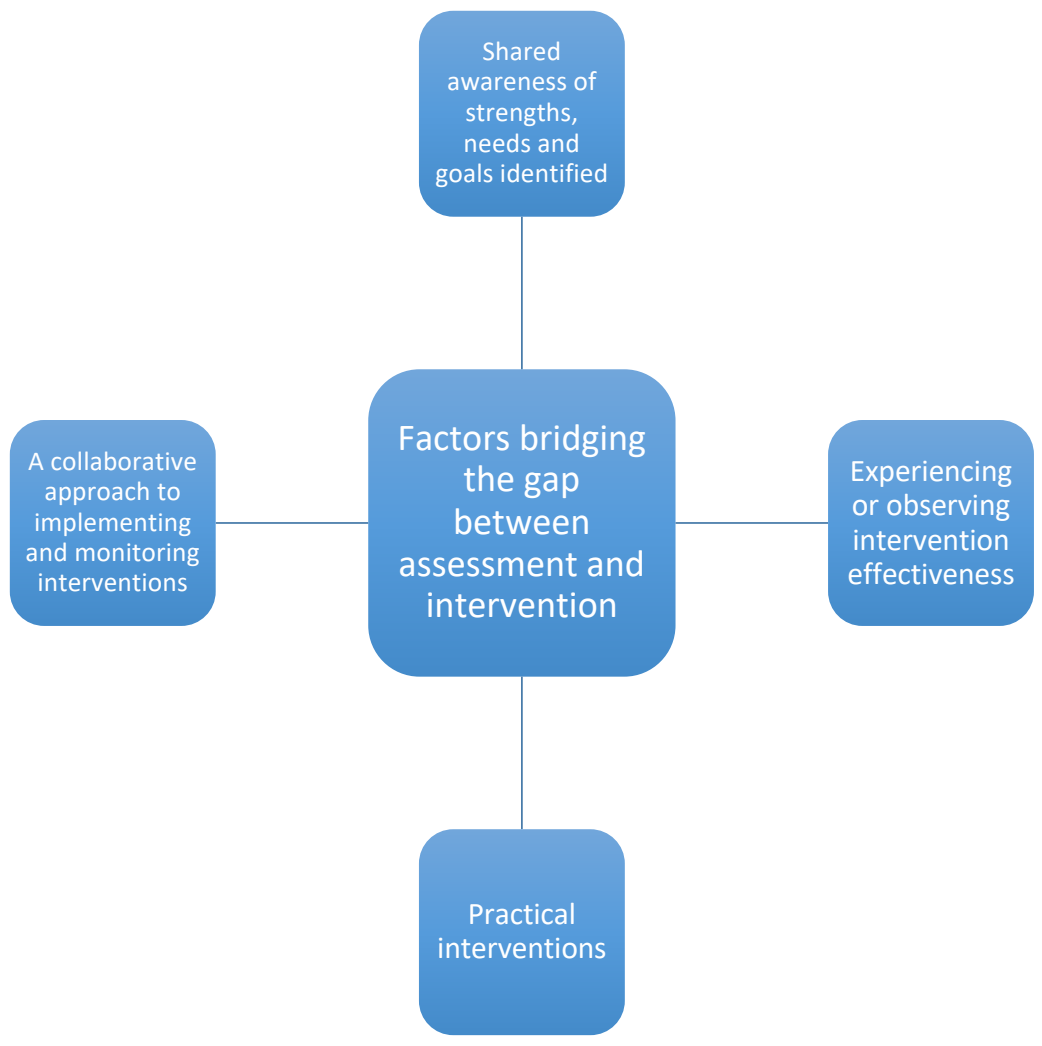

Factors bridging the gap between assessment and intervention (Theme 3)

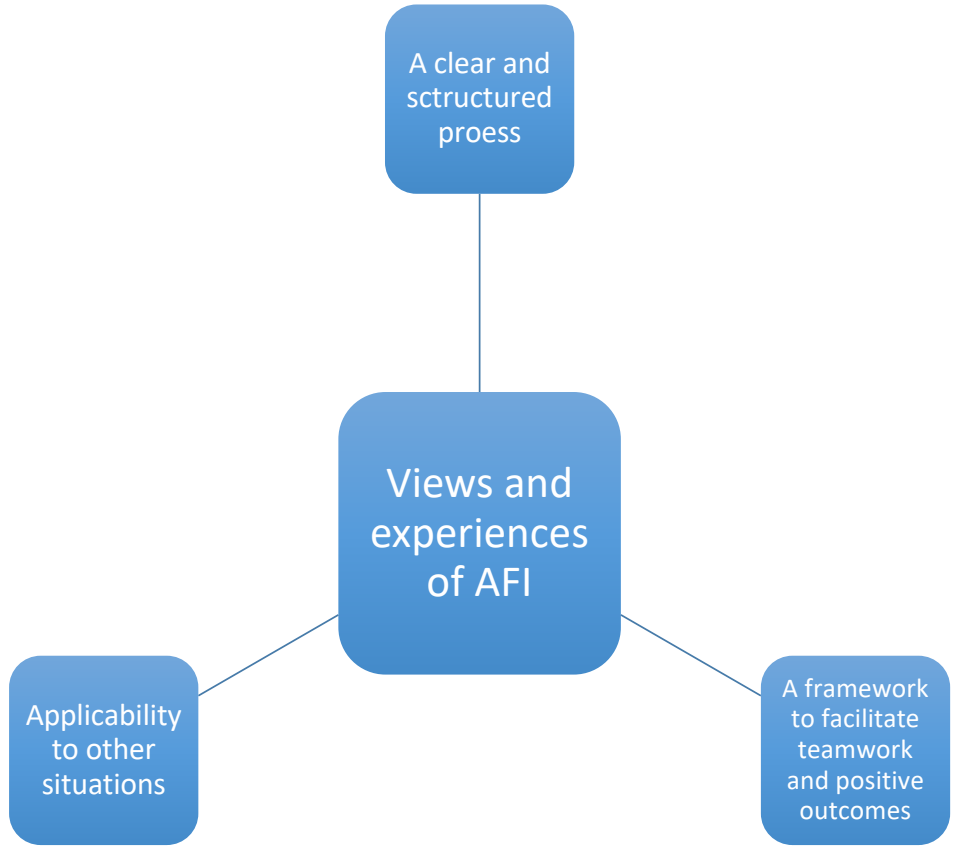

Views and experiences of AFI (Theme 4) 


\begin{tabular}{l} 
Initial Categories \\
\hline Team approach to assessment at the meso \\
and micro system levels \\
- Roles and responsibilities \\
- Dialogue and active participation \\
- Child's voice \\
- Missing team players
\end{tabular}

Outcomes of a team approach to assessment at the meso and micro system levels

- Strengthening relationships Codes

TA

TA R

TA DA

TA CV

TA MP

- Knowledge and awareness of specific needs

- Insight into home/school situation

- Empowerment of team members

- Awareness of everyone's intentions and activities

Perspectives on barriers to engage in a team approach to assessment

- Belief systems and values

$\mathrm{BE}$

- Unrealistic expectations

BE BV

BE UE

- Power of the referrer

- Opportunity structures

BE PR

- Past experiences of limited

BE OS communication

Factors that bridge the gap between assessment and intervention

- Awareness of strengths and needs

- Observing the effectiveness of intervention recommendations

- Practical interventions that are useful to all

- Shared decision making

- Team approach to monitoring intervention recommendations

- Allowing time for change to occur

BE PEC

BG

BG ASN

BG OE

BG PI

BG SD

BG TAM

BG TC

The role of language in assessment

- Clear, accessible language

- Language to promote

LA understanding

- Consistency of language use across systems

- Language that evokes feelings

LA CA

LA U

LA CS

LA EF

LA SNP 
- Language of strengths, needs and potential

Views and experiences of AFI

\section{VE}

- Structured model

VE SM

- Framework to facilitate team work VE FT and resulting outcomes

- A positive assessment experience

VE PE

- Applicability to other situations

VE AS 


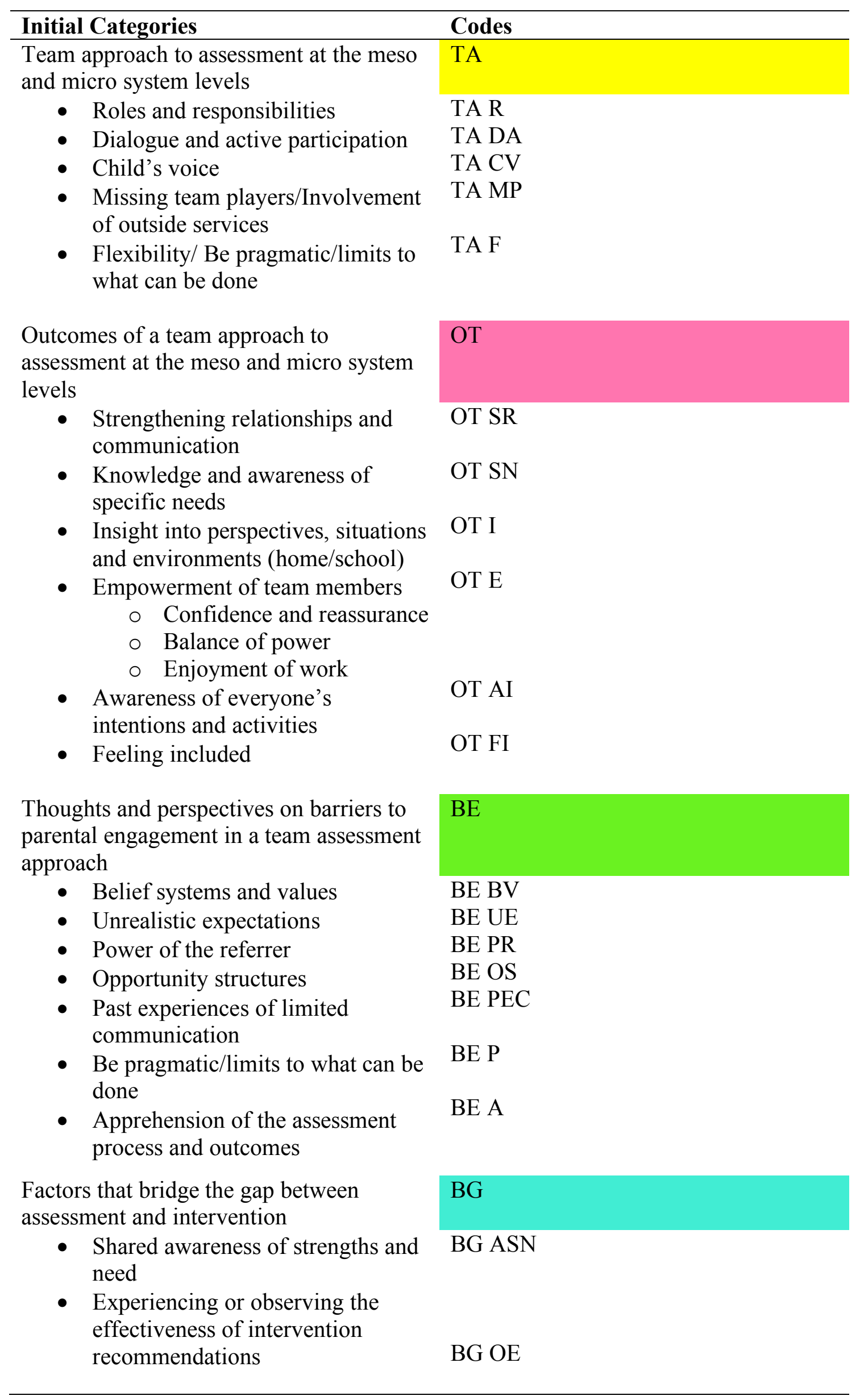


- Practical interventions that are useful to all

BG PI

- Shared decision making

- Team approach to implementing

BG SD and monitoring intervention BG TAIM recommendations

- Allowing time for change to occur

- Strengthened relationships

BG TC

BG SR

The role of language in assessment

- Clear, accessible language

- Language to promote

LA CA understanding

- Consistency of language use across LA CS systems

- Language that evokes feelings LA EF

- Language of strengths, needs and potential

Views and experiences of AFI

VE

- Structured model

VE SM

- Framework to facilitate team work

VE FT and resulting outcomes

- A positive assessment experience

- Applicability to other situations

- Time consuming 
Devising Main Themes and Subthemes

\begin{tabular}{|c|c|c|}
\hline Theme 1 & Subthemes & Codes \\
\hline \multirow[t]{10}{*}{$\begin{array}{l}\text { A team approach to } \\
\text { assessment }\end{array}$} & $\begin{array}{l}\text { Active roles and } \\
\text { communication }\end{array}$ & $\begin{array}{l}\text { Roles and responsibilities (TA } \\
\text { R) }\end{array}$ \\
\hline & & $\begin{array}{l}\text { Dialogue and active } \\
\text { participation (TA DA) }\end{array}$ \\
\hline & & Child's voice (TA CV) \\
\hline & & $\begin{array}{l}\text { Inclusion and empowerment of } \\
\text { the child (TA IE) }\end{array}$ \\
\hline & & $\begin{array}{l}\text { Flexibility and co-operation (TA } \\
\text { F) }\end{array}$ \\
\hline & & Outside services (TA O) \\
\hline & $\begin{array}{l}\text { Strengthening } \\
\text { Relationships }\end{array}$ & $\begin{array}{l}\text { Relationships and } \\
\text { communication (TA RC) }\end{array}$ \\
\hline & & $\begin{array}{l}\text { Awareness of everyone's } \\
\text { intentions and activities (TA AI) }\end{array}$ \\
\hline & $\begin{array}{l}\text { Insight into strengths, } \\
\text { needs and perspectives }\end{array}$ & $\begin{array}{l}\text { Knowledge and awareness of } \\
\text { specific needs (TA SN) }\end{array}$ \\
\hline & & $\begin{array}{l}\text { Insight into perspectives and } \\
\text { situations (TA I) }\end{array}$ \\
\hline Theme 2 & Subthemes & Codes \\
\hline \multirow[t]{5}{*}{$\begin{array}{l}\text { Perspectives on } \\
\text { barriers to parental } \\
\text { engagement }\end{array}$} & $\begin{array}{l}\text { Judgements, belief } \\
\text { systems, practices and } \\
\text { values }\end{array}$ & $\begin{array}{l}\text { Values (BE V) } \\
\text { Belief systems (BE BS) }\end{array}$ \\
\hline & & Practices (BE P) \\
\hline & & Judgements (BE J) \\
\hline & $\begin{array}{l}\text { Apprehension of the } \\
\text { assessment process and } \\
\text { outcomes }\end{array}$ & $\begin{array}{l}\text { Language of assessment (BE } \\
\text { LA) }\end{array}$ \\
\hline & & $\begin{array}{l}\text { Language that evokes feelings } \\
\text { (BE EF) }\end{array}$ \\
\hline Theme 3 & Subthemes & Codes \\
\hline $\begin{array}{l}\text { Factors bridging the } \\
\text { gap between } \\
\text { assessment and } \\
\text { intervention }\end{array}$ & $\begin{array}{l}\text { Shared awareness of } \\
\text { strengths, needs and goals } \\
\text { identified }\end{array}$ & $\begin{array}{l}\text { Shared awareness of strengths } \\
\text { and need (BG ASN) } \\
\text { Monitoring (BG M) }\end{array}$ \\
\hline
\end{tabular}


Experiencing or observing Experiencing interventions (BG intervention effectiveness E)

Observing interventions (BG O)

Practical interventions

Practical interventions (BG PI)

Useful interventions (BG UI)

A collaborative approach to implementing and Team approach to implementing monitoring interventions and monitoring interventions (BG TAIM)

\begin{tabular}{lll}
\hline Theme 4 & Subthemes & Codes \\
\hline $\begin{array}{l}\text { Views and } \\
\text { experiences of AFI }\end{array}$ & $\begin{array}{l}\text { A clear and structured } \\
\text { process }\end{array}$ & $\begin{array}{l}\text { Structured model (VE SM) } \\
\text { Clear accessible language (VE } \\
\text { CA) }\end{array}$ \\
& $\begin{array}{l}\text { Language to promote } \\
\text { understanding (VE U) }\end{array}$ \\
$\begin{array}{l}\text { A framework to facilitate } \\
\text { teamwork and positive } \\
\text { outcomes }\end{array}$ & $\begin{array}{l}\text { Framework to facilitate } \\
\text { teamwork and resulting } \\
\text { outcomes (VE FT) }\end{array}$ \\
& $\begin{array}{l}\text { Language of strengths, needs } \\
\text { and potential (VE SNP) }\end{array}$ \\
& $\begin{array}{l}\text { Applicability to other } \\
\text { situations }\end{array}$ & $\begin{array}{l}\text { Applicability to other situations } \\
\text { (VE AS) }\end{array}$ \\
\hline
\end{tabular}




\title{
Appendix 47 Example of Coding Applied to a Participant's Interview Transcript
}

\author{
(Michael-class teacher)
}

Can you describe your experience of working with the Assessment for Intervention model?

I found it a very worthwhile experience. I think getting the voice of the pupil, cur voice and the TA CV parent was really good and am, just helps us get a better understanding of the specific needs TA DA and then the various stages of it made it very clear and we saw the progress then and it was TA SN really beneficial. $\backslash V E S M$ V S O

OK and from your perspective what was my role throughout the Assessment for Intervention process?

\section{TA R}

Hmm, well I think you were really a facilitator, you know you facilitated the opportunity for us, me the class teacher, the learning support teacher to sit down and you know to voice our needs and am you know to share them with you and then obviously then you went back and you did your research and you interviewed the parent as well so it was really good that you got all the $-T A I$ different sides of the situation and the pupil voice was important too so, and then you were the person that took back that feedback and put it into action, so am I think) ( was just someone that was a facilitator and then someone that created the opportunity to...

$$
\text { TACV }
$$

Ok and what was the parent's role, Jim's mom's role throughout the process?

Hmm, well I guess her role was to give us a sort of broader picture of Jim and his home life and /

A SN her particular concerns and needs and things that work well, what he responds well to at home and am, I think as well she just wanted to get some ideas from school and have a better

understanding of his specific needs, you know so...I think the role was to strengthen - TA RC communication too so it's like to be the educator at home, because she's the primary educator and I think it was a very important role what she had, she was an equal partner in that modes $\backslash$ TA $R$ so...

OK and I suppose your role and the other teacher's role in the process?

Well like that I guess we had our concerns at the start and that's why we referred Jlm for the assessment project and am...then it was our role to observe initially, to voice our concerns and $\angle$ TA DA then maybe to garnish some feedback and then going through the different steps and then we got some recommendations from you of some particular tasks and interventions we could use VE SM and supports in class, so It was just really following the different steps and giving our feedback. our observations and then trying things and just again from the interviews with you after each phase, am..,it's just really taking on board all the different recommendations and then just trying to move forward with them and so that was it.

OK and lastly what was Jim's role throughout the process?

$$
\text { TA PA }
$$

Yeah well Jim played a very active role in the process. I suppose initially being introduced to you and us explaining together and with Tina his mum as well and Sarah and I, what the project 


\section{TA IE}

was about the model, and just making aware of us things he might be having difficulty with so TA R his role was to be able to share his areas of concern and areas where he felt he needed a bit of TACV strengthening and support. And then am, he had a very central role, he was honest in his feedback and he was telling us what works well and what doesn't and what he finds difficult so I TACV think it was really important, like his voice was heard and I think he really appreciated that and then he enjoyed getting the positive feedback at the end and his certificate and all about him and so it was good, it felt like he was ust as important in the whole process as anyone so it was good yeah.

$$
\text { TA CV TA IE }
$$

So you think his experience...

I think itil be a worthwhile experience for him yeah...so it wasn't just something that was imposed on him, it was something that he was really you know, he was involved in...so yeah I think it was a good experience.

$$
\text { TA A I E }
$$

Ok do you feel your understanding of Jim's situation has changed after working through the five stages of the Assessment for Intervention model?

Yeah I definitely think it has, am I suppose I would have had concerns about his numeracy and oral language skills but now I can see that he has very good like visual skills and it's just possibly the difficulty in, maybe the difficulty and the weakness in his working memory and trying to implement, you know, skills into processing and you know, processing information, so I think that's the biggest thing and even ideas to work with him and things that will help train the muscles and improve possibly his working memory will be good, so I think I do have a better understanding, definitely. Initially it was like, we were just wondering what is it but now, you know, it's better, like there's possibly Specific Difficulties but it's something that we're more aware of and $/$ guess when we have these recommendations and implementations and then - BGM come back to it, see how they go and then in a year or two, we will be more aware and sort of cognizant of what are his needs but definitely more aware for now so...

Yeah and following that response to intervention approach

Yeah definitely following the steps was really helpfuly - VE SM

Did the intervention recommendations address your questions at the intake stage? VE U

They did yeah they were very clear and they helped focus me on you know my particular concerns and just to sit down and have a little think about it and I guess they were very good difecting questions to focus you and help your understanding so...

$\checkmark E C A$ /VE $U$

And do you think then the recommendations at the end, did they address your questions that you had at the intake stage? 
Appendix 48 Devising Main Themes, Subthemes, Codes and Relevant Statements

\section{Theme 1: A Team Approach to Assessment}

\begin{tabular}{ll}
\hline $\begin{array}{l}\text { Subtheme } \\
\text { 1. Active roles and } \\
\text { communication }\end{array}$ & $\begin{array}{l}\text { Codes } \\
\text { Roles and } \\
\text { responsibilities }\end{array}$ \\
& $\begin{array}{l}\text { Dialogue and active } \\
\text { participation }\end{array}$
\end{tabular}

Child's voice

\section{Relevant Statements}

Pilot Case

Am, I saw kind of your role [TEP] as facilitator, that the questions you asked kind of guided us in a way that got us thinking about things, that maybe if we were writing a report on a piece of paper, we mightn't have actually thought of (Grace, Pilot Case teacher, lines 26-28)

I think having the parents and children involved really actually... gave another dimension to the whole intervention process that it's not just me talking about school (Grace, Pilot Case teacher, lines 3-5)

I think kind of highlighting to the parents as well that there was issues (Grace, Pilot Case teacher, line 47)

It was lovely to actually just to meet her [parent] in this situation and to be able to hear her voice (Grace, Pilot Case teacher, lines 74-75)

Any time we've met [team], we've had huge amounts of information shared between everyone (Grace, Pilot Case teacher, line 276)

And then having the child himself involved am...it's interesting to see their perspective like I think as teachers we do guess a lot about what kids are thinking and what they mean when they say something, am but hearing their own actual voice and hearing what's going on in their head and how they perceive something, I think is really really important (Grace, Pilot Case teacher, lines 36-39) 
I suppose your [TEP] role fundamentally was kind of, teaching us the model of

assessment, how to implement it in the home environment and in the school environment (Louise, Pilot Case parent, lines 15-17)

You [TEP] needed collectively engagement from the parents, the teachers and from the child involved (Louise, Pilot Case parent, lines 17-18)

Someone looking in [TEP] and they can bring other ideas and kind of thoughts to the table and things that you might not have thought of before so...that would have been for me anyhow, the benefit of it (Louise, Pilot Case parent, lines 113-115)

It [the process] was kind of open disclosure, and any of the processes and interventions discussed were openly discussed to John in child friendly language (Louise, Pilot Case parent, lines 21-22)

He [child] probably am...was kind of a little bit taken aback, when I said to him on Wednesday and on yesterday evening that you know, we couldn't spend as much time in the activity that he likes spending most, he was taken a little bit back by that (Louise, Pilot Case parent, lines 29-32)

Part of the time it would be hard, part of the time it could be easy but most of the time its hard [working together with parent, teacher and TEP] (John, Pilot Case child, line 7)

Just doing hard decisions [response to what was hard] (John, Pilot Case child, line 9)

Case 1

Well I think you [TEP] were really a facilitator (Michael, Case 1 teacher, line 9)

His role [child] was to be able to share his areas of concern and areas where he felt he needed a bit of strengthening and support (Michael, Case 1 teacher, lines 37-38) 
I think getting the voice of the pupil, our voice and the parent was really good (Michael, Case 1 teacher, lines 3-4)

To voice our [teachers]concerns and then maybe to garnish some feedback and then going through the different steps and then we got some recommendations from you of some particular tasks and interventions we could use and supports in class (Michael, Case 1 teacher, lines 26-29)

It [teachers' role] was just really following the different steps and giving our feedback, our observations and then trying things (Michael, Case 1 teacher, lines 29-30)

He [child] was honest in his feedback and he was telling us what works well and what doesn't and what he finds difficult so I think it was really important, like his voice was heard and I think he really appreciated that (Michael, Case 1 teacher, lines 38-40)

He [child] had a very central role (Michael, Case 1 teacher, line 38)

You [TEP] explained each part of the model to us, what we were going to do at each step of the model and to make it clear to us what was involved, what we had to do, what you were going to do, what the child, the parent, what everyone was going to do throughout the model (Sarah, Case 1 SET, lines 22-25)

She [parent] is the one that would have the most insight into his [child] needs both at home and I know in school (Sarah, Case 1 SET, lines 37-38)

Well...from the beginning I suppose we were all involved from the very initial, the outset, trying to decide... and like that when we spoke with his mother, trying to decide what we felt his [child] needs were (Sarah, Case 1 SET, lines 28-30)

I suppose like that from the beginning, trying to discuss to see where we felt that he [child] may have problems, where his problems might lie, where his needs might be and 
then just having our observations in class also, and having any of the specific things that we might, any observations that we might have...done, maybe to write them down but also anything that we found that worked, with regards our observations, anything specific, like if there was praise or less homework or anything that might have helped him (Sarah, Case 1 SET, lines 44-49)

Discussing things like you know, about Jim at home and his work (Tina, Case 1 parent, line 5)

It [AFI] helped me to see more and stuff you know (Tina, Case 1 parent, line 239)

Case 2

Just facilitating the whole process [TEP's role] and am, guiding us through it all, all the different people that were involved (Anne, Case 2 teacher, lines 9-11)

I felt that just through talking through everything (Anne, Case 2 teacher, lines 57-58)

Again kind of information but that can be a little bit challenging because they [child] might be reluctant to give you that information so am, I think maybe it's harder to get it from the child, it's more the adults in the situation (Anne, Case 2 teacher, lines 21-23)

And those observations are there all the time, you're just doing that as a teacher anyway, you're always looking but just having to hone in on one particular child and really analyse that situation (Anne, Case 2 teacher, lines 58-60)

And just to have the opportunity to do that and analyse a situation so in-depth and with such support, I think is really good, am it's a very hard thing to do on your own or sort of (Anne, Case 2 teacher, lines 171-173) 
There's only so much thinking you can do about an individual child on your own,

whereas like when you have such great support and you can talk about it and sort of tease out ideas and I think it's really good (Anne, Case 2 teacher, lines 174-176)

Inclusion and empowerment of the child

\section{Pilot Case}

[The child] was kind of going well actually, I would try...(Grace, Pilot Case teacher, line 81)

It's just to help him [child] understand and give him strategies to cope with it himself (Grace, Pilot Case teacher, line 179)

So for him [child] I think seeing someone else involved, that his parents were involved, I was involved and that we were all working together I think kind of showed him that there was a team effort and that he was part of that team and I think that's what he needed to see as well, it wasn't us vs. him that it was us all together (Grace, Pilot Case teacher, lines 64-68)

And they're [the child] not being isolated out or being singled out for... maybe having a need voice but we're trying to support them within the class as well I think that's a huge thing for them to be able to understand as well...(Grace, Pilot Case teacher, lines 259-

262)

Case 1

It felt like he [child] was just as important in the whole process as anyone so it was good yeah (Michael, Case 1 teacher, lines 42-43)

It wasn't just something that was imposed on him [the child], it was something that he was really you know, he was involved in (Michael, Case 1 teacher, lines 45-46)

And that they [the child] were part of this process (Sarah, Case 1 SET, line 17) 
He [child] was able to discuss it even with us in class, do you know his role, and what, if he struggled even after having his talk with you, like it was now... he felt more willing to let us know, oh that worked, or that didn't work, or I found that a little bit hard or I struggle when you say it this way or If you... it gave him that voice which I felt he certainly didn't do it before this process (Sarah, Case 1 SET, lines 58-62)

Like teachers never do that we don't ever ask the children, do you know I mean we don't, 'well what do you think', we don't give them a voice, so we don't, we just presume (Sarah, Case 1 SET, lines 213-214)

In Maths like I said even he [child] was more willing now to tell us 'Miss that didn't work right' or 'I like when you say it that was Miss', which was great (Sarah, Case 1 SET, lines 139-140)

So he's [child] more open, more putting his hand up (Tina, Case 1 parent, line 212)

Case 2

I think, like when a child like Michelle can even just start writing, straight away they just feel a little bit more involved and a little bit more confidence about things so again just encouraging that bit of independence as well (Anne, Case 2 teacher, lines 132-134)

Flexibility and cooperation

\section{Pilot Case}

Yeah or maybe just after school [timing for meetings] I know that's awkward then for parents trying to get kids minded and stuff but maybe even an afternoon or something. I'm not quite sure how you'd go about it but I definitely think there's huge merit to it (Grace, Pilot Case teacher, lines 17-19)

I have been thinking because the information is brilliant, and you need time to get information but just maybe the time of day would be one area to look at maybe...but 
again like I said you can't help if parents are only available at one time in the morning, then it has to be the morning (Grace, Pilot Case teacher, lines 283-286)

Case 1

You cannot define or determine how engaged everyone will be and if they'll attend all the different stages, so I guess that's the only limitation but that's like in any setting, you know, you have to persevere with it and sometimes it can take longer than possibly you would have hoped and anticipated, but am, it's not really a limitation it's just possibly one drawback but sure that's what happens in life you just have to am, make the best of it (Michael, Case 1 teacher)

... again I suppose you're going to have those limitations with everything and that's...you have to work around it and do you know (Sarah, Case 1 SET, lines 292-293)

Case 2

Yes so I think obviously we did the very best that we could with the situation we had (Anne, Case 2 teacher, line 188)

Outside services

\section{Pilot Case}

But no definitely the reality and fantasy is a huge thing but that's a much bigger issue than school like, that's beyond us now so, but that would be the one thing I wouldn't feel confident in supporting, like I'd chat around it but I know that my message wouldn't get through as much (Grace, Pilot Case teacher, lines 164-167)

So hopefully when they [outside services] get involved and they have things that maybe can help, I will feel a bit more confident because there could be strategies that they can even give me that will help in class but am... (Grace, Pilot Case teacher, lines 172-174) 
Case 1

Absences role players or the participants to be engaged and I guess, you cannot define or determine how engaged everyone will be (Michael, Case 1 teacher, lines 145-147)

Case 2

Missing a lot of time and then you know when I had planned to do lessons or planned to implement some of the little tips and things like that, not being here was obviously do you know, a big problem (Anne, Case 2 teacher, lines 27-29)

\section{Strengthening} relationships

\section{Relationships and communication}

\section{Awareness of} everyone's intentions and activities

\section{Pilot Case}

So I think in a way that's another big positive as well that he [child] actually was opening up (Grace, Pilot Case teacher, lines 80-81)

Am, so I think the supports that I can try and provide to him in class and trying to use some of the strategies and even to support the parents (Grace, Pilot Case teacher, lines $52-54)$

I think to understand that was a big part of it that there was this kind of opening up of communication between home and school (Grace, Pilot Case teacher, lines 75-76)

So but I think that even he [child] does seem to be much more responsive now to things we've been saying (Grace, Pilot Case teacher, lines 116-117)

So definitely the other things have come up like, responding to me and working class, he [child] definitely seems to have taken well to that (Grace, Pilot Case teacher, lines 130131) 
As I said to you even in the couple of weeks I've seen a change in his [child] behaviour even so far that he is more responsive, and kind of more positive towards me... (Grace, Pilot Case teacher, lines 150-151)

And that they're [parents] aware that I am trying to help because I know sometimes with kids, they might go home and they might tell the negative things that happened or they mightn't talk about school at all... and for me to be able to go well actually they did really well this year or this did happen but this is how we resolved it or this is how we approached it (Grace, Pilot Case teacher, lines 254-258)

So them [the child] being aware that we are actually helping and that there are ways that we can help, I think is a huge relief for the child as well as everyone involved, they can all see that we're working together (Grace, Pilot Case teacher, lines 40-42)

I think the fact that he [child] knew that people were actually taking an interest in him and that we were looking at trying to help him (Grace, Pilot Case teacher, lines 59-60)

He [child] is actually aware that teacher is trying to help here (Grace, Pilot Case teacher, line 99)

I suppose like maybe just a simple thing that he [child] identified that he likes having a chat so it's to sit down with him and actually say to him am...Do you know why we had to put other little activities on his agenda and the reason for it is $\mathrm{X}, \mathrm{Y}$ and $\mathrm{Z}$ (Louise, Pilot Case parent, lines 68-71)

Working with you [TEP] (John, Pilot Case child, line 12) [on favourite part of process]

Am...at least I know a little bit about working with you but it's hard to do with the teachers and mom and yeah (John, Pilot Case child, lines 24-25) 
Case 1

This whole process has strengthened my relationship with Jim and you know (Michael, Case 1 teacher, lines 99-100)

Knowing [the child] that we were going to address them together as a team (Sarah, Case 1 SET, lines 17-18)

I think it was great that he [child] knew that he was going to get help because he wants to achieve (Sarah, Case 1 SET, lines 65-66)

Doing more with Jim, I kind of got more involved with Jim do you know, he let me do more with him, with his homework and stuff (Tina, Case 1 parent, lines 26-27)

Like letting me sit beside him [child] and do the homework, do you know what I mean (Tina, Case 1 parent, line 29)

Because he'd [child] go home and he'd be telling me what he was doing with you [TEP] and learning stuff (Tina, Case 1 parent, lines 40-41)

Yeah, he's [child] talking more (Tina, Case 1 parent, line 168)

He [child] goes he likes [TEP] (Tina, Case 1 parent, line 41)

He's [child] using the scrapbook now and putting pictures in it and stuff but he won't let me show anyone else, only me (Tina, Case 1 parent, lines 53-54)

He [child] was happy coming in here and doing work with you (Tina, Case 1 parent, line 96) 
Talking to the teachers more on stuff about Jim and Jim's more open now (Tina, Case 1 parent, line 206)

I can talk to the teachers more and if I've a problem I can go to them now and Jim can as well (Tina, Case 1 parent, line 246)

\section{Case 2}

You know, she [child] would be saying at the start anyway, 'do I go to see that lady today?' You know and then she obviously was calling you by your name and that so she loved that I suppose... (Anne, Case 2 teacher, lines 36-37)

I feel both of us [class teacher and TEP] were extremely approachable, we have a very good relationship with her [parent] (Anne, Case 2 teacher, lines 212-213)

\section{Insight into strengths, needs and perspectives}

Knowledge and awareness of specific needs

\section{Pilot Case}

I think now after doing the behaviour functional assessment that that was kind of highlighting areas that I mightn't have necessarily thought about, like the triggers and stuff that these behaviours just happened and it was trying to control the situation so it wasn't getting everyone involved...am, so I think I'd be more aware now to go ok, well this happened at 12 o' clock, the [trigger for restricted interest occurs at 12 o' clock], I think I'd just definitely be more aware (Grace, Pilot Case teacher, lines 205-210)

And academically then just reassuring him [child] that he does have the ability and that he doesn't have issues that way, that will reassure him with his question (Grace, Pilot Case teacher, lines 163-164)

Like you have understanding and insight into his [child] need for the obsessional interest that he has but I think, I suppose you have...greater understanding as to why he does participate in these activities (Louise, Pilot Case parent, lines 48-50) 
And I suppose the other thing is, to try and do is to kind of get him [child] to reason so that he might participate in the idea of spending less time [engaging in restricted interest], and so look if he understands, he might buy into it more readily (Louise, Pilot Case parent, lines 70-73)

Case 1

Just helps us [the process] get a better understanding of the specific needs [child's] (Michael, Case 1 teacher, line 4)

I can see that he [child] has very good like visual skills and it's just possibly the difficulty in, maybe the difficulty and the weakness in his working memory (Michael, Case 1 teacher, lines 51-52)

And it was good for them [child] to recognise, any needs that they might have (Sarah, Case 1 SET, lines 16-17)

Now I know exactly what his [child] needs are, I know what his needs are in literacy and in numeracy and with regard to working memory (Sarah, Case 1 SET, lines 110-111)

I understand more about Jim (Tina, Case 1 parent, line 3)

And helping to find out what his [child's] strengths were [role of TEP], you know what he's capable of (Tina, Case 1 parent, line 11)

You know kind of where he [child] is now (Tina, Case 1 parent, line 14)

Am, drawing and reading and a little bit of maths (Jim, Case 1 child, line 68)-on his strengths

Maths and spelling (Jim, Case 1 child, line 89)-on his needs 
Case 2

At the start looking at right well what do I know and what can we do about this and then so many weeks later really feeling a lot more confident about...maybe you knew it all along but it's just sort of, I don't know it just sort of confirms it for you and you understand things a lot better (Anne, Case 2 teacher, lines 62-65)

Insight into perspectives and situations

\section{Pilot Case}

I actually saw what was happening at home and that kind of reassured that behaviours that I had noticed were actually happening at home as well, that it wasn't just schoolbased (Grace, Pilot Case teacher, lines 5-7)

I think having the parents involved like I said earlier getting their experience because sure we all know there's things going on at home that we're not always aware of. And having that relationship that could be built up, that we could actually discuss what was going on or if they were having issues at home that maybe were falling into school. Or for, I know for kids that are on the spectrum and stuff I know that they can hold it all in at school and when they go home it's an outburst, so even hearing if that was a factor at home was a huge benefit to me because at least I know then if he was going home in a bad mood that I could ring the mum and go look something happened today and just to let you know (Grace, Pilot Case teacher, lines 28-36)

I think I do understand the situation a bit more, I can see his [child] side, I can see the parent's side and I think they see my side of it as well... that we're all just, I think we're just more aware, I think would be the word I would use (Grace, Pilot Case teacher, lines 83-85)

So hopefully even if we can get him [child] to see it slightly, that maybe it will start to click (Grace, Pilot Case teacher, lines 129-130) 
Definitely hearing his [child] situation at home is a huge eye opener as well because they're having serious difficulty with him at home (Grace, Pilot Case teacher, lines 234235)

Cause sometimes you might feel there's certain problems in the home environment and they might be different to the problems that are going on inside in the school and sometimes when you have an outsider [TEP] coming in, and kind of collectively looking at it as a stranger from the outside, I think that sometimes can be beneficial (Louise, Pilot Case parent, lines 107-111)

Case 1

It was really good that you [TEP] got all the different sides of the situation (Michael,

Case 1 teacher, lines 12-13)

Her [parent's] role was to give us a sort of broader picture of Jim and his home life and her particular concerns and needs and things that work well, what he responds well to at home (Michael, Case 1 teacher, lines 17-18)

Maybe anything that might be happening at home that we could take on board in school or things the school could do that she [parent] might find helpful or things that school might find if she did at home that we might find helpful (Sarah, Case 1 SET, lines 33-35)

I know more about Jim, about his work and his homework and stuff (Tina, Case 1 parent, lines 5-6)

Case 2

Am obviously the parent had to provide information that we wouldn't be aware of, am so to add that other dimension to things and I suppose to be supportive in the process and to understand...I suppose why everything was being done and the benefits of it all (Anne, Case 2 teacher, lines 13-15) 
Yeah again I suppose providing the information that I could [teacher's role] provide

based on what I know about the child and my everyday observations, am so providing all of that information and then just kind of working together to try to come up with the best plan moving forward, really (Anne, Case 2 teacher, lines 17-19)

Again kind of information but that can be a little bit challenging because they [child] might be reluctant to give you that information so am, I think maybe it's harder to get it from the child, it's more the adults in the situation (Anne, Case 2 teacher, lines 21-23)

Just being way more aware of the situation (Anne, Case 2 teacher, line 58)

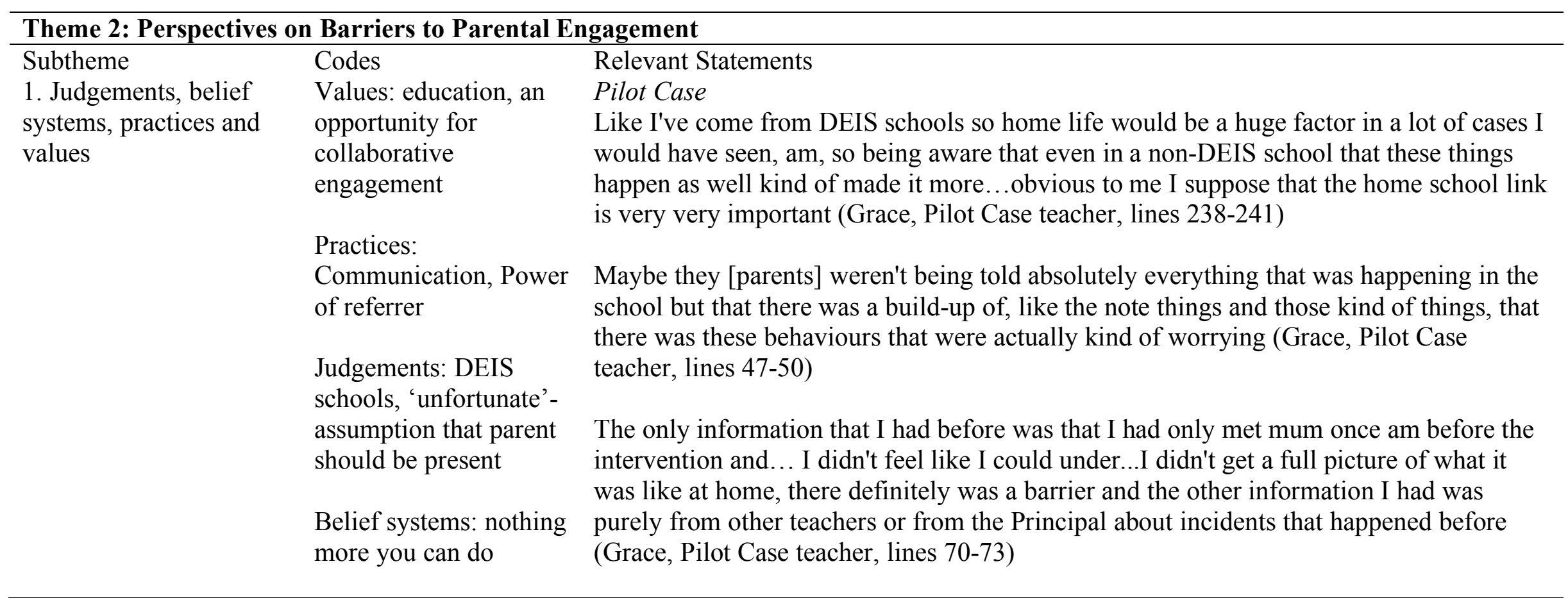


Like we only get to meet them [parents] maybe once a year with parent teacher meetings and beyond that it's maybe a phone call or the odd meeting (Grace, Pilot Case teacher, lines 243-244)

Case 1

Again I suppose from a parent's perspective as well and also from a teacher's perspective, but it's such a pity that...yeah do you know when these [assessment process] are available for you and your child, that you don't avail of them (Sarah, Case 1 SET, lines 264-267)

I feel that I could access it [assessment process] no problem, again when the child was in school again something that he could do no problem as well, he was happy to do it and then the parents... it's a pity (Sarah, Case 1 SET, lines 271-273)

Even like the communication with the parents like...I find that hugely important but do we ever try to make that time to do it no (Sarah, Case 1 SET, lines 214-216)

I don't think there is any other way you could help that [parent involvement]...I really don't think there is, I really don't think so...but maybe other parents would be happy for you to come into their home (Sarah, Case 1 SET, lines 287-289)

Well like that I guess we [teachers] had our concerns at the start and that's why we referred Jim for the assessment (Michael, Case 1 teacher, lines 25-26)

Yeah cause I didn't know anything about Jim's learning (Tina, Case 1 parent, line 102)

You know I kinda feel like I've wasted a year of his [child] life, that's what I feel...of learning, not now, last year all last year...yeah (Tina, Case 1 parent, lines 107-108) 
Yeah cause I didn't know he [child] was...he needed that much help, no I didn't think he needed it (Tina, Case 1 parent, line 138)

I'm kinda thinking sure I didn't do homework at home, I had nothing to do with him, I'd no books, I'd no books you see... so I didn't know what was happening (Tina, Case 1 parent, lines 145-147)

Case 2

It's just unfortunate she [child] didn't get more of it from not being here (Anne, Case 2 teacher, line 40)

It's just really unfortunate because it could have been something that she [child] could have got much more out of (Anne, Case 2 teacher, lines 44-45)

You know that's unfortunately after you know kind of, it's just I don't think they're [parent and child] going to get the benefit out of it as a result of all of that so that's just unfortunate but am... (Anne, Case 2 teacher, lines 192-194)

The parent's input as well was positive at the start but then as time went on, am just trying to get hold of her and trying to get her in to speak for different meetings (Anne, Case 2 teacher, lines 190-192)

I mean, I feel that everyone else involved in the situation and like both of us would have had, we knew all of this and we would have gone into the situation being very mindful of the mother and we would have am... been thinking about her and how she felt about everything and dealing with that accordingly (Anne, Case 2 teacher, lines 203-207)

But there's only so much you can do and then she [parent] has to come forward and put herself forward so it's just unfortunate that she just couldn't...couldn't do that (Anne, Case 2 teacher, lines 207-208) 
I really don't think so and that is my genuine, I absolutely don't think so. I can't see how there's anything else that we could have done because I feel both of us were extremely approachable, we have a very good relationship with her and she was always very comfortable in our company am...from what I could see so I think there is nothing more we could have done, I really do believe that (Anne, Case 2 teacher, lines 211-215)

2. Apprehension of the assessment process and outcomes

\section{Language of} assessment

Language that evokes feelings

\section{Pilot Case}

Hopefully it's not going to be an expectation that it's going to happen [change and progress] overnight, that hopefully it's going to be gradual do you know, and that's the way I'm kind of thinking (Louise, Pilot Case parent, lines 125-127)

\section{Case 1}

Do you know where sometimes you can think of an assessment, the child is thinking in their mind, oh gosh, do you know is there something wrong (Sarah, Case 1 SET, lines 72-74)

There was no like 'assessment', you know the word 'assessment' we're being tested, do you know, there was none of that aspect to it, there was none of that feel to it, where you might feel apprehensive and worried and upset and even as a parent myself, there wasn't... I didn't get that from the parent when she was here (Sarah, Case 1 SET, lines 238-241)

He's [child] a bit of a fairy I didn't like that, that's still in my brain, he doesn't want to work...but like he's not going to if you're not going to help him like. But now it's different this year so...(Tina, Case 1 parent, lines 148-150)

Case 2

I do honestly believe that she [parent] genuinely wants that help, but I think as time goes on if there is any input that she has to put in, it could be something that's just a little bit, a 
stretch too far for her, even small little things like making changes, the small changes that she has to make she might be thinking of them as much bigger and it's just finding it hard to break it down, so I think that's her own issues and her own abilities really (Anne, Case 2 teacher, lines 198-201)

And am, then there could be a slight fear of the unknown [for parent] as well, sometimes in these situations (Anne, Case 2 teacher, lines 202-203)

\begin{tabular}{ll}
\hline Theme 3: Factors Bridging the Gap between Assessment and Intervention \\
\hline $\begin{array}{l}\text { Subtheme } \\
\begin{array}{l}\text { 1. Shared awareness of } \\
\text { strengths, needs and } \\
\text { goals identified }\end{array}\end{array} \quad \begin{array}{l}\text { Relevant Statements } \\
\text { Strengths and need }\end{array}$ & $\begin{array}{l}\text { Pilot Case } \\
\text { The information that he [child] gave you on the pie chart and stuff that was very } \\
\text { informative because I can always refer back (Grace, Pilot Case teacher, lines 189-190) }\end{array}$ \\
& $\begin{array}{l}\text { I think in terms of the intervention and the recommendations that if he's [child] aware } \\
\text { that I know of the things that he's been talking about and... the interest he said that he } \\
\text { was willing to try out and that he's willing to reduce his time spent on [the restricted } \\
\text { interest], I think that's going to be a huge support as well, just having that knowledge } \\
\text { (Grace, Pilot Case teacher, lines 195-198) }\end{array}$ \\
& $\begin{array}{l}\text { That was really really assuring for me that I was actually on the right track and I wasn't } \\
\text { causing damage if that's the word...that I was actually helping the situation (Grace, Pilot } \\
\text { Case teacher, lines 105-107) }\end{array}$ \\
& $\begin{array}{l}\text { Am, but the reassurance of it has really helped so my confidence has gone, like I know } \\
\text { this is how I speak to him (Grace, Pilot Case teacher, lines 140-142) }\end{array}$ \\
& $\begin{array}{l}\text { He [child] probably am...was kind of a little bit taken aback, when I said to him on } \\
\text { Wednesday and on yesterday evening that you know, we couldn't spend as much time in }\end{array}$ \\
\end{tabular}


the activity that he likes spending most, he was taken a little bit back by that (Louise,

Pilot Case parent, lines 29-32)

I probably feel a bit more restrictive about feeding into it [restricted interest]

am...(Louise, Pilot Case parent, line 41)

What about your new activities? Yeah that would be one (John, Pilot Case child, line 43)

Study my best in school (John, Pilot Case child, line 41)

Case 1

And even ideas to work with him [child] and things that will help train the muscles and improve possibly his working memory will be good (Michael, Case 1 teacher, lines 5455)

Ideas for working on his oral language and his memory and auditory skills, so definitely recommendations that have addressed and I feel moving forward they will be a very good tool to help support Jim's needs (Michael, Case 1 teacher, lines 70-72)

How you could bring those strengths and his [child] personal interests into the school setting to give an opportunity to, maybe work on his oral language skills (Michael, Case 1 teacher, lines 137-139)

Trying to work his [child] strengths on his weaknesses also, do you know, trying to see if they helped in developing his weaknesses (Sarah, Case 1 SET, lines 53-54)

Like we would have kept on trying whatever interventions we had, and overloading him [child] constantly (Sarah, Case 1 SET, lines 85-86) 
We know how we can work on that to get him [child] to where he needs to be and to expand and to get him to where he can be (Sarah, Case 1 SET, lines 92-94)

But now we know what the situation is, we know, we have recommendations to address those problems, and how to constantly to keep on improving, step-by-step how we can build and develop Jim to the child that he can possibly be (Sarah, Case 1 SET, lines 100102)

But now I know with the spellings which I suppose you need to (Sarah, Case 1 SET, line 187)

Yeah, I can now [support needs], cause I know what to do now (Tina, Case 1 parent, line 163)

I get him [child] books and stuff do you know, and getting him to do it and yeah (Tina, Case 1 parent, line 165)

I definitely can because I didn't know how bad things were so now I know. I know now to get him [child] to do a bit more, do more with him (Tina, Case 1 parent, lines 172173)

\section{Case 2}

Because it's at her [child] level, so she's able to get started on something, she's not just looking because it can be daunting looking at a blank page or a copy page or something like that whereas at least you feel like you know you can write down something (Anne, Case 2 teacher, lines 129-132)

And sometimes as well it's good even things that you have been doing in the past but just to sort of know that we had similar ideas on that, so that you know I'm working towards 
the right way of doing it, do you know what I mean? (Anne, Case 2 teacher, lines 114116)

Ideas and sometimes you'll be wondering are you doing the right thing or is this going to work and I found that really beneficial (Anne, Case 2 teacher, lines 183-184)

Monitoring

Pilot Case

Because I think I'm aware of the situation and because the behaviours they were, I think it's something that I'd be extra tuned into anyway (Grace, Pilot Case teacher, lines 222223)

\section{Case 1}

I can now [monitor needs] that I know what the needs are (Sarah, Case 1 SET, line 151)

And even like that I suppose, when we're talking about spellings, like starting, the class would get 20 spellings, which was totally out of the Jim's ability, so really going starting at 10 and again with his spelling trick to remember his words, how to spell his words, even if needs be we'll start at 8 and then we'll go up one every week depending on how he's achieving and not overloading and then again seeing if that trick works, it will help him, even if he's only doing half of the words with the trick and then the other half learning them rotely, am but again another way of monitoring to see if the amount of spellings that he's able to do has increased (Sarah, Case 1 SET, lines 172-178)

Case 2

I would feel I would be a lot more mindful now and I'd probably pick up on things (Anne, Case 2 teacher, lines 138-139)

More clued into the specific but specifically what she [child] needs (Anne, Case 2 teacher, line 142) 
2. Experiencing or observing intervention effectiveness
Experiencing

interventions

Observing

interventions

\section{Pilot Case}

And having the other strategies like the close proximity, the behaviour specific praise like they're all things I think are... they're really useful and are useful for any other child in the class as well but for him especially because he [child] does seem to be responding to them (Grace, Pilot Case teacher, lines 142-144)

I'd say the checklist definitely, his [child] own self-assessment because I would be trying that with the whole class anyway but making it specific for him I think he'd really respond to that because again it's the one-to-one attention that's just for him but yet it's beneficial to himself and for me, that we can both work at it (Grace, Pilot Case teacher, lines 198-202)

I do think I'll be able to help his [child] needs, I know from when we have those one-toone chats, kind of bringing him back to the topic, I think that he would be actually willing to give information and to talk about it Grace, Pilot Case teacher, lines 211-213)

I found the little pie chart very very useful because the little pie chart for him [child], the fact that he participated in the pie chart himself and sometimes when he gets distracted and he wants to go back to talking about [the restricted interest], you can kind of bring the pie chart back into it and it makes him think and pause for a minute, am I think the pie chart has helped in our situation somewhat, yeah (Louise, Pilot Case parent, lines 7882)

Case 1

We saw the progress (Michael, Case 1 teacher, line 5)

I suppose definitely the recommendations, you know appraisal (Michael, Case 1 teacher, lines 69-70)

His one that he [child] produced in class on Friday, he said for the word 'said', so how he was going to remember this and do you know he presented it to the class and it was a 
confidence builder and he stood up in front of the class beaming and he said Miss I know how we're going to remember that and he had his own little strategy, he had that strategy for the 'said', the S and the A and the I and the D, what words he was going to put in place so that he would remember that so 'Swimming Alone Is Dangerous', I remember It so clearly because he was so good and he was beaming which was, such a simple strategy that he loved and that all of the class wanted to take on, which was great for Jim because it gave him ownership of this new game and now all the class, they ask 'Miss can we all have one each night?' (Sarah, Case 1 SET, lines 121-130)

And it's great because it [mnemonic aid] works (Sarah, Case 1 SET, line 135)

And then that trick is going to help him immensely (Sarah, Case 1 SET, line 188)

Yeah because at home with the spellings and stuff...like if I told him [child] to do spellings at home like, if I was doing it like you were doing, he would do it after me, he would do it like (Tina, Case 1 parent, lines 129-130)

I feel it [mnemonic aid] working (Tina, Case 1 parent, lines 133-134)

Spellings like...just make up silly sentences like and then for maths, like use counters and you'll get better (Jim, Case 1 child, lines 93-94)

Just like 'said', you know, 'swimming alone is dangerous' and like if you get stuck on it all you have to do is just remember the words and then you know how to spell 'said' then (Jim, Case 1 child, lines 136-137)

Case 2

Obviously put in place these extra you know ideas, especially say along the lines for English and mapping out things ahead of a lesson and again with the maths and checklists and just I suppose making things a little bit easier for her [child] at the start of 
the lesson and then obviously then when I get a chance I can do a little bit more one to one with her but she is encouraged to get started independently (Anne, Case 2 teacher, lines 101-105)

We have the little checklist now that she [child] puts into her pencil case, do you know what I mean, they're similar but just additional and anything extra is a help, you know any extra ideas and same with the English and just sort of writing out the words and doing, again we would be doing it anyway with brainstorming but sometimes just if a template looks different or is different it can also help, you know which is great (Anne, Case 2 teacher, lines 121-125)

Just a lot more aware of what's going to work for her [child], you know so it might make it [monitoring] a bit easier (Anne, Case 2 teacher, lines 142-143)

\section{Practical interventions Practical interventions}

\section{Pilot Case}

They'll be a huge support in class especially when you work with 26 other kids, that Useful interventions there are things that I can actually implement that won't detract from the others and that can be easily implemented and that they could all actually even try (Grace, Pilot Case teacher, lines 92-95)

That they can actually be used with all the class cause in a large class group like I don't have the same amount of one-to-one work that you might have with smaller numbers (Grace, Pilot Case teacher, lines 182-184)

I think kind of giving him [child] the time to have his own little free time, but yet still have that he has a choice, but yet still in a controlled appropriate way, am, I think is very very manageable (Grace, Pilot Case teacher, lines 184-186) 
Little steps that you can take in the home environment as a mother and just little steps just about listening, because you identified that he likes a little chat on the little pyramid. (Louise, Pilot Case parent, lines 76-78)

Case 1

That all of the class wanted to take on [intervention], which was great for Jim because it gave him ownership of this new game and now all the class, they ask 'Miss can we all have one each night?' (Sarah, Case 1 SET, lines 128-130)

And again very clear, easy, specific recommendations, simple like again you don't have to buy a shop to implement them, do you know? (Sarah, Case 1 SET, lines 232-233)

Case 2

One of the areas that isn't just kind of cut and dry is obviously your social skills and all of that so I think that's really the practical tips and the 'Talkabout' and am, the cognitivebehavioral therapy, all of that like that's what works, it's practical, it's very doable, it's very user-friendly, am that's what works and that's what you need to get your hands on, so I think that's very good (Anne, Case 2 teacher, lines 84-87)

All of those tips are brilliant and can be used for everybody, which is great as well and again that's again getting back to the practicalities of things, a class of 25 it is very hard to get the one to one time or even sort of small groups or whatever so when you have something that you can use with everybody but that's going to be beneficial for everybody, that's really important you know (Anne, Case 2 teacher, lines 89-93)

As I said probably repeating myself again here but practical and that's what it's all about just getting it done (Anne, Case 2 teacher, lines 155-156) [on intervention recommendations] 
4. A collaborative approach to

implementing and

monitoring interventions
Team approach to

implementing and

monitoring

interventions

\section{Pilot Case}

Yeah, I think your suggestion of the role plays will definitely be one thing because he [child] does have a part in the Christmas Show so even supporting that at home and in school (Grace, Pilot Case teacher, lines 110-111)

I can see that there's definitely things that we can put in place, just to... support him [child] a little bit with whatever extra support he gets elsewhere (Grace, Pilot Case teacher, lines 133-135)

And for the parents to see that they have changed as well it would be fantastic for them to see as well because it's proof that there is something happening (Grace, Pilot Case teacher, lines 229-230)

Case 1

Now I feel like I've a specific task to do and I know what activities and what areas would help Jim (Michael, Case 1 teacher, lines 89-90)

I know his [child] needs and I know that the recommendations that you have given us, I know that we can work on those recommendations (Sarah, Case 1 SET, lines 111-112)

It's him [child] taking it on which I think If it was us imposing it on him, it would be very much 'Oh gosh no, I don't want to do it' (Sarah, Case 1 SET, lines 132-133)

He's [child] aware of strategies that he can do and that he can put in place as well for him to achieve, he knows what he needs to achieve in (Sarah, Case 1 SET, lines 144-145)

I definitely feel more prepared and equipped to support them [needs] now, so am I think yeah for sure having a very strong relationship with the learning support teacher and with the parents and obviously the recommendations here (Michael, Case 1 teacher, lines 7476) 
Areas where he [child] needs support and just to talk to him and ask him the questions

like what worked well or you know, where he is struggling, so I think monitoring for sure and between having a good relationship with the learning support teacher, that we can monitor his progress (Michael, Case 1 teacher, lines 100-103)

I can monitor them [needs]. We can assess, and again him [child] being involved, seeing from, we know where he's at at the moment, with those recommendations in place, building on them and monitoring and making sure that they're working do you know, to the best that they can for him (Sarah, Case 1 SET, lines 151-154)

To complete them (Jim, Case 1 child, line 125) [on parent and teacher to help achieve goals]

Case 2

At the start looking at right well what do I know and what can we do about this (Anne, Case 2 teacher, lines 62-63)

\section{Theme 4: Views and Experiences of AFI}

\section{Subtheme \\ Codes \\ 1. A clear and structured Structured model} process

Clear accessible language

Language to promote understanding

\section{Relevant Statements}

\section{Pilot Case}

Am and I thought that the couple of steps that were in it that they were very easy to understand, yet we're still kind of getting through it and that we were still looking at the same things in a normal intervention model but yet there was that bit more detail in it, which I found really good (Grace, Pilot Case teacher)

I think the 5 steps were very beneficial and it was a very clear process to follow (Grace, Pilot Case teacher, lines 267-268)

It was simple and written in a language that was easy to understand and it wasn't in highfalutin language so that made it easier to follow it and to try and implement some of the recommendations then (Louise, Pilot Case parent, lines 9-11) 
And interventions discussed were openly discussed to John in child friendly language am, so that he could kind of engage with the process as well (Louise, Pilot Case parent, lines 21-23)

I think it would have been very difficult not to be able to identify what the problem was because most of the questions were kind of, I won't say bullet points but they were kind of descriptive type questions, identifying what the problems was (Louise, Pilot Case parent, lines 61-63)

Case 1

The fact that it's [AFI process] clearly laid out as well (Michael, Case 1 teacher, line 120)

It's just like gradual, step-by-step, so it's like sometimes when we have a difficult situation and we try and jump right into it, so I think going with the gradual approach (Michael, Case 1 teacher, lines 123-124)

And it was lovely to see it broken down into the steps and to be so clear and so precise of what we had to do at each step, what our involvement was, what the parent's involvement was, what the child's involvement was (Sarah, Case 1 SET, lines 12-14)

And you [TEP] explained it in detail, all the five steps that was involved in the model (Sarah, Case 1 SET, lines 4-5)

No it $[\mathrm{AFI}]$ helped me a lot now it did, it did now it helped me to see more and stuff you know (Tina, Case 1 parent, lines 238-239)

Yeah it [AFI] was good like, it was interesting as well you know and I understand more about Jim (Tina, Case 1 parent, line 3) 
Case 2

In terms of the structure of everything and the whole process and, am very sort of like user-friendly (Anne, Case 2 teacher, lines 3-4)

Just very structured [AFI process] (Anne, Case 2 teacher, line 75)

In terms of the way everything was explained (Anne, Case 2 teacher, lines 4-5)

I really understood the whole process, am...there was no kind of, you know the way sometimes things can get a little bit wordy or too much information (Anne, Case 2 teacher, lines 72-73)

Yeah it's very much just bullet points, you know this is what we're going through rather than sometimes things can be very wordy and you can't actually see what the point is, am whereas I just found all of that very practical and to the point (Anne, Case 2 teacher, lines 77-79)

2. A framework to facilitate teamwork and positive outcomes
Framework to facilitate Pilot Case

teamwork and resulting outcomes

Language of strengths, needs and potential
So I think having that channel of communication is definitely important... and that's a huge part of the model as well, having that communication so I think that was really beneficial (Grace, Pilot Case teacher, lines 245-246)

That if the language they were using at home was copied in school and vice a versa, that we could actually am, really really support him and he'd understand that we were looking out for his best interests as well (Grace, Pilot Case teacher, lines 54-56)

Yeah am, I think awareness of the situation at home and having the voice of the parent and the child as well. Like my only prior experience with these kind of interventions was purely me filling in the report and then getting a report back...or maybe seeing a note that the parents had sent in but not actually getting to speak to them, so I think having 
that interaction and then hearing my voice and seeing what I've been experiencing and then vice versa me seeing them and hearing their experiences I think that was a huge benefit of it (Grace, Pilot Case teacher, lines 249-254)

I lost out on some class teaching time with it (line 266)...but in the long run, it's going to be beneficial because we have so much information and we have so much line of discussion between home and school (Grace, Pilot Case teacher, lines 270-271)

Am, I suppose the benefits was it was kind of a team approach (Louise, Pilot Case parent, line 107)

Case 1

It's a team and a shared approach (Michael, Case 1 teacher, line 119)

Getting to know the child, the student better, his specific needs, am strengthening the relationship with the parents, you know, having that communication, those am communication lines strengthens you know (Michael, Case 1 teacher, lines 134-136)

I think the whole joint approach to it and the collaborative approach is so beneficial (Michael, Case 1 teacher, lines 141-142)

But I think the whole concept and the model I think it's much better than you know, a one on one, very formalised setting, getting your feedback, this you feel like... it is a real process and you're involved in it and there's much more consultation and communication which I think was key moving forward so (Michael, Case 1 teacher, lines 151-154)

It was a tool for him to use, to express his opinion on how his learning might be improved (Sarah, Case 1 SET, lines 74-75)

To ensure that that child can achieve to the best that they can (Sarah, Case 1 SET, lines $10-11)$ 
Yeah and trying to see what works well, where his strengths lie and trying to play strengths (Sarah, Case 1 SET, line 52)

But now we know what the situation is, we know, we have recommendations to address those problems, and how to constantly to keep on improving, step-by-step how we can build and develop Jim to the child that he can possibly be (Sarah, Case 1 SET, lines 100102)

Even when you explained am from his report and when you explained that it was so clear, that I would never have had someone explain something so clearly to me before. Now I understand, how... when we get to the recommendations, yeah we were given a report before and here's the recommendations, but now I understand why these are in place and how the different attainments and how it works, it makes sense to me now and it's just everything is so much more clear (Sarah, Case 1 SET, lines 202-207)

Again, I know Rome wasn't built in a day but one recommendation at a time, do you know when we achieve or get to the best where Jim can on that recommendation, we can move on and we can keep building and building on those recommendations, and get him to where he should be (Sarah, Case 1 SET, lines 113-115)

Helping Jim [role of TEP], yeah and helping to find out what his strengths were, you know what he's capable of (Tina, Case 1 parent, lines 11-12)

No it helped me a lot now it did, it did now it helped me to see more and stuff you know, because if this didn't happen, next year would have been too late I'd say in fifth class like, so I feel a lot happier (Tina, Case 1 parent, lines 238-240)

I can talk to the teachers more and if I've a problem I can go to them now and Jim can as well (Tina, Case 1 parent, line 246) 
Case 2

So I think that's really beneficial, to have that opportunity you know what I mean, to deal with somebody and am...to come together and come up with a plan together, you know when everyone is sort of working together, it just really makes it a lot easier (Anne, Case 2 teacher, lines 179-181)

At the start looking at right well what do I know and what can we do about this (Anne, Case 2 teacher, lines 62-63)

Yeah, absolutely I think even though you're dealing with one specific child and their needs (Anne, Case 2 teacher, line 159)

Applicability to other situations
Applicability to other situations
Pilot Case

So I think in other situations that maybe if there's a child who isn't getting their homework done that maybe there is something else happening at home, so being aware of home situations is definitely a huge advantage (Grace, Pilot Case teacher, lines 235238)

Am...well look you'd be using it with maybe regards to you know, probably planning if you were bringing him somewhere, am and he was going to visit someone and just to prep him before he goes so that the visit is more enjoyable for his siblings as well (Louise, Pilot Case parent, lines 92-94)

It'll help you when he's going into secondary school to be able to identify what subjects he might like to study and take when he goes into first year (Louise, Pilot Case parent, lines 101-102)

Case 1

Now I see the huge benefit of getting the parent in, trying to get their voice, getting the child in, going through the steps, do you know step by step (Sarah, Case 1 SET, lines 216-217) 
The child's voice yeah...I keep coming back to that but that was definitely my favourite because I've seen first-hand the impact it has had on the child (Sarah, Case 1 SET, lines 221-223)

I found the model...the process hugely beneficial, not only to the child but to me as a teacher, in my learning and in the things that I have learned from the process, about how to address the needs, how to assess the needs of the children in the first place and then how I'm going to, as the teacher address those needs, and to ensure that that child can achieve to the best that they can (Sarah, Case 1 SET, lines 7-11)

Yeah well definitely I think am, I think like parental attitude and for anything even for like behaviour contracts or anything because...it's basically like, giving a voice and hearing different people's perspectives (Michael, Case 1 teacher, lines 117-119)

\section{Case 2}

Yeah, absolutely I think even though you're dealing with one specific child and their needs, it does crossover into so many other things, so yeah, no I definitely think I would and also in terms of the resources and in terms of the information that's come from all of this, I could see how it would work across the board, you know am, with Michelle obviously but also there are definitely things there that would work with other children and just has given me greater insight into everything (Anne, Case 2 teacher, lines 159164) 
Appendix 49 Excerpt from Researcher Diary Demonstrating Progressing Understanding of Student's Situation (Pilot Case)

\begin{tabular}{|c|c|c|}
\hline $\begin{array}{l}\text { 3c. I translated these hypotheses into questions for } \\
\text { investigation }\end{array}$ & 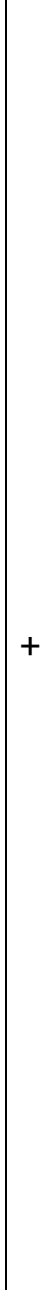 & $\begin{array}{l}\text { Are certain activities/times of the day triggering or } \\
\text { maintaining John's engagement in his restricted interest? } \\
\text { What interactions between John and his teacher are } \\
\text { positively and negatively impacting upon his behaviour? } \\
\text { What types of interactions work well between John and his } \\
\text { parents and what interactions worked well with his previous } \\
\text { class teacher? } \\
\text { What type of praise and feedback does John like to receive in } \\
\text { the classroom? } \\
\text { If John engages in other activities that give him a sense of } \\
\text { calm, will his need to engage in the restricted interest } \\
\text { decrease? (change oriented hypothesis) } \\
\text { What new activity/interest outside of the restricted interest } \\
\text { would John be willing to try? } \\
\text { If John's parents and teacher provide him with choices and } \\
\text { compromise, will he be more willing to try activities outside } \\
\text { of his restricted interest? (change oriented hypothesis) }\end{array}$ \\
\hline
\end{tabular}




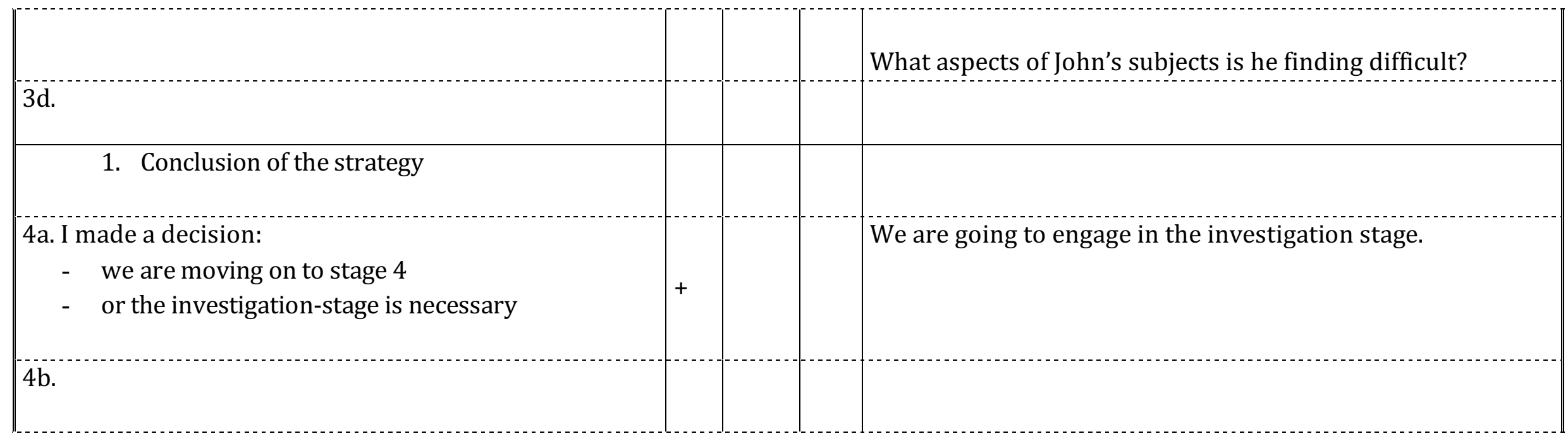

\section{Conclusions Strategy, the impact of applying elements of this stage were:}

Applying elements of this stage allowed me to continually refer back to the questions of the clients, to ensure investigation would address their questions and personal theory. It also ensured that I followed the 'need to know' v. 'nice to know' approach. I realised that I had enough information gathered for some intervention recommendations but not enough for others, making the stage more goal oriented. It took time, but I feel as I become more competent in applying the model, and in the development of my skills as a school psychologist, I will become more efficient. It was a very worthwhile process. 
Appendix 50 Excerpt from Researcher Diary Demonstrating Researcher's Knowledge of Intervention Supports (Pilot Case)

1. Estimation of the most appropriate recommendation

5a. I know what the most suitable intervention for this student is

5b. I know what the most suitable intervention for this teacher is

c. I know what the most suitable intervention for these

5c. I know what the most suitable intervention for these parents is

$5 \mathrm{~d}$. It is clear whether a combined intervention is necessary

$5 \mathrm{e}$.

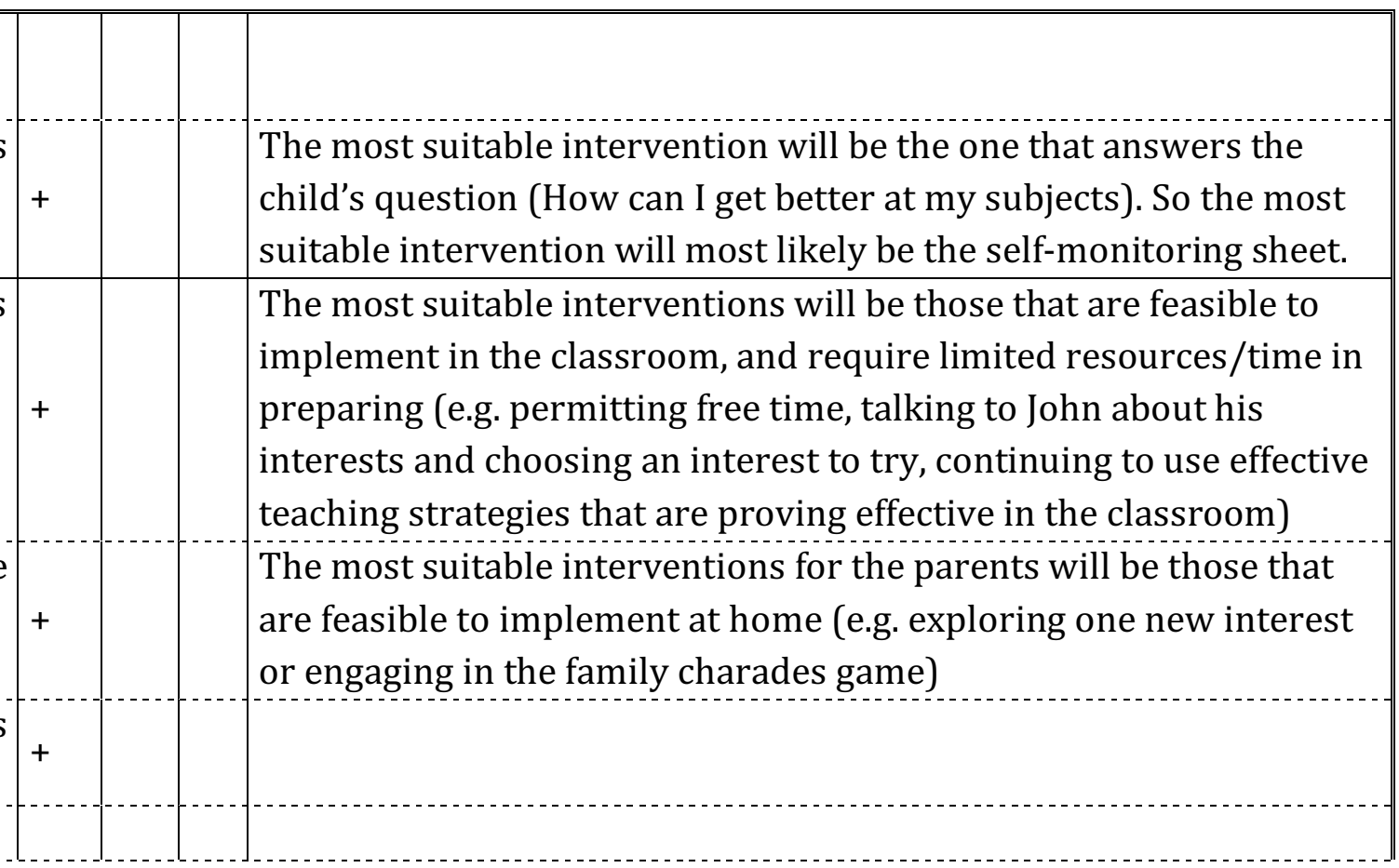

Conclusions stage 4, the impact of applying elements of this stage were: I found it difficult to make SMARTI goals. I will present to the clients goals and needs that can be adapted, and will discuss during stage 5, how they can be made SMARTI-collaborate with the parents/teachers/child, they can adapt to their own situation. 
Appendix 51 Excerpt from Researcher Diary Demonstrating Progressing Understanding of Student's Situation (Case 1)

\begin{tabular}{|c|c|c|}
\hline $\begin{array}{l}\text { 1. I consulted the clients: can they participate as co- } \\
\text { researchers? }\end{array}$ & + & $\begin{array}{l}\text { Yes, each client has agreed to be a co-investigator and has } \\
\text { been briefed on the tasks to be completed (see methods of } \\
\text { investigation above). }\end{array}$ \\
\hline $\begin{array}{l}\text { 2. I gathered the data necessary for testing the } \\
\text { hypotheses }\end{array}$ & + & $\begin{array}{l}\text { Yes, clients and TEP have completed tasks for investigation, } \\
\text { to the best of their ability given time constraints and within } \\
\text { the demands of home and school life. It has been difficult to } \\
\text { meet parent in person, as she has started a new job but } \\
\text { efforts have been made to keep her included in the process } \\
\text { (calls and offers of alternative meeting times). }\end{array}$ \\
\hline 3. I interpreted the data and answered the questions & + & $\begin{array}{l}\text { 1. How is Jim currently performing in the areas of literacy } \\
\text { and numeracy (e.g. reading, comprehension, written work } \\
\text { and numeracy/problem solving skills)? What is his learning } \\
\text { potential in these areas?* } \\
\text { 2. What are Jim's cognitive strengths and weaknesses? } \\
\text { 3. What teaching methodologies, strategies and supports } \\
\text { does Jim respond well to at home and at school? (e.g. } \\
\text { prompts, visual aids, mnemonic aids, concrete materials, } \\
\text { additional time to complete tasks). Are there any strategies } \\
\text { that need to be changed or modified? }\end{array}$ \\
\hline
\end{tabular}




\begin{tabular}{|l|l|l|l|l||}
\hline & & & & $\begin{array}{l}\text { * Assessment information was interpreted under these } \\
\text { questions and was kept confidential. }\end{array}$ \\
\hline $\begin{array}{l}\text { Extra: I investigated the potential for change with a } \\
\text { change-oriented hypothesis }\end{array}$ & & & & \\
\hline
\end{tabular}

Conclusions Investigation, the impact of applying elements of this stage were: Applying elements of this stage (e.g. operationalising concepts and interpreting the data under each question for investigation) has ensured questions can actually be investigated and the data can now be triangulated to form an ecologically valid case formulation. 
Appendix 52 Excerpt from Researcher Diary Demonstrating Researcher's Knowledge of Intervention Supports (Case 1)

\begin{tabular}{|c|c|c|}
\hline $\begin{array}{l}\text { 4d. I know what interventions are desirable and what } \\
\text { (scientific) arguments support or oppose these options }\end{array}$ & + & $\begin{array}{l}\text { Yes, I have researched the recommendations to make sure } \\
\text { they are evidence informed, evidence-based. }\end{array}$ \\
\hline $\begin{array}{l}\text { 4e. I know which of the options seem achievable in this } \\
\text { case }\end{array}$ & & $\begin{array}{l}\text { Yes, the intervention that can be practicably be applied in } \\
\text { the classroom and at home, that don't take up too much time. }\end{array}$ \\
\hline \multicolumn{3}{|l|}{ 4d. } \\
\hline $\begin{array}{l}\text { 5a. I know what the most suitable intervention for this } \\
\text { student is }\end{array}$ & & $\begin{array}{l}\text { The most suitable intervention will be the one that answers } \\
\text { the child's question (How can I get better at spelling and } \\
\text { maths). So the most suitable intervention will most likely be } \\
\text { the self-checking and spelling trick. }\end{array}$ \\
\hline $\begin{array}{l}5 \mathrm{c} \text {. I know what the most suitable intervention for these } \\
\text { parents is }\end{array}$ & & $\begin{array}{l}\text { The most suitable interventions for the parents will be those } \\
\text { that are feasible to implement at home (e.g. require limited } \\
\text { resources/time) }\end{array}$ \\
\hline
\end{tabular}




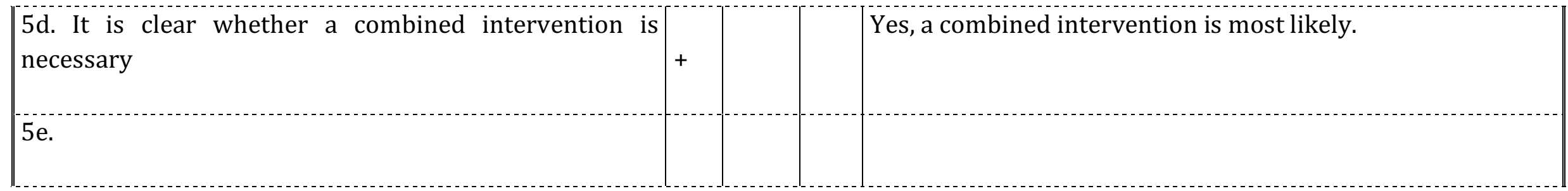

Conclusions stage 4, the impact of applying elements of this stage were: I found the structure of this stage for forming goals, needs and recommendations for Jim, his parents and teachers very useful and report writing for the feedback stage was straightforward as a result. 
Appendix 53 Excerpt from Researcher Diary Demonstrating Researcher's Understanding of the Student's Situation and Knowledge of Intervention Supports (Case 2)

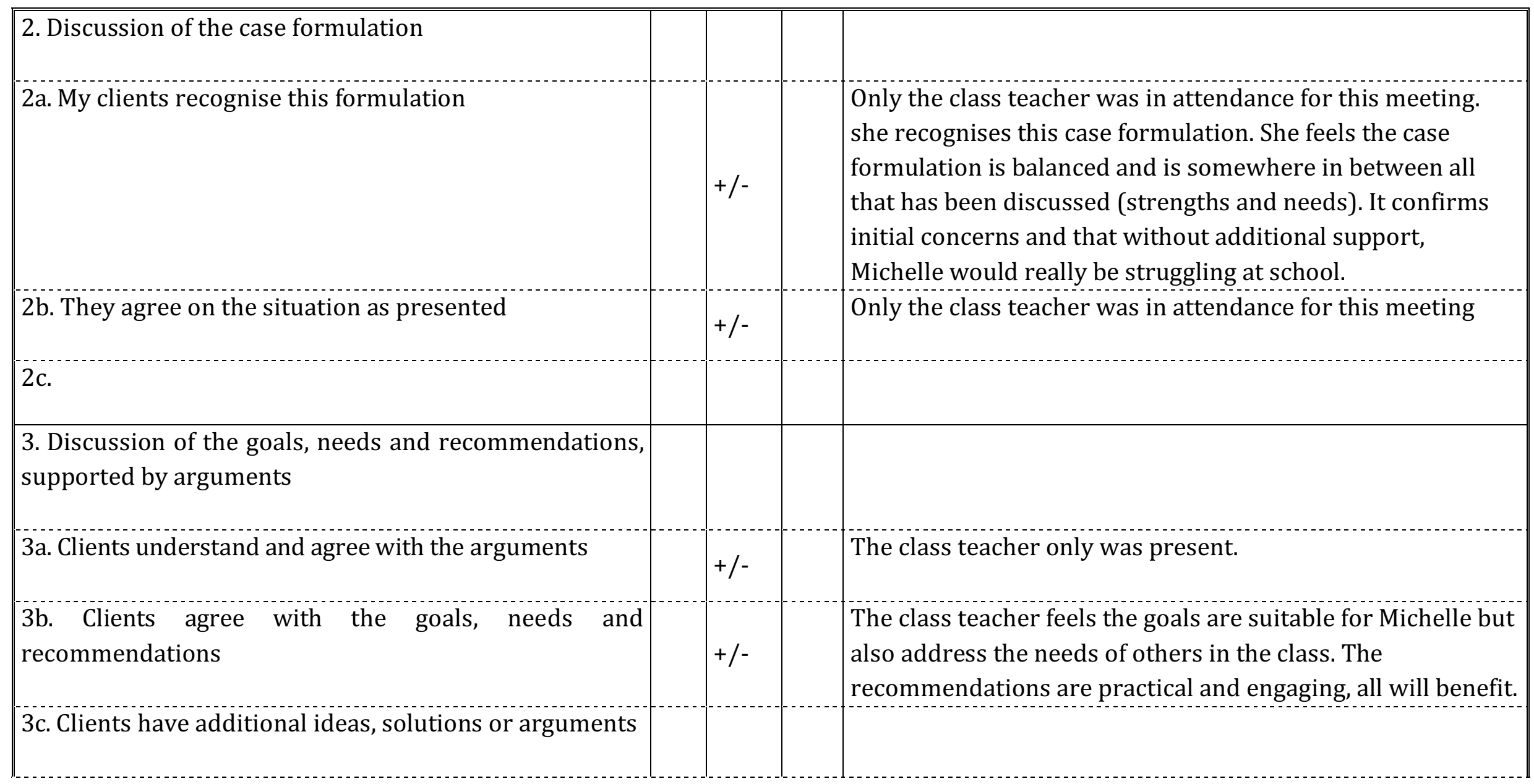




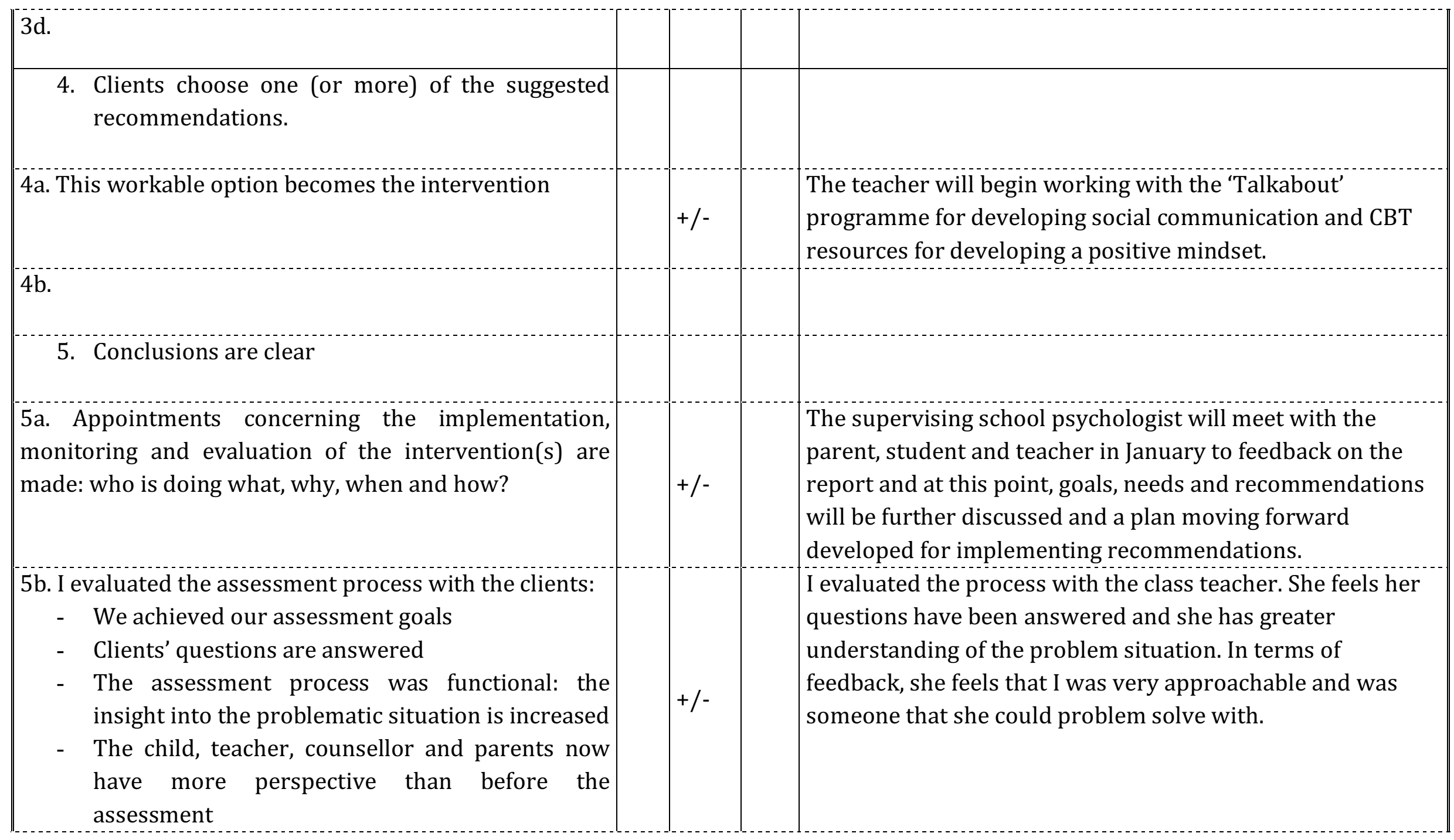




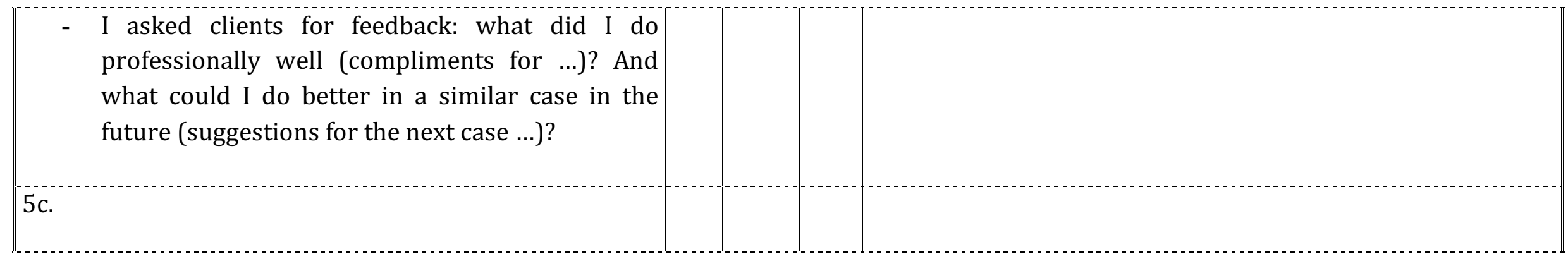

Conclusions the impact of applying elements of this stage were: Applying elements of this stage ensured that I understood the situation and interactions between the risk and protective factors for Michelle, her school and home environment. Consideration of these factors allowed me to present a valid, transactional case formulation and devise feasible goals, needs and intervention recommendations. As with the previous cases, the structure of this stage ensured that I followed and implemented a systematic approach to delivering feedback to clients. 
Appendix 54 Empirical Paper

'An exploration of the Assessment for Intervention model in an Irish Educational Psychological context'

Carol Slatterya ${ }^{\mathrm{a}}$ Dr. Margaret Egan ${ }^{\mathrm{a}}$

${ }^{a}$ Mary Immaculate College, Limerick, Ireland

Contact: Carol Slattery, 00656003@micstudent.mic.ul.ie 


\section{'An exploration of the Assessment for Intervention model in an Irish Educational Psychological context'}

The current research sought to explore the utility of the Assessment for Intervention (AFI) model in bridging the gap between assessment and intervention. Ecological Systems Theory (EST) and Social Constructivist Theory (SCT) were adopted as the conceptual framework for analysis. An exploratory 'two case' case study was employed to answer the research question 'Can the Assessment for Intervention model bridge the gap between assessment and intervention, through activity and interaction at the meso and microsystem levels?'. The AFI framework was applied to two case referrals made to a school psychological service in Ireland and a pilot study was conducted to inform the research design. Participants rated Likert Statements exploring case propositions, before and after application of the AFI model. Semi-structured interviews were also conducted with participants $(\mathrm{n}=8)$ and AFI templates served as a researcher diary. Thematic analysis and pattern-matching were employed to analyse the interview data. Changes in ratings on Likert Statements were presented in tabular format and excerpts from the researcher diary were included in the discussion of findings. Results suggest some interesting implications for policy and practice, particularly for those in educational psychology. The findings suggest the AFI model can address the gap between assessment and intervention discussed in academic discourse (Resing et al., 2017; VanDerHeyden, 2018).

Keywords AFI model, assessment, intervention, exploratory case study, Ecological Systems Theory, Social Constructivist Theory, Circular 0013/2017. 


\section{Introduction}

Traditionally, the educational psychologist's (EP) distinctive role involved psychometric testing and diagnosis of a Special Educational Need (SEN) for categorisation and special education provision purposes (Farrell, 2010; Filter et al., 2013). Over the decades, a global shift has occurred in assessment practices, from a traditional focus on within-child factors reflective of the medical model, towards consideration of social and environmental factors reflective of an interactionist, ecological approach (Davis \& Deponio, 2013; Kennedy et al., 2008). International policy and legislative initiatives have been somewhat consistent with this reported shift in practice. In 2007, the European Agency for Special Needs and Inclusive Education commissioned a project examining assessment that informs teaching and learning in inclusive settings (Watkins, 2007). The concluding comments of the report state:

There has been a move from looking at individual pupils in isolation, to considering the context of pupil's learning. At the same time, the assessment process has moved away from a 'snapshot' approach involving professionals from outside the mainstream classroom, to an on-going process of mainstream teachers, parents and pupils themselves developing an understanding of not just what pupils learn, but also how they learn it. (p. 61)

The report endorses 'inclusive assessment', whereby policy and practice are designed to support the learning of all pupils as far as possible and the allocation of support, placement and additional resourcing to meet a student's needs should be informed by, but not be solely based upon initial identification or diagnostic procedures (Watkins, 2007).

In Ireland, the traditional resource allocation system within mainstream schools reflected a medical model of practice, as assessment and diagnosis of disability and SEN were prerequisite to accessing limited resources (National Council for Special Education (NCSE), 2014). In 2013, a NCSE policy advice paper exposed several inequities within the system, including delays in accessing assessments and resource allocation contingent upon labelling and categorisation (NCSE, 2013). In 2017, the new model for allocation of special education teaching resources in mainstream schools was launched and stipulates resource allocation based on identified needs rather than diagnosis (Department of Education and Skills (DES), 2017). Additional support should enhance the child's performance and participation in all school activities (DES, 2017), consistent with the aims of the European Agency for Special Needs and Inclusive Education (Watkins, 2007). To ensure children's needs are being addressed, schools are 
encouraged to answer three pivotal questions; how can we identify needs? how can we meet needs? and how can we monitor and report on progress? Relevant to educational psychological practice, Circular 0013/2017 affirms that the focus of professional assessment under the new allocation model is on providing understanding of a child's needs, the nature of difficulties, and informing appropriate interventions (DES, 2017).

The stipulations for professional assessment outlined in Circular 0013/2017 (DES, 2017) directly impact upon educational psychological practice in Ireland, specifically the shift in focus from assessment for diagnosis to a needs-based approach that informs appropriate interventions. Linking assessment to intervention in educational psychological practice is a presenting challenge, according to academic discourse (Resing et al., 2017) and is described as "the next frontier for school psychology" (VanDerHeyden, 2018, p. 51). A systematic review of assessment approaches that EPs are currently applying in practice and whether these approaches are informing appropriate interventions for clients was conducted and a summary of findings is provided in the following section.

\section{Systematic review of EP assessment approaches}

The systematic review was conducted to explore the following review questions:

(1) What assessment approaches are educational psychologists applying in practice?

(2) Are the assessment approaches informing appropriate interventions for clients?

An electronic database search of Academic Search Complete, PsycINFO and ERIC was conducted through EBSCO host in August, 2018. Keywords pertaining to review question 1 (See Table 1) and review question 2 (See Table 2) were searched. If available, a filter was applied so that only peer reviewed studies and studies written in English would be included. A limit on year of publication was also applied (2013-2018) to yield the most current assessment approaches applied in practice. An initial search yield of 2,027 titles was generated. Titles were screened against inclusion and exclusion criteria outlined in Appendix 1 and twenty-one articles were included for review. Each of these studies were critically appraised using Gough's (2007) Weight of Evidence Framework. Studies were allocated a 'High', 'Medium' or 'Low' rating according to coding protocols and review criteria set by the researcher. 
Table 1. Database search items (Search 1: 1, 298)

\begin{tabular}{ll}
\hline Databases: & Search terms: \\
Academic Search, ERIC, PsycINFO & "education* psycholog*" OR "school \\
& psycholog*" AND "assessment practice" \\
& OR "assessment approach" OR \\
& "assessment model" OR "assessment \\
& framework" \\
\hline
\end{tabular}

Table 2. Database search items (Search 2: 729)

\begin{tabular}{ll}
\hline Databases: & Search terms: \\
Academic Search, ERIC, PsycINFO & "education* psycholog*" OR "school \\
& psychology*" AND "assessment \\
& practice" OR "assessment approach" OR \\
& "assessment model” OR "assessment \\
& framework" AND intervention OR \\
& recommendation OR strateg* OR \\
& treatment \\
Academic Search, ERIC, PsycINFO & "education* psycholog* assessment" OR \\
& "school psycholog* assessment" AND \\
& "intervention" OR "recommendation" OR \\
& "strateg* OR treatment" \\
\hline
\end{tabular}

\section{Participants and design}

According to the stipulated inclusion and exclusion criteria, participants must be parents, teachers, children or EPs and the students referred for assessment attend a mainstream primary or secondary school with or without a previously diagnosed SEN. Appendix 2 provides summary information on participants in each of the studies, the reason for referral/purpose of assessment, age range of students assessed and participants who reported on the appropriateness of interventions arising from the assessment where relevant. Eleven studies specified the purpose of assessment was to determine eligibility of a diagnosis/label including ADHD, ASD, ED, SLD and Writing Disorder, limiting generalisability of findings to these specified populations. Three studies did not outline reasons for referral (Bahr et al., 2017; Filter et al., 2013; SoteloDynega \& Dixon, 2014). Participants reporting on whether the assessment approach informed appropriate interventions for clients included EPs (Bozic, 2013; Tobias, 2017), teachers, counsellors, parents, students and assessors (Pameijer, 2017), students, parents and teachers (Lawrence \& Cahill, 2014), parent and teachers (Parker et al., 2016) and student and school staff (Cane, 2016). Studies with a quantitative, qualitative or mixed-methods design were reviewed. Twelve were survey studies, seven were case studies and two of the studies utilised interview methods to explore the views of 
participants. The findings relative to review question 1 and 2 are discussed in the following section. Appendix 3 provides a summary table outlining the assessment approach, context under which the assessment was undertaken, evidence of whether the approach informed appropriate interventions for clients and theories or models that resonated with the assessment and/or intervention approach.

\section{Systematic review findings}

This systematic review sought to investigate the assessment approaches currently being applied by EPs in practice (review question 1) and the appropriateness of intervention recommendations arising from these assessments for clients (review question 2). Twenty-one studies were reviewed and all provided empirical evidence of current assessment approaches used by EPs in practice. Twelve studies were consistent with the medical model of practice. Nine of the studies provided evidence of alternative forms of assessment and resonated with theories including Ecological Systems Theory (EST) (Bronfenbrenner, 1979, 1989), Social Constructivist Theory (SCT) (Palinscar, 1998; Vygotsky, 1978) and Positive Psychology (Seligman, 2002). Only six studies provided evidence to suggest the approach informed appropriate interventions for clients, indicating a gap exists in EP practice between the assessments being conducted and interventions that clients are receiving. This finding is consistent with the assertions of Resing et al. (2017) on the challenge of linking assessment to intervention in EP practice and VanDerHeyden (2018) who desrcibed this challenge as the "next frontier for school spychology" (p.51). Assessment approaches informing appropriate intervention for clients included strength-based assessment (Bozic, 2013), solutionfocused assessment (Cane, 2016), the genogram (Tobias, 2017) and a multi- modal assessment/RTI approach (Parker et al., 2016), the Assessment for Intervention model (Pameijer, 2017) and Dynamic Assessment (Lawrence \& Cahill, 2014). An additional finding across the studies reviewed was the inclusion or exclusion of the voice of the child during the assessment and intervention process. Students reported on the appropriateness of intervention recommendations in three studies (Cane, 2016; Lawrence \& Cahill, 2014; Pameijer, 2017). However, there was also an acknowledged absence of the voice of the child in studies. Hanchon and Allen (2013) reported only $31 \%$ of respondents conducted diagnostic student interviews in their ED evaluations and participants in Hill and Turner (2016) reported few children being involved in decision making about their treatment for ADHD. Of all the assessment approaches reviewed, the AFI model was considered most consistent with the aims of Circular 0013/2017 
(DES, 2017), as it works to offer a better understanding of the child and the support he/she needs. A description of the AFI model is provided in the next section.

\section{The Assessment for Intervention model (Pameijer, 2016, 2017)}

AFI is a five-stage model of assessment and intervention and its aim is to bridge the gap between assessment and intervention in order to provide recommendations that are both scientifically sound and useful for the student, teacher and parent/guardian (Pameijer, 2017). This aim addresses the findings of the systematic review and assertions of Resing, Lauchlan, \& Elliott (2017) and VanDerHeyden (2018). Appendix 4 provides a detailed outline of the five stages (Intake; Strategy Development; Investigation; Integration; Recommendations, Appointments and Feedback). The Pameijer (2017) questionnaire evaluation study was allocated a 'High' overall Weight of Evidence rating in the current systematic review. The findings revealed the stages of AFI structured the assessment process from beginning to end, all involved worked in cooperation towards a shared goal and outcomes were meaningful to clients (Pameijer, 2017). From a theoretical perspective, discussion of findings in the systematic review indicated alignment of the AFI model with EST and SCT. A second phase to the literature review was prompted to explore how EST and SCT can inform educational psychological assessment practices generally, and more specifically, the AFI model (Pameijer, 2016, 2017). The concepts within these theories were analysed in accordance with AFI principles and informed the researcher's conceptual framework, outlined in the following section.

\section{Conceptual framework development}

A conceptual framework offers a logical structure of connected concepts that helps to provide a picture or visual display of how ideas in a study are associated to one another (Grant \& Osanloo, 2014). The seven AFI principles describe the theoretical context and rationale that underlie the five stages of the AFI model (Pameijer, 2017). The current research explored the applicability of EST and SCT to the AFI model, to further strengthen its theoretical underpinnings and the combination of theories informed the analysis of findings. In accordance with EST, it was proposed that the issuance of Circular 0013/2017 (DES, 2017) at the macro level instigated a period of ecological transition for the EP, school staff, parents and child with SEN. The five stages of the AFI model were applied to case referrals at the meso and microsystem levels, with parent/guardians, school staff and child. Figure 1 presents a diagram of the conceptual 
framework outlining the proposed ecological transition at the macro, exo, meso and microsystem levels. The diagram also delineates the AFI model and EST and SCT concepts at the meso and micro system levels and is followed by a theoretical statement for the current study and the research question.

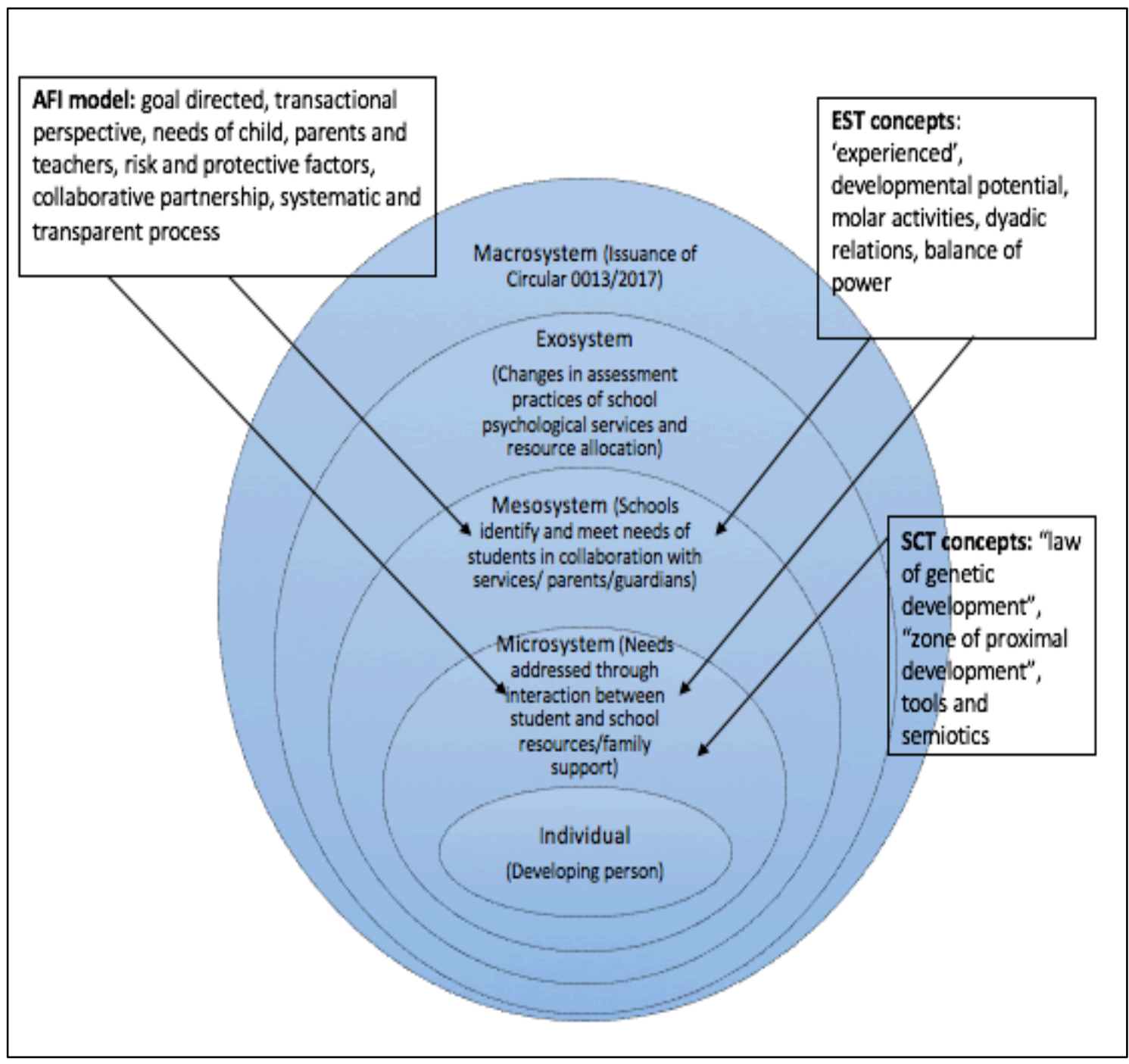

Figure 1. Diagram of conceptual framework

\section{Theoretical statement and research question}

The conceptual framework was refined into a theoretical statement and proposition for the current research project:

During a time of ecological transition with the issuance of Circular 0013/2017 (DES, 2017), the EP applies the five stages of the AFI model to a case referral. By interacting with the child, parents/guardians and teachers in accordance with AFI principles at the meso and microsystem levels, the assessment process leads to intervention recommendations that are meaningful to teachers, parents/guardians and child. Engaging together in the five-stage process exposes EST and SCT concepts. 
Subsequently, the research question outlined below evolved from this theoretical statement and literature review findings:

'Can the Assessment for Intervention model bridge the gap between assessment and intervention, through activity and interaction ${ }^{4}$ at the meso and microsystem levels?'

\section{Research design}

The assumptions of the researcher's conceptual framework was considered to resonate most with the constructivist paradigm. Qualitative methods utilising an exploratory 'two case' case study were employed to explore the application of the AFI model in an Irish educational psychological context. Case study designs are most suited to research questions that seek to provide explanation to a present circumstance and require an extensive, thorough description of a particular phenomenon (e.g. "how" or "why" a phenomenon works) (Yin, 2009, p. 4). When conducting a case study, the goal is to expand and generalise theories (analytic generalisation) as opposed to enumerate frequencies (statistical generalisation) (Yin, 2009). With regards the current study, the conceptual framework served as the vehicle for generalising to other cases and in the generation of case propositions. Within the current context of an existing gap between assessment and intervention in educational psychological practice, identified in the findings of the systematic review and deliberated in academic discourse (Resing et al., 2017; VanDerHeyden, 2018), a case study design was considered appropriate for an indepth exploration into the utility of the AFI model in 'bridging the gap'. Applying the case study method facilitated theorising of EST and SCT concepts, in the analysis of data gathered.

\section{Case propositions}

Case study propositions were used to guide data collection and relevant analytic strategies (Yin, 2009, p. 130). The propositions outlined below arose from the conceptual framework, research question and the aims of AFI (Pameijer, 2016, 2017). Following engagement with the five stages of the AFI model:

(1) Parents, teachers and Trainee Educational Psychologist (TEP) will have a better understanding of the child's situation, as a result of interactions and activities that have occurred at the meso and microsystem levels.

\footnotetext{
${ }^{4}$ Activity and interaction are those activities and interactions that resonate with EST and SCT concepts, including molar activities, dyadic relations, "law of genetic development", ZPD and tools and semiotics.
} 
(2) Parents, teachers and TEP will know what intervention supports the child needs at home and at school, as a result of interactions and activities that have occurred at the meso and microsystem levels.

(3) Teachers and parents will have greater perceived competence in supporting the child's needs at home and at school, as a result of interactions and activities that have occurred at the meso and microsystem levels.

(4) The child will have a better understanding of their strengths and needs and what to do to improve their needs, as a result of interactions and activities that have occurred at the meso and microsystem levels.

(5) At a time of ecological transition, teachers will feel competent in their ability to work according to the aims of Circular 0013/2017, in meeting and monitoring the needs of the child.

\section{Participants}

Case 1 included a student in $4^{\text {th }}$ class (Jim), his mother (Tina), class teacher (Michael) and Special Education Teacher (SET-Sarah) $(n=4)$. Jim is a ten-year old boy and is attending $4^{\text {th }}$ class in a mainstream primary school. He was referred to the school psychological service to provide insight into difficulties in literacy and numeracy and information on specific supports and strategies to target theses needs.

Case 2 comprised of a student in $6^{\text {th }}$ class (Michelle), her mother (Mary) and class teacher (Anne) $(n=3)$. Michelle is a twelve-year old girl and is attending $6^{\text {th }}$ class in a mainstream primary school. Michelle's mother and school requested the involvement of the school psychological service to provide insight into learning difficulties in English and Maths and support around social-emotional and communication skills.

A pilot study which included a student in $4^{\text {th }}$ class (John), his mother (Louise) and class teacher (Grace) $(n=3)$ was also conducted. John is a ten-year old boy and is attending $4^{\text {th }}$ class in a mainstream primary school. He has a diagnosis of Autism Spectrum Disorder (ASD) and presents with a fixated, restricted interest. John was referred to the school psychological service to provide insight into how to address his engagement in the restricted interest and to broaden his interests. Due to an incomplete data set for Case 2, the pilot study data was also analysed and presented as a case. Table 3 below outlines the pseudonyms applied to each participant. 
Table 3. Participants

\begin{tabular}{lll}
\hline Case & Participant/Role & Pseudonym \\
Pilot Case & $\begin{array}{l}\text { Class teacher } \\
\text { Parent }\end{array}$ & Louise \\
Student & John \\
Case 1 & Class teacher & Michael \\
& SET & Sarah \\
& Parent & Tina \\
& Student & Jim \\
& Class teacher & \\
Case 2 & Parent & Anne \\
& Student & Mary \\
& & Michelle \\
\hline
\end{tabular}

\section{Measures and analysis}

Qualitative measures were employed to address the research question and case propositions. Participants rated Likert statements before and after application of the AFI model. Semi-structured interviews were also conducted following engagement with the AFI model to explore the research question and case propositions. AFI templates served as a researcher diary and were recorded before, during and after application of the model. A reflective journal was also used to elucidate actions recorded and to reflect upon the process. Thematic analysis (Braun \& Clarke, 2006) and pattern-matching (Yin, 2009) were employed to analyse the interview data. Changes in ratings on Likert statements were presented in tabular format and excerpts from the researcher diary were included in the discussion of findings. Analysis of data was informed by the researcher's conceptual framework.

\section{Pilot study}

A pilot study was conducted initially, to inform and refine the research design, measures and data collection procedures (Mertens, 2015; Yin, 2009). The pilot case study was carried out with a case referral on the researcher's school psychology placement. A case selection protocol and case study protocol were adhered to in carrying out the pilot case study (Appendices 5 and 6). The student, the student's class teacher and one of his parents participated in the pilot study $(n=3)$. The findings from this phase of the study were used to inform and revise measures and the development of an in-depth procedure for conducing the proceeding cases. To enhance reliability, a procedural template was devised and was based on the case study protocol, the AFI templates and instructional guidance provided in Pameijer (2016). 


\section{Data collection procedures}

Case 1 and Case 2 were conducted in parallel due to time constraints. Upon receipt of informed consent and assent from participants, procedural phases for data collection were implemented as outlined below:

(1) Parents, teachers and students rated Likert scale statements before engaging with the AFI model. Space for comments was provided. Instruction and clarification was provided as needed. The researcher documented the process and reflections in the researcher diary (AFI templates) and reflective journal.

(2) The researcher applied the five-stage AFI model to the case referral and participants engaged in a range of activities and interactions including dialogue between team members, co-investigator observation tasks and 1:1 assessments. The five-stage process and reflections were documented to demonstrate transparency and reliability.

(3) Parents, teachers and student rated the same Likert scale statements from Phase 1, following engagement with the stages of the AFI model. Space for comments was provided. The researcher also conducted semi-structured interviews with parents and teachers to explore their views on the utility of the AFI model in bridging the gap between assessment and intervention (research question) and to address the case propositions. Interviews were also conducted with the students to explore their views on the process and to address case propositions. Interviews were recorded on a recording device borrowed from the Mary Immaculate College library. Reflections were documented in the reflective journal.

\section{Ethical considerations}

Ethical approval was obtained from Mary Immaculate College in May 2018 and approval to conduct the research project was granted by the school psychology service in June 218. Transparency and credibility of researcher actions and intentions were made clear to the participants at all times. The Board of Management, teachers and parents were briefed on the aims and goals of the research project and provided with an information sheet. The Board of Management, teachers and parents also signed an informed consent form with explicit information indicating that they could withdraw at any time and for any reason. Parents were also informed of their right to withdraw their 
child from the study at any time. The students participating were considered vulnerable as they were under the age of 18 and referred for behaviour, social-emotional and/or learning needs. The researcher took careful steps to ensure the students fully understood the aims of the project, that their participation was voluntary and of their right to withdraw at any time. A child friendly information sheet and assent form was presented to and signed by the students.

\section{Findings}

This section presents the findings of the current research project. Likert scale rating scores are presented in tabular format in the section below. This is followed by the pattern-matching logic applied to interview responses, outlined under the case propositions. Four themes and associated subthemes were generated through thematic analysis, including 'A team approach to assessment', 'Perspectives on barriers to parental engagement', 'Factors bridging the gap between assessment and intervention' and 'Views and experiences of AFI'. The theme 'Factors bridging the gap between assessment and intervention' is presented in the current research paper.

\section{Likert scale ratings}

Average parent and teacher Likert scale ratings outlined in Table 4 indicate greater understanding of the situation, knowledge of intervention supports and greater perceived competence in supporting the child's needs following engagement with the AFI model. Averages were rounded to the nearest decimal point, consistent with the Pameijer (2017) evaluation study and are reported for participants who completed the Likert scale statements before and after engaging with the AFI model. With regards Proposition 4, Likert scale ratings provided by John in the Pilot Study indicate that he was "not sure" of his strengths following engagement with the AFI model. His ratings indicate that he now knows what he needs help with at school and still does not know what he can do to improve on needs at school. Likert scale ratings provided by Jim in Case 1 indicate he knew what his strengths and needs were before and after engaging with the AFI model. Jim's rating changed from not knowing what he can do to improve on needs at school to being "not sure" following engagement with the AFI model. 
Table 4. Average Likert scale ratings

\begin{tabular}{|c|c|c|c|}
\hline$\underline{\text { Likert Statement }}$ & $\underline{\text { Participant }}$ & $\begin{array}{l}\text { Before engagement } \\
\text { with the AFI model }\end{array}$ & $\begin{array}{l}\text { After engagement } \\
\text { with the AFI model }\end{array}$ \\
\hline $\begin{array}{l}\text { I understand the } \\
\text { student's situation }\end{array}$ & Teacher $(n=4)$ & 5.5 & 8.9 \\
\hline $\begin{array}{l}\text { I understand my } \\
\text { child's situation }\end{array}$ & Parent $(n=2)$ & 6 & 6.5 \\
\hline $\begin{array}{l}\text { I know what } \\
\text { support the student } \\
\text { needs at school }\end{array}$ & Teacher $(n=4)$ & 5.5 & 8.9 \\
\hline $\begin{array}{l}\text { I know what } \\
\text { support my child } \\
\text { needs at home }\end{array}$ & Parent $(n=2)$ & 3.5 & 9 \\
\hline $\begin{array}{l}\text { I am able to the } \\
\text { student's needs at } \\
\text { school }\end{array}$ & Teacher $(n=4)$ & 4.8 & 9 \\
\hline $\begin{array}{l}\text { I am able to } \\
\text { support my child's } \\
\text { needs at home }\end{array}$ & Parent $(n=2)$ & 5 & 9 \\
\hline
\end{tabular}

\section{Pattern-matching logic}

Pattern-matching logic was applied to the responses of participants who engaged in interview following engagement with the AFI model in each of the three cases. Responses are presented with resonating AFI principles (Pameijer, 2017) and are discussed in the context of the conceptual framework and literature in the discussion section.

\section{Proposition 1}

Pattern-matching logic was applied to parent and teacher responses to questions exploring change in understanding and experience of the AFI model. Responses across all three studies suggest interactions and activities that occurred at the meso and microsystem levels (See Figure 1) during the five stages were connected to greater understanding of the situation. Activities and interactions described by participants during interview included information taking, a team approach to identifying the issues and hearing each other's voices (Pilot Case); getting the voice of the student, parent and teachers and establishing working memory as an area of cognitive weakness (Case 1); talking through everything and analysing the situation (Case 2). These activities and interactions resonated with several AFI principles, including applying a transactional perspective to the presenting situation (Principle 2) and engaging in tasks as 'coassessors' (Principle 6) (Pameijer, 2017). 


\section{Proposition 2}

To address Proposition 2, parents and teachers were asked questions on the intervention recommendations and supporting the child's needs. Patterns across responses indicate interactions and activities that occurred at the meso and microsystem levels generated knowledge of intervention supports that the child needed. These included intervention recommendations observed and discussed during the AFI process such as close proximity praise, behaviour specific praise and exploring alternative interests (Pilot Case); appraisal, a spelling acronym and ideas to address memory and auditory skills (Case 1); social communication and CBT interventions, checklists and graphic organisers (Case 2). Consistent with Proposition 1, these activities and interactions were in accordance with AFI Principles 6 and additionally Principles 3 and 4, identifying teacher and parent approaches that would address the child's needs (Pameijer, 2017)

\section{Proposition 3}

Proposition 3 was addressed with a question exploring ability to support the child's needs. Patterns within parent and teacher interview responses suggest interactions and activities that occurred at the meso and microsystem levels during the AFI process were connected to greater perceived competence to support the child's needs. These activities and interactions included gaining knowledge of alternative interests and developing understanding of the situation and triggers for behaviour (Pilot Case); developing knowledge of student's needs, recommendations to address needs and strengthening relationships between home and school (Case 1); gaining knowledge around extra interventions and continuing with strategies that are working well (Case 2). Akin to Proposition 2, these activities and interactions were consistent with AFI Principles 3 and 6 and additionally, Principle 1 participating in a goal directed and functional assessment, with the development of feasible intervention recommendations (Pameijer, 2017).

\section{Proposition 4}

To address Proposition 4, John (child in Pilot Study) and Jim (child in Case 1) were asked interview questions exploring their thoughts on their strengths, needs and what they need to do to improve on needs. They were also asked a question on how they found the process of working together with their parent, teachers and the TEP. Patterns identified within their responses connected interactions and activities that occurred at the microsystem levels during the AFI process, including dialogue between team 
members, co-investigator observation tasks and 1:1 assessments/joint activity tasks to knowledge of strengths, needs and what to do to address needs. John responded "kind of" to questions exploring if working together helped him to find out about his strengths and needs and that it was more difficult to find out about his strengths and needs with his mother and teacher, than with the TEP. His responses indicated that the assessment process was a challenging experience in "making hard decisions". With regard to addressing needs, John commented that he needs to "study my best in school". When asked about making time for new activities and interests, John commented "yeah that would be one". In contrast, Jim responded "yes" to questions exploring if working together helped him to find out about his strengths and needs. When asked what he needs to do to improve on needs, Jim mentioned strategies that had been practiced during 1:1 dynamic assessment. Activities and interactions that Jim and John engaged in at the microsystem level are considered consistent with AFI Principle 6 (Pameijer, 2017) as they were both considered 'co-assessors' when completing 'co-investigator tasks' and their opinions and feedback contributed to intervention planning.

\section{Proposition 5}

As regards Proposition 5, teachers were asked a question exploring their perceived levels of competence to monitor the student's needs moving forward. Patterns within their responses associated ability to monitor needs to interactions and activities that occurred at the meso and microsystem levels during the AFI process. In Case 1, patterns within Michael's response connected competence to his strengthened relationship with Jim and having a good relationship with the learning support teacher. Sarah attributed competency to her awareness of Jim's learning needs following engagement with the AFI process. Similarly in Case 2, patterns within Anne's response linked competence to greater awareness and insight into needs following the AFI process. Patterns within Anne and Grace's response also linked competence to monitor needs to their general teaching skills. These activities and interactions were considered consistent with AFI Principle 3, focusing on the educational needs of the child and AFI Principle 6, engaging in tasks as 'co-assessors'. The theme 'Factors bridging the gap between assessment and intervention' substantiates Proposition finding, presented in the section below. 


\section{Thematic analysis}

The theme 'Factors bridging the gap between assessment and intervention' identified in the current study had four associated subthemes; 'Experiencing or observing intervention effectiveness', 'Practical interventions', 'Shared awareness of strengths, needs and perspectives' and 'A collaborative approach to implementing and monitoring interventions'. These subthemes are expanded upon in the sections below. Figure 2 delineates the themes and subthemes in a thematic map of analysis.

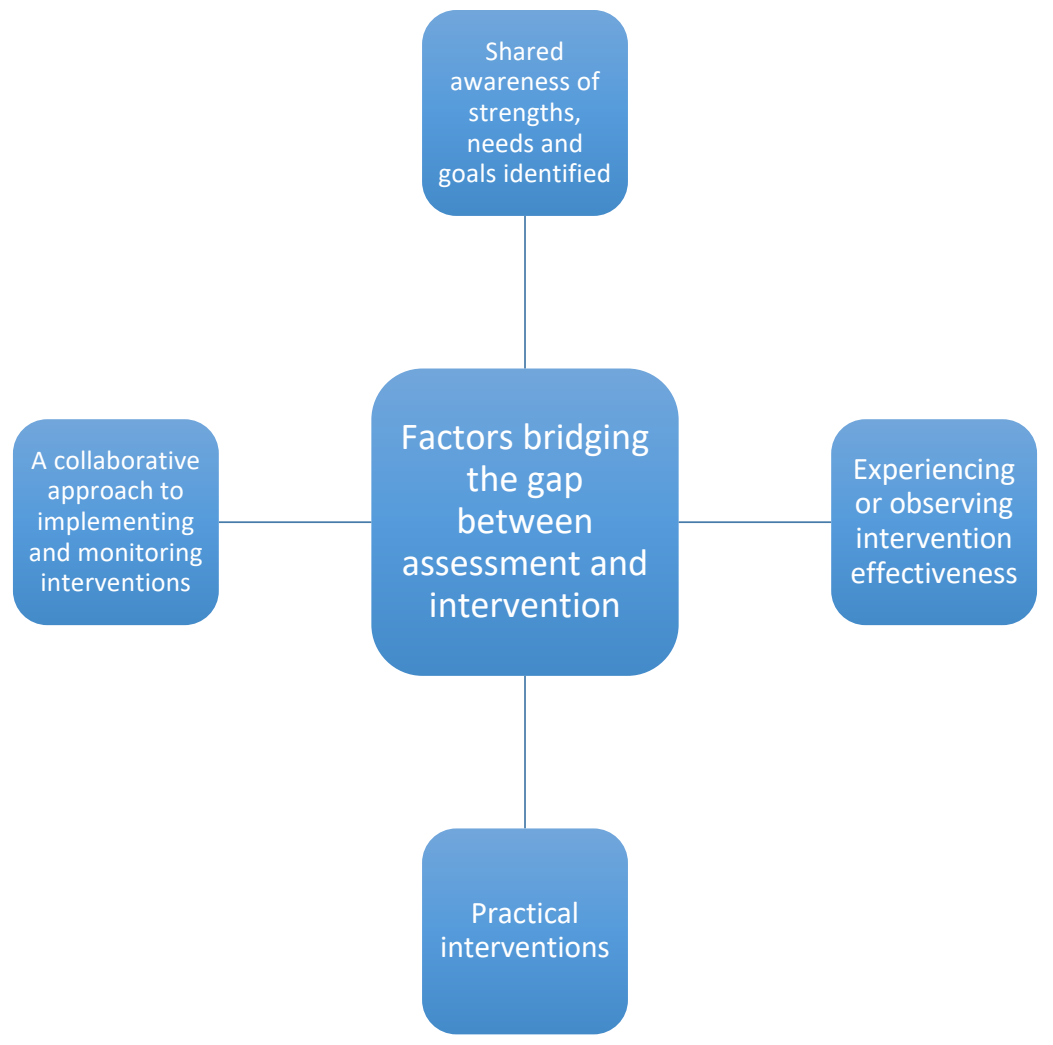

Figure 2. Factors bridging the gap between assessment and intervention

Shared awareness of strengths, needs and goals identified

Relevant to Proposition 2, thematic analysis of interview responses suggests shared knowledge of strengths and needs identified may have contributed to knowledge of appropriate intervention supports. For example, Grace referred to the benefit of the student being 'aware' that needs and goals identified were shared amongst the team. The teachers in Case 1 discussed how they would incorporate strengths identified and shared between the team, to target areas of weakness: 
...trying to work his strengths on his weaknesses also, do you know, trying to see if they helped in developing his weaknesses (Sarah)

\section{Experiencing or observing the effectiveness of intervention recommendations}

Pertaining to Proposition 2, experiencing or observing intervention effectiveness during the Investigation stage may have also contributed to knowledge of intervention supports. Anne observed the effectiveness of the self-monitoring checklist which encouraged Michelle "to get started independently". Grace observed John's responsiveness to one-to-one behaviour strategies and may have applied this knowledge when considering the recommendation of a self-monitoring checklist:

I think he'd really respond to that because again it's the one-to-one attention that's just for him (Grace)

Sarah and Tina commented on the effectiveness of a mnemonic spelling aid during the Investigation stage at school and at home, which was translated into an intervention recommendation at the Feedback stage:

....and it's great because it works (Sarah)

I feel it (spelling trick) working (Tina)

\section{Practical interventions}

Regarding Proposition 3, practical intervention recommendations may have contributed to perceived competence to support the child's needs. In the Pilot Case, Grace and Louise indicated that intervention recommendations were straightforward to implement, taking "little steps" in the home environment and "easily implemented" interventions in the classroom. Sarah (Case 1) commented that recommendations were "very clear, easy, specific" and the spelling intervention is a strategy "that all of the class wanted to take on". Anne (Case 2) also noted the "tips" could be used with everybody:

...all of those tips are brilliant and can be used for everybody, which is great as well and again that's again getting back to the practicalities of things... (Anne)

In line with AFI, researcher reflections recorded at the Feedback stage documented that parents and teachers were able to choose recommendations that they would be willing to start 'tomorrow' (Pameijer, 2017): 
Tina wants to try the recommendation for the spelling trick, encouraging selfmonitoring of work in maths and to keep a journal diary at home of interests that he can share during news time (December $12^{\text {th }}$, Feedback stage)

\section{A collaborative approach to implementing and monitoring interventions}

The teachers in Case 1 commented on how they would work together with Jim to monitor interventions by asking "questions like what worked well or you know, where he is struggling" (Michael) and "making sure that they're working do you know, to the best that they can for him" (Sarah). Jim also commented that he would need his parents and teachers to help "complete" his goals, reflective of a collaborative approach. Grace referred to parental involvement in the monitoring of intervention recommendations, in terms of observing changes in behaviour and potential progress:

....and for the parents to see that they (behaviours) have changed as well it would be fantastic (Grace)

\section{Synthesis of Findings}

In summary, the collective findings of the case studies suggest the AFI model can bridge the gap between assessment and intervention through activity and interaction at the meso and microsystem levels (Bronfenbrenner, 1979). Average parent and teacher Likert scale ratings indicated greater understanding of the situation, knowledge of intervention supports and greater perceived competence in supporting the child's needs following engagement with the AFI model. This is consistent with findings presented in Pameijer (2017), where $90 \%$ of teachers and $65 \%$ of parents indicated the assessment offered a better understanding of the student, $70 \%$ of teachers and $52 \%$ of parents reported the assessment offered them recommendations and $65 \%$ of teachers felt they could apply the recommendations in their class. The current research extended these findings with the application of pattern-matching and thematic analysis to participant interview responses. Pattern-matching logic provided supportive evidence for Propositions 1, 2, 3, 4 and 5 in each of the three cases ${ }^{5}$ and responses indicate activities and interactions that occurred at the meso and microsystem levels, including dialogue between team members, co-investigator observation tasks and 1:1 assessments/joint activity tasks, were connected to:

(1) Greater understanding of the child's situation (Proposition 1)

(2) Knowledge of intervention supports the child needed (Proposition 2)

\footnotetext{
${ }^{5}$ Data were collected from the teacher only post engagement in the AFI process in Case 2, therefore there was limited evidence that could be drawn upon for analysis in this case.
} 
(3) Greater perceived competence in supporting the child's needs (Proposition 3)

(4) Greater understanding of strengths, needs and what to do to address needs (Proposition 4-child only)

(5) Greater perceived competence to meet and monitor needs moving forward (Proposition 5-teachers only)

An in-depth analysis of findings demonstrated that these activities and interactions were in accordance with AFI principles, EST and SCT concepts, further strengthening the theoretical underpinnings of the AFI model. Findings are further discussed in the context of the conceptual framework in the section below. The assessment approach applied contrasts with the traditional, medical model assessment practices evident in twelve of the studies in the systematic review, where determination of a diagnosis or special education provision was one of the primary objectives of the assessment. It is proposed that the collective findings of this exploratory research project, expanded upon in the sections below, address the gap identified in the current systematic review, between assessments being conducted and the interventions that clients receive in EP practice. The findings satisfy the stipulations for professional assessment outlined in Circular 0013/2017 in providing an understanding of the child's needs, the nature of difficulties, and in informing appropriate interventions (DES, 2017).

\section{Discussion of Factors Bridging the Gap Between Assessment and Intervention}

Relevant to Proposition 2, the findings presented in each case indicate that assessment informed appropriate interventions for participants. These finding are consistent with six of the twenty-one studies reviewed in the systematic review, that provided evidence of assessment informing appropriate interventions for clients (Bozic, 2013; Cane, 2016; Lawrence \& Cahill, 2014; Pameijer, 2017; Parker et al., 2016; Tobias, 2017). There were several factors identified as bridging the gap between assessment and intervention including shared awareness of strengths and needs identified, experiencing or observing intervention effectiveness, practical intervention recommendations and a collaborative approach to implementing and monitoring interventions. Each of these factors are discussed in accordance with the literature and conceptual framework in the sections that follow. 


\section{Shared awareness of strengths and needs identified}

In Case 1 and Case 2, shared awareness of students' learning potential, achieved through observations conducted by team members at home and in the classroom as 'coassessors' (Pameijer, 2017), and engaging in 1:1 assessment of actual and learning potential with the TEP, helped to form realistic and achievable goals and intervention recommendations, consistent with Vygotsky's ZAD and ZPD (Vygotsky, 1978, pp. 8586). This awareness is consistent with social constructivist assessment approaches outlined by Green and Gredler (2002) and Thomas and Oldfather (1997), where learning is considered a reciprocal process as the student takes on an increasingly independent role and observation of how a student solves problems and constructs meaning informs intervention planning

\section{Experiencing or observing intervention effectiveness}

Experiencing or observing intervention effectiveness occurred during the Investigation stage in each of the three cases and these interventions were later translated into recommendations. For example in Case 1, Sarah, Tina and Jim commented on how they tried the mnemonic aid for remembering tricky spelling words during the Investigation stage and observed it to be effective. In Case 2, Anne and Michelle observed or experienced the effectiveness of interventions during the Investigation stage, including the self-monitoring checklist and graphic organiser, which became intervention recommendations at the Feedback stage. This is in agreement with findings presented in Lawrence and Cahill (2014), where student and teacher interview responses provided evidence of the utility of interventions in the classroom context, and parent feedback indicated intervention effectiveness in the home setting.

\section{Practical intervention recommendations}

Pertinent to Proposition 3 and competency to support student needs, practical intervention recommendations were provided and were described by teachers and parents as being feasible to implement and beneficial to other children in the class, or "little steps" that could be taken in the home environment. Consistent with AFI (Pameijer, 2017), researcher reflections indicated that parents and teachers in the Pilot Study and Case 1 were able to identify an intervention recommendation that they were willing to start "tomorrow". 


\section{A collaborative approach to implementing and monitoring interventions}

Relevant to Proposition 5 and monitoring of needs, Grace's responses in the Pilot Study indicated parental inclusion in the monitoring of goals and interventions and Michael commented that he planned to consult with Jim in this process in Case 1. Jim also commented that he would need his parents and teachers to help him "complete" his goals, reflective of his inclusion in the process (Pameijer, 2017). These finding are consistent with the aims of Circular 0013/2017 (DES, 2017) and findings presented by Cane (2016) in the systematic review, where student and school staff input was sought during assessment, to directly inform a solution-focused intervention. Student and staff were involved in the monitoring of the intervention and scaling and qualitative feedback indicated improvements in the student's learning and behaviour. Collectively, it is proposed the factors presented in the current study have implications for EP practice and address the gap between assessments being conducted and the interventions that clients receive, identified in the current systematic review.

\subsection{Implications of research findings}

The research presents significant implications for the discipline of educational psychology in the context of Circular 0013/2017 (DES, 2017). The first key implication for EP practice is the presentation of the AFI model (Pameijer, 2017) as an assessment framework consistent with ecological and social constructivist perspectives, that can satisfy the aims of Circular 0013/2017 (DES, 2017). A second implication is the consideration of factors that bridged the gap between assessment and intervention in the current research project, in EP practice. These implications are discussed further in the sections below.

\section{Implications for Policy}

Parents, teachers and TEP found AFI (Pameijer, 2017) to be a clear, structured process that facilitated engagement in team assessment, consistent with ecological and social constructivist assessment approaches (Burns, 2013; Thomas \& Oldfather, 1997). Findings presented indicate parents and teachers had a greater understanding of the child's situation, knowledge of intervention supports and greater perceived competence in supporting the child's needs following engagement with the AFI model, consistent with the aims of Circular 0013/2017 (DES, 2017). Teachers in Case 1 and the Pilot Study compared their experience of AFI to traditional, formalised assessment approaches and commented on their preference for AFI (Pameijer, 2017). The 
theoretical statement of the current research project proposed that communication and interaction at the meso and micro system levels would be necessary to successfully meet the aims of Circular 0013/2017 in identifying, meeting and monitoring needs (DES, 2017), and is supported by the findings presented. AFI provides a structured framework for communication and interaction at the meso and microsystem levels, between school psychological service, school, home and student and strengthens the developmental potential (Bronfenbrenner, 1979, p. 216) of these settings to work together to meet the aims of Circular 0013/2017 (DES, 2017). Furthermore, the students' voice and their active participation was facilitated and encouraged throughout the process, shifting the balance of power towards the child as the developing person (Bronfenbrenner, 1979, p. 58 ) and contrasting with studies in the systematic review that acknowledged the absence of the voice of the child during the assessment process (Hanchon \& Allen, 2013; Hill \& Turner, 2016). AFI provides a framework for the inclusion of the voice of the child, which is consistent with the 'inclusive assessment' approaches promoted by the European Agency for Special Needs and Inclusive Education (Watkins, 2007). The AFI model could be applied in EP practice to facilitate the child's active involvement in working towards meeting the aims of Circular 0013/2017 (DES, 2017).

\section{Implications for EP practice}

The researcher's systematic review identified a gap between assessment being conducted by EPs and the interventions that clients receive, consistent with assertions by Resing et al. (2017) and VanDerHeyden (2018). Stipulations for professional assessment in Circular 0013/2017 (2017) outline the role of the EP in providing understanding of a child's needs, the nature of difficulties, and informing appropriate interventions. The overall aim of the AFI model is to bridge the gap between assessment and intervention in order to provide recommendations that are both scientifically sound and useful for the student, teacher and parent (Pameijer, 2017). Factors identified as helping to bridge the gap in the current research project included shared awareness of strengths and needs identified between child, parents, teachers and TEP; experiencing or observing intervention effectiveness during the Investigation stage; practical intervention recommendations and a collaborative approach to implementing and monitoring interventions. These factors could be considered by EPs in practice as they work in accordance to the aims of Circular 0013/2017 (DES, 2017). 


\section{Implications for educational contexts}

The AFI model was discussed as having practical applicability to educational settings by teachers in each of the case studies, reflecting the model's developmental validity (Bronfenbrenner, 1979, p. 29). Teachers indicated the model could be applied to consider the home situation when a child is having difficulties at school (Pilot Study), to assess and meet the needs of other children in the class and in any situation where communication is required (Case 1) and its applicability "across the board" as information gathered and interventions were useful to other children (Case 2). In terms of monitoring needs, teachers in two of the cases discussed involving the parent (Pilot Study) and student (Case 1) in monitoring the student's needs moving forward. The child in Case 1 also stated that he would need his parents and teachers to help him "complete" his goals, reflective of his inclusion in the process. This feedback on the applicability of the AFI model in educational practice suggests its relevance in this field and specific recommendations for practice are outlined in the following section.

\section{Summary of recommendations for practice}

In summary, it is recommended that training and input on the AFI model (Pameijer, 2017) be considered on a national level by school psychological services and professional training bodies as the unique role of the EP shifts away from a medical model of practice, towards an ecological, interactionist approach. Additionally, in consideration of the aims of Circular 0013/2017 (DES, 2017) and the endorsement of inclusive assessment by the European Agency for Special Needs and Inclusive Education (Watkins, 2007), training and input on the AFI model (Pameijer, 2017) could be considered for teachers and school staff. Working together at a systems level to apply the aims and principles of AFI would support the developmental potential of home, school and school psychological services in identifying, meeting and monitoring the needs of students (Bronfenbrenner, 1979, p. 216). Furthermore, factors identified by the current research project as bridging the gap between assessment and intervention could be considered by EPs in practice as they work in accordance to the aims of Circular 0013/2017 (DES, 2017).

\section{Limitations of the research}

While the findings of this study can be used to inform future practice, the exploratory case study design would suggest generalisability of findings is limited to theory, similar settings, population and age group (Yin, 2009). The researcher adhered to a case 
selection protocol with specified selection criteria and a larger, more varied group of participants may have provided greater variation and depth of findings. Due to the referral of participants through the school psychological service, participant and researcher bias could have influenced responses on the Likert scale statements, interviews and researcher diary and journal. Although the constructivist paradigm promotes the co-construction of knowledge between researcher and participants (Mertens, 2015), the potential for social desirability bias in responses should be considered as a possible limitation of findings. With regard to interviews, teachers in Case 1 and Case 2 expressed their disappointment that the parents were not as present during the assessment process, reflective perhaps of their own belief systems as parents and teachers, and the value they had placed on the AFI process. In the context of the current study, belief systems and values were not directly addressed during interview, which may have provided for a richer data set. An additional limitation is that data were collected from the teacher only in Case 2 following engagement with the AFI model and therefore there was limited evidence in this case that could be drawn upon for analysis. The time of year in which the study was conducted (October-December) presented a challenge as data was collected right up until the Christmas holidays, which is a very busy time for schools and parents. Finally, although the researcher had received training on the AFI model, level of experience and competency as a TEP applying the model in practice should be considered in the interpretation of findings. Despite limitations presented, this study may be the starting point for a more extended exploration of the AFI model in an Irish Educational Psychological context, discussed further in recommendations for future research.

\section{Future research}

There are a number of opportunities for further research to consolidate and enhance the findings presented. The study could be replicated for a more extended exploration of the AFI model in an Irish Educational Psychological context and also to evaluate the effectiveness of intervention recommendations following a specified period of implementation. For a richer data set, the research could include the views and perspectives of additional stakeholders, including School Principals and Inclusion Support Assistants. In accordance with recommendations for future research presented in Pameijer (2017, p. 80), further research is warranted to investigate if AFI produces more ecologically valid case formulations and effective interventions than 'assessment as usual'. 


\section{Conclusion}

To conclude, the current research project provided original insight into the application of the AFI model in educational psychological practice, through the conceptual lens of EST (Bronfenbrenner, 1979) and SCT (Palinscar, 1998; Vygotsky, 1978). The presented findings suggest the AFI model can bridge the gap between assessment and intervention, through activity and interaction at the meso and microsystem levels. The findings address assertions for professional assessment outlined in Circular 0013/2017 (DES, 2017), indicating the scholarly significance of this doctoral research with practical implications for EP and educational practice (Yin, 2009). Limitations include the exploratory case study design and limited generalisability of findings to theory, similar settings, population and age group (Yin, 2009). However, this study may be the foundation for a more extended exploration of the AFI model in Irish Educational Psychological practice, as detailed in the recommendations for future research.

\section{Disclosure statement}

No potential conflict of interest was reported by the authors. 


\section{References}

Aganza, J. S., Godinez, A., Smith, D., Gonzalez, L. G., \& Robinson-Zañartu, C. (2014). Using cultural assets to enhance assessment of Latino students. Contemporary School Psychology, 19(1), 30-45. doi:10.1007/s40688-014-0041-7

Aiello, R., Ruble, L., \& Esler, A. (2016). National study of school psychologists' use of evidence-based assessment in autism spectrum disorder. Journal of Applied School Psychology, 33(1), 67-88. doi:10.1080/15377903.2016.1236307

Alegra, M. (2013). Assessment for intervention: Second evaluation of a pilot in Unita, a centre for inclusive education. Rotterdam, NL: CED-groep.

American Psychiatric Association. (2000). Diagnostic and statistical manual of mental disorders (4th ed.). Washington, DC: Author.

Bahr, M. W., Leduc, J. D., Hild, M. A., Davis, S. E., Summers, J. K., \& McNeal, B. (2017). Evidence for the Expanding Role of Consultation in the Practice of School Psychologists. Psychology in the Schools, 54(6), 581-595. doi:10.1002/pits.22020

Barker, C., Pistrang, N., \& Elliott, R. (2002). Research Methods in Clinical Psychology: An Introduction for Students and Practitioners (2nd ed.). West Sussex, England: John Wiley \& Sons, Ltd.

Bourke, R., \& Dharan, V. (2015). Assessment practices of educational psychologists in Aotearoa/New Zealand: from diagnostic to dialogic ways of working. Educational Psychology in Practice, 31(4), 369-381. doi:10.1080/02667363.2015.1070709

Bowles, T., Scull, J., Clinton, J. H. a. J., Larkins, G., Cicconi, V., Kumar, D., \& Arnup, J. L. (2016). Conducting psychological assessments in schools: Adapting for converging skills and expanding knowledge. Issues in Educational Reserach, 26(1), 10-28.

Bozic, N. (2013). Developing a strength-based approach to educational psychology practice: A multiple case study. Educational \& Child Psychology, 30(4), 18-29.

Bozic, N., Lawthom, R., \& Murray, J. (2017). Exploring the context of strengths - a new approach to strength-based assessment. Educational Psychology in Practice, 34(1), 26-40. doi:10.1080/02667363.2017.1367917

Brantlinger, E., Jimenez, R., Klingner, J., Pugach, M., \& Richardson, V. (2005). Qualitative studies in special education. Exceptional Children, 71(2), 195-207.

Braun, V., \& Clarke, V. (2006). Using thematic analysis in psychology. Qualitative Research in Psychology, 3(2), 77-101. doi:10.1191/1478088706qp063oa 
Bronfenbrenner, U. (1979). The ecology of human development: Experiments by nature and design. Boston, MA: Harvard College.

Bronfenbrenner, U. (1989). Ecological systems theory. Annals of Child Development, 6, 187-249.

Burns, M. K. (2013). Contextualizing school psychology practice: Introducing featured research commentaries. School Psychology Review, 42(3), 334-342.

Cane, F. (2016). Everyone's solution: A case study of a systemic and solution-focused approach to therapeutic intervention in a secondary school. Educational \& Child Psychology, 33(4), 66-79.

Christenson, S. L., \& Anderson, A. R. (2002). Commentary: The centrality of the learning context for students' academic enabler skills. School Psychology Review, 31(3), 378-393.

Chun, H., \& Dickson, G. (2011). A psychoecological model of academic performance among Hispanic adolescents. Journal of Youth Adolescence, 40(12), 1581-1594. doi:10.1007/s10964-011-9640-z

Cottrell, J. M., \& Barrett, C. A. (2015). Job satisfaction among practicing school psychologists: The impact of SLD identification. Contemporary School Psychology, 20(1), 21-30. doi:10.1007/s40688-015-0076-4

Cresswell, J. W., \& Poth, C. N. (2017). Qualitative inquiry research design: Choosing among ve approaches (4th ed.). London: Sage Publications.

Davis, J. M., \& Deponio, P. (2013). Analysing conflicting approaches to dyslexia on a European project: moving to a more strategic, participatory, strength-based and integrated approach. International Journal of Inclusive Education, 18(5), 515534. doi:10.1080/13603116.2013.802023

Dean, V. J., Bums, M. K., Grialou, T., \& Varro, P. (2006). Comparison of ecological validity of leaming disabilities diagnostic models. Psychology in the Schools, $43,157-168$.

Deci, E. L., \& Ryan, R. M. (2008). Self-determination theory: A macrotheory of human motivation, development and health. Canadian Psychology, 49(3), 182-185.

Decker, S. L., Hale, J. B., \& Flanagan, D. P. (2013). Professional practice issues in the assessment of cognitive functioning for educational applications. Psychology in the Schools, 50(3), 300-313. doi:10.1002/pits.2167510.1002/pits

Department of Children and Youth Affairs. (2011). Children First: National Guidance for the Protection and Welfare of Children. Dublin: Government Publications. 
Department of Children and Youth Affairs. (2016). Access \& inclusion model: A new model for supporting access to early childhood care and education (ECCE) programme for children with a disability. Retrieved from http://aim.gov.ie/wpcontent/uploads/2016/06/AIM-Policy.pdf.

Department of Education and Skills. (2017). Guidelines for primary schools supporting pupils with special educational needs in mainstream schools. Athlone: Department of Education and Skills.

Department of Education and Skills. (2017). Special education teaching allocation circular 0013/2017. Retrieved from http://www.sess.ie/sites/default/files/inlinefiles/c10013 2017.pdf.

Department of Education and Skills. (2017). Special education teaching allocation circular 0014/2017. Retrieved from https://www.education.ie/en/Circulars-andForms/Active-Circulars/c10014_2017.pdf.

Desforges, M., \& Lindsay, G. (2010). Procedures used to diagnose a disability and to assess special educational needs: An international review (5). Retrieved from http://ncse.ie/wp-content/uploads/2014/10/5 NCSE_Diag_Ass.pdf

Elliott, J. G., Resing, W. C. M., \& Beckmann, J. F. (2018). Dynamic assessment a case of unfulfilled potential. Educational Review, 70(1), 7-17.

doi:10.1080/00131911.2018.1396806

Farrell, P. (2010). School psychology: Learning lessons from history and moving forward. School Psychology International, 31(6), 581-598.

Feuerstein, R., Rand, Y., \& Hoffman, M. (1979). The DA of retarded performers: The learning potential assessment device. Baltimore, MD: University Park Press.

Filter, K. J., Ebsen, S., \& Dibos, R. (2013). School psychology crossroads in America: Discrepancies between actual and preferred discrete practices and barriers to preferred practice. International Journal of Special Education, 28(1), 88-100.

Franzen, R. (1920). The accomplishment quotient: A school mark in terms of individual capacity. Teachers College Record, 21, 432-440.

Frenzel, A. C., Goetz, T., Pekrun, R., \& Watt, H. M. G. (2010). Development of mathematics interest in adolescence: Influences of gender, family, and school context. Journal of Research on Adolescence, 20(2), 507-537.

doi:10.1111/j.1532-7795.2010.00645.x

Geffken, G. R., Keeley, M. L., Kellison, I., Storch, E. A., \& Rodrigue, J. R. (2006). Parental adherence to child psychologists' recommendations from psychological testing. Professional Psychology: Research and Practice, 37(5), 499-505. doi:10.1037/0735-7028.37.5.499

Gough, D. (2007). Weight of Evidence: a framework for the appraisal 
of the quality and relevance of evidence. Research Papers in Education, 22(2), 213228. doi:10.1080/02671520701296189

Gough, D., Oliver, S., \& Thomas, J. (2013). Learning from research: Systematic reviews for informing policy decisions. University of London: EPPI-Centre, Social Science Reserach Unit, Institute of Education.

Grant, C., \& Osanloo, A. (2014). Understanding, selecting, and integrating a theoretical framework in dissertation research: Creating the blueprint for your "House". Administrative Issues Journal Education Practice and Research. doi:10.5929/2014.4.2.9

Green, S. K., \& Gredler, M. E. (2002). A review and analysis of constructivism for school-based practice. School Psychology Review, 31(1), 53-70.

Guest, G., Bunce, A., \& Johnson, L. (2016). How many interviews are enough? Field Methods, 18(1), 59-82. doi:10.1177/1525822x05279903

Hanchon, T. A., \& Allen, R. A. (2013). Identifying students with emotional disturbance: School psychologists' practices and perceptions. Psychology in the Schools, 50(2), 193-208. doi:10.1002/pits.21668

Harrison, G. L., \& McManus, K. L. (2016). Clinical reasoning in the assessment and intervention planning for writing disorder. Canadian Journal of School Psychology, 32(1), 73-86. doi:10.1177/0829573516658997

Hill, V., \& Turner, H. (2016). Educational psychologists' perspectives on the medicalisation of childhood behaviour: A focus on Attention Deficit Hyperactive Disorder (ADHD). Educational \& Child Psychology, 33(2), 12-29.

Horner, R. H., Carr, E. G., Halle, J., McGee, G., Odom, S., \& Wolery, M. (2005). The use of single-subject research to identify evidence-based practice in special education. Exceptional Children, 71(2), 165-179.

Hyett, N., Kenny, A., \& Dickson-Swift, V. (2014). Methodology or method? A critical review of qualitative case study reports. International Journal of Qualitative Studies on Health and Well-being, 9, 23606. doi:10.3402/qhw.v9.23606

Iida, M., Shrout, P. E., Laurenceau, J.-P., \& Bolger, N. (2012). Using diary methods in psychological research. In H. Cooper (Ed.), APA Handbook of Research Methods in Psychology: Vol. 1. Foundations, Planning, Measures, and Psychometrics, (pp. 277-305): American Psychological Association.

Jose, M. C., Michael, J. C., Chappel, A., \& Cunningham, J. (2013). School psychologists' continuing professional development preferences and practices. Psychology in the Schools, 50(4), 415-432. doi:10.1002/pits.2168410.1002/pits 
Kennedy, E. K., Frederickson, N., \& Monsen, J. (2008). Do educational psychologists "walk the talk" when consulting? Educational Psychology in Practice, 24(3), 169-187. doi:10.1080/02667360802256733

Krosnick, J., A. (1999). Survey research. Annual Review of Psychology, 50, 537-567.

Laing, R. D. (1971). The politics of the family and other essays. London: Tavistock.

Lawrence, N., \& Cahill, S. (2014). The impact of dynamic assessment: an exploration of the views of children, parents and teachers. British Journal of Special Education, 41(2), 191-211. doi:10.1111/1467-8578.12060

McCrimmon, A. W., \& Yule, A. E. (2016). Clinical reasoning in the assessment and planning for intervention for autism spectrum disorder. Canadian Journal of School Psychology, 32(1), 59-72. doi:10.1177/0829573516658564

Mertens, D. M. (2015). Research and evaluation in education and psychology. London: Sage.

Monsen, J., Graham, B., Frederickson, N., \& Cameron, R. J. (1998). An accountable model of practice. Educational Psychology in Practice, 13(4), 234-249. doi:10.1080/0266736980130405

National Council for Special Education. (2013). Supporting students with special educational needs in schools. Trim: National Council for Special Education.

National Council for Special Education. (2014). Delivery for students with special educational needs: A better and more equitable way. Retrieved from http://ncse.ie/wpcontent/uploads/2014/09/Allocating_resources_1_5_14_Web_accessible_versio n_FINAL.pdf.

Odom, S. L., Vitztum, J., Wolery, R., Lieber, J., Sandall, S., Hanson, M. J., . . Horn, E. (2004). Preschool inclusion in the United States: A review of research from an ecological systems perspective. Journal of Research in Special Educational Needs, 4(1), 17-49. doi:10.1111/J.1471-3802.2004.00016.x

Ogg, J., Fefer, S., Sundman-Wheat, A., McMahan, M., Stewart, T., Chappel, A., \& Bateman, L. (2013). School-based assessment of ADHD: Purpose, alignment with best practice guidelines, and training. Journal of Applied School Psychology, 29(4), 305-327. doi:10.1080/15377903.2013.836775

Palinscar, A. S. (1998). Social constructivist perspectives on teaching and learning. Annual Review of Psychology, 49, 345-375.

Pameijer, N. (2006). Towards needs-based assessment: Bridging the gap between assessment and practice. Educational \& Child Psychology, 23(3), 12-24. 
Pameijer, N. (2016). Assessment for intervention: A practice-based model.

Pameijer, N. (2017). Assessment and intervention: Bridging the gap with a practicebased model. Educational \& Child Psychology, 34(1), 66-82.

Parker, J., Zaboski, B., \& Joyce-Beaulieu, D. (2016). School-based cognitive-behavioral therapy for an adolescent presenting with ADHD and explosive anger: A case study. Contemporary School Psychology, 20(4), 356-369. doi:10.1007/s40688016-0093-y

Passenger, T. (2013). Introduction to educational psychology ractice. In A. J. Holliman (Ed.), The Routledge International Companion to Educational Psychology. London: Routledge. Retrieved from https://www.routledgehandbooks.com/doi/10.4324/9780203809402.ch3. doi:10.4324/9780203809402.ch3

Resing, W., Lauchlan, F., \& Elliott, J. (2017). Guest editorial: Bridging the gap between psychological assessment and educational instruction. Educational \& Child Psychology, 34(1), 6-8.

Ring, E., Sullivan, L. O., Ryan, M., \& Burke, P. (2018). A melange or a mosaic of theories?: How theoretical perspectives on children's learning and development can inform a responsive pedagogy in a redeveloped primary school curriculum. Limerick: Mary Immaculate College.

Robson, C. (2011). Real world research 3e (3rd ed.). Chichester: John Wiley \& Sons.

Sansosti, F. J., \& Sansosti, J. M. (2013). Effective school-based service delivery for students with autism spectrum disorders: Where we are and where we need to go. Psychology in the Schools, 50(3), 229-244. doi:10.1002/pits.21669

Schunk, D. H. (2012). Learning theories an educational perspective (6th ed.). London: Pearson.

Schwandt, T. A. (2000). Three epistemological stances for qualitative inquiry: Interpretivism, hermeneutics, and social constructionism. In N. K. Denzin \& Y. S. Lincoln (Eds.), Handbook of qualitative reserach (Vol. 2, pp. 189-214). Thousand Oaks: CA: Sage.

Seligman, M. E. P. (2002). Positive psychology, positive prevention, and positive therapy. In C. R. Snyder \& S. J. Lopez (Eds.), Handbook of positive psychology. Oxford: University Press.

Sotelo-Dynega, M., \& Dixon, S. G. (2014). Cognitive assessment practices: A survey of school psychologists. Psychology in the Schools, 51(10), 1031-1045.

doi:10.1002/pits.21802 
Stothard, J., Woods, K., \& Innoue, A. (2018). An exploration of practitioner educational psychologists' understandings and practice in relation to dyslexia. Educational \& Child Psychology, 35(1), 13-26.

Strong, T. O. M. (2005). Constructivist ethics? let's talk about them: an introduction to the special issue on ethics and constructivist psychology. Journal of Constructivist Psychology, 18(2), 89-102. doi:10.1080/10720530590914752

The Teaching Council. (2017). Cosán: Framework for Teacher's Learning Development Process (2016-2020. Retrieved from https://www.teachingcouncil.ie/en/Publications/Teacher-Education/CosanDevelopment-Process.pdf.

Thomas, S., \& Oldfather, P. (1997). Intrinsic motivations, literacy, and assessment practices: "That's my grade. That's me.". Educational Psychologist, 32(2), 107123.

Tilly, W. D. (2008). The Evolution of School Psychology to Science-Based Practice: Problem Solving and the Three-Tiered Model. In A. Thomas \& J. Grimes (Eds.), Best Practices in School Psychology (Vol. 5). Bethesda: NASP.

Tobias, A. (2017). The use of genograms in educational psychology practice. Educational Psychology in Practice, 34(1), 89-104. doi:10.1080/02667363.2017.1411787

U.S. Department of Education. (2004). Individuals with Disabilities Education Improvement Act (IDEIA). Retrieved from https://sites.ed.gov/idea/.

VanDerHeyden, A. M. (2018). Why do school psychologists cling to ineffective practices? Let's do what works. School Psychology Forum: Research in Practice, 12(1), 44-52.

Vega, D., Lasser, J., \& Afifi, A. F. M. (2015). School psychologists and the assessment of culturally and linguistically diverse students. Contemporary School Psychology, 20(3), 218-229. doi:10.1007/s40688-015-0075-5

Vygotsky, L. (1978). Mind in Society: The Development of Higher Psychological Processes. Cambridge: Harvard University Press.

Vygotsky, L. (1981). The instrumental method in society. In J. Wertsch (Ed.), The concept of activity in Soviet psychology. Armonk, NY: Sharpe.

Wagner, P. (2008). Consultation as a framework for practice. In B. Kelly, L. Woolfson, \& J. Boyle (Eds.), Frameworks for practice in eduactional psychology. London: Jessica Kingsley Publishers.

Watkins, A. (2007). Assessment in Inclusive Settings: Key Issues for Policy and Practice. Odense, Denmark: European Agency for Development in Special Needs Education. 
Wertsch, J. (1991). Voices of the mind: A socio-cultural approach to mediated action. Cambridge: Harvard University Press.

Yeomans, J. (2008). Dynamic Assessment practice: some suggestions for ensuring follow up. Educational Psychology in Practice, 24(2), 105-114. doi:10.1080/02667360802076107

Yin, R. K. (2009). Case study research: Design and methods (4th ed.). London: Sage Publications Inc. 
Appendix 1 Inclusion and Exclusion Criteria

\begin{tabular}{|c|c|c|c|}
\hline Criteria & Inclusion & Exclusion & Rationale \\
\hline $\begin{array}{l}1 \text { Type of } \\
\text { publication }\end{array}$ & Peer reviewed paper & $\begin{array}{l}\text { Not a peer } \\
\text { reviewed paper }\end{array}$ & $\begin{array}{l}\text { Peer reviewed papers have } \\
\text { been independently } \\
\text { assessed for quality }\end{array}$ \\
\hline 2 Language & $\begin{array}{l}\text { Study must be } \\
\text { written in English }\end{array}$ & $\begin{array}{l}\text { Study is not } \\
\text { written in English }\end{array}$ & $\begin{array}{l}\text { In order for the study to be } \\
\text { understood, it must be } \\
\text { written in English }\end{array}$ \\
\hline $\begin{array}{l}3 \\
\text { Assessment } \\
\text { Approach }\end{array}$ & $\begin{array}{l}\text { An assessment } \\
\text { approach, practice, } \\
\text { model or framework } \\
\text { applied by a } \\
\text { qualified EP }\end{array}$ & $\begin{array}{l}\text { Assessment } \\
\text { approaches that } \\
\text { are not applied by } \\
\text { an EP }\end{array}$ & $\begin{array}{l}\text { The current review is } \\
\text { situated in an educational } \\
\text { psychological context } \\
\text { therefore assessments } \\
\text { carried out by EPs only } \\
\text { were considered. }\end{array}$ \\
\hline $\begin{array}{l}4 \\
\text { Intervention }\end{array}$ & $\begin{array}{l}\text { An intervention, } \\
\text { recommendation, } \\
\text { strategy or treatment } \\
\text { arising from an EP's } \\
\text { assessment }\end{array}$ & $\begin{array}{l}\text { Interventions, } \\
\text { recommendations, } \\
\text { strategies or } \\
\text { treatments that do } \\
\text { not arise from an } \\
\text { EP's assessment }\end{array}$ & $\begin{array}{l}\text { The current review is } \\
\text { situated in an educational } \\
\text { psychological context } \\
\text { therefore interventions } \\
\text { arising from an EP's } \\
\text { assessment only were } \\
\text { considered. }\end{array}$ \\
\hline 5 Data & $\begin{array}{l}\text { The study provides } \\
\text { primary, empirical } \\
\text { data }\end{array}$ & $\begin{array}{l}\text { The study does } \\
\text { not provide } \\
\text { empirical data } \\
\text { (e.g. reviews, } \\
\text { commentaries) }\end{array}$ & $\begin{array}{l}\text { Empirical data allows the } \\
\text { reviewer to investigate the } \\
\text { assessment approaches } \\
\text { currently being applied by } \\
\text { EPs (review question 1) } \\
\text { and the appropriateness of } \\
\text { intervention } \\
\text { recommendations (review } \\
\text { question 2) arising from } \\
\text { these assessments. A } \\
\text { variety of measures may } \\
\text { be employed in studies } \\
\text { including questionnaire, } \\
\text { interviews and self- } \\
\text { reflection. }\end{array}$ \\
\hline $\begin{array}{l}6 \\
\text { Participants }\end{array}$ & $\begin{array}{l}\text { Participants in } \\
\text { studies must be } \\
\text { parents, teachers, } \\
\text { children or EPs. The } \\
\text { child may or may } \\
\text { not have a diagnosed } \\
\text { SEN and attends a } \\
\text { mainstream primary } \\
\text { or secondary school. }\end{array}$ & $\begin{array}{l}\text { Participants in the } \\
\text { study are not } \\
\text { parents, teachers, } \\
\text { children or EPs } \\
\text { and the child does } \\
\text { not attend a } \\
\text { mainstream } \\
\text { primary or } \\
\text { secondary school. }\end{array}$ & $\begin{array}{l}\text { The new model provides } \\
\text { for school age children in } \\
\text { mainstream schools } \\
\text { therefore studies of } \\
\text { students attending primary } \\
\text { or secondary school, their } \\
\text { parents/guardians, } \\
\text { teachers, EP were } \\
\text { considered. }\end{array}$ \\
\hline
\end{tabular}


Appendix 2 Participant summary table

\begin{tabular}{|c|c|c|c|c|}
\hline Study & $\begin{array}{l}\text { Who were the } \\
\text { participants in the study } \\
\text { and where were they } \\
\text { from? }\end{array}$ & $\begin{array}{l}\text { Who carried out the } \\
\text { assessment? }\end{array}$ & $\begin{array}{l}\text { What was the reason for } \\
\text { referral/purpose of } \\
\text { assessment and age range of } \\
\text { students assessed? }\end{array}$ & $\begin{array}{l}\text { Who reported on the } \\
\text { utility of the intervention? }\end{array}$ \\
\hline \multirow[t]{2}{*}{ Pameijer, N. (2017) } & $\begin{array}{l}\text { Teachers }(n=104), \\
\text { counsellors }(n=87), \\
\text { parents }(n=96) \text { and } \\
\text { assessors }(n=99)\end{array}$ & $\begin{array}{l}\text { School psychologists and } \\
\text { external school-coaches }\end{array}$ & $\begin{array}{l}\text { Children with learning and/or } \\
\text { behaviour difficulties in } \\
\text { primary school (ages 4-12). }\end{array}$ & $\begin{array}{l}\text { Teachers, counsellors, } \\
\text { parents, students and } \\
\text { assessors }\end{array}$ \\
\hline & $\begin{array}{l}\text { Netherlands } \\
\text { (geographical area not } \\
\text { specified) }\end{array}$ & & & \\
\hline $\begin{array}{l}\text { Ogg, Fefer, Sundman- } \\
\text { Wheat, McMahan, } \\
\text { Stewart, Chappel \& } \\
\text { Bateman (2013) }\end{array}$ & $\begin{array}{l}217 \text { school psychologists } \\
\text { United States (nationally } \\
\text { representative sample } \\
\text { reported) }\end{array}$ & School psychologists & $\begin{array}{l}\text { Assessment of children } \\
\text { presenting with symptoms of } \\
\text { Attention Deficit } \\
\text { Hyperactivity Disorder } \\
\text { (ADHD) in Pre-K, } \\
\text { Elementary and Middle } \\
\text { Schools (ages 4-15 approx.) }\end{array}$ & N/A \\
\hline $\begin{array}{l}\text { Sotelo-Dynega, \& } \\
\text { Dixon (2014) }\end{array}$ & $\begin{array}{l}323 \text { school psychologists } \\
\text { United States ( } 42 / 50 \\
\text { states represented) }\end{array}$ & School psychologists & $\begin{array}{l}\text { Children referred for } \\
\text { cognitive assessment in Pre- } \\
\text { K, Elementary, Middle } \\
\text { School, High School and } \\
\text { College (ages 3-21 approx.) }\end{array}$ & N/A \\
\hline $\begin{array}{l}\text { Bourke \& Dharan } \\
(2015)\end{array}$ & $\begin{array}{l}34 \text { psychologists } \\
\text { working in Education }\end{array}$ & $\begin{array}{l}\text { Psychologists working in } \\
\text { Education }\end{array}$ & $\begin{array}{l}\text { Children with behavioural, } \\
\text { emotional, social, and }\end{array}$ & N/A \\
\hline
\end{tabular}


New Zealand

(geographical area not

specified)

Bahr, Leduc, Hild,

Davis, Summers \&

McNeal (2017)

(2013)

Sansosti \& Sansost

175 school psychology

practitioners

Illinois, and Iowa)

United States (24/50

states)

Hill \& Turner (2016)

136 EPs

United Kingdom

(geographically

representative sample

reported)
United States (Missouri,

214 school psychologists

978 school psychologists School psychologists and 93 trainers of school psychology

United States (20/50

states represented)
School psychologists

School psychology practitioners

EPs

Assessment of children

N/A presenting with symptoms of Autism Spectrum Disorder (ASD) in Pre-K, Elementary, Middle School, High School 
and alternative educational centres.

Aiello, Ruble \& Esler (2016)

Vega, Lasser \& Afifi (2015)

Cottrell \& Barrett

(2015)

Filter, Ebsen \& Dibos (2013)

Lawrence \& Cahill (2014)
402 school psychologists

United States $(43 / 50$

states represented)

140 school psychologists School psychologists

United States (states not specified)

471 school psychologists School psychologists

United States (South,

West, Mid-Western

States)

216 school psychologists School psychologists

United States $(41 / 50$

states)

Students (9), parents (8)

and teachers (7)

United Kingdom

(London)
Assessment of children presenting with symptoms of ASD ages 0-21.

Assessment of culturally and linguistically diverse (CLD)

students (age range not

specified) for special education eligibility.

Assessment of children

N/A presenting with symptoms of Specific Learning Disabilities (SLD) in Elementary, Middle School and High School.

Reason for referral and age range is not specified. N/A

Assessment of the learning potential of children with special educational needs in primary and secondary schools (ages 7-14).
N/A

N/A
Students, parents and teachers




\begin{tabular}{|c|c|c|c|c|}
\hline $\begin{array}{l}\text { Stothard, Woods \& } \\
\text { Innoue (2018) }\end{array}$ & $\begin{array}{l}6 \text { EPs } \\
\text { North of England } \\
\text { (geographical area not } \\
\text { reported) }\end{array}$ & EPs & $\begin{array}{l}\text { Assessment of children } \\
\text { presenting with symptoms of } \\
\text { literacy difficulties/Dyslexia. } \\
\text { Age range is not specified. }\end{array}$ & N/A \\
\hline Tobias (2017) & $\begin{array}{l}\text { Student and her family } \\
\text { United Kingdom } \\
\text { (Brighton) }\end{array}$ & EP & $\begin{array}{l}\text { Assessment of child } \\
\text { presenting with school } \\
\text { refusal, aged } 11 .\end{array}$ & EP \\
\hline $\begin{array}{l}\text { Bozic, Lawthom \& } \\
\text { Murray (2017) }\end{array}$ & $\begin{array}{l}8 \text { children and young } \\
\text { people } \\
\text { United Kingdom } \\
\text { (geographical area not } \\
\text { specified) }\end{array}$ & EP & $\begin{array}{l}\text { Referrals included learning, } \\
\text { social and behaviour } \\
\text { difficulties (ages 6.9-19.2 } \\
\text { years). }\end{array}$ & N/A \\
\hline Bozic (2013) & $\begin{array}{l}6 \text { children and young } \\
\text { people } \\
\text { United Kingdom } \\
\text { (geographical area not } \\
\text { specified) }\end{array}$ & EP & $\begin{array}{l}\text { Referrals included emotional } \\
\text { and behaviour difficulties } \\
\text { (ages } 10.7-14.9 \text { years). }\end{array}$ & EP \\
\hline $\begin{array}{l}\text { Harrison \& McManus } \\
\text { (2016) }\end{array}$ & $\begin{array}{l}\text { Student, parents, } \\
\text { teachers }\end{array}$ & School psychologist & $\begin{array}{l}\text { Assessment of child } \\
\text { presenting with symptoms of } \\
\text { Writing Disorder, aged } 13 \text {. }\end{array}$ & N/A \\
\hline
\end{tabular}




\section{Canada (Victoria, British}

\section{Columbia)}

Parker, Zaboski \&

Joyce-Beaulieu (2016)
Student, parent and School psychologist

teachers

United States (South-

eastern region)

McCrimmon \& Yule (2016)

Cane (2016)
Student, parent, teachers

Canada (Calgary)

Student and school staff

(SENCO and two

members of pastoral

staff)

United Kingdom

(Surrey)
Assessment of child with symptoms of ADHD,

oppositional behaviours, and

explosive anger

symptomology, aged 14.

\section{Assessment of child}

presenting with symptoms of

ASD, aged 12.

Assessment of child

presenting with externalising

behavioural difficulties, aged

12.
N/A

Parent and teachers

Student and school staff 


\begin{tabular}{|c|c|c|c|c|}
\hline $\begin{array}{l}\text { Study/ } \\
\text { Geographical } \\
\underline{\text { Location }}\end{array}$ & $\begin{array}{l}\text { What was the assessment approach/model } \\
\text { applied? }\end{array}$ & $\begin{array}{l}\text { In what context was the } \\
\text { assessment carried out? }\end{array}$ & $\begin{array}{l}\text { Was there evidence of } \\
\text { whether the assessment } \\
\text { was informing } \\
\text { appropriate } \\
\text { intervention? }\end{array}$ & $\begin{array}{l}\text { What theories or } \\
\text { models resonate with } \\
\text { this approach? }\end{array}$ \\
\hline $\begin{array}{l}\text { Pameijer } \\
\text { (2017) } \\
\text { Netherlands }\end{array}$ & $\begin{array}{l}\text { The Assessment for Intervention (AFI) } \\
\text { Model. AFI is a five-stage model of } \\
\text { assessment and intervention. The stages of the } \\
\text { model provided structure to the assessment } \\
\text { from beginning to end, all involved worked } \\
\text { towards a shared goal and outcomes were } \\
\text { meaningful to counsellors, teachers and } \\
\text { parents as their questions led the assessment. }\end{array}$ & $\begin{array}{l}\text { Twenty schools } \\
\text { participated in a three } \\
\text { year pilot in the } \\
\text { Netherlands, } \\
\text { implementing the AFI } \\
\text { model, to assess the } \\
\text { needs of children with } \\
\text { learning and/or } \\
\text { behaviour difficulties. }\end{array}$ & $\begin{array}{l}\text { Yes- The majority of } \\
\text { teachers reported that } \\
\text { not only did they better } \\
\text { understand the student, } \\
\text { they also knew how to } \\
\text { adjust their teaching } \\
\text { more to their student's } \\
\text { specific educational } \\
\text { needs. }\end{array}$ & $\begin{array}{l}\text { EST (Bronfenbrenner, } \\
1979,1989)\end{array}$ \\
\hline $\begin{array}{l}\text { Lawrence \& } \\
\text { Cahill (2014) }\end{array}$ & $\begin{array}{l}\text { Dynamic assessment was reported to impact } \\
\text { positively upon the child's emotional well- }\end{array}$ & $\begin{array}{l}\text { Assessment of the } \\
\text { learning potential of }\end{array}$ & $\begin{array}{l}\text { Yes- Student and } \\
\text { teacher comments }\end{array}$ & SCT (Palinscar, 1998) \\
\hline $\begin{array}{l}\text { United } \\
\text { Kingdom }\end{array}$ & $\begin{array}{l}\text { being, self-perceptions, approach to learning } \\
\text { and social relationships, both directly, and } \\
\text { through the subsequent intervention of parents } \\
\text { and educators. Dynamic assessment, as well as } \\
\text { providing instructionally useful information } \\
\text { for parents and teachers, encouraged them to } \\
\text { move beyond locating the problem within the } \\
\text { child, to reconceptualising their special } \\
\text { educational needs in context. }\end{array}$ & $\begin{array}{l}\text { children with special } \\
\text { educational needs. }\end{array}$ & $\begin{array}{l}\text { provided evidence of } \\
\text { utility of intervention in } \\
\text { the classroom context. } \\
\text { Parents also reported } \\
\text { that DA resulted in } \\
\text { attempts to manage } \\
\text { their child's behaviour } \\
\text { in a different and more } \\
\text { positive way. }\end{array}$ & $\begin{array}{l}\text { Structural Cognitive } \\
\text { Modifiability and } \\
\text { Mediated Learning } \\
\text { Experience (Feuerstein } \\
\text { et al., 1979) }\end{array}$ \\
\hline
\end{tabular}




\begin{tabular}{|c|c|c|c|c|}
\hline $\begin{array}{l}\text { Tobias }(2017 \\
\text { United } \\
\text { Kingdom }\end{array}$ & $\begin{array}{l}\text { As an assessment tool, the genogram was able } \\
\text { to gather an extremely rich source of } \\
\text { information, and its co-construction with the } \\
\text { family facilitated a much deeper discussion } \\
\text { than would have normally been possible within } \\
\text { the constraints of an initial consultation. The } \\
\text { genogram is described as an assessment tool } \\
\text { for identifying behavioural patterns and } \\
\text { psychological aspects of family relationships, } \\
\text { as well as being part of the therapeutic } \\
\text { intervention itself. It is commonly used in } \\
\text { systemic family therapy. }\end{array}$ & $\begin{array}{l}\text { Assessment of child } \\
\text { presenting with school } \\
\text { refusal. }\end{array}$ & $\begin{array}{l}\text { Yes- The EP reported } \\
\text { the genogram was } \\
\text { helpful as part of an } \\
\text { initial intervention, } \\
\text { because it drew the } \\
\text { family's attention to } \\
\text { some of the repeated } \\
\text { patterns of behaviour } \\
\text { and unresolved, or } \\
\text { unvoiced, issues. }\end{array}$ & $\begin{array}{l}\text { EST (Bronfenbrenner, } \\
\text { 1979, 1989) } \\
\text { SCT (Vygotsky, 1978) }\end{array}$ \\
\hline $\begin{array}{l}\text { Bozic (2013) } \\
\text { United } \\
\text { Kingdom }\end{array}$ & $\begin{array}{l}\text { A strength-based assessment approach was } \\
\text { applied. In each case, the child/young person } \\
\text { was interviewed using either the Child and } \\
\text { Adolescent Strengths Assessment (Lyons et } \\
\text { al., 2000) or Assets Interview (Morrison et al., } \\
\text { 2006). Information gained from the assessment } \\
\text { was then used to inform an intervention plan. } \\
\text { Findings indicated that strengths could be } \\
\text { identified at a range of ecological levels. } \\
\text { Strengths influenced action plans by affecting } \\
\text { the choice of strategy and/or target of } \\
\text { interventions. }\end{array}$ & $\begin{array}{l}\text { Assessments of } \\
\text { children with emotional } \\
\text { and behaviour } \\
\text { difficulties. }\end{array}$ & $\begin{array}{l}\text { Yes-There was } \\
\text { evidence of positive } \\
\text { change in a high } \\
\text { proportion of cases. In } \\
\text { four out of the five } \\
\text { cases where strength- } \\
\text { based information } \\
\text { influenced the } \\
\text { development of an } \\
\text { action plan, there was } \\
\text { evidence of positive } \\
\text { change when repeated } \\
\text { measures were taken. }\end{array}$ & $\begin{array}{l}\text { Positive Psychology } \\
\text { (Seligman, 2002) } \\
\text { EST (Bronfenbrenner, } \\
\text { 1979, 1989) }\end{array}$ \\
\hline $\begin{array}{l}\text { Parker, } \\
\text { Zaboski \& } \\
\text { Joyce- }\end{array}$ & $\begin{array}{l}\text { This case demonstrated a multi-modal } \\
\text { assessment approach to inform intervention, } \\
\text { including a developmental history; parent, }\end{array}$ & $\begin{array}{l}\text { Assessment of child } \\
\text { presenting with } \\
\text { symptoms of attention }\end{array}$ & $\begin{array}{l}\text { Yes- Treatment } \\
\text { included CBT sessions } \\
\text { over } 6 \text { months and a 9- }\end{array}$ & $\begin{array}{l}\text { Response to } \\
\text { Intervention Model }\end{array}$ \\
\hline
\end{tabular}




\begin{tabular}{|c|c|c|c|c|}
\hline $\begin{array}{l}\text { Beaulieu } \\
(2016) \\
\text { United States }\end{array}$ & $\begin{array}{l}\text { teacher, and student interviews; systematic } \\
\text { classroom observations; and BASC-2 and } \\
\text { Conners parent, teacher, and student social, } \\
\text { emotional, and behaviour rating reports. An } \\
\text { RTI approach indicated ratings of ADHD-type } \\
\text { behaviours and severe problematic behaviours } \\
\text { that no longer fell in the clinically significant } \\
\text { range. }\end{array}$ & $\begin{array}{l}\text { deficit hyperactivity } \\
\text { disorder (ADHD), } \\
\text { oppositional } \\
\text { behaviours, and } \\
\text { explosive anger } \\
\text { symptomology. }\end{array}$ & $\begin{array}{l}\text { week classroom Daily } \\
\text { Behaviour Report Card } \\
\text { plan. Outcome data } \\
\text { revealed a decrease in } \\
\text { office discipline } \\
\text { referrals, lower levels } \\
\text { of behaviour symptoms, } \\
\text { and an increase in } \\
\text { prosocial classroom } \\
\text { behaviours with } \\
\text { maintained } \\
\text { improvement into the } \\
\text { following school year. }\end{array}$ & $\begin{array}{l}\text { (U.S. Department of } \\
\text { Education, 2004) } \\
\text { EST (Bronfenbrenner, } \\
1979,1989)\end{array}$ \\
\hline $\begin{array}{l}\text { Cane (2016) } \\
\text { United } \\
\text { Kingdom }\end{array}$ & $\begin{array}{l}\text { A solution-focused assessment approach } \\
\text { was applied. The solution-focused model } \\
\text { adopted a strength-based approach, which } \\
\text { seeks to identify and foster the conditions } \\
\text { necessary to achieve the preferred state. } \\
\text { Readiness to change was assessed using the } \\
\text { Sheffield Motivational Interviewing card-sort } \\
\text { activity. School staff and student views were } \\
\text { sought and intervention goals devised } \\
\text { accordingly. }\end{array}$ & $\begin{array}{l}\text { Assessment of child } \\
\text { presenting with } \\
\text { externalising } \\
\text { behavioural difficulties. }\end{array}$ & $\begin{array}{l}\text { Yes-The use of scaling } \\
\text { illustrated how, over the } \\
\text { course of the SFBT } \\
\text { intervention, the child } \\
\text { rated himself as } \\
\text { progressing and } \\
\text { maintained positive } \\
\text { changes at follow up. } \\
\text { Qualitative feedback } \\
\text { from school staff } \\
\text { indicated improvements } \\
\text { in behaviour and } \\
\text { learning. }\end{array}$ & $\begin{array}{l}\text { Positive Psychology } \\
\text { (Seligman, 2002) } \\
\text { SCT (Palinscar, 1998) }\end{array}$ \\
\hline
\end{tabular}




\section{Ogg, Fefer,}

Sundman-

Wheat,

McMahan,

Stewart,

Chappel \&

Bateman

(2013)

United States

Sotelo-

Dynega, \&

Dixon (2014)

United States

The majority of school psychologists sampled continue to engage in traditional assessment

practices (i.e. IQ testing). However, the

majority also reported that they were able to

translate the findings of their cognitive

assessments into specific, individualised

interventions for the examinees.

Bourke \&

Dharan

(2015)

New Zealand

A holistic approach to assessment and a preference towards more dialogic and ecological ways of working was reported with interviews, observation and collaboration as

key to assessment practices. Assessment data is used for three primary reasons: to understand the child, inform decision-making,
Assessment of children No

presenting with

symptoms of ADHD.

Cognitive assessment

practices of school

No

psychologists.

Assessment of children

No with behavioural, emotional, social, and cognitive difficulties.
Medical Model (Laing, 1971)

Problem Solving

Model (Monsen et al., 1998; Tilly, 2008)

Response to

Intervention Model

(U.S. Department of

Education, 2004)

Medical Model (Laing, 1971)

Problem Solving Model (Monsen et al., 1998; Tilly, 2008)

ECT (Bronfenbrenner, 1979, 1989)

Problem Solving

Model (Monsen et al., 1998; Tilly, 2008)

SCT (Palinscar, 1998) 
and contribute to discussions around

appropriate interventions.

Bahr, Leduc, School psychologists reported spending the Hild, Davis,

Summers \&

McNeal

(2017)

United States

Hanchon \&

Allen (2013)

United States greatest amount of their time on problem-

solving consultation, as opposed to diagnostic assessment. Most participants, 112 (or 64\%), indicated problem-solving consultation as one of their top five preferences.

\section{Inconsistent assessment practices are} reported. School psychologists report that they value a multimethod, multisource assessment model when ED is a classification consideration. However, in many instances, their actual assessments are missing commonly recommended sources of data for making eligibility decisions, including classroom observations; parent, teacher, and student interviews; and behaviour rating scales.

Hill \& Turner The data suggests that in current practice the (2016)

United Kingdom medical model dominates. Responses highlighted how currently EPs are rarely engaged in the assessment of ADHD. Where Local Authorities have developed standardised pathways or protocols governing the diagnostic process, EPs are involved in the assessment process and children are more likely to access

$\begin{array}{lll}\text { General assessment } & \text { No } & \text { Problem Solving } \\ \text { practices of school } & & \text { Model (Monsen et al., } \\ \text { psychologists. } & & 1998 ; \text { Tilly, 2008) }\end{array}$

Assessment of children No

Medical Model (Laing, presenting with

symptoms of ED.
Assessment of children No presenting with symptoms of ADHD.
Medical Model (Laing, 1971) 
psychological interventions, and for contextual factors to be considered.

Sansosti \&

Sansosti

(2013)

United States

Aiello, Ruble

\& Esler

(2016)

United States
Findings suggest that practitioners engage in some of the best practice assessment methods embraced by researchers and policy advocates (National Autism Center), including use of adaptive behaviour scales, autism checklists/rating scales, direct observations, developmental/health histories, interviews, and standardised intelligence tests. $54 \%$ of school psychologists reported engaging either frequently or very frequently in developing interventions for students with ASD during the prior school year. However, the researchers conclude findings indicate that assessment may be viewed more from the perspective of eligibility determination rather than for the development of interventions.

402 school psychologists were surveyed for their knowledge of and training and experience with ASD on assessment practices. The majority of school psychologists reported that they did not engage in comprehensive assessment of ASD, which was defined as assessments that consider all areas of development in addition to the use of ASDspecific instruments.
Assessment of children No presenting with

Medical Model (Laing, 1971)

symptoms of ASD.

Assessment of children No presenting with

Medical Model (Laing, 1971) 


\begin{tabular}{|c|c|c|c|c|}
\hline $\begin{array}{l}\text { Vega, Lasser } \\
\text { \& Afifi } \\
(2015) \\
\text { United States }\end{array}$ & $\begin{array}{l}\text { Participants reported conducting } \\
\text { comprehensive assessments to determine } \\
\text { eligibility for special education among CLD } \\
\text { students. The majority of the participants ( } 75.5 \\
\text { \%) reported assessing CLD students' } \\
\text { cognitive, achievement, and social-emotional } \\
\text { functioning when conducting an evaluation to } \\
\text { determine eligibility for special education } \\
\text { services. Other areas reportedly assessed } \\
\text { included racial and ethnic identity } \\
\text { development, acculturation, language } \\
\text { proficiency, environmental impact, } \\
\text { neuropsychological functioning, curriculum- } \\
\text { based measurement, and motor functioning. }\end{array}$ & $\begin{array}{l}\text { Assessment of } \\
\text { culturally and } \\
\text { linguistically diverse } \\
\text { (CLD) students for } \\
\text { special education } \\
\text { eligibility. }\end{array}$ & No & $\begin{array}{l}\text { Medical Model (Laing, } \\
\text { 1971) }\end{array}$ \\
\hline $\begin{array}{l}\text { Cottrell \& } \\
\text { Barrett (2015) }\end{array}$ & \multirow{2}{*}{$\begin{array}{l}\text { The Ability-Achievement discrepancy } \\
\text { method was the assessment method most used } \\
(\mathrm{M}=2.68, \mathrm{SD}=1.19) \text {, followed by RTI } \\
(\mathrm{M}=2.42, \mathrm{SD}=1.05) \text { and pattern of strengths } \\
\text { and weaknesses }(\mathrm{M}=1.86, \mathrm{SD}=1.03) .\end{array}$} & \multirow{2}{*}{$\begin{array}{l}\text { Assessment of children } \\
\text { presenting with } \\
\text { symptoms of SLD. }\end{array}$} & \multirow[t]{2}{*}{ No } & $\begin{array}{l}\text { Medical Model (Laing, } \\
\text { 1971) }\end{array}$ \\
\hline United States & & & & $\begin{array}{l}\text { Ability-Achievement } \\
\text { Discrepancy Model } \\
\text { (Franzen, 1920) }\end{array}$ \\
\hline $\begin{array}{l}\text { Filter, Ebsen } \\
\text { \& Dibos }\end{array}$ & \multirow{2}{*}{$\begin{array}{l}\text { The three most common activities reported } \\
\text { were report writing }(\mathrm{M}=7.46 ; \mathrm{SD}=5.42) \text {, IQ } \\
\text { testing }(\mathrm{M}=5.69 ; \mathrm{SD}=4.99) \text {, and staff } \\
\text { consultation }(\mathrm{M}=5.47 ; \mathrm{SD}=5.94) \text {. School } \\
\text { psychologists reported spending } 5.69 \text { hours per } \\
\text { week administering IQ tests but prefer to spend } \\
1.83 \text { hours per week less in this discrete } \\
\text { practice. Further, IQ tests traditionally } \\
\text { comprised significant portions of reports }\end{array}$} & \multirow{2}{*}{$\begin{array}{l}\text { The general practice of } \\
\text { school psychologists } \\
\text { including assessment, } \\
\text { intervention, meetings, } \\
\text { and continuing } \\
\text { education }\end{array}$} & \multirow[t]{2}{*}{ No } & $\begin{array}{l}\text { Medical Model (Laing, } \\
\text { 1971) }\end{array}$ \\
\hline $\begin{array}{l}(2013) \\
\text { United States }\end{array}$ & & & & $\begin{array}{l}\text { Ability-Achievement } \\
\text { Discrepancy Model } \\
\text { (Franzen, 1920) }\end{array}$ \\
\hline
\end{tabular}


written by school psychologists and the present

sample reported wanting to spend 3.34 hours

less per week writing reports.

Stothard,

Woods \&

Innoue (2018)

United

Kingdom

Bozic,

Lawthom \&

Murray

(2017)

United

Kingdom

Harrison \&

McManus

(2016)

Canada

\section{Inconsistent assessment practices are} reported. A narrow range of EP assessment and intervention practice for Dyslexia was described, suggesting a need for professional clarification and development. Several EPs reported using psychometric tests, some discussed using unspecified assessments of reading and/or spelling and some discussed taking a strengths and needs approach.

\section{A contextualised strength-based assessment} approach was applied. By engaging with the 'Context Strength Finder' (CSF), all children/young people identified situations or contexts which they associated with the presence of specific strengths. In some cases, they highlighted aspects of a situation which might be hypothesised to have pedagogical value.

\section{This case highlighted a multimodal}

assessment approach to inform diagnosis and intervention, situated within a contemporary scientist-practitioner framework. This approach involved the following steps: (a) problem analysis and development of hypotheses based on the collection of
Assessment of children

No presenting with symptoms of literacy difficulties/Dyslexia.

Assessments of

No children with learning, social and behaviour difficulties.

Assessment of child presenting with symptoms of Writing Disorder.

No
Ability-Achievement Discrepancy Model

(Franzen, 1920)

EST (Bronfenbrenner, 1979, 1989)

EST (Bronfenbrenner, 1979, 1989)

SCT (Palinscar, 1998)

Positive Psychology

(Seligman, 2002)

Medical Model (Laing, 1971)

Problem Solving

Model (Monsen et al., 1998; Tilly, 2008) 
background data (b) collection of assessment

data to test hypotheses; (c) synthesis and interpretation of all assessment data in relation to hypotheses; (d) intervention development; and (e) intervention evaluation.

McCrimmon Given the presenting issues and concerns, a \& Yule (2016)

Canada

\section{comprehensive assessment approach was}

applied to examine the child's cognitive, academic, behavioural, and attentional functioning in addition to a specific assessment for symptoms of ASD. The practitioners' approach to assessment and case

conceptualisation was driven by advances in the clinical conceptualisation of diagnostic features of childhood disorders and effective and efficient approaches to obtain evidence about the presence or absence of symptoms necessary to yield a clinical diagnosis. The child's intellectual, adaptive, academic, and behavioural challenges were all important considerations in determining the nature and context of potential interventions.
Assessment of child

No presenting with symptoms of ASD.
Medical Model (Laing, 1971)

Problem Solving Model (Monsen et al., 1998; Tilly, 2008) 
Appendix 4 The Five Stages of the AFI Model (Adapted from Pameijer, 2016, 2017)

Stage

1. Intake, how can we collaborate?

2. Strategy, how to proceed in this particular case?
3: Investigation, answering the selected answers

4: Integration, goals and needs

\section{Description}

The first goal is to collect information so that the school psychologist can determine a strategy for a particular case. Another key objective is to achieve compatibility with the school, student and parents/guardians, in order to create a constructive partnership. In the first meeting the questions, aims, expectations and requests of those involved are discussed: what do they intend to accomplish (or avoid), why and how? How do they explain the situation? Which case formulation and recommendation are most likely to help them? These questions shed light on their frame of reference and support the school psychologist in tailoring the process to their personal theory and needs. Appointments for cooperation are made, e.g. who collects which information?; when will one meet again to discuss the findings?

The input of this stage is the information collected in the intake and the output is the strategy that best fits a specific situation. First, the relevant information is organised in four sections: student, instructional environment, parental support of learning and relevant history. Then the school psychologist decides what more needs to be known to answer the clients' and his questions. Is the investigation stage (Stage 3) necessary? This is the case when more information is needed in order to formulate recommendations. Or can he already move on to stage 4 (Integration stage)? The bottom line is: no investigation will be conducted unless its outcomes will influence the choice of the intervention. Each question is justified with the 'ifthen-rationale': if we know ..., then we can recommend... However, if we don't know..., we then cannot recommend ... This way, collecting data is goal-directed and directly linked to intervention. If stage 3 is required, alternate hypotheses from a transactional frame of reference are formulated, relevant hypotheses are selected, based on their impact on the choice of an intervention and these hypotheses are translated into questions for investigation.

This stage involves a goal-directed rather than a routine collection of data. The selected hypotheses determine the information to be gathered. The content of this stage thus varies in each case, ranging from using one instrument to several different tools.

The information is integrated into a specific case formulation: how can the situation be understood? This summary is translated into goals for the student, teaching strategies and parental support, educational needs of the student and support needs of his teacher and/or 
parents/guardians. As several interventions focus on the same target, choices have to be made. The AFI-model prefers interventions that have been proven to be effective. The school psychologist can benefit from several metaanalyses when deciding in this.

5: Recommendations, In this stage the clients are informed about the outcomes of appointments the assessment. By providing them with clear and meaningful information, related to their personal theory, hopes and worries, they can choose for themselves which option is both desirable and achievable. An important aim is to arrive at a feasible intervention, supported by all parties. The school psychologist therefore asks if those involved are willing and able to 'start tomorrow'. If the answer is affirmative, the child, teacher and parent/guardian are encouraged to change their behaviour. If this is not yet the case, the assessment process continues with further consultation. 


\section{Criteria 1}

The initial criteria for participant selection will be a student in a senior class in primary school $\left(4^{\text {th }}, 5^{\text {th }}\right.$ or $\left.6^{\text {th }}\right)$ referred to the school psychology service to address behaviour, socialemotional and/or learning needs. Students in senior classes are being sought so as to maximise the voice of the child in the research project, in line with article 12 of the International Convention on the Rights of the Child (2013) (Pameijer, 2017).

\section{Criteria 2}

The referral form will preferably outline a complex case in terms of learning, behaviour and/or social-emotional needs, that will be suited to the stages of the AFI model. The researcher's placement supervisor will help decide on the level of complexity.

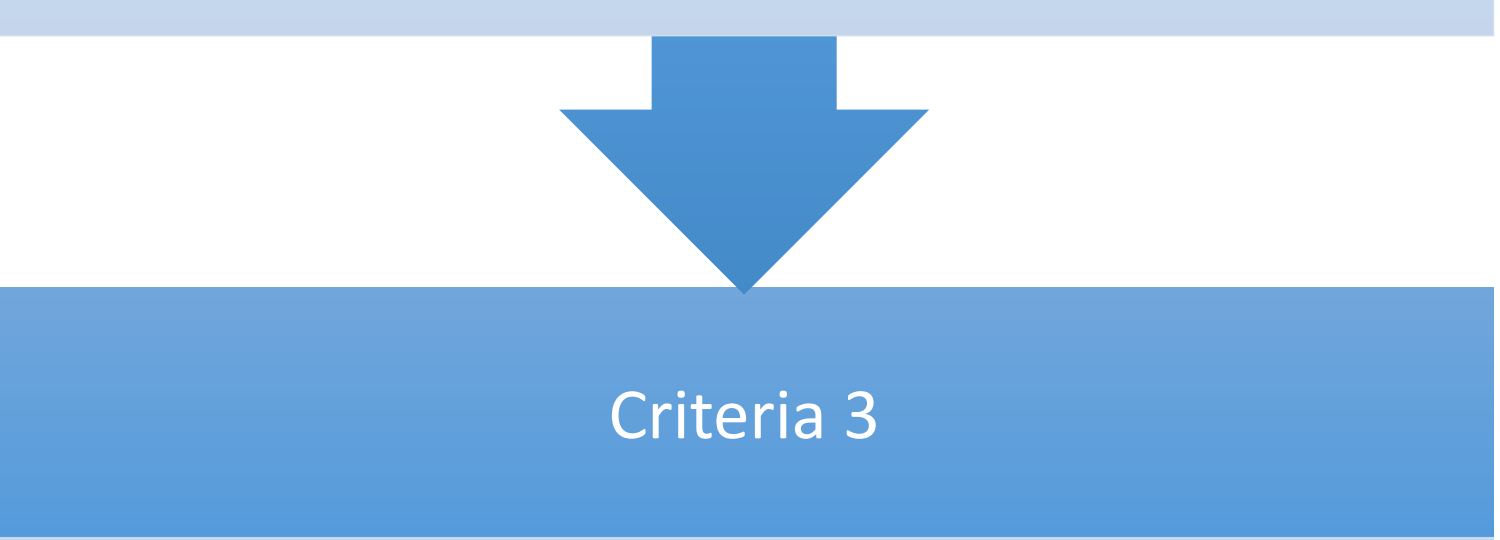

The parents/guardians and school staff (teachers and SNA) will preferably show interest in the AFI model and five-stage process, and motivation to take part in the study. 
Appendix 6 Case Study Protocol (Devised in accordance with Yin, 2009, p. 80 guidelines) and Yin (2018)

A. Introduction to the Case Study and Purpose of Protocol

4. Case study questions, hypotheses and propositions

a. Research question: 'Can the Assessment for Intervention model bridge the gap between assessment and intervention, through activity and interaction* at the meso and microsystem levels?'

*Activity and interaction are those activities and interactions that resonate with EST and SCT concepts, including but not limited to molar activities, dyadic relations, "law of genetic development", ZPD and tools and semiotics.

b. Case study propositions: Five propositions arising from from the conceptual framework and theoretical statement, research question and the aims of AFI (Pameijer, 2016, 2017).

Following application of the five stages of the AFI model:

1. Parents/guardians, school staff and TEP will have a better understanding of the child's situation, as a result of interactions and activities that have occurred at the meso and microsystem levels.

2. Parents/guardians, school staff and TEP will know what intervention supports the child needs at home and at school, as a result of interactions and activities that have occurred at the meso and microsystem levels.

3. School staff and parents/guardians will have greater perceived competence in supporting the child's needs at home and at school, as a result of interactions and activities that have occurred at the meso and microsystem levels.

4. The child will have a better understanding of their strengths and needs and what to do to improve their needs, as a result of interactions and activities that have occurred at the meso and microsystem levels. 
5. At a time of ecological transition, school staff will feel competent in their ability to work according to the aims of Circular 0013/2017, in meeting and monitoring the needs of the child.

5. Conceptual framework for the case study: The current research is conducted through the theoretical lens of EST and SCT, because these were the theories that emerged in the discussion of findings in the literature review. To design a conceptual framework for the current study, the concepts within these theories were analysed in accordance with AFI principles. The conceptual framework was refined into a theoretical statement, or proposition for the current study:

'During a time of ecological transition with the issuance of Circular 0013/2017, the EP applies the five stages of the AFI model to a case referral. By interacting with the child, parents/guardians and teachers in accordance with AFI principles at the meso and microsystem levels, the assessment process leads to intervention recommendations that are meaningful to teachers, parents/guardians and child. Engaging together in the five-stage process exposes EST and SCT concepts'.

6. Role of protocol in guiding the case study investigator: The protocol guides the researcher in the data collection process and enhance the reliability of the research. It is considered a standardised agenda for the researcher's line of inquiry (Yin, 2009).

\section{B. Data Collection Procedures}

4. Names of sites to be visited, including contact persons: Schools 1 and 2 and their respective SENCOs/principals

5. Data Collection Plan:

a. October/November 2018: Complete pilot study. Revise interview questions/consent forms/printing etc. following pilot study. *See separate document outlining revisions made.

b. October 2018: Choose suitable case referrals with placement supervisor 
c. October 2018: Distribute information sheets to schools, parents/guardians and student. Informed consent/ assent of school staff, parents/guardians and student.

d. November 2018: Set up database of participants (School 1 and School 2)

e. November 2018: Cases are conducted in parallel due to time constraints. Participants in School 1 and 2 complete Likert scale statements before application of AFI model and researcher records reflections in researcher diary.

f. November/December 2018: Apply the five-stage AFI model to the case referral in School 1 and 2 and record researcher reflections in the researcher diary*.

g. December 2018: Participants complete Likert scale statements and semistructured interviews after application of AFI model and researcher records reflections in researcher diary

* See detailed outline of data collection procedures and researcher actions at each stage of the AFI model in a separate appendix.

6. Expected preparation prior to school visits: Print information and consent/assent sheets, distribute and collect signed informed consent/assent sheets, print researcher diary (AFI templates), Likert scale questionnaires, interview questions and interview protocol. Borrow voice recording device from MIC library.

\section{Protocol Questions}

The main purpose of the protocol's questions is to keep the researcher on track as data collection proceeds and serves as the researcher's line of inquiry (Yin, 2018 p. 99).

7. Does the AFI model link assessment to meaningful intervention for clients? If so, how? What activities and interactions lead to meaningful intervention recommendations for clients?

- Source of evidence: Researcher reflections, interviews with clients, Likert scale statements

8. Do clients feel competent in meeting and monitoring the needs of the child identified?

- Source of evidence: Researcher reflections, interviews with clients, Likert scale statements 
9. To what extent are all clients actively involved in the five-stage process?

- Source of evidence: Researcher reflections, interviews with clients

10. What factors promote and hinder client involvement in the five-stage process?

- Source of evidence: Researcher reflections, interviews with clients

11. Can the model be feasibly applied by EPs in practice?

- Source of evidence: Researcher reflections

12. What are the benefits and limitations of the AFI model?

- Source of evidence: Researcher reflections, interviews with clients,

Likert scale statements

D. Outline of Case Study Report

13. Results under each proposition (Pilot Case)

a. Participant ratings on the Likert scale statements, before and after implementation of the AFI model presented in tabular format

b. Pattern-matching of interview responses

c. Thematic analysis of interview responses

d. Researcher diary and reflective journal

14. Summary of findings addressing the research question (Pilot Case)

15. Discussion of findings under related themes in accordance with the literature and conceptual framework (Pilot Case)

16. Repeat steps 1-3 for Case 1 and 2

17. Cross-case analysis and discussion

18. Conclusions and directions for future research 